Scheduled Outage: The shutdown of a generating unit, transmission line, or other facility, for inspection or maintenance, in accordance with an advance schedule.

Short Ton: A unit of weight equal to 2,000 pounds.

Spot Purchases: A single shipment of fuel or volumes of fuel, purchased for delivery within 1 year. Spot purchases are often made by a user to fulfill a certain portion of energy requirements, to meet unanticipated energy needs, or to take advantage of low-fuel prices.

Standby Facility: A facility that supports a utility system and is generally running under no-load. It is available to replace or supplement a facility normally in service.

Standby Service: Support service that is available, as needed, to supplement a consumer, a utility system, or to another utility if a schedule or an agreement authorizes the transaction. The service is not regularly used.

Steam-Electric Plant (Conventional): A plant in which the prime mover is a steam turbine. The steam used to drive the turbine is produced in a boiler where fossil fuels are burned.

Stocks: A supply of fuel accumulated for future use. This includes coal and fuel oil stocks at the plant site, in coal cars, tanks, or barges at the plant site, or at separate storage sites.

Subbituminous Coal: Subbituminous coal, or black lignite, is dull black and generally contains 20 to 30 percent moisture. The heat content of subbituminous coal ranges from 16 to 24 million Btu per ton as received and averages about 18 million Btu per ton. Subbituminous coal, mined in the western coal fields, is used for generating electricity and space heating.

Substation: Facility equipment that switches, changes, or regulates electric voltage.

Sulfur: One of the elements present in varying quantities in coal which contributes to environmental degradation when coal is burned. In terms of sulfur content by weight, coal is generally classified as low (less than or equal to 1 percent), medium (greater than 1 percent and less than or equal to 3 percent), and high (greater than 3 percent). Sulfur content is measured as a percent by weight of coal on an "as received" or a "dry" (moisture-free, usually part of a laboratory analysis) basis.
Switching Station: Facility equipment used to tie together two or more electric circuits through switches. The switches are selectively arranged to permit a circuit to be disconnected, or to change the electric connection between the circuits.

System (Electric): Physically connected generation, transmission, and distribution facilities operated as an integrated unit under one central management, or operating supervision.

Transformer: An electrical device for changing the voltage of alternating current.

Transmission: The movement or transfer of electric energy over an interconnected group of lines and associated equipment between points of supply and points at which it is transformed for delivery to consumers, or is delivered to other electric systems. Transmission is considered to end when the energy is transformed for distribution to the consumer.

Transmission System (Electric): An interconnected group of electric transmission lines and associated equipment for moving or transferring electric energy in bulk between points of supply and points at which it is transformed for delivery over the distribution system lines to consumers, or is delivered to other electric systems.

Turbine: A machine for generating rotary mechanical power from the energy of a stream of fluid (such as water, steam, or hot gas). Turbines convert the kinetic energy of fluids to mechanical energy through the principles of impulse and reaction, or a mixture of the two.

Watt: The electrical unit of power. The rate of energy transfer equivalent to 1 ampere flowing under a pressure of 1 volt at unity power factor.

Watthour (Wh): An electrical energy unit of measure equal to 1 watt of power supplied to, or taken from, an electric circuit steadily for 1 hour.

Wheeling Service: The movement of electricity from one system to another over transmission facilities of intervening systems. Wheeling service contracts can be established between two or more systems.

Year to Date: The cumulative sum of each month's value starting with January and ending with the current month of the data. 


\section{Electronic Publishing System EPTB}

EPUB is an electronic publishing system maintained by the Energy Information Administration (EIA) of the U.S. Department of Energy. EPUB allows the general public to electronically access selected energy data from many of EIA's statistical reports. The system is a menu-driven, bulletin-board-type system with extensive online help capabilities that can be accessed free of charge 24 hours a day by using a terminal or PC with an asynchronous modem. (EPUB will be taken down briefly at midnight for backup.)

PC users must provide the following information to their communications software in order to successfully access the EPUB system:

Communications Parameters:

Baud Rate: $300-2400$ bps

Data Bits: 8 ; Stop Bits: 1

Parity: None; Duplex: Full

Terminal Type: ANSI, ANSI-BBS, VT100, etc.

Once communications software and/or hardware have been configured, EPUB can be accessed by dialing (202)586-2557. When a connection to the system has been made, some users may find that the menu-driven instructions and the online capabilities will provide enough information to effectively use EPUB. If needed, more extensive information may be found in the EPUB User's Guide, which is available online from the EPUB system or from:

National Energy Information Center, EI-231

Energy Information Administration

Forrestal Building, Room 1F-048

Washington, DC 20585

(202)586-8800

TTY: For people who are deaf or

hard of hearing: (202)586-1181

Hours: 9 a.m. to 5 p.m., M-F, eastern time
For communications or technical assistance, call (202)586-8959, 8 a.m. to 5 p.m. eastern time, Monday through Friday. For questions about the contents of EPUB reports and data, call (202)5868800,9 a.m. to 5 p.m., M-F, eastern time.

EPUB provides statistical information, as well as data from selected EIA publications, such as:

Heating fuel data

Updated the 2nd week of the month.

Oxygenates data

Updated approximately the 25 th of the month.

Weekly Petroleum Status Report

Updated on Wednesdays (Thursdays in the event of a holiday) at 5 p.m.

Petroleum Supply Monthly

Updated on the 20th of the month.

Petroleum Marketing Monthly

Updated on the 20th of the month.

Natural Gas Monthly

Updated on the 20th of the month.

Weekly Coal Production

Updated on Fridays at 5 p.m.

Quarterly Coal Report

Updated 60 days after the end of the quarter.

Electric Power Monthly

Updated on the 1st of the month.

Monthly Energy Review

Updated the last week of the month.

Short-Term Energy Outlook

Updated 60 days after the end of the quarter.

Winter Fuels Report (October through April) Updated every Thursday at 5 p.m.

W

Periodically, new items are added to the electronic bulletin board. EIA is now completing implementation of two new subject areas on this system. The first area, "End-Use Consumption Surveys," provides electronic access to selected data and analyses from the residential, commercial, manufacturing, and residential transportation consumption surveys. The second area, "Monthly Energy Review (MER) Features," provides access to special articles from the most recent publication, with a listing of all previous articles. 


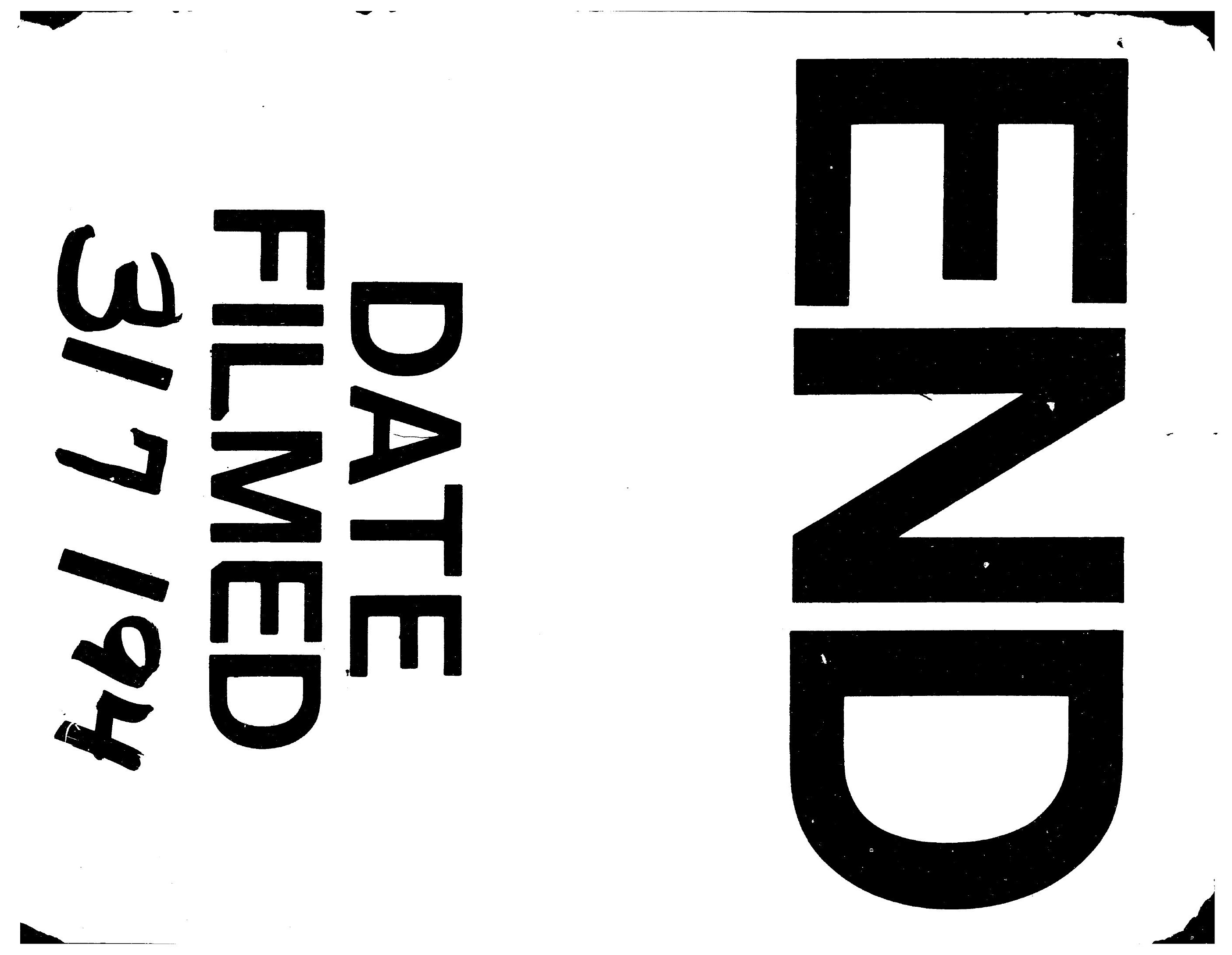





\section{Preface}

The Electric Power Monthly (EPM) presents monthly electricity statistics. The purpose of this publication is to provide energy decisionmakers with accurate and timely information that may be used in forming various perspectives on electric issues that lie ahead. Data in this report are presented for a wide audience including Congress, Federal and State agencies, the electric utility industry, and the general public. The EIA collected the information in this report to fulfill its data collection and dissemination responsibilities as specified in the Federal Energy Administration Act of 1974 (Public Law 93-275) as amended.

\section{Background}

The $E P M$ is prepared by the Survey Management Division; Office of Coal, Nuclear, Electric and Alternate Fuels, Energy Information Administration (EIA), Department of Energy. This publication provides monthly statistics at the U.S., Census division, and State levels for net generation, fossil fuel consumption and stocks, quantity and quality of fossil fuels, cost of fossil fuels, electricity sales, revenue, and average revenue, per kilowatthour of electricity sold. Data on net generation, fuel consumption, fuel stocks, quantity and cost of fossil fuels are also displayed for the North American Electric Reliability Council (NERC) regions.

Statistics by company and plant are published in the $E P M$ on the capability of new generating units, net generation, fuel consumption, fuel stocks, quantity and quality of fuel, and cost of fossil fuels.

Data on quantity, quality, and cost of fossil fuels lag data on net generation, fuel consumption, fuel stocks, electricity sales, and average revenue per kilowatthour by 1 month. This difference in reporting appears in the U.S., Census division, and State level tables. However, for purposes of comparison, plant-level data are presented for the earlier month.

\section{Coverage of Sources}

The EPM contains information from six data sources: the Form EIA-759, "Monthly Power Plant Report"; the Federal Energy Regulatory Commission (FERC) Form 423, "Monthly Report of Cost and Quality of
Fuels for Electric Plants"; the Form EIA-826, "Monthly Electric Utility Sales and Revenue Report with State Distributions"; the Form EIA-861, "Annual Electric Utility Report"; the Form EIA-860, "Annual Electric Generator Report"; and a form collected by the Office of Emergency Planning and Operations (OE), the Form OE-417R, "Electric Power System Emergency Report." Copies of these forms and their instructions may be obtained from the National Energy Information Center. A brief summary of these forms follows; a more detailed description of the forms is contained in Appendix C, "Technical Notes."

The Form EIA-759 is used to collect monthly data on net generation; consumption of coal, petroleum, and natural gas; and end-of-the-month stocks of coal and petroleum for each plant by prime mover and fuel-type combination. Data are collected from all operators of electric utility generating plants in the United States--approximately 800 (except those having plants solely on standby).

The FERC Form 423, which is a restricted-universe census, is used to collect data from electric generating plants with a total steam-electric and combined-cycle nameplate capacity of 50 or more megawatts (approximately 230 electric utilities). The threshold of 50 or more megawatts was established by FERC. Data collected on the FERC Form 423 include quantity, quality, delivered cost, origin, mine type, fuel type, supplier, and purchase type of fossil fuel receipts.

The Form EIA-826 is used to collect sales and revenue data for the residential, commercial, industrial, and other sectors. Other sales and revenue data collected include public street and highway lighting, other sales and revenue to public authorities, sales to railroads and railways, and interdepartmental sales. Respondents to the Form EIA-826 are based on a statistically chosen sample and include 238 investor-owned and publicly owned electric utilities from a universe of approximately 3,250 utilities. The sample, which is evaluated annually, was designed to obtain estimates of electricity sales, revenue, and revenue per kilowatthour for all U.S. electric utilities by end-use sector. These estimates are provided at the State, Census division, and U.S. levels. Estimates of coefficients of variation, which indicate possible error due to sampling, are also published at each level. 
The Form EIA-860 is used to collect data annually from all electric utilities in the United States and Puerto Rico that operate power plants or plan to operate a power plant within 10 years of the reporting year. Generator-specific information is reported by approximately 900 respondents.

The Form EIA-861 is a survey of electric utilities in the United States, its territories, and Puerto Rico. The survey is used to collect information from the universe of electric utilities (approximately 3,250). Data collected on the Form EIA-861 include information on the production, sales, revenue from sales, and trade of electricity.

The Form OE-417R is used to collect information on major electric utility system emergencies, including the type of emergency, the utility and area affected, the date and time of the event, a cescription of the event, and expected time of restoration of service. The responsibility of collecting these data has been delegated to the Office of Emergency Planning and Operations within the Department of Energy. 


\section{Contents}

Page

U.S. Electric Power At A Glance $\ldots \ldots \ldots \ldots \ldots \ldots \ldots \ldots \ldots \ldots \ldots \ldots \ldots \ldots \ldots \ldots$

U.S. Electric Utility Net Generation $\ldots \ldots \ldots \ldots \ldots \ldots \ldots \ldots \ldots \ldots \ldots \ldots \ldots \ldots \ldots \ldots$

U.S. Electric Utility Consumption of Fossil Fuels $\ldots \ldots \ldots \ldots \ldots \ldots \ldots \ldots \ldots \ldots \ldots \ldots$

Fossil-Fuel Stocks at U.S. Electric Utilities $\ldots \ldots \ldots \ldots \ldots \ldots \ldots \ldots \ldots \ldots \ldots \ldots \ldots \ldots$

Receipts and Cost of Fossil Fuels at U.S. Electric Utilities $\ldots \ldots \ldots \ldots \ldots \ldots \ldots \ldots \ldots \ldots \ldots$

U.S. Electric Utility Sales, Revenue, and Average Revenue per Kilowatthour . . . . . . . . . 61

Monthly Plant Aggregates: U.S. Electric Utility Net Generation, Fuel Consumption, and Fuel Stocks 75

Monthly Plant Aggregates: U.S. Electric Utility Receipts, Cost, and Quality of Fossil Fuels . . . . . 149

Appendices

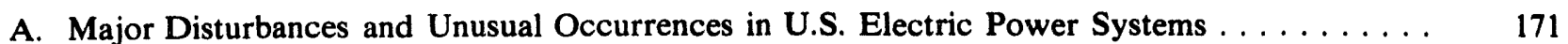

B. References $\ldots \ldots \ldots \ldots \ldots \ldots \ldots \ldots \ldots \ldots \ldots \ldots \ldots \ldots \ldots \ldots \ldots \ldots$

C. Technical Notes $\ldots \ldots \ldots \ldots \ldots \ldots \ldots \ldots \ldots \ldots \ldots \ldots \ldots \ldots \ldots \ldots \ldots$

Glossary

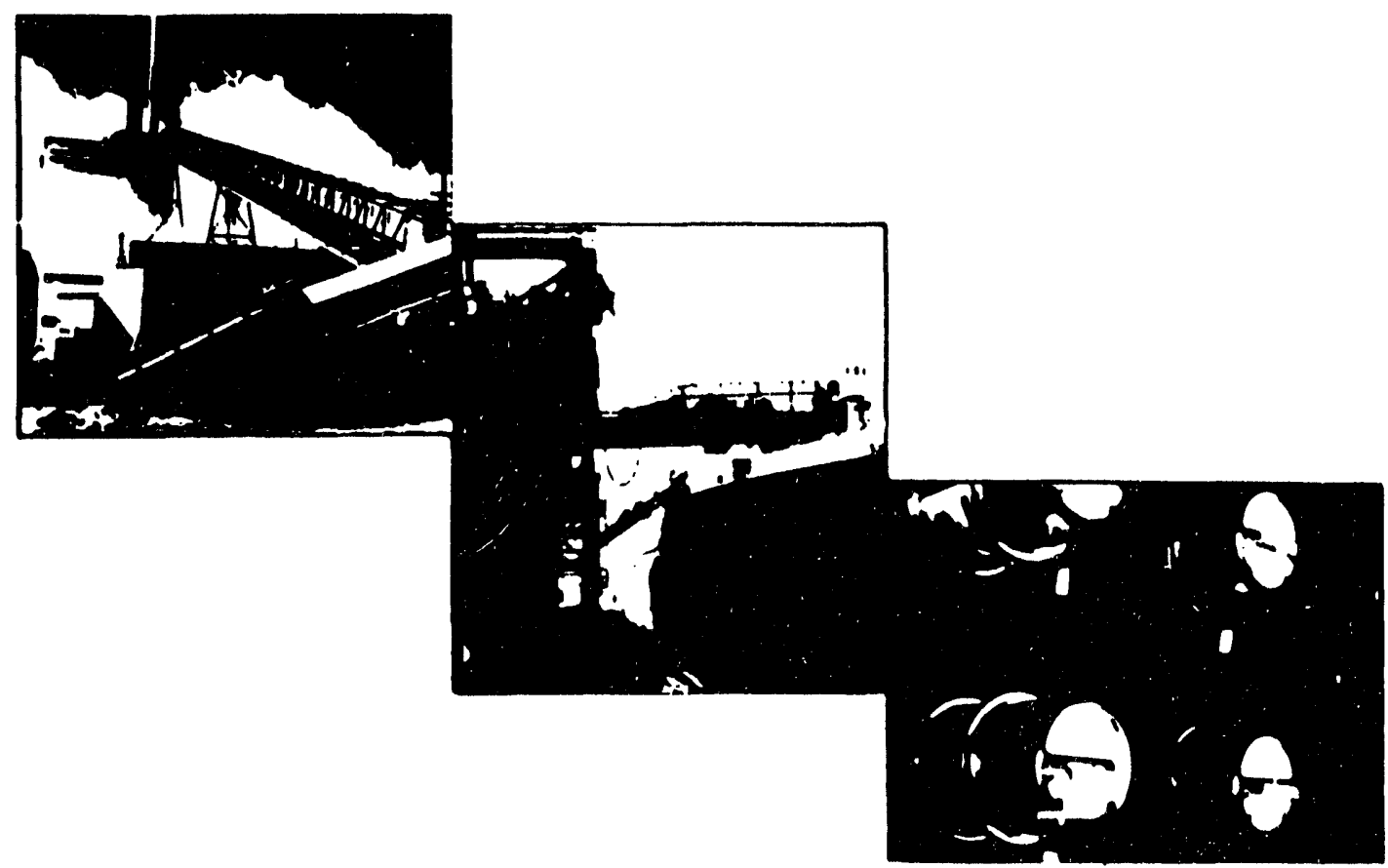


1. Newly Added Units by Company, Plant, and State $\ldots \ldots \ldots \ldots \ldots \ldots \ldots \ldots$

2. Electric Power Summary Statistics for the United States . . . . . . . . . . . . . . . . U.S. Electric Utility Net Generation by Month and Energy Source, January 1992 Through

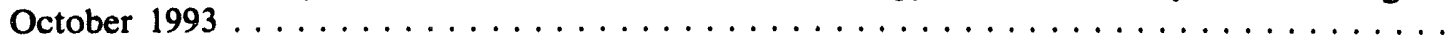

U.S. Electric Utility Net Generation by Energy Source, 1983 Through October $1993 \ldots \ldots$

5. Electric Utility Net Generation by NERC Region and Hawaii $\ldots \ldots \ldots \ldots \ldots \ldots \ldots$

Electric Utility Net Generation by Census Division and State $\ldots \ldots \ldots \ldots \ldots \ldots$ Electric Utility Net Generation from Coal by Census Division and State $\ldots \ldots \ldots \ldots \ldots$ Electric Utility Net Generation from Petroleum by Census Division and State . . . . . . . . Electric Utility Steam Net Generation from Petroleum by Census Division and State . . . . Electric Utility GT/IC Net Generation from Petroleum by Census Division and State . . . . . Electric Utility Net Generation from Gas by Census Division and State . . . . . . . . . . . Electric Utility Steam Net Generation from Gas by Census Division and State . . . . . . . . Electric Utility GT/IC Net Generation from Gas by Census Division and State . . . . . . Electric Utility Hydroelectric Net Generation by Census Division and State . . . . . . . . . Electric Utility Nuclear-Powered Net Generation by Census Division and State . . . . . . . Electric Utility Net Generation from Other Energy Sources by Census Division and State . . U.S. Electric Utility Consumption of Fossil Fuels, 1983 Through October $1993 \ldots \ldots \ldots \ldots$. . . Electric Utility Consumption of Coal by NERC Region and Hawaii $\ldots \ldots \ldots \ldots \ldots$ Electric Utility Consumption of Petroleum by NERC Region and Hawaii $\ldots \ldots \ldots \ldots \ldots$ Electric Utility Consumption of Gas by NERC Region and Hawaii $\ldots \ldots \ldots \ldots \ldots \ldots$ Electric Utility Consumption of Coal by Census Division and State $\ldots \ldots \ldots \ldots \ldots \ldots$ Electric Utility Consumption of Petroleum by Census Division and State . . . . . . . . Consumption of Petroleum at Steam-Fired Electric Utility Plants by Census Division and

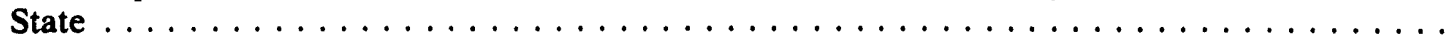
Consumption of Petroleum at GT/IC Electric Utility Plants by Census Division and State Electric Utility Consumption of Gas by Census Division and State . . . . . . . . . . Consumption of Gas at Steam-Fired Electric Utility Plants by Census Division and State . . Consumption of Gas at GT/IC Electric Utility Plants by Census Division and State . . . . . U.S. Electric Utility Stocks of Coal and Petroleum, 1983 Through October $1993 \ldots \ldots \ldots$

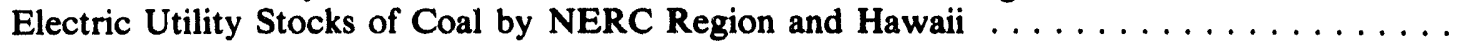
Electric Utility Stocks of Petroleum by NERC Region and Hawaii $\ldots \ldots \ldots \ldots \ldots \ldots$ Electric Utility Stocks of Coal by Census Division and State $\ldots \ldots \ldots \ldots \ldots \ldots \ldots$ Electric Utility Stocks of Petroleum by Census Division and State . . . . . . . . . . . U.S. Electric Utility Receipts of and Average Cost for Fossil Fuels, 1983 Through September 1993 Electric Utility Receipts of Coal by NERC Region and Hawaii . . . . . . . . . . . . Average Cost of Coal Delivered to Electric Utilities by NERC Region and Hawaii . . . . . Electric Utility Receipts of Petroleum by NERC Region and Hawaii . . . . . . . . . . Average Cost of Petroleum Delivered to Electric Utilities by NERC Region and Hawaii . . Electric Utility Receipts of Gas by NERC Region and Hawaii . . . . . . . . . . . 
49. Receipts and Average Cost of Gas Delivered to Electric Utilities by Census Division and

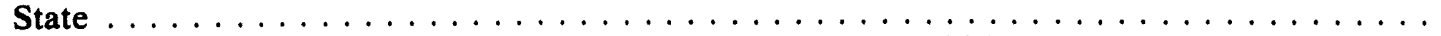

50. Receipts and Average Cost of Gas Delivered to Electric Utilities by Type of Purchase,

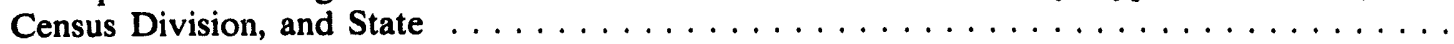

51. U.S. Electric Utility Sales of Electricity by Sector, 1983 Through October $1993 \ldots \ldots$. . . . .

52. Electric Utility Sales of Electricity to Ultimate Consumers by Sector, Census Division, and

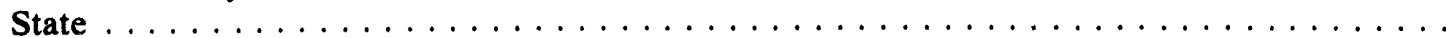

53. Estimated Coefficients of Variation for Electric Utility Sales of Electricity by Census Divi-

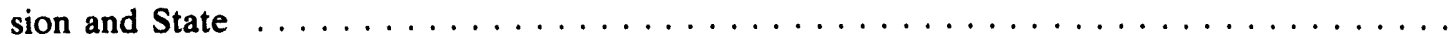

54. Electric Utility Sales of Electricity to Ultimate Consumers by Sector, Census Division, and State, January Through September 1992 and $1993 \ldots \ldots \ldots \ldots \ldots \ldots \ldots \ldots$

55. Revenue From U.S. Electric Utility Sales of Electricity to Ultimate Consumers by Sector,

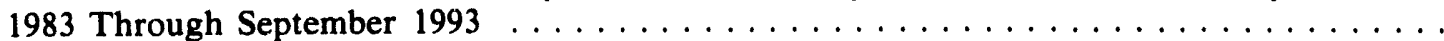

56. Electric Utility Revenue From Sales of Electricity to Ultimate Consumers by Sector, Cen-

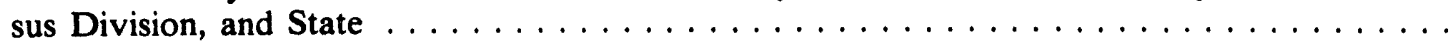

57. Estimated Coefficients of Variation of Revenue from Electric Utility Sales of Electricity by

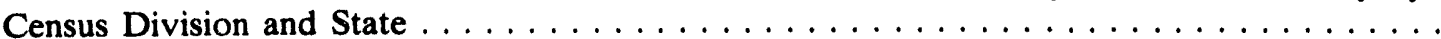

58. Electric Utility Revenue From Sales to Ultimate Consumers by Sector, Census Division, and State, January Through October 1992 and $1993 \ldots \ldots \ldots \ldots \ldots \ldots$

59. U.S. Electric Utility Average Revenue per Kilowatthour by Sector, 1983 Through October

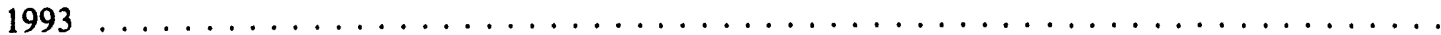

60. Electric Utility Average Revenue per Kilowatthour by Sector, Census Division, and State

61. Estimated Coefficients of Variation for Electric Utility Average Revenue per Kilowatthour by Sector, Census Division, and State $\ldots \ldots \ldots \ldots \ldots \ldots \ldots$

62. U.S. Electric Utility Net Generation, Fuel Consumption, and Fuel Stocks by Company and

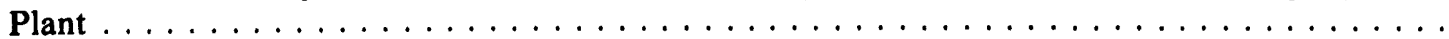

63. Receipts, Average Cost, and Quality of Fossil Fuels Delivered to U.S. Electric Utilities by

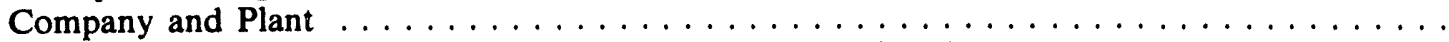

A1. Major Disturbances and Unusual Occurrences in U.S. Electric Power Systems . . . . . . .

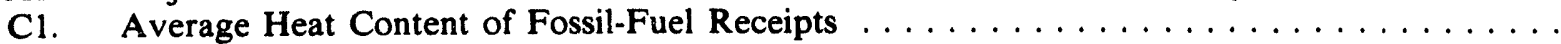

C2. Comparison of Preliminary Versus Final Published Data at the U.S. Level . . . . . . . . . . .

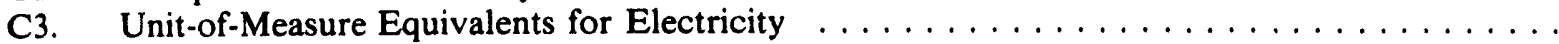

C4. Comparison of Published Data at the State Level, 1990 and 1991, by End-use Sector .... .

\section{Illustrations}

1. Net Generation by Energy Source at the National Level . . . . . . . . . . . . . . 


\section{U.S. Electric Power At A Glance}

Electricity is the most convenient, clean, and accessible form of useable energy.

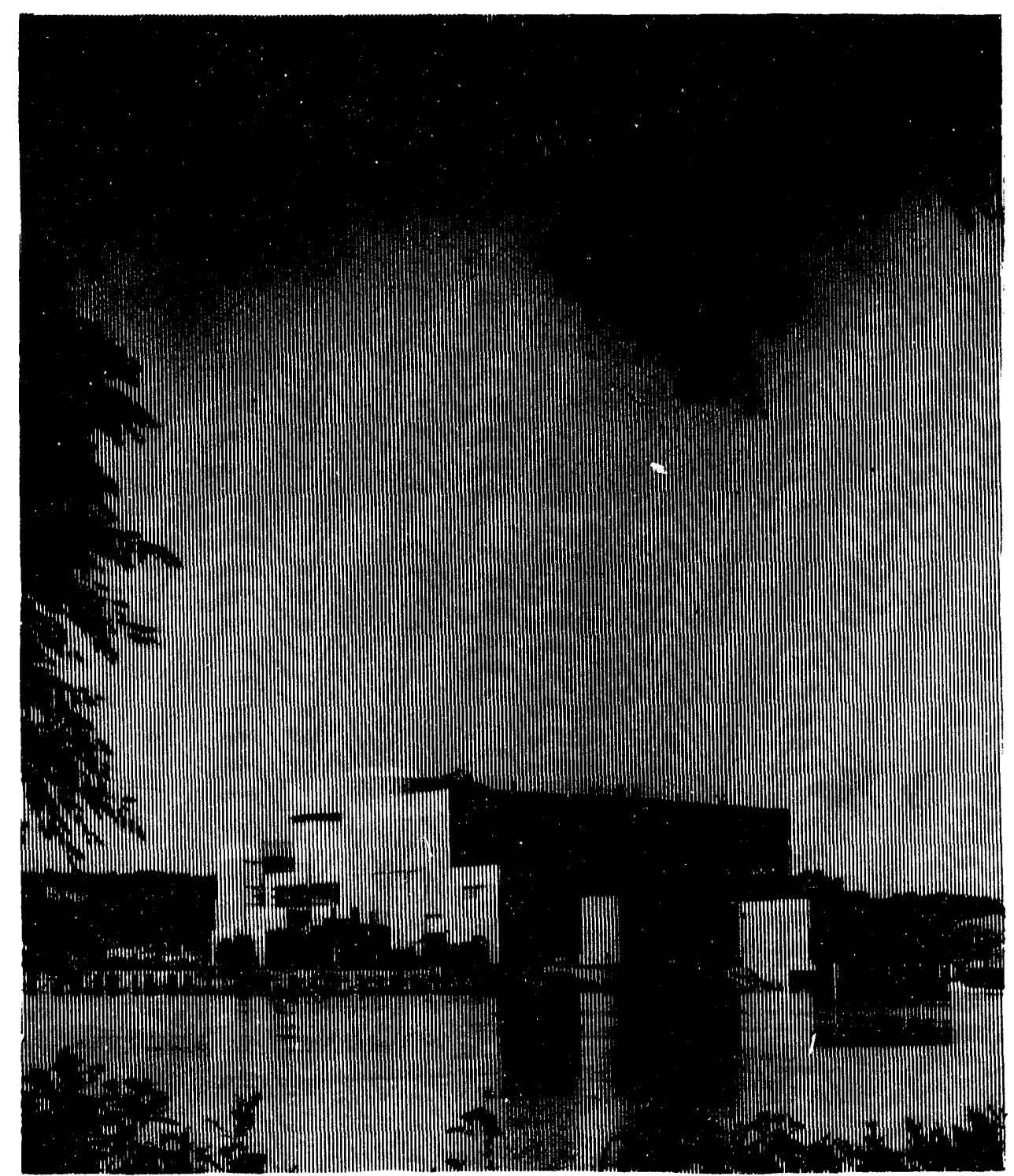


Monthly Update

\section{September 1993}

Receipts and Fuel Costs. Receipts of coal delivered to electric utilities in September 1993 totaled 65 million short tons, a decrease of 1 million short tons from September 1992 and nearly unchanged from August 1993. Events in September 1993 that affected coal receipts and costs include the United Mine Workers' of America (UMWA) strike, continued higher levels of hydroelectric generation in the western United States, compared with the prior year's levels, and the after effects of the Midwest flood.

The UMWA strike, which began in early. May, continued through September. The strike has led to lower coal receipts at several electric utilities and has resulted in an increase in the cost of spot-market coal from the Appalachian and Interior coal-producing regions.

The floodwaters in the Midwest, which had disrupted fuel deliveries to electric utilities in July and August, began to recede in September. This led to an improvement in rail and barge traffic. Illinois, Iowa, Missouri, and Wisconsin (the States most affected by the flood) each reported increases in coal receipts, compared with August.

The increase in hydroelectric generation in September, compared with last year, continued a year-to-date upward trend and contributed to limiting fossil-fuel requirements in several regions of the United States. The continued shutdown of the Tennessee Valley Authority's 2,440-megawatt Sequoyah Nuclear Plant in Tennessee and the 2,700-megawatt South Texas Nuclear Project in Texas favored higher fossil-fuel receipts in these States.

The average cost of coal delivered to electric utilities during September was $\$ 28.44$ per short ton, an increase of $\$ 0.21$ per short ton from August 1993 and a decrease of $\$ 1.09$ per short ton from September 1992. The average cost of spot-market coal delivered to electric utilities during September 1993 was $\$ 28.66$ per short ton, an increase of $\$ 0.61$ per short ton from August 1993. The cost of spot-market coal mined in the Appalachian and Interior coal-producing regions and delivered to electric utilities in September increased by \$0.64 and \$0.15 per short ton, respectively, from August. Spot-market coal mined in the Western coalproducing region and delivered to electric utilities in September increased by $\$ 0.61$ per short from August. The UMWA strike was the most important factor affecting delivered coal costs in September.

Electric utilities received a record 25 million short tons of subbituminous coal during September. Factors leading to increased receipts of subbituminous coal include replenishment of stockpiles drawn down by record coal consumption in July and August, electric utilities replacing eastern bituminous coal from UMWA struck mines with western subbituminous coal, an increase in test burns of subbituminous coal, and a shift toward subbituminous coal by some electric utilities in preparation for the January 1995 deadline for compliance with the Clean Air Act Amendments of 1990.

Receipts of petroleum in September 1993 were 16 million barrels, an increase of 6 million barrels from September 1992 petroleum receipts. This increase in petroleum receipts was due to a combination of low onhand stocks of fuel oil at the start of the month and the low cost of petroleum reported by electric utilities for September. The average cost of petroleum was $\$ 14.66$ per barrel, a decrease of $\$ 2.91$ per barrel from September 1992.

Total U.S. receipts of gas delivered to electric utilities during September 1993 were 250 billion cubic feet (Bcf), a decrease of $10 \mathrm{Bcf}$, compared with last year at this time. The cost of gas delivered to electric utilities in September 1993 was $\$ 2.70$ per thousand cubic feet (Mcf), \$0.17 per Mcf higher than that of a year ago.

On a cents-per-million-Btu basis, gas was the most expensive fossil fuel delivered to electric utilities in September 1993. The average cost of gas was 263.6 cents per million Btu, compared with 231.0 cents for petroleum and 138.5 cents for coal.

\section{October 1993}

Generation. Total U.S. net generation of electricity during October 1993 was 224 billion kilowatthours, 1 percent above the amount reported in October 1992. The energy source with the largest quantitative increase in generation, compared with October of last year, was coal (higher by 3 billion kilowatthours). Electric utilities used more coal to compensate for the decline in nuclear-powered generation ( 9 percent below October 1992). Generation from petroleum- and gas-fired plants also had higher production levels, compared with the same period in 1992.

During October 1993, electricity produced from coalfired plants was 131 billion kilowatthours, 2 percent more than the amount produced during October 1992. Electric utilities in the East North Central Census Division, the largest consumer of coal in the Nation, produced 31 billion kilowatthours of electricity from coalfired plants during the month, 3 percent above the level in October 1992. The coal-fired generation from this division represented 24 percent of the total U.S. coal-fired generation in October 1993.

Generation from petroleum-fired plants was 8 billion kilowatthours, 11 percent above the corresponding period in 1992. Generation from gas-fired plants was 23 billion kilowatthours, 12 percent more than the level reported during the same period in 1992. Production 
of electricity from gas-fired plants in the West South Central Census Division (the largest consumer of gas by electric utilities at 56 percent of the total U.S. gasfired generation) was 20 percent above the amount reported during October 1992. Cooler temperatures in this Census division, compared with a year ago and normal levels, may have contributed to this large increase in gas-fired generation. Based on the number of heating degree days, temperatures were cooler than normal levels and last year at this time by 190 and 264 percent, respectively.

Sales. Total sales of electricity to ultimate consumers in October 1993 were 229 billion kilowatthours, an increase of 4 billion kilowatthours ( 2 percent), compared with October 1992. Retail sales of electricity in all end-use sectors increased, compared with a year ago. The largest increase occurred in the residential sector, where sales reached 72 billion kilowatthours in October, an increase of 2 billion kilowatthours ( 3 percent), compared with last year. These sales accounted for 44 percent of the 4-billion-kilowatthour increase in total sales to ultimate consumers in the United States.

Sales of electricity to residential consumers increased the most, compared with last year, in the West South Central Census Division. During the month, sales of electricity to residential consumers in this Census division reached 12 billion kilowatthours, increasing by 1 billion kilowatthours (13 percent), compared with a year ago. Residential sales in the State of Texas accounted for 69 percent of the increase in this Census division and 47 percent of the 2 billion kilowatthour increase in total U.S. residential electricity sales. Cooler temperatures in Texas and Louisiana, compared with a year ago and normal levels, may have contributed to these increases in residential electricity sales. Based on the number of heating degree days, temperatures in these States were cooler by 343 and 189 percent, respectively, compared with last year, and cooler than normal by 114 and 165 percent, respectively.

Figure 1. Net Generation by Energy Source at the Natlonal Level

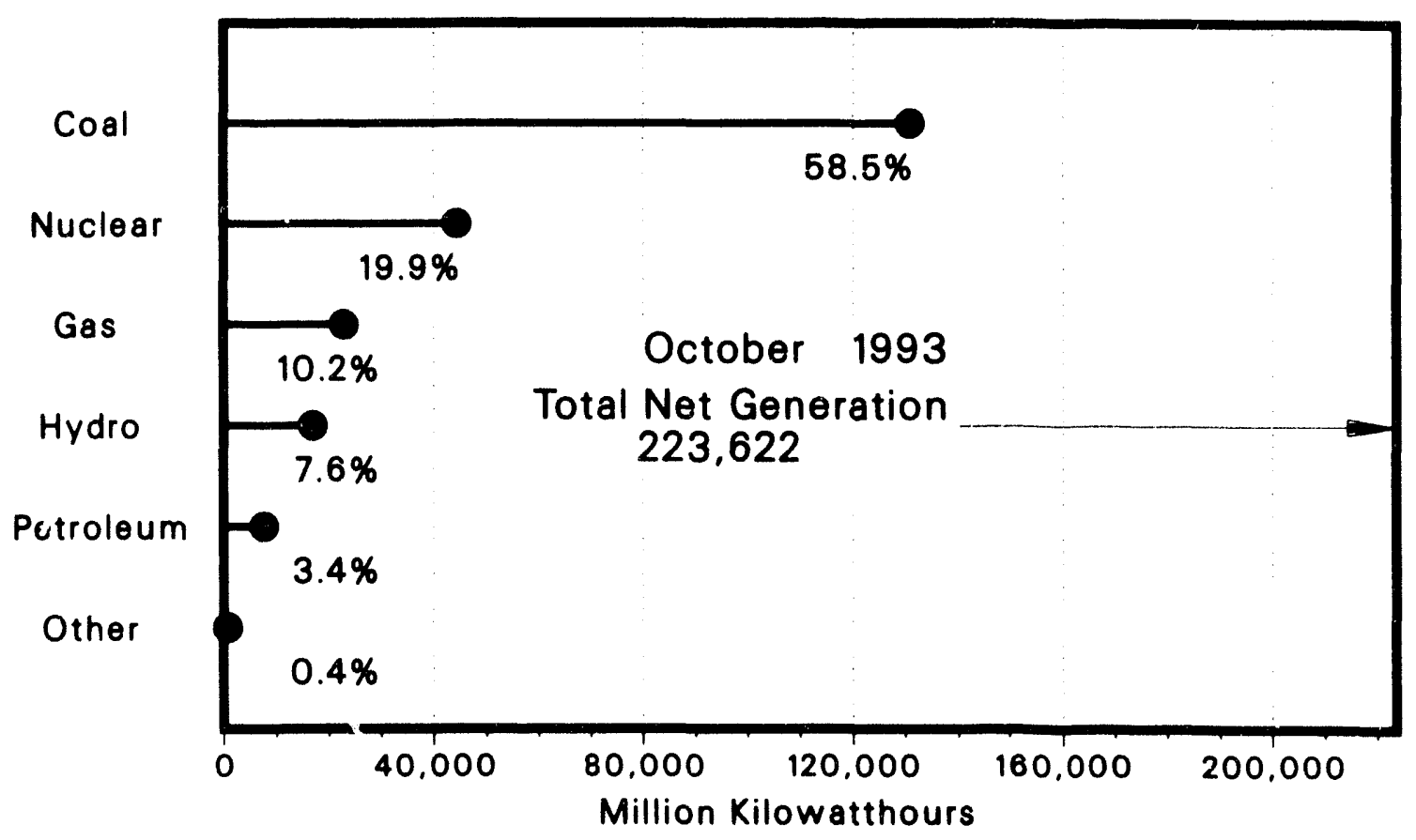

Note: Other energy sources include geothermal, wood, wind, waste, and solar. Data for 1993 are proliminary.

Source: Energy Information Administration, Form ElA-759, "Monthly Power Plant Report." 
Table 1. New Electric Generating Units by Operating Company, Plant, and State, and Retirements and Total Capabllity at U.S. Electric Utilities, 1993

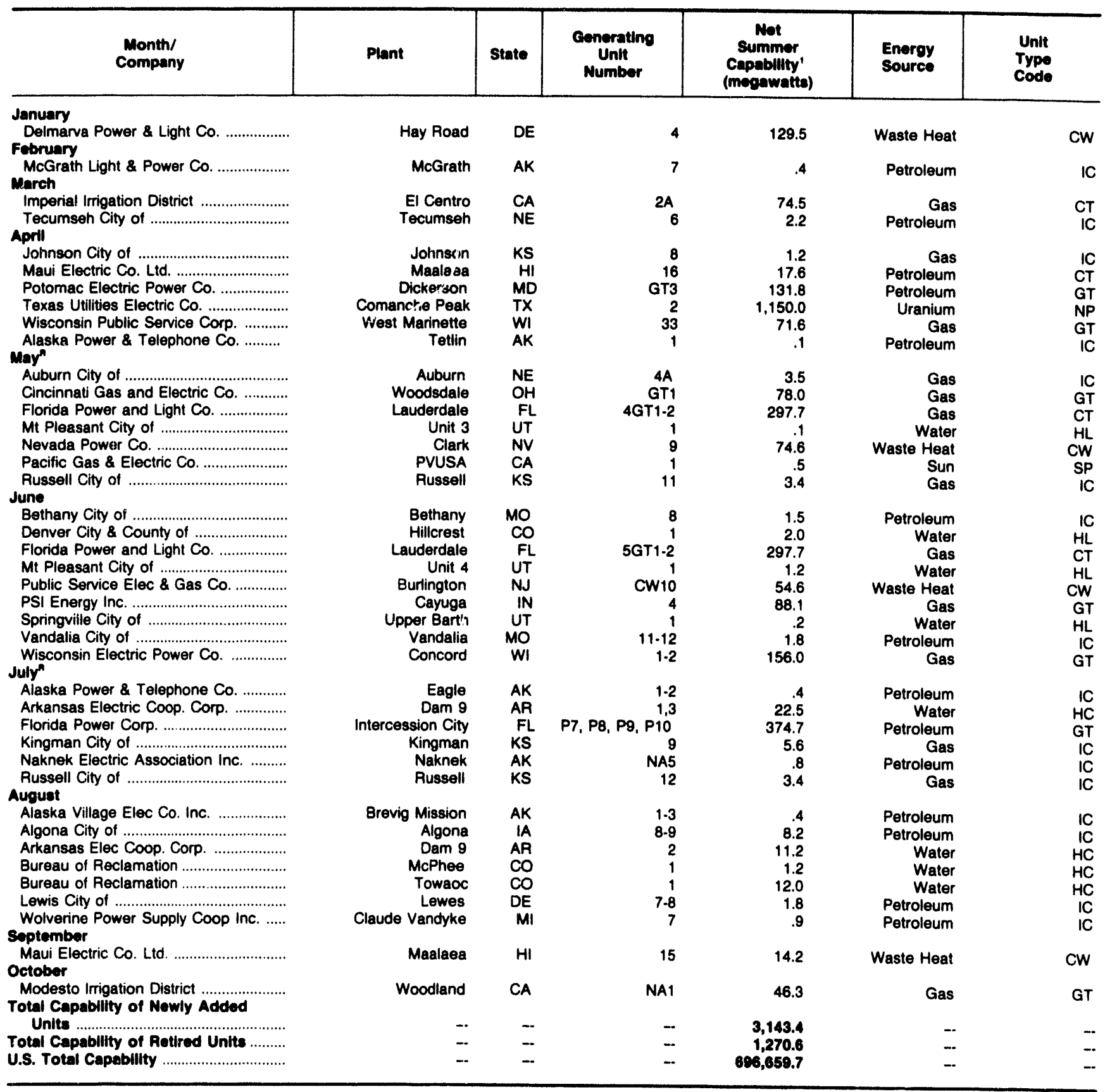

- Net summer capability is estimated.

- Revised.

Notes: -Totals may not equal sum of components because of independent rounding. Data are preliminary. Final data for the year are to be released in the Electric Power Annual (DOE/EIA - 0348(92)). Unit Type Codes are: IC = Internal Combustion, GT=Combustion (Gas) Turbine, CT =Combined-Cycle Combustion Turbine, $\mathrm{CW}=$ Combined Cycle Steam Turbine with only waste heat capability. $\mathrm{HC}=\mathrm{Hydraulic}$ Turbine - conventional, HR=Hydraulic Turbine reversible (pumped storage), ST = Steam Turbine - Boiler, HL=Hydraulic Turbine - Pipeline, NP=Steam Turbine - Pressurized Water Nuclear Reactor. Source: Energy Information Administration, Form EIA-860, "Annual Electric Generator Report." 


\begin{tabular}{|c|c|c|c|c|c|c|}
\hline \multirow[b]{2}{*}{ Items } & \multirow{2}{*}{$\begin{array}{l}\text { October } \\
1993^{\prime}\end{array}$} & \multirow{2}{*}{$\begin{array}{l}\text { September } \\
\text { I993' }^{\prime}\end{array}$} & \multirow{2}{*}{$\begin{array}{l}\text { October } \\
\text { 1992' }\end{array}$} & \multicolumn{3}{|c|}{ Year to Date } \\
\hline & & & & $1993^{\prime}$ & 1992 & $\begin{array}{c}\text { Difference } \\
\text { (Percent) }\end{array}$ \\
\hline \multicolumn{7}{|l|}{ Net Generation (Million kWh) } \\
\hline 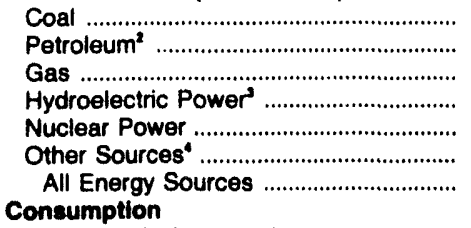 & $\begin{array}{r}130,926 \\
7,659 \\
22,906 \\
16,899 \\
44,434 \\
799 \\
223,622\end{array}$ & $\begin{array}{r}133,856 \\
9,759 \\
25,020 \\
17,089 \\
49,989 \\
804 \\
236,516\end{array}$ & $\begin{array}{r}127,940 \\
6,908 \\
20,420 \\
16,375 \\
48,784 \\
862 \\
221,289\end{array}$ & $\begin{array}{r}1,362,736 \\
81,774 \\
221,022 \\
226,149 \\
510,321 \\
7,958 \\
2,409,960\end{array}$ & $\begin{array}{r}1,312,126 \\
75,688 \\
229,096 \\
196,458 \\
509,975 \\
8,486 \\
2,331,830\end{array}$ & $\begin{array}{r}3.9 \\
8.0 \\
-3.5 \\
15.1 \\
.1 \\
-6.2 \\
3.4\end{array}$ \\
\hline 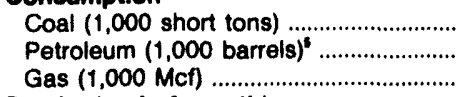 & $\begin{array}{r}64,909 \\
12,221 \\
233,192\end{array}$ & $\begin{array}{r}66,504 \\
15,760 \\
258,812\end{array}$ & $\begin{array}{r}62,806 \\
11,246 \\
212,640\end{array}$ & $\begin{array}{r}676,595 \\
133,615 \\
2,297,532\end{array}$ & $\begin{array}{r}647,883 \\
125,264 \\
2,400,704\end{array}$ & $\begin{array}{r}4.4 \\
6.7 \\
-4.3\end{array}$ \\
\hline 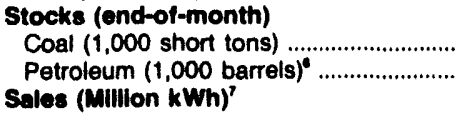 & $\begin{array}{r}115,206 \\
61,256\end{array}$ & $\begin{array}{r}112,773 \\
60,673\end{array}$ & $\begin{array}{r}156,859 \\
68,269\end{array}$ & - & - & - \\
\hline 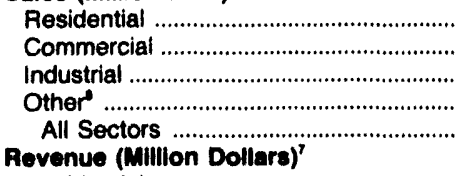 & $\begin{array}{r}71,770 \\
65,242 \\
83,377 \\
8,270 \\
228,660\end{array}$ & $\begin{array}{r}88,884 \\
71,708 \\
83,839 \\
8,699 \\
253,130\end{array}$ & $\begin{array}{r}69,838 \\
63,985 \\
82,678 \\
7,766 \\
224,267\end{array}$ & $\begin{array}{r}834,174 \\
664,909 \\
820,476 \\
81,335 \\
2,400,894\end{array}$ & $\begin{array}{r}776,696 \\
640,451 \\
807,577 \\
78,816 \\
2,303,539\end{array}$ & $\begin{array}{l}7.4 \\
3.8 \\
1.6 \\
3.2 \\
4.2\end{array}$ \\
\hline 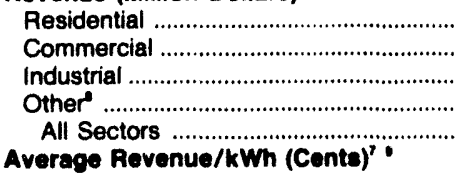 & $\begin{array}{r}6,290 \\
5,276 \\
4,187 \\
601 \\
16,354\end{array}$ & $\begin{array}{r}7,823 \\
5,770 \\
4,307 \\
615 \\
18,516\end{array}$ & $\begin{array}{r}5,912 \\
5,030 \\
4,051 \\
533 \\
15,525\end{array}$ & $\begin{array}{r}69,998 \\
51,586 \\
40,159 \\
5,576 \\
167,318\end{array}$ & $\begin{array}{r}64,321 \\
49,096 \\
39,356 \\
5,249 \\
158,022\end{array}$ & $\begin{array}{l}8.8 \\
5.1 \\
2.0 \\
6.2 \\
5.9\end{array}$ \\
\hline 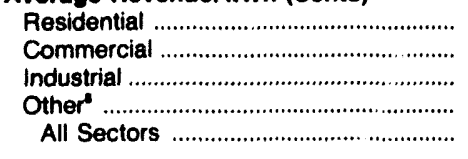 & $\begin{array}{l}8.8 \\
8.1 \\
5.0 \\
7.3 \\
7.2\end{array}$ & $\begin{array}{l}8.8 \\
8.0 \\
5.1 \\
7.1 \\
7.3\end{array}$ & $\begin{array}{l}8.5 \\
7.9 \\
4.9 \\
6.9 \\
6.9\end{array}$ & $\begin{array}{l}8.4 \\
7.8 \\
4.9 \\
6.9 \\
7.0\end{array}$ & $\begin{array}{l}8.3 \\
7.7 \\
4.9 \\
6.7 \\
6.9\end{array}$ & $\begin{array}{l}1.2 \\
1.3 \\
- \\
3.0 \\
1.4\end{array}$ \\
\hline \multirow{2}{*}{ 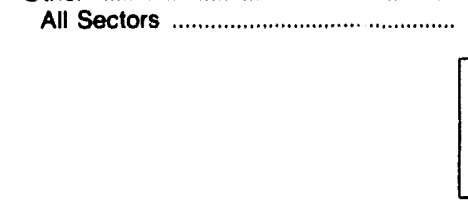 } & \multirow{2}{*}{$\begin{array}{l}\text { September }_{1993^{10}} \\
\text {. }\end{array}$} & \multirow{2}{*}{$\begin{array}{l}\text { Auguat } \\
1993^{\text {to }}\end{array}$} & \multirow{2}{*}{$\begin{array}{l}\text { septe mber } \\
\text { 190: }^{10}\end{array}$} & \multicolumn{3}{|c|}{ Year to Date } \\
\hline & & & & $1993^{10}$ & $1892^{10}$ & $\begin{array}{c}\text { Difierence } \\
\text { (Percent) }\end{array}$ \\
\hline \multicolumn{7}{|l|}{ Recelpts" } \\
\hline 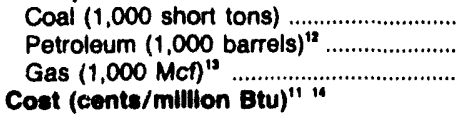 & $\begin{array}{r}65,358 \\
15,766 \\
249,708\end{array}$ & $\begin{array}{r}65,739 \\
15,624 \\
339,454\end{array}$ & $\begin{array}{r}66,503 \\
9,319 \\
259,771\end{array}$ & $\begin{array}{r}569,406 \\
105,407 \\
1,977,873\end{array}$ & $\begin{array}{r}579,054 \\
107,436 \\
2,081,221\end{array}$ & $\begin{array}{l}-1.7 \\
-1.0 \\
-5.0\end{array}$ \\
\hline 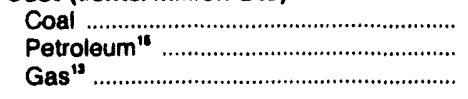 & $\begin{array}{l}138.5 \\
231.0 \\
263.6\end{array}$ & $\begin{array}{l}137.4 \\
232.2 \\
252.5\end{array}$ & $\begin{array}{l}142.0 \\
277.6 \\
246.3\end{array}$ & $\begin{array}{l}138.6 \\
251.9 \\
256.6\end{array}$ & $\begin{array}{l}141.5 \\
246.9 \\
218.5\end{array}$ & $\begin{array}{r}-2.0 \\
2.0 \\
17.4\end{array}$ \\
\hline
\end{tabular}

Values for net generation, consumption, stocks, sales, revenue, and average revenue per kllowatthour are final for 1992 and are preliminary for 1993 .

Inciudes petroleum coke.

3 Pumping energy used at pumped storage plants for October 1993 was 2.012 million kilowatthours.

- includes geothermal, wood, wind, waste, and solar.

- The October 1993 petroleum coke consumption was 111526 short tons.

- The October 1993 petroleum coke stocks were 68,801 short tons.

7 Estimates for sales and net generation may not correspond exactly for a particular month. Net generation data are for the caleridar month. Sales and associated revenue data accumulated from bills collected for periods of time (28 to 35 days) that vary dependent upon customer class, represent consumption occurring in and outside of the calendar month. This among other reasons (i.e., sales data may include purchases of electricity from nonutilities or imported electricity), is why the monthly sales and generation data are not directly comparable.

- Includes public street and highway lighting, other sales to public authorities, sales to railroads and railways, and interdepartmental sales.

- Based on unrounded values. (See technical notes for an explanation of the modification to the sample design as of January 1993 estimates.)

to Data for 1992 are final. Data for 1993 are preliminary.

"As of 1991, receipt and cost data are for electric generating plants with a total steam-electric and combined-cycle nameplate capacity of 50 or more megawatts.

17. The September 1983 petroleum coke receipts were 131,082 short tons.

13 Includes small amounts of coke-oven, refinery, and blast-furnace gas.

11 Average cost of fuel delivered to electric generating plants; cost values are weighted values.

1. September 1993 petroleum coke cost was 70.3 cents per million Btu.

Notes: - Totals may not equal sum of components because of independent rounding. - Percent difference is calculated before rounding.

$\bullet \mathrm{kWh}=$ kilowatthours, and Mcf = thousand cubic feet. $\bullet N M=$ Percent difference calculation not meaningful. $\bullet$ Monetary values are expressed in nominal terms. -Revenue nd average revenue per kilowatthour do not include sales and excise taxes.

Sources: •Energy Intormation Administration, Form ElA-759, "Monthly Power Plant Report." ofederal Energy Regulatory Commission, FERC Form 423. "Monthly Report of Cost and Quality of Fuels for Electric Plants." •Energy information Administration, Form EIA-826, "Monthly Electric Utility Sales and Revenue Report with State Distributions." 


\section{U.S. Electric Utility Net Generation}

These power lines are part of America's network that supplies electricity for the United States.

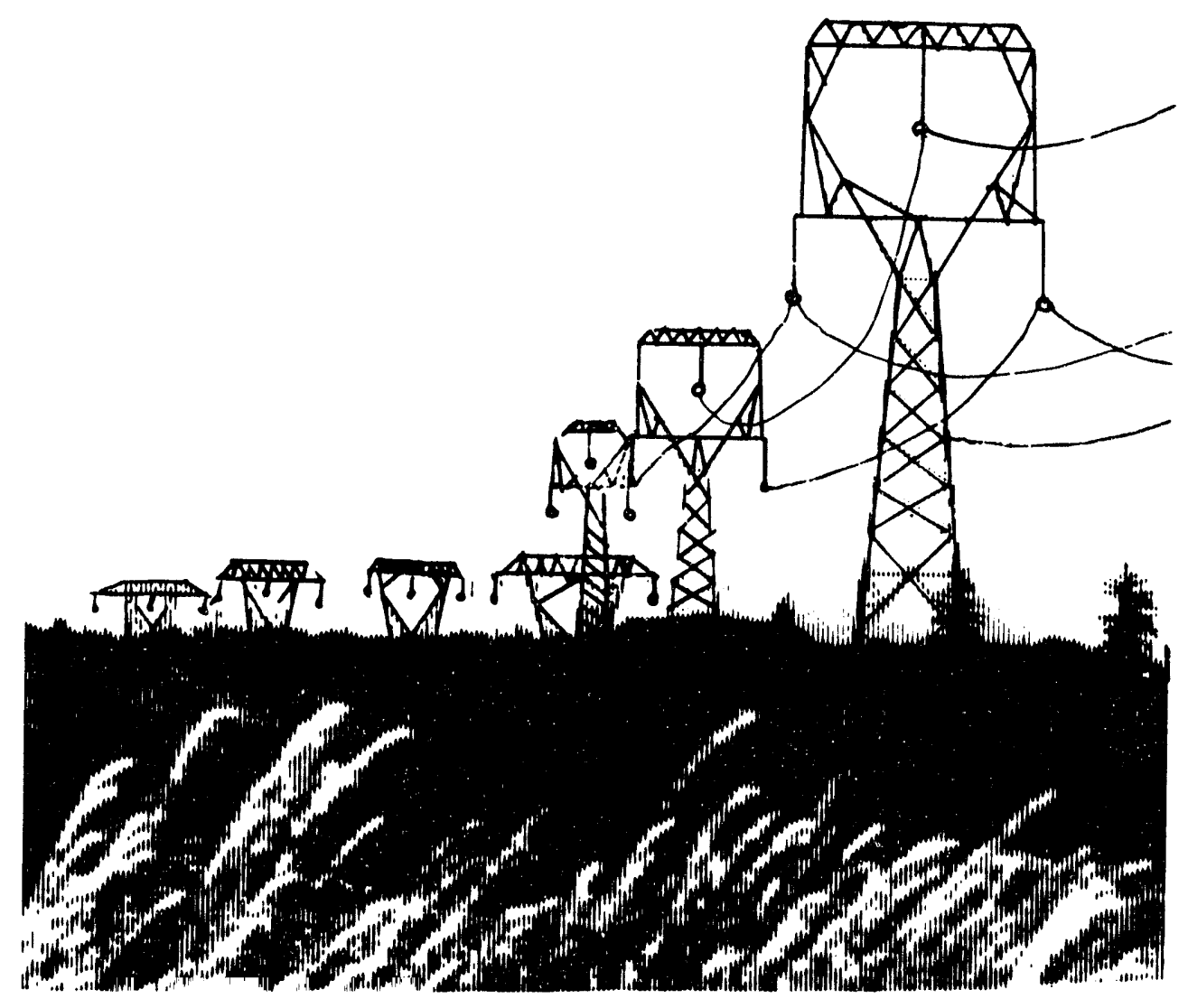


Table 3. U.S. Electric Utillty Net Generation by Month and Energy Source, January 1992 Through October 1993

\begin{tabular}{|c|c|c|c|c|c|c|c|}
\hline \multirow{2}{*}{ Period } & \multirow{2}{*}{$\begin{array}{c}\text { All Energy } \\
\text { Sources } \\
\text { (Million) } \\
\text { (Kilowatthours) }\end{array}$} & \multicolumn{6}{|c|}{ Share of Total U.S. Net Generation (Percent) } \\
\hline & & Coal' & Petroleum ${ }^{2}$ & Gas & Hydroelectric & Nuclear & Other \\
\hline \multicolumn{8}{|l|}{$1992^{4}$} \\
\hline 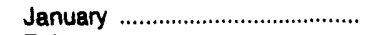 & 243,970 & 56.3 & 4.2 & 6.6 & 8.8 & 23.7 & 0.4 \\
\hline 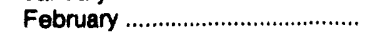 & 217,761 & 55.9 & 3.8 & 7.4 & 8.3 & 24.2 & .4 \\
\hline 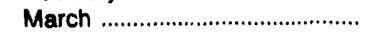 & 224,665 & 56.8 & 3.9 & 8.9 & 9.6 & 20.4 & .4 \\
\hline 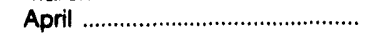 & 210,837 & 56.9 & 3.1 & 10.4 & 9.2 & 20.0 & .4 \\
\hline 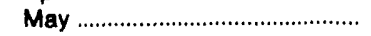 & 220,355 & 56.2 & 2.3 & 10.3 & 10.1 & 20.7 & .4 \\
\hline 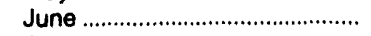 & 236,842 & 54.7 & 3.2 & 10.6 & 9.6 & 21.6 & .4 \\
\hline July . & 266,148 & 56.0 & 3.2 & 12.0 & 7.4 & 21.1 & .3 \\
\hline 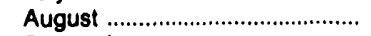 & 255,203 & 55.6 & 2.7 & 11.3 & 7.1 & 23.0 & .3 \\
\hline 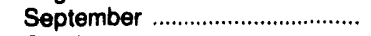 & 234,760 & 56.8 & 2.9 & 11.1 & 7.2 & 21.7 & .4 \\
\hline 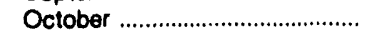 & 221,289 & 57.8 & 3.1 & 9.2 & 7.4 & 22.0 & .4 \\
\hline 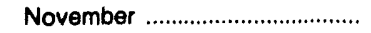 & 221,263 & 56.7 & 3.1 & 8.1 & 8.7 & 22.9 & .4 \\
\hline 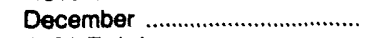 & 244,126 & 56.6 & 2.6 & 6.9 & 9.8 & 23.8 & .4 \\
\hline $1993^{s}$ Total & $2,797,219$ & $5 f, .3$ & 3.2 & 9.4 & 8.6 & 22.1 & .4 \\
\hline 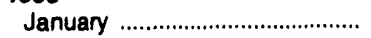 & 245,797 & 56.3 & 2.9 & 6.4 & 10.0 & 24.0 & .3 \\
\hline 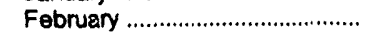 & 224,663 & 57.9 & 3.1 & 7.0 & 8.8 & 22.8 & .4 \\
\hline 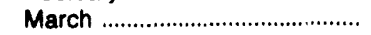 & 234,630 & 58.1 & 3.7 & 8.0 & 10.1 & 19.9 & .4 \\
\hline 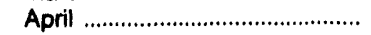 & 211,292 & 56.9 & 2.5 & 7.9 & 11.9 & 20.4 & .4 \\
\hline 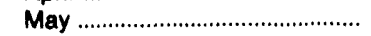 & 222,396 & 54.4 & 2.4 & 7.1 & 13.2 & 22.6 & .3 \\
\hline 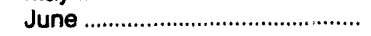 & 249,625 & 55.1 & 3.1 & 9.8 & 10.7 & 21.1 & .3 \\
\hline 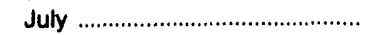 & 282,270 & 56.1 & 4.0 & 11.2 & 8.4 & 20.0 & .3 \\
\hline 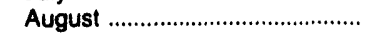 & 279,147 & 56.0 & 4.3 & 12.3 & 7.1 & 20.1 & .3 \\
\hline 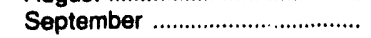 & 236,516 & 56.6 & 4.1 & 10.6 & 7.2 & 21.1 & .3 \\
\hline 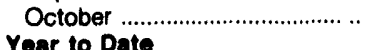 & 223,622 & 58.5 & 3.4 & 10.2 & 7.6 & 19.9 & .4 \\
\hline 1993 ; 1992 & $\begin{array}{l}2,409,960 \\
2,331,830\end{array}$ & $\begin{array}{l}56.5 \\
56.3\end{array}$ & $\begin{array}{l}3.4 \\
3.2\end{array}$ & $\begin{array}{l}9.2 \\
9.8\end{array}$ & $\begin{array}{l}9.4 \\
8.4\end{array}$ & $\begin{array}{l}21.2 \\
21.9\end{array}$ & .3 \\
\hline
\end{tabular}

' Includes lignite, bituminous coal, subbituminous coal, and anthracite.

Includes fuel oil Nos. 2, 4, 5, and 6, crude oil, kerosene, and petroleum coke.

- Includes geothermal, wood, wind, waste, and solar.

- Data for 1992 are final.

- Data for 1993 are preliminary.

Notes: - Totals may not equal sum of components because of independent rounding.

Source: Energy Information Administration, Form ElA-759, "Monthly Power Plant Report."

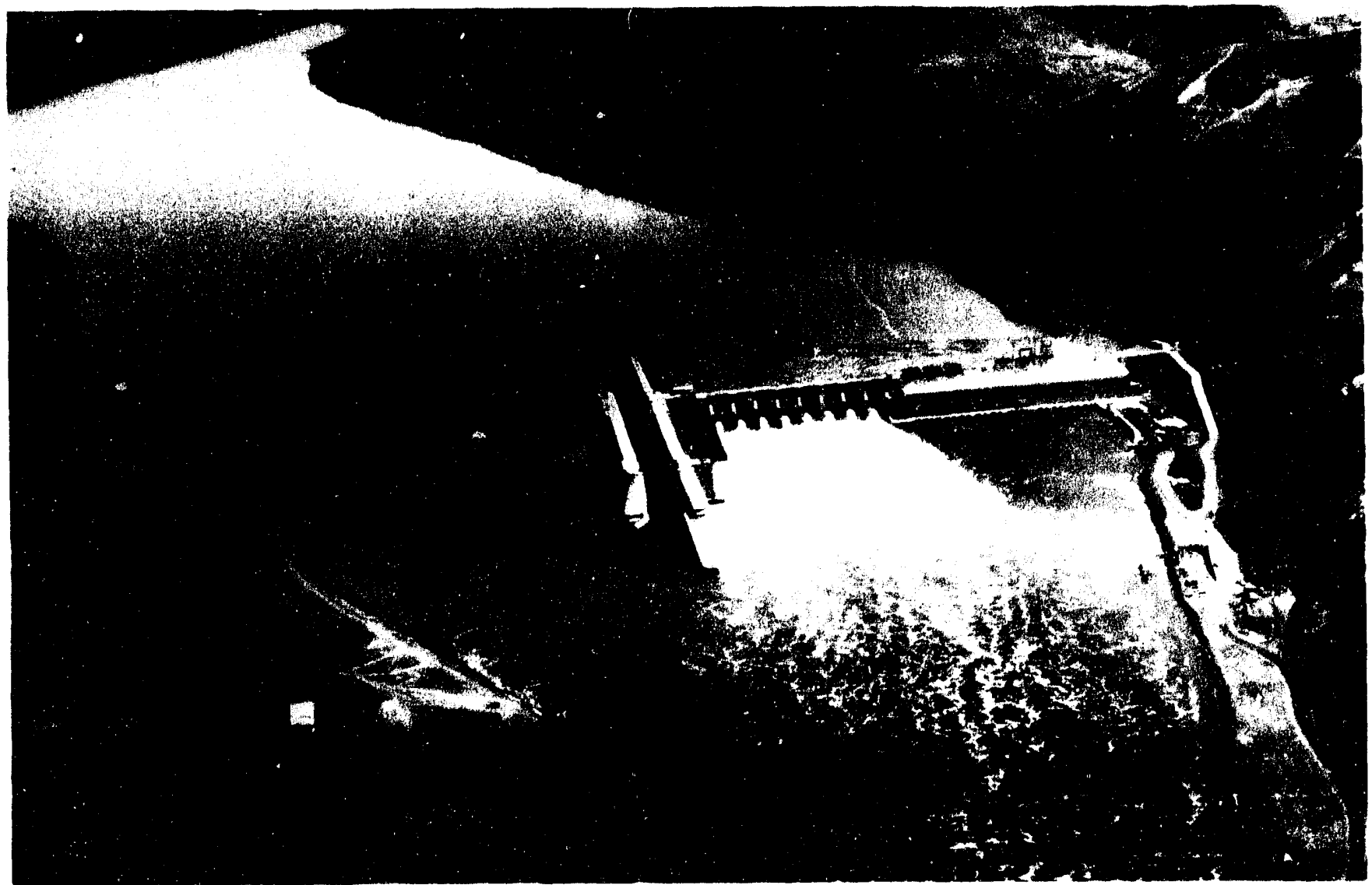

The Lower Granite Lock and Dam is a hydroelectric facility, located in Snake River, Washington. 
Table 4. U.S. Electric Utility Net Generation by Energy Source, 1983 Through October 1993 (Million Kilowatthours)

\begin{tabular}{|c|c|c|c|c|c|c|c|}
\hline Period & $\begin{array}{l}\text { All Energy } \\
\text { Sources }\end{array}$ & Coal' & Petroleum ${ }^{2}$ & Gas & Hydroelectric ${ }^{3}$ & Nuclear & Other \\
\hline $\begin{array}{l}1983 \\
1984 \\
1985 \\
1986 \\
1987 \\
1988 \\
1989 \\
1990 \\
1991 \\
1992\end{array}$ & $\begin{array}{l}2,310,285 \\
2,416,304 \\
2,469,841 \\
2,487,310 \\
2,572,127 \\
2,704,250 \\
2,784,304 \\
2,808,151 \\
2,825,023\end{array}$ & $\begin{array}{l}1,259,424 \\
1,341,681 \\
1,402,128 \\
1,385,831 \\
1,463,781 \\
1,540,653 \\
1,553,661 \\
1,559,606 \\
1,551,167\end{array}$ & $\begin{array}{l}144,499 \\
119,808 \\
100,202 \\
136,585 \\
118,493 \\
148,900 \\
158,318 \\
117,017 \\
111,463\end{array}$ & $\begin{array}{l}274,098 \\
297,394 \\
291,948 \\
248,508 \\
272,621 \\
252,801 \\
266,598 \\
264,089 \\
264,172\end{array}$ & $\begin{array}{l}332,130 \\
321,150 \\
281,149 \\
290,844 \\
249,695 \\
222,940 \\
265,063 \\
279,926 \\
275,519\end{array}$ & $\begin{array}{l}293,677 \\
327,634 \\
383,691 \\
414,038 \\
455,270 \\
526,973 \\
529,355 \\
576,862 \\
612,565\end{array}$ & $\begin{array}{r}6,456 \\
8,638 \\
10,724 \\
11,503 \\
12,267 \\
11,984 \\
11,309 \\
10,651 \\
10,137\end{array}$ \\
\hline 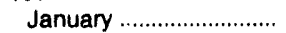 & 243,970 & 137,327 & 10,202 & 16,178 & 21,502 & 57.849 & 912 \\
\hline 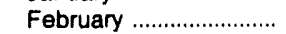 & 217,761 & 121,731 & 8,296 & 16,165 & 17,966 & 52,804 & 798 \\
\hline 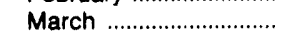 & 224,665 & 127,678 & 8,809 & 19,906 & 21,566 & 45,835 & 871 \\
\hline 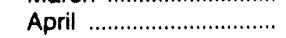 & 210,837 & 119,909 & 6,505 & 21,913 & 19,454 & 42,268 & 788 \\
\hline 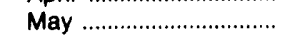 & 220,355 & 123,768 & 5,156 & 22,689 & 22,285 & 45,627 & 830 \\
\hline 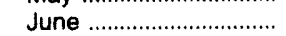 & 236,842 & 129,607 & 7,508 & 24,997 & 22,698 & 51,185 & 846 \\
\hline 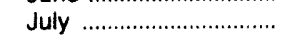 & 266,148 & 149,028 & 8,540 & 31,950 & 19,711 & 56,049 & 869 \\
\hline 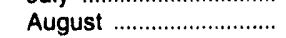 & 255,203 & 141,900 & 6,923 & 28,778 & 18,062 & 58,656 & 885 \\
\hline 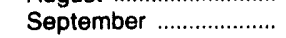 & 234,760 & 133,239 & 6,841 & 26,099 & 16,838 & 50,919 & 825 \\
\hline 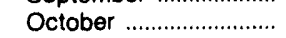 & 221,289 & 127,940 & 6,908 & 20,420 & 16,375 & 48,784 & 862 \\
\hline 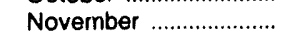 & 221,263 & 125,535 & 6,838 & 18,031 & 19,294 & 50,726 & 840 \\
\hline 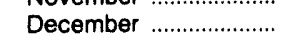 & 244,126 & 138,234 & 6,390 & 16,744 & 23,808 & 58,075 & 874 \\
\hline 1992 Total ....................... & $2,797,219$ & $1,575,895$ & 88,916 & 263,872 & 239,559 & 618,776 & 10,200 \\
\hline 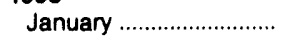 & 245,797 & 138,357 & 7,226 & 15,811 & 24,474 & 59,076 & 853 \\
\hline 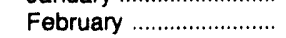 & 224,663 & 130,078 & 6,950 & 15,773 & 19,743 & 51,319 & 800 \\
\hline March ............................. & 234,630 & 136,280 & 8,569 & 18,740 & 23,583 & 46,606 & 852 \\
\hline April & 211,292 & 120,325 & 5,205 & 16,591 & 25,171 & 43,199 & 802 \\
\hline 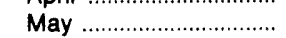 & 222,396 & 120,878 & 5,268 & 15,843 & 29,323 & 50,367 & 716 \\
\hline 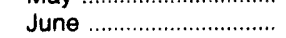 & 249,625 & 137,464 & 7,819 & 24,391 & 26,606 & 52,620 & 725 \\
\hline 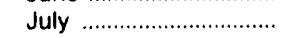 & 282,270 & 158,380 & 11,341 & 31,684 & 23,575 & 56,502 & 788 \\
\hline August & 279,147 & 156,193 & 11,978 & 34,262 & 19,685 & 56,209 & 820 \\
\hline September ........................ & 236,516 & 133,856 & 9,759 & 25,020 & 17,089 & 49,989 & 804 \\
\hline $\begin{array}{l}\text { October .................................. } \\
\text { Year to Date }\end{array}$ & 223,622 & 130,926 & 7,659 & 22,906 & 16,899 & 44,434 & 799 \\
\hline 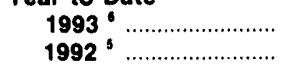 & $\begin{array}{l}2,409,960 \\
2,331,830\end{array}$ & $\begin{array}{l}1,362,736 \\
1,312,126\end{array}$ & $\begin{array}{l}81,774 \\
75,688\end{array}$ & $\begin{array}{l}221,022 \\
229,096\end{array}$ & $\begin{array}{l}226,149 \\
196,458\end{array}$ & $\begin{array}{l}510,321 \\
509,975\end{array}$ & $\begin{array}{l}7,958 \\
8,486\end{array}$ \\
\hline
\end{tabular}

- Includes lignite, bituminous coal, subbituminous coal, and anthracite.

2 Includes fuel oil Nos. 2, 4, 5, and 6, crude oil, kerosene, and petroleum coke.

- Pumping energy used for pumped storage plants for October 1993 was 2,012 million kilowatthours

- Includes geothermal, wood, wind, waste, and solar.

- Data for 1992 and prior years are final.

- Data for 1993 are preliminary.

Notes: - Totals may not equal sum of components because of independent rounding.

Source: Energy Information Administration, Form EIA-759, "Monthly Power Plant Report."

Table 5. Electric Utility Net Generation by NERC Region and Hawali (Million Kilowatthours)

\begin{tabular}{|c|c|c|c|c|c|c|}
\hline \multirow{2}{*}{$\begin{array}{l}\text { NERC Region } \\
\text { and Hawall }\end{array}$} & \multirow{2}{*}{$\begin{array}{l}\text { October } \\
1993^{1}\end{array}$} & \multirow{2}{*}{$\begin{array}{c}\text { September } \\
1993^{\prime}\end{array}$} & \multirow{2}{*}{$\begin{array}{l}\text { October } \\
1992^{2}\end{array}$} & \multicolumn{3}{|c|}{ Year to Date } \\
\hline & & & & $1993^{\prime}$ & $1992^{2}$ & $\begin{array}{l}\text { Difference } \\
\text { (Percent) }\end{array}$ \\
\hline 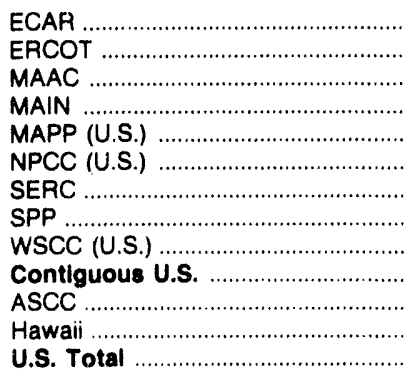 & $\begin{array}{r}38,785 \\
15,516 \\
15,015 \\
16,087 \\
11,403 \\
14,466 \\
50,700 \\
20,890 \\
39,783 \\
222,646 \\
387 \\
589 \\
223,622\end{array}$ & $\begin{array}{r}38,015 \\
18,290 \\
16,513 \\
16,518 \\
10,977 \\
14,810 \\
57,324 \\
22,467 \\
40,698 \\
235,612 \\
355 \\
549 \\
236,516\end{array}$ & $\begin{array}{r}39,242 \\
15,043 \\
14,690 \\
16,289 \\
10,968 \\
15,576 \\
49,115 \\
19,900 \\
39,569 \\
220,393 \\
361 \\
535 \\
221,289\end{array}$ & $\begin{array}{r}408,282 \\
168,689 \\
171,584 \\
179,414 \\
118,225 \\
163,407 \\
551,917 \\
227,489 \\
412,139 \\
2,401,146 \\
3,718 \\
5,096 \\
2,409,960\end{array}$ & $\begin{array}{r}399,054 \\
162,551 \\
160,649 \\
163,887 \\
115,696 \\
169,370 \\
526,746 \\
215,508 \\
409,192 \\
2,322,653 \\
3,340 \\
5,837 \\
2,331,830\end{array}$ & $\begin{array}{r}2.3 \\
3.8 \\
6.8 \\
9.5 \\
2.2 \\
-3.5 \\
4.8 \\
5.6 \\
.7 \\
3.4 \\
11.3 \\
-12.7 \\
3.4\end{array}$ \\
\hline
\end{tabular}

'Data for 1993 are preliminary

2 Data for 1992 are final.

Notes: - Totals may not equal sum of components because of independent rounding. • See Glossary for explanation of acronyms. • Percent difference is calculated before rounding.

Source: Energy Information Administration, Form ElA-759, "Monthly Power Plant Report." 
Table 6. Electric Utility Net Generation by Census Division and State (Million Kilowatthours)

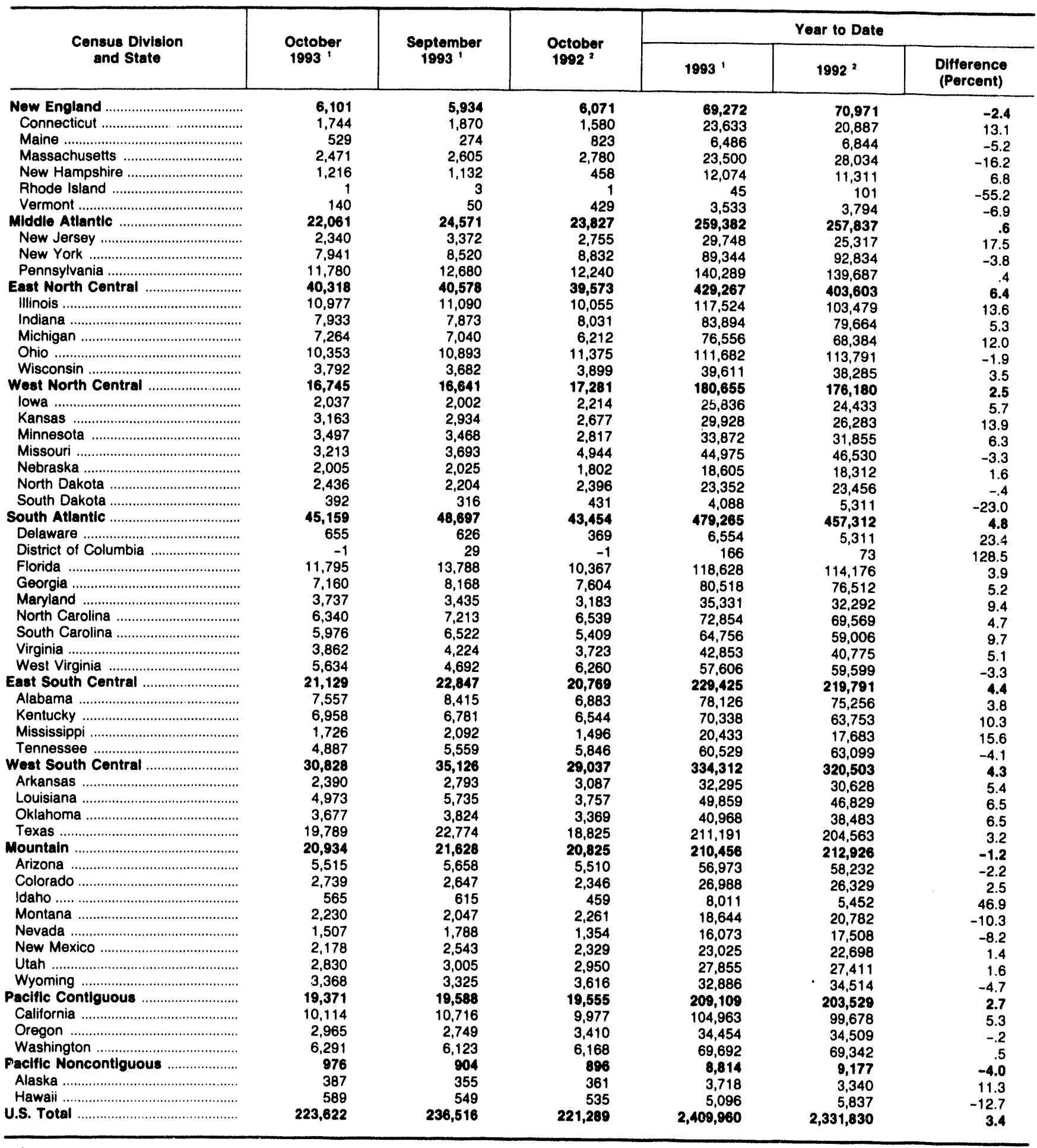

Data for 1993 are preliminary

Data for 1992 are final.

Notes: - Totals may not equal sum of components because of independent rounding. •Percent difference is calculated before rounding.

Source: Energy Information Administration, Form ElA-759, "Monthly Power Plant Report." 
Table 7. Electric Utility Net Generation from Coal by Census Division and State (Million Kilowatthours)

\begin{tabular}{|c|c|c|c|c|c|c|c|c|}
\hline \multirow{3}{*}{$\begin{array}{l}\text { Census Division } \\
\text { and State }\end{array}$} & \multirow{3}{*}{$\begin{array}{l}\text { October } \\
1993^{\prime}\end{array}$} & \multirow{3}{*}{$\begin{array}{l}\text { September } \\
1993^{1}\end{array}$} & \multirow{3}{*}{$\begin{array}{l}\text { October } \\
1992^{2}\end{array}$} & \multicolumn{5}{|c|}{ Yoar to Date } \\
\hline & & & & \multicolumn{3}{|c|}{ Coal Ceneration } & \multicolumn{2}{|c|}{ Share of Total (Percent) } \\
\hline & & & & $1993^{\prime}$ & $1992^{2}$ & $\begin{array}{l}\text { Difference } \\
\text { (Percent) }\end{array}$ & $1993^{\prime}$ & $1902^{2}$ \\
\hline 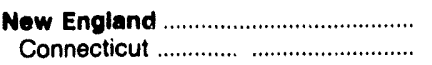 & $\begin{array}{r}1,232 \\
5\end{array}$ & $\begin{array}{r}1,411 \\
233\end{array}$ & $\begin{array}{r}1,497 \\
257\end{array}$ & $\begin{array}{r}12,566 \\
1,818\end{array}$ & $\begin{array}{r}13,539 \\
1,900\end{array}$ & $\begin{array}{l}-7.2 \\
-4.4\end{array}$ & $\begin{array}{r}18.1 \\
7.7\end{array}$ & $\begin{array}{r}19.1 \\
9.1\end{array}$ \\
\hline 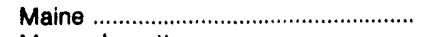 & - & -- & $-\overline{-1}$ & $\overline{-i n}$ & $-\infty$ & - & -- & - \\
\hline $\begin{array}{l}\text { Massachusetts } \\
\text { New Hampshire }\end{array}$ & $\begin{array}{l}936 \\
290\end{array}$ & $\begin{array}{l}891 \\
288\end{array}$ & $\begin{array}{r}1,027 \\
213\end{array}$ & $\begin{array}{l}8,043 \\
2,706\end{array}$ & $\begin{array}{l}9,101 \\
2,537\end{array}$ & $\begin{array}{r}-11.6 \\
6.6\end{array}$ & $\begin{array}{l}34.2 \\
22.4\end{array}$ & $\begin{array}{l}32.5 \\
22.4\end{array}$ \\
\hline 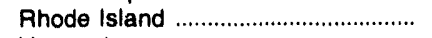 & - & -- & -- & - & $-\infty$ & - & - & - \\
\hline 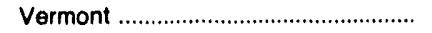 & -- & - & - & - & - & - & - & - \\
\hline Middle Atlantic . ....................................... & 10,339 & 9,620 & 10,428 & 107,003 & 111,595 & -4.1 & 41.3 & 43.3 \\
\hline 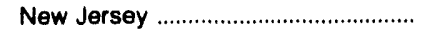 & 317 & 306 & 670 & 4,517 & 4,315 & 4.7 & 15.2 & 17.0 \\
\hline 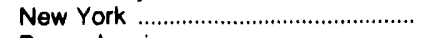 & 1,767 & 1,543 & 1,770 & 17,894 & 20,897 & -14.4 & 20.0 & 22.5 \\
\hline 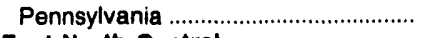 & 8,255 & 7,770 & 7,989 & 84,591 & 86,383 & -2.1 & 60.3 & 61.8 \\
\hline East North Central ................................ & 30,815 & 29,306 & 29,906 & 313,082 & 299,106 & 4.7 & 72.9 & 74.1 \\
\hline 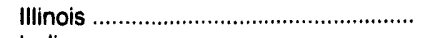 & 5,525 & 4,504 & 3,500 & 47,859 & 41,449 & 15.5 & 40.7 & 40.1 \\
\hline 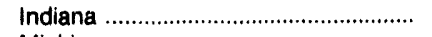 & 7,833 & 7,796 & 7,949 & 82,916 & 78,378 & 5.8 & 98.8 & 98.4 \\
\hline 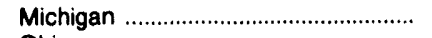 & 5,081 & 5,140 & 5,512 & 51,205 & 51,191 & • & 66.9 & 74.9 \\
\hline Ohio & 9,642 & 9,390 & 9,997 & 103,099 & 101,145 & 1.9 & 92.3 & 88.9 \\
\hline 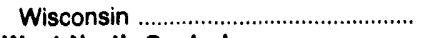 & 2,834 & 2,476 & 2,948 & 28,003 & 26,944 & 3.9 & 70.7 & 70.4 \\
\hline West North Central .............................. & 12,795 & 11,954 & 12,653 & 136,914 & 131,957 & 3.8 & 75.8 & 74.9 \\
\hline lowa & 1,761 & 1,925 & 1,749 & 22,471 & 20,734 & 8.4 & 87.0 & 84.9 \\
\hline 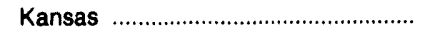 & 2,209 & 1,975 & 1,776 & $22 \mid 21$ & 18,389 & 20.3 & 73.9 & 70.0 \\
\hline Minnesota & 2,227 & 2,113 & 1,813 & $21,4 \div 3$ & 19,505 & 11.9 & 64.4 & 61.2 \\
\hline 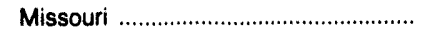 & 2,805 & 2,654 & 4,001 & 34,123 & 38,985 & -12.5 & 75.9 & 83.8 \\
\hline 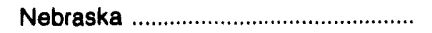 & 1,320 & 1,120 & 800 & 12,167 & 10,296 & 18.2 & 65.4 & 56.2 \\
\hline 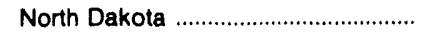 & 2,337 & 2,096 & 2,308 & 22,135 & 21,971 & .7 & 94.8 & 93.7 \\
\hline South Dakota & 136 & 70 & 205 & 2,073 & 2,077 & -.2 & 50.7 & 39.1 \\
\hline 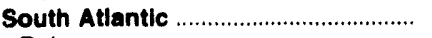 & 25,757 & 28,260 & 25,439 & 281,067 & 268,002 & 4.9 & 58.6 & 58.6 \\
\hline 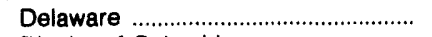 & 375 & 417 & 283 & 4,230 & 3,270 & 29.4 & 64.5 & 61.6 \\
\hline 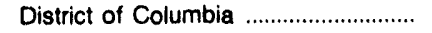 & - & - & -- & - & $-\infty$ & - & - & - \\
\hline Florida & 4,826 & 5,493 & 4,856 & 52,739 & 52,056 & 1.3 & 44.5 & 45.6 \\
\hline 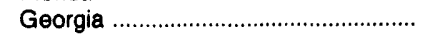 & 4,894 & 5,963 & 5,025 & 53,675 & 49,463 & 8.5 & 66.7 & 64.6 \\
\hline 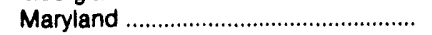 & 2,170 & 1,814 & 1,668 & 20,716 & 19,622 & 5.6 & 58.6 & 60.8 \\
\hline 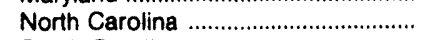 & 4,204 & 5,530 & 4,357 & 49,621 & 45,933 & 8.0 & 68.1 & 66.0 \\
\hline 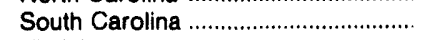 & 1,805 & 2,367 & 1.597 & 22,362 & 19,670 & 13.7 & 34.5 & 33.3 \\
\hline Virginia & $1, R B 1$ & 2,011 & 1,416 & 20,592 & 18,909 & 8.9 & 48.1 & 46.4 \\
\hline 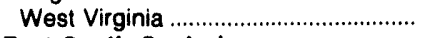 & 5,601 & 4,665 & 6,237 & 57,130 & 59,079 & -3.3 & 99.2 & 99.1 \\
\hline 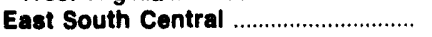 & 17,865 & 18,708 & 15,290 & 181,212 & 161,447 & 12.2 & 79.0 & 73.5 \\
\hline Alabama & 5,818 & 6,225 & 4,998 & 55,120 & 50,961 & 8.2 & 70.6 & 67.7 \\
\hline 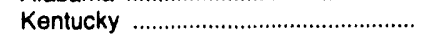 & 6.753 & 6,546 & 6,228 & 67,609 & 60,574 & 11.6 & 96.1 & 95.0 \\
\hline 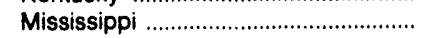 & 827 & 955 & 526 & 7,828 & 6,932 & 12.9 & 38.3 & 39.2 \\
\hline 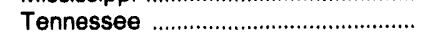 & 4,467 & 4,983 & 3,538 & 50,655 & 42,980 & 17.9 & 83.7 & 68.1 \\
\hline 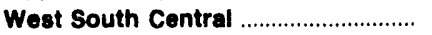 & 14,241 & 16,325 & 15,042 & 162,907 & 154,425 & 5.5 & 48.7 & 48.2 \\
\hline 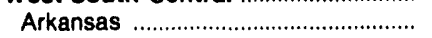 & 1,005 & 1,600 & 2,094 & 15,873 & 16,462 & -3.6 & 49.1 & 53.7 \\
\hline 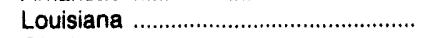 & 1,252 & 1,677 & 1,209 & 16,128 & 16,266 & -.9 & 32.3 & 34.7 \\
\hline 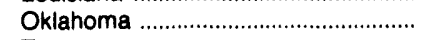 & 2,118 & 2,227 & 2,386 & 23,788 & 22,822 & 4.2 & 58.1 & 59.3 \\
\hline 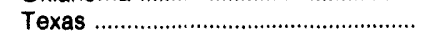 & 9,866 & 10,821 & 9,353 & 107,119 & 98,875 & 8.3 & 50.7 & 48.3 \\
\hline 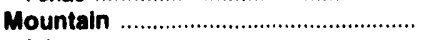 & 16,434 & 16,945 & 16,384 & 158,193 & 161,071 & -1.8 & 75.2 & 75.6 \\
\hline 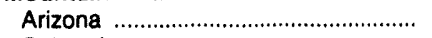 & 3,360 & 3,320 & 3,089 & 30,371 & 28,163 & 7.8 & 53.3 & 48.4 \\
\hline 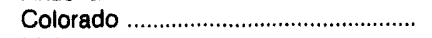 & 2,599 & 2,425 & 2,201 & 24,925 & 24,700 & .9 & 92.4 & 93.8 \\
\hline 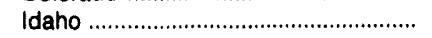 & - & -- & -- & - & - & -- & -- & -- \\
\hline 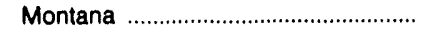 & 1,301 & 1,277 & 1,495 & 11,125 & 13,962 & -20.3 & 59.7 & 67.2 \\
\hline Nevada & 1,151 & 1,467 & 1,019 & 12,513 & 13,549 & -7.6 & 77.9 & 77.4 \\
\hline 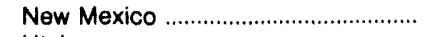 & 1,986 & 2,315 & 2,145 & 20,534 & 20,622 & -.4 & 89.2 & 90.9 \\
\hline Utah & 2,710 & 2,889 & 2,849 & 26,600 & 26,194 & 1.6 & 95.5 & 95.6 \\
\hline 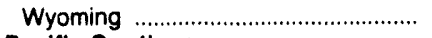 & 3,326 & 3,252 & 3,585 & 32,125 & 33,880 & -5.2 & 97.7 & 98.2 \\
\hline Paciflc Contiguous ................................ & 1,318 & 1,304 & 1,271 & 8,528 & 10,752 & -11.4 & 4.6 & 5.3 \\
\hline 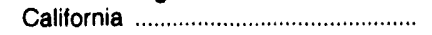 & - & $-\infty$ & -- & - & -- & -- & $-\infty$ & -- \\
\hline Oregon & 377 & 360 & 366 & 2,640 & 2,945 & -10.4 & 7.7 & 8.5 \\
\hline Washington & 942 & 944 & 905 & 6,888 & 7,806 & -11.8 & 9.9 & 11.3 \\
\hline Pacific Noncontiguous ....................... & 30 & 23 & 29 & 263 & 233 & 13.0 & 3.0 & 2.5 \\
\hline Alaska & 30 & 23 & 29 & 263 & 233 & 13.0 & 7.1 & 7.0 \\
\hline 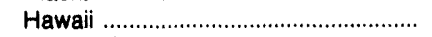 & - & - & - & -- & - & - & - & $-\infty$ \\
\hline 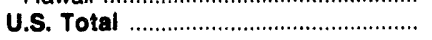 & 130,926 & 133,856 & 127,940 & $1,362,736$ & $1,312,126$ & 3.9 & 58.5 & 56.3 \\
\hline
\end{tabular}

Data for 1993 are preliminary.

Data for 1992 are final.

- For detailed data, the absolute value is less than 0.5 ; for percentage calculations, the absolute value is less than 0.05 percent

Notes: -Negative generation denotes that electric power consumed for plant use exceeds gross generation. - Totals may not equal sum of compo. nents because of independent rounding. •Percent difference is calculated before rounding. $\bullet$ Coal includes lignite, bituminous coal, subbituminous coal, and anthracite

Source: Energy Information Administration, Form EIA-759, "Monthly Power Plant Report." 
Table 8. Electric Utility Net Generation from Petroleum by Census Division and State (Million Kilowatthours)

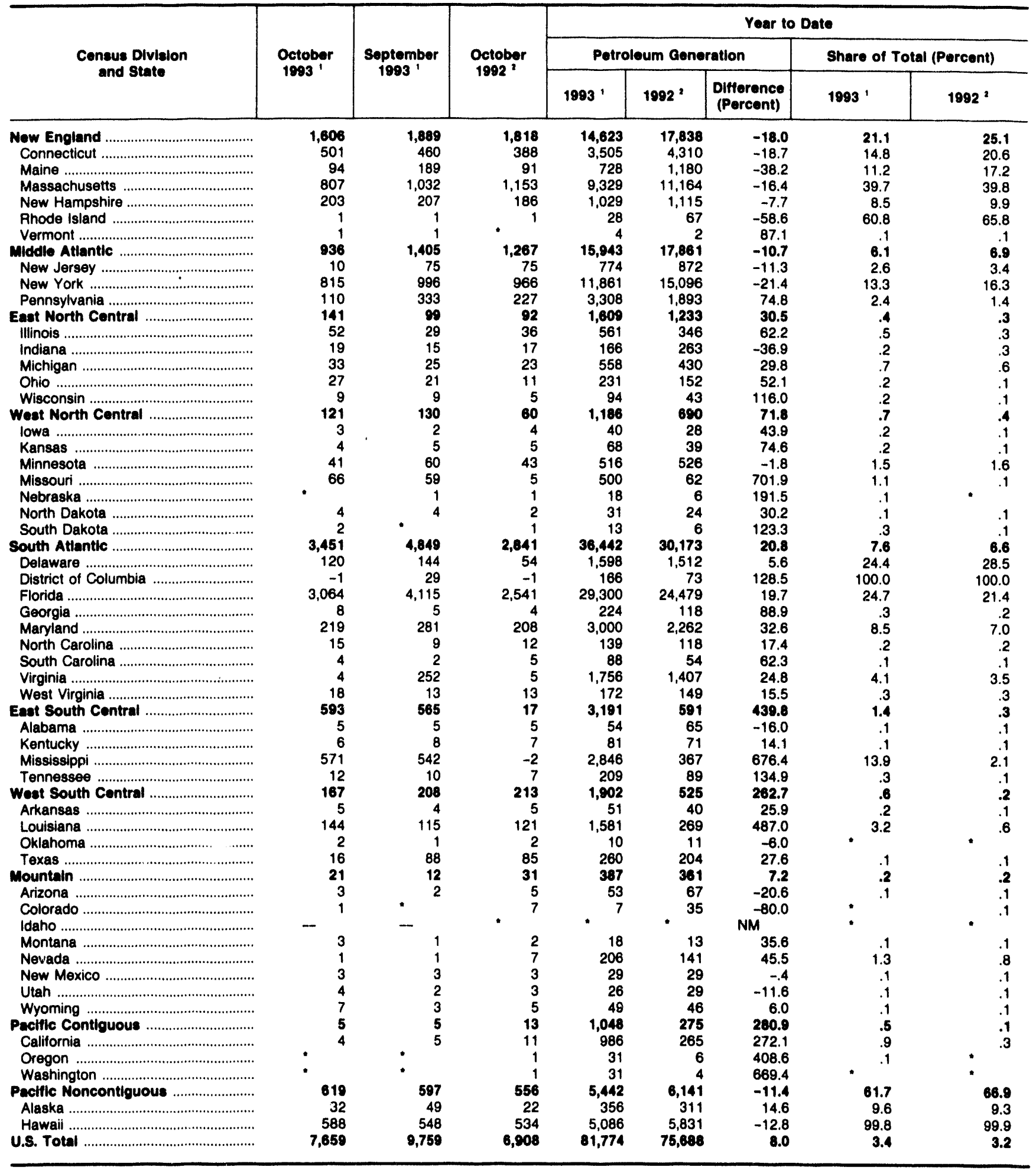

Data for 1993 are preliminary.

Data for 1992 are final.

- For detailed data, the absolute value is less than 0.5 ; for percentage calculations, the absolute value is less than 0.05 percent.

- Calculation not meaningful.

Notes: -Negative generation denotes that electric power consumed for plant use exceeds gross generation. •Totals may not equal sum of components because of independent rounding. •Percent difference is calculated before rounding. $\bullet$ Includes fuel oil Nos. 2, 4, 5, and 6, crude oil, kerosene, and petroleum coke.

Source: Energy Information Administration, Form EIA-759, "Monthly Power Plant Peport." 
Table 9. Electric Utility Steam Net Generation from Petroleum by Census Division and State (Million Kilowatthours)

\begin{tabular}{|c|c|c|c|c|c|c|c|c|}
\hline \multirow{3}{*}{$\begin{array}{l}\text { Census Division } \\
\text { and State }\end{array}$} & \multirow{3}{*}{$\begin{array}{l}\text { October } \\
1993^{1}\end{array}$} & \multirow{3}{*}{$\begin{array}{l}\text { September } \\
1993\end{array}$} & \multirow{3}{*}{$\begin{array}{l}\text { October } \\
1992^{2}\end{array}$} & \multicolumn{5}{|c|}{ Year to Date } \\
\hline & & & & \multicolumn{3}{|c|}{ Petroleum (Steam) } & \multicolumn{2}{|c|}{ Share of Total (Percent) } \\
\hline & & & & $1993^{\prime}$ & $1992^{2}$ & $\begin{array}{l}\text { Difference } \\
\text { (Percent) }\end{array}$ & $1993^{\prime}$ & $1992^{2}$ \\
\hline 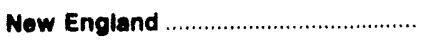 & 1,591 & 1,872 & 1,809 & 14,448 & 17,657 & -18.2 & 20.9 & 24.9 \\
\hline 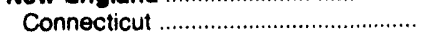 & 500 & 459 & 388 & 3,495 & 4,301 & -18.7 & 14.8 & 20.6 \\
\hline 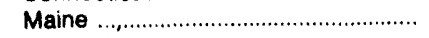 & 94 & 188 & 91 & 726 & 1,179 & -38.4 & 11.2 & 17.2 \\
\hline 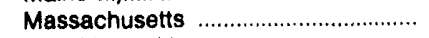 & 794 & 1,018 & 1,145 & 9,178 & 11,002 & -16.6 & 39.1 & 39.2 \\
\hline 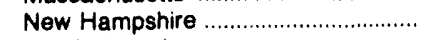 & 203 & 207 & 186 & 1,029 & 1,115 & -7.7 & 8.5 & 9.9 \\
\hline Rhode Island & - & -- & $\cdot$ & 20 & 60 & -67.2 & 43.2 & 59.0 \\
\hline 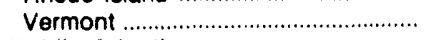 & $\cdot$ & -- & - & $\cdot$ & 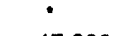 & NM & $*$ & - \\
\hline Middle Atlantic & 927 & 1,370 & 1,262 & 15,588 & 17,622 & -11.5 & 6.0 & 6.8 \\
\hline 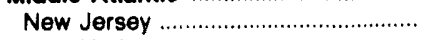 & 7 & 68 & 72 & 662 & 777 & -14.8 & 2.2 & 3.1 \\
\hline 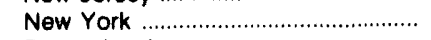 & 810 & 975 & 963 & 11,699 & 14,997 & -22.0 & 13.1 & 16.2 \\
\hline 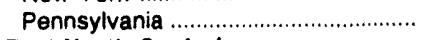 & 109 & 326 & 227 & 3,227 & 1,848 & 74.6 & 2.3 & 1.3 \\
\hline East North Central ................................ & 125 & 90 & 91 & 1,520 & 1,214 & 25.2 & .4 & .3 \\
\hline 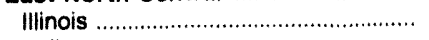 & 52 & 28 & 36 & 545 & 341 & 59.9 & .5 & .3 \\
\hline 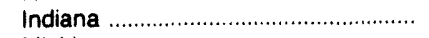 & 11 & 11 & 17 & 148 & 261 & -43.3 & .2 & .3 \\
\hline 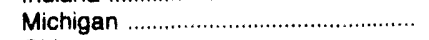 & 32 & 24 & 23 & 542 & 424 & 28.0 & .7 & .6 \\
\hline Ohio & 25 & 18 & 11 & 206 & 146 & 41.0 & .2 & .1 \\
\hline 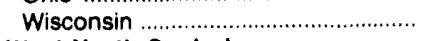 & 6 & 9 & 5 & 79 & 42 & 87.6 & .2 & .1 \\
\hline West North Central ............................. & 118 & 125 & 55 & 1,077 & 656 & 64.3 & .6 & .4 \\
\hline 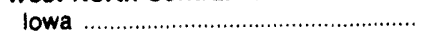 & 3 & 1 & 2 & 21 & 21 & -.8 & .1 & 1 \\
\hline 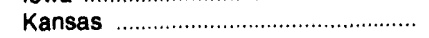 & 3 & 3 & 4 & 46 & 24 & 95.2 & .2 & .1 \\
\hline 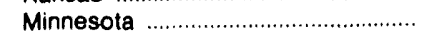 & 41 & 59 & 43 & 507 & 523 & -3.1 & 1.5 & 1.6 \\
\hline 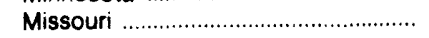 & 66 & 58 & 5 & 452 & 57 & 687.6 & 1.0 & .1 \\
\hline 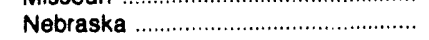 & - & 1 & - & 14 & 3 & 343.6 & .1 & * \\
\hline 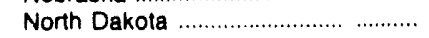 & 4 & 3 & 2 & 30 & 24 & 28.2 & .1 & .1 \\
\hline 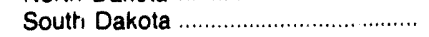 & 1 & $\cdot$ & & 6 & 3 & 75.1 & .1 & .1 \\
\hline 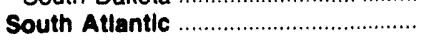 & 3,423 & 4,767 & 2,797 & 35,683 & 29,541 & 20.3 & 7.4 & 6.5 \\
\hline Delaware & 120 & 142 & 53 & 1,585 & 1,507 & 5.2 & 24.2 & 28.4 \\
\hline 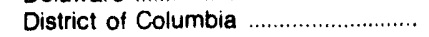 & -1 & 28 & -1 & 162 & 59 & 174.9 & 97.8 & 81.3 \\
\hline Florida & 3,044 & 4,054 & 2,501 & 28,832 & 24,028 & 20.0 & 24.3 & 21.0 \\
\hline 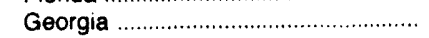 & 4 & 3 & 4 & 132 & 85 & 54.3 & .2 & 1 \\
\hline 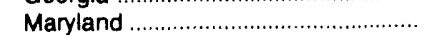 & 218 & 274 & 207 & 2,902 & 2,225 & 30.4 & 8.2 & 6.9 \\
\hline 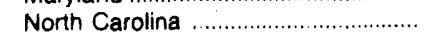 & 13 & 7 & 11 & 105 & 100 & 5.6 & .1 & .1 \\
\hline 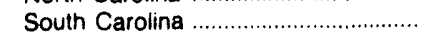 & 3 & 1 & 5 & 66 & 49 & 35.0 & .1 & 1 \\
\hline Virginia & 4 & 246 & 4 & 1,727 & 1,340 & 28.9 & 4.0 & 3.3 \\
\hline 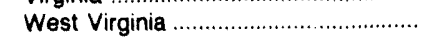 & 18 & 13 & 13 & 172 & 149 & 15.5 & .3 & 3 \\
\hline 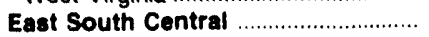 & 588 & 561 & 13 & 3,037 & $\mathbf{5 5 0}$ & 452.5 & 1.3 & .3 \\
\hline Alabama & 4 & 4 & 5 & 51 & 54 & -5.6 & .1 & .1 \\
\hline 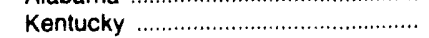 & 6 & 8 & 7 & 79 & 70 & 12.6 & .1 & .1 \\
\hline 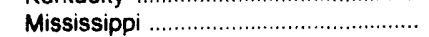 & 571 & 542 & -2 & 2,837 & 367 & 673.9 & 13.9 & 2.1 \\
\hline Tennessee & 7 & 7 & 3 & 70 & 59 & 19.1 & .1 & .1 \\
\hline 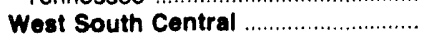 & 165 & 206 & 211 & 1,884 & 513 & 267.2 & 6 & .2 \\
\hline 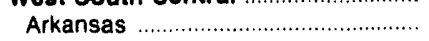 & 5 & 3 & 5 & 41 & 40 & 2.2 & .1 & .1 \\
\hline Louisiana & 144 & 115 & 121 & 1,581 & 272 & 481.4 & 3.2 & 6 \\
\hline 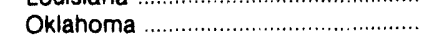 & 2 & 1 & 2 & 9 & 10 & -9.8 & - & - \\
\hline Texas & 15 & 87 & 83 & 252 & 191 & 32.2 & .1 & .1 \\
\hline 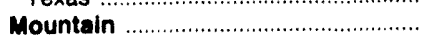 & 21 & 12 & 30 & 381 & 342 & 11.4 & .2 & .2 \\
\hline Arizona & 2 & 2 & 4 & 52 & 62 & -16.0 & .1 & .1 \\
\hline 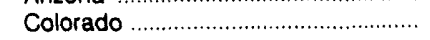 & 1 & - & 6 & 8 & 32 & -76.7 & $\cdot$ & 1 \\
\hline 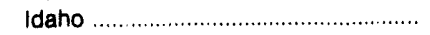 & - & - & -- & -- & -- & -- & -- & -- \\
\hline Montana & 3 & 1 & 2 & 18 & 13 & 36.2 & .1 & .1 \\
\hline 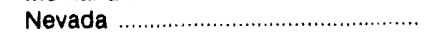 & 1 & 1 & 7 & 203 & 135 & 51.0 & 1.3 & .8 \\
\hline New Mexico ........................................ & 3 & 3 & 3 & 28 & 28 & -.1 & .1 & .1 \\
\hline Utah & 4 & 2 & 3 & 23 & 26 & -9.1 & .1 & .1 \\
\hline 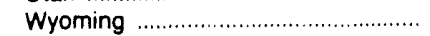 & 7 & 3 & 5 & 49 & 46 & 6.0 & .1 & .1 \\
\hline 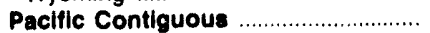 & 1 & 1 & 10 & 959 & 228 & 320.2 & .5 & .1 \\
\hline California & 1 & 1 & 9 & 948 & 219 & 331.8 & 9 & .2 \\
\hline Oregon & $\cdot$ & $\cdot$ & 1 & 4 & 6 & -21.6 & $"$ & • \\
\hline Washington & $*$ & - & - & 7 & 3 & 119.7 & $\cdot$ & $\cdot$ \\
\hline 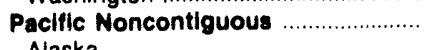 & .490 & .445 & . 439 & 4,165 & 4,961 & $\mathrm{NM}^{-16.0}$ & .47 .3 & 54.1 \\
\hline Alaska & 490 & 445 & 439 & 4,165 & $\begin{array}{r}1 \\
4,960\end{array}$ & $\begin{array}{l}\text { NM } \\
-16.0\end{array}$ & 81.7 & 85.0 \\
\hline & 7,450 & 9,449 & 6,718 & 78,742 & 73,284 & 7.4 & 3.3 & 3.1 \\
\hline
\end{tabular}

Data for 1993 are preliminary.

- Data for 1992 are final.

- = For detailed data, the absolute value is less than 0.5 ; for percentage calculations, the absolute value is less than 0.05 percent

- Calculation not meaningful.

Notes: - Negative generation denotes that electric power consumed for plant use exceeds gross generation. $\bullet$ Totals may not equal sum of compo. nents because of independent rounding. Percent difference is calculated before rounding. $\bullet$ includes fuel oil Nos. 2, 4, 5, and 6, crude oil, and kerosene.

Source: Energy Information Administration, Form ElA-759, "Monthly Power Plant Report." 
Table 10. Electric Utility GT/IC Net Generation from Petroleum by Census Division and State (Million Kilowatthours)

\begin{tabular}{|c|c|c|c|c|c|c|c|c|}
\hline \multirow{3}{*}{$\begin{array}{l}\text { Consus Division } \\
\text { and State }\end{array}$} & \multirow{3}{*}{$\begin{array}{l}\text { October } \\
1993^{\prime}\end{array}$} & \multirow{3}{*}{$\begin{array}{l}\text { September } \\
1993\end{array}$} & \multirow{3}{*}{$\begin{array}{l}\text { October } \\
1992^{2}\end{array}$} & \multicolumn{5}{|c|}{ Year to Date } \\
\hline & & & & \multicolumn{3}{|c|}{ Petroleum (GT/IC) } & \multicolumn{2}{|c|}{ Share of Total (Percent) } \\
\hline & & & & $1993^{\prime}$ & $1992^{2}$ & $\begin{array}{l}\text { Difference } \\
\text { (Percent) }\end{array}$ & 1993 ' & $1992^{2}$ \\
\hline Now Enguand ................... & 15 & 17 & 9 & 175 & 180 & -3.1 & 0.3 & 0.3 \\
\hline $\begin{array}{l}\text { Connecticut } \\
\text { Maine }\end{array}$ & : & 1 & : & 9 & 8 & 10.3 & : & • \\
\hline 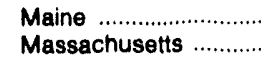 & 13 & 1 & • & 2 & 1 & 288.2 & & \\
\hline $\begin{array}{l}\text { Massachusetts ................. } \\
\text { New Hampshire .......... }\end{array}$ & . 13 & - 14 & 8 & ${ }^{151}$ & $\begin{array}{r}162 \\
1\end{array}$ & $\mathrm{NM}^{-6.5}$ & . & .6 \\
\hline & 1 & 1 & 1 & 8 & 7 & 15.6 & 17.7 & 6.8 \\
\hline Vermont ......................... & 1 & 1 & - & 4 & 2 & 101.5 & .1 & - \\
\hline Middlo Atlantic .............. & 8 & 35 & 6 & 355 & 239 & 48.9 & .1 & .1 \\
\hline New Jersey ...................... & 3 & 7 & 3 & 112 & 94 & 18.1 & .4 & .4 \\
\hline New York ......................... & 4 & 21 & 3 & 163 & 99 & 63.8 & .2 & .1 \\
\hline Pennsylvania ................ & 1 & 7 & • & 81 & 45 & 80.6 & .1 & • \\
\hline East North Contral ..... & 16 & 9 & 1 & 89 & 19 & 363.1 & " & - \\
\hline 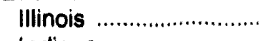 & 1 & 1 & 1 & 16 & 5 & 213.9 & : & - \\
\hline Indiana ............................. & 8 & 4 & * & 18 & 1 & 1449.8 & • & - \\
\hline 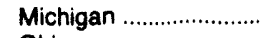 & 2 & 1 & • & 15 & 6 & 159.9 & : & - \\
\hline Ohio & 2 & 3 & 1 & 25 & 6 & 329.8 & •" & - \\
\hline Wisconsin ........................ & 3 & • & • & 14 & 1 & 1278.5 & - & - \\
\hline West North Central .... & 2 & 5 & 4 & 109 & 35 & 214.4 & .1 & * \\
\hline lowa ................................ & 1 & 1 & 2 & 19 & 7 & 189.0 & .1 & - \\
\hline Kansas ……....................... & 1 & 2 & 1 & 22 & 15 & 42.6 & .1 & .1 \\
\hline Minnesota ....................... & " & 1 & 1 & 9 & 3 & 261.1 & • & - \\
\hline Missouri ............................ & • & 1 & • & 48 & 5 & 867.6 & .1 & • \\
\hline 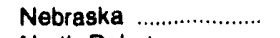 & : & • & 1 & 3 & 3 & 21.0 & * & - \\
\hline North Dakota ................ & : & • & - & 1 & • & NM & • & • \\
\hline South Dakota .................. & • & • & - & 7 & 2 & 193.9 & .2 & • \\
\hline South Atlantic ................. & 28 & 82 & 43 & 759 & 631 & 20.3 & .2 & .1 \\
\hline Delaware ............................... & • & 2 & * & 13 & 6 & 123.2 & .2 & .1 \\
\hline District of Columbia ... & • & 1 & • & 4 & 14 & -73.0 & 2.2 & 18.7 \\
\hline Florida & 21 & 61 & 40 & 468 & 451 & 3.7 & .4 & .4 \\
\hline Georgia ........................... & 4 & 1 & • & 92 & 33 & 179.1 & .1 & - \\
\hline Maryland ......................... & 1 & 7 & 1 & 98 & 37 & 164.8 & .3 & .1 \\
\hline North Carolina ................. & 1 & 2 & 1 & 34 & 19 & 79.9 & • & - \\
\hline South Carolina ............ & • & • & - & 22 & 5 & 327.4 & • & \\
\hline Virginia & : & 7 & 1 & 29 & 67 & -56.1 & .1 & .2 \\
\hline West Virginia ................. & • & • & • & - & • & NM & • & •" \\
\hline East South Central ..... & 5 & 3 & 4 & 154 & 41 & 271.4 & .1 & * \\
\hline Alabama & : & 1 & 1 & 3 & 11 & -69.7 & : & • \\
\hline 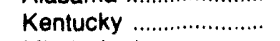 & $\cdot$ & • & • & 1 & • & NM & • & - \\
\hline Mississippi ........................ & • & - & -- & 10 & - & NM & - & • \\
\hline Tennessee ...................... & 5 & 3 & 3 & 140 & 31 & 357.1 & .2 & • \\
\hline West South Central ... & 2 & 2 & 2 & 19 & 12 & 62.0 & • & - \\
\hline Arkansas ......................... & ; & & : & 10 & • & NM & : & : \\
\hline Louisiana ........................ & : & : & : & •" & -3 & NM & : & - \\
\hline Oklahoma ......................... & • & • & - & 1 & 1 & 43.1 & - & - \\
\hline Texas & 1 & 1 & 2 & 8 & 13 & -39.7 & - & • \\
\hline Mountain ............................ & • & •" & 1 & 6 & 19 & -67.4 & • & - \\
\hline Arizona & - & - & • & 1 & 5 & -73.2 & • & - \\
\hline Colorado ........................... & • & • & 1 & : & 3 & NM & • & - \\
\hline Idaho & - & -- & : & : & : & NM & : & : \\
\hline Montana ............................. & : & : & : & •" & • & NM & : & : \\
\hline 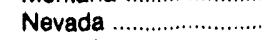 & : & : & : & 2 & 7 & -65.8 & : & * \\
\hline New Mexico ..................... & : & " & * & • & - & NM & • & • \\
\hline Utah .......................................... & $\cdot$ & • & • & 3 & 4 & -29.6 & * & • \\
\hline Wyoming ............................ & -- & -- & -- & -- & • & NM & $=$ & • \\
\hline Pacific Contiguous ..... & 3 & 1 & 3 & 88 & 47 & 89.2 & $"$ & • \\
\hline California & 3 & 4 & 2 & 38 & 45 & -16.1 & • & - \\
\hline Oregon ....…………....... & - & • & - & 26 & • & NM & .1 & - \\
\hline 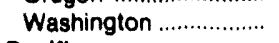 & - & " & • & 24 & 1 & 2615.6 & • & - \\
\hline Pacific & & & & & & & & \\
\hline Noncontiguous ........ & 129 & 152 & 117 & 1,277 & 1,180 & 8.2 & 14.5 & 12.9 \\
\hline Alaska & 32 & 49 & 22 & 356 & 310 & 14.7 & 9.6 & 9.3 \\
\hline Hawaii .............................. & 98 & 103 & 95 & 921 & 870 & 5.9 & 18.1 & 14.9 \\
\hline U.S. Total ................. & 209 & 309 & 190 & 3,032 & 2,404 & 26.1 & .1 & .1 \\
\hline
\end{tabular}

Data for 1993 are preliminary.

2 Data for 1992 are final.

- For detailed data, the absolute value is less than 0.5 ; for percentage calculations, the absolute value is less than 0.05 percent

= Calculation not meaningful.

Notes: $\bullet G T / I C=$ Gas Turbine/Internal Combustion. - Negative generation denotes that electric power consumed for plant use exceeds gross generation. -Totals may not equal sum of components because of independent rounding. • Percent difference is calculated before rounding. $\bullet$ Includes fuel oil Nos. $2,4,5$, and 6, crude oil, and kerosene.

Source: Energy Information Administration, Form ElA-759, "Monthly Power Plant Report." 
Table 11. Electric Utility Net Generation from Gas by Census Division and State (Million Kilowatthours)

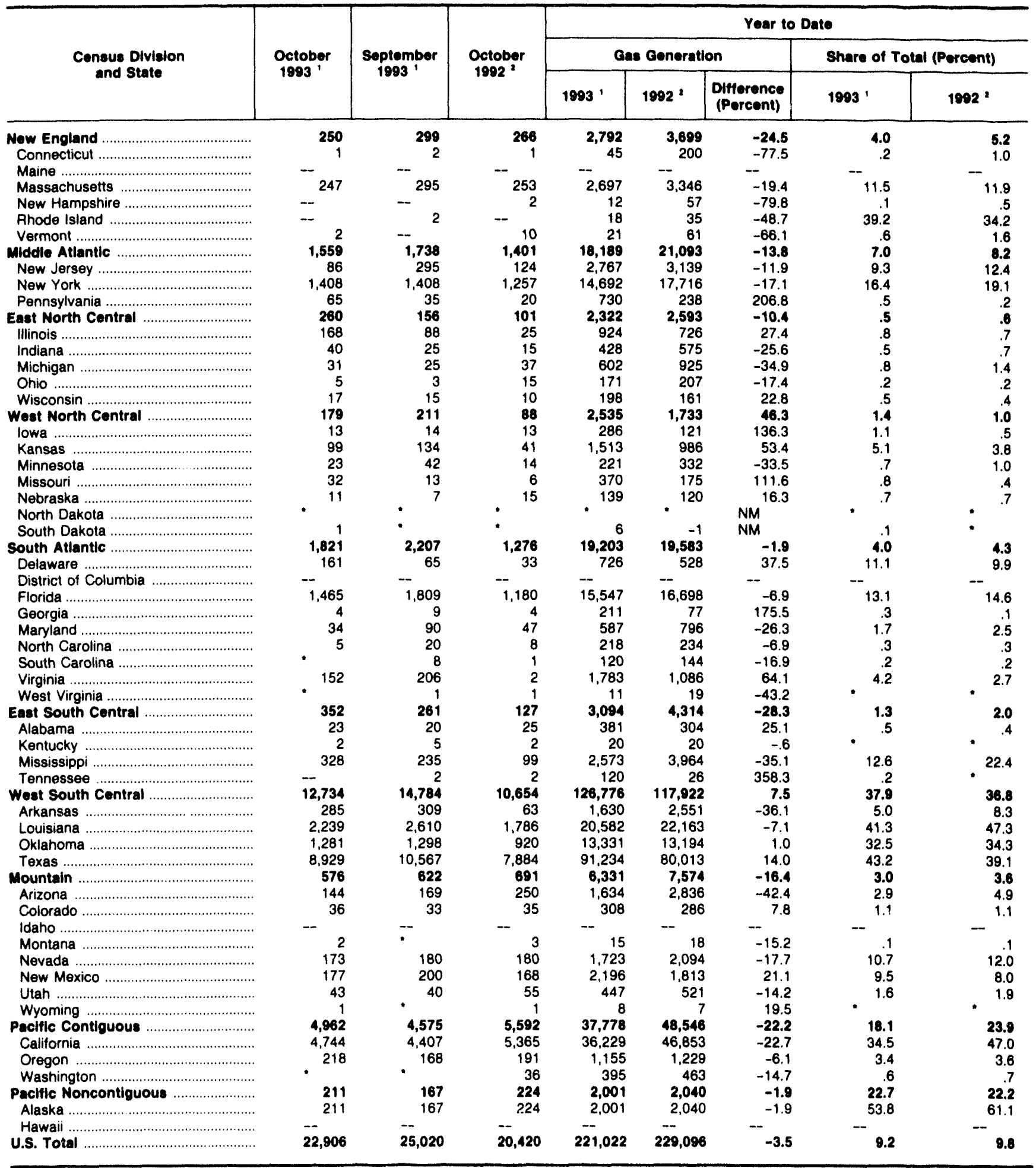

Data for 1993 are preliminary.

- Data for 1992 are final.

- For detailed data, the absolute value is less than 0.5 ; for percentage calculations, the absolute value is less than 0.05 percent.

= Calculation not meaningful.

Notes: -Negative generation denotes that electric power consumed for plant use exceeds gross generation. - Totals may not equal sum of components because of independent rounding. $\bullet$ Percent difference is calculated betore rounding

Source: Energy Information Administration, Form ElA.759, "Monthly Power Plant Report." 
Table 12. Electric Utility Steam Net Generation from Gas by Census Division and State (Million Kilowatthours)

\begin{tabular}{|c|c|c|c|c|c|c|c|c|}
\hline \multirow{3}{*}{$\begin{array}{c}\text { Census Division } \\
\text { and State }\end{array}$} & \multirow{3}{*}{$\begin{array}{l}\text { October } \\
1993\end{array}$} & \multirow{3}{*}{$\begin{array}{c}\text { September } \\
1993\end{array}$} & \multirow{3}{*}{$\begin{array}{l}\text { October } \\
1092^{2}\end{array}$} & \multicolumn{5}{|c|}{ Year to Data } \\
\hline & & & & \multicolumn{3}{|c|}{ Cas (8toam) } & \multicolumn{2}{|c|}{ Shere of Toted (Pereent) } \\
\hline & & & & $1993^{\prime}$ & $1002^{2}$ & $\begin{array}{l}\text { Ditiorence } \\
\text { (Porcent) }\end{array}$ & $1003^{\prime}$ & $1622^{\prime}$ \\
\hline 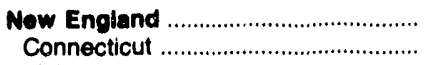 & $\begin{array}{r}233 \\
1\end{array}$ & $\begin{array}{r}242 \\
2\end{array}$ & $\begin{array}{r}249 \\
1\end{array}$ & $\begin{array}{r}2,530 \\
45\end{array}$ & $\begin{array}{r}3,381 \\
200\end{array}$ & $\begin{array}{l}-26.2 \\
-77.5\end{array}$ & $\begin{array}{r}3.7 \\
.2\end{array}$ & $\begin{array}{l}4.8 \\
1.0\end{array}$ \\
\hline 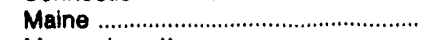 & $-\infty$ & - & - & - & - & - & - & - \\
\hline 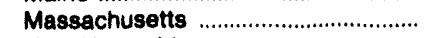 & 230 & 238 & 235 & 2,435 & 3,028 & -19.6 & 10.4 & 10.8 \\
\hline New Hampshire ..................................... & - & - & 2 & 12 & 57 & -79.8 & .1 & .5 \\
\hline 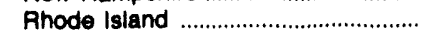 & -- & 2 & $-\infty$ & 18 & 35 & -48.7 & 39.2 & 34.2 \\
\hline 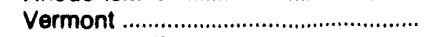 & 2 & - & 10 & 21 & 61 & -68.1 & .6 & 1.6 \\
\hline Middle Atlantic .......................................... & 1,487 & 1,604 & 1,345 & 17,144 & 20,310 & -15.6 & 8.6 & 7.0 \\
\hline $\begin{array}{l}\text { New Jersey } \\
\text { New York }\end{array}$ & & $\begin{array}{r}209 \\
1,363\end{array}$ & $\begin{array}{r}71 \\
1,254\end{array}$ & $\begin{array}{r}2,052 \\
14,391\end{array}$ & $\begin{array}{r}2,569 \\
17,524\end{array}$ & $\begin{array}{l}-20.1 \\
-17.8\end{array}$ & 6.9 & 10.1 \\
\hline $\begin{array}{l}\text { New York } \\
\text { Pennsylvania }\end{array}$ & $\begin{array}{r}1,402 \\
63\end{array}$ & 32 & $\begin{array}{r}1,204 \\
20\end{array}$ & $\begin{array}{r}14,391 \\
701\end{array}$ & $\begin{array}{r}17.524 \\
218\end{array}$ & $\begin{array}{l}-77.8 \\
221.8\end{array}$ & $\begin{array}{r}16.1 \\
5\end{array}$ & 18.9 \\
\hline East North Central .................................. & 243 & 149 & 86 & 2,075 & 2,320 & -10.8 & .0 & .2 \\
\hline 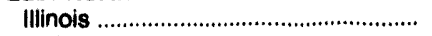 & 168 & 87 & 24 & 906 & 710 & 27.6 & .8 & .7 \\
\hline 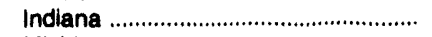 & 30 & 21 & 14 & 364 & 559 & -34.9 & .4 & .7 \\
\hline 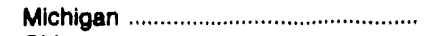 & 29 & 24 & 25 & 530 & 726 & -27.0 & .7 & 1.1 \\
\hline Ohio & 2 & 2 & 14 & 101 & 183 & -44.9 & .1 & .2 \\
\hline 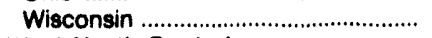 & 15 & 15 & 9 & 174 & 148 & 17.8 & .4 & 4 \\
\hline Weat North Central ................................. & 160 & 186 & 71 & 2,064 & 1,446 & 42.8 & 1.1 & ه \\
\hline lowa & 13 & 14 & 9 & 206 & 96 & 113.4 & .8 & 4 \\
\hline 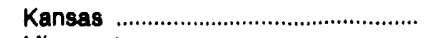 & 87 & 116 & 30 & 1,276 & 823 & $\mathbf{5 5 . 0}$ & 4.3 & 3.1 \\
\hline 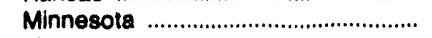 & 22 & 42 & 14 & 213 & 302 & -29.5 & .6 & .8 \\
\hline 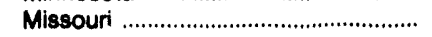 & 31 & 8 & 5 & 268 & 129 & 109.3 & 6 & .3 \\
\hline 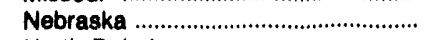 & $?$ & 6 & 13 & 97 & 98 & -.7 & .5 & .5 \\
\hline 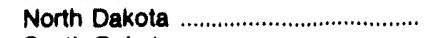 & -- & - & -- & $\cdot$ & & NM & - & - \\
\hline South Dakota & $\cdot$ & & 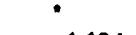 & 3 & -1 & NM & .1 & $\bullet$ \\
\hline 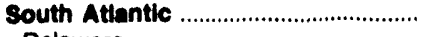 & 1,454 & 1,751 & 1,164 & 15,267 & 17,224 & -11.4 & 3.2 & 3.8 \\
\hline 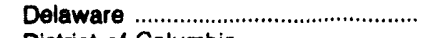 & 31 & 6 & 12 & 158 & 321 & -50.8 & 2.4 & 6.1 \\
\hline 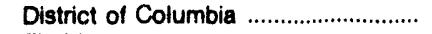 & - & - & - & - & - & - & - & - \\
\hline Florida & 1,406 & 1,665 & 1,117 & 14,504 & 15,937 & -8.0 & 12.2 & 14.0 \\
\hline 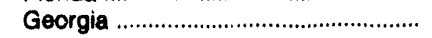 & 2 & $\theta$ & 3 & 183 & 72 & 155.2 & .2 & .1 \\
\hline 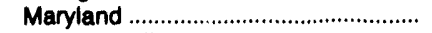 & 10 & 57 & 30 & 305 & 596 & -48.8 & .9 & 1.8 \\
\hline 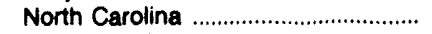 & 1 & 5 & 1 & 49 & 63 & -21.6 & .1 & .1 \\
\hline 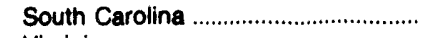 & - & 1 & : & 24 & 103 & -76.9 & - & .2 \\
\hline 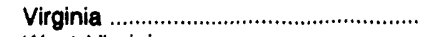 & 5 & 8 & - & 33 & 113 & -70.9 & .1 & .3 \\
\hline 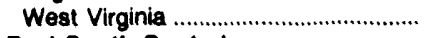 & & 1 & 1 & 11 & 18 & -43.2 & - & - \\
\hline Eest South Central .................................. & 301 & 200 & 88 & 2,327 & 3,705 & -37.2 & 1.0 & 1.7 \\
\hline 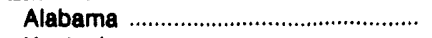 & 22 & 20 & 22 & 246 & 260 & -5.6 & .3 & .3 \\
\hline 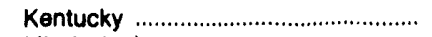 & 2 & 5 & 1 & 17 & 18 & -5.8 & $\cdot$ & $\cdot$ \\
\hline 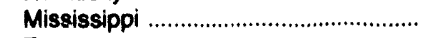 & 277 & 184 & 62 & 2,065 & 3,427 & -39.7 & 10.1 & 19.4 \\
\hline 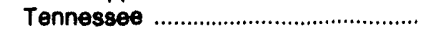 & -- & - & - & - & - & NM & - & - \\
\hline 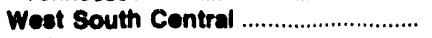 & 12,218 & 14,169 & 10,075 & 121,647 & 112,667 & 8.0 & 38.4 & 35.2 \\
\hline 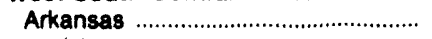 & 285 & 309 & 63 & 1,630 & 2,551 & $-36,1$ & 5.0 & 8.3 \\
\hline 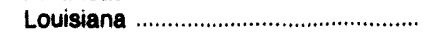 & 2,207 & 2,553 & 1,738 & 20,023 & 21,703 & -7.7 & 40.2 & 48.3 \\
\hline 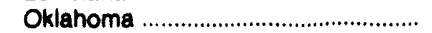 & 1,026 & 1,097 & 803 & 11,047 & 10,782 & 2.5 & 27.0 & 28.0 \\
\hline Texas & 8,700 & 10,210 & 7,472 & 88,947 & 77,621 & 14.6 & 42.1 & 37.8 \\
\hline 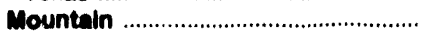 & 458 & 470 & 590 & 5,027 & 8,512 & -22.8 & 2.4 & 3.1 \\
\hline 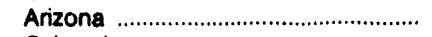 & 59 & 67 & 183 & 774 & 2,017 & -61.6 & 1.4 & 3.5 \\
\hline 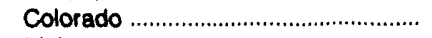 & 34 & 32 & 32 & 277 & 251 & 10.2 & 1.0 & 1.0 \\
\hline 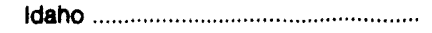 & - & - & - & - & - & - & - & - \\
\hline 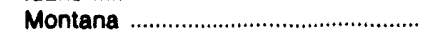 & 1 & • & $\cdot$ & 7 & 13 & -49.2 & - & .1 \\
\hline 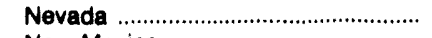 & 155 & 139 & 177 & 1,458 & 2,012 & -27.5 & 9.1 & 11.5 \\
\hline 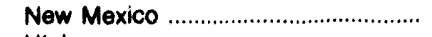 & 176 & 197 & 166 & 2,163 & 1,791 & 20.8 & 9.4 & 7.8 \\
\hline 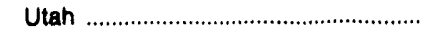 & 33 & 35 & 39 & 341 & 422 & -19.1 & 1.2 & 1.5 \\
\hline 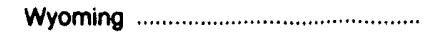 & 1 & & 1 & 8 & 7 & 18.5 & • & - \\
\hline Pectific Contiguous ................................. & 4,479 & 4,244 & 5,159 & 35,200 & 45,407 & -22.5 & 18.0 & 22.3 \\
\hline 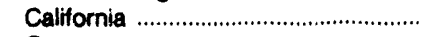 & 4,479 & 4,244 & 5,159 & 35,208 & 45,402 & -22.5 & 33.5 & 45.6 \\
\hline 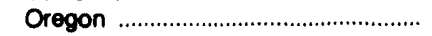 & - & - & - & - & - & $\mathbf{N M}$ & - & - \\
\hline 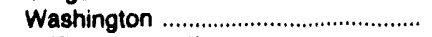 & - & " & - & 1 & 5 & -81.9 & $\cdot$ & - \\
\hline Pactile Noncontiguous ........................ & 36 & 18 & 35 & 331 & 330 & .3 & 3.8 & 3.0 \\
\hline 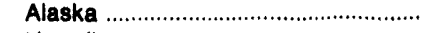 & 36 & 18 & 35 & 331 & 330 & .3 & 8.9 & 9.9 \\
\hline 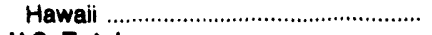 & - & - & - & - & - & - & - & - \\
\hline 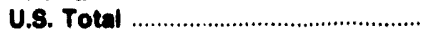 & 21,071 & 23,041 & 18,860 & 203,620 & 213,290 & -4.6 & 8.4 & 9.1 \\
\hline
\end{tabular}

- Data for 1983 are preliminary.

- Data for 1892 are final.

- - For detailed data, the absolute value is less than 0.5 ; for percentage calculations, the absolute value is lese than 0.05 percent.

= Calculation not meaningtul.

Notes: -Negative generation denotes that electric power consumed for plant use exceeds gross generation. -Totale may not equal eum of components because of independent rounding. PPercent difference is calculated belore rounding.

Source: Energy Information Administration, Form EIA-759, "Monthly Power Plant Report." 
Table 13. Electric Utility GT/IC Net Generation from Gas by Census Division and State (Million Kilowatthours)

\begin{tabular}{|c|c|c|c|c|c|c|c|c|}
\hline \multirow{3}{*}{$\begin{array}{c}\text { Census Division } \\
\text { and State }\end{array}$} & \multirow{3}{*}{$\begin{array}{l}\text { October } \\
1993^{\prime}\end{array}$} & \multirow{3}{*}{$\begin{array}{l}\text { September } \\
1993\end{array}$} & \multirow{3}{*}{$\begin{array}{l}\text { October } \\
1992^{2}\end{array}$} & \multicolumn{5}{|c|}{ Year to Date } \\
\hline & & & & \multicolumn{3}{|c|}{ Gas (GT/IC) } & \multicolumn{2}{|c|}{ Share of Total (Percent) } \\
\hline & & & & $1993^{\prime}$ & $1992^{2}$ & $\begin{array}{c}\text { Difference } \\
\text { (Percent) }\end{array}$ & $1893^{\prime}$ & $1992^{\prime}$ \\
\hline 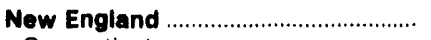 & 17 & 57 & 17 & 262 & 318 & -17.5 & 0.4 & 0.4 \\
\hline 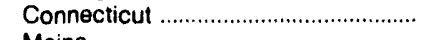 & -- & -- & -- & -- & -- & -- & - & -- \\
\hline Maine & $\rightarrow$ & -- & -- & $-\cdots$ & - & $\cdots$ & -- & -- \\
\hline 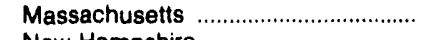 & 17 & 57 & 17 & 262 & 318 & -17.5 & 1.1 & 1.1 \\
\hline 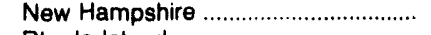 & -- & - & -- & -- & -- & NM & -- & -- \\
\hline Rhode Island & - & - & -- & -- & -- & -- & -- & \\
\hline 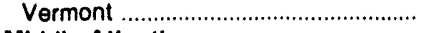 & - & - & -- & - & - & -- & -- & - \\
\hline 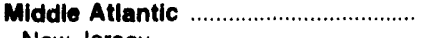 & 73 & 134 & 55 & 1,045 & 783 & 33.5 & .4 & .3 \\
\hline 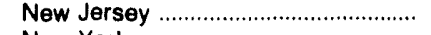 & 65 & 86 & 53 & 715 & 571 & 25.3 & 2.4 & 2.3 \\
\hline 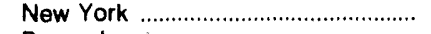 & 7 & 45 & 2 & 300 & 192 & 56.4 & .3 & .2 \\
\hline 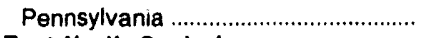 & 1 & 3 & - & 29 & 20 & 45.0 & - & - \\
\hline & 17 & 7 & 15 & 248 & 268 & -7.5 & .1 & .1 \\
\hline 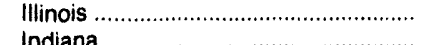 & " & * & 1 & 18 & 16 & 15.7 & $\cdot$ & - \\
\hline & 10 & 3 & 1 & 64 & 16 & 298.6 & .1 & $\bullet$ \\
\hline Michigan .............................................. & 2 & 1 & 12 & 72 & 199 & -63.8 & .1 & .3 \\
\hline Ohio & 3 & 2 & • & 70 & 24 & 191.2 & .1 & • \\
\hline 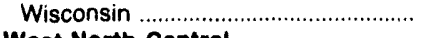 & 2 & 1 & 1 & 23 & 13 & 84.6 & .1 & • \\
\hline West North Central ............................. & 19 & 25 & 17 & 470 & 285 & 64.9 & .3 & .2 \\
\hline lowa & 1 & 1 & 4 & 80 & 25 & 226.4 & .3 & .1 \\
\hline Kansas & 13 & 18 & 11 & 237 & 163 & 45.0 & .8 & .6 \\
\hline 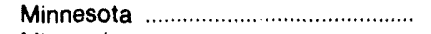 & * & 1 & * & 8 & 30 & -74.0 & * & .1 \\
\hline 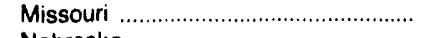 & 1 & 5 & 1 & 100 & 46 & 118.2 & .2 & .1 \\
\hline 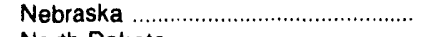 & 3 & 1 & 2 & 42 & 22 & 93.0 & .2 & 1 \\
\hline 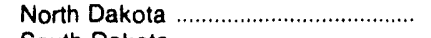 & * & : & * & * & : & NM & - & * \\
\hline South Dakota & 1 & • & • & 3 & * & NM & .1 & $\cdot$ \\
\hline South Atlantic & 367 & 456 & 112 & 3,936 & 2,359 & 66.8 & .8 & .5 \\
\hline Delaware & 130 & 59 & 21 & 568 & 207 & 174.8 & 8.7 & 3.9 \\
\hline District of Columbia ................................ & -- & -- & -- & -- & -- & -- & - & -- \\
\hline 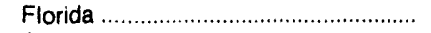 & 59 & 144 & 63 & 1,043 & 762 & 37.0 & .9 & .7 \\
\hline 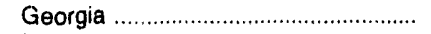 & 2 & * & * & 28 & 5 & 460.9 & - & * \\
\hline 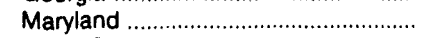 & 24 & 33 & 18 & 282 & 201 & 40.7 & 8 & 6 \\
\hline 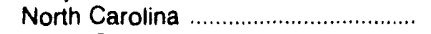 & 4 & 15 & 7 & 169 & 171 & -1.6 & .2 & .2 \\
\hline South Carolina & $\star$ & 7 & 1 & 96 & 41 & 134.3 & .1 & .1 \\
\hline 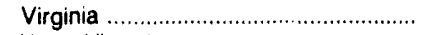 & 147 & 198 & 2 & 1,750 & 973 & 79.8 & 4.1 & 2.4 \\
\hline 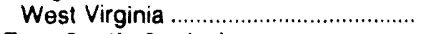 & - & -- & -- & -- & - & - & - & - \\
\hline East South Central ................................ & 52 & 53 & 41 & 767 & 609 & 25.9 & .3 & . 3 \\
\hline Alabama & 1 & -- & 3 & 135 & 44 & 205.6 & .2 & .0 \\
\hline 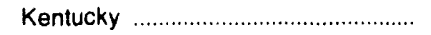 & • & * & • & 2 & 2 & 61.2 & $\bullet$ & - \\
\hline 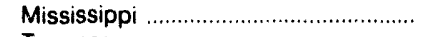 & 50 & 51 & 36 & 509 & 537 & -5.3 & 2.5 & 3.0 \\
\hline 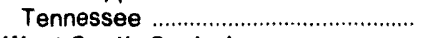 & - & 2 & 2 & 120 & 26 & 358.3 & .2 & * \\
\hline West South Central ............................... & 516 & 615 & 578 & 5,130 & 5,264 & -2.5 & 1.5 & 1.6 \\
\hline 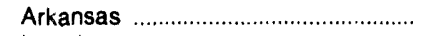 & -- & * & -- & & - & NM & $\cdot$ & - \\
\hline 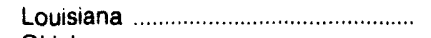 & 32 & 56 & 49 & 559 & 460 & 21.4 & 1.1 & 1.0 \\
\hline Oklahoma & 255 & 201 & 117 & 2,284 & 2,412 & -5.3 & 5.6 & 6.3 \\
\hline 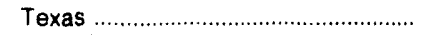 & 229 & 358 & 412 & 2,287 & 2,392 & -4.4 & 1.1 & 1.2 \\
\hline 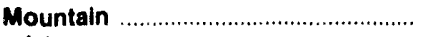 & 118 & 152 & 93 & 1,304 & 1,062 & 22.8 & .6 & .5 \\
\hline 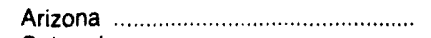 & 85 & 102 & 67 & 860 & 819 & 5.1 & 1.5 & 1,4 \\
\hline 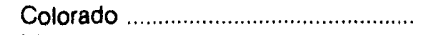 & 2 & 1 & 3 & 31 & 35 & -10.2 & .1 & .1 \\
\hline Idaho & -- & -- & - & -- & -- & -- & - & -- \\
\hline 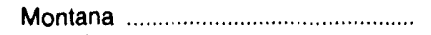 & 1 & * & 2 & 9 & 5 & 69.2 & * & * \\
\hline 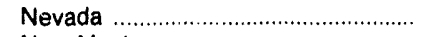 & 19 & 42 & 3 & 265 & 82 & 223.4 & 1.6 & .5 \\
\hline 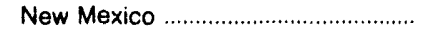 & 1 & 3 & 1 & 33 & 22 & 49.2 & .1 & .1 \\
\hline 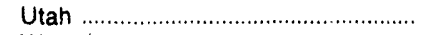 & 10 & 4 & 16 & 106 & 99 & 6.7 & .4 & .4 \\
\hline 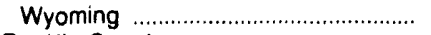 & - & -- & -- & -- & -- & - & - & - \\
\hline Pacific Contiguous .............................. & 483 & 332 & 433 & 2,569 & 3,138 & -18.1 & 1.2 & 1.5 \\
\hline 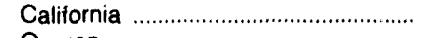 & 264 & 164 & 206 & 1,020 & 1,451 & -29.7 & 1.0 & 1.5 \\
\hline 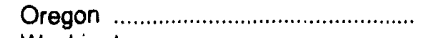 & 218 & 168 & 191 & 1,155 & 1,229 & -6.1 & 3.4 & 3.6 \\
\hline 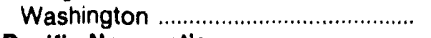 & * & $*$ & 36 & 394 & 458 & -13.9 & .6 & .7 \\
\hline Pacific Noncontiguous ........................ & 175 & 140 & 188 & 1,670 & 1,710 & -2.3 & 19.0 & 18.6 \\
\hline Alaska & 175 & 149 & 188 & 1,670 & 1,710 & -2.3 & 44.9 & 51.2 \\
\hline 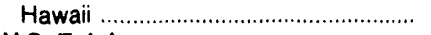 & $\rightarrow$ & -- & - & -- & -- & - & - & - \\
\hline U.S. Total & 1,835 & 1,980 & 1,551 & 17,402 & 15,797 & 10.2 & .7 & .7 \\
\hline
\end{tabular}

- Data for 1993 are preliminary.

- Data for 1992 are final.

* = For detailed data, the absolute value is less than 0.5 ; for percentage calculations, the absolute value is less than 0.05 percent.

= Calculation not meaningful.

Notes: $\bullet G T / I C=$ Gas Turbine/Internal Combustion. -Negative generation denotes that electric power consumed for plant use exceeds gross genera tion. - Totals may not equal sum of components because of independent rounding. $\bullet$ Percent difference is calculated before rounding.

Source: Energy Information idministration, Form EIA-759, "Monthly Power Plant Report." 
Table 14. Electric Utillty Hydroelectric Net Generation by Census Division and State (Million Kilowatthours)

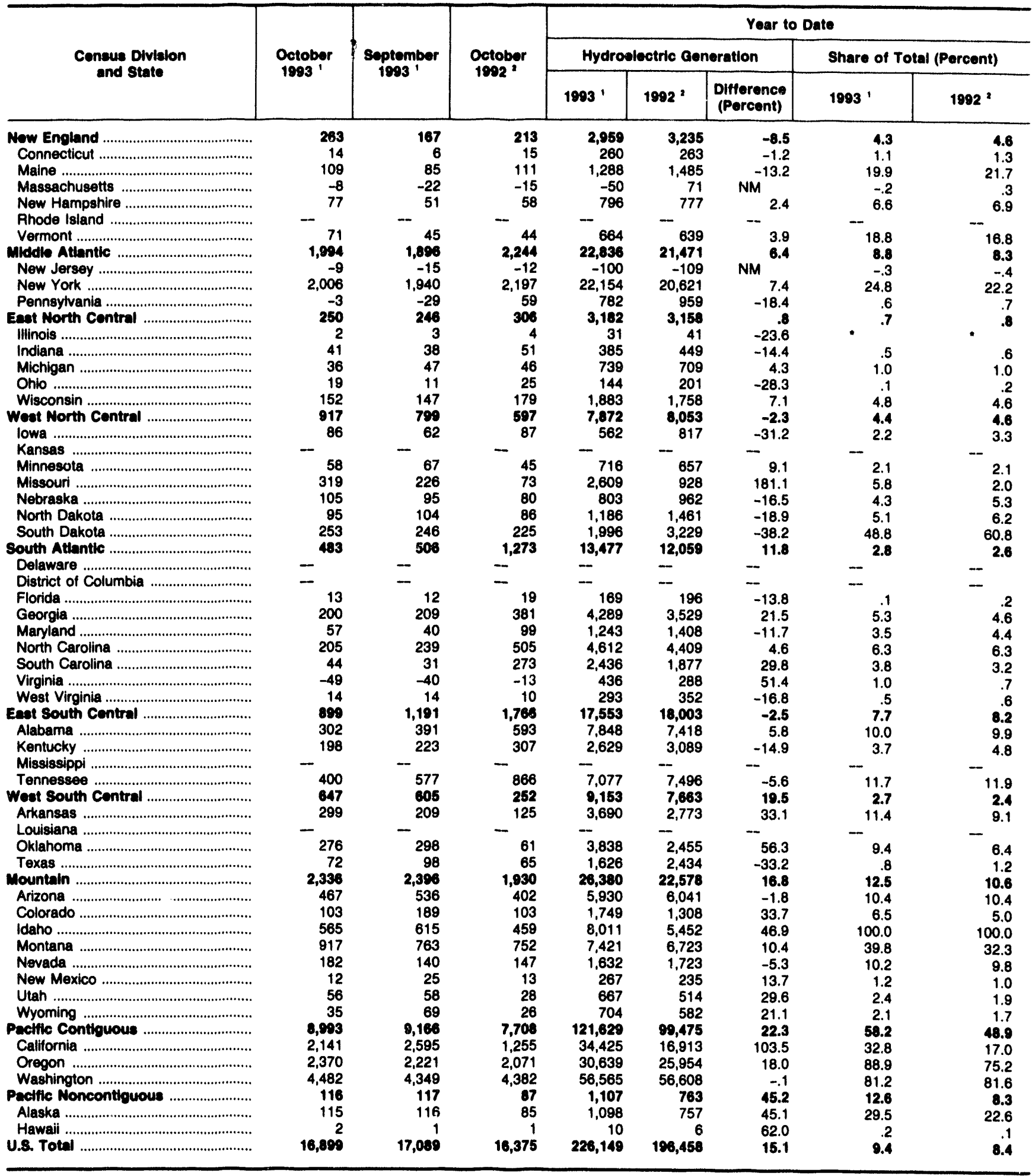

1. Data for 1993 are preliminary.

Data for 1992 are final.

* For detailed data, the absolute value is less than 0.5 ; for percentage calculations, the absolute value is less than 0.05 percent.

- Calculation not meaningtul.

Notes: -Negative generation denotes that electric power consumed for plant use exceeds gross generation. • Pumping energy used at pumped storage plants for October 1993 was 2,012 million kilowatthours. - Totals may not equal sum of components because of independent rounding. •Percent difference is calculated before rounding.

Source: Energy Information Administration, Form ElA-759, "Monthly Power Plant Report." 
Table 15. Electric Uttlity Muclear-Powered Not Generation by Census Division and State (Million Kilowatthours)

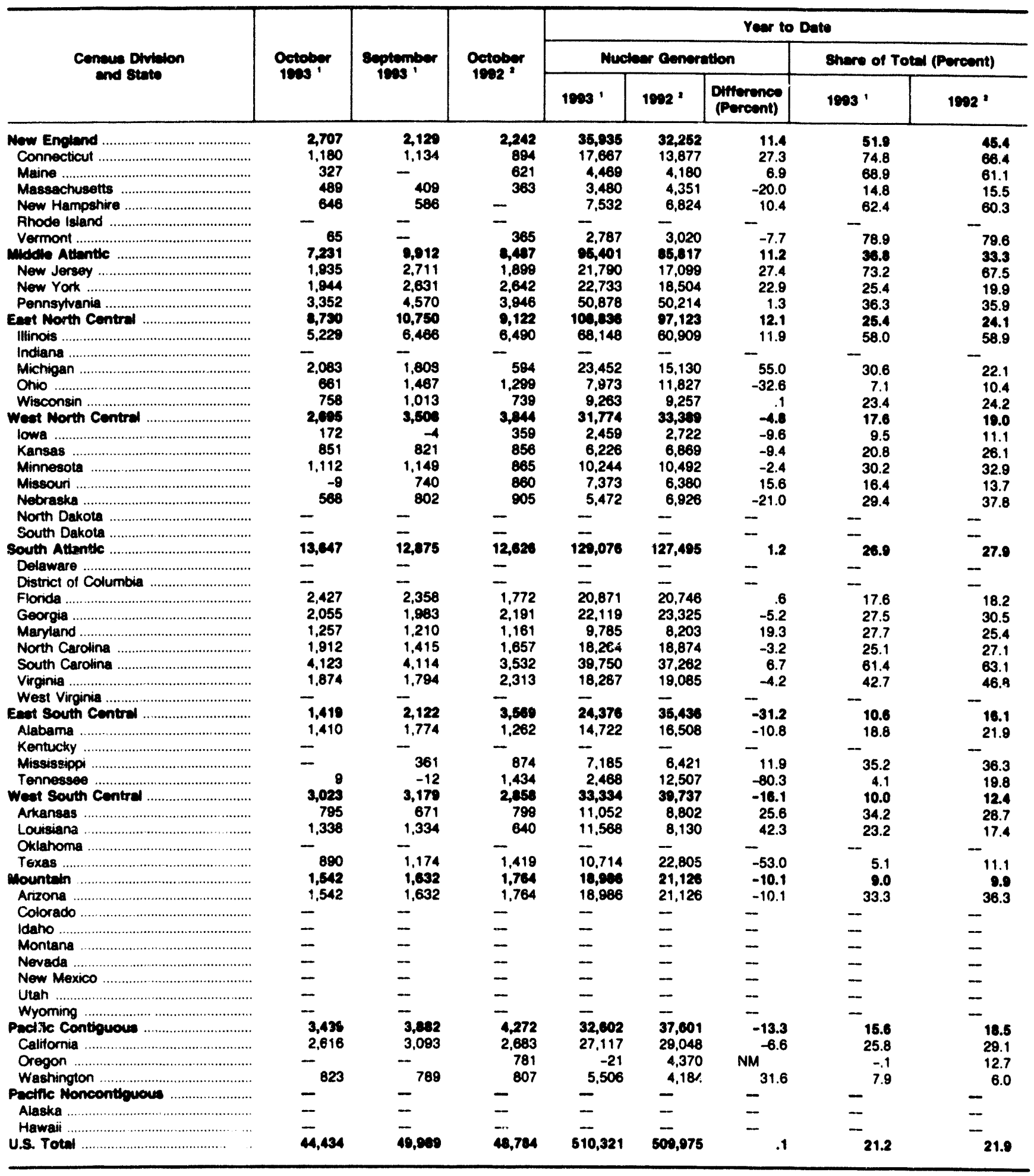

- Data for 1983 are preliminary.

- Data for 1992 are final.

- Calculation not meaningtul.

Notes: -Negative generation denotes that electric power consumed for plant use exceeds gross generation. -Totals may not equal sum of components because of independent rounding. -Percent difference is calculated before rounding.

Source: Energy Information Administration, Form ElA-759. "Monthly Power Plant Report." 
Table 16. Electric Utility Net Generation from Other Energy Sources by Census Division and State (Million Kilowatthours)

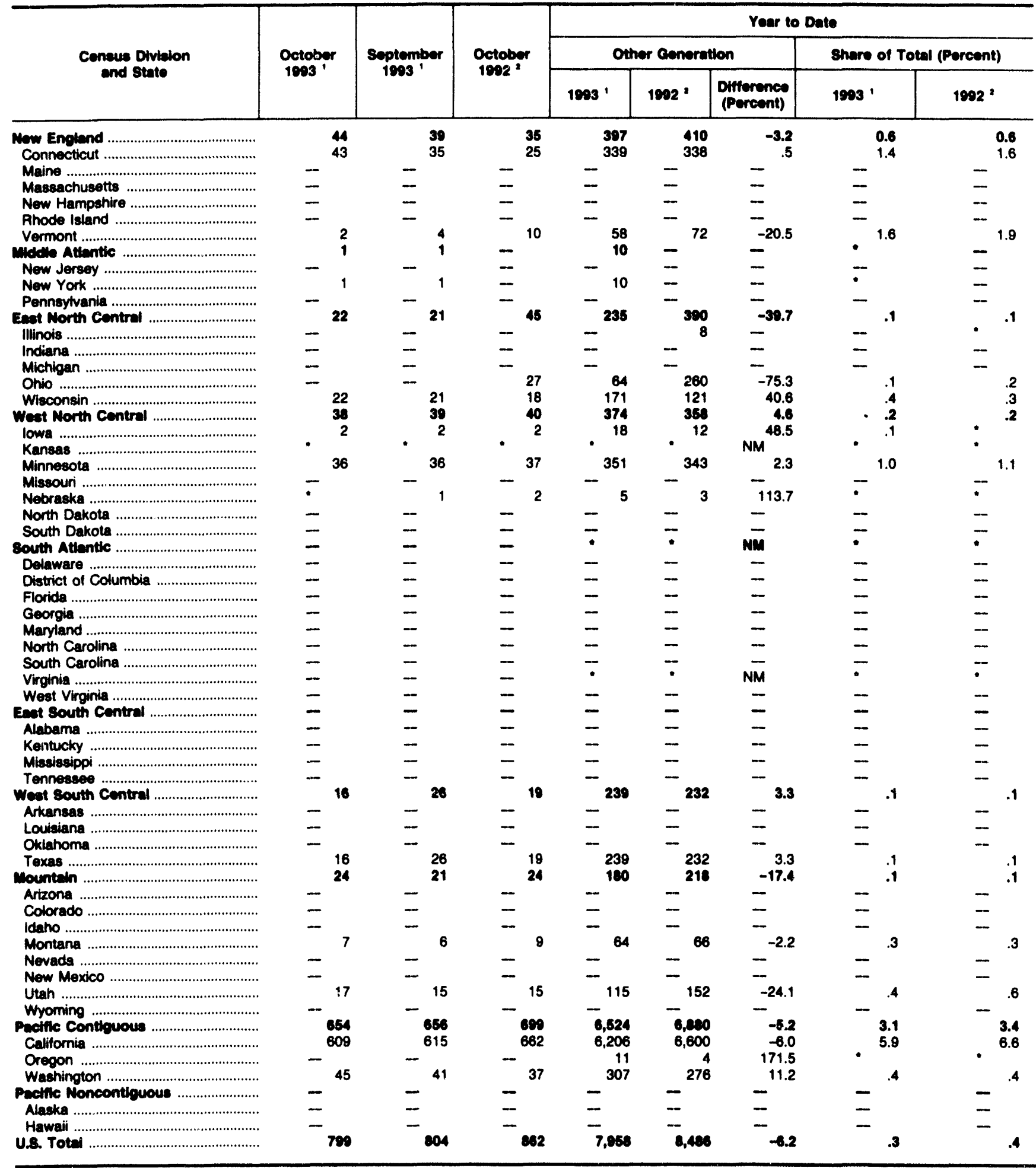

- Data for 1993 are preliminary.

- Data for 1992 are final.

- = For detailed data, the absolute value is less than 0.5: for percentage calculations, the absolute value is less than 0.05 percent.

= Calculation not meaningful.

Notes: - Neostive generation denotes that electric power consumed for plant use exceeds gross generation. -Tolals may not equal sum of compo nents because of independent rounding. PPercent difference is calculated before rounding. •Nonutility sources are not included. $\bullet$ Other energy sources include geothermal, wood, wind, waste, and solar.

Source: Energy Information Administration, Form ElA-759, "Monthly Power Plant Report." 


\section{U.S. Electric Utility Consumption of Fossil Fuels}

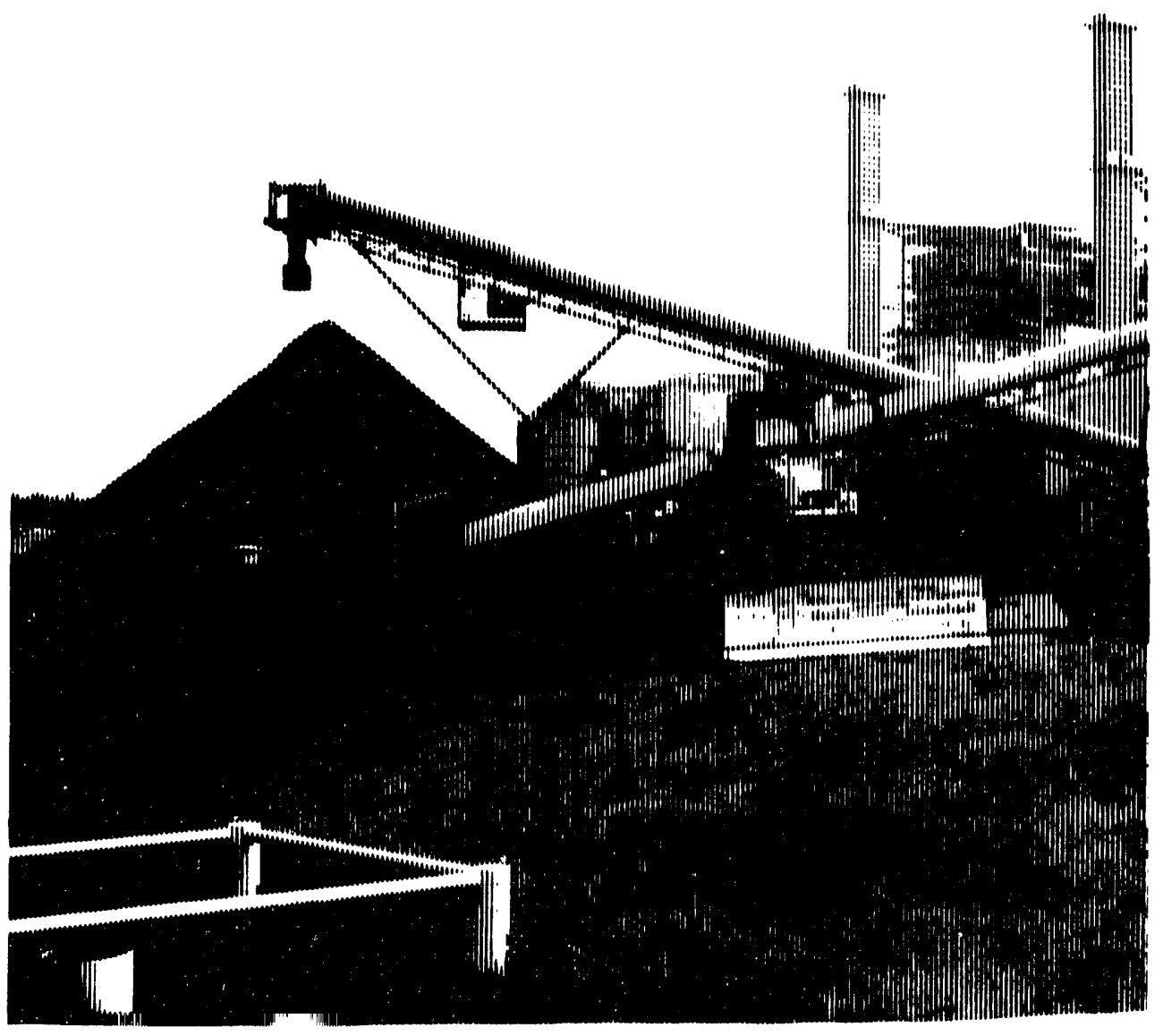


Table 17. U.S. Electric Utility Consumption of Fossil Fuels, 1983 Through October 1993

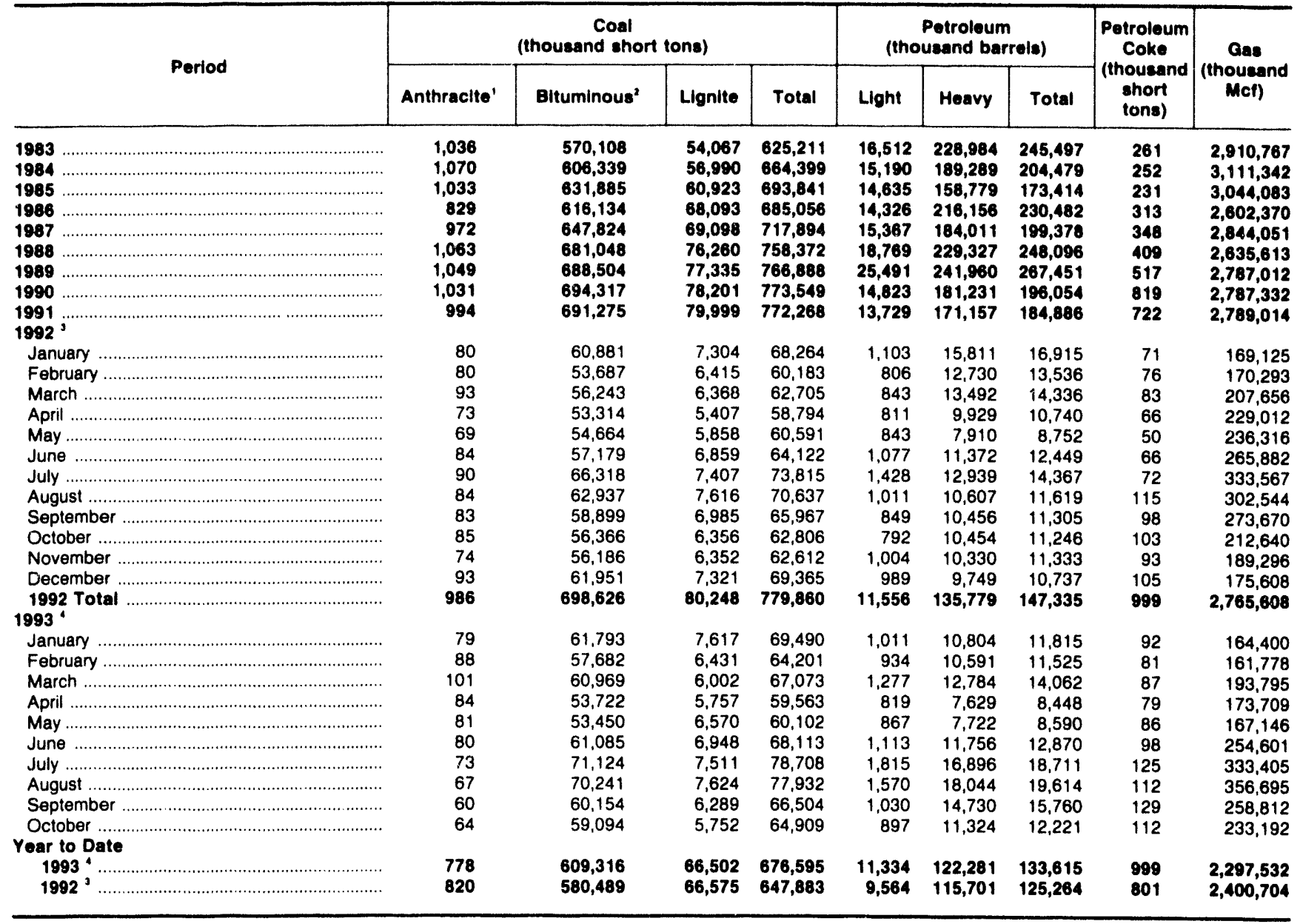

1 Includes anthracite silt stored off-site.

2 Includes subbituminous coal.

- Data for 1992 and prior years are final.

- Data for 1993 are preliminary.

Notes: Totals may not equal sum of components because of independent rounding. $\bullet$ Mcf $=$ thousand cubic feet.

Source: Energy Information Administration. Form ElA-759, "Monthly Power Plant Report," and predecessor forms.

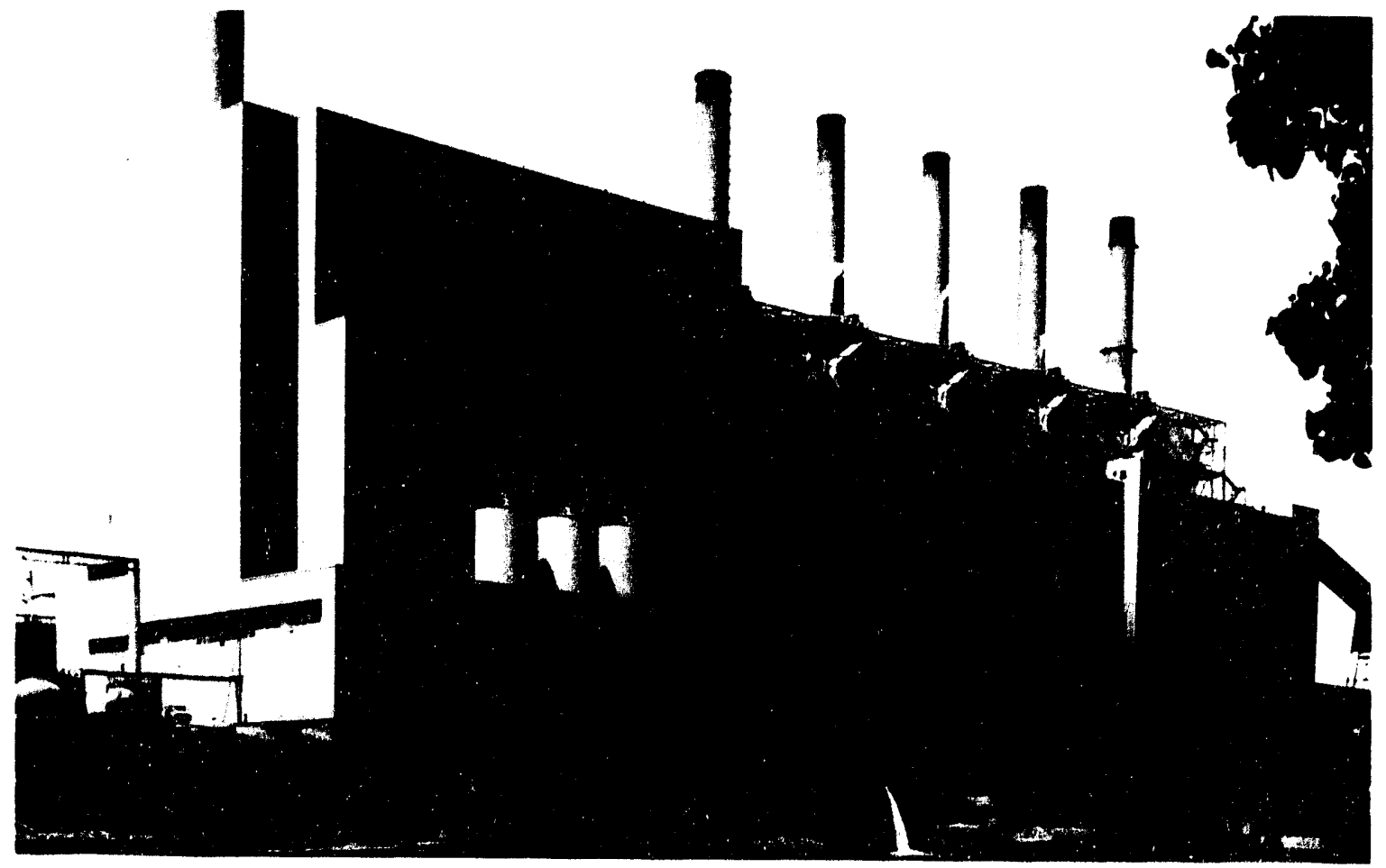

The Wabash River Generating Station is a coal-fired facility operated by Public Service Company of Indiana. 
Table 18. Electric Utility Consumption of Coal by NERC Region and Hawall (Thousand Short Tons)

\begin{tabular}{|c|c|c|c|c|c|c|}
\hline \multirow{2}{*}{$\begin{array}{l}\text { NERC Reglon } \\
\text { and Hawall }\end{array}$} & \multirow{2}{*}{$\begin{array}{l}\text { October } \\
1993\end{array}$} & \multirow{2}{*}{$\begin{array}{c}\text { September } \\
1993\end{array}$} & \multirow{2}{*}{$\begin{array}{l}\text { October } \\
1902 ?\end{array}$} & \multicolumn{3}{|c|}{ Year to Date } \\
\hline & & & & $1993^{\prime}$ & $1992^{2}$ & $\begin{array}{c}\text { Difference } \\
\text { (Percent) }\end{array}$ \\
\hline $\begin{array}{l}\text { ECAR } \\
\text { ERCOT } \\
\text { MAAC } \\
\text { MAIN } \\
\text { MAPP (U.S.) } \\
\text { NPCC (U.S.) } \\
\text { SERC } \\
\text { SPP } \\
\text { WSCC (U.S.) } \\
\text { Contiguous U.S. } \\
\text { ASCC } \\
\text { Hawaii } \\
\text { U.S. Total }\end{array}$ & $\begin{array}{r}15,486 \\
5,195 \\
3,264 \\
5,355 \\
5,636 \\
1,186 \\
12,226 \\
6,781 \\
9,758 \\
64,886 \\
23 \\
-\quad \\
64,909\end{array}$ & $\begin{array}{r}14,933 \\
5,840 \\
3,142 \\
4,592 \\
5,188 \\
1,195 \\
14,366 \\
7,411 \\
9,816 \\
66,483 \\
21 \\
-\quad \\
66,504\end{array}$ & $\begin{array}{r}15,554 \\
5,133 \\
3,130 \\
4,196 \\
5,035 \\
1,268 \\
11,400 \\
7,547 \\
9,515 \\
62,779 \\
2 \\
-\quad \\
62,806\end{array}$ & $\begin{array}{r}159,364 \\
60,232 \\
34,214 \\
49,949 \\
57,878 \\
11,996 \\
134,948 \\
76,380 \\
91,393 \\
676,353 \\
242 \\
-- \\
676,595\end{array}$ & $\begin{array}{r}154,608 \\
56,877 \\
34,037 \\
44,588 \\
54,472 \\
13,428 \\
123,236 \\
73,168 \\
93,246 \\
647,660 \\
224 \\
-- \\
647,883\end{array}$ & $\begin{array}{r}3.1 \\
5.9 \\
.5 \\
12.0 \\
6.3 \\
-10.7 \\
9.5 \\
4.4 \\
-2.0 \\
4.4 \\
8.1 \\
- \\
\end{array}$ \\
\hline
\end{tabular}

1 Data for 1993 are preliminary.

Data for 1992 are tinal.

Notes: $\bullet$ Totals may not equal sum of components because of independent rounding. $\bullet$ Percent difference is calculated before rounding. $\bullet$ Coal includes lignite, bituminous coal, subbituminous coal, and anthracite.

Source: Energy Information Administration, Form EIA-759, "Monthly Power Plant Report."

Table 19. Electric Utility Consumption of Petroleum by NERC Region and Hawail (Thousand Barrels)

\begin{tabular}{|c|c|c|c|c|c|c|}
\hline \multirow{2}{*}{$\begin{array}{c}\text { NERC Region } \\
\text { and Hawall }\end{array}$} & \multirow{2}{*}{$\begin{array}{l}\text { October } \\
1993\end{array}$} & \multirow{2}{*}{$\begin{array}{l}\text { September } \\
1993\end{array}$} & \multirow{2}{*}{$\begin{array}{l}\text { October } \\
1992^{2}\end{array}$} & \multicolumn{3}{|c|}{ Year to Date } \\
\hline & & & & $1993^{\prime}$ & $1992^{2}$ & $\begin{array}{l}\text { Difference } \\
\text { (Percent) }\end{array}$ \\
\hline $\begin{array}{l}\text { ECAR } \\
\text { ERCOT } \\
\text { MAAC } \\
\text { MAIN } \\
\text { MAPP (U.S.) } \\
\text { NPCC (U.S.) } \\
\text { SERC } \\
\text { SPP } \\
\text { WSCC (U.S.) } \\
\text { Contiguous U.S. } \\
\text { ASCC } \\
\text { Hawaii } \\
\text { U.S. Total }\end{array}$ & $\begin{array}{r}217 \\
27 \\
763 \\
158 \\
23 \\
4,087 \\
4,913 \\
901 \\
51 \\
11,139 \\
58 \\
1,024 \\
12,221\end{array}$ & $\begin{array}{r}197 \\
14 \\
1,560 \\
108 \\
20 \\
4,884 \\
7,009 \\
892 \\
35 \\
14,718 \\
91 \\
952 \\
15,760\end{array}$ & $\begin{array}{r}154 \\
149 \\
997 \\
178 \\
28 \\
4,554 \\
4,083 \\
41 \\
86 \\
10,289 \\
42 \\
935 \\
11,246\end{array}$ & $\begin{array}{r}2,541 \\
225 \\
15,982 \\
2,001 \\
325 \\
44,067 \\
50,852 \\
5,554 \\
2,464 \\
124,010 \\
659 \\
8,947 \\
133,615\end{array}$ & $\begin{array}{r}2,050 \\
350 \\
11,427 \\
2,018 \\
214 \\
54,024 \\
42,225 \\
992 \\
1,195 \\
114,493 \\
580 \\
10,191 \\
125,264\end{array}$ & $\begin{array}{r}23.9 \\
-35.8 \\
39.9 \\
-.8 \\
52.1 \\
-18.4 \\
20.4 \\
460.0 \\
106.3 \\
8.3 \\
13.6 \\
-12.2 \\
6.7\end{array}$ \\
\hline
\end{tabular}

1 Data for 1993 are preliminary.

- Data for 1992 are final.

Note: Totals may not equal sum of components because of independent rounding.

Source: Energy Information Administration. Form EIA-759, "Monthly Power Plant Report." 
Table 20. Electric Utility Consumption of Gas by NERC Reglon and Hawall (Million Cubic Feet)

\begin{tabular}{|c|c|c|c|c|c|c|}
\hline \multirow{2}{*}{$\begin{array}{l}\text { NERC Roglon } \\
\text { and Hawall }\end{array}$} & \multirow{2}{*}{$\begin{array}{l}\text { October } \\
1903\end{array}$} & \multirow{2}{*}{$\begin{array}{l}\text { September } \\
1903^{1}\end{array}$} & \multirow{2}{*}{$\begin{array}{l}\text { October } \\
1902^{2}\end{array}$} & \multicolumn{3}{|c|}{ Year to Date } \\
\hline & & & & $1993^{\prime}$ & $1992^{2}$ & $\begin{array}{l}\text { Difterence } \\
\text { (Percent) }\end{array}$ \\
\hline $\begin{array}{l}\text { ECAR } \\
\text { ERCOT } \\
\text { MAAC (MAIN } \\
\text { MAPP (U.S.) } \\
\text { NPCC (U.S.) } \\
\text { SERC } \\
\text { SPP } \\
\text { WSCC (U.S.) } \\
\text { Contiguous U.S. } \\
\text { ASCC } \\
\text { Hawaii } \\
\text { U.S. Total }\end{array}$ & $\begin{array}{r}2,063 \\
73,632 \\
3,348 \\
2,568 \\
619 \\
17,274 \\
16,046 \\
59,525 \\
55,710 \\
230,785 \\
2,407 \\
\overline{233}, 192\end{array}$ & $\begin{array}{r}1,260 \\
88,082 \\
5,558 \\
1,573 \\
811 \\
18,337 \\
22,201 \\
66,084 \\
52,888 \\
256,785 \\
2,017 \\
-\overline{258,812}\end{array}$ & $\begin{array}{r}2,086 \\
65,021 \\
3,041 \\
459 \\
654 \\
17,147 \\
14,202 \\
43,760 \\
63,756 \\
210,126 \\
2,514 \\
\overline{212,640}\end{array}$ & $\begin{array}{r}23,501 \\
755,897 \\
54,134 \\
17,474 \\
9,372 \\
184,051 \\
200,019 \\
578,066 \\
452,924 \\
2,275,438 \\
22,094 \\
-- \\
2,297,532\end{array}$ & $\begin{array}{r}30,811 \\
641,258 \\
57,265 \\
11,206 \\
8,187 \\
224,541 \\
216,376 \\
616,647 \\
570,937 \\
2,377,228 \\
23,476 \\
-\overline{2,400,704}\end{array}$ & $\begin{array}{r}-23.7 \\
17.9 \\
-5.5 \\
55.9 \\
14.5 \\
-18.0 \\
-7.6 \\
-6.3 \\
-20.7 \\
-4.3 \\
-5.9 \\
-- \\
-4.3\end{array}$ \\
\hline
\end{tabular}

- Data for 1993 are preliminary.

- Data for 1992 are final.

Note: Totals may not equal sum of components because of independent rounding

Source: Energy Information Administration, Form ElA-759, "Monthly Power Plant Report." 
Table 21. Electric Utility Consumption of Coal by Census Division and State (Thousand Short Tons)

\begin{tabular}{|c|c|c|c|c|c|c|}
\hline \multirow{2}{*}{$\begin{array}{l}\text { Census Divialon } \\
\text { and State }\end{array}$} & \multirow{2}{*}{$\begin{array}{l}\text { October } \\
1993^{1}\end{array}$} & \multirow{2}{*}{$\begin{array}{l}\text { Soptomber } \\
1993\end{array}$} & \multirow{2}{*}{$\begin{array}{l}\text { October } \\
1992^{2}\end{array}$} & \multicolumn{3}{|c|}{ Year to Date } \\
\hline & & & & $1993^{\prime}$ & $1092^{2}$ & $\begin{array}{l}\text { Difference } \\
\text { (Percent) }\end{array}$ \\
\hline 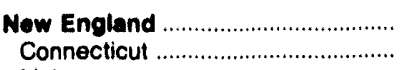 & $\begin{array}{r}477 \\
2\end{array}$ & $\begin{array}{r}533 \\
89\end{array}$ & $\begin{array}{r}555 \\
97\end{array}$ & $\begin{array}{r}4,818 \\
695\end{array}$ & $\begin{array}{r}5,068 \\
718\end{array}$ & $\begin{array}{l}-4.9 \\
-3.1\end{array}$ \\
\hline 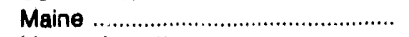 & - & -- & -- & -- & -- & -- \\
\hline 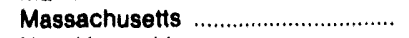 & 339 & 326 & 374 & 3,012 & 3,355 & -10.2 \\
\hline 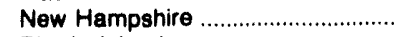 & 136 & 117 & 84 & 1,110 & 895 & 11.6 \\
\hline 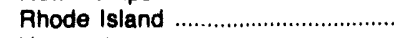 & $-\infty$ & -- & -- & - & -- & - \\
\hline 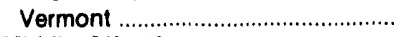 & -- & -- & -- & - & -- & - \\
\hline Middle Atlantic ................................... & 4,158 & 3,948 & 4,042 & 42,849 & 44,197 & $-\mathbf{3 . 0}$ \\
\hline 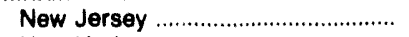 & 129 & 122 & 246 & 1,744 & 1,683 & 3.6 \\
\hline 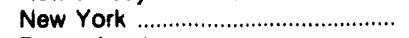 & 708 & 662 & 713 & 7,178 & 8,360 & -14.1 \\
\hline 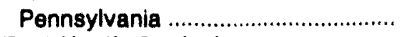 & 3,320 & 3,164 & 3,083 & 33,927 & 34,154 & -.7 \\
\hline East North Central ............................... & 14,842 & 14,168 & 13,895 & 149,484 & 140,191 & 6.6 \\
\hline 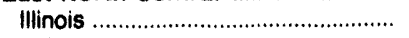 & 2,998 & 2,471 & 1,746 & 25,492 & 20,913 & 21.9 \\
\hline 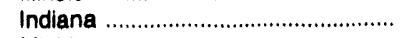 & 3,868 & 3,913 & 3,881 & 41,042 & 38,439 & 6.8 \\
\hline 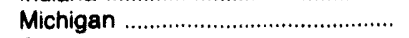 & 2,382 & 2,386 & 2,510 & 23,885 & 23,513 & 1.6 \\
\hline 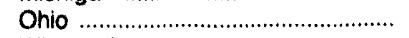 & 4,005 & 3,983 & 4,145 & 43,205 & 42,325 & 2.1 \\
\hline 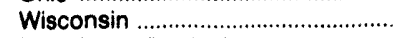 & 1,589 & 1,415 & 1,612 & 15,860 & 15,002 & 5.7 \\
\hline West North Central ............................. & 8,464 & 7,886 & 8,054 & 88,586 & 84,212 & 5.2 \\
\hline 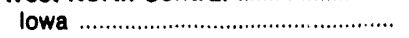 & 1,075 & 1,205 & 1,066 & 14,033 & 12,789 & 9.7 \\
\hline Kansas & 1,419 & 1,272 & 1,122 & 14,192 & 11,664 & 21.7 \\
\hline 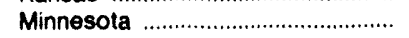 & 1,501 & 1,307 & 1,199 & 13,433 & 12,667 & 6.0 \\
\hline 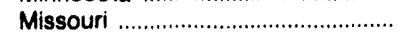 & 1,549 & 1,550 & 2,028 & 18,323 & 19,696 & -7.0 \\
\hline 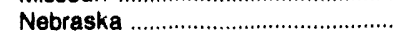 & 826 & 706 & 499 & 7,678 & 6,545 & 17.3 \\
\hline 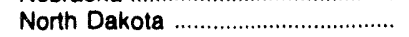 & 1,970 & 1,780 & 1,942 & 19,096 & 18,960 & .7 \\
\hline South Dakota & 125 & 65 & 197 & 1,840 & 1,891 & -2.7 \\
\hline South Atiantic & 10,102 & 11,210 & 10,033 & 111,071 & 105,963 & 4.8 \\
\hline Dela'ware & 162 & 179 & 117 & 1,828 & 1,400 & 30.6 \\
\hline District of Columbia ............................. & - & - & -- & - & - & $\ldots$ \\
\hline Florida & 1,974 & 2,224 & 1,975 & 21,405 & 21,128 & 1.3 \\
\hline Georgia & 1,920 & 2,372 & 2,017 & 21,507 & 20,075 & 7.1 \\
\hline 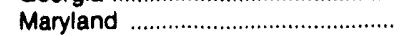 & 830 & 701 & 622 & 7,922 & 7,457 & 6.2 \\
\hline North Carolina & 1,637 & 2,157 & 1,707 & 19,273 & 17,846 & $\begin{array}{l}0.2 \\
8.0\end{array}$ \\
\hline 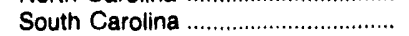 & 673 & 937 & 630 & 8,750 & 7,732 & 13.2 \\
\hline Virginia & 702 & 772 & 539 & 7,861 & 7,215 & $\begin{array}{r}13.2 \\
8.9\end{array}$ \\
\hline West Virginia & 2,204 & 1,869 & 2,425 & 22,525 & 23,109 & $\begin{array}{r}0.8 \\
-2.5\end{array}$ \\
\hline 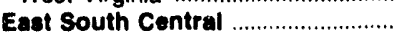 & 7,345 & 7,792 & 6,342 & 75,588 & 67,422 & 12.1 \\
\hline Alabama & 2,358 & 2,557 & 2,048 & 22,766 & 20,931 & 8.8 \\
\hline 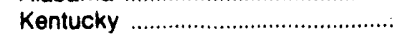 & 2,887 & 2,850 & 2,645 & 29,215 & 26,198 & 11.5 \\
\hline 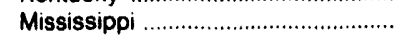 & 318 & 399 & 216 & 3,349 & 2,906 & 15.2 \\
\hline 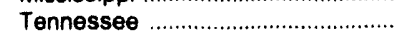 & 1,781 & 1,986 & 1.433 & 20,259 & 17,387 & 16.5 \\
\hline West South Central ............................... & 9,721 & 11,111 & 10,329 & 112,397 & 107,325 & 4.7 \\
\hline Arkansas & 628 & 988 & 1,266 & 9,785 & 10,047 & -2.6 \\
\hline 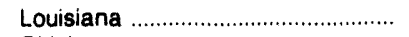 & 832 & 1,143 & 855 & 10,922 & 10,702 & 2.1 \\
\hline Oklahoma & 1,305 & 1,367 & 1,447 & 14,525 & 13,679 & 6.2 \\
\hline 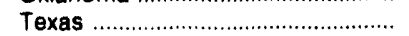 & 6,957 & 7,613 & 6,762 & 77,166 & 72,897 & 5.9 \\
\hline 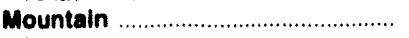 & 8,963 & 9,021 & 8,791 & 85,560 & 86,818 & -1.2 \\
\hline Arizona & 1,656 & 1,653 & 1,527 & 15,120 & 14,121 & 7.1 \\
\hline 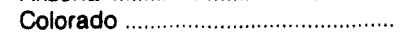 & 1,372 & 1,265 & 1,158 & 13,282 & 13,072 & 1.6 \\
\hline Idaho & -- & -- & - & - & - & - \\
\hline Montana & 819 & 805 & 944 & 7,121 & 8,771 & -18.8 \\
\hline 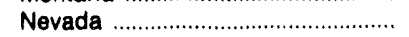 & 737 & 688 & 485 & 6,537 & 6,529 & .1 \\
\hline 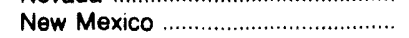 & 1,154 & 1,364 & 1,227 & 12,068 & 12,038 & .3 \\
\hline Utah & 1,191 & 1,262 & 1,279 & 11,669 & 11,447 & 1.9 \\
\hline Wyoming & 2,034 & 1,984 & 2,172 & 19,763 & 20,641 & -4.3 \\
\hline Pacific Contiguous .............................. & 815 & 815 & 739 & 5,990 & 6,663 & -10.1 \\
\hline 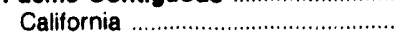 & -- & -- & -- & -- & -- & -- \\
\hline 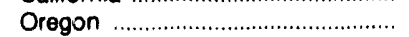 & 218 & 212 & 186 & 1,557 & 1,631 & -4.6 \\
\hline Washington & 596 & 603 & 553 & 4,434 & 5,032 & -11.8 \\
\hline Pacific Noncontiguous .................... & 23 & 21 & 27 & 242 & 224 & 8.1 \\
\hline Alaska & 23 & 21 & 27 & 242 & 224 & 8.1 \\
\hline 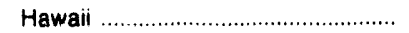 & -- & -- & - & - & - & - \\
\hline 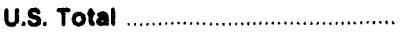 & 64,909 & 66,504 & 62,806 & 676,595 & 647,883 & 4.4 \\
\hline
\end{tabular}

- Data for 1993 are preliminary.

Data for 1992 are final.

Notes: - Totals may not equal sum of components because of independent rounding. •Percent difference is calculated before rounding. • Coal includes lignite, bituminous coal, subbituminous coal, and anthracite.

Source: Energy Information Administration, Form ElA-759, "Monthly Power Plant Report." 
Table 22. Electric Utility Consumption of Petroleum by Census Division and State (Thousand Barrels)

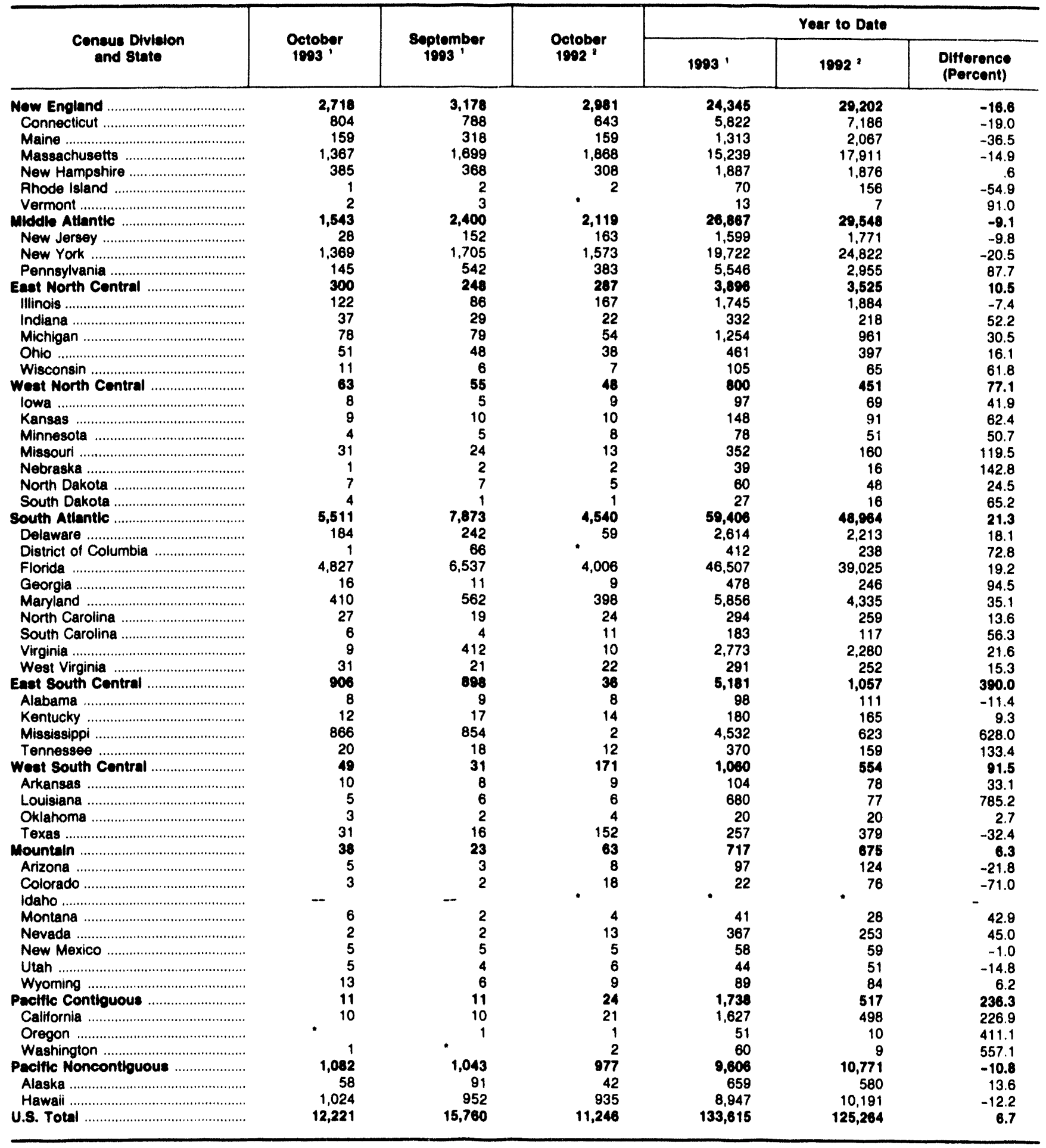

- Data for 1993 are preliminary.

- Data for 1992 are final.

- = For detailed data, the absolute value is less than 0.5 ; for percentage calculations, the absolute value is less than 0.05 percent. Notes: - Totals may not equal sum of components because of independent rounding. $\bullet$ Percent difference is calculated belore rounding. $\bullet$ Data do not include petroleum coke. The October 1993 petroleum coke consumption was 111526 short tons. Source: Energy Information Administration, Form ElA-759, "Monthly Power Plant Report." 
Table 23. Consumption of Petroleum at Steam-Fired Electric Utility Plants by Census Division and State

(Thousand Barrels)

\begin{tabular}{|c|c|c|c|c|c|c|}
\hline \multirow{2}{*}{$\begin{array}{c}\text { Census Division } \\
\text { and State }\end{array}$} & \multirow{2}{*}{$\begin{array}{l}\text { October } \\
1993\end{array}$} & \multirow{2}{*}{$\begin{array}{l}\text { Soptomber } \\
1903\end{array}$} & \multirow{2}{*}{$\begin{array}{l}\text { October } \\
1902^{2}\end{array}$} & \multicolumn{3}{|c|}{ Year to Date } \\
\hline & & & & $1903^{\prime}$ & $1992^{2}$ & $\begin{array}{l}\text { Difterence } \\
\text { (Percent) }\end{array}$ \\
\hline 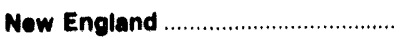 & 2,687 & 3,141 & 2,962 & 23,962 & 28,838 & -16.9 \\
\hline 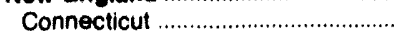 & 803 & 785 & 642 & 5,795 & 7,161 & -19.1 \\
\hline 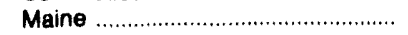 & 159 & 317 & 159 & 1,306 & 2,063 & -36.7 \\
\hline 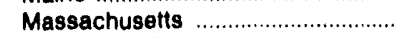 & 1,340 & 1,671 & 1,852 & 14,919 & 17,595 & -15.2 \\
\hline 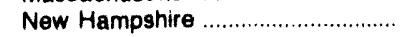 & 385 & 368 & 308 & 1,885 & 1,874 & .6 \\
\hline 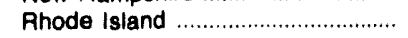 & - & - & 1 & 55 & 141 & -61.4 \\
\hline 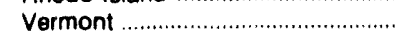 & $\cdot$ & $\cdot$ & $\cdot$ & 2 & 1 & 88.7 \\
\hline 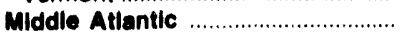 & 1,512 & 2,312 & 2,098 & 25,906 & 28,898 & -10.4 \\
\hline 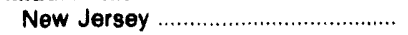 & 19 & 139 & 155 & 1,306 & 1,533 & -14.8 \\
\hline 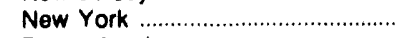 & 1,356 & 1,653 & 1,564 & 19,283 & 24,555 & -21.5 \\
\hline 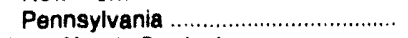 & 137 & 520 & 379 & 5,317 & 2,810 & 89.2 \\
\hline East North Central ............................ & 201 & 216 & 279 & 3,627 & 3,419 & 6.1 \\
\hline 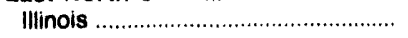 & 120 & 82 & 165 & 1,698 & 1,866 & -9.0 \\
\hline 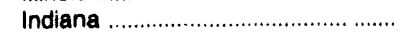 & 22 & 21 & 21 & 288 & 211 & 36.6 \\
\hline 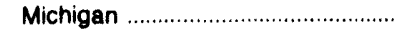 & 72 & 73 & 51 & 1,202 & 927 & 29.6 \\
\hline Ohio & 44 & 34 & 36 & 382 & 365 & 4.7 \\
\hline 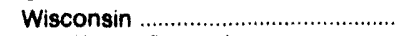 & 3 & 5 & 5 & 57 & 51 & 12.9 \\
\hline West North Central ............................ & 52 & 38 & 31 & 469 & 312 & 50.2 \\
\hline lowa & 6 & 2 & 4 & 42 & 42 & -.1 \\
\hline 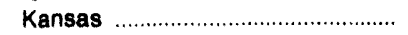 & 6 & 7 & 7 & 97 & 55 & 75.3 \\
\hline 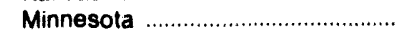 & 4 & 3 & 4 & 36 & 31 & 14.8 \\
\hline 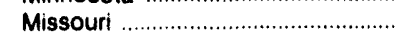 & 29 & 19 & 11 & 200 & 124 & 61.4 \\
\hline 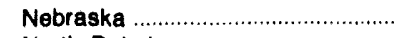 & & 1 & • & 27 & 6 & 323.6 \\
\hline 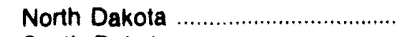 & 6 & 6 & 5 & 57 & 47 & 21.5 \\
\hline South Dakota & 2 & - & 1 & 10 & 6 & 62.5 \\
\hline 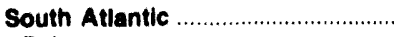 & $\mathbf{5 , 4 4 2}$ & 7,695 & 4,458 & 57,634 & 47,530 & 21.3 \\
\hline 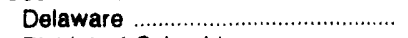 & 183 & 237 & 59 & 2,582 & 2,196 & 17.6 \\
\hline District of Columbia & 1 & 62 & $\cdot$ & 395 & 192 & 105.6 \\
\hline Florida & 4,777 & 6,413 & 3,939 & 45,477 & 38,068 & 19.5 \\
\hline 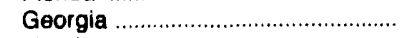 & 8 & 7 & 7 & 265 & 165 & 60.3 \\
\hline 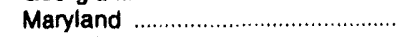 & 406 & 543 & 396 & 5,625 & 4,243 & 32.6 \\
\hline 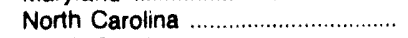 & 23 & 12 & 18 & 176 & 173 & 1.9 \\
\hline 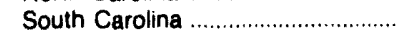 & 6 & 2 & 8 & 113 & 88 & 29.4 \\
\hline 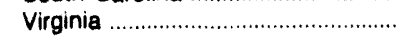 & 7 & 398 & 8 & 2,711 & 2,155 & 25.8 \\
\hline West Virginia & 31 & 21 & 22 & 290 & 252 & 15.4 \\
\hline East South Central & 897 & 891 & 30 & 4,830 & 982 & 392.1 \\
\hline Alabama & 8 & 7 & 8 & 89 & 95 & -6.6 \\
\hline Kentucky & 12 & 17 & 14 & 176 & 163 & 8.1 \\
\hline 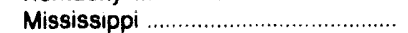 & 866 & 854 & 2 & 4,450 & 622 & 615.0 \\
\hline 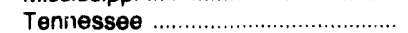 & 12 & 13 & 6 & 115 & 101 & 14.0 \\
\hline West South Central & 46 & 27 & 168 & 1,010 & 521 & 93.7 \\
\hline Arkansas & 10 & 6 & 9 & 80 & 77 & 3.7 \\
\hline Louisiana & 5 & 6 & 5 & 679 & 76 & 797.5 \\
\hline Oklahoma & 3 & 1 & 4 & 17 & 18 & -2.2 \\
\hline Texas & 28 & 14 & 148 & 234 & 351 & $\begin{array}{r}-2.2 \\
-33.4\end{array}$ \\
\hline 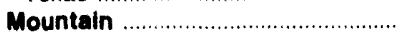 & 37 & 22 & 60 & 692 & 625 & 10.6 \\
\hline Arizona & 4 & 3 & 7 & 93 & 111 & -16.3 \\
\hline Colorado & 3 & 1 & 16 & 19 & 66 & -71.4 \\
\hline Idaho & -- & -- & -- & - & -- & - \\
\hline 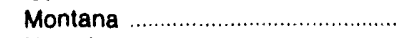 & 6 & 2 & 4 & 40 & 28 & 44.1 \\
\hline 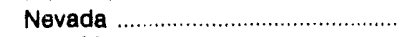 & 2 & 2 & 13 & 356 & 235 & 51.6 \\
\hline 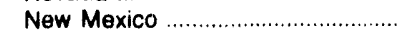 & 5 & 5 & 5 & 58 & 58 & -.6 \\
\hline Utah & 5 & 3 & 5 & 38 & 44 & -14.2 \\
\hline 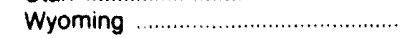 & 13 & 6 & 9 & 89 & 84 & 6.2 \\
\hline Pacific Contiguous ..................... & 2 & 2 & 17 & 1,557 & 405 & 284.0 \\
\hline California & 2 & 2 & 15 & 1,535 & 389 & 294.4 \\
\hline Oregon & • & 1 & 1 & 8 & 9 & -8.9 \\
\hline 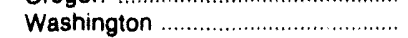 & 1 & -- & 1 & 14 & 7 & 98.6 \\
\hline Pacific Noncontiguous .................. & 842 & 766 & 786 & 7,264 & 8,576 & -15.3 \\
\hline Alaska & $\cdot-\dot{n}$ & & 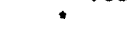 & 2 & 2 & -31.7 \\
\hline 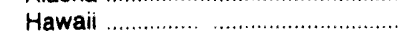 & 842 & 766 & 766 & 7,263 & 8,573 & -15.3 \\
\hline U.S. Total & 11,777 & 15,111 & 10,867 & 126,951 & 120,105 & 5.7 \\
\hline
\end{tabular}

- Data for 1993 are preliminary

- Data for 1992 are final.

- = For detailed data, the absolute value is less than 0.5 ; for percentage calculations, the absolute value is less than 0.05 percent.

Notes: - Totals may not equal sum of components because of independent rounding. $\bullet$ Percent difference is calculated before rounding. $\bullet$ Data do not include petroleum coke. The October 1993 petroleum coke consumption was 111526 short tons. Source: Energy Information Administration, Form EIA.759, "Monthly Power Plant Report." 
Table 24. Consumption of Petroloum at GT/IC Electric Utility Plants by Census Division and State

(Thousand Barrels)

\begin{tabular}{|c|c|c|c|c|c|c|}
\hline \multirow{2}{*}{$\begin{array}{c}\text { Consus Division } \\
\text { and State }\end{array}$} & \multirow{2}{*}{$\begin{array}{l}\text { October } \\
1903\end{array}$} & \multirow{2}{*}{$\begin{array}{l}\text { september } \\
1803\end{array}$} & \multirow{2}{*}{$\begin{array}{l}\text { October } \\
1902\end{array}$} & \multicolumn{3}{|c|}{ Year to Date } \\
\hline & & & & $1903^{\prime}$ & $1992^{2}$ & $\begin{array}{l}\text { Difference } \\
\text { (Percent) }\end{array}$ \\
\hline 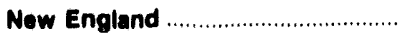 & 31 & 37 & 19 & 383 & 366 & 4.5 \\
\hline Connecticut ........................................ & 2 & 3 & 1 & 26 & 25 & 7.4 \\
\hline 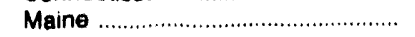 & $\cdot$ & 1 & 1 & 7 & 3 & 93.6 \\
\hline 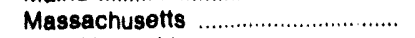 & 27 & 28 & 16 & 320 & 316 & 1.4 \\
\hline New Hampshire ................................ & - & & • & 2 & 2 & 33.0 \\
\hline 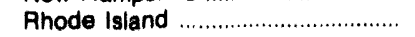 & 1 & 2 & 1 & 16 & $1 \overline{4}$ & 8.5 \\
\hline 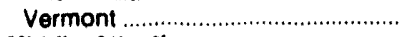 & 2 & 2 & $\bullet$ & 11 & 6 & 91.4 \\
\hline Middle Atlantic ............................... & 31 & 88 & 21 & 800 & 650 & 47.6 \\
\hline 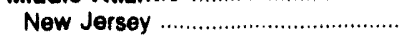 & 9 & 14 & 8 & 292 & 238 & 22.7 \\
\hline 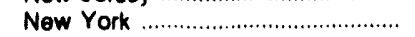 & 14 & 52 & 9 & 439 & 268 & 64.2 \\
\hline Pennsylvania ......................................... & 8 & 22 & 4 & 229 & 144 & 58.3 \\
\hline East North Central ............................. & 40 & 32 & 8 & 289 & 108 & 154.6 \\
\hline 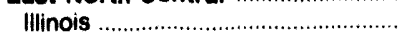 & 2 & 4 & 2 & 47 & 18 & 152.6 \\
\hline Indiana & 15 & 8 & 1 & 44 & 7 & 530.2 \\
\hline 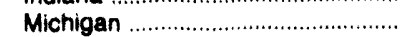 & 6 & 5 & 2 & 52 & 34 & 54.7 \\
\hline 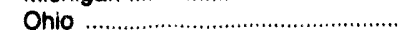 & 7 & 14 & 2 & 79 & 33 & 143.6 \\
\hline 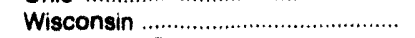 & 9 & 1 & 1 & 47 & 14 & 237.1 \\
\hline West North Central .......................... & 11 & 16 & 17 & 330 & 139 & 137.7 \\
\hline lowa & 3 & 3 & 6 & 55 & 26 & 108.7 \\
\hline Kansas & 3 & 4 & 3 & 50 & 35 & 42.1 \\
\hline 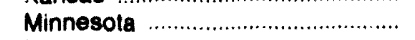 & 1 & 2 & 4 & 42 & 20 & 106.8 \\
\hline 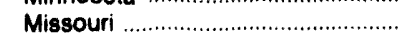 & 1 & 5 & 2 & 152 & 36 & 317.9 \\
\hline 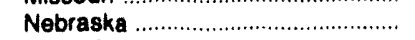 & 1 & 1 & 2 & 12 & 10 & 25.9 \\
\hline North Dakota & - & 1 & - & 3 & 1 & 156.5 \\
\hline South Dakota & 2 & 1 & - & 16 & 10 & 67.0 \\
\hline 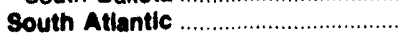 & 69 & 178 & 82 & 1,772 & 1,434 & 23.6 \\
\hline 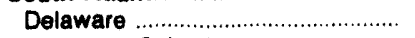 & & 5 & $\cdot$ & 32 & 17 & 91.3 \\
\hline District of Columbia ............................ & • & 4 & - & 17 & 46 & -64.0 \\
\hline Florida & 49 & 124 & 67 & 1,030 & 957 & 7.6 \\
\hline 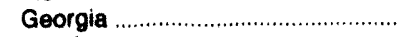 & 9 & 3 & 2 & 213 & 81 & 164.3 \\
\hline 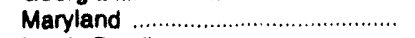 & 4 & 18 & 2 & 231 & 92 & 150.9 \\
\hline North Carolina ..................................... & 4 & 7 & 5 & 118 & 86 & 37.3 \\
\hline 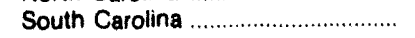 & 1 & 1 & 3 & 69 & 29 & 137.6 \\
\hline 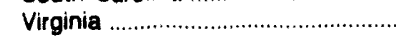 & 1 & 14 & 2 & 62 & 126 & -50.6 \\
\hline 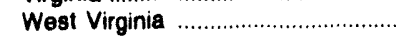 & • & $\cdot$ & * & • & $\cdot$ & - \\
\hline East South Central ............................ & 10 & 7 & 6 & 351 & 78 & 363.6 \\
\hline 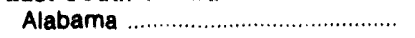 & 1 & 2 & 1 & 9 & 16 & -40.5 \\
\hline Kentucky & * & - & • & 4 & 2 & 119.2 \\
\hline 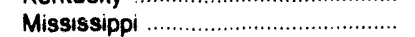 & • & • & -- & 82 & - & - \\
\hline 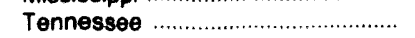 & 9 & 5 & 6 & 255 & 58 & 342.0 \\
\hline West South Central .......................... & 3 & 4 & $\mathbf{5}$ & 50 & 32 & 56.1 \\
\hline 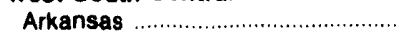 & 1 & 2 & • & 24 & 1 & 2250.1 \\
\hline 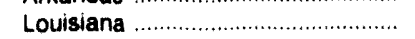 & - & • & • & 1 & 1 & -4.6 \\
\hline 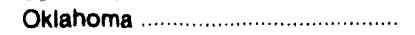 & • & - & • & 3 & 2 & 48.8 \\
\hline Texas & 2 & 2 & 4 & 23 & 28 & -20.1 \\
\hline 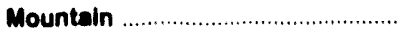 & 1 & 1 & 3 & 28 & 50 & -48.4 \\
\hline 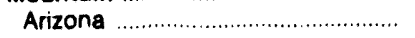 & & : & 1 & 5 & 14 & -66.4 \\
\hline 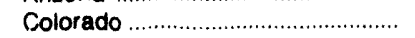 & - & * & 1 & 3 & 9 & -68.7 \\
\hline 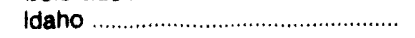 & - & - & : & • & • & - \\
\hline 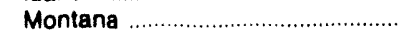 & - & $n$ & : & 1 & 1 & -.1 \\
\hline 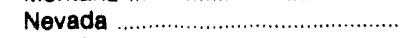 & - & • & : & 11 & 18 & -39.1 \\
\hline New Mexico ........................................ & • & • & - & 1 & 1 & -28.3 \\
\hline Utah & 1 & 1 & 1 & 6 & 7 & -18.6 \\
\hline 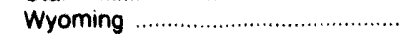 & -- & - & - & $-\infty$ & -- & - \\
\hline Pacific Contiguous & 9 & 9 & 6 & 181 & 111 & 62.5 \\
\hline California & 8 & 9 & 5 & 92 & 109 & -14.8 \\
\hline Oregon & * & - & - & 42 & 1 & 6554.6 \\
\hline Washington & • & - & 1 & 46 & 2 & 2060.0 \\
\hline Pacific Noncontiguous ................... & 240 & 276 & 211 & 2,342 & 2,195 & 6.7 \\
\hline Alaska & 58 & 91 & 42 & 657 & 578 & 13.8 \\
\hline 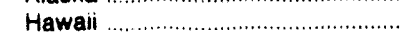 & 182 & 186 & 169 & 1,684 & 1,618 & 4.1 \\
\hline 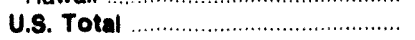 & 444 & 649 & 379 & 6,684 & 5,160 & 29.2 \\
\hline
\end{tabular}

- Data for 1993 are preliminary.

- Data for 1992 are final.

- For detailed data, the absolute value is less than 0.5 ; for percentage calculations, the absolute value, is less than 0.05 percent Notes: $\cdot G T / I C=$ Gas Turbine/Internal Combustion. - Totals may not equal sum of components because of independent rounding. •Percent difference is calculated betore rounding. -Data do not include petroleum coke.

Source: Energy Information Administration, Form ElA-759, "Monthly Power Plant Report." 
Table 25. Electric Utility Consumption of Gas by Census Division and State (Million Cubic Feet)

\begin{tabular}{|c|c|c|c|c|c|c|}
\hline \multirow{2}{*}{$\begin{array}{l}\text { Cenaus Division } \\
\text { and State }\end{array}$} & \multirow{2}{*}{$\begin{array}{l}\text { October } \\
1993\end{array}$} & \multirow{2}{*}{$\begin{array}{l}\text { Soptember } \\
1903\end{array}$} & \multirow{2}{*}{$\begin{array}{l}\text { October } \\
1992^{2}\end{array}$} & \multicolumn{3}{|c|}{ Year to Date } \\
\hline & & & & $1993^{\prime}$ & $1992^{\prime}$ & $\begin{array}{l}\text { Ditference } \\
\text { (Percent) }\end{array}$ \\
\hline 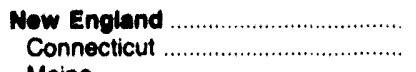 & $\begin{array}{r}2,487 \\
16\end{array}$ & $\begin{array}{r}2,808 \\
22\end{array}$ & $\begin{array}{r}3,840 \\
19\end{array}$ & $\begin{array}{r}27,890 \\
520\end{array}$ & $\begin{array}{r}38,138 \\
2,054\end{array}$ & $\begin{array}{l}-26.8 \\
-74.7\end{array}$ \\
\hline 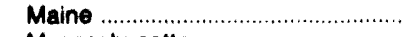 & -- & - & - & -- & $-\infty$ & -- \\
\hline 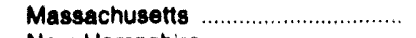 & 2,450 & 2,854 & 3,683 & 26,717 & 34.211 & -21.9 \\
\hline Now Hampshire .................................. & +- & -- & 16 & 136 & 633 & -78.6 \\
\hline 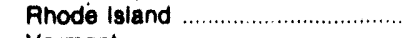 & -- & 30 & -- & 251 & 469 & -46.5 \\
\hline 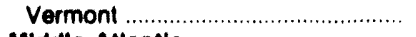 & 21 & -- & 122 & 266 & 772 & -65.5 \\
\hline 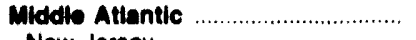 & 16,545 & 19,279 & 15,131 & 196,772 & 225,975 & -12.9 \\
\hline 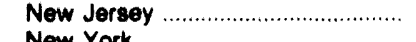 & 1.059 & 3,479 & 1,562 & 32,996 & 36,881 & -10.5 \\
\hline 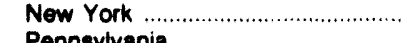 & 14,786 & 15.431 & 13,307 & 156.161 & 186,403 & -16.2 \\
\hline Pennsylvania ..................................... & 700 & 369 & 262 & 7,614 & 2,690 & 183.0 \\
\hline Eest North Central .......................... & 4,530 & 2,736 & 2,485 & 38,380 & 41,297 & -7.1 \\
\hline 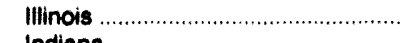 & 2,274 & 1,272 & 301 & 12,165 & 8,644 & 40.7 \\
\hline 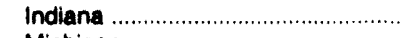 & 424 & 250 & 158 & 4,594 & 6,422 & -28.5 \\
\hline 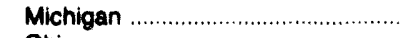 & 1.506 & 930 & 1,675 & 16,300 & 21,187 & -23.1 \\
\hline Ohio & 86 & 76 & 217 & 2,617 & 2,813 & -7.0 \\
\hline Wisconsin & 240 & 207 & 134 & 2,705 & 2,232 & 21.2 \\
\hline Wost North Central .......................... & 2,408 & 2,685 & 1,234 & 33,140 & 22,634 & 46.5 \\
\hline lowa & 200 & 191 & 200 & 3,973 & 1.866 & 113.0 \\
\hline Kansas & 1,406 & 1,708 & 546 & 19,540 & 12,638 & 54.6 \\
\hline 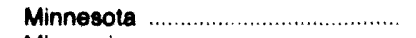 & 281 & 524 & 210 & 3,144 & 4,340 & -27.6 \\
\hline 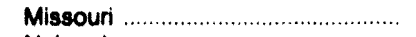 & 392 & 176 & 67 & 4,666 & 2,200 & 112.1 \\
\hline 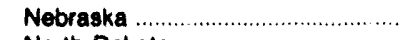 & 120 & 82 & 210 & 1,718 & 1,576 & 9.0 \\
\hline 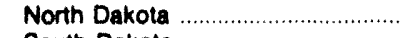 & $\cdot$ & • & -- & 1 & 1 & -47.3 \\
\hline South Dakota & 9 & 4 & 2 & 108 & 13 & 750.6 \\
\hline 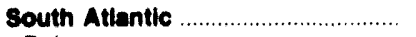 & 15,516 & 21,836 & 14,015 & 189,613 & 213,630 & -11.2 \\
\hline 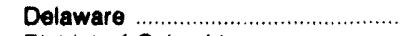 & 1,290 & 531 & 581 & 6,167 & 7,218 & -14.6 \\
\hline District of Columbia & -- & -- & $-\overline{-1}$ & - & - & -- \\
\hline Florida & 12,328 & 17,684 & 12,525 & 151,907 & 179,797 & -15.5 \\
\hline 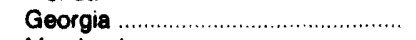 & 50 & 103 & 65 & 2,964 & 1,137 & 160.7 \\
\hline 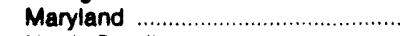 & 365 & 1,194 & 665 & 7.576 & 10,675 & -29.0 \\
\hline 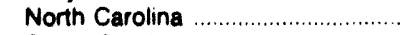 & 90 & 229 & 127 & 2,639 & 2,825 & -6.6 \\
\hline 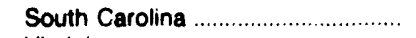 & 6 & 132 & 22 & 1,824 & 1,707 & 6.8 \\
\hline Virginia & 1,383 & 1,956 & 25 & 16,430 & 10,087 & 62.9 \\
\hline 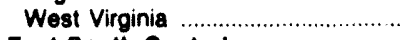 & 4 & 6 & 6 & 107 & 184 & -41.9 \\
\hline East South Central ........................... & 4,277 & 3,521 & 1,887 & 40,353 & 54,008 & -25.3 \\
\hline 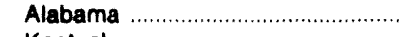 & 237 & 213 & 234 & 4,095 & 2,816 & 45.4 \\
\hline 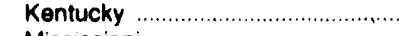 & 18 & 54 & 19 & 225 & 225 & $\cdot-1.4$ \\
\hline 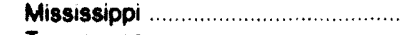 & 4,022 & 3,238 & 1,616 & 34.509 & 50,712 & -32.0 \\
\hline 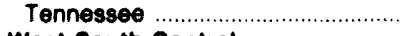 & $-\overline{-}$ & 15 & 18 & 1,524 & 255 & 496.5 \\
\hline West South Central ............................ & 129,998 & 151,559 & 108,692 & $1,295,035$ & $1,214,010$ & 6.7 \\
\hline Arkansas & 3,088 & 3,349 & 733 & 17,959 & 26.561 & -32.4 \\
\hline Louisiana .......................................... & 23,055 & 27,395 & 18,382 & 211,948 & 229.707 & -7.7 \\
\hline Oklahoma & 12,320 & 13.054 & 9,300 & 132,588 & 130,346 & 1.7 \\
\hline 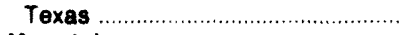 & 91,536 & 107,761 & 80,276 & 932,540 & 827,396 & 12.7 \\
\hline 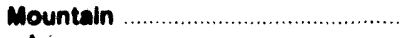 & 6,440 & 6,825 & 7,601 & 69,920 & 82,989 & -15.7 \\
\hline 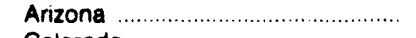 & 1,540 & 1,816 & 2,631 & 17,732 & 30,014 & -40.9 \\
\hline Colorado & 448 & 378 & 493 & 4,104 & 4,097 & .2 \\
\hline Idaho & -- & -- & -- & -- & - & - \\
\hline 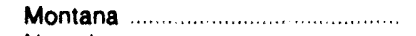 & 19 & 2 & 32 & 172 & 165 & 4.1 \\
\hline 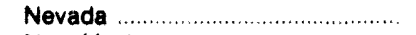 & 1,935 & 1,977 & 1,968 & 18,664 & 23.111 & -19.2 \\
\hline 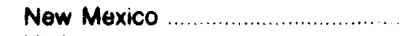 & 1,954 & 2,239 & 1,803 & 23,983 & 19,638 & 22.1 \\
\hline 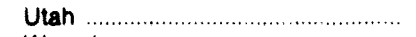 & 539 & 414 & 665 & 5,187 & 5,897 & -12.0 \\
\hline 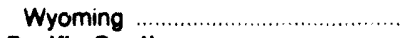 & 5 & 1 & 8 & 78 & 68 & 15.1 \\
\hline Pacific Contiguous & 48,583 & 45,448 & 55,241 & 384,327 & 484,547 & -20.7 \\
\hline 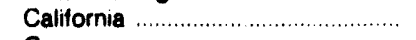 & 46,514 & 43,805 & 53,026 & 368,617 & 467,260 & -21.1 \\
\hline 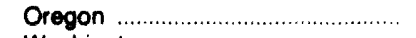 & 2,065 & 1,638 & 1,789 & 11,129 & 11,915 & -6.6 \\
\hline 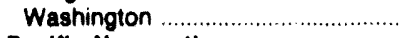 & 4 & 6 & 426 & 4,580 & 5,372 & -14.7 \\
\hline Pacific Noncontiguous ..................... & 2,407 & 2,017 & 2,514 & 22,094 & 23,476 & -5.9 \\
\hline Alaska & 2,407 & 2,017 & 2,514 & 22,094 & 23,476 & -5.9 \\
\hline 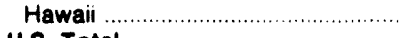 & $-\bar{x}$ & -- & -- & -- & -- & -- \\
\hline U.S. Total & 233,192 & 258,812 & 212,640 & $2,297,532$ & $2,400,704$ & -4.3 \\
\hline
\end{tabular}

' Data for 1993 are preliminary

- Data for 1992 are tinal

- For detailed data, the absolute value is less than 0.5 ; for percentage calculations, the absolute value is less than 0.05 percent. Notes: - Totals may not equal sum of components because of independent rounding.

Source: Energy Information Administration, Form ElA.759, "Monthly Power Plant Report." 
Table 26. Consumption of Gas at Steam-Fired Electric Utillty Plants by Census Division and State (Million Cubic Feet)

\begin{tabular}{|c|c|c|c|c|c|c|}
\hline \multirow{2}{*}{$\begin{array}{l}\text { Census Division } \\
\text { and State }\end{array}$} & \multirow{2}{*}{$\begin{array}{l}\text { October } \\
1893 \text { ' }\end{array}$} & \multirow{2}{*}{$\begin{array}{l}\text { September } \\
1903\end{array}$} & \multirow{2}{*}{$\begin{array}{l}\text { October } \\
1992^{2}\end{array}$} & \multicolumn{3}{|c|}{ Year to Date } \\
\hline & & & & $1903^{\prime}$ & $1902:$ & $\begin{array}{l}\text { Oitterence } \\
\text { (Pereont) }\end{array}$ \\
\hline $\begin{array}{l}\text { Now England } \\
\text { Connecticut }\end{array}$ & $\begin{array}{r}2,325 \\
16\end{array}$ & $\begin{array}{r}2,394 \\
22\end{array}$ & $\begin{array}{r}3,888 \\
18\end{array}$ & $\begin{array}{r}25,381 \\
520\end{array}$ & $\begin{array}{r}34,009 \\
2,054\end{array}$ & $\begin{array}{l}-27.2 \\
-74.7\end{array}$ \\
\hline Maine & - & $-m$ & - & - & - & - \\
\hline 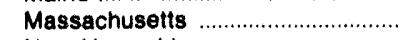 & 2,288 & 2,343 & 3,531 & 24,209 & 30,942 & -21.8 \\
\hline 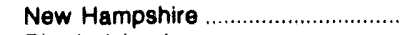 & $-\infty$ & $-\infty$ & 16 & 136 & 633 & -78.6 \\
\hline 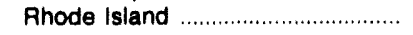 & - & 30 & - & 251 & 469 & -46.5 \\
\hline 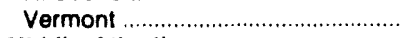 & 21 & $-\overline{-}$ & 122 & 268 & 772 & -65.5 \\
\hline & $\begin{array}{r}15,724 \\
382\end{array}$ & $\begin{array}{r}17,602 \\
2,447\end{array}$ & $\begin{array}{r}14,405 \\
879\end{array}$ & 182,583 & 216,171 & -16.1 \\
\hline $\begin{array}{l}\text { New Jersey } \\
\text { New York }\end{array}$ & $\begin{array}{r}382 \\
14,660\end{array}$ & $\begin{array}{r}2,447 \\
14,728\end{array}$ & $\begin{array}{r}879 \\
13,266\end{array}$ & $\begin{array}{r}23,912 \\
151,465\end{array}$ & $\begin{array}{r}29,316 \\
183,440\end{array}$ & $\begin{array}{l}-18.4 \\
-17.4\end{array}$ \\
\hline 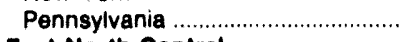 & 682 & 327 & 261 & 7,206 & 2,415 & 198.3 \\
\hline East North Central .............................. & 4,285 & 2,592 & 2,260 & 34,840 & 37,915 & -8.6 \\
\hline 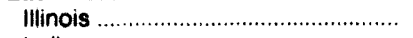 & 2,264 & 1.258 & 287 & 11,862 & 8,388 & 41.4 \\
\hline 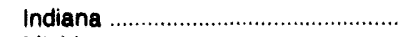 & 314 & 203 & 139 & 3,728 & 6,171 & -39.6 \\
\hline 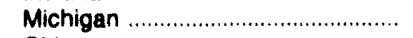 & 1,481 & 910 & 1,530 & 15,399 & 18,008 & -18.0 \\
\hline Ohio & 27 & 28 & 192 & 1,318 & 2,346 & -43.8 \\
\hline 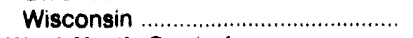 & 199 & 193 & 121 & 2,341 & 2,002 & 16.9 \\
\hline 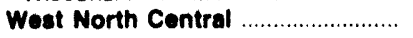 & 2,147 & 2,333 & 981 & 28,375 & 18,717 & 40.0 \\
\hline 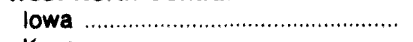 & 183 & 172 & 146 & 2,681 & 1,386 & 93.4 \\
\hline 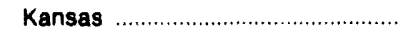 & 1,242 & 1,480 & 404 & 16,365 & 10,572 & 54.8 \\
\hline 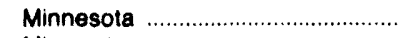 & 274 & 510 & 209 & 2,904 & 3,899 & -27.4 \\
\hline 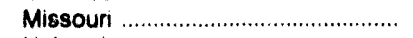 & 363 & 100 & 39 & 3,209 & 1,479 & 116.9 \\
\hline Nebraska & 84 & 71 & 182 & 1,158 & 1,271 & -8.9 \\
\hline 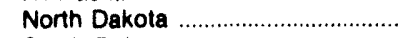 & $\cdot$ & • & - & . & 1 & NM \\
\hline South Dakota & 1 & $\cdot$ & 1 & 59 & 9 & 565.1 \\
\hline 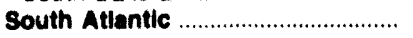 & 12,052 & 18,746 & 12,488 & 146,037 & 188,200 & -20.8 \\
\hline Delaware & 307 & 62 & 342 & 1,114 & 5,277 & -78.9 \\
\hline District of Columbia ............................. & - & -- & -- & - & - & - \\
\hline Florida & 11,539 & 15,697 & 11,594 & 138,237 & 188,468 & -17.8 \\
\hline Georgia & 25 & 102 & 57 & 2,582 & 1,052 & 145.3 \\
\hline 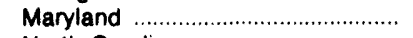 & 125 & 789 & 460 & 4,235 & 8,194 & -48.3 \\
\hline North Carolina ................................... & -- & -- & - & - & - & NM \\
\hline South Carolina & 2 & 9 & 5 & 243 & 1,019 & -76.1 \\
\hline 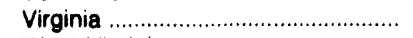 & 48 & 81 & 2 & 319 & 1,086 & -70.9 \\
\hline 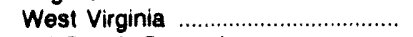 & 4 & 6 & 6 & 107 & 184 & -41.9 \\
\hline East South Central ............................ & 3,032 & 2,318 & 938 & 25,323 & 40,003 & -38.2 \\
\hline Alabama & 226 & 213 & 211 & 2,678 & 2,514 & 6.6 \\
\hline 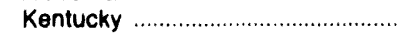 & 16 & 52 & 14 & 178 & 188 & -9.1 \\
\hline 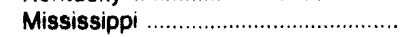 & 2,790 & 2,053 & 713 & 22,466 & 38,273 & -41.3 \\
\hline 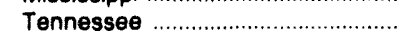 & - & -- & - & - & - & $N M^{-4.0}$ \\
\hline 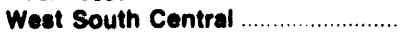 & 124,071 & 144,333 & 101,964 & $1,234,005$ & $1,164,115$ & $\mathbf{7 . 0}$ \\
\hline 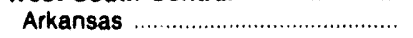 & 3,088 & 3,349 & 733 & 17,958 & 26,561 & -32.4 \\
\hline 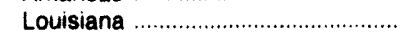 & 22,637 & 26,674 & 17,769 & 205,495 & 224,234 & -8.4 \\
\hline Oklahoma & 9,686 & 10.955 & 7,970 & 107,857 & 104,239 & 3.5 \\
\hline Texas & 88,659 & 103,355 & 75,492 & 803,375 & 799,080 & 13.1 \\
\hline 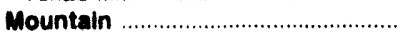 & 5,083 & 5,255 & 6,477 & 56,414 & 70,484 & -21.3 \\
\hline 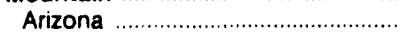 & 613 & 797 & 1,916 & 8,713 & 21,157 & -58.8 \\
\hline Colorado & 417 & 364 & 438 & 3,625 & 3,580 & 1.3 \\
\hline Idaho & - & - & -- & - & - & - \\
\hline 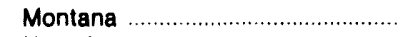 & 5 & 1 & 3 & 53 & 86 & -44.3 \\
\hline Nevada & 1,735 & 1,538 & 1,918 & 15,667 & 21,667 & -27.7 \\
\hline New Mexico & 1,945 & 2,179 & 1,785 & 23,548 & 19,358 & 21.6 \\
\hline Utah & 363 & 375 & 408 & 3,729 & 4,528 & -17.7 \\
\hline 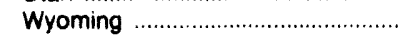 & 5 & 1 & 8 & 78 & 68 & 15.1 \\
\hline Pacific Contiguous ............................... & 44,135 & 42,623 & 50,916 & 369,450 & 461,737 & -20.4 \\
\hline 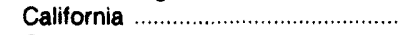 & 44,133 & 42,621 & 50,916 & 358,435 & 451,694 & -20.4 \\
\hline Oregon & - & -- & -- & - & - & NM \\
\hline Washington & 2 & 2 & $\cdot$ & 16 & 43 & -63.3 \\
\hline Pacifle Noncontiguous ................... & - & - & - & - & - & NM \\
\hline Alaska & -- & -- & - & - & - & NM \\
\hline 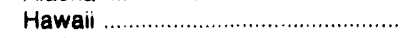 & -- & - & - & -- & - & - \\
\hline 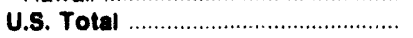 & 212,854 & 236,097 & 194,104 & $2,000,695$ & $2,200,250$ & -5.4 \\
\hline
\end{tabular}

Data for 1993 are preliminary.

- Data for 1992 are final.

- For detailed data, the absolute value is less than 0.5 ; for percentage calculations, the absolute value is less than 0.05 percent.

= Calculation not meaningful.

Notes: - Totals may not equal sum of components because of independent rounding.

Source: Energy Intormation Administration, Form ElA.759, "Monthly Power Plant Report." 
Table 27. Consumption of Gas at GT/IC Electric Utility Plants by Census Division and State (Million Cubic Feet)

\begin{tabular}{|c|c|c|c|c|c|c|}
\hline \multirow{2}{*}{$\begin{array}{c}\text { Census Divialon } \\
\text { and State }\end{array}$} & \multirow{2}{*}{$\begin{array}{l}\text { October } \\
1993^{1}\end{array}$} & \multirow{2}{*}{$\begin{array}{l}\text { September } \\
1903^{1}\end{array}$} & \multirow{2}{*}{$\begin{array}{l}\text { October } \\
1902^{2}\end{array}$} & \multicolumn{3}{|c|}{ Year to Date } \\
\hline & & & & $1993^{\prime}$ & $1992^{2}$ & $\begin{array}{l}\text { Difference } \\
\text { (Percent) }\end{array}$ \\
\hline Now England & 162 & 512 & 152 & 2,509 & 3,289 & -23.3 \\
\hline 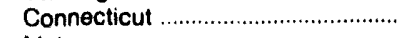 & -- & - & - & - & - & - \\
\hline Maine & - & - & -- & - & - & - \\
\hline 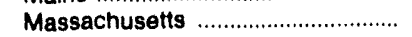 & 162 & 512 & 152 & 2,509 & 3,269 & -23.3 \\
\hline New Hampshire ................................. & - & - & -- & - & - & NM \\
\hline Rhode Island & - & - & - & - & -- & - \\
\hline 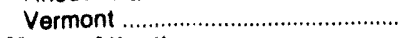 & - & - & - & - & $\overline{-i n}$ & - \\
\hline Middle Attantic ..................................... & 821 & 1,777 & 725 & 14,189 & 10,804 & 31.3 \\
\hline 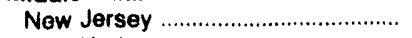 & 677 & 1,031 & 683 & 9,085 & 7,566 & 20.1 \\
\hline New York & 126 & 704 & 41 & 4,697 & 2,963 & 58.5 \\
\hline Pennsylvania ...................................... & 18 & 42 & 1 & 408 & 275 & 48.4 \\
\hline East North Central ............................. & 245 & 144 & 216 & 3,732 & 3,382 & 10.4 \\
\hline 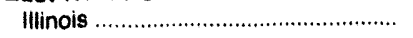 & 10 & 14 & 15 & 303 & 255 & 18.6 \\
\hline 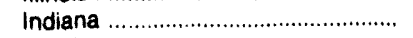 & 110 & 48 & 18 & 866 & 251 & 245.4 \\
\hline 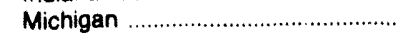 & 25 & 20 & 145 & 900 & 2,179 & -58.7 \\
\hline 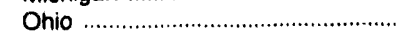 & 59 & 47 & 25 & 1,299 & 467 & 178.1 \\
\hline 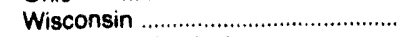 & 41 & 15 & 13 & 364 & 230 & 58.4 \\
\hline Weat North Central ........................... & 280 & 352 & 254 & 6,774 & 3,917 & 72.9 \\
\hline 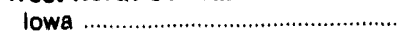 & 17 & 19 & 54 & 1,293 & 480 & 169.4 \\
\hline 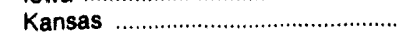 & 163 & 228 & 142 & 3,175 & 2,067 & 53.6 \\
\hline 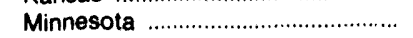 & 7 & 14 & 2 & 239 & 341 & -29.8 \\
\hline 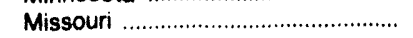 & 29 & 76 & 28 & 1,457 & 720 & 102.3 \\
\hline 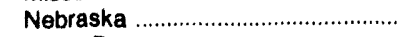 & 36 & 11 & 27 & 559 & 305 & 83.5 \\
\hline 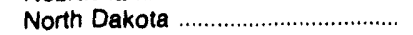 & - & - & - & $\cdot$ & $\cdot$ & NM \\
\hline 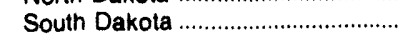 & 8 & 4 & 1 & 50 & 4 & 1165.9 \\
\hline 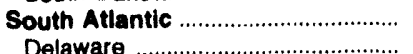 & $\begin{array}{r}3,464 \\
983\end{array}$ & $\begin{array}{r}5,080 \\
469\end{array}$ & $\begin{array}{r}1,549 \\
239\end{array}$ & $\begin{array}{r}42,777 \\
5,054\end{array}$ & $\begin{array}{r}28,341 \\
1,941\end{array}$ & $\begin{array}{r}50.9 \\
160.4\end{array}$ \\
\hline $\begin{array}{l}\text { Delaware } \\
\text { District of Columbia }\end{array}$ & - & $-{ }^{408}$ & $-{ }^{200}$ & - & $\begin{array}{l}1,741 \\
-\end{array}$ & 100.4 \\
\hline 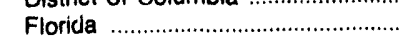 & 789 & 1,987 & 930 & 13,670 & 11,330 & 20.6 \\
\hline Georgia & 25 & 1 & 7 & 383 & 85 & 351.5 \\
\hline 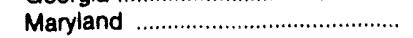 & 239 & 405 & 205 & 3,340 & 2,481 & 34.7 \\
\hline 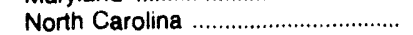 & 90 & 229 & 127 & 2,639 & 2,825 & -6.6 \\
\hline 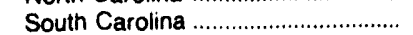 & 4 & 124 & 17 & 1,580 & 688 & 129.8 \\
\hline Virginia & 1,334 & 1,875 & 23 & 16,111 & 8,990 & 79.2 \\
\hline West Virginia & - & - & -- & -- & $\ldots$ & -- \\
\hline 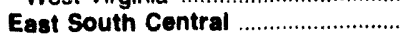 & 1,245 & 1,203 & 949 & 15,030 & 13,026 & 15.4 \\
\hline Alabama & 11 & - & 23 & 1,417 & 302 & 368.7 \\
\hline 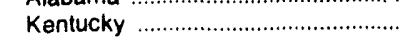 & 2 & 2 & 5 & 47 & 29 & 61.4 \\
\hline 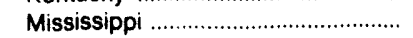 & 1,233 & 1,186 & 903 & 12,043 & 12,439 & -3.2 \\
\hline 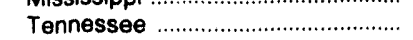 & $-\infty$ & 15 & 18 & 1,524 & 255 & 496.5 \\
\hline 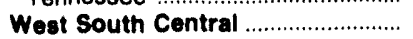 & $\mathbf{5 , 9 2 8}$ & 7,226 & 6,728 & 60,350 & 59,895 & .8 \\
\hline 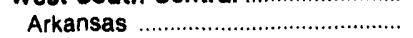 & - & $\cdot$ & - & 1 & -- & NM \\
\hline 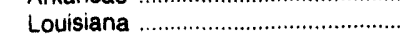 & 418 & 721 & 613 & 6,453 & 5,473 & 17.9 \\
\hline Oklahoma & 2,634 & 2,099 & 1,330 & 24,731 & 26,107 & -5.3 \\
\hline Texas & 2,876 & 4,405 & 4,785 & 29,165 & 28,316 & 3.0 \\
\hline 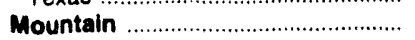 & 1,357 & 1,571 & 1,124 & 14,508 & 12,535 & 15.7 \\
\hline Arizona & 926 & 1,018 & 715 & 9,020 & 8,856 & 1.8 \\
\hline 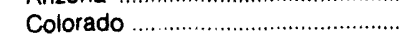 & 30 & 14 & 55 & 478 & 517 & -7.5 \\
\hline Idaho & -- & - & -- & - & - & - \\
\hline 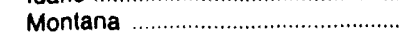 & 14 & $\cdot$ & 29 & 119 & 70 & 70.4 \\
\hline 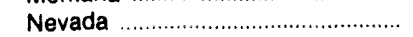 & 200 & 439 & 50 & 2,996 & 1,444 & 107.5 \\
\hline 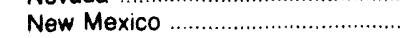 & 10 & 60 & 18 & 435 & 280 & 55.4 \\
\hline Utah & 177 & 39 & 257 & 1,458 & 1,368 & 6.6 \\
\hline 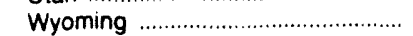 & -- & - & - & -- & - & - \\
\hline Pacific Contiguous ........................... & 4,448 & 2,825 & 4,326 & 24,876 & 32,810 & -24.2 \\
\hline California & 2,382 & 1,183 & 2,111 & 9,182 & 15,566 & -41.0 \\
\hline Oregon & 2,065 & 1,638 & 1,789 & 11,129 & 11,915 & -6.6 \\
\hline Washington & 1 & 4 & 426 & 4,565 & 5,329 & -14.3 \\
\hline Pacific Noncontiguous & 2,407 & 2,017 & 2,514 & 22,094 & 23,476 & -5.9 \\
\hline Alaska & 2,407 & 2,017 & 2,514 & 22,094 & 23,476 & -5.9 \\
\hline 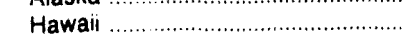 & - & - & - & - & - & - \\
\hline U.S. Total & 20,339 & 22,715 & 18,537 & 206,837 & 191,454 & 8.0 \\
\hline
\end{tabular}

- Data for 1993 are preliminary.

- Data for 1992 are final.

- For detailed data, the absolute value is less than 0.5 ; for percentage calculations, the absolute value is less than 0.05 percent.

$\omega$ = Calculation not meaningful.

Notes: $\bullet G T / I C=$ Gas Turbine/Internal Combustion. $\bullet$ Totals may not equal sum of components because of independent rounding Source: Energy Information Administration, Form EIA-759, "Monthly Power Plant Report." 


\section{Fossil-Fuel Stocks at U.S. Electric Utilities}

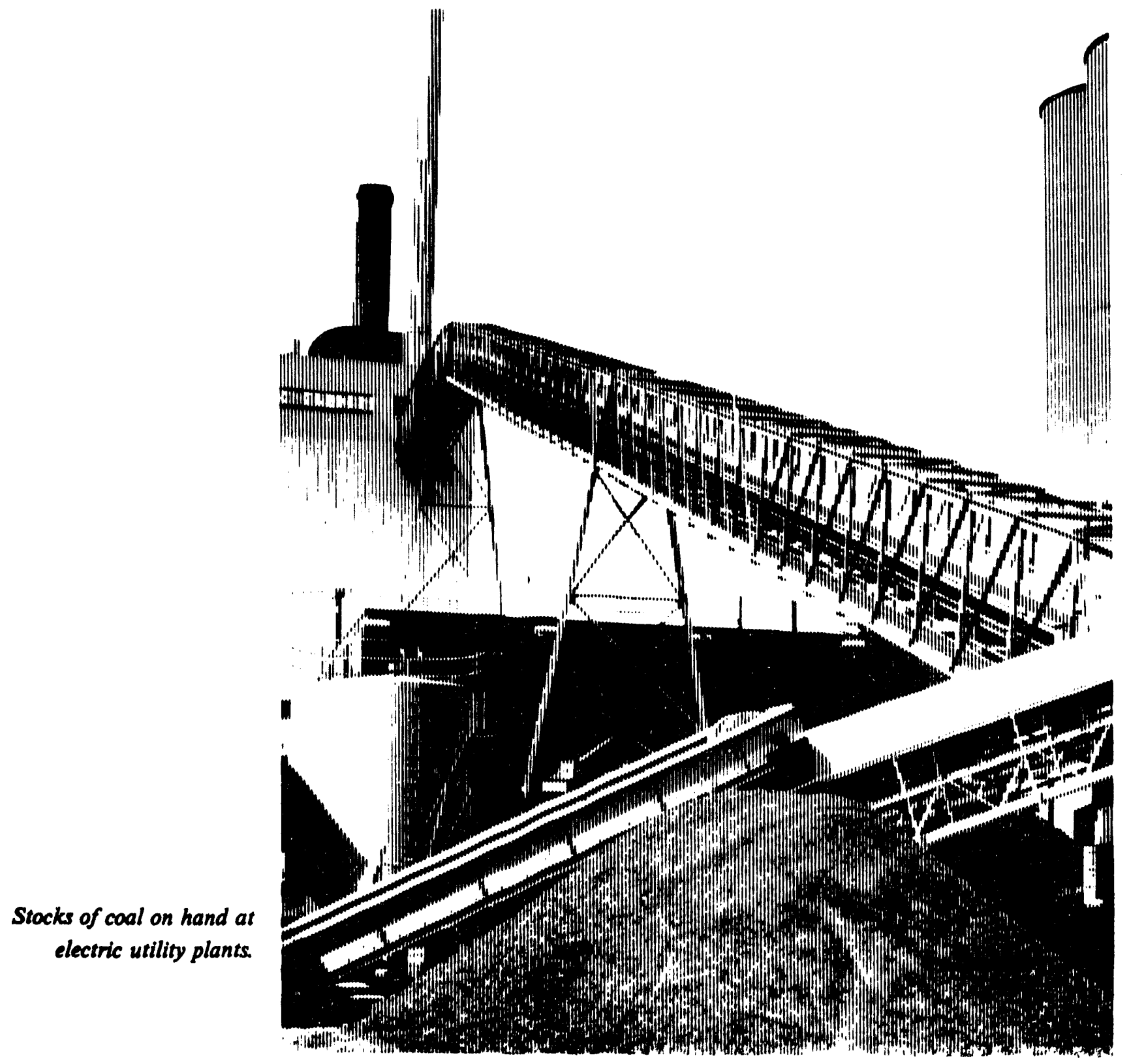


Table 28. U.S. Electric Utility Stocks of Coal and Patribum, 1983 Through October 1993

\begin{tabular}{|c|c|c|c|c|c|c|c|c|}
\hline \multirow{2}{*}{ Putiod } & \multicolumn{4}{|c|}{$\begin{array}{c}\text { Coel } \\
\text { (thousend enent tono) }\end{array}$} & \multicolumn{3}{|c|}{$\begin{array}{l}\text { Pciroleum } \\
\text { (thousend barrela) }\end{array}$} & \multirow{2}{*}{$\begin{array}{l}\text { Potroinen } \\
\text { Colte } \\
\text { (thousend } \\
\text { chort } \\
\text { tone) }\end{array}$} \\
\hline & Anthrecte' & Dhuminous" & Uentit & Total & Uem & Heary & Totel & \\
\hline 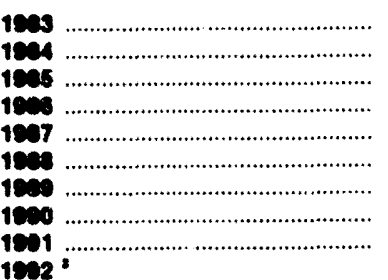 & $\begin{array}{l}6,507 \\
6,710 \\
7,199 \\
7,000 \\
6,940 \\
6,531 \\
6,403 \\
6,490 \\
6,513\end{array}$ & $\begin{array}{l}145,260 \\
167,110 \\
142,144 \\
144,695 \\
158,670 \\
135,434 \\
122,097 \\
142,060 \\
146,397\end{array}$ & $\begin{array}{l}3,041 \\
5,001 \\
7,043 \\
6,042 \\
7,187 \\
6,512 \\
8,400 \\
7,016 \\
5,008\end{array}$ & $\begin{array}{l}155,600 \\
170,727 \\
184,370 \\
181,003 \\
170,717 \\
149,607 \\
135,100 \\
185,163 \\
157,070\end{array}$ & $\begin{array}{l}18,101 \\
10,116 \\
18,913 \\
18,240 \\
18,760 \\
18,010 \\
18,924 \\
18,471 \\
18,367\end{array}$ & $\begin{array}{l}70,573 \\
62,605 \\
57,304 \\
63,041 \\
85,000 \\
64,107 \\
47,449 \\
67,050 \\
84,053\end{array}$ & $\begin{array}{l}00,375 \\
07,610 \\
73,010 \\
73,111 \\
70,127 \\
60,295 \\
01,270 \\
03,601 \\
74,603\end{array}$ & $\begin{array}{r}85 \\
50 \\
40 \\
40 \\
51 \\
10 \\
105 \\
4 \\
70\end{array}$ \\
\hline 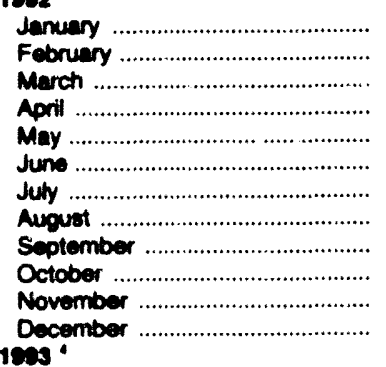 & $\begin{array}{l}6,488 \\
6,455 \\
6,388 \\
6,379 \\
6,370 \\
6,355 \\
6,341 \\
6,343 \\
6,329 \\
6,304 \\
6,273 \\
6,215\end{array}$ & $\begin{array}{l}143,466 \\
146,338 \\
147,978 \\
149,824 \\
152,275 \\
151,224 \\
141,613 \\
140,166 \\
140,409 \\
144,068 \\
145,406 \\
142,156\end{array}$ & $\begin{array}{l}5,683 \\
5,352 \\
5,658 \\
6,387 \\
6,867 \\
6,598 \\
6,449 \\
6,071 \\
5,946 \\
6,487 \\
6,169 \\
5,759\end{array}$ & $\begin{array}{l}155,637 \\
158,145 \\
160,032 \\
162,591 \\
165,512 \\
164,176 \\
154,403 \\
152,580 \\
152,685 \\
156,859 \\
157,849 \\
154,130\end{array}$ & $\begin{array}{l}15,712 \\
15,655 \\
15,589 \\
15,371 \\
15,214 \\
15,117 \\
14,995 \\
15,456 \\
15,251 \\
15,351 \\
15,302 \\
15,714\end{array}$ & $\begin{array}{l}\mathbf{5 3 , 1 3 6} \\
\mathbf{5 4 , 7 5 0} \\
\mathbf{5 4 , 5 1 3} \\
\mathbf{5 2 , 9 1 5} \\
\mathbf{5 5 , 1 4 4} \\
\mathbf{5 3 , 7 9 4} \\
\mathbf{5 3 , 4 4 5} \\
\mathbf{5 4 , 4 3 4} \\
\mathbf{5 2 , 7 3 1} \\
\mathbf{5 2 , 8 1 8} \\
\mathbf{5 3 , 6 3 2} \\
\mathbf{5 6 , 1 3 5}\end{array}$ & $\begin{array}{l}68,849 \\
70,408 \\
70,103 \\
68,186 \\
70,358 \\
68,910 \\
68,440 \\
69,890 \\
67,982 \\
68,289 \\
68,934 \\
71,849\end{array}$ & $\begin{array}{l}75 \\
62 \\
56 \\
47 \\
63 \\
67 \\
56 \\
48 \\
51 \\
55 \\
59 \\
67\end{array}$ \\
\hline 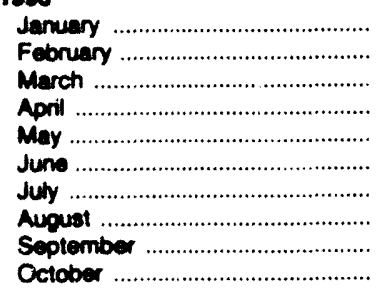 & $\begin{array}{l}6,166 \\
6,107 \\
6,036 \\
5,802 \\
5,773 \\
5,786 \\
5,755 \\
5,745 \\
5,735 \\
5,718\end{array}$ & $\begin{array}{l}138,685 \\
134,674 \\
132,183 \\
136,159 \\
138,165 \\
133,673 \\
115,194 \\
102,612 \\
100,891 \\
102,801\end{array}$ & $\begin{array}{l}5,521 \\
5,357 \\
5,758 \\
6,088 \\
6,132 \\
6,008 \\
5,688 \\
5,651 \\
6,147 \\
6,687\end{array}$ & $\begin{array}{l}150,371 \\
146,139 \\
143,978 \\
148,049 \\
150,070 \\
145,448 \\
126,685 \\
114,008 \\
112,773 \\
115,208\end{array}$ & $\begin{array}{l}15,856 \\
15,205 \\
15,001 \\
14,835 \\
14,682 \\
14,923 \\
14,605 \\
14,830 \\
14,760 \\
14,883\end{array}$ & $\begin{array}{l}53,781 \\
50,008 \\
45,313 \\
47,858 \\
50,422 \\
48,294 \\
47,401 \\
43,843 \\
45,913 \\
46,293\end{array}$ & $\begin{array}{l}69,736 \\
65,213 \\
60,314 \\
62,793 \\
65,103 \\
64,217 \\
62,007 \\
58,772 \\
60,673 \\
61,256\end{array}$ & $\begin{array}{l}65 \\
60 \\
68 \\
77 \\
82 \\
82 \\
73 \\
90 \\
62 \\
69\end{array}$ \\
\hline
\end{tabular}

- Anthracite includes anthracite silt stored off-site.

- Bituminous cosl includes subbituminous coll.

- Dat for 1992 and prior years are final.

- Data for 1893 are preliminary.

Notes: Totals may not equal sum of components because of independent rounding. eStocks are end-ol-month stocks at electric utilities. Source: Eneroy Information Administration, Form EIA-758. "Monthly Power Plant Report," and predeceseco forms. 
Table 29. Electric Utility Stocks of Coal by NERC Region and Hawali (Thousand Short Tons)

\begin{tabular}{|c|c|c|c|c|c|}
\hline $\begin{array}{c}\text { NERC Region } \\
\text { and Hawall }\end{array}$ & $\begin{array}{l}\text { October } \\
1993\end{array}$ & $\begin{array}{l}\text { September } \\
1993\end{array}$ & $\begin{array}{l}\text { October } \\
1992^{2}\end{array}$ & $\begin{array}{c}\text { Monthly Difference } \\
\text { (Percent) }\end{array}$ & $\begin{array}{c}\text { Yoarty Difference } \\
\text { (Percent) }\end{array}$ \\
\hline $\begin{array}{l}\text { ECAR } \\
\text { ERCOT } \\
\text { MAAC } \\
\text { MAIN } \\
\text { MAPP (U.S.) } \\
\text { NPCC (U.S.) } \\
\text { SERC } \\
\text { SPP } \\
\text { WSCC (U.S.) } \\
\text { COntiguous U.S. } \\
\text { ASCC } \\
\text { Hawaii } \\
\text { U.S. Total }\end{array}$ & $\begin{array}{r}29,582 \\
5,105 \\
12,228 \\
8,930 \\
9,673 \\
2,272 \\
19,021 \\
11,791 \\
16,600 \\
115,201 \\
-\quad 5 \\
115,206\end{array}$ & $\begin{array}{r}30,154 \\
4,702 \\
11,975 \\
8,702 \\
9,129 \\
2,411 \\
18,384 \\
10,592 \\
16,719 \\
112,768 \\
-\quad 5 \\
-112,773\end{array}$ & $\begin{array}{r}43,880 \\
6,688 \\
15,296 \\
15,292 \\
12,727 \\
3,231 \\
23,288 \\
17,498 \\
18,952 \\
156,851 \\
7 \\
-\quad 7 \\
156,859\end{array}$ & $\begin{array}{r}-1.9 \\
8.6 \\
2.1 \\
2.6 \\
6.0 \\
-5.8 \\
3.5 \\
11.3 \\
-.7 \\
2.2 \\
-1.8 \\
-\quad 2.2\end{array}$ & $\begin{array}{r}-32.6 \\
-23.7 \\
-20.1 \\
-41.6 \\
-24.0 \\
-29.7 \\
-18.3 \\
-32.6 \\
-12.4 \\
-28.6 \\
-32.4 \\
- \\
-26.6\end{array}$ \\
\hline
\end{tabular}

Data for 1993 are preliminary

2 Data for 1992 are final.

Notes: - Totals may not equal sum of components because of independent rounding. • Percent difference is calculated betore rounding. $\bullet$ Coal includes lignite, bituminous coal, subbituminous coal, and anthracite. - Stocks are end-of-month stocks at electric utilities.

Source: Energy Information Administration. Form EIA-759, "Monthly Power Plant Report."

Table 30. Electric Utility Stocks of Petroleum by NERC Region and Hawail (Thousand Barrels)

\begin{tabular}{|c|c|c|c|c|c|}
\hline $\begin{array}{c}\text { NE'AC Region } \\
\text { and Hawall }\end{array}$ & $\begin{array}{l}\text { October } \\
1993^{1}\end{array}$ & $\begin{array}{c}\text { September } \\
1993\end{array}$ & $\begin{array}{l}\text { October } \\
1992^{2}\end{array}$ & $\begin{array}{c}\text { Monthly Difierence } \\
\text { (Percent) }\end{array}$ & $\begin{array}{l}\text { Yearty Difterence } \\
\text { (Percent) }\end{array}$ \\
\hline $\begin{array}{l}\text { ECAR } \\
\text { MACOT } \\
\text { MAIN } \\
\text { MAPP (U.S.) } \\
\text { NPCC (U.S.) } \\
\text { SERC } \\
\text { SPP } \\
\text { WSCC (U.S.) } \\
\text { Contiguous U.S. } \\
\text { ASCC } \\
\text { Hawaii .... } \\
\text { U.S. Total }\end{array}$ & $\begin{array}{r}1,531 \\
5,150 \\
5,538 \\
1,011 \\
734 \\
12,316 \\
11,092 \\
4,256 \\
18,420 \\
60,047 \\
296 \\
913 \\
61,256\end{array}$ & $\begin{array}{r}1,545 \\
5,165 \\
5,226 \\
975 \\
742 \\
11,579 \\
11,124 \\
4,691 \\
18,494 \\
59,542 \\
291 \\
841 \\
60,673\end{array}$ & $\begin{array}{r}1,738 \\
5,236 \\
5,534 \\
1,475 \\
753 \\
16,236 \\
11,259 \\
4,472 \\
20,437 \\
67,139 \\
275 \\
854 \\
68,269\end{array}$ & $\begin{array}{r}-0.9 \\
-.3 \\
6.0 \\
3.7 \\
-1.1 \\
6.4 \\
-.3 \\
-9.3 \\
-.4 \\
.8 \\
1.7 \\
8.6 \\
1.0\end{array}$ & $\begin{array}{r}-11.9 \\
-1.6 \\
.1 \\
-31.5 \\
-2.5 \\
-24.1 \\
-1.5 \\
-4.8 \\
-9.9 \\
-10.8 \\
7.3 \\
6.9 \\
-10.3\end{array}$ \\
\hline
\end{tabular}

' Data for 1993 are preliminary.

2 Data for 1992 are final.

Notes: - Totals may not equal sum of components because of independent rounding. •Percent difference is calculated before rounding. $\bullet$ Data do not include petroleum coke. Stocks are end-of-month stocks at electric utilities.

Source: Energy Information Administration, Form ElA.759. "Monthly Power Plant Feport." 
Table 31. Electric Utility Stocks of Coal by Census Division and State (Thousand Short Tons)

\begin{tabular}{|c|c|c|c|c|c|}
\hline $\begin{array}{l}\text { Ceneus Division } \\
\text { and State }\end{array}$ & $\begin{array}{l}\text { October } \\
1993\end{array}$ & $\begin{array}{l}\text { Soptember } \\
1993\end{array}$ & $\begin{array}{l}\text { October } \\
1992 ?\end{array}$ & $\begin{array}{c}\text { Monthly Difference } \\
\text { (Percent) }\end{array}$ & $\begin{array}{l}\text { Yearly Difference } \\
\text { (Percent) }\end{array}$ \\
\hline 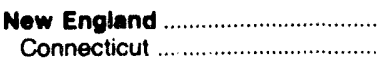 & $\begin{array}{r}1,126 \\
168\end{array}$ & $\begin{array}{r}1,245 \\
116\end{array}$ & $\begin{array}{r}1,246 \\
139\end{array}$ & $\begin{array}{l}-9.5 \\
45.0\end{array}$ & $\begin{array}{r}\mathbf{- 9 . 6} \\
21.0\end{array}$ \\
\hline 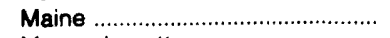 & - & - & - & -- & -- \\
\hline 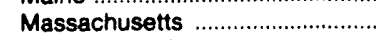 & 577 & 762 & 682 & -24.3 & -15.5 \\
\hline New Hampshire ............................. & 381 & 367 & 425 & 3.9 & -10.1 \\
\hline 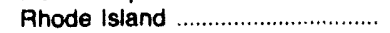 & - & - & - & - & - \\
\hline 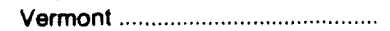 & - & -- & - & - & -- \\
\hline Middle Attantic ................................. & 13,090 & 13,331 & 17,476 & -1.8 & -25.1 \\
\hline 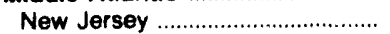 & 498 & 548 & 762 & -9.2 & -34.7 \\
\hline 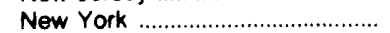 & 1.145 & 1,166 & 1,985 & -1.8 & -42.3 \\
\hline 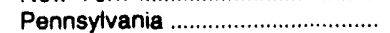 & 11,447 & 11,616 & 14,728 & -1.5 & -22.3 \\
\hline East North Central ........................ & 28,070 & 27,754 & 42,701 & 1.1 & -34.3 \\
\hline 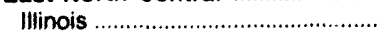 & 4,616 & 4,780 & 7,748 & -3.4 & -40.4 \\
\hline 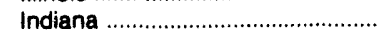 & 6.589 & 6,735 & 12,391 & -2.2 & -46.8 \\
\hline 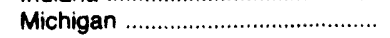 & 6,399 & 6,171 & 8,106 & 3.7 & -21.1 \\
\hline 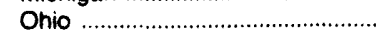 & $7 ; 458$ & 7,390 & 10,141 & .9 & -26.5 \\
\hline Wisconsin & 3,007 & 2,678 & 4,314 & 12.3 & -30.3 \\
\hline West North Central ....................... & 14,382 & 13,546 & 21,008 & 6.2 & -31.5 \\
\hline lowa & 3,526 & 3,209 & 4,810 & 9.9 & -26.7 \\
\hline Kansas & 2,112 & 2,073 & 3,025 & 1.9 & -30.2 \\
\hline Minnesota & 1,299 & 1,214 & 2,180 & 7.0 & -40.4 \\
\hline 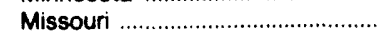 & 3,416 & 3,061 & 6,485 & 11.6 & -47.3 \\
\hline 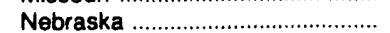 & 1,251 & 1,267 & 1,838 & -1.3 & -32.0 \\
\hline North Dakota & 2,501 & 2,460 & 2,386 & 1.7 & 4.8 \\
\hline South Dakota & 276 & 262 & 284 & 5.2 & -2.8 \\
\hline 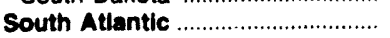 & 19,550 & 18,848 & 28,110 & 3.7 & -25.1 \\
\hline 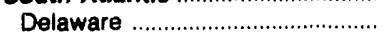 & 330 & 220 & 365 & 50.1 & -9.7 \\
\hline District of Columbia .......................... & - & - & - & - & $\ldots$ \\
\hline Florida & 3,119 & 3,032 & 4,108 & 2.9 & -24.1 \\
\hline Georgia & 2,907 & 2,861 & 3,676 & 1.6 & -20.9 \\
\hline 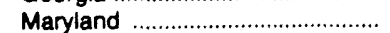 & 1,687 & 1.742 & 2,219 & -3.1 & -24.0 \\
\hline North Carolina & 3,025 & 2.594 & 3,612 & 16.6 & -16.3 \\
\hline 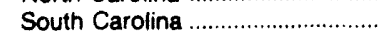 & 1,596 & 1,339 & 1,915 & 19.2 & -16.6 \\
\hline Virginia & 1,465 & 1,361 & 1,930 & 7.7 & -24.1 \\
\hline West Virginia & 5,421 & 5,699 & 8,284 & -4.9 & -34.6 \\
\hline East South Central .......................... & 10,000 & 10,310 & 11,686 & -3.0 & -14.4 \\
\hline Alabama & 3,168 & 3,231 & 3,837 & -2.0 & -17.4 \\
\hline 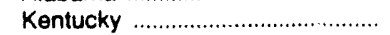 & 4,472 & 4,636 & 4,808 & -3.5 & -7.0 \\
\hline 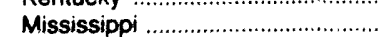 & 349 & 415 & 732 & -15.9 & -52.3 \\
\hline 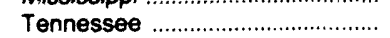 & 2,011 & 2,028 & 2,309 & -.8 & $-12,9$ \\
\hline 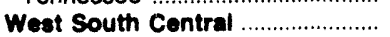 & 12,371 & 11,004 & 17,665 & 12.4 & -30.0 \\
\hline Arkansas & 1,220 & 981 & 1,661 & 24.3 & -26.6 \\
\hline 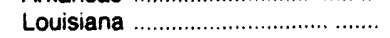 & 1,608 & 1,316 & 2,299 & 22.2 & -30.0 \\
\hline Oklahoma & 2,087 & 1,772 & 2,865 & 17.8 & -27.2 \\
\hline 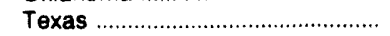 & 7,456 & 6,935 & 10,840 & 7.5 & -31.2 \\
\hline 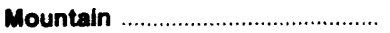 & 15,438 & 15,384 & 17,565 & .3 & -12.1 \\
\hline Arizona & 3,707 & 3,624 & 4,146 & 2.3 & -10.6 \\
\hline 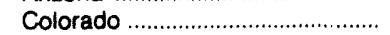 & 3,445 & 3,402 & 3,912 & 1.2 & -11.9 \\
\hline 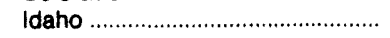 & - & - & - & - & - \\
\hline 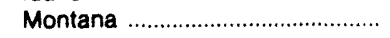 & 795 & 816 & 781 & -2.6 & 1.8 \\
\hline 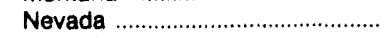 & 1,094 & 1,125 & 1,485 & -2.8 & -26.4 \\
\hline 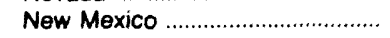 & 1,431 & 1,369 & 1,379 & 4.6 & 3.8 \\
\hline Utah & 3,222 & 3,321 & 3,352 & -3.0 & -3.9 \\
\hline 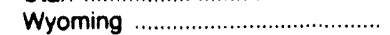 & 1,745 & 1,727 & 2,511 & 1.0 & -30.5 \\
\hline Pacific Contiguous & 1,175 & 1,346 & 1,394 & -12.7 & -15.7 \\
\hline California & - & - & - & - & - \\
\hline Oregon & 509 & 586 & 618 & -13.2 & -17.7 \\
\hline 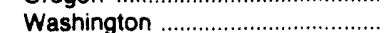 & 667 & 760 & 776 & -12.3 & -14.1 \\
\hline Pactic Noncontiguous .................. & 5 & 5 & 7 & -1.8 & -32.4 \\
\hline Alaska & 5 & 5 & 7 & -1.8 & -32.4 \\
\hline 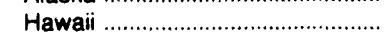 & -- & -- & $\rightarrow$ & - & $-.-0<-4$ \\
\hline U.S. Total & 115,208 & 112,773 & 156,859 & 2.2 & -26.6 \\
\hline
\end{tabular}

Data for 1993 are preliminary.

2 Data for 1992 are final.

Notes: - Totals may not equal sum of components because of independent rounding. •Percent difference is calculated before rounding. $\bullet$ Coal includes lignite, bituminous coal, subbituminous coal, and anthracite. - Stocks are end-of-month stocks at electric utilities.

Source: Energy Information Administration, Form ElA-759, "Monthly Power Plant Report." 
Table 32. Electric Utility Stocks of Petroleum by Census Division and State (Thousand Barrels)

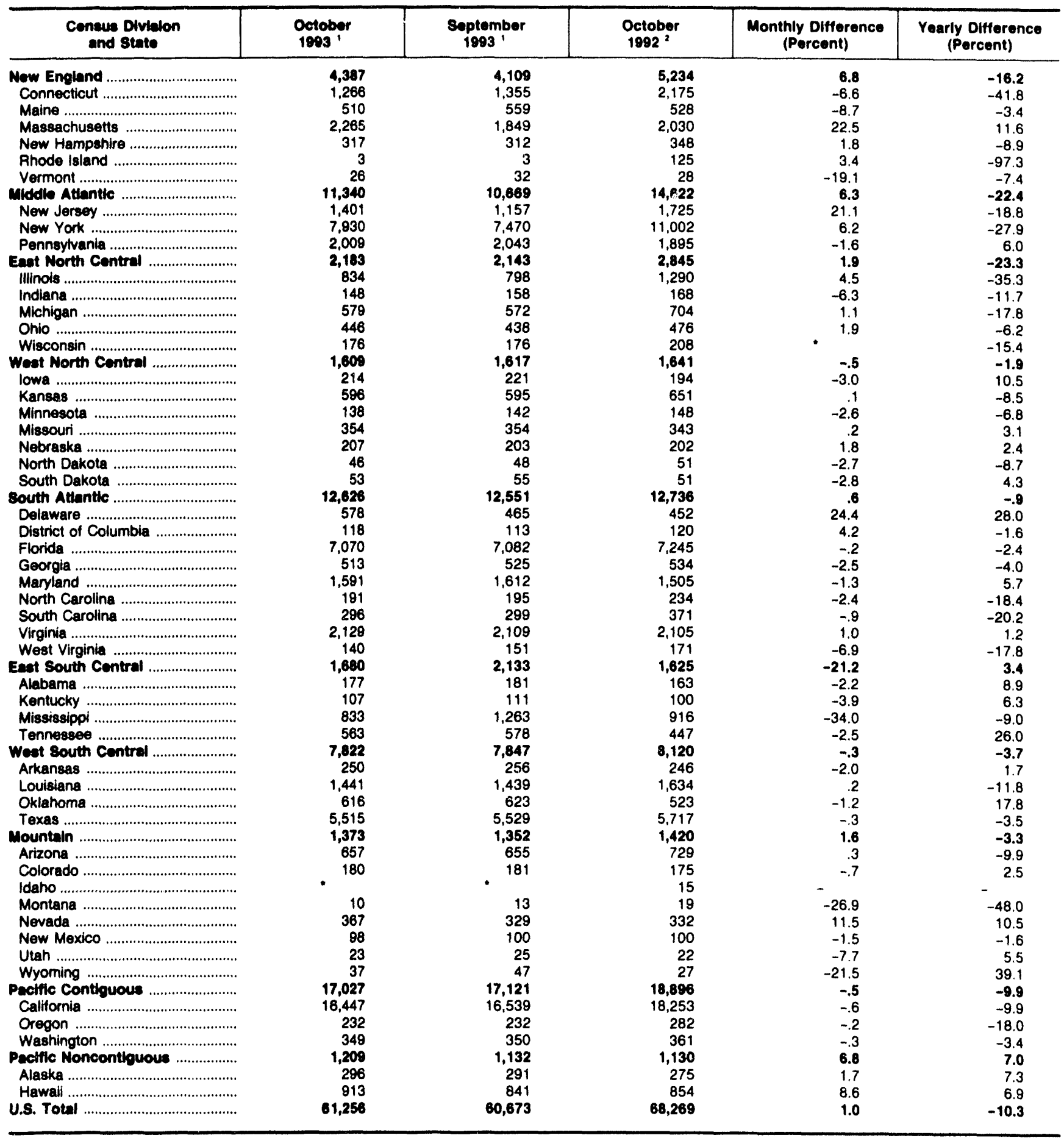

- Data for 1993 are preliminary.

2 Data for 1992 are final.

- = For detailed data, the absolute value is less than 0.5 ; for percentage calculations, the absolute value is less than 0.05 percent.

Notes: - Totais may not equal sum of components because of independent rounding. $\cdot$ Percent difference is calculated before rounding. $\bullet$ Data do not include petroteum coke. -The October 1893 petroleum coke stocks were 68,801 short tons. $\bullet$ Stocks are end-of-month stocks at electric utilities. Source: Energy Information Administration, Form ElA-759, "Monthly Power Plant Report." 


\section{Receipts and Cost of Fossil Fuels at U.S. Electric Utilities}

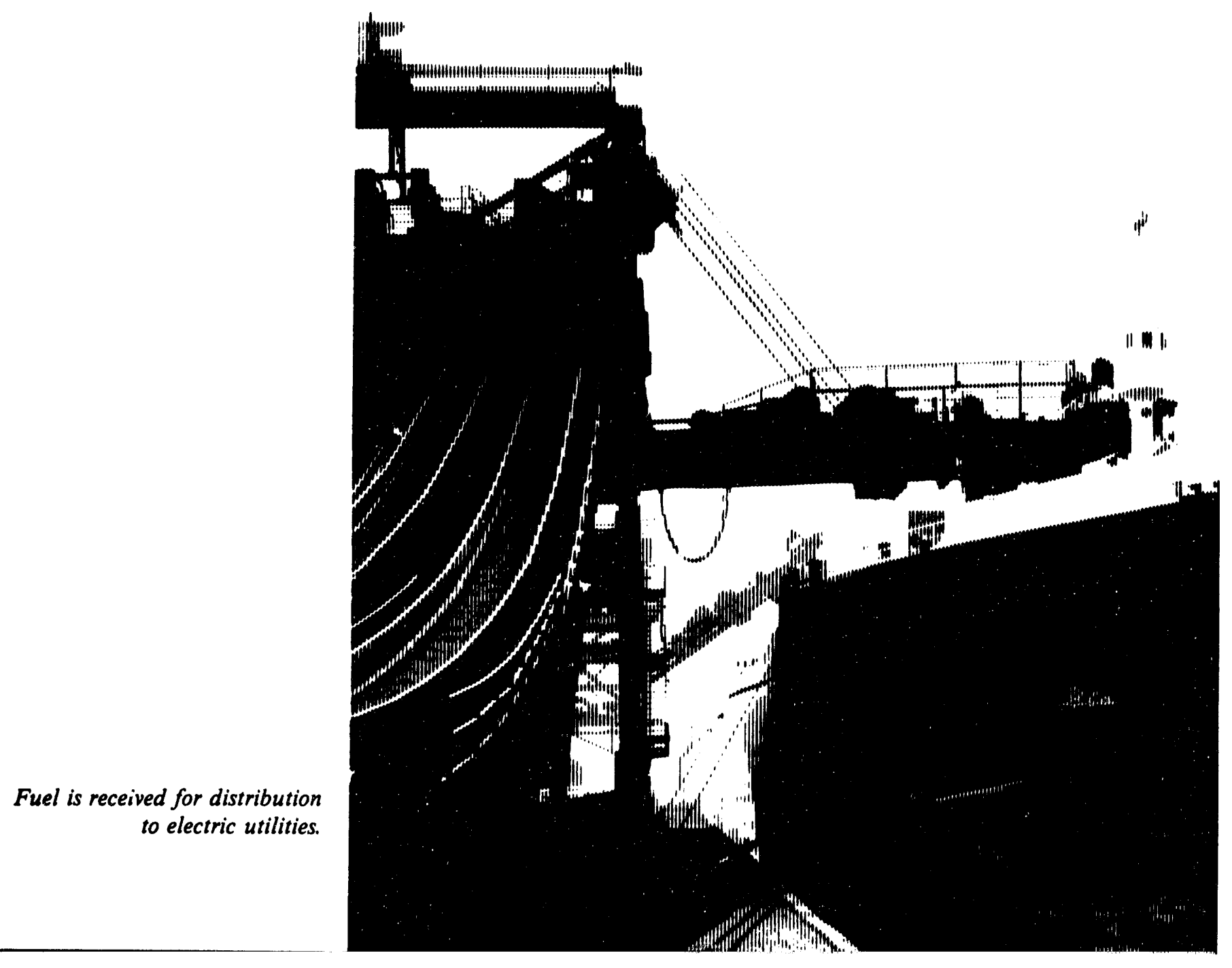


Table 33. U.S. Electric Utility Recelpts of and Average Cost for Fossil Fuels, 1983 Through September 1993

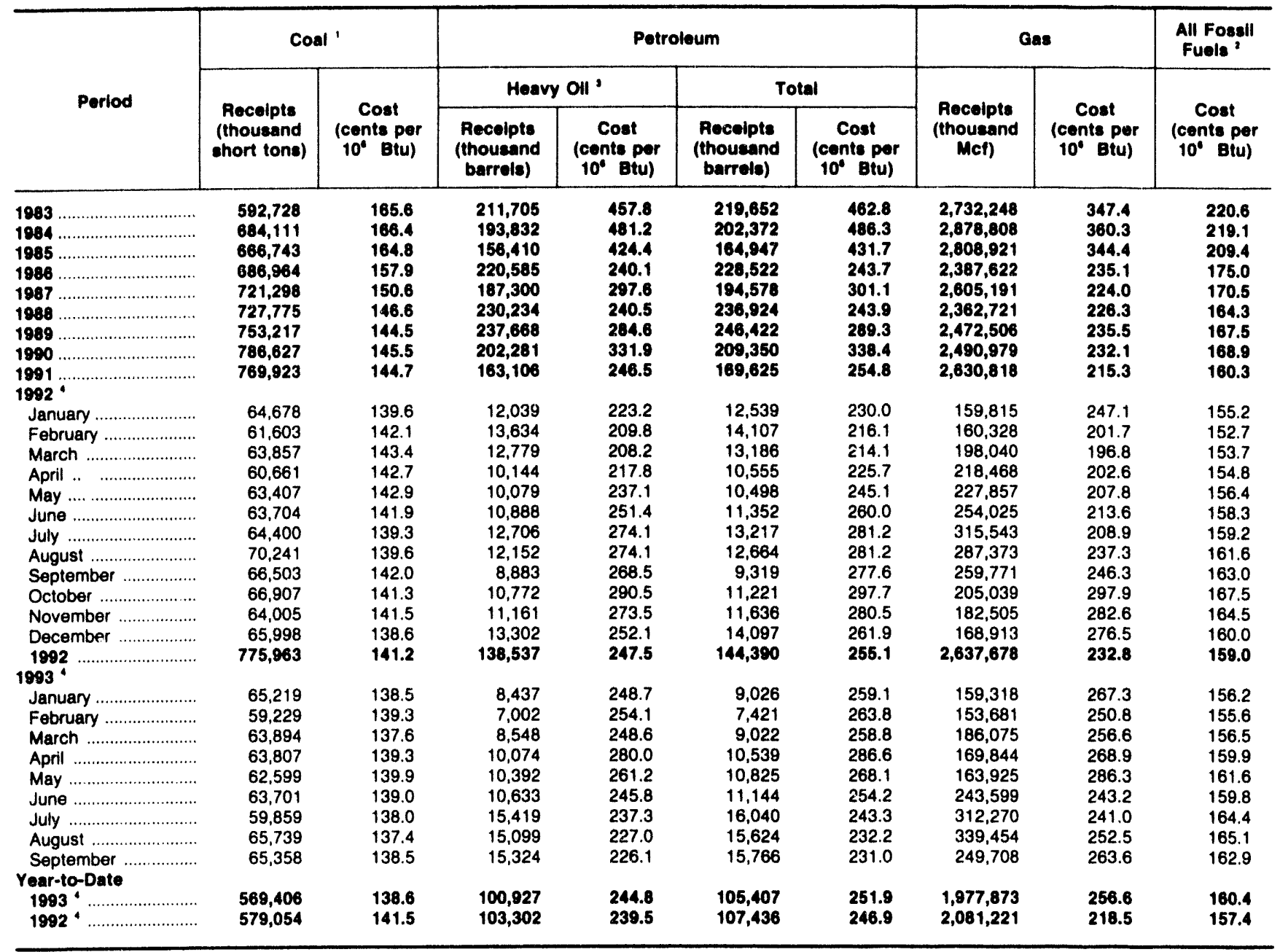

1 Includes lignite, bituminous coal, subbituminous coal, and anthracite.

2 The weighted average for all fossil fuels includes both heavy oil and light oil (Fuel Oil No. 2, kerosene, and jet fuel) prices. Data do not include petroleum coke.

Heavy oil includes Fuel Oil Nos. 4, 5, and 6, and topped crude fuel oil.

- Data for 1992 and prior years are final. Data for 1993 are preliminary

Notes: - Totals may not equal sum of components because of independent rounding. - As of 1991, data are for electric generating plants with a total steam-electric and combined-cycle nameplate capacity of 50 or more megawatts. -Data for $1983-1990$ are for steam-electric plants with a generator nameplate capacity of 50 or more megawatts. Prior to January 1983, data are for plants with a capacity of 25 or more megawatts and include peaking units - Mcf = thousand cubic feet. $\bullet$ Monetary values are expressed in nominal terms. forms.

Source: Federal Energy Regulatory Commission, FERC Form 423, "Monthly Report of Cost and Quality of Fuels for Electric Plants," and predecessor 
Table 34. Electric Utility Recelpts of Coal by NERC Region and Hawail (Thousand Short Tons)

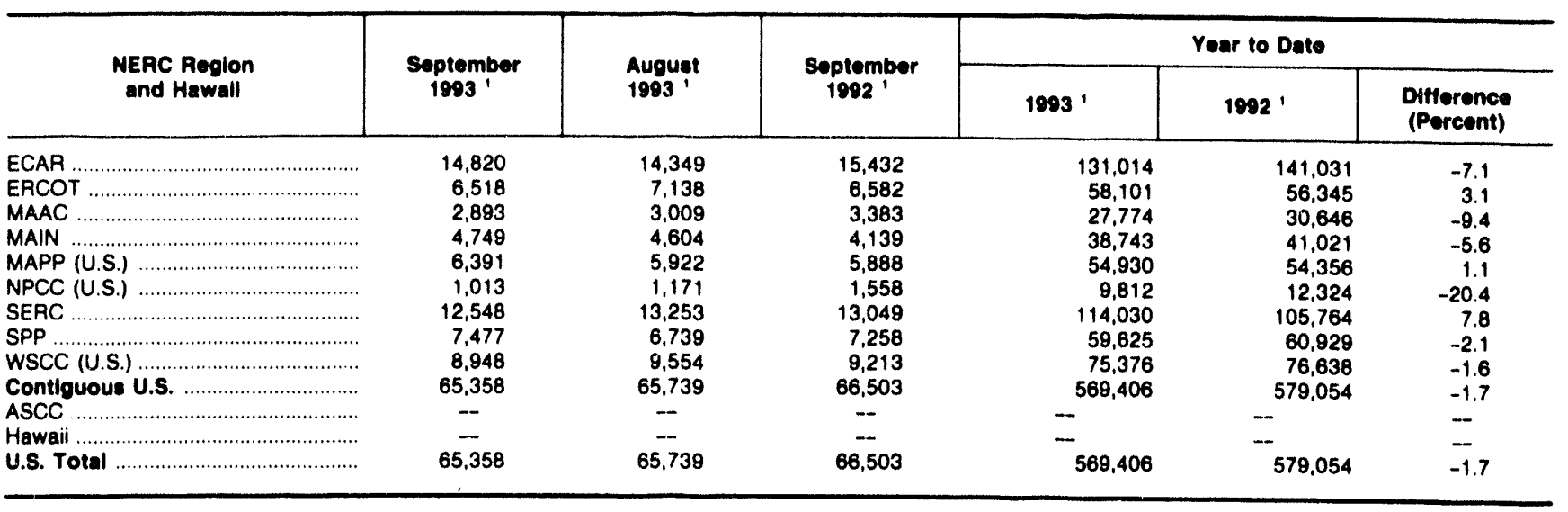

- Data for 1992 are final. Data for 1993 are preliminary.

Notes: - Totals may not equal sum of components because of independent rounding. $\bullet A s$ of 1891 , data are for electric generating plants with a total steam-electric and combined-cycle nameplate capacity of 50 or more megawatts. Includes lignite, bituminous coal, subbituminous coal, and anthracite.

Source: Federal Energy Regulatory Commission, FERC Form 423, "Monthly Report of Cost and Quality of Fuels for Electric Plants."

Table 35. Average Cost of Coal Dellvered to Electric Utilities by NERC Region and Hawall (Cents per Million Btu)

\begin{tabular}{|c|c|c|c|c|c|c|}
\hline \multirow{2}{*}{$\begin{array}{l}\text { NERC Reglon } \\
\text { and Hawail }\end{array}$} & \multirow{2}{*}{$\begin{array}{l}\text { September } \\
1993\end{array}$} & \multirow{2}{*}{$\begin{array}{l}\text { Auguet } \\
1993^{1}\end{array}$} & \multirow{2}{*}{$\begin{array}{c}\text { Soptember } \\
1992\end{array}$} & \multicolumn{3}{|c|}{ Year to Date } \\
\hline & & & & $1993^{\prime}$ & $1982^{\prime}$ & $\begin{array}{l}\text { Ditterence } \\
\text { (Percent) }\end{array}$ \\
\hline ECAR & 138.6 & 136.4 & 141.4 & 136.7 & 140.0 & -2.4 \\
\hline ERCOT & 132.0 & 120.5 & 139.3 & 135.9 & 140.3 & -3.1 \\
\hline MAAC & 148.2 & 145.2 & 146.6 & 146.6 & 154.1 & -4.9 \\
\hline 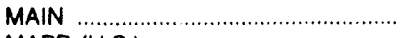 & 151.2 & 155.9 & 163.1 & 153.2 & 157.5 & -2.7 \\
\hline 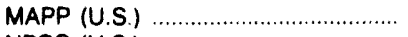 & 91.8 & 88.3 & 98.4 & 90.5 & 85.6 & -5.3 \\
\hline NPCC (U.S.) . . . . . & 156.7 & 155.9 & 152.9 & 155.6 & 158.9 & -2.0 \\
\hline 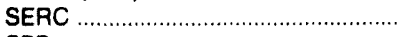 & 159.8 & 161.1 & 161.1 & 159.7 & 163.0 & -2.0 \\
\hline SPP & 137.8 & 138.8 & 141.9 & 137.7 & 141.4 & -2.7 \\
\hline 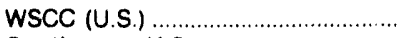 & 118.7 & 115.1 & 119.0 & 118.6 & 115.3 & 2.9 \\
\hline Contiguous U.S. & 138.5 & 137.4 & 142.0 & 138.6 & 141.5 & -2.0 \\
\hline ASCC $\ldots \ldots \ldots \ldots \ldots \ldots \ldots \ldots \ldots \ldots$ & -- & -- & -- & - & - & - \\
\hline 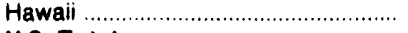 & - & - & - & - & - & - \\
\hline U.S. Total & 138.5 & 137.4 & 142.0 & 138.6 & 141.5 & -2.0 \\
\hline
\end{tabular}

Data for 1992 are final. Data for 1993 are preliminary

Notes: - Totals may not equal sum of components because of independent rounding. •As of 1991, data are for electric generating plants with a total steam-electric and combined-cycle nameplate capacity of 50 or more megawatts. Includes lignite, bituminous coal, subbituminous coal, and anthracite. - Monetary values are expressed in monetary terms.

Source: Federal Energy Regulatory Commission, FERC Form 423, "Monthly Report of Cost and Quality of Fuels for Electric Plants." 
Table 36. Electric Utility Recelpts of Petroleum by NERC Reglon and Hawall (Thousand Barrels)

\begin{tabular}{|c|c|c|c|c|c|c|}
\hline \multirow{2}{*}{$\begin{array}{l}\text { NERC Region } \\
\text { and Hawall }\end{array}$} & \multirow{2}{*}{$\begin{array}{l}\text { September } \\
1093^{i}\end{array}$} & \multirow{2}{*}{$\begin{array}{l}\text { August } \\
1003 \text { ! }\end{array}$} & \multirow{2}{*}{$\begin{array}{c}\text { Soptember } \\
1092\end{array}$} & \multicolumn{3}{|c|}{ Year to Date } \\
\hline & & & & $1003^{\prime}$ & $1992^{\prime}$ & $\begin{array}{l}\text { Difterence } \\
\text { (Porcent) }\end{array}$ \\
\hline $\begin{array}{l}\text { ECAR } \\
\text { EACOT MAC } \\
\text { MAIN } \\
\text { MAPP (U.S.) } \\
\text { NPCC (U.S.) } \\
\text { SERC } \\
\text { SPP } \\
\text { WSCC (U.S.) } \\
\text { Contiguous U.S. } \\
\text { ASCC } \\
\text { Hawai ............ } \\
\text { U.S. Total }\end{array}$ & $\begin{array}{r}257 \\
4 \\
2,141 \\
168 \\
16 \\
5,367 \\
6,430 \\
877 \\
10 \\
15,269 \\
-\quad 497 \\
15,766\end{array}$ & $\begin{array}{r}268 \\
4 \\
2,379 \\
90 \\
53 \\
4,168 \\
7,129 \\
946 \\
14 \\
15,052 \\
-\quad 572 \\
15,624\end{array}$ & $\begin{array}{r}228 \\
22 \\
1,065 \\
161 \\
13 \\
3,712 \\
3,748 \\
29 \\
19 \\
8,996 \\
-\quad 323 \\
, 319\end{array}$ & $\begin{array}{r}1,841 \\
98 \\
14,667 \\
1,426 \\
248 \\
34,899 \\
41,972 \\
4,405 \\
613 \\
100,269 \\
-\quad 5,137 \\
105,407\end{array}$ & $\begin{array}{r}1,636 \\
177 \\
10,311 \\
1,899 \\
151 \\
50,506 \\
35,055 \\
873 \\
380 \\
100,987 \\
-\quad \\
6,448 \\
107,436\end{array}$ & $\begin{array}{r}18.7 \\
-44.1 \\
42.2 \\
-24.9 \\
64.2 \\
-30.9 \\
10.7 \\
404.6 \\
61.0 \\
-.7 \\
-- \\
-20.3 \\
-1.9\end{array}$ \\
\hline
\end{tabular}

- Data for 1992 are final. Data for 1993 are preliminary.

Notes: - Totals may not equal sum of components because of independent rounding. • As of 1991, data are for electric generating plents with a total steam-electric and combined-cycle nameplate capacity of 50 or more megawatts.

Source: Federal Energy Regulatory Commission, FERC Form 423, "Monthly Report of Cost and Quality of Fuels for Electric Plants."

Table 37. Average Cost of Petroleum Dellvered to Electric Utllitles by NERC Reglon and Hawall (Cents per Million Btu)

\begin{tabular}{|c|c|c|c|c|c|c|}
\hline \multirow{2}{*}{$\begin{array}{l}\text { NERC Region } \\
\text { and Hawall }\end{array}$} & \multirow{2}{*}{$\begin{array}{l}\text { Soptember } \\
1903\end{array}$} & \multirow{2}{*}{$\begin{array}{l}\text { August } \\
1993 !\end{array}$} & \multirow{2}{*}{ Septomber } & \multicolumn{3}{|c|}{ Year to Date } \\
\hline & & & & $1003^{\prime}$ & $1002^{\prime}$ & $\begin{array}{l}\text { Ditference } \\
\text { (Porcent) }\end{array}$ \\
\hline $\begin{array}{l}\text { ECAR } \\
\text { ERCOT } \\
\text { MAAC } \\
\text { MAIN } \\
\text { MAPP (U.S.) } \\
\text { NPCC (U.S.) } \\
\text { SERC } \\
\text { SPP } \\
\text { WSCC (U.S.) } \\
\text { Contiguous U.S. }\end{array}$ & $\begin{array}{l}359.6 \\
347.3 \\
248.4 \\
295.8 \\
475.1 \\
237.6 \\
215.3 \\
178.8 \\
476.3 \\
229.2\end{array}$ & $\begin{array}{l}322.3 \\
382.4 \\
235.3 \\
350.6 \\
398.0 \\
239.3 \\
221.1 \\
174.0 \\
431.9 \\
228.7\end{array}$ & $\begin{array}{l}381.0 \\
461.0 \\
296.0 \\
324.0 \\
507.9 \\
274.1 \\
261.6 \\
329.3 \\
509.8 \\
276.3\end{array}$ & $\begin{array}{l}377.2 \\
394.5 \\
250.6 \\
320.5 \\
431.2 \\
261.8 \\
230.5 \\
180.5 \\
394.1 \\
248.1\end{array}$ & $\begin{array}{l}378.5 \\
405.1 \\
252.6 \\
305.3 \\
451.8 \\
238.6 \\
238.3 \\
236.9 \\
416.4 \\
244.5\end{array}$ & $\begin{array}{r}-0.3 \\
-2.6 \\
-.8 \\
5.0 \\
-4.6 \\
9.7 \\
-3.3 \\
-19.6 \\
-5.4 \\
1.5\end{array}$ \\
\hline ASCC & - & -- & - & - & - & - \\
\hline 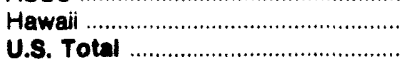 & $\begin{array}{l}283.9 \\
231.0\end{array}$ & $\begin{array}{l}326.8 \\
232.2\end{array}$ & $\begin{array}{l}315.3 \\
277.6\end{array}$ & $\begin{array}{l}328.3 \\
251.9\end{array}$ & $\begin{array}{l}285.6 \\
246.9\end{array}$ & $\begin{array}{r}15.0 \\
2.0\end{array}$ \\
\hline
\end{tabular}

- Data for 1992 are final. Data for 1993 are preliminary.

Notes: - Totals may not equal sum of components because of independent rounding. -As of 1991, data are for electric generating plants with a total steam-electric and combined-cycle nameplate capacity of 50 or more megawatts. Monetary values are expressed in monetary terms.

Source: Federal Energy Regulatory Commission, FERC Form 423, "Monthly Report of Cost and Quality of Fuels for Electric Plants." 
Table 38. Electric Utility Recelpts of Gas by NERC Reglon and Hawall (Million Cubic Feet)

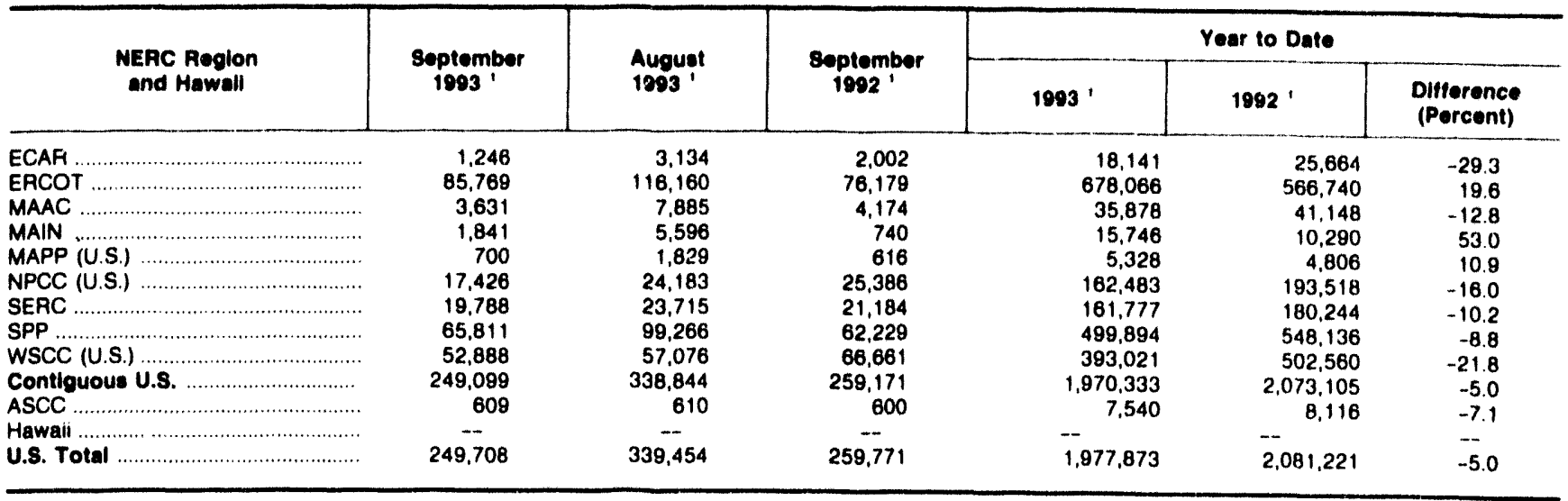

- Data for 1992 are final. Data for 1993 are preliminary.

Notes: - Totals may not equal sum of components because of independent rounding. • As of 1991, data are for electric generating plants with a total steam-electric and combined-cycle nameplate capacity of 50 or more megawatts.

Source: Federal Energy Regulatory Commission. FERC Form 423, "Monthly Report of Cost and Quality of Fuels for Electric Plants."

Table 39. Average Cost of Gas Delivered to Electric Utilities by NERC Region and Hawail (Cents per Million Btu)

\begin{tabular}{|c|c|c|c|c|c|c|}
\hline \multirow{2}{*}{$\begin{array}{l}\text { NERC Reglon } \\
\text { and Hawall }\end{array}$} & \multirow{2}{*}{$\begin{array}{c}\text { September } \\
1993\end{array}$} & \multirow{2}{*}{$\begin{array}{l}\text { August } \\
1993^{1}\end{array}$} & \multirow{2}{*}{$\begin{array}{c}\text { Soptomber } \\
1992^{+}\end{array}$} & \multicolumn{3}{|c|}{ Year to Date } \\
\hline & & & & $1903^{\prime}$ & $19922^{\prime}$ & $\begin{array}{l}\text { Difference } \\
\text { (Percent) }\end{array}$ \\
\hline $\begin{array}{l}\text { ECAR } \\
\text { ERCOT } \\
\text { MAAC } \\
\text { MAIN (U.S.) } \\
\text { NPCC (U.S.) } \\
\text { SERC } \\
\text { SPP } \\
\text { WSCC (U.S.) } \\
\text { Contiguous U.S. } \\
\text { ASCC } \\
\text { Hawaii } \\
\text { U.S. Total }\end{array}$ & $\begin{array}{r}276.0 \\
252.1 \\
269.8 \\
258.0 \\
287.0 \\
274.1 \\
238.4 \\
267.5 \\
284.7 \\
264.1 \\
63.3 \\
-- \\
263.6\end{array}$ & $\begin{array}{l}267.5 \\
241.0 \\
234.8 \\
233.0 \\
267.3 \\
250.7 \\
235.9 \\
250.6 \\
292.3 \\
252.8 \\
64.0 \\
252.5\end{array}$ & $\begin{array}{l}237.7 \\
230.4 \\
248.0 \\
247.5 \\
231.8 \\
251.5 \\
253.6 \\
238.0 \\
269.8 \\
246.8 \\
56.8 \\
-- \\
246.3\end{array}$ & $\begin{array}{l}262.4 \\
240.7 \\
240.8 \\
247.8 \\
279.4 \\
265.8 \\
244.4 \\
253.9 \\
293.5 \\
257.3 \\
63.8 \\
-- \\
256.6\end{array}$ & $\begin{array}{l}211.7 \\
209.5 \\
216.2 \\
212.5 \\
213.2 \\
228.5 \\
220.2 \\
203.4 \\
244.0 \\
219.1 \\
57.9 \\
- \\
218.5\end{array}$ & $\begin{array}{l}24.0 \\
14.9 \\
11.4 \\
16.7 \\
31.1 \\
16.3 \\
11.0 \\
24.9 \\
20.3 \\
17.4 \\
10.1 \\
-- \\
17.4\end{array}$ \\
\hline
\end{tabular}

- Data for 1992 are final. Data for 1993 are preliminary.

Notes: - Totals may not equal sum of components because of independent rounding. $\bullet$ As of 1991, data are for electric generating plants with a total steam-electric and combined-cycle nameplate capacity of 50 or more megawatts. Monetary values are expressed in monetary terms.

Source: Federal Energy Regulatory Commission, FERC Form 423, "Monthly Report of Cost and Quality of Fuels for Electric Plants." 
Table 40. Electric Utility Recelpts of Coal by Type, Census Division, and State, September 1993

\begin{tabular}{|c|c|c|c|c|c|c|c|c|c|c|}
\hline \multirow[b]{2}{*}{$\begin{array}{l}\text { Consuse Division } \\
\text { and state }\end{array}$} & \multicolumn{2}{|c|}{ Anthractte } & \multicolumn{2}{|c|}{ Bltuminous } & \multicolumn{2}{|c|}{ subbltuminous } & \multicolumn{2}{|c|}{ Lanite } & \multicolumn{2}{|c|}{ Total } \\
\hline & $\begin{array}{c}\text { (thourand } \\
\text { short } \\
\text { tons) }\end{array}$ & $\begin{array}{l}\text { (billion } \\
\text { Dtu) }\end{array}$ & $\begin{array}{l}\text { (thousand } \\
\text { chort } \\
\text { tona) }\end{array}$ & $\begin{array}{l}\text { (billion } \\
\text { Btu) }\end{array}$ & $\begin{array}{c}\text { (thousend } \\
\text { chort } \\
\text { lons) }\end{array}$ & $\begin{array}{l}\text { (billtion } \\
\text { Btu) }\end{array}$ & $\begin{array}{c}\text { (thousand } \\
\text { short } \\
\text { tono) }\end{array}$ & $\begin{array}{l}\text { (billion } \\
\text { Btu) }\end{array}$ & $\begin{array}{c}\text { (thousand } \\
\text { short } \\
\text { tona) }\end{array}$ & $\begin{array}{l}\text { (billion } \\
\text { Bfu) }\end{array}$ \\
\hline Now England ............................... & - & - & 470 & 12,174 & - & - & - & - & 470 & 12,174 \\
\hline 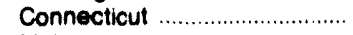 & - & - & 68 & 1,794 & - & - & -- & $\cdots$ & 68 & 1,794 \\
\hline 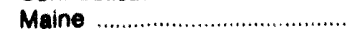 & -- & - & - & - & - & - & - & - & $\ldots$ & - \\
\hline Massachusetts & -- & - & 295 & 7,572 & - & - & - & $\cdots$ & 295 & 7,572 \\
\hline 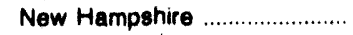 & - & - & 107 & 2,808 & - & - & - & - & 107 & 2,808 \\
\hline Rhode Island & - & -- & - & - & -- & - & - & -- & - & $\ldots$ \\
\hline 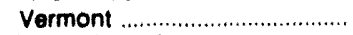 & - & - & - & - & -- & -- & - & - & - & -. \\
\hline 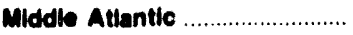 & 44 & 730 & 3,874 & 69,663 & - & - & - & - & 3,618 & $\$ 0,293$ \\
\hline 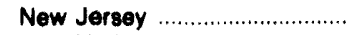 & - & - & 58 & 1.544 & - & - & - & - & 58 & 1,544 \\
\hline 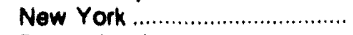 & -- & - & 543 & 14,003 & - & - & -- & -- & 543 & 14,003 \\
\hline 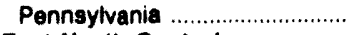 & 44 & 730 & 2.972 & 74.016 & $=$ & - & - & - & 3,016 & 74.747 \\
\hline East North Central ........................ & - & - & 9,480 & 223,230 & 8,031 & 89,919 & - & - & 14,401 & 313,157 \\
\hline 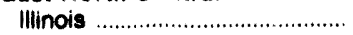 & -- & - & 1,436 & 32.185 & 1,180 & 21,102 & - & - & 2,616 & 53,287 \\
\hline 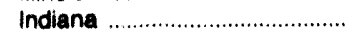 & - & - & 2,575 & 57,959 & 1.068 & 18,163 & - & - & 3,634 & 76,121 \\
\hline 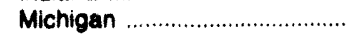 & - & - & 1,372 & 35,069 & 1,607 & 30,010 & - & -- & 2,979 & 65,079 \\
\hline Ohio & - & - & 3,676 & 88,405 & - & - & - & -- & 3,676 & 88,405 \\
\hline Wisconsin & -- & - & 391 & 9,620 & 1,186 & 20,644 & -- & -. & 1,576 & 30,264 \\
\hline Weat North Central .................. & - & - & 691 & 15,967 & 6,003 & 105,028 & 2,095 & 27,600 & 8,879 & 146,405 \\
\hline lowa & -- & - & 112 & 2,527 & 1,318 & 22,399 & - & - & 1,430 & 24,925 \\
\hline Kansas & - & - & 88 & 2,001 & 1,371 & 23,253 & - & -- & 1,459 & 25,254 \\
\hline 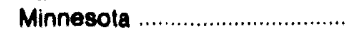 & -- & - & 6 & 140 & 1,380 & 24,334 & -- & - & 1,386 & 24,474 \\
\hline 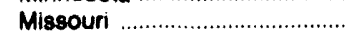 & -- & -- & 485 & 11,289 & 1,270 & 22,160 & -- & -- & 1,756 & 33,455 \\
\hline 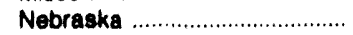 & - & - & - & - & 754 & 12,876 & - & - & 754 & 12,876 \\
\hline North Dakota & -- & - & - & - & - & - & 2,061 & 27,077 & 2,061 & 27,077 \\
\hline South Dakota & - & - & - & - & - & - & 34 & 424 & 34 & 424 \\
\hline South Attantic & - & - & 10,084 & 252,587 & - & - & - & - & 10,004 & 252,507 \\
\hline Delaware & - & - & 108 & 2,745 & - & - & - & - & 106 & 2,745 \\
\hline District of Columbia & -- & - & - & - & - & - & - & - & - & - \\
\hline Florida & - & - & 1,806 & 46,844 & - & - & - & - & 1,896 & 46,944 \\
\hline 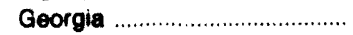 & - & - & 1,880 & 46,530 & - & - & -- & - & 1,880 & 46,530 \\
\hline 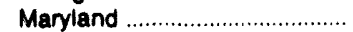 & -- & - & 698 & 17,806 & - & - & -- & -- & 698 & 17,806 \\
\hline North Carolina & -- & - & 1,979 & 49,388 & - & - & -- & -- & 1,979 & 49,388 \\
\hline South Carolina & -- & - & 772 & 19.781 & -- & - & - & - & 772 & 19,781 \\
\hline Virginia & -- & - & 823 & 21,284 & - & - & - & - & 823 & 21,284 \\
\hline West Virginia & - & -- & 1,831 & 48,108 & - & -- & -- & - & 1,931 & 48,108 \\
\hline Eaot South Central ....................... & - & - & 6,968 & 166,711 & 72 & 1,415 & - & - & 7,030 & 168,126 \\
\hline Alabama & -- & - & 2,058 & 50.119 & - & - & -- & - & 2,058 & 50,119 \\
\hline 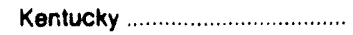 & - & - & 3,057 & 71,155 & 72 & 1,415 & - & - & 3,129 & 72,570 \\
\hline Mississippi ……....................... & - & - & 298 & 7,369 & - & -- & - & - & 299 & 7,369 \\
\hline Tennessee .................................... & - & - & 1,545 & 38,068 & - & - & - & -- & 1,545 & 38,068 \\
\hline Weet South Central ..................... & - & - & 227 & 4,064 & 6,622 & 113,296 & 4,535 & 66,067 & 11,384 & 175,117 \\
\hline 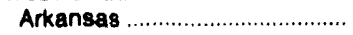 & -- & - & - & - & 907 & 15,733 & - & - & 907 & 15,733 \\
\hline Louisiana & - & - & - & - & 939 & 15,910 & 287 & 3,990 & 1,226 & 19,900 \\
\hline Oklanoma & - & - & -- & - & 1,581 & 27,173 & - & - & 1,581 & 27,173 \\
\hline Texas & - & -- & 227 & 4,854 & 3,185 & 54,480 & 4.248 & 52,877 & 7,670 & 112,311 \\
\hline 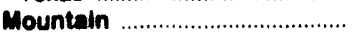 & - & - & 2,682 & 50,003 & 0,123 & 113,592 & 18 & 232 & 8,803 & 171,007 \\
\hline 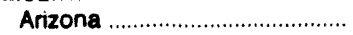 & -- & - & 317 & 5,830 & 1,381 & 29,306 & -- & - & 1,698 & 35,136 \\
\hline Colorado ...................................... & - & - & 472 & 10,134 & 804 & 15,015 & -. & - & 1,278 & 25,149 \\
\hline Idano & - & - & - & - & - & -- & - & - & - & - \\
\hline Montana & -- & -- & - & -- & 801 & 13,764 & 18 & 232 & 819 & 13,996 \\
\hline 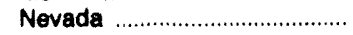 & - & -- & 597 & 13,268 & 82 & 1,576 & -- & - & 679 & 14,844 \\
\hline New Mexico & - & - & - & - & 1,314 & 23,584 & - & - & 1,314 & 23,584 \\
\hline Utah & - & - & 1,156 & 26,497 & - & - & - & - & 1,156 & 26,497 \\
\hline Wyoming & -- & - & 120 & 2,333 & 1,741 & 30,348 & - & - & 1,861 & 32,681 \\
\hline Pactife & - & - & - & - & 610 & 9,970 & - & - & 610 & 9,970 \\
\hline 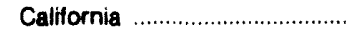 & - & - & - & - & - & - & -- & -- & - & - \\
\hline Oregon & - & - & - & - & 167 & 2.814 & - & - & 167 & 2,814 \\
\hline 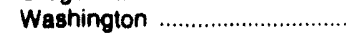 & - & - & -- & - & 443 & 7,156 & - & -- & 443 & 7,156 \\
\hline Pactific Noncontiguous ............. & - & - & - & - & - & - & - & - & - & - \\
\hline Alaska & -- & - & - & - & - & - & -- & -- & -- & -- \\
\hline 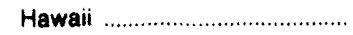 & -- & - & - & - & - & - & -- & - & - & - \\
\hline U.S. Total & 44 & 730 & 34,117 & 823,247 & 24,651 & 433,220 & 6,647 & 84,600 & 65,358 & $1,341,797$ \\
\hline
\end{tabular}

Notes: - Totals may not equal sum of components because of independent rounding. Data are for electric generating plants with total steam-electric and combined-cycle nameplate capacity of 50 or more megawatts. - Data for 1893 are preliminary.

Source: Federal Energy Regulatory Commission, FERC Form 423, "Monthly Report of Cost and Quality of Fuels for Electric Plants." 


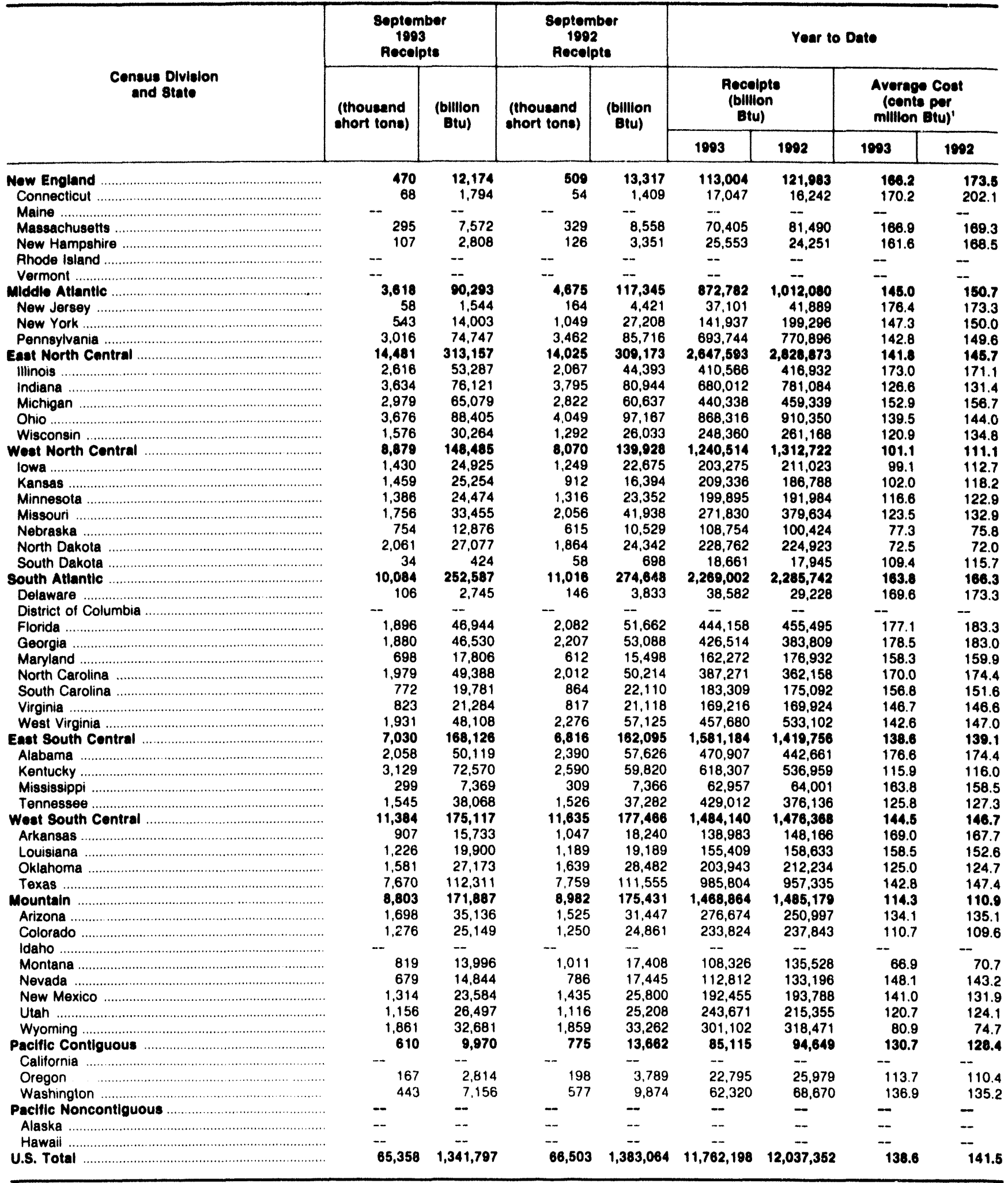

- Monetary values are expressed in nominal terms.

Notes: Data for 1992 are final. Data for 1993 are preliminary. - Totals may not equal sum of components because of independent rounding. -Data are for electric generating plants with a total steam-electric and combined-cycle nameplate capacity of 50 or more megawatts. $\cdot$ Coal includes lignite, bituminous coal. subbituminous coal, and anthracite.

Source: Federal Energy Regulatory Commission, FERC Form 423, "Monthly Peport of Cost and Quality of Fuels lor Electric Plants." 
Table 42. Recelpts and Average Cost of Coal Dellvered to Electric Utilities by Type of Purchase, Mining Method, Census Division, and State, September 1993

\begin{tabular}{|c|c|c|c|c|c|c|c|c|c|c|c|c|}
\hline \multirow{4}{*}{$\begin{array}{l}\text { Census Division } \\
\text { and State }\end{array}$} & \multicolumn{6}{|c|}{ Type of Purchase } & \multicolumn{6}{|c|}{ Type of Mining } \\
\hline & \multicolumn{3}{|c|}{ Contract } & \multicolumn{3}{|c|}{ 8pot } & \multicolumn{3}{|c|}{ Strip and Auger } & \multicolumn{3}{|c|}{ Underground } \\
\hline & \multirow{2}{*}{\begin{tabular}{|c|} 
Recelpts \\
$\begin{array}{c}(1,000 \\
\text { short } \\
\text { tons })\end{array}$
\end{tabular}} & \multicolumn{2}{|c|}{$\begin{array}{c}\text { Average } \\
\text { Cost' }\end{array}$} & \multirow{2}{*}{\begin{tabular}{|c|} 
Recolpts \\
$\begin{array}{c}(1,000 \\
\text { hort } \\
\text { tons) }\end{array}$ \\
\end{tabular}} & \multicolumn{2}{|c|}{$\begin{array}{l}\text { Average } \\
\text { Cost' }\end{array}$} & Pocelpts & $\begin{array}{l}\text { Avere } \\
\text { Cost }\end{array}$ & & Recolpte & $\begin{array}{l}\text { Avere } \\
\text { Cost }\end{array}$ & \\
\hline & & $\begin{array}{l}\text { (Cents } \\
\text { per } \\
10^{\circ} \\
\text { Btu) }\end{array}$ & $\begin{array}{c}\text { (\$ } \\
\text { per } \\
\text { short } \\
\text { ton) }\end{array}$ & & $\begin{array}{l}\text { (Cents } \\
\text { per } \\
10^{6} \\
\text { Btu) }\end{array}$ & $\begin{array}{c}\text { (8 } \\
\text { per } \\
\text { short } \\
\text { ton) }\end{array}$ & $\begin{array}{l}(1,000 \\
\text { chort } \\
\text { tons) }\end{array}$ & $\begin{array}{l}\text { (Conts } \\
\text { per } \\
10^{6} \\
\text { Btu) }\end{array}$ & $\begin{array}{c}\text { (8 } \\
\text { por } \\
\text { ehort } \\
\text { ton) }\end{array}$ & $\begin{array}{l}(1,000 \\
\text { shont } \\
\text { tons) }\end{array}$ & $\begin{array}{l}\text { (Cents } \\
\text { per } \\
10^{6} \\
\text { Btu) }\end{array}$ & $\begin{array}{l}\text { (8 } \\
\text { per } \\
\text { ehort } \\
\text { ton) }\end{array}$ \\
\hline 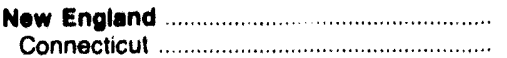 & $\begin{array}{r}417 \\
68\end{array}$ & $\begin{array}{l}162.2 \\
171.4\end{array}$ & $\begin{array}{l}42.15 \\
45.22\end{array}$ & 52 & 164.7 & $\begin{array}{l}41.88 \\
--\end{array}$ & 119 & 150.7 & 40.11 & $\begin{array}{r}351 \\
68\end{array}$ & $\begin{array}{l}183.7 \\
171.4\end{array}$ & $\begin{array}{l}42.00 \\
45.22\end{array}$ \\
\hline 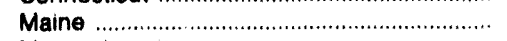 & -- & - & $\ldots$ & -- & -- & -- & - & - & -- & $-\infty$ & -- & - \\
\hline $\begin{array}{l}\text { Massachusetts ........................................... } \\
\text { New Hampshire }\end{array}$ & $\begin{array}{l}242 \\
107\end{array}$ & $\begin{array}{l}162.3 \\
156.3\end{array}$ & $\begin{array}{l}41.80 \\
40.97\end{array}$ & $-{ }_{-}^{52}$ & $\begin{array}{ll}164.7 \\
-\end{array}$ & $\begin{array}{c}41.88 \\
--\end{array}$ & $\begin{array}{l}81 \\
27\end{array}$ & $\begin{array}{l}161.8 \\
148.9\end{array}$ & $\begin{array}{l}40.57 \\
38.56\end{array}$ & $\begin{array}{r}203 \\
80\end{array}$ & $\begin{array}{l}163.1 \\
158.8\end{array}$ & $\begin{array}{l}42.37 \\
41.80\end{array}$ \\
\hline 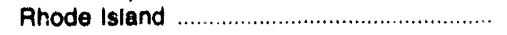 & -- & -- & -- & - & -- & - & -- & -- & - & - & - & - \\
\hline Vermont ............... & -- & - & - & -- & - & -- & - & - & - & - & - & - \\
\hline 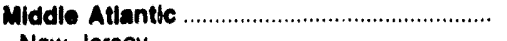 & 2,219 & 152.7 & 38.56 & 1,400 & 135.8 & 33.27 & 1,562 & 140.1 & 34.14 & 2,050 & 180.9 & 38.32 \\
\hline $\begin{array}{l}\text { Now Jersey } \\
\text { New York }\end{array}$ & 58 & 167.5 & 44.27 & - & - & - & 31 & 164.4 & 42.85 & 27 & 170.9 & 45.86 \\
\hline $\begin{array}{l}\text { New York } \\
\text { Pennsylvania }\end{array}$ & $\begin{array}{r}238 \\
1.922\end{array}$ & $\begin{array}{l}162.2 \\
151.1\end{array}$ & $\begin{array}{l}42.25 \\
37.93\end{array}$ & $\begin{array}{r}305 \\
1.095\end{array}$ & $\begin{array}{l}143.4 \\
133.6\end{array}$ & $\begin{array}{l}36.65 \\
32.33\end{array}$ & $\begin{array}{r}98 \\
1.433\end{array}$ & $\begin{array}{l}141.4 \\
139.4\end{array}$ & $\begin{array}{l}33.84 \\
33.98\end{array}$ & $\begin{array}{r}445 \\
1583\end{array}$ & 153.8 & $\begin{array}{l}40.27 \\
37.64\end{array}$ \\
\hline 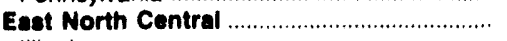 & 9,045 & 150.6 & 32.06 & 4,536 & 128.6 & 28.80 & 0,981 & 140.7 & $28.8 \pi$ & 4,500 & $\begin{array}{l}149.6 \\
140.7\end{array}$ & $\begin{array}{l}37.64 \\
35.90\end{array}$ \\
\hline Illinois & 2,071 & 174.2 & 34.53 & 544 & 140.5 & 31.57 & 1,649 & 184.8 & 35.24 & 967 & 140.1 & 31.65 \\
\hline Indiana ....... & 2,525 & 128.9 & 26.96 & 1.109 & 113.8 & 23.93 & 2,812 & 118.4 & 24.2 & 822 & & 32.26 \\
\hline Michigan .... & 2,046 & 155.3 & 32.31 & 933 & 156.4 & 37.73 & 2,053 & 151.1 & 30. & 926 & & 42. \\
\hline Ohio ................. & 2.473 & 159.2 & 38.36 & 1,203 & 118.5 & 28.38 & 2,170 & 144.9 & 34.21 & 1,506 & 147 & 36.37 \\
\hline 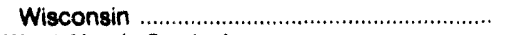 & 831 & 116.9 & 21.97 & 746 & 118.5 & 23.48 & 1,297 & 108.9 & 19.50 & 279 & 148.6 & 37.44 \\
\hline Woat North Contral ....... & 7,195 & 102.8 & 16.94 & 1,684 & 91.9 & 16.33 & 8,393 & 93.6 & 15.29 & 486 & 185.1 & 43.32 \\
\hline 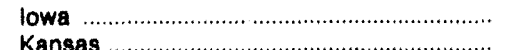 & 842 & 119.8 & $\begin{array}{l}20.59 \\
19.89\end{array}$ & $\begin{array}{l}588 \\
455\end{array}$ & 91.8 & 16.34 & 1,396 & 105.9 & 18.31 & 34 & 176.6 & 40.62 \\
\hline Kansas ....... & 1.004 & 114.7 & 19.89 & 45 & 79.7 & 13.75 & 1,419 & 97.8 & 16. & 39 & & 60.42 \\
\hline . & 1,074 & 120.4 & 21.2 & 31 & 98.0 & 17.34 & 1,38 & 115.0 & 20. & 6 & 167 & 39.67 \\
\hline 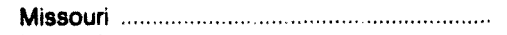 & 1,605 & 119.8 & 22.6 & 151 & 134.6 & 27.82 & 1,349 & 98 & 17. & 407 & 178.9 & 41.94 \\
\hline Nebraska ........ & 576 & 71.2 & 12.25 & 178 & 68.2 & 11.34 & 754 & 70.5 & 12.0 & - & - & - \\
\hline$\ldots \ldots \ldots \ldots \ldots \ldots \ldots$ & 2,061 & 65.9 & 8.66 & - & -- & -- & 2,061 & 65.8 & 8.66 & - & - & - \\
\hline South Dakola . & 34 & 115.3 & 14.37 & - & - & - & 34 & 115.3 & 14.37 & - & - & - \\
\hline . & 7,392 & 170.2 & 42.67 & 2,692 & 146.3 & 36.31 & 4,102 & 162.8 & 40.40 & 5,922 & 164.1 & 41.36 \\
\hline 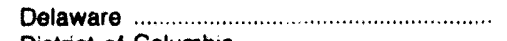 & & 167.3 & 43.39 & -- & - & -- & 21 & 160.4 & 40.93 & & 168.0 & 44.00 \\
\hline . & - & -- & - & -- & $-\bar{x}$ & -- & $=$ & - & - & - & - & -- \\
\hline 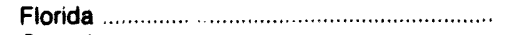 & 1.483 & 184.1 & 45.83 & 413 & 161.5 & 39.21 & 785 & 173.7 & 42.07 & 1,111 & 183.0 & 46.03 \\
\hline (1) & 1,453 & 180.3 & 44.55 & 427 & 165.1 & 41.08 & 810 & 170.5 & 42. & 1,070 & 181 & 45.02 \\
\hline Maryl & 433 & 162.7 & 41.44 & 265 & 153.9 & 39.41 & 38 & 162 & 41. & & & 40.22 \\
\hline 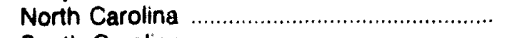 & 1,378 & 180.4 & 44.89 & 601 & 153.9 & 38.65 & 891 & 169.0 & 42.8 & 1,088 & 175 & 43.76 \\
\hline 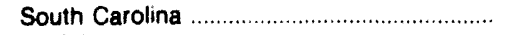 & 567 & 158.6 & 40.78 & 20 & 153.4 & 39.01 & 11 & 157.1 & 40. & 653 & & 40.26 \\
\hline$\ldots$ & 653 & 147.1 & 37.99 & 17 & 147.2 & 38.31 & 31 & 145.3 & 37. & 508 & 148 & 38.37 \\
\hline . & 1.319 & 152.7 & 38.18 & 61 & 104.8 & 25.92 & 78 & 145.4 & 35. & 1,149 & & 33.23 \\
\hline East South Contral ........................................ & 4,167 & 146.5 & 34.97 & 2,86 & 125.8 & 30.16 & 3,43 & 139.6 & 33. & 3,5 & & 32.68 \\
\hline Alabama & 1,430 & 191.2 & 46.28 & 628 & 132.9 & 32.84 & 1,012 & 179.1 & 43.7 & 1,0 & & 40.66 \\
\hline$\ldots$ & 1,88 & & & 1,24 & 117.7 & & 1,933 & & & 1,18 & & 25.65 \\
\hline$\ldots \ldots \ldots+\ldots \ldots \ldots-1$. & 190 & 165.7 & 42.06 & 108 & 157.7 & 37.02 & 77 & 136.4 & 34.6 & 222 & 172.5 & 42.18 \\
\hline Tennessee & 665 & 124.1 & 30.8 & 880 & 127.8 & 31.26 & 417 & & & 1,128 & 124 & 30.85 \\
\hline 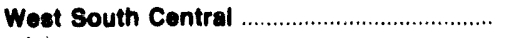 & 11,002 & 144.7 & 22.0 & 382 & 119.6 & 22.76 & 11,31 & 143 & 22. & 72 & 157.8 & 33.50 \\
\hline 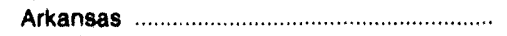 & 907 & 178.8 & 31.03 & - & - & - & 90 & & 31. & - & - & - \\
\hline 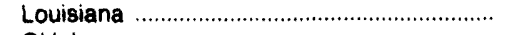 & 1,226 & 158.4 & 25.70 & -- & - & -- & & & & - & - & -- \\
\hline . & 1,376 & 128.9 & 22. & 205 & 93.3 & 15.90 & & & 21. & - & - & - \\
\hline$\ldots$ & 7,493 & 140.7 & 20.3 & 17 & 143.9 & 30.7 & 7,5 & 140 & 20. & 72 & 157.8 & 33.58 \\
\hline 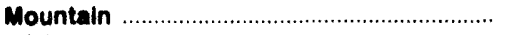 & 8,209 & 118.5 & 23.12 & 593 & 78.1 & 15.38 & 7,2 & & & 1,538 & 129.3 & 29.59 \\
\hline 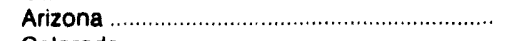 & 1,595 & 141.3 & 29.28 & 103 & 119.1 & 24.07 & 1,69 & 140.0 & 28.9 & - & $\overline{100}$ & - \\
\hline$\ldots+\ldots \ldots \ldots \ldots \ldots \ldots$ & 1,219 & 111.8 & 22.08 & 57 & 76.2 & 14.48 & 1,089 & 111.9 & 21.51 & 187 & 102.2 & 23.07 \\
\hline$\ldots+\ldots+1-\ldots$ & - & 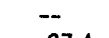 & -- & -- & -- & -- & - & - & - & - & - & - \\
\hline$\ldots$ & 81 & & & - & - & - & 819 & & & - & - & - \\
\hline$\ldots \ldots \ldots \ldots \ldots \ldots \ldots \ldots \ldots$ & 679 & & & - & -- & -- & 484 & 130.0 & 27.8 & 195 & 195.9 & 44.99 \\
\hline . & 1,314 & & 23.38 & - & $-\overline{-}$ & $-\overline{-}$ & 1,314 & 130.2 & 23.38 & - & - & - \\
\hline 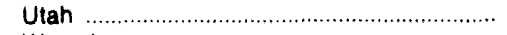 & 1,060 & 127.0 & 29.00 & 96 & 73.3 & 17.49 & - & - & - & 1.156 & 122.3 & 28.04 \\
\hline & 1.524 & 87.2 & 15.14 & 337 & 66.4 & 12.27 & 1.861 & 83.3 & 14.62 & - & - & - \\
\hline 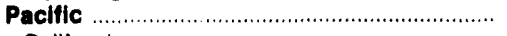 & 409 & 131.5 & 20.86 & 201 & 116.5 & 20.18 & 610 & 126.3 & 20.64 & - & - & - \\
\hline$\ldots$ & - & -- & -- & -- & $=$ & - & - & - & - & - & - & - \\
\hline (1) & -- & - & - & 167 & 113.8 & 19.17 & 167 & 113.8 & 19.17 & -- & - & - \\
\hline 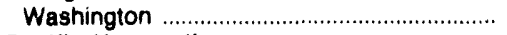 & 409 & 131.5 & 20.86 & 34 & 127.9 & 25.15 & 443 & 131.2 & 21.18 & - & - & - \\
\hline 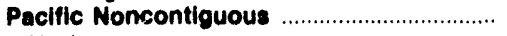 & -- & - & - & - & - & - & - & - & - & - & - & - \\
\hline Alaska & - & - & - & - & - & - & - & - & - & - & - & - \\
\hline Hawaii & nose & $-\bar{n}$ & - & $-\bar{n}$ & $-\overline{0}$ & - & - & -- & - & - & - & $=$ \\
\hline U. S. Total & 50,955 & 142.3 & 28.38 & 14,403 & 126.8 & 28.66 & 46,782 & 131.9 & 25.01 & 10,576 & 151.8 & 37.00 \\
\hline
\end{tabular}

- Monetary values are expressed in nominal terms

Notes: - Totals may not equal sum of components because of independent rounding. -Data are for electric generating plants with a total steamelectric and combined-cycle nameplate capacity of 50 or more megawatts. -Data for 1993 are preliminary.

Source: Federal Energy Regulatory Commission, FERC Form 423, "Monthly Report of Cost and Quality of Fuels for Electric Plants." 
Table 43. Recelpts and Average Cost of Coal Dellvered to Electric Utilities by Sulfur Content, Census Division, and State, September 1993

\begin{tabular}{|c|c|c|c|c|c|c|c|c|c|}
\hline \multirow{3}{*}{$\begin{array}{c}\text { Census Division } \\
\text { and State }\end{array}$} & \multicolumn{3}{|c|}{$0.5 \%$ or Lese } & \multicolumn{3}{|c|}{ More than $0.5 \%$ up to $1.0 \%$} & \multicolumn{3}{|c|}{ More than $1.0 \%$ up to $1.5 \%$} \\
\hline & \multirow{2}{*}{$\begin{array}{c}\text { Recelpts } \\
\text { (1,000 } \\
\text { short } \\
\text { tons) }\end{array}$} & \multicolumn{2}{|c|}{$\begin{array}{l}\text { Averape } \\
\text { Cost' }\end{array}$} & \multirow{2}{*}{$\begin{array}{c}\text { Aecelpts } \\
\begin{array}{c}(1,000 \\
\text { chort } \\
\text { tons) }\end{array}\end{array}$} & \multicolumn{2}{|c|}{$\begin{array}{l}\text { Average } \\
\text { Coot' }\end{array}$} & \multirow{2}{*}{$\begin{array}{c}\text { Receipts } \\
\begin{array}{c}\text { (1,000 } \\
\text { chort } \\
\text { tons) }\end{array}\end{array}$} & \multicolumn{2}{|c|}{$\begin{array}{l}\text { Avernge } \\
\text { Cost }\end{array}$} \\
\hline & & $\begin{array}{c}\text { (Cents } \\
\text { per } \\
10^{\circ} \text { Btu) }\end{array}$ & $\begin{array}{l}\text { (6 por } \\
\text { chort } \\
\text { ton) }\end{array}$ & & $\begin{array}{c}\text { (Cents } \\
\text { per } \\
10^{\circ} \text { Btu) }\end{array}$ & $\begin{array}{l}\text { (S por } \\
\text { chort } \\
\text { ton) }\end{array}$ & & $\begin{array}{c}\text { (Cents } \\
\text { per } \\
10^{\circ} \text { Btu) }\end{array}$ & $\begin{array}{l}\text { (8 por } \\
\text { ehort } \\
\text { ton) }\end{array}$ \\
\hline New England ....... & - & - & - & 251 & 162.6 & 41.84 & 180 & 164.3 & 42.93 \\
\hline Connecticut . & - & -- & - & 68 & 171.4 & 45.22 & $-\infty$ & -- & -- \\
\hline 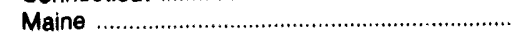 & - & -- & -- & -- & - & -- & -- & -- & -- \\
\hline 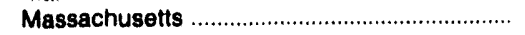 & - & -- & -- & 156 & 161.0 & 40.94 & 139 & 164.5 & 42.80 \\
\hline 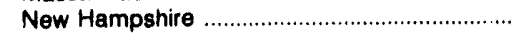 & - & - & -- & 27 & 148.9 & 38.56 & 41 & 163.5 & 43.39 \\
\hline Rhode Island & - & - & -- & - & - & -- & -- & -- & $-\infty$ \\
\hline 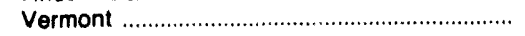 & - & -- & - & - & - & -- & - & -- & -- \\
\hline 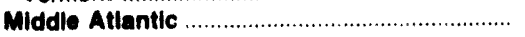 & 4 & 90.8 & 14.21 & 383 & 165.3 & 40.79 & 541 & 141.1 & 36.13 \\
\hline 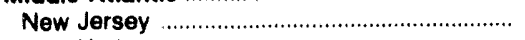 & -- & -- & -- & 12 & 178.8 & 49.75 & - & - & $\ldots$ \\
\hline 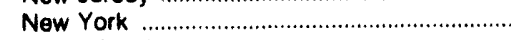 & - & -- & -- & 186 & 180.0 & 45.40 & 149 & 134.4 & 34.85 \\
\hline 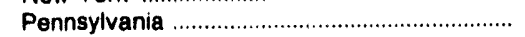 & 4 & 90.8 & 14.21 & 184 & 148.6 & 35.55 & 392 & 143.7 & 36.62 \\
\hline 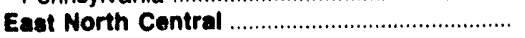 & 4,806 & 141.6 & 25.64 & 3,231 & 156.3 & 37.46 & 1,149 & 149.8 & 36.75 \\
\hline Illinois & 1,203 & 203.8 & 36.68 & 276 & 159.4 & 39.98 & 1 & 157.5 & 34.64 \\
\hline 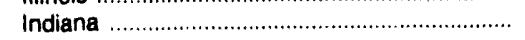 & 1,143 & 113.6 & 19.95 & 159 & 168.4 & 38.45 & 387 & 150.4 & 33.51 \\
\hline 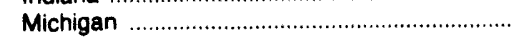 & 1,356 & 143.5 & 26.92 & 1,308 & 166.6 & 40.10 & 281 & 151.2 & 39.65 \\
\hline Ohio & - & - & -- & 1,287 & 144.1 & 34.65 & 409 & 144.8 & 36.40 \\
\hline Wisconsin & 1,205 & 103.3 & 18.56 & 200 & 154.5 & 33.96 & 70 & 170.7 & 45.05 \\
\hline 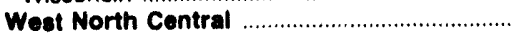 & 5,274 & 97.4 & 16.90 & 2,959 & 86.7 & 12.75 & 290 & 153.4 & 27.93 \\
\hline lowa & 1,156 & 105.3 & 17.95 & 162 & 84.3 & 14.07 & - & - & - \\
\hline 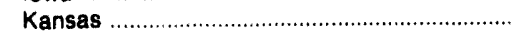 & 1,402 & 97.8 & 16.69 & -- & - & -- & -- & - & - \\
\hline 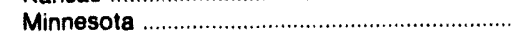 & 709 & 108.0 & 19.20 & 671 & 122.5 & 21.42 & 4 & 169.3 & 40.06 \\
\hline 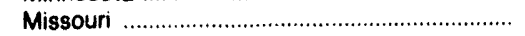 & 1,253 & 99.6 & 17.80 & 192 & 117.4 & 23.43 & 126 & 210.0 & 49.15 \\
\hline 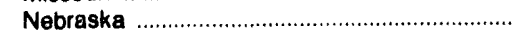 & 754 & 70.5 & 12.03 & - & - & - & -- & - & - \\
\hline 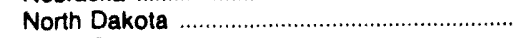 & - & $\rightarrow$ & $\rightarrow$ & 1,901 & 64.8 & 8.47 & 160 & 77.9 & 10.87 \\
\hline 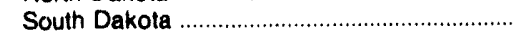 & - & - & - & 34 & 115.3 & 14.37 & -- & - & -- \\
\hline 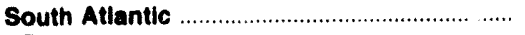 & - & - & - & 4,069 & 171.4 & 43.02 & $\mathbf{3 , 3 8 7}$ & 161.8 & 40.72 \\
\hline 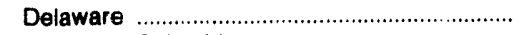 & -- & -- & - & 83 & 169.6 & 43.87 & 23 & 159.4 & 41.68 \\
\hline District of Columbia ......................................... & -- & -- & $\cdots$ & -- & - & - & - & - & - \\
\hline 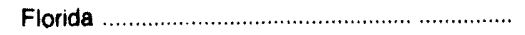 & -- & -- & $-\infty$ & 841 & 169.8 & 42.29 & 492 & 189.3 & 47.74 \\
\hline 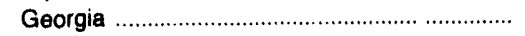 & - & -- & -- & 678 & 190.4 & 47.80 & 615 & 163.6 & 40.42 \\
\hline 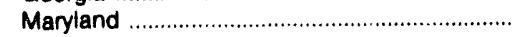 & -- & -- & -- & 293 & 155.8 & 39.56 & 128 & 145.6 & 37.19 \\
\hline 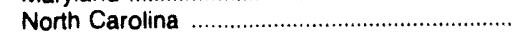 & -- & -- & -- & 1,004 & 178.3 & 44.35 & 967 & 166.2 & 41.60 \\
\hline South Carolina & -- & -- & - & 179 & 162.9 & 42.50 & 539 & 155.2 & 39.44 \\
\hline Virginia & - & -- & - & 452 & 147.1 & 37.95 & 334 & 143.6 & 37.28 \\
\hline West Virginia & -- & - & - & 540 & 170.1 & 41.85 & 289 & 138.1 & 34.28 \\
\hline 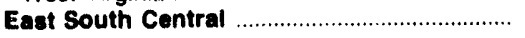 & 118 & 128.5 & 33.60 & 1,879 & 169.2 & 40.59 & 1,033 & 129.0 & 31.93 \\
\hline Alabama & 79 & 125.2 & 33.75 & 912 & 201.0 & 48.80 & 284 & 148.5 & 36.53 \\
\hline 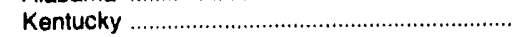 & 14 & 107.2 & 27.38 & 720 & 129.3 & 30.52 & 227 & 112.5 & 27.20 \\
\hline 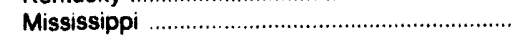 & 22 & 156.2 & 36.93 & 173 & 180.6 & 44.06 & 16 & 145.6 & 35.89 \\
\hline 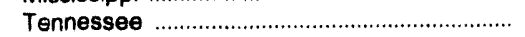 & - & - & - & 74 & 126.4 & 29.12 & 507 & 124.9 & 31.35 \\
\hline 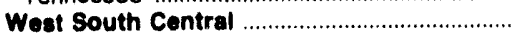 & 7,528 & 154.6 & 25.59 & 2,414 & 119.7 & 16.48 & 785 & 99.4 & 13.51 \\
\hline Arkansas & 907 & 178.8 & 31.03 & - & - & - & -- & - & - \\
\hline 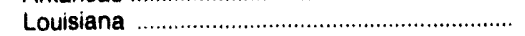 & 939 & 164.6 & 27.88 & 287 & 133.5 & 18.56 & - & - & -- \\
\hline 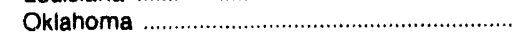 & 1,581 & 124.3 & 21.36 & - & - & - & -- & - & -- \\
\hline 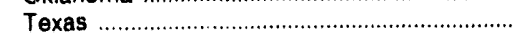 & 4,101 & 158.9 & 25.48 & 2,127 & 117.8 & 16.18 & 785 & 99.4 & 13.51 \\
\hline 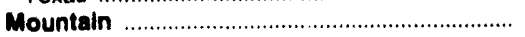 & 3,780 & 125.7 & 24.83 & 5,009 & 108.2 & 20.94 & 14 & 74.9 & 15.40 \\
\hline Arizona & 678 & 172.2 & 34.83 & 1,020 & 119.4 & 25.07 & - & - & - \\
\hline 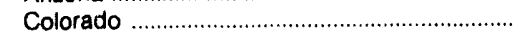 & 1,148 & 111.2 & 21.72 & 120 & 104.6 & 22.24 & 8 & 83.5 & 16.77 \\
\hline Idaho & -- & - & -- & -- & -- & - & - & -- & - \\
\hline 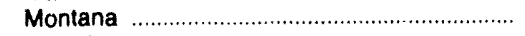 & - & - & -- & 819 & 67.4 & 11.52 & -- & -- & -- \\
\hline 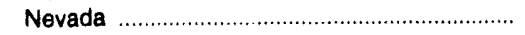 & 277 & 196.6 & 42.96 & 402 & 117.7 & 25.74 & -- & -- & - \\
\hline 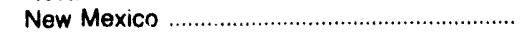 & -- & -- & -- & 1,314 & 130.2 & 23.38 & - & - & - \\
\hline Utah & 779 & 139.2 & 31.49 & 377 & 88.9 & 20.93 & -- & - & - \\
\hline 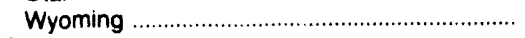 & 899 & 59.9 & 9.89 & 957 & 103.0 & 19.08 & 5 & 60.8 & 12.99 \\
\hline 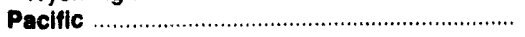 & 194 & 116.5 & 19.93 & 416 & 131.2 & 20.96 & - & - & - \\
\hline 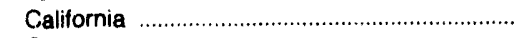 & - & - & - & -- & $-\infty$ & - & - & - & - \\
\hline Oregon & 167 & 113.8 & 19.17 & -- & -- & - & -- & -- & $\cdots$ \\
\hline Washington & 27 & 131.3 & 24.65 & 416 & 131.2 & 20.96 & - & - & -- \\
\hline Pacific Noncontiguous ..................................... & - & - & - & - & - & - & - & - & - \\
\hline 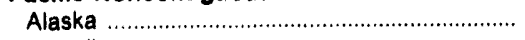 & - & -- & -- & -- & - & - & -- & - & - \\
\hline 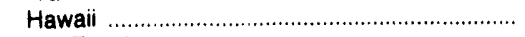 & -- & - & $-\infty$ & - & -- & - & -- & - & -- \\
\hline U. S. Total & 21,802 & 131.9 & 23.36 & 20,611 & 140.1 & 28.60 & 7,378 & 149.2 & 35.14 \\
\hline
\end{tabular}

1 Monetary values are expressed in nominal terms.

Notes: - Totals may not equal sum of components because of independent rounding. -Data are for electric generating plants with a total steam. electric and combined-cycle nameplate capacity of 50 or more megawatts. -Data for 1993 are preliminary.

Source: Federal Energy Regulatory Commission, FERC Forrn 423, "Monthly Report of Cost and Quality of Fuels for Electric Plants." 
Table 43. Recelpts and Average Cost of Coal Delivered to Electric Utilities by Sulfur Content, Census Division, and State, September 1993 (Continued)

\begin{tabular}{|c|c|c|c|c|c|c|c|c|c|c|c|}
\hline \multirow{3}{*}{$\begin{array}{l}\text { Conaus Divialon } \\
\text { and State }\end{array}$} & \multicolumn{3}{|c|}{ More than $1.5 \%$ up to $2.0 \%$} & \multicolumn{3}{|c|}{ More than $2.0 \%$ up to $3.0 \%$} & \multicolumn{3}{|c|}{ More than $3.0 \%$} & \multicolumn{2}{|c|}{ All Purchases } \\
\hline & \multirow{2}{*}{$\begin{array}{c}\text { Rocelpte } \\
\text { (1,000 } \\
\text { shont } \\
\text { tons) }\end{array}$} & \multicolumn{2}{|c|}{$\begin{array}{l}\text { Average } \\
\text { Cost' }\end{array}$} & \multirow{2}{*}{$\begin{array}{c}\text { Recolpte } \\
\begin{array}{c}\text { (1,000 } \\
\text { chort } \\
\text { tons) }\end{array}\end{array}$} & \multicolumn{2}{|c|}{$\begin{array}{l}\text { Average } \\
\text { Cost' }\end{array}$} & \multirow{2}{*}{$\begin{array}{l}\text { Accelpts } \\
\text { (1,000 } \\
\text { short } \\
\text { tons) }\end{array}$} & \multicolumn{4}{|c|}{$\begin{array}{l}\text { Average } \\
\text { Cost' }\end{array}$} \\
\hline & & $\begin{array}{c}\text { (Cents } \\
\text { por } \\
10^{\circ} \text { Btu) }\end{array}$ & $\begin{array}{l}\text { (s per } \\
\text { chort } \\
\text { ton) }\end{array}$ & & $\begin{array}{c}\text { (Cents } \\
\text { per } \\
10^{\circ} \text { Btu) }\end{array}$ & $\begin{array}{l}\text { (8 por } \\
\text { chort } \\
\text { (on) }\end{array}$ & & $\begin{array}{c}\text { (Cents } \\
\text { per } \\
10^{\circ} \\
\text { Btu) }\end{array}$ & $\begin{array}{c}(8 \\
\text { per } \\
\text { chort } \\
\text { ton) }\end{array}$ & $\begin{array}{l}\text { (Gents } \\
\text { per } \\
10^{\circ} \\
\text { Btu) }\end{array}$ & $\begin{array}{l}\text { (8) } \\
\text { per } \\
\text { chort } \\
\text { tonis) }\end{array}$ \\
\hline 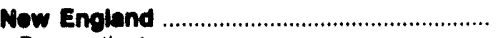 & - & - & - & 39 & 153.8 & 40.13 & - & - & - & 162.5 & 42.12 \\
\hline 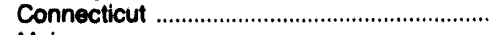 & -- & - & - & -- & - & - & -- & - & -- & 171.4 & 45.22 \\
\hline Maine & -- & -- & - & - & - & - & - & - & - & $\ldots$ & -- \\
\hline 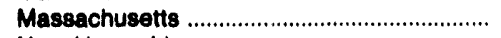 & -- & -- & -- & -- & - & - & - & - & -- & 162.7 & 41.82 \\
\hline 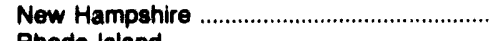 & - & - & - & 39 & 153.8 & 40.13 & - & - & - & 156.3 & 40.97 \\
\hline Rhode Island & - & - & - & - & - & - & - & - & - & - & -- \\
\hline Vermont ......... - & - & $\overline{s e n}_{10}$ & $\bar{m}$ & - & $-\overline{10}$ & $-\overline{c o s}$ & - & - & $\overline{-}$ & - & - \\
\hline Middle Attantlc & 834 & 151.4 & 37.21 & 1,840 & 141.9 & 36.20 & 7 & 112.8 & 26.24 & 146.3 & 36.51 \\
\hline 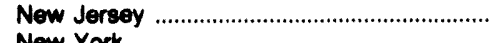 & - & $-\bar{x}_{0}$ & $=-$ & 46 & 164.4 & 42.85 & -- & - & - & 167.5 & 44.27 \\
\hline $\begin{array}{l}\text { Now York } \\
\text { Pennsytvania }\end{array}$ & 128 & 145.8 & 37.92 & 79 & 129.7 & 34.23 & - & $-\overline{10}$ & - & 151.7 & 39.10 \\
\hline 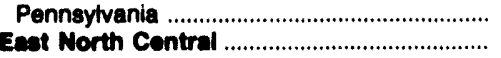 & $\begin{array}{l}706 \\
552\end{array}$ & $\begin{array}{l}152.5 \\
128.1\end{array}$ & $\begin{array}{l}37.91 \\
29.59\end{array}$ & $\begin{array}{l}1,723 \\
1,790\end{array}$ & $\begin{array}{l}141.8 \\
120.2\end{array}$ & $\begin{array}{l}35.04 \\
20.24\end{array}$ & 2,894 & $\begin{array}{l}112.8 \\
140.7\end{array}$ & $\begin{array}{l}26.24 \\
32.14\end{array}$ & $\begin{array}{l}144.9 \\
143.5\end{array}$ & $\begin{array}{l}35.90 \\
31.03\end{array}$ \\
\hline 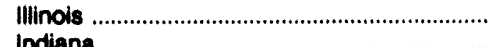 & $\begin{array}{r}70 \\
251\end{array}$ & 109.1 & 24.06 & 517 & 141.8 & 31.58 & 548 & 133.2 & 28.24 & 166.5 & 33.91 \\
\hline $\begin{array}{l}\text { Indiana } \\
\text { Michigan }\end{array}$ & & 121.0 & 27.29 & - 865 & 119.7 & 27.02 & & 120.9 & 27.13 & 124.3 & 26.03 \\
\hline & & $\overline{-}$ & $\overline{0-21}$ & - & - & - & 34 & 158.8 & 36.01 & 155.7 & 34.01 \\
\hline Ohio & $\begin{array}{r}166 \\
65\end{array}$ & $\begin{array}{l}148.3 \\
120.6\end{array}$ & $\begin{array}{l}35.74 \\
28.67\end{array}$ & $\begin{array}{r}371 \\
36\end{array}$ & 123.1 & 29.80 & 1,443 & 153.7 & 36.41 & 145.9 & 35.09 \\
\hline $\begin{array}{l}\text { Wisconsin } \\
\text { Weat North Central }\end{array}$ & $\begin{array}{r}60 \\
1\end{array}$ & 165.0 & 40.19 & $\begin{array}{r}36 \\
200\end{array}$ & $\begin{array}{l}194.2 \\
192.1\end{array}$ & $\begin{array}{l}43.44 \\
44.27\end{array}$ & 84 & 123.0 & 20.23 & $\begin{array}{l}118.2 \\
100.6\end{array}$ & $\begin{array}{l}22.68 \\
16.82\end{array}$ \\
\hline 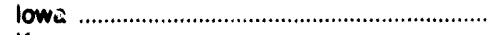 & - & -- & -- & 106 & 155.7 & 35.25 & 6 & 142.0 & 30.16 & 108.1 & 18.84 \\
\hline Kansas & - & - & - & 29 & 307.0 & 71.97 & 28 & 110.3 & 25.61 & 103.8 & 17.97 \\
\hline Minnesota & 1 & 165.0 & 40.19 & - & - & - & - & - & $\cdots$ & 115.3 & 20.37 \\
\hline Missourt & - & - & - & 134 & 194.7 & 45.31 & 50 & 127.7 & 29.41 & 121.2 & 23.10 \\
\hline Nebraska & - & -- & - & - & -- & - & - & - & - & 70.5 & 12.03 \\
\hline North Dakota & - & - & - & -- & - & - & - & - & - & 65.9 & 8.66 \\
\hline 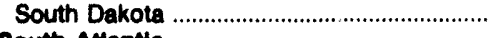 & $-\bar{n}$ & 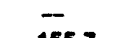 & $\overline{-}$ & - & $\overline{-}$ & $\bar{n}$ & - & - & - & 115.3 & 14.37 \\
\hline 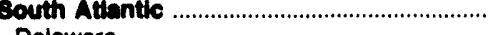 & 1,344 & 155.7 & 30.84 & 844 & 162.0 & 40.54 & 440 & 120.0 & 31.24 & 163.6 & 40.97 \\
\hline Delaware & - & - & - & - & - & - & - & - & - & 167.3 & 43.39 \\
\hline District of Columbia & $-\infty$ & 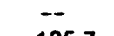 & - & - & $=$ & - & - & - & -- & - & -- \\
\hline $\begin{array}{l}\text { Florida } \\
\text { Goorgia }\end{array}$ & $\begin{array}{l}184 \\
408\end{array}$ & $\begin{array}{l}185.7 \\
180.5\end{array}$ & $\begin{array}{l}45.14 \\
45.04\end{array}$ & $\begin{array}{l}324 \\
119\end{array}$ & $\begin{array}{l}181.8 \\
144.2\end{array}$ & $\begin{array}{l}44.21 \\
33.95\end{array}$ & $\begin{array}{l}54 \\
60\end{array}$ & $\begin{array}{l}199.0 \\
196.6\end{array}$ & $\begin{array}{l}44.99 \\
43.31\end{array}$ & $\begin{array}{l}179.2 \\
176.8\end{array}$ & $\begin{array}{l}44.39 \\
43.76\end{array}$ \\
\hline 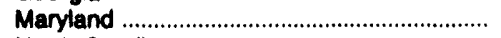 & 161 & 175.9 & 44.61 & 116 & 160.5 & 41.84 & $-\infty$ & $\ldots$ & - & 159.3 & 40.67 \\
\hline North Carolina & 8 & 163.0 & 41.06 & - & - & - & - & -- & - & 172.3 & 42.99 \\
\hline South Carolina & 54 & 158.5 & 41.64 & o & 154.2 & 41.78 & - & - & - & 157.2 & 40.31 \\
\hline Virginia & $=$ & - & - & 36 & 180.0 & 46.68 & - & - & - & 147.1 & 38.06 \\
\hline West Virginia & 529 & 119.6 & 29.60 & 248 & 143.7 & 37.4 & 326 & 108.2 & 26.71 & 137.6 & 34.29 \\
\hline Enat South Central ........................................... & 1,199 & 146.1 & 35.48 & 1,691 & 119.4 & 28.5 & 1,112 & 112.8 & 25.30 & 138.0 & 33.01 \\
\hline Alabama & 580 & 164.5 & 39.72 & 115 & 135.0 & 33.6 & & $\$ 18.8$ & 27.61 & 173.2 & 42.18 \\
\hline 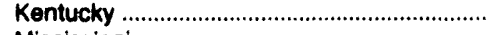 & 258 & 133.9 & 32.10 & 885 & 109. & 25.6 & 1,024 & 112.3 & 25.11 & 117.4 & 27.23 \\
\hline 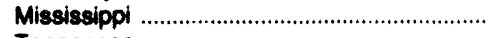 & - & -- & - & 88 & 134.2 & 34.2 & -- & - & -- & 163.0 & 40.23 \\
\hline 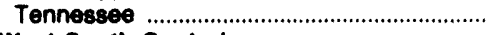 & 360 & 125.7 & 31.11 & 603 & 127.6 & 31.13 & - & - & - & 126.2 & 31.10 \\
\hline Weat South Centrai ......................................... & 373 & 155.5 & 15.10 & 285 & 20.9 & 10.63 & - & - & - & 143.7 & 22.10 \\
\hline 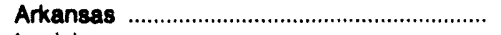 & -- & - & - & -- & - & - & - & - & - & 178.8 & 31.03 \\
\hline 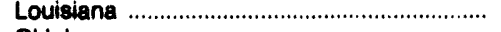 & - & - & - & - & -- & - & - & $\cdots$ & -- & 158.4 & 25.70 \\
\hline 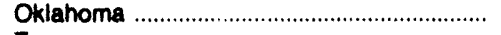 & -- & -- & - & - & - & - & - & -- & - & 124.3 & 21.36 \\
\hline Texas ……1- & 373 & 155.5 & 15.10 & 285 & 98.8 & 10.63 & - & - & -- & 140.8 & 20.62 \\
\hline Mountaln & - & - & - & - & - & - & - & - & - & 115.7 & 22.60 \\
\hline Arizona & - & - & - & - & - & - & - & - & - & & 28.97 \\
\hline Colorado & - & - & - & - & - & -- & - & - & -- & 110.3 & 21.74 \\
\hline (1) & - & - & - & - & - & - & - & - & - & $-\infty$ & -- \\
\hline (1) & - & - & - & - & - & - & - & - & -- & 67.4 & 11.52 \\
\hline Nevada & - & -- & - & - & - & - & - & - & -- & 149.8 & 32.77 \\
\hline 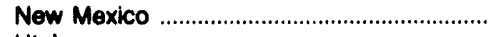 & -- & - & - & -- & - & - & - & - & - & 130.2 & 23.38 \\
\hline - & - & - & - & - & - & - & - & -- & - & 122.3 & 28.04 \\
\hline 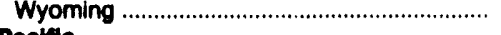 & -- & - & - & - & - & - & -- & - & - & 83.3 & 14.62 \\
\hline 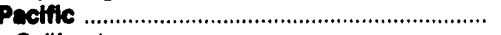 & - & - & - & - & - & - & - & - & - & 126.3 & 20.64 \\
\hline 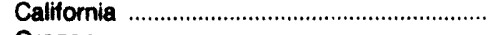 & - & - & $\cdots$ & - & - & - & - & - & - & - & - \\
\hline 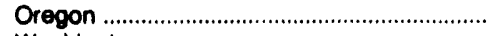 & - & - & - & - & - & - & $\cdots$ & - & - & 113.8 & 18.17 \\
\hline Washington & - & -- & - & - & -- & - & - & - & - & 131.2 & 21.19 \\
\hline Pactific Moncontiguous ......................................... & - & - & - & - & - & - & - & - & - & - & - \\
\hline 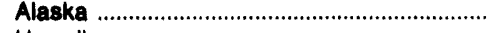 & - & - & - & - & - & - & - & - & - & - & _. \\
\hline Hawaii & -- & - & - & - & - & - & - & - & - & - & - \\
\hline U. 8. Total & 4,303 & 148.5 & 34.48 & 6,768 & 138.5 & 31.00 & 4,497 & 132.5 & 30.28 & 138.5 & 28.44 \\
\hline
\end{tabular}

- Monetary values are expressed in nominal terms.

- = Less than 0.05 .

Notes: - Totals may not equal sum of components because of independent rounding. -Data are for electric generating plants with a total steamelectric and combined-cycle nameplate capacity of 50 or more megawatts. -Data for 1893 are preliminary.

Source: Federal Energy Regulatory Commission, FERC Form 423, "Monthly Report of Cost and Quality of Fuels for Electric Plants." 
Table 44. Electric Utility Receipts of Petroleum by Type, Census Division, and State, September 1993

\begin{tabular}{|c|c|c|c|c|c|c|c|c|c|c|}
\hline \multirow{2}{*}{$\begin{array}{l}\text { Consus Divialon } \\
\text { and Stale }\end{array}$} & \multicolumn{2}{|c|}{ Nu. 2 Fuat OII } & \multicolumn{2}{|c|}{ No. 4 Fuel OII' } & \multicolumn{2}{|c|}{ No. 5 Fuel OII' } & \multicolumn{2}{|c|}{ No. 6 Fuel Oll } & \multicolumn{2}{|c|}{ Total } \\
\hline & $\begin{array}{l}\text { (thouvend } \\
\text { berrels) }\end{array}$ & $\begin{array}{l}\text { (blllon } \\
\text { Btu) }\end{array}$ & $\begin{array}{l}\text { (thousand } \\
\text { barrels) }\end{array}$ & $\begin{array}{l}\text { (billon } \\
\text { Btu) }\end{array}$ & $\begin{array}{l}\text { (thousand } \\
\text { barrets) }\end{array}$ & $\begin{array}{l}\text { (billlon } \\
\text { Btu) }\end{array}$ & $\begin{array}{c}\text { (thoueand } \\
\text { barrels) }\end{array}$ & $\begin{array}{l}\text { (billion } \\
\text { Btu) }\end{array}$ & $\begin{array}{c}\text { (thousand } \\
\text { barrele) }\end{array}$ & $\begin{array}{l}\text { (billion } \\
\text { Btu) }\end{array}$ \\
\hline New England & - & 49 & - & - & - & - & 2,095 & 19,120 & 3,004 & 19,169 \\
\hline Connecticut & 2 & 13 & - & - & - & - & 705 & 4.484 & 707 & 4,497 \\
\hline Maine & 1 & 5 & - & - & - & - & 444 & 2,815 & 445 & 2,820 \\
\hline Massachusetts …….............. & 2 & 11 & - & - & - & - & 1,490 & 9,477 & 1,492 & $\mathbf{9 , 4 8 8}$ \\
\hline New Hampshire ................................ & 2 & 14 & -- & - & - & - & 356 & 2,343 & 359 & 2,357 \\
\hline Rhode Island & - & - & - & - & -- & -- & - & - & - & - \\
\hline 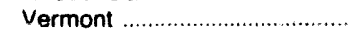 & 1 & 5 & - & - & - & - & -_ & -- & 1 & 5 \\
\hline 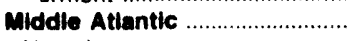 & $\infty 0$ & 468 & - & - & - & - & 3,374 & 21,414 & 3,454 & 21,880 \\
\hline 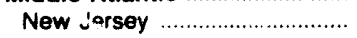 & 37 & 214 & - & - & - & -- & 452 & 2,840 & 489 & 3,054 \\
\hline New York & 5 & 31 & - & - & - & -- & 2,358 & 14,965 & 2,363 & 14,896 \\
\hline 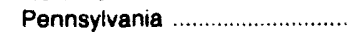 & 38 & 222 & - & - & - & - & 564 & 3,608 & 602 & 3,830 \\
\hline East North Central ...................... & 117 & 677 & - & - & - & - & 238 & 1,519 & 355 & 2,196 \\
\hline Illinois ................................ & 29 & 168 & - & - & - & - & 129 & $8 r^{4}$ & 158 & 993 \\
\hline Indiana & 42 & 243 & - & - & - & -- & -- & - & 42 & 243 \\
\hline Michigan & 24 & 140 & - & -- & - & - & 109 & 695 & 134 & 835 \\
\hline Ohio … & 21 & 124 & -- & - & -- & -- & -- & -- & 21 & 124 \\
\hline Wisconsin & $\cdot$ & 1 & - & - & - & - & -. & -- & $\cdot$. & 1 \\
\hline West North Central ...................... & 22 & 128 & - & - & - & - & 13 & 83 & 34 & 200 \\
\hline lowa & 2 & 10 & - & - & -- & - & -- & -- & 2 & 10 \\
\hline Kansas & - & - & - & - & - & - & $-\ldots$ & - & - & -- \\
\hline 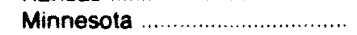 & 1 & 7 & - & - & - & - & -- & -- & 1 & 7 \\
\hline 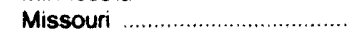 & 11 & 63 & - & - & - & - & 13 & 83 & 24 & 146 \\
\hline Nebraska & 2 & 14 & - & - & - & - & -- & -- & 2 & 14 \\
\hline North Dakota & 6 & 33 & - & - & - & - & - & -- & 6 & 33 \\
\hline 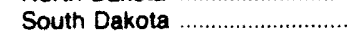 & - & - & - & - & - & -- & -- & - & - & -- \\
\hline South Atiantic & 152 & 808 & 83 & 500 & - & - & 7,272 & 46,284 & 7,507 & $\$ 7,672$ \\
\hline Delaware & 7 & 40 & - & - & - & - & 354 & 2,255 & 360 & 2,295 \\
\hline District of Columbia & 2 & 12 & 83 & 500 & - & -- & -- & -- & 85 & 511 \\
\hline Florida ................................. & 25 & 147 & - & - & - & -- & 5,612 & 35,744 & 5,637 & 35,892 \\
\hline Georgia & 12 & 69 & -- & - & - & - & -- & -- & 12 & 69 \\
\hline 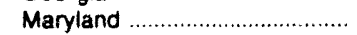 & 28 & 160 & - & - & - & -- & 574 & 3,638 & 601 & 3,799 \\
\hline North Carolina & 11 & 64 & - & - & -- & -- & $\ldots$ & - & 11 & 64 \\
\hline South Carolina ........................ & 1 & 6 & - & - & - & - & -- & -. & 1 & 6 \\
\hline Virginia & 22 & 127 & - & - & -- & -- & 733 & 4,646 & 754 & 4,773 \\
\hline West Virginia .................................. & 45 & 264 & -- & - & - & $\ldots$ & -- & $-\infty$ & 45 & 264 \\
\hline East South Central & 34 & 197 & - & - & - & - & 851 & 5,487 & 885 & 5,684 \\
\hline Alabama & 8 & 47 & -- & - & - & -- & -- & $-\infty$ & 8 & 47 \\
\hline 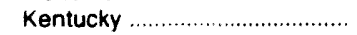 & 13 & 74 & - & - & -- & -- & -- & -- & 13 & 74 \\
\hline Mississippi & 2 & 12 & - & - & -- & -- & 851 & 5,487 & 854 & 5,499 \\
\hline 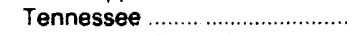 & 11 & 63 & -- & - & -- & -- & - & - & 11 & 63 \\
\hline West South Central ................... & 14 & 82 & - & - & - & - & - & - & 14 & 82 \\
\hline Arkansas & 4 & 25 & - & - & - & -- & -- & -- & 4 & 25 \\
\hline 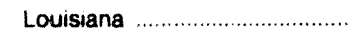 & 6 & 33 & - & - & -- & -- & -- & - & 6 & 33 \\
\hline OHlahoma & - & - & - & -- & -- & -- & - & - & -- & -- \\
\hline Texas & 4 & 23 & - & - & - & - & - & -- & 4 & 23 \\
\hline 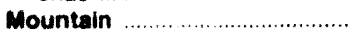 & 12 & 71 & - & - & - & - & - & - & 12 & 71 \\
\hline Arizona & 1 & 4 & - & - & - & - & -- & -- & 1 & 4 \\
\hline 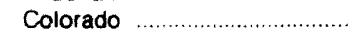 & - & - & -- & - & -- & -- & - & -- & - & - \\
\hline Idaho & - & - & - & -- & - & -- & -- & -- & -- & -- \\
\hline Montana & - & - & -- & - & -- & -- & -- & - & -. & -. \\
\hline Nevada & - & - & - & - & -- & - & - & -- & - & -- \\
\hline New Mexico & 6 & 34 & -- & - & -- & -- & -- & - & 6 & 34 \\
\hline Utan & - & - & -. & -- & -- & -- & -. & -. & - & -_ \\
\hline Wyoming & 6 & 33 & -- & - & -. & -- & -- & - & 6 & 33 \\
\hline Pacific & 2 & 12 & - & - & - & - & - & - & 2 & 12 \\
\hline California & -- & - & -- & - & -- & -- & -- & - & -- & -- \\
\hline Oregon & -- & -- & -- & - & - & -. & -- & -- & - & -- \\
\hline Washington & 2 & 12 & -- & - & - & -- & - & -- & 2 & 12 \\
\hline Pacific Noncontiguous & - & - & - & - & - & - & 497 & 3,124 & 497 & 3,124 \\
\hline Alaska & -- & -- & -- & - & - & - & - & - & -- & $\ldots$ \\
\hline 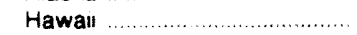 & - & -- & - & - & - & -- & 497 & 3,124 & 497 & 3,124 \\
\hline U.S. Total & 442 & 2,569 & 83 & 500 & - & - & 15,241 & 97,029 & 15,766 & 100,098 \\
\hline
\end{tabular}

- Blend of No. 2 Fuel Dil and No. 6 Fuel Oil.

Less than 0.5

Notes: -Totals mey not equal sum of components because of independent rounding. $\bullet$ Data are for electric generating plants with total steam-electric and combined-cycle nameplate capacity of 50 or more megawatts. Data for 1993 are preliminary.

Source: Federal Energy Regulatory Commission. FERC Form 423, "Monthly Report of Cost and Quality of Fuals for Electric Plants." 
Table 45. Receipts and Average Cost of Petroleum Dellvered to Electric Utilities by Census Division and State

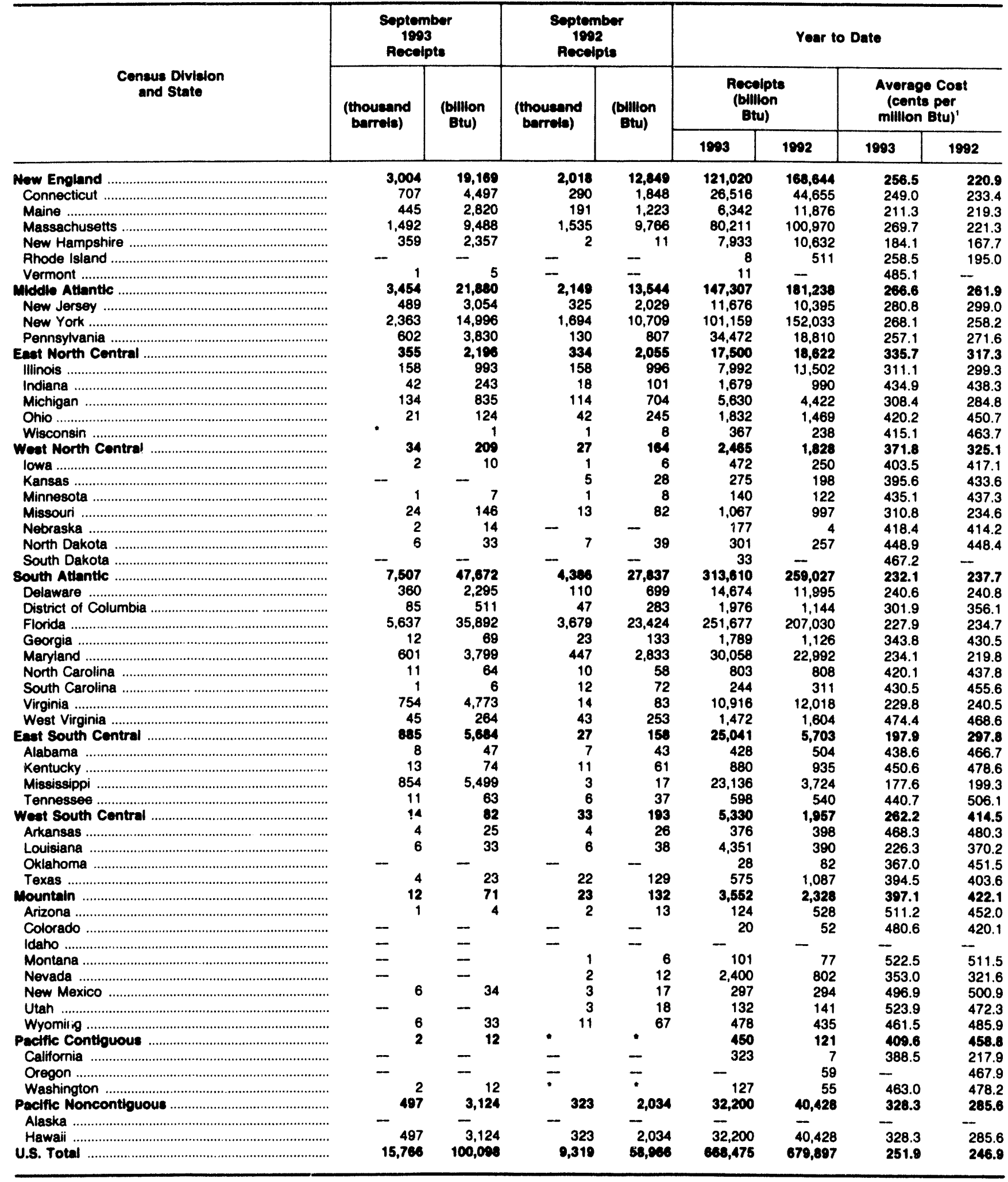

Monetary values are expressed in nominal terms.

Less than 0.5 .

Notes: -Data for 1992 are final. Data for 1993 are preliminary. - Totals may not equal sum of components because of independent rounding. - Data are for electric generating plants with a total steam-electric and combined-cycle nameplate capacity of 50 or more megawatts. $\bullet$ The September 1993 petroleum coke receipts were 131,082 short tons and the cost was 70.3 cents per million Btu.

Source. Federal Energy Regulatory Commission, FERC Form 423, "Monthly Report of Cost and Quality of Fuels for Electric Plants." 
Table 46. Recelpts and Average Cost of Petroleum Delivered to Electric Utilities by Type, Census Division, and State, September 1993

\begin{tabular}{|c|c|c|c|c|c|c|c|c|c|c|c|c|}
\hline \multirow{4}{*}{$\begin{array}{l}\text { Consus Divilion } \\
\text { and State }\end{array}$} & \multicolumn{6}{|c|}{ Fuel Oll No. 6 by Type of Purchaee } & \multicolumn{6}{|c|}{ Averaged Cost of Fuel Olls' } \\
\hline & \multicolumn{3}{|c|}{ Contract } & \multicolumn{3}{|c|}{ Spot } & \multicolumn{2}{|c|}{ No. 2} & \multicolumn{2}{|c|}{ No. 4 -No. 5} & \multicolumn{2}{|c|}{ No. 6} \\
\hline & \multirow{2}{*}{\begin{tabular}{|c|} 
Recelpts \\
$\begin{array}{c}(1,000 \\
\text { bble })\end{array}$ \\
\end{tabular}} & \multicolumn{2}{|c|}{$\begin{array}{l}\text { Averape } \\
\text { Cost' }\end{array}$} & Recelpte & $\begin{array}{l}\text { Avers } \\
\text { Coot }\end{array}$ & & ents & & & & & \\
\hline & & $\begin{array}{c}\text { (Cents } \\
\text { per } \\
10^{\circ} \text { Btu) }\end{array}$ & $\begin{array}{c}\text { (8) } \\
\text { per } \\
\text { bot) }\end{array}$ & $\begin{array}{c}(1,000 \\
\text { bblo })\end{array}$ & $\begin{array}{c}\text { (Cents } \\
\text { per } \\
10^{\circ} \text { Btu) }\end{array}$ & $\begin{array}{c}18 \\
\text { por } \\
\text { boti) }\end{array}$ & $10^{\mathrm{Por}} \mathrm{Btu}$ & $\begin{array}{l}\text { per } \\
\text { bbl) }\end{array}$ & $10^{\circ}$ Btu) & $\begin{array}{l}\text { por } \\
\text { bbl) }\end{array}$ & $10^{\text {per }}$ Btu) & $\begin{array}{l}\text { por } \\
\text { bbd) }\end{array}$ \\
\hline Now England ........... & 2,241 & 224.1 & 14.32 & 754 & 253.1 & 16.10 & 404.0 & 23.44 & - & - & 231.4 & 14.77 \\
\hline 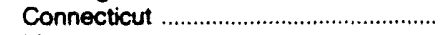 & 144 & 251.4 & 16.04 & 561 & 237.8 & 15.12 & 403.3 & 23.63 & - & -- & 240.6 & 15.31 \\
\hline Maine & 444 & 206.5 & 13.09 & - & - & - & 402.3 & 23.44 & -- & - & 206.5 & 13.08 \\
\hline Massachusetts & 1,297 & 242.4 & 15.41 & 193 & 297.4 & 18.94 & 385.0 & 22.64 & - & - & 249.5 & 15.87 \\
\hline 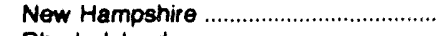 & 356 & 170.2 & 11.19 & - & -- & - & 396.8 & 23.12 & - & - & 170.2 & 11.18 \\
\hline Rhode Istand & - & -- & - & - & - & - & - & - & - & - & $-\infty$ & - \\
\hline Vermont & -- & - & - & - & - & - & 465.2 & 25.31 & - & - & - & -_ \\
\hline Middie Attantic . & 2,777 & 252.1 & 16.02 & 597 & 228.3 & 14.50 & 404.2 & 23.44 & - & - & 248.1 & 15.75 \\
\hline 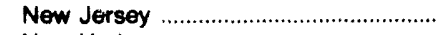 & 452 & 260.4 & 16.37 & - & - & - & 393.9 & 22.72 & -- & -- & 260.4 & 16.37 \\
\hline 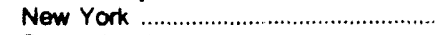 & 1,830 & 249.5 & 15.86 & 528 & 227.2 & 14.34 & 460.8 & 26.64 & -- & - & 244.5 & 15.52 \\
\hline 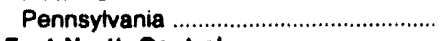 & 495 & 254.5 & 16.28 & 69 & 244.8 & 15.69 & 406.2 & 23.69 & - & - & 253.4 & 16.21 \\
\hline East North Central & 129 & 266.6 & 17.04 & 109 & 278.1 & 17.65 & 406.1 & 23.50 & - & - & 271.9 & 17.32 \\
\hline Illinois & 129 & 266.6 & 17.04 & - & - & - & 403.3 & 23.50 & - & -- & 266.6 & 17.04 \\
\hline Indiana & - & - & - & - & $=$ & - & 409.9 & 23.57 & - & - & - & - \\
\hline Michigan & -- & - & - & 109 & 278.1 & 17.65 & 385.4 & 22.33 & - & - & 278.1 & 17.65 \\
\hline Ohio & - & - & - & - & - & - & 425.3 & 24.65 & - & -- & - & - \\
\hline Wisconsin & - & -- & -- & - & -- & - & 437.9 & 25.75 & -- & - & - & - \\
\hline West North Central ............................. & - & - & - & 13 & 175.5 & 11.50 & 426.7 & 24.73 & - & - & 175.5 & 11.50 \\
\hline lowa & - & - & - & - & - & - & 404.9 & 23.84 & - & -. & - & - \\
\hline Kansas & - & - & -- & - & - & - & - & - & -- & - & -- & - \\
\hline Minnesola & -- & -- & -- & - & - & - & 443.7 & 25.62 & -- & -- & -- & _- \\
\hline 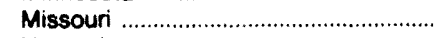 & - & - & -- & 13 & 175.5 & 11.50 & 400.4 & 23.09 & - & - & 175.5 & 11.50 \\
\hline Nebraska & - & - & -- & - & - & - & 426.9 & 24.65 & - & - & - & - \\
\hline North Dakota & - & - & - & - & - & -- & 480.6 & 28.05 & -. & - & -- & - \\
\hline 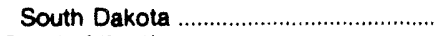 & - & - & -- & - & - & -- & - & - & - & - & -- & - \\
\hline 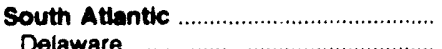 & 3,433 & 214.1 & 13.64 & 3,839 & 213.1 & 13.55 & 416.7 & 24.30 & 303.9 & 18.30 & 213.6 & 13.59 \\
\hline $\begin{array}{l}\text { Delaware } \\
\text { District of Columbia }\end{array}$ & 354 & 227.5 & 14.50 & $-\infty$ & - & - & 394.1 & 23.22 & - & - & 227.5 & 14.50 \\
\hline $\begin{array}{l}\text { District of Columbia } \\
\text { Florida }\end{array}$ & - & - & - & - & - & - & 386.6 & 22.44 & 303.9 & 18.30 & - & - \\
\hline Florida & 2,506 & 212.5 & 13.56 & 3,106 & 212.3 & 13.50 & 415.3 & 24.22 & - & - & 212.4 & 13.53 \\
\hline Georgia & - & - & - & - & - & - & 421.9 & 24.53 & - & - & - & - \\
\hline 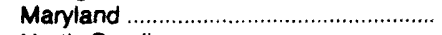 & 574 & 212.4 & 13.47 & - & - & - & 391.8 & 22.78 & - & - & 212.4 & 13.47 \\
\hline North Carolina & - & -- & - & - & - & - & 408.5 & 23.70 & - & - & - & -- \\
\hline South Carolina & - & - & - & - & - & - & 389.2 & 22.78 & - & -- & -- & - \\
\hline Virginia & - & - & - & 733 & 216.8 & 13.75 & 384.1 & 22.47 & - & -- & 216.8 & 13.75 \\
\hline 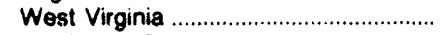 & - & -- & -- & - & - & - & 454.4 & 26.50 & - & -. & - & - \\
\hline East South Central & - & - & - & 851 & 175.6 & 11.32 & 437.5 & 25.39 & - & - & 175.6 & 11.32 \\
\hline Alabama & - & - & - & - & - & - & 436.1 & 25.15 & - & - & $\ldots$ & - \\
\hline Kentucky & - & - & - & - & - & - & 452.8 & 26.41 & - & - & -- & - \\
\hline Mississippi & -- & -- & -- & 851 & 175.6 & 11.32 & 454.5 & 26.51 & - & -. & 175.6 & 11.32 \\
\hline 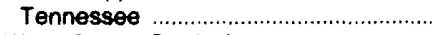 & - & - & - & - & - & - & 417.5 & 24.18 & - & -- & - & - \\
\hline West South Central & - & - & - & - & - & - & 392.9 & 22.84 & - & - & - & $\overline{-}$ \\
\hline Arkansas & - & - & - & - & - & - & 435.9 & 25.03 & - & - & - & - \\
\hline 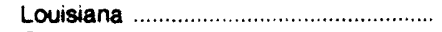 & - & - & - & - & - & - & 392.0 & 23.05 & - & - & -- & -- \\
\hline Oklahoma & - & - & - & - & -- & -- & - & - & - & -- & - & - \\
\hline Texas & - & -- & -- & - & - & - & 347.3 & 20.13 & - & - & -- & -_ \\
\hline Mountaln & - & - & - & - & - & - & 497.2 & 28.69 & - & - & - & - \\
\hline Arizona & - & - & - & - & - & - & 497.3 & 29.85 & - & - & - & - \\
\hline Colorado & - & -- & - & - & -- & - & - & - & - & -- & - & -.. \\
\hline Idaho & - & -- & -- & - & -- & - & - & -- & - & - & -- & - \\
\hline Montana & - & -- & -- & - & - & - & - & - & - & - & - & - \\
\hline . & - & - & -- & - & -- & - & -- & - & - & - & - & - \\
\hline 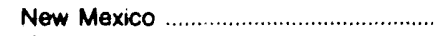 & - & - & -- & - & - & - & 482.1 & 27.54 & - & - & - & -- \\
\hline Utah & - & - & -- & -- & - & - & - & - & -- & - & - & - \\
\hline 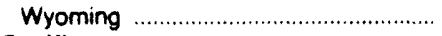 & - & - & - & - & - & - & 512.9 & 29.78 & -- & -- & -- & - \\
\hline Pacticic … & - & - & - & - & - & - & 467.5 & 27.48 & - & - & - & - \\
\hline California & - & -- & - & - & - & - & -- & -- & - & -- & - & -- \\
\hline Oregon & - & - & -- & - & -- & - & - & - & - & - & -- & - \\
\hline 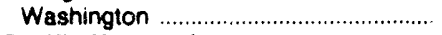 & - & - & - & - & - & - & 467.5 & 27.48 & - & -- & - & - \\
\hline 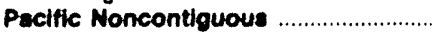 & 497 & 283.9 & 17.85 & - & - & - & - & - & - & - & 283.9 & 17.85 \\
\hline Alaska & - & - & - & -- & -- & - & - & -- & -- & - & - & - \\
\hline 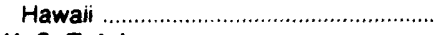 & 497 & 283.9 & 17.85 & - & - & - & -- & - & - & - & 283.9 & 17.85 \\
\hline U. S. Total & 9,077 & 232.7 & 14.81 & 6,164 & 215.4 & 13.71 & 415.2 & 24.11 & 303.9 & 18.30 & 225.7 & 14.37 \\
\hline
\end{tabular}

- Monetary values are expressed in nominal terms.

Notes: - Totals may not equal sum of components because of independent rounding. - Data are for electric generating plants with a total steamelectric and combined-cycle nameplate capacity of 50 or more megawatts. Data for 1993 ars preliminary.

Source: Federal Energy Regulatory Commission, FERC Form 423, "Monthly Report of Cost and Quality of Fuels for Electric Plants." 
Table 47. Receipts and Average Cost of Heavy OIl Delivered to Electric Utilities by Sulfur Content, Census Division, and State, September 1993

\begin{tabular}{|c|c|c|c|c|c|c|c|c|c|}
\hline \multirow{3}{*}{$\begin{array}{c}\text { Census Division } \\
\text { and State }\end{array}$} & \multicolumn{3}{|c|}{$0.3 \%$ or Less } & \multicolumn{3}{|c|}{ More than $0.3 \%$ up to $0.5 \%$} & \multicolumn{3}{|c|}{ More than $0.5 \%$ up to $1.0 \%$} \\
\hline & \multirow{2}{*}{$\begin{array}{c}\text { Recelpts } \\
\begin{array}{c}(1,000 \\
\text { bbls })\end{array}\end{array}$} & \multicolumn{2}{|c|}{$\begin{array}{l}\text { Average } \\
\text { Cost }^{\prime}\end{array}$} & \multirow{2}{*}{$\frac{\text { Recelpts }}{\left(\begin{array}{c}(1,000 \\
\text { bbls })\end{array}\right.}$} & \multicolumn{2}{|c|}{$\begin{array}{l}\text { Average } \\
\text { Cost' }\end{array}$} & \multirow{2}{*}{$\begin{array}{c}\text { Receipts } \\
\begin{array}{c}(1,000 \\
\text { bbis })\end{array}\end{array}$} & \multicolumn{2}{|c|}{$\begin{array}{l}\text { Average } \\
\text { Cost }^{\prime}\end{array}$} \\
\hline & & $\begin{array}{c}\text { (Cents } \\
\text { per } \\
10^{\circ} \text { Btu) }\end{array}$ & $\begin{array}{c}\text { (\$ per } \\
\text { bbl) }\end{array}$ & & $\begin{array}{c}\text { (Cents } \\
\text { per } \\
10^{6} \text { Btu) }\end{array}$ & $\begin{array}{l}\text { (S per } \\
\text { bbl) }\end{array}$ & & $\begin{array}{c}\text { (Cents } \\
\text { per } \\
10^{\circ} \text { Btu) }\end{array}$ & $\begin{array}{c}\text { (\$ per } \\
\text { bbl) }\end{array}$ \\
\hline 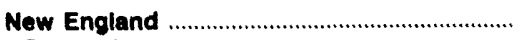 & - & - & - & 156 & 265.3 & 16.60 & 1,590 & 228.1 & 14.56 \\
\hline 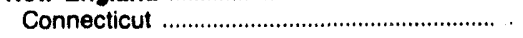 & -- & -- & - & 128 & 267.2 & 16.69 & 576 & 234.8 & 15.00 \\
\hline 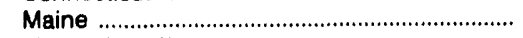 & -- & -- & -- & - & -- & - & 199 & 226.5 & 14.42 \\
\hline 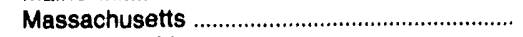 & - & -- & - & 28 & 256.7 & 16.20 & 814 & 223.8 & 14.29 \\
\hline 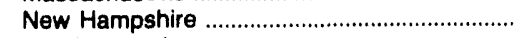 & -- & -- & - & -- & - & -- & -- & - & - \\
\hline 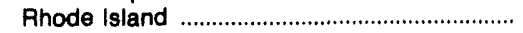 & -- & -- &.$- \cdot$ & -- & -- & - & -- & -- & -- \\
\hline 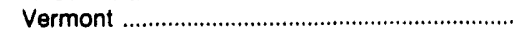 & - & - & - & -- & -- & - & -- & - & -- \\
\hline Middle Attantic & 1,071 & 254.6 & 15.94 & 441 & 247.6 & 15.66 & 1,304 & 263.9 & 16.88 \\
\hline 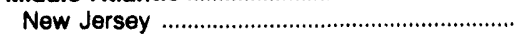 & 395 & 260.3 & 16.34 & -- & - & - & 57 & 261.6 & 16.55 \\
\hline 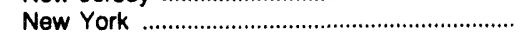 & 676 & 251.3 & 15.71 & 140 & 254.3 & 15.71 & 984 & 264.2 & 16.90 \\
\hline 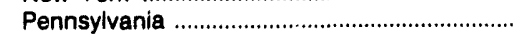 & - & -- & -- & 301 & 244.6 & 15.63 & 263 & 263.4 & 16.87 \\
\hline 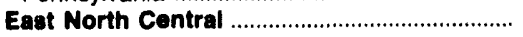 & - & - & - & - & - & - & 238 & 271.9 & 17.32 \\
\hline 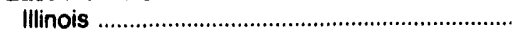 & - & -- & -- & -- & -- & -- & 129 & 266.6 & 17.04 \\
\hline 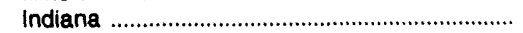 & -- & -- & -- & -- & - & -- & - & - & $\ldots$ \\
\hline 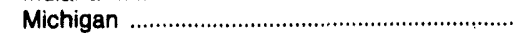 & - & -- & -- & -- & -- & -- & 109 & 278.1 & 17.65 \\
\hline Ohio & -- & -- & -- & - & - & -- & - & -- & $-\infty$ \\
\hline Wisconsin & -- & -- & -- & -- & -- & - & - & -- & -- \\
\hline 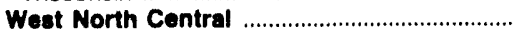 & - & - & - & - & - & - & - & - & - \\
\hline lowa & -- & - & - & -- & -- & -- & -- & -- & - \\
\hline 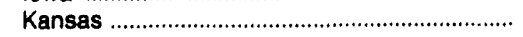 & - & -- & - & -- & - & -- & -- & - & -- \\
\hline 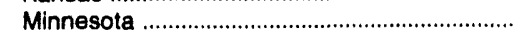 & -- & -- & -- & -- & - & -- & -- & -- & -- \\
\hline 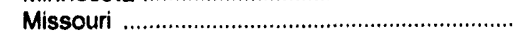 & - & -- & -- & - & - & - & -- & - & -- \\
\hline 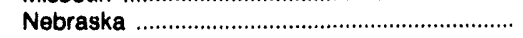 & - & -- & - & - & - & -- & -- & -- & - \\
\hline 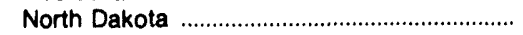 & -- & -- & -- & -- & -- & - & -- & -- & -- \\
\hline South Dakota & -- & -- & - & -- & - & -- & -- & -- & - \\
\hline South Atiantic & - & - & - & - & - & - & 4,072 & 222.6 & 14.20 \\
\hline Delaware & - & -- & - & -- & - & -- & 312 & 221.7 & 14.16 \\
\hline District of Columbia & -- & - & - & -- & -- & -- & 83 & 303.9 & 18.30 \\
\hline Florida & - & -- & - & - & -- & - & 3,087 & 219.8 & 14.08 \\
\hline Georgia & -- & -- & - & - & - & - & - & -- & - \\
\hline Maryland & -- & - & - & -- & - & -- & 121 & 233.9 & 14.77 \\
\hline North Carolina & -- & -- & - & - & - & -- & - & - & - \\
\hline 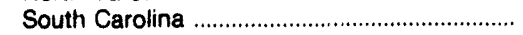 & -- & -- & -- & -- & -- & -- & - & - & -- \\
\hline 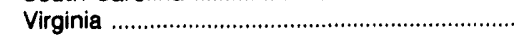 & -- & - & - & - & - & $\rightarrow$ & 470 & 225.5 & 14.16 \\
\hline 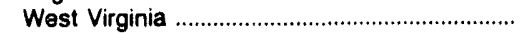 & -- & -- & - & -- & -- & $\cdots$ & - & -- & - \\
\hline East South Central ............................................ & - & - & - & - & - & - & - & - & - \\
\hline 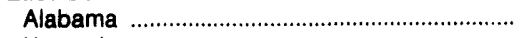 & -- & -- & -- & -- & -- & -- & - & -- & -- \\
\hline 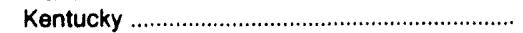 & -- & - & - & -- & - & -- & - & - & -- \\
\hline 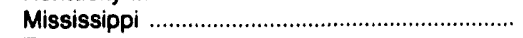 & -- & - & - & - & - & -- & - & -- & -- \\
\hline 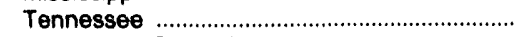 & - & -- & - & -- & - & -- & - & -- & -- \\
\hline 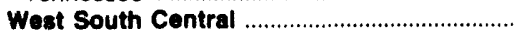 & - & - & - & - & - & - & - & - & - \\
\hline Arkansas & - & -- & -- & - & - & - & - & - & $\rightarrow$ \\
\hline 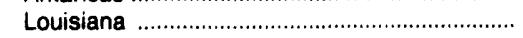 & - & -- & - & -- & - & - & - & - & - \\
\hline 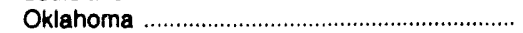 & -- & -- & - & -- & -- & - & - & -- & - \\
\hline 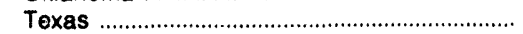 & -- & - & - & - & -- & - & - & -- & - \\
\hline 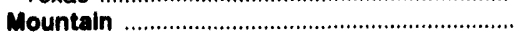 & - & - & - & - & - & - & - & - & - \\
\hline 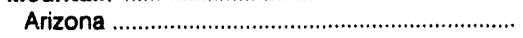 & - & - & -- & - & - & - & - & - & - \\
\hline Colorado & -- & - & - & - & - & - & - & -- & $\cdots$ \\
\hline 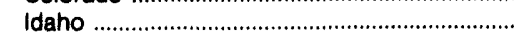 & - & -- & - & -- & -- & - & - & -- & - \\
\hline 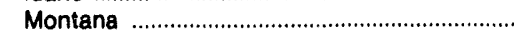 & - & - & - & -- & - & - & - & -- & -- \\
\hline Nevada & - & -- & - & - & - & -- & - & -- & -- \\
\hline 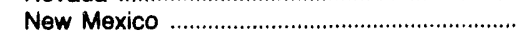 & -- & -- & - & - & -- & -- & - & -- & - \\
\hline Utah & -- & -- & - & $-\infty$ & - & -- & - & -- & - \\
\hline 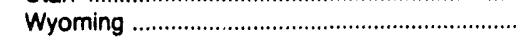 & -- & - & -- & - & -- & - & -- & -- & - \\
\hline Paclfic & - & - & - & - & - & - & - & - & - \\
\hline California & - & -- & - & -- & -- & -- & - & - & -- \\
\hline 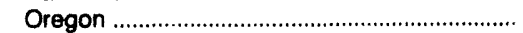 & - & -- & - & -- & - & -- & -- & -- & - \\
\hline Washington & -- & -- & -- & - & - & - & - & -- & -- \\
\hline 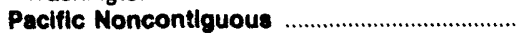 & - & - & - & 497 & 283.8 & 17.85 & - & - & - \\
\hline Alaska & -- & -- & - & - & - & - & - & - & - \\
\hline 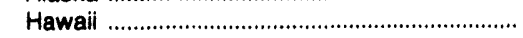 & - & - & - & 497 & 283.9 & 17.85 & - & - & -- \\
\hline U. S. Total & 1,071 & 254.6 & 15.94 & 1,094 & 266.5 & 16.79 & 7,205 & 233.0 & 14.87 \\
\hline
\end{tabular}

- Monetary values are expressed in nominal terms.

Notes: - Totals may not equal sum of components because of independent rounding. - Data are for electric generating plants with a total steamelectric and combined-cycle nameplate capacity of 50 or more megawatts. •Fuel Oil No. 2 has been omitted from this table. $\bullet$ Oil and petroleum are used interchangeably in this report. Data for 1993 are preliminary.

Source: Federal Energy Regulatory Commission, FERC Form 423, "Monthly Report of Cost and Quality of Fuels for Electric Plants." 
Table 47. Receipts and Average Cost of Heavy OII Dellvered to Electric Utilities by Sulfur Content, Census Division, and State, September 1993 (Continued)

\begin{tabular}{|c|c|c|c|c|c|c|c|c|c|c|c|}
\hline \multirow{3}{*}{$\begin{array}{c}\text { Census Division } \\
\text { and State }\end{array}$} & \multicolumn{3}{|c|}{ More than $1.0 \%$ up to $2.0 \%$} & \multicolumn{3}{|c|}{ More than $2.0 \%$ up to $3.0 \%$} & \multicolumn{3}{|c|}{ More than $3.0 \%$} & \multicolumn{2}{|c|}{ All Purchases } \\
\hline & \multirow{2}{*}{$\begin{array}{c}\text { Recelpts } \\
\begin{array}{c}(1,000 \\
\text { bbls })\end{array}\end{array}$} & \multicolumn{2}{|c|}{$\begin{array}{l}\text { Average } \\
\text { Cost' }\end{array}$} & \multirow{2}{*}{\begin{tabular}{|c|} 
Recelpts \\
$(1,000$ \\
bbls $)$
\end{tabular}} & \multicolumn{2}{|c|}{$\begin{array}{l}\text { Average } \\
\text { Cost' }^{\prime}\end{array}$} & \multirow{2}{*}{\begin{tabular}{|c|} 
Recoipts \\
$(1,000$ \\
bbls $)$
\end{tabular}} & \multicolumn{4}{|c|}{$\begin{array}{l}\text { Average } \\
\text { Cost }^{\prime}\end{array}$} \\
\hline & & $\begin{array}{c}\text { (Cents } \\
\text { per } \\
10^{6} \text { Btu) }\end{array}$ & $\begin{array}{c}\text { (5 per } \\
\text { bbl) }\end{array}$ & & $\begin{array}{c}\text { (Cents } \\
\text { per } \\
10^{\circ} \text { Btu) }\end{array}$ & $\begin{array}{c}\text { (5 per } \\
\text { bbl) }\end{array}$ & & $\begin{array}{c}\text { (Cents } \\
\text { per } \\
10^{\circ} \\
\text { Btu) }\end{array}$ & $\begin{array}{l}\text { (\$ } \\
\text { per } \\
\text { bbl) }\end{array}$ & $\begin{array}{c}\text { (Cents } \\
\text { per } \\
10^{\circ} \\
\text { Btu) }\end{array}$ & $\begin{array}{l}\text { (s } \\
\text { per } \\
\text { bbl) }\end{array}$ \\
\hline 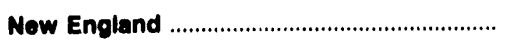 & 793 & 231.1 & 14.88 & 456 & 231.7 & 14.66 & - & - & - & 231.4 & 14.77 \\
\hline 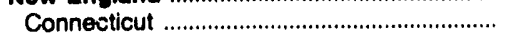 & - & - & - & - & - & - & -- & -- & -- & 240.6 & 15.31 \\
\hline Maine & 245 & 190.1 & 12.01 & -- & - & - & -- & -- & -- & 206.5 & 13.09 \\
\hline 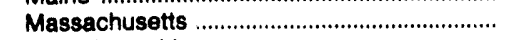 & 192 & 400.3 & 25.40 & 456 & 231.7 & 14.66 & -- & - & -- & 249.5 & 15.87 \\
\hline 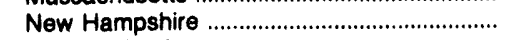 & 356 & 170.2 & 11.19 & - & - & -- & -- & -- & - & 170.2 & 11.19 \\
\hline 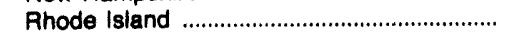 & -- & - & -- & - & - & - & -- & -- & - & - & - \\
\hline 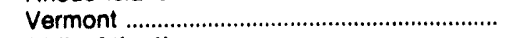 & -- & -- & -- & -- & -- & -- & - & - & -- & - & -- \\
\hline 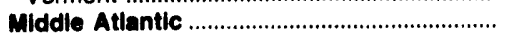 & 558 & 199.5 & 12.81 & - & - & - & - & - & - & 248.1 & 15.75 \\
\hline 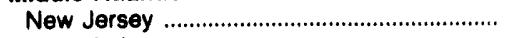 &.-- & - & - & -- & -- & -- & -- & - & - & 260.4 & 16.37 \\
\hline 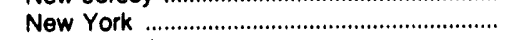 & 558 & 199.5 & 12.81 & - & - & - & - & -- & - & 244.5 & 15.52 \\
\hline 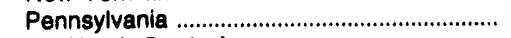 & - & $-r$ & -- & - & - & - & -- & -- & -- & 253.4 & 16.21 \\
\hline Eest North Contral & - & - & - & -- & - & - & -- & - & - & 271.9 & 17.32 \\
\hline 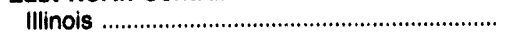 & -- & - & -- & -- & - & -- & -- & - & - & 266.6 & 17.04 \\
\hline 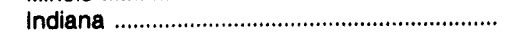 & - & -- & -- & -- & -- & -- & - & -- & -- & + & - \\
\hline 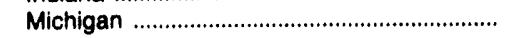 & - & -- & - & -- & - & - & - & -- & - & 278.1 & 17.65 \\
\hline Ohio & - & -- & -- & -- & - & -- & - & -- & -- & - & - \\
\hline 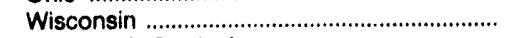 & - & -- & -- & -- & -- & -- & -- & -- & - & -- & - \\
\hline West North Central ........................................ & - & - & - & 13 & 175.5 & 11.50 & - & - & - & 175.5 & 11.50 \\
\hline lowa & - & - & -- & - & -- & -- & -- & - & - & - & - \\
\hline 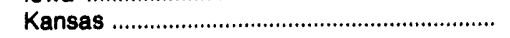 & -- & -- & -- & -- & -- & -- & -- & -- & - & -- & -- \\
\hline 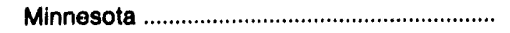 & - & - & -- & -- & - & - & - & - & -- & -- & -- \\
\hline 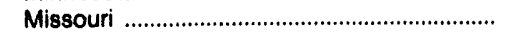 & -- & -- & - & 13 & 175.5 & 11.50 & -- & - & -- & 175.5 & 11.50 \\
\hline 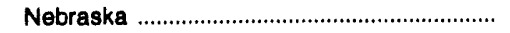 & -- & -- & - & -- & -- & - & -- & -- & -- & -- & -- \\
\hline 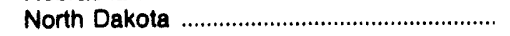 & - & - & -- & -- & -- & -- & -- & -- & -- & -- & - \\
\hline South Dakota & - & -- & -- & -- & - & -- & -- & -- & - & $-\infty$ & -- \\
\hline 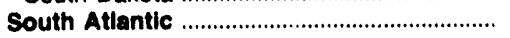 & 1,812 & 212.9 & 13.49 & 1,470 & 194.0 & 12.31 & -- & - & - & 214.5 & 13.65 \\
\hline 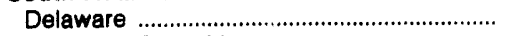 & 42 & 271.0 & 17.05 & - & - & - & -- & - & -- & 227.5 & 14.50 \\
\hline District of Columbia & - & -- & - & -- & -- & -- & -- & - & - & 303.9 & 18.30 \\
\hline Florida & 1,055 & 216.1 & 13.62 & 1,470 & 194.0 & 12.31 & -- & -- & - & 212.4 & 13.53 \\
\hline Georgla & - & - & - & - & - & - & -- & -- & - & $-\infty$ & -- \\
\hline 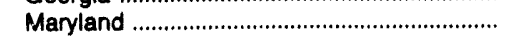 & 453 & 206.7 & 13.12 & - & -- & -- & - & -- & - & 212.4 & 13.47 \\
\hline 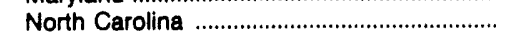 & - & -- & -- & -- & -- & -- & -- & - & - & -- & - \\
\hline 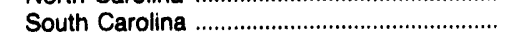 & - & -- & -- & -- & -- & -- & -- & -- & -- & - & - \\
\hline Virginia & 263 & 201.7 & 13.02 & -- & -- & -- & - & -- & -- & 216.8 & 13.75 \\
\hline 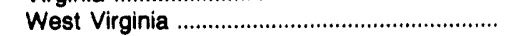 & - & - & - & - & - & -- & -- & -- & -- & - & -- \\
\hline East South Central & - & - & - & 851 & 175.6 & 11.32 & - & - & - & 175.6 & 11.32 \\
\hline 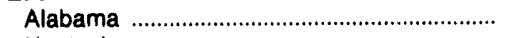 & - & -- & -- & -- & - & -- & -- & -- & -- & -- & -- \\
\hline 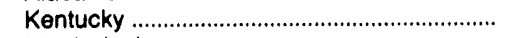 & - & -- & - & - & $-\overline{-}$ & - & -- & -- & -- & -- & - \\
\hline 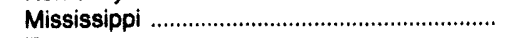 & -- & -- & - & 851 & 175.6 & 11.32 & -- & -- & -- & 175.6 & 11.32 \\
\hline 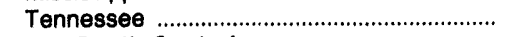 & - & - & - & -- & -- & - & -- & -- & -- & -- & - \\
\hline 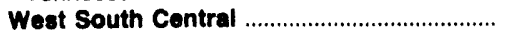 & - & - & - & - & - & - & - & - & - & - & - \\
\hline 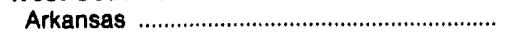 & -- & -- & -- & -- & - & -- & $\rightarrow$ & -- & -- & -- & -- \\
\hline 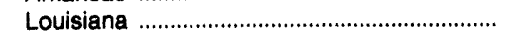 & - & -- & -- & -- & $\cdots$ & -- & -- & -- & -- & -- & -- \\
\hline Oklahoma & - & -- & - & -- & -- & -- & - & -- & -- & -- & -- \\
\hline Texas & - & - & - & -- & - & - & - & $-\infty$ & -- & -- & -- \\
\hline 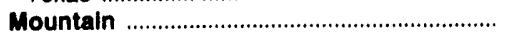 & - & - & - & -- & -- & -- & -- & -- & - & - & -- \\
\hline 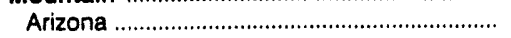 & - & - & - & - & -- & -- & -- & -- & -- & -- & -- \\
\hline Colorado & - & -- & -- & -- & -- & - & -- & -- & -- & -- & - \\
\hline Idaho & -- & -- & -- & - & -- & -- & -- & -- & - & -- & -- \\
\hline 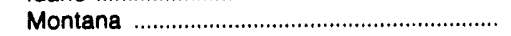 & -- & -- & $\cdots$ & -- & -- & -- & - & -- & -- & -- & - \\
\hline 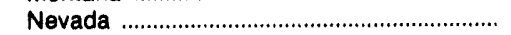 & -- & -- & -- & -- & -- & - & -- & -- & - & -- & -- \\
\hline 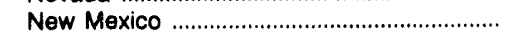 & - & -- & -- & -- & -- & -- & -- & -- & - & -- & -- \\
\hline Utah & -- & - & -- & -- & -- & -- & -- & -- & - & -- & -- \\
\hline Wyoming & -- & -- & -- & - & -- & - & -- & -- & -- & -- & - \\
\hline 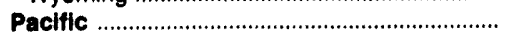 & - & -- & - & -- & - & - & - & - & - & - & -- \\
\hline 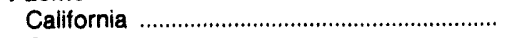 & - & -- & -- & -- & -- & -- & -- & -- & -- & -- & -- \\
\hline 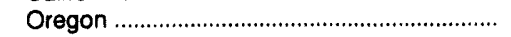 & - & -- & -- & -- & -- & -- & - & - & -- & -- & -- \\
\hline 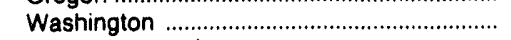 & -- & -- & -- & - & -- & - & -- & - & -- & -- & - \\
\hline 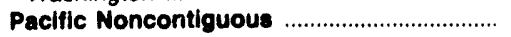 & - & - & - & - & - & - & - & - & - & 283.9 & 17.85 \\
\hline Alaska & -- & - & -- & -- & -- & -- & -- & -- & -- & - & - \\
\hline 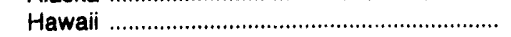 & - & $\rightarrow$ & - & -- & -- & -- & -- & -- & $-\infty$ & 283.9 & 17.85 \\
\hline U. S. Total & 3,163 & 215.2 & 13.72 & 2,790 & 194.3 & 12.39 & - & - & -- & 226.1 & 14.39 \\
\hline
\end{tabular}

1 Monetary values are expressed in nominal terms.

Notes: - Totals may not equal sum of components because of independent rounding. Data are for electric generating plants with a total steamelectric and combined-cycle nameplate capacity of 50 or more megawatts. - Fuel Oil No. 2 has been omitted from this table. $\bullet$ Oil and petroleum are used interchangeably in this report. Data for 1993 are preliminary.

Source: Federal Energy Regulatory Commission. FERC Form 423, "Monthly Report of Cost and Quality of Fuels for Electric Plants." 
Table 48. Electric Utility Recelpts of Gas by Type, Census Division, and State, September 1993

\begin{tabular}{|c|c|c|c|c|c|c|c|c|}
\hline \multirow{2}{*}{$\begin{array}{c}\text { Census Division } \\
\text { and State }\end{array}$} & \multicolumn{2}{|c|}{ Natural } & \multicolumn{2}{|c|}{ Blast-Furnance' } & \multicolumn{2}{|c|}{ Refinery } & \multicolumn{2}{|c|}{ Total } \\
\hline & $\begin{array}{l}\text { (thousand } \\
\text { Mcf) }\end{array}$ & $\begin{array}{c}\text { (billion } \\
\text { Btu) }\end{array}$ & $\begin{array}{l}\text { (thousand } \\
\text { Mct) }\end{array}$ & $\begin{array}{c}\text { (blllion } \\
\text { Btu) }\end{array}$ & $\begin{array}{l}\text { (thoueand } \\
\text { Mcf) }\end{array}$ & $\begin{array}{c}\text { (blllion } \\
\text { Btu) }\end{array}$ & $\begin{array}{c}\text { (thousand } \\
\text { Mcf) }\end{array}$ & $\begin{array}{c}\text { (blllion } \\
\text { Btu) }\end{array}$ \\
\hline 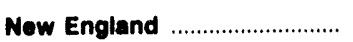 & 2,842 & 2,945 & - & - & - & - & 2,842 & 2,945 \\
\hline 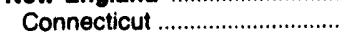 & 21 & 21 & -- & - & - & - & 21 & 21 \\
\hline Maine & - & -- & -- & - & -- & - & -- & -- \\
\hline 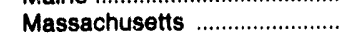 & 2,789 & 2,891 & -- & -- & - & - & 2,789 & 2,891 \\
\hline New Hampshire ...................... & - & - & - & - & - & - & - & - \\
\hline 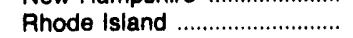 & 32 & 33 & - & -- & - & -- & 32 & 33 \\
\hline 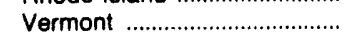 & - & -- & - & - & - & - & -- & $-\infty$ \\
\hline Middle Atlantic & 17,376 & 17,930 & - & - & - & - & 17,376 & 17,930 \\
\hline 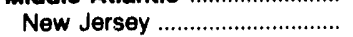 & 2,517 & 2,602 & - & - & - & - & 2,517 & 2,602 \\
\hline 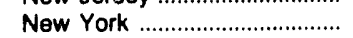 & 14,584 & 15,044 & - & - & - & - & 14,584 & 15,044 \\
\hline 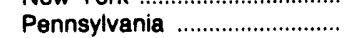 & 275 & 284 & -- & - & - & - & 275 & 284 \\
\hline East North Central ..................... & 2,259 & 2,286 & 759 & 135 & - & - & 3,018 & 2,431 \\
\hline 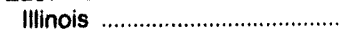 & 1,543 & 1,567 & -- & -- & -- & -- & 1,543 & 1,567 \\
\hline 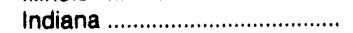 & 220 & 224 & - & - & -- & - & 220 & 224 \\
\hline 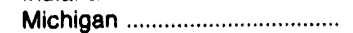 & 268 & 273 & 759 & 135 & - & -- & 1,027 & 408 \\
\hline Ohio & 34 & 35 & - & - & -- & - & 34 & 35 \\
\hline 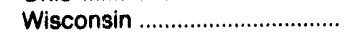 & 194 & 196 & -- & - & - & - & 194 & 196 \\
\hline West North Central .................. & 2,117 & 2,095 & - & - & - & - & 2,117 & 2,095 \\
\hline lowa & 217 & 218 & -- & - & -- & -- & 217 & 218 \\
\hline 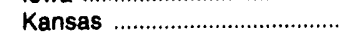 & 1,264 & 1,242 & - & - & -- & - & 1,264 & 1,242 \\
\hline Minnesota & 411 & 413 & -- & -- & - & - & 411 & 413 \\
\hline 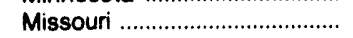 & 153 & 154 & - & -- & - & - & 153 & 154 \\
\hline 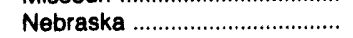 & 71 & 69 & - & -- & - & -- & 71 & 69 \\
\hline 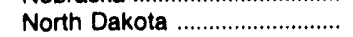 & $*$ & $\cdot$ & - & -- & - & - & 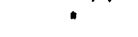 &. \\
\hline South Dakota & - & -- & - & - & - & - & -- & - \\
\hline 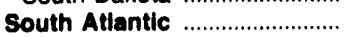 & 19,604 & 19,887 & - & - & 75 & 75 & 19,679 & 19,982 \\
\hline Delaware & 65 & 67 & - & - & - & -- & 65 & 67 \\
\hline 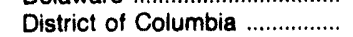 & - & -- & - & - & - & - & -- & - \\
\hline Florida & 16,495 & 16,643 & - & -- & -- & - & 16,495 & 16,643 \\
\hline 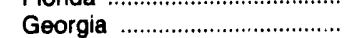 & 107 & 110 & - & - & - & - & 107 & 110 \\
\hline 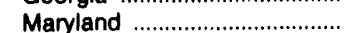 & 789 & 823 & - & - & - & - & 789 & 823 \\
\hline 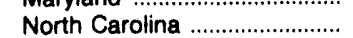 & 414 & 427 & - & - & - & - & 414 & 427 \\
\hline 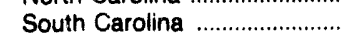 & 12 & 12 & -- & - & -- & -- & 12 & 12 \\
\hline Virginia & 1,714 & 1,796 & - & - & 75 & 75 & 1,789 & 1,871 \\
\hline West Virginia & 9 & 9 & - & - & -- & -- & 9 & 9 \\
\hline 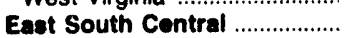 & 2,444 & 2,509 & - & - & - & - & 2,444 & 2,509 \\
\hline Alabama & 213 & 215 & - & - & - & - & 213 & 215 \\
\hline Kentucky & 28 & 29 & -- & -- & - & - & 28 & 29 \\
\hline 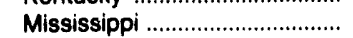 & 2,203 & 2,265 & - & - & - & - & 2,203 & 2,265 \\
\hline 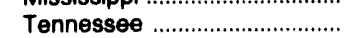 & $\ldots$ & - & - & - & - & - & - & -- \\
\hline Wost South Central ..................... & 149,364 & 153,970 & - & - & - & - & 149,364 & 153,970 \\
\hline Arkansas & 3,223 & 3,292 & - & - & - & - & 3,223 & 3,292 \\
\hline Louisiana & 28,364 & 29,575 & - & - & - & - & 28,364 & 29,575 \\
\hline Oklahoma & 12,687 & 13,227 & -- & - & - & - & 12,687 & 13,227 \\
\hline 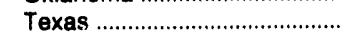 & 105,090 & 107,875 & -- & - & - & - & 105,090 & 107,875 \\
\hline 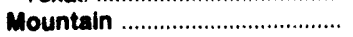 & 6,402 & 6,555 & - & - & - & - & 6,402 & 6,555 \\
\hline Arizona & 1,765 & 1,809 & - & - & - & - & 1,765 & 1,809 \\
\hline Colorado & 142 & 146 & - & - & - & - & 142 & 146 \\
\hline Idaho & - & - & - & - & - & - & $\ldots$ & - \\
\hline Montana & $\bullet$ & $\cdot$ & -- & - & - & - & $\cdot$ & $\cdot$ \\
\hline 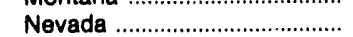 & 1,912 & 1.951 & - & - & - & - & 1,912 & 1,951 \\
\hline 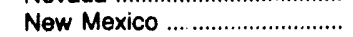 & 2,202 & 2,236 & - & - & - & - & 2,202 & 2,236 \\
\hline Utah & 375 & 407 & -- & - & $\ldots$ & - & 375 & 407 \\
\hline Wyoming & 5 & 5 & - & - & - & - & 5 & 5 \\
\hline Paciflc & 45,588 & 46,861 & - & - & - & - & 45,588 & 46,861 \\
\hline California & 43,948 & 45,203 & - & - & - & - & 43,948 & 45,203 \\
\hline Oregon & $\begin{array}{r}4,630 \\
1,638\end{array}$ & 1,656 & - & - & - & - & 1,638 & 1,656 \\
\hline 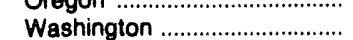 & $\begin{array}{r}1,000 \\
2\end{array}$ & 2 & - & - & - & - & $\begin{array}{r}1,000 \\
2\end{array}$ & 1,000 \\
\hline Peciflc Noncontiguous .......... & 878 & 877 & - & - & - & - & 878 & 877 \\
\hline Alaska & 878 & 877 & - & - & - & - & 878 & 877 \\
\hline 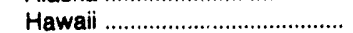 & - & - & - & - & - & - & - & -_ \\
\hline 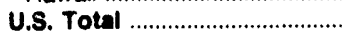 & 248,874 & 255,924 & 759 & 135 & 75 & 75 & 249,708 & 256,134 \\
\hline
\end{tabular}

- Includes coke oven gas.

Less than 0.5 .

Notes: - Totals may not equal sum of components because of independent rounding. - Data are for electric generating plants with total steam-electric and combined-cycle nameplate capacity of 50 or more megawatts. Data for 1993 are preliminary. $\bullet$ Mcf $=$ thousand cubic feet.

Source: Federal Energy Regulatory Commission, FERC Form 423, "Monthly Report of Cost and Qually of Fuels for Electric Plants." 
Table 49. Receipts and Average Cost of Gas Delivered to Electric Utilities by Census Division and State

\begin{tabular}{|c|c|c|c|c|c|c|c|c|}
\hline \multirow{3}{*}{$\begin{array}{l}\text { Census Division } \\
\text { and State }\end{array}$} & \multicolumn{2}{|c|}{$\begin{array}{l}\text { Septomber } \\
1993 \\
\text { Recolpte }\end{array}$} & \multicolumn{2}{|c|}{$\begin{array}{l}\text { Septomber } \\
1902 \\
\text { Recelpte }\end{array}$} & \multicolumn{4}{|c|}{ Year to Date } \\
\hline & \multirow[t]{2}{*}{$\begin{array}{l}\text { (thousand } \\
\text { Mcf) }\end{array}$} & \multirow[t]{2}{*}{$\begin{array}{l}\text { (billlon } \\
\text { Btu) }\end{array}$} & \multirow[t]{2}{*}{$\begin{array}{l}\text { (thousand } \\
\text { Mcf) }\end{array}$} & \multirow[t]{2}{*}{$\begin{array}{l}\text { (billion } \\
\text { Btu) }\end{array}$} & \multicolumn{2}{|c|}{$\begin{array}{l}\text { Recelpts } \\
\text { (billion } \\
\text { Btu) }\end{array}$} & \multicolumn{2}{|c|}{$\begin{array}{l}\text { Average Cost } \\
\text { (cents per } \\
\text { million Btu)' }\end{array}$} \\
\hline & & & & & 1993 & 1992 & 1993 & 1992 \\
\hline $\begin{array}{l}\text { Now England } \\
\text { Connecticut }\end{array}$ & $\begin{array}{r}2,842 \\
21\end{array}$ & $\begin{array}{r}2,945 \\
21\end{array}$ & $\begin{array}{r}5,092 \\
15\end{array}$ & $\begin{array}{r}5,255 \\
15\end{array}$ & $\begin{array}{r}25,778 \\
517\end{array}$ & $\begin{array}{r}35,141 \\
1,999\end{array}$ & $\begin{array}{l}265.4 \\
348.7\end{array}$ & $\begin{array}{l}240.8 \\
254.7\end{array}$ \\
\hline 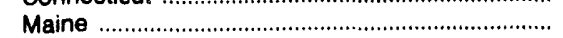 & - & -- & - & -- & - & - & -- & -- \\
\hline 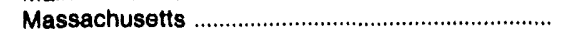 & 2,789 & 2,891 & 4,574 & 4,727 & 24,605 & 31,096 & 264.6 & 242.3 \\
\hline New Hampshire & -- & & 500 & 509 & 138 & 933 & 217.2 & 205.9 \\
\hline 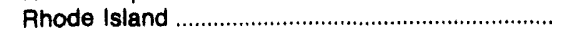 & 33 & 33 & 1 & 1 & 275 & 472 & 257.3 & 213.4 \\
\hline Vermont & - & $=$ & 2 & 2 & 244 & 641 & 201.5 & 194.3 \\
\hline Middle Atlantic . & 17,376 & 17,930 & 23,509 & 24,238 & 173,811 & 196,747 & 259.9 & 222.9 \\
\hline 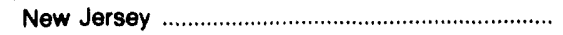 & 2,517 & 2,602 & 3,025 & 3,130 & 26,057 & 31,155 & 229.1 & 205.3 \\
\hline 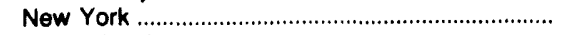 & 14,584 & 15,044 & 20,293 & 20,911 & 141,685 & 164,067 & 265.9 & 225.8 \\
\hline 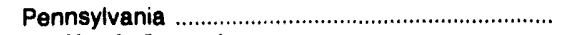 & 275 & 284 & 191 & 197 & 6,069 & 1,525 & 252.7 & 268.3 \\
\hline East North Central ...................................................... & 3,018 & 2,431 & 2,685 & 2,045 & 23,464 & 26,196 & 253.5 & 210.4 \\
\hline 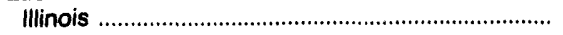 & 1,543 & 1,567 & 533 & 541 & 11,182 & 8,192 & 248.8 & 211.3 \\
\hline Indiana & 220 & 224 & 269 & 269 & 3,520 & 6,036 & 275.0 & 235.8 \\
\hline Michigan & 1,027 & 408 & 1,529 & 873 & 5,232 & 7,969 & 240.8 & 187.2 \\
\hline 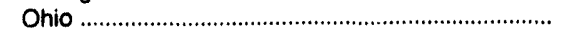 & 34 & 35 & 166 & 170 & 1,366 & 2,115 & 279.4 & 215.7 \\
\hline Wisconsin & 194 & 196 & 189 & 192 & 2,165 & 1,884 & 256.4 & 217.8 \\
\hline West North Central & 2,117 & 2,095 & 1,773 & 1,734 & 21,993 & 14,926 & 246.0 & 198.7 \\
\hline lowa & 217 & 218 & 107 & 108 & 2,679 & 1,306 & 306.7 & 289.9 \\
\hline 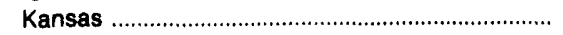 & 1,264 & 1,242 & 1,099 & 1.064 & 13,029 & 8,773 & 235.6 & 194.5 \\
\hline Minnesota & 412 & 413 & 309 & 311 & 1,625 & 2,634 & 242.7 & 170.2 \\
\hline 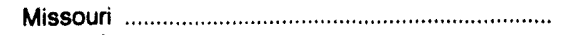 & 153 & 154 & 59 & 60 & 3,628 & 1,362 & 234.4 & 175.4 \\
\hline 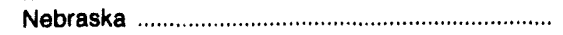 & 71 & 69 & 199 & 191 & 980 & 844 & 267.9 & 227.2 \\
\hline 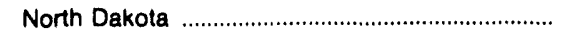 & • & • & 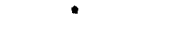 & - & • & * & 423.3 & 398.5 \\
\hline South Dakota & - & - & - & - & 52 & 7 & 237.8 & 256.6 \\
\hline South Atiantic . & 19,679 & 19,962 & 21,284 & 21,615 & 160,312 & 184,925 & 246.4 & 223.4 \\
\hline 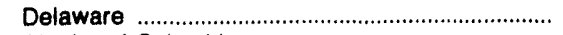 & & 67 & 130 & 135 & 850 & 2,203 & 313.9 & 253.3 \\
\hline District of Columbia & - & - & $\because-$ & $-\overline{10}$ & $\overline{2}$ & $-\overline{s e n}$ & - & -- \\
\hline Florida & 16,495 & 16,643 & 19,238 & 19,474 & 134,604 & 159,600 & 237.6 & 220.8 \\
\hline Georgia & 107 & 110 & 58 & 59 & 2,938 & 1,143 & 321.3 & 271.9 \\
\hline 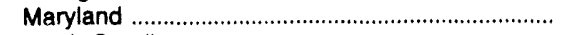 & 789 & 823 & 845 & 891 & 4,285 & 7,941 & 284.2 & 240.0 \\
\hline North Carolina & 414 & 427 & 334 & 345 & 2,230 & 2,587 & 348.8 & 270.3 \\
\hline 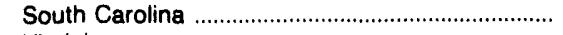 & 12 & 12 & 10 & 10 & 475 & 1,285 & 289.2 & 161.5 \\
\hline Virginia . . & 1,789 & 1,871 & 662 & 693 & 14,836 & 10,044 & 278.0 & 233.4 \\
\hline 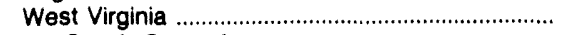 & & & 9 & 9 & 95 & 123 & 428.0 & 367.1 \\
\hline East South Contral & 2,444 & 2,509 & 4,368 & 4,524 & 22,608 & 40,351 & 246.9 & 178.4 \\
\hline Alabama & 213 & 215 & 259 & 266 & 1,966 & 2,237 & 257.6 & 202.2 \\
\hline 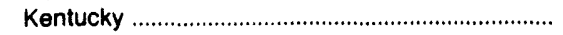 & 28 & 29 & 16 & 16 & 165 & 186 & 301.5 & 257.2 \\
\hline 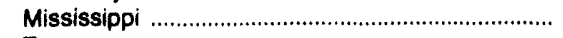 & 2,203 & 2,265 & 4,094 & 4,241 & 20,477 & 37,928 & 245.4 & 176.6 \\
\hline 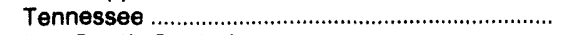 & - & $=-$ & $-\overline{c o s}(20)$ & $-\overline{-}$ & - & - & - & - \\
\hline West South Central & 149,364 & 153,970 & 134,374 & 138,489 & $1,184,106$ & $1,109,825$ & 246.4 & 207.4 \\
\hline Arkansas & 3,223 & 3,292 & 2,331 & 2,399 & 14.072 & 26,532 & 218.1 & 152.5 \\
\hline Louisiana & 28,364 & 29,575 & 22,786 & 23,875 & 189,872 & 205,984 & 238.1 & 170.5 \\
\hline Oklahoma & 12,687 & 13,227 & 15,495 & 16,081 & 121,273 & 122,974 & 312.0 & 297.6 \\
\hline Texas & 105,090 & 107,875 & 93,762 & 96,134 & 858,889 & 754,335 & 239.5 & 204.7 \\
\hline 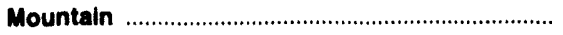 & 6,402 & 6,555 & 0,411 & 9,683 & 59,050 & 70,321 & 242.8 & 193.2 \\
\hline Arizona & 1,765 & 1,809 & 4,437 & 4,581 & 15,770 & 26,891 & 289.0 & 216.1 \\
\hline Colorado & 142 & 146 & 103 & 104 & 1,529 & 992 & 250.1 & 195.2 \\
\hline Idaho & - & - & -- & -- & - & -- & -- & -- \\
\hline Montana & & & 2 & 2 & 51 & 112 & 430.0 & 356.8 \\
\hline Nevada & 1,912 & 1,951 & 2,489 & 2,539 & 16,572 & 20,248 & 233.9 & 176.2 \\
\hline New Mexico & 2,202 & 2,236 & 1,892 & 1,914 & 21,489 & 17,584 & 219.9 & 182.0 \\
\hline Utah & 375 & 407 & 481 & 527 & 3,560 & 4,433 & 210.5 & 169.8 \\
\hline 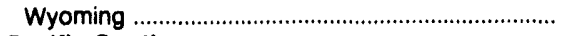 & & & 6 & 6 & 78 & 63 & 325.0 & 322.3 \\
\hline Pacific Contiguous & 45,588 & 46,861 & 56,165 & 58,151 & 341,922 & 439,562 & 302.5 & 252.6 \\
\hline California & 43,948 & 45,203 & 54,155 & 56,119 & 332,791 & 428,957 & 304.9 & 254.4 \\
\hline Oregon & 1,638 & 1,656 & 2,007 & 2,029 & 9,117 & 10,576 & 216.4 & 179.0 \\
\hline 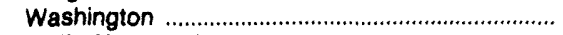 & 2 & 2 & 3 & & 14 & 30 & 384.3 & 303.5 \\
\hline 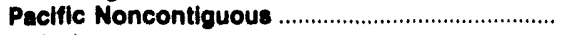 & 878 & 877 & 1,108 & 1,109 & 12,064 & 12,902 & 129.0 & 122.5 \\
\hline Alaska & 878 & 877 & 1,108 & 1,109 & 12,064 & 12,902 & 129.0 & 122.5 \\
\hline 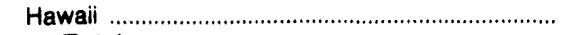 & -- & - & - & & -- & -- & -- & -- \\
\hline U.S. Total & 249,708 & 256,134 & 259,771 & 266,843 & $2,025,108$ & $2,130,896$ & 256.6 & 218.5 \\
\hline
\end{tabular}

- Monetary values are expressed in nominal terms.

Less than 0.5 .

Notes: -Data for 1992 are final. Data for 1993 are preliminary. - Totals may not equal sum of components because of independent rounding. -Data are for electric generating plants with a total steam-electric and combined-cycle nameplate capacity of 50 or more megawatts. -Includes small quantities of coke-oven, refinery, and blast-furnace gas. $\bullet M c t=$ thousand cubic feet.

Source: Federal Energy Regulatory Commission, FERC Form 423, "Monthly Report of Cost and Quality of Fuels for Electric Plants." 
Table 50. Recelpts and Average Cost of Gas Delivered to Electric Utilities by Type of Purchase, Census Division, and State, September 1993

\begin{tabular}{|c|c|c|c|c|c|c|c|c|c|c|c|c|}
\hline \multirow{3}{*}{$\begin{array}{l}\text { Census Division } \\
\text { and State }\end{array}$} & \multicolumn{3}{|c|}{ Firm Gas } & \multicolumn{3}{|c|}{ Interruptible Gas } & \multicolumn{3}{|c|}{ Spot Gas } & \multicolumn{3}{|c|}{ Total Gas } \\
\hline & \multirow{2}{*}{\begin{tabular}{|c|} 
Recolpts \\
$\begin{array}{c}(1,000 \\
\text { Mcn }\end{array}$
\end{tabular}} & \multicolumn{2}{|c|}{$\begin{array}{l}\text { Average } \\
\text { Cost' }\end{array}$} & \multirow{2}{*}{\begin{tabular}{|c|} 
Aecoiple \\
$\begin{array}{c}(1,000 \\
\text { Mon }\end{array}$
\end{tabular}} & \multicolumn{2}{|c|}{$\begin{array}{l}\text { Average } \\
\text { Coot' }\end{array}$} & \multirow{2}{*}{$\begin{array}{c}\text { Recelpts } \\
\begin{array}{c}(1,000 \\
\text { Mef })\end{array}\end{array}$} & \multicolumn{2}{|c|}{$\begin{array}{l}\text { Average } \\
\text { Cost' }\end{array}$} & Recelpte & $\begin{array}{l}\text { Avere } \\
\text { Cost }\end{array}$ & \\
\hline & & $\begin{array}{l}\text { (Cents } \\
\text { per } \\
10^{\circ} \\
\text { Btu) }\end{array}$ & $\begin{array}{c}\text { (s } \\
\text { por } \\
\text { Met) }\end{array}$ & & $\begin{array}{l}\text { (Cents } \\
\text { per } \\
10^{\circ} \\
\text { Btu) }\end{array}$ & $\underset{\text { Mer }}{\text { por }}$ & & $\begin{array}{l}\text { (Cents } \\
\text { per } \\
10^{0} \\
\text { Btu) }\end{array}$ & $\begin{array}{c}\text { (\$ } \\
\text { por } \\
\text { Mef) }\end{array}$ & $\begin{array}{l}(1,000 \\
\text { Mct })\end{array}$ & $\begin{array}{l}\text { (Cents } \\
\text { per } \\
10^{\circ} \\
\text { Btu) }\end{array}$ & $\begin{array}{l}\text { (\$ } \\
\text { por } \\
\text { Mcf) }\end{array}$ \\
\hline $\begin{array}{l}\text { Now England } \\
\text { Connecticut }\end{array}$ & $\begin{array}{l}13 \\
13\end{array}$ & $\begin{array}{l}736.1 \\
736.1\end{array}$ & $\begin{array}{l}7.58 \\
7.58\end{array}$ & $\begin{array}{r}2,829 \\
8\end{array}$ & $\begin{array}{l}269.3 \\
212.0\end{array}$ & $\begin{array}{l}2.79 \\
2.18\end{array}$ & $\overline{-}$ & $\overline{-}$ & $\overline{-}$ & $\begin{array}{r}2,842 \\
21\end{array}$ & $\begin{array}{l}271.5 \\
544.0\end{array}$ & $\begin{array}{l}2.61 \\
5.60\end{array}$ \\
\hline 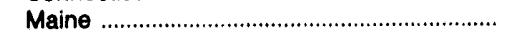 & -- & - & - & - & - & - & -- & - & - & $\ldots$ & - & -- \\
\hline 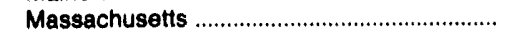 & - & - & -- & 2,789 & 269.5 & 2.79 & - & -- & - & 2,789 & 269.5 & 2.79 \\
\hline New Hampshire ......... & - & -- & -- & - & - & - & -- & - & -. & $-\infty$ & - & -- \\
\hline Rhode Island .............. & -- & - & -- & 32 & 271.4 & 2.76 & -- & -. & -- & 32 & 271.4 & 2.76 \\
\hline 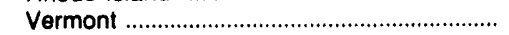 & -- & -- & -- & - & - & -- & -- & -- & - & -- & - & $\ldots$ \\
\hline 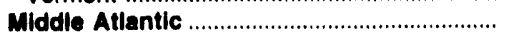 & 3,315 & 317.8 & 3.29 & $\mathbf{9 , 9 3 9}$ & 264.1 & 2.73 & 4,122 & 253.6 & 2.59 & 17,376 & 271.9 & 2.81 \\
\hline 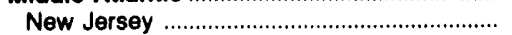 & - & - & -- & 2,517 & 255.0 & 2.64 & - & - & - & 2,517 & & 2.64 \\
\hline 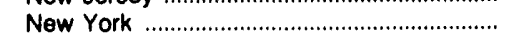 & 3,302 & 317.9 & 3.29 & 7,160 & 266.6 & 2.76 & 4.122 & 253.6 & 2.59 & 14,584 & 274.6 & 2.83 \\
\hline 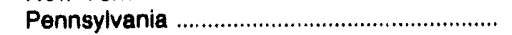 & 13 & 293.9 & 3.03 & 262 & 285.2 & 2.94 & - & - & - & 275 & 285.6 & 2.94 \\
\hline East North Contral ............................................... & 442 & 321.4 & 3.27 & 1,194 & 254.2 & 1.23 & 1,382 & 244.5 & 2.48 & 3,018 & 261.1 & 2.10 \\
\hline 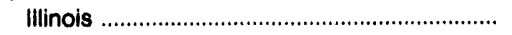 & 128 & 358.1 & 3.63 & 33 & 325.7 & 3.33 & 1,382 & 244.5 & 2.48 & 1,543 & 255.7 & 2.60 \\
\hline 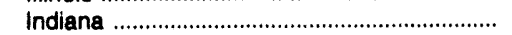 & 123 & 282.7 & 2.88 & 06 & 258.9 & 2.64 & -- & - & -- & 220 & & 2.78 \\
\hline 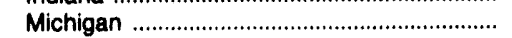 & 151 & 295.1 & 3.01 & 876 & 235.5 & .68 & -- & - & -- & 1,027 & & 1.03 \\
\hline 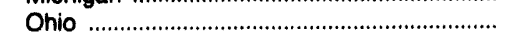 & 21 & 395.3 & 4.07 & 13 & 335.8 & 3.45 & -- & - & -- & 34 & 372 & 3.84 \\
\hline Wisconsin ....... & 19 & 453.1 & 4.61 & 175 & 258.5 & 2.62 & -- & -- & -- & 194 & 27 & 2.81 \\
\hline Wost North Central ....……………………..... & 445 & 286.4 & 2.56 & 1,671 & 248.3 & 2.48 & 1 & 246.9 & 2.47 & 2,117 & 252.0 & 2.49 \\
\hline 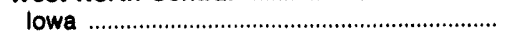 & 25 & 403.7 & 4.14 & 193 & 332.1 & 3.32 & -- & - & - & 217 & 340.4 & 3.41 \\
\hline 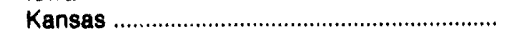 & 411 & 257.9 & 2.46 & 853 & 220.8 & 2.20 & 1 & 246.9 & 2.47 & 1,264 & 232.5 & 2.28 \\
\hline Minnesota . & -- & - & $-\ldots$ & 411 & 257.1 & 2.58 & - & - & - & 411 & 257.1 & 2.58 \\
\hline 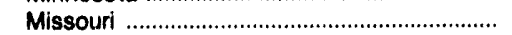 & - & -- & - & 153 & 250.3 & 2.51 & -- & - & -- & 153 & 1.3 & 2.51 \\
\hline 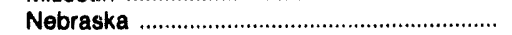 & 10 & 252.0 & 2.52 & 61 & 303.8 & 2.82 & - & -- & -- & 71 & & 2.87 \\
\hline 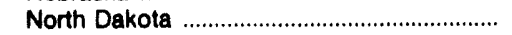 & - & -- & - & - & 425.5 & 4.69 & -- & - & - & $\cdot$ & 425.5 & 4.69 \\
\hline 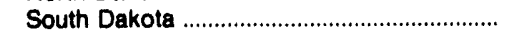 & -- & -- & - & - & - & - & - & - & -- & -- & -- & - \\
\hline 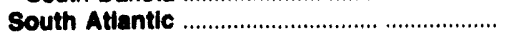 & 14,770 & 223.8 & 2.25 & 3,172 & 293.4 & 3.03 & 1,737 & 285.3 & 2.99 & 19,679 & 240.8 & 2.44 \\
\hline 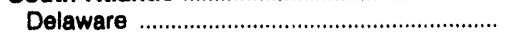 & & 297. & 3.07 & - & - & - & - & $\ldots$ & $\ldots$ & 65 & 297.3 & 3.07 \\
\hline 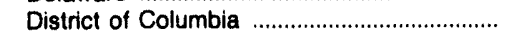 & -- & - & -- & -- & -- & -- & - & -- & -. & - & - & - \\
\hline 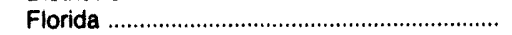 & 14,705 & 223.4 & 2.25 & 1,760 & 271.3 & 2.80 & 31 & 263.8 & 2.74 & 16,495 & 228.7 & 2.31 \\
\hline 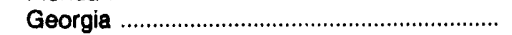 & - & -- & - & 107 & 362 & 3.72 & -- & - & - & 107 & .0 & 3.72 \\
\hline 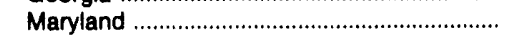 & -- & - & - & 789 & 309.7 & 3.23 & - & - & -- & 789 & 9.7 & 3.23 \\
\hline 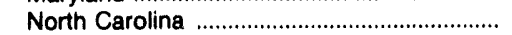 & -- & - & -- & 414 & 354 & 3.66 & - & -- & -- & 414 & & 3.66 \\
\hline South Carolina .............................................. & -- & -- & - & 12 & 317.1 & 3.26 & - & - & -. & 12 & 317.1 & 3.26 \\
\hline 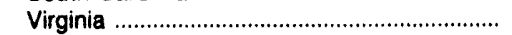 & - & -- & - & 82 & 194.9 & 1.95 & 1,706 & 285.7 & 2.99 & 1,789 & & 2.95 \\
\hline Virginia & -- & - & - & 9 & 406.4 & 4.06 & - & - & - & 9 & 406.4 & 4.06 \\
\hline 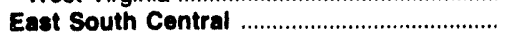 & 1,001 & 273.2 & 2.82 & 1,419 & 255.1 & 2.60 & 24 & 297.1 & 3.05 & 2,444 & 268 & 2.70 \\
\hline Alabama & - & - & - & 213 & 283.8 & 2.87 & - & - & -- & 213 & 28 & 2.87 \\
\hline 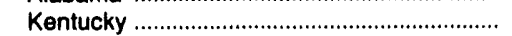 & - & -- & - & 4 & 358.2 & 3.58 & 24 & 297.1 & 3.05 & 28 & & 3.12 \\
\hline 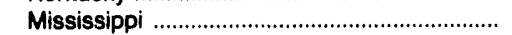 & 1,001 & 273.2 & 2.82 & 1,202 & 248.7 & 2.55 & - & - & -- & 2,203 & 260.4 & 2.68 \\
\hline 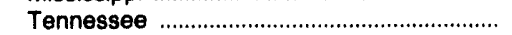 & -- & - & - & - & - & - & - & -- & -- & - & - & - \\
\hline 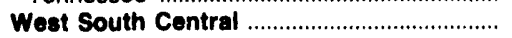 & 112,643 & 263.8 & 2.72 & 26,317 & & 2.45 & 10,404 & 254.0 & 2.65 & 149,364 & 258.9 & 2.67 \\
\hline 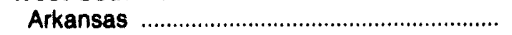 & 282 & 143 & 1.61 & 2,941 & 252.7 & 2.56 & - & - & - & 3,223 & & 2.47 \\
\hline 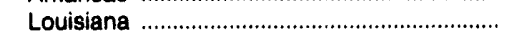 & 14,726 & 265.8 & & 4,394 & 252 & 2.63 & 9,244 & 254.1 & 2.65 & & 259.9 & 2.71 \\
\hline Oklahoma ……………………………... & 11,984 & 324 & 3.39 & 703 & 223 & 2.26 & - & $\ldots$ & - & 12,687 & 319.3 & 3.33 \\
\hline 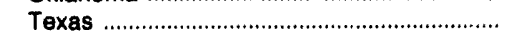 & 85,651 & 255.2 & 2.62 & 18,278 & 235. & 2.40 & 1,160 & 253.2 & 2.60 & 105,090 & 251.7 & 2.58 \\
\hline 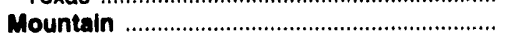 & 2,260 & 285.2 & 2.90 & 3,562 & 237. & 2.44 & 580 & 258.3 & 2.66 & 6,402 & 256.3 & 2.62 \\
\hline 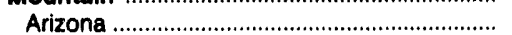 & 1,394 & 321 & 3.2 & 351 & 271. & 2.79 & 20 & 215.6 & 2.22 & 1,765 & 310.0 & 3.18 \\
\hline 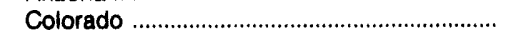 & 26 & 228. & 2.24 & 116 & 244.1 & 2.53 & - & - & - & 142 & 241.5 & 2.48 \\
\hline 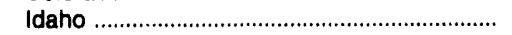 & -- & - & -- & -- & -- & - & -- & - & -- & - & - & $-\infty$ \\
\hline 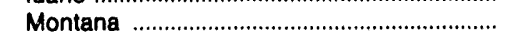 & -- & - & -- & & 450.3 & 5.07 & - & - & -- & - & 450.3 & 5.07 \\
\hline 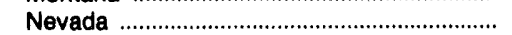 & - & $-\infty$ & - & 1,352 & 230 & 2.34 & 560 & 259.8 & 2.67 & 1,912 & & 2.44 \\
\hline New Mexico ………………………….... & 840 & 226.1 & 2.28 & 1,362 & 229 & 2.34 & - & - & - & 2,202 & 228.2 & 2.32 \\
\hline 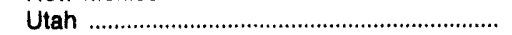 & - & - & - & 375 & 257 & 2.80 & -- & -- & -- & 375 & 257.6 & 2.80 \\
\hline 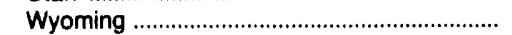 & -- & -- & - & & 360.2 & 3.71 & - & - & - & & 360.2 & 3.71 \\
\hline 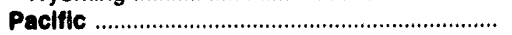 & - & - & - & 11,458 & 315.7 & 3.23 & 34,130 & 280.8 & & & 289.5 & 2.98 \\
\hline 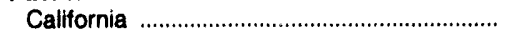 & - & - & - & 9,818 & 332.4 & 3.40 & 34,130 & 280.8 & 2.89 & 43,948 & 292.3 & 3.01 \\
\hline 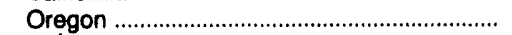 & -- & -- & -- & 1,638 & 214.1 & 2.16 & - & - & $\ldots$ & 1,638 & 214.1 & 2.16 \\
\hline Washington .......................................... & - & -- & - & 2 & 381.0 & 4.00 & - & -- & - & 2 & 381.0 & 4.00 \\
\hline Pacific Noncontiguous …………………........... & 878 & 121.3 & 1.21 & - & - & - & - & - & - & 878 & 121.3 & 1.21 \\
\hline 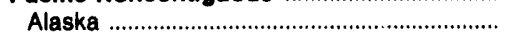 & 878 & 121.3 & 1.21 & - & - & - & - & -- & -- & 878 & 121.3 & 1.21 \\
\hline 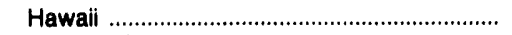 & - & - & -- & - & - & -- & - & - & -- & - & - & - \\
\hline U. S. Total & 135,766 & 280.6 & 2.68 & 61,561 & 262.8 & 2.67 & 52,381 & 272.3 & 2.81 & 249,708 & 263.6 & 2.70 \\
\hline
\end{tabular}

Monetary values are expressed in nominal terms.

- = Less than 0.05 .

Notes: - Totals may not equal sum of components because of independent rounding. -Data are for electric generating plants with a total steamelectric and combined-cycle nameplate capacity of 50 or more megawatts. -Data for 1993 are preliminary. -Mcf $=$ thousand cubic feet.

Source: Federal Energy Regulatory Commission, FERC Form 423, "Monthly Report of Cost and Quality of Fuels for Electric Plants." 
U.S. Electric Utility Sales, Revenue, and Average Revenue per Kilowatthour 
Table 51. U.S. Electric Utillty Sales of Electriclty by Sector, 1983 Through October 1993 (Million Kilowatthours)

\begin{tabular}{|c|c|c|c|c|c|c|c|c|c|c|}
\hline \multirow[b]{2}{*}{ Perlod } & \multicolumn{2}{|c|}{ Resldential } & \multicolumn{2}{|c|}{ Commorelal } & \multicolumn{2}{|c|}{ Industrial } & \multicolumn{2}{|c|}{ Other' } & \multicolumn{2}{|c|}{ All Sectore } \\
\hline & $\begin{array}{l}\text { Monthly } \\
\text { Series" }\end{array}$ & $\begin{array}{l}\text { Annual } \\
\text { sertes' }\end{array}$ & $\begin{array}{l}\text { Monthly } \\
\text { Serlese }\end{array}$ & $\begin{array}{l}\text { Annual } \\
\text { Eeries: }\end{array}$ & $\begin{array}{l}\text { Monthly } \\
\text { Sertes' }\end{array}$ & $\begin{array}{l}\text { Annual } \\
\text { Serles: }\end{array}$ & $\begin{array}{l}\text { Monthly } \\
\text { sorles' }\end{array}$ & $\begin{array}{l}\text { Annual } \\
\text { Series' }\end{array}$ & $\begin{array}{l}\text { Monthly } \\
\text { Serles? }\end{array}$ & $\begin{array}{l}\text { Annual } \\
\text { series' }\end{array}$ \\
\hline 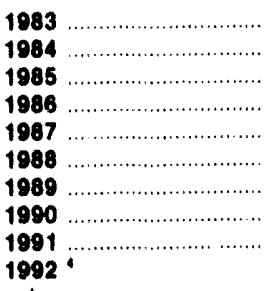 & $\begin{array}{l}750,948 \\
777,654 \\
790,977 \\
817,683 \\
849,613 \\
892,125 \\
903,979 \\
921,473 \\
957,801\end{array}$ & $\begin{array}{c}-\overline{780,092} \\
793,934 \\
819,008 \\
850,410 \\
892,806 \\
905,526 \\
924,019 \\
856,417\end{array}$ & $\begin{array}{l}543,708 \\
578,261 \\
609,968 \\
641,409 \\
673,707 \\
697,711 \\
728,220 \\
760,635 \\
765,476\end{array}$ & $\begin{array}{c}-\overline{682,621} \\
605,900 \\
680,620 \\
640,433 \\
690,100 \\
725,861 \\
751,027 \\
785,684\end{array}$ & $\begin{array}{l}775,900 \\
840,898 \\
824,523 \\
809,292 \\
845,286 \\
895,751 \\
924,376 \\
934,428 \\
944,084\end{array}$ & $\begin{array}{c}-\overline{\mathbf{8 3 7}} \mathbf{8 3 6} \\
\mathbf{8 3 6 , 7 7 2} \\
\mathbf{8 3 0 , 5 3 1} \\
\mathbf{8 5 0 , 2 3 3} \\
\mathbf{8 0 0 , 4 9 6} \\
925, \mathbf{0 5 0} \\
\mathbf{9 4 5 , 5 2 2} \\
\mathbf{9 4 6 , 5 8 3}\end{array}$ & $\begin{array}{l}80,219 \\
81,849 \\
05,075 \\
83,409 \\
86,054 \\
82,302 \\
91,068 \\
95,936 \\
90,513\end{array}$ & $\begin{array}{c}-\overline{85,246} \\
87,279 \\
88,615 \\
89,196 \\
89,598 \\
89,768 \\
91,988 \\
94,339\end{array}$ & $\begin{array}{l}2,150,958 \\
2,278,372 \\
2,309,843 \\
2,350,835 \\
2,455,440 \\
2,567,949 \\
2,646,651 \\
2,704,672 \\
2,764,474\end{array}$ & $\begin{array}{c}- \\
2,285,796 \\
2,323,974 \\
2,386,753 \\
2,467,272 \\
2,578,082 \\
2,646,009 \\
2,712,558 \\
2,762,003\end{array}$ \\
\hline January ........................... & 91,310 & - & 62,441 & - & 76,760 & - & 7,725 & - & 238,235 & -- \\
\hline February ....................... & 82,022 & - & 59,876 & - & 76,312 & - & 7,507 & - & 225,717 & -- \\
\hline March ............................ & 73,635 & -- & 59,574 & - & 78,741 & - & 7,542 & - & 219,491 & -- \\
\hline April ................................ & 68,322 & - & 58,081 & - & 77,607 & - & 7,448 & - & 211,458 & - \\
\hline May …........................... & 64,662 & - & 60,559 & - & 80,181 & -- & 7,767 & -- & 213,179 & -- \\
\hline June & 70,745 & -- & 65,209 & - & 82,800 & - & 7,901 & -- & 226,755 & -- \\
\hline July & 88,510 & -- & 71,445 & - & 84,195 & - & 8,392 & - & 252,541 & -- \\
\hline August ........................... & 88,251 & - & 70,844 & - & 85,013 & - & 8,327 & - & 252,435 & - \\
\hline September ..................... & 79,400 & -- & 68,437 & - & 83,182 & - & 8,441 & - & 239,460 & -- \\
\hline October ...................... & 69,838 & - & 63,985 & - & 82,678 & - & 7,766 & - & 224,267 & -- \\
\hline November .................... & 69,970 & - & 60,131 & - & 80,421 & - & 7,462 & -- & 217,984 & - \\
\hline December .................... & 87,378 & - & 63,082 & - & 77,358 & - & 7,725 & - & 235,543 & -- \\
\hline $\begin{array}{l}1992 \text { Total } \\
1993\end{array}$ & 934,044 & - & 783,684 & - & 905,356 & - & 94,003 & - & $2,757,067$ & - \\
\hline 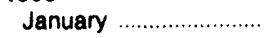 & 93,739 & -- & 63,930 & - & 78,074 & - & 8,113 & - & 243,856 & -- \\
\hline February ..................... & 83.416 & -- & 60,624 & - & 77,017 & - & 7,940 & -- & 228,897 & -- \\
\hline March ........................... & 83,023 & - & 62,169 & - & 79,504 & -- & 7,919 & - & 232,615 & - \\
\hline April & 69,668 & - & 59,389 & - & 79,593 & - & 7,588 & - & 216,238 & - \\
\hline 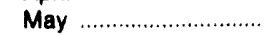 & 63,852 & -- & 61,420 & - & 82,100 & - & 7,602 & - & 214,975 & - \\
\hline 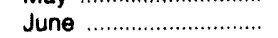 & 76,584 & - & 68,171 & - & 84,768 & - & 8,138 & - & 237,662 & - \\
\hline July & 101,023 & - & 75,704 & - & 85,370 & - & 8,457 & - & 270,555 & - \\
\hline August & 102,214 & - & 76,551 & - & 86,832 & - & 8,609 & -- & 274,206 & - \\
\hline 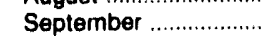 & 88,884 & - & 71,708 & - & 83,839 & - & 8,699 & -- & 253.131 & -- \\
\hline $\begin{array}{l}\text { October ......................... } \\
\text { Year to Date }\end{array}$ & 71,770 & - & 65,242 & - & 83,377 & -- & 8,270 & - & 228,680 & - \\
\hline 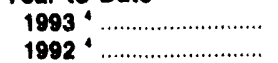 & $\begin{array}{l}834,174 \\
776,696\end{array}$ & - & $\begin{array}{l}684,909 \\
640,451\end{array}$ & $\overline{-}$ & $\begin{array}{l}820,476 \\
807,577\end{array}$ & - & $\begin{array}{l}\mathbf{8 1 , 3 3 5} \\
78,816\end{array}$ & - & $\begin{array}{l}2,400,894 \\
2,303,539\end{array}$ & - \\
\hline
\end{tabular}

1 Includes public street and highway lighting, other sales to public authorities, sales to railroads and railways, and interdepartmental sales.

2 See technical notes for an explanation of the modification to the sample design as of January 1893 estimates.

- As of 1984, national sales values are based on data reported on the Form ElA-861, "Annual Electric Utility Report."

- Estimates for 1992 and prior years are final and 1993 are preliminary.

Notes: - Totals may not equal sum of components because of independent rounding. • Estimates for sales and net generation may not correspond exactly for a particular month. Net generation data are for the calendar month. Sales and associated revenue data accumulated from bills collected for periods of time (28 to 35 days) that vary dependent upon customer class, represent consumption occurring in and outside of the calendar month. This, among other reasons (i.e., sales data may include purchases of electricity from nonutilities or imported electricity), is why the monthly sales and generation data are not directly comparable.

Sources: -Monthly Serles: Energy Information Administration, Form ElA-826, "Monthly Electric Utilly Sales and Revenue Report with State Distributions," formerly the "Electric Utility Company Monthly Statement," and predecessor forms. •Ainual Sertea: Energy Information Administration, Form ElA. 861. "Annual Electric Utility Report." 
Table 52. Electric Utility Sales of Electricity to Uitimate Consumers by Sector, Census Division, and State, October 1992 and 1993

(Million Kilowatthours)

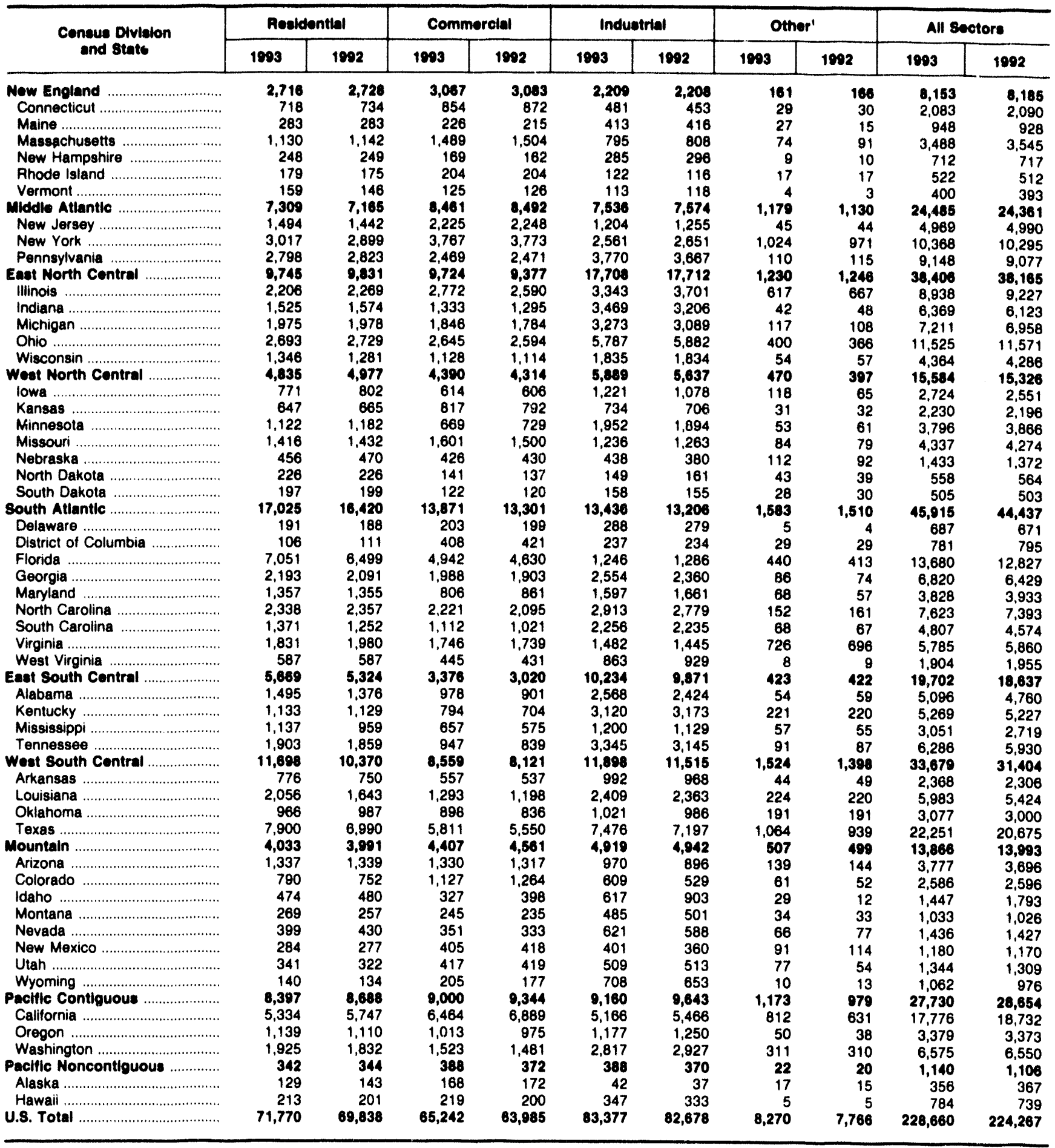

1 Includes public street and highway lighting, other sales to public authorities, sales to railroads and railways, and interdepartmental sales.

Notes: Estimates for 1992 are final and 1993 are preliminary. - Totals may not equal sum of components because of independent rounding. • Estimated sales are based on the sales by utilities in the sample. - See technical notes for an explanation of the modification to the sample design as of January 1993 estimates. Estimates for sales and net generation may not correspond exactly for a particular month. Net generation data are for the calendar month. Sales and associated revenue data accumulated from bills collected for periods of time (28 to 35 days) that vary dependent upon customer class, represent consumption occurring in and outside of the calendar month. This, among other reasons (i.e., sales data may include purchases of electricity from nonutilities or imported electricity), is why the monthly sales and generation data are not directly comparable.

Source: Energy Information Administration, Form ElA-826, "Monthly Electric Utility Sales and Revenue Report with State Distributions." 
Table 53. Estimated Coefficlents of Varlation for Electric Utility Sales of Electriclty by Census Division and State, October 1993

(Percent)

\begin{tabular}{|c|c|c|c|c|c|}
\hline $\begin{array}{c}\text { Conaus Divialon } \\
\text { and state }\end{array}$ & Resldential & Commerclal & Induetrial & Other' & All sectors \\
\hline 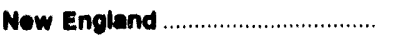 & 0.5 & 0.2 & 0.4 & 6.3 & 0.2 \\
\hline 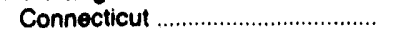 & .1 & .1 & .6 & 1.3 & .2 \\
\hline 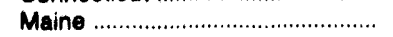 & .8 & .1 & .1 & 8.4 & .7 \\
\hline 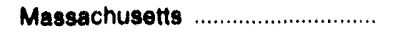 & 1.1 & .4 &.$\theta$ & 13.4 & .4 \\
\hline New Hampshire ............................ & .7 & 6 & 1.5 & .5 & .9 \\
\hline 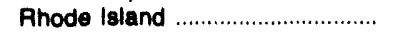 & .4 & .1 & .1 & .1 & .1 \\
\hline 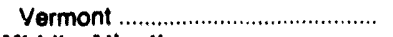 & .6 & 2 & 1.0 & 16.3 & .7 \\
\hline Middle Attantic .................................. & 1.0 & .5 & 1.0 & 2.4 & .3 \\
\hline 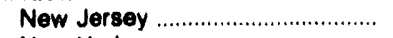 & .7 & .2 & .3 & .6 & .2 \\
\hline 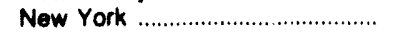 & 2.0 & 1.1 & .8 & 2.2 & .3 \\
\hline 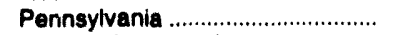 & 1.6 & .4 & 1.9 & 15.7 & .8 \\
\hline Eat North Contral ........................... & .9 & 1.0 & 1.1 & 2.0 & .6 \\
\hline 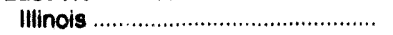 & 1.0 & 3.4 & 1.5 & .2 & 1.1 \\
\hline 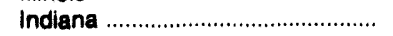 & 1.3 & 1.8 & 1.1 & 16.5 & 1.4 \\
\hline Michigan ........................................... & 2.0 & .5 & 1.3 & .8 & .0 \\
\hline 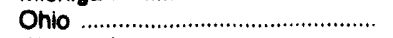 & 2.6 & 1.0 & 3.0 & 6.0 & 1.7 \\
\hline 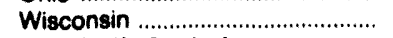 & 1.1 & 8 & 2.5 & 1.6 & .8 \\
\hline Woot North Central ........................ & 1.4 & 1.0 & 1.6 & 6.0 & .0 \\
\hline lowa & 3.8 & 3.2 & 4.9 & 17.8 & 3.7 \\
\hline 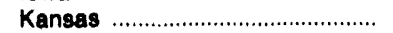 & 3.2 & 2.8 & 8.1 & 11.1 & 4.0 \\
\hline 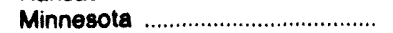 & 4.0 & 4.2 & 1.8 & 4.3 & .3 \\
\hline 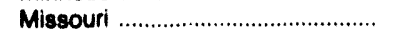 & 2.5 & .7 & .5 & .7 & .8 \\
\hline 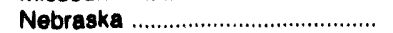 & 2.3 & 1.5 & 3.1 & 16.0 & 1.1 \\
\hline 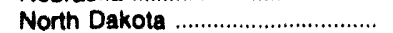 & 2.6 & 2.5 & 2.6 & 5.8 & 1.2 \\
\hline South Dakota & 1.6 & 2.2 & 1.0 & 10.2 & 2.3 \\
\hline 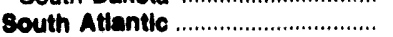 & 1.2 & .3 & .5 &. & .5 \\
\hline Delaware & 1.4 & .6 & .4 & 3.4 & .1 \\
\hline District of Columbia & .0 & .0 & .0 & .0 & .0 \\
\hline Florida & 1.3 & .2 & 2.6 & 2.4 & 1.1 \\
\hline 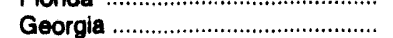 & 7.6 & 1.5 & 1.5 & 3.6 & 2.3 \\
\hline 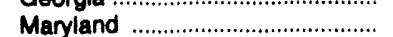 & .6 & 1.2 & .5 & 4.5 & .8 \\
\hline 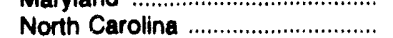 & 2.1 & 1.1 & .6 & 2.4 & .0 \\
\hline South Carolina & 2.6 & .6 & .8 & .2 & .6 \\
\hline 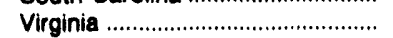 & 2.9 & .4 & .8 & .2 & 1.5 \\
\hline 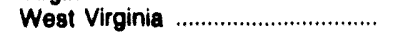 & 1.8 & .6 & 3.4 & 3.5 & .6 \\
\hline 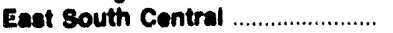 & .8 & 2.1 & 1.1 & 2.7 & .9 \\
\hline Alabama & .7 & 3.9 & 1.5 & 6.2 & .8 \\
\hline 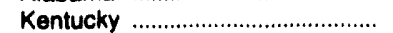 & 2.5 & 1.8 & 1.7 & 1.8 & 2.8 \\
\hline 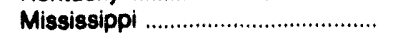 & 1.9 & 1.4 & 4.8 & 17.3 & 2.7 \\
\hline 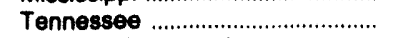 & 1.1 & 6.3 & 1.9 & 1.2 & 1.0 \\
\hline West South Contral ....................... & 2.3 & 1.1 & 1.1 & 1.6 & 1.1 \\
\hline 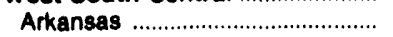 & 1.8 & .9 & 1.0 & 4.5 & .5 \\
\hline 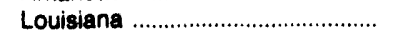 & 1.8 & 1.5 & .3 & 2.9 & .8 \\
\hline Oklahoma & 6.7 & .7 & 3.5 & 1.4 & .4 \\
\hline 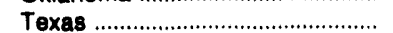 & 3.2 & 1.5 & 1.6 & 2.2 & 1.7 \\
\hline 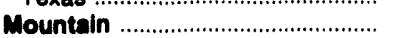 & 1.1 & 1.7 & .8 & 14.4 & 1.1 \\
\hline 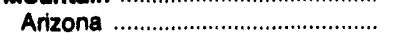 & .7 & .7 & 2.1 & 4.5 & .5 \\
\hline Colorado & 5.6 & 6.4 & 3.6 & 27.3 & 5.7 \\
\hline Idaho & .4 & 5.2 & 2.7 & 3.3 & 2.6 \\
\hline 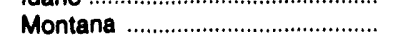 & 1.2 & .7 & 2.1 & 9.3 & 3.1 \\
\hline 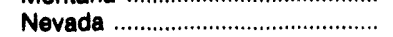 & 1.2 & 2.1 & .5 & 1.0 & .6 \\
\hline 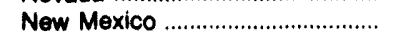 & 6 & .8 & 2.9 & 23.2 & .6 \\
\hline Utah & .1 & .2 & 2.3 & 88.0 & .4 \\
\hline 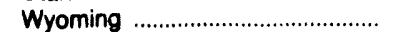 & 1.6 & 4.9 & .3 & 13.2 & .5 \\
\hline Pacific Contiguous .......................... & 1.3 & 1.7 & 2.9 & 8.0 & .8 \\
\hline 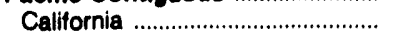 & 1.7 & 2.3 & 3.8 & 12.7 & .8 \\
\hline Oregon & 4.0 & .9 & 7.1 & 25.6 & 2.7 \\
\hline 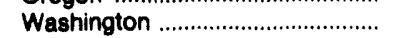 & 2.2 & 1.7 & 5.6 & 4.8 & 2.4 \\
\hline Paclfic Noncontiguous ................. & .3 & .5 & .7 & 10.2 & .3 \\
\hline Alaska & .9 & 1.2 & 2.7 & 13.4 & .7 \\
\hline 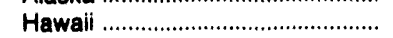 & .2 & .1 & .7 & .1 & .3 \\
\hline 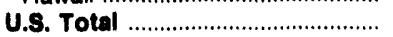 & .5 & .4 & .5 & 1.7 & .3 \\
\hline
\end{tabular}

1 Includes public street and highway lighting, other sales to public authorities, sales to railroads and railways, and interdepartmental sales. Notes: -For an explanation of coefficients of variation, see the technical notes. -Estimates for 1993 are preliminary.

Sources: Energy Information Administration, Form EIA-826, "Monthly Electric Utility Sales and Revenue Report with State Distributions." 
Table 54. Electric Utility Sales of Electriclty to Ultimate Consumers by Sector, Census Division, and State, January Through October 1992 and 1993

(Million Kilowatthours)

\begin{tabular}{|c|c|c|c|c|c|c|c|c|c|c|}
\hline \multirow{2}{*}{$\begin{array}{c}\text { Census Division } \\
\text { and State }\end{array}$} & \multicolumn{2}{|c|}{ Resldential } & \multicolumn{2}{|c|}{ Commerclal } & \multicolumn{2}{|c|}{ Industrial } & \multicolumn{2}{|c|}{ Other' } & \multicolumn{2}{|c|}{ All Sectors } \\
\hline & 1993 & 1902 & 1993 & 1992 & 1993 & 1992 & 1993 & 1992 & 1903 & $199 \%$ \\
\hline New England ...................... & 31,421 & 30,934 & 32,414 & 31,677 & 21,819 & 21,694 & 1,733 & 1,659 & 87,388 & 85,964 \\
\hline 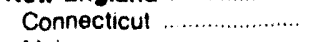 & 8.791 & 8.557 & 8,958 & 8,844 & 4,637 & 4,695 & 297 & 305 & 22,684 & 22,402 \\
\hline 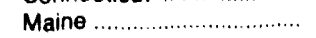 & 3,094 & 3,176 & 2,306 & 2,256 & 4.118 & 3,970 & 270 & 141 & 9.787 & 9,543 \\
\hline Massachusetts .................... & 13,057 & 12,859 & 15,938 & 15,532 & 7,913 & 7,884 & 886 & 921 & 37,794 & 37.206 \\
\hline Now Hampshire ............... & 2,841 & 2,812 & 1,812 & 1.722 & 2,824 & 2,817 & 92 & 99 & 7,568 & 7,451 \\
\hline Rhode Island ..................... & 2,014 & 1,951 & 2,125 & 2,070 & 1,186 & 1,131 & 151 & 155 & 5,476 & 5,307 \\
\hline Vermont & 1,624 & 1,578 & 1,275 & 1,253 & 1.142 & 1,187 & 38 & 38 & 4,079 & 4,056 \\
\hline Middle Atiantic ................ & 86,463 & 82,040 & 91,082 & 87,777 & 76,143 & 76,288 & 11,598 & 11,441 & 285,286 & 257,532 \\
\hline 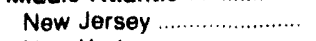 & 18,672 & 17,078 & 24,011 & 22.800 & 12,216 & 12,391 & 398 & 394 & 55,296 & 52,664 \\
\hline 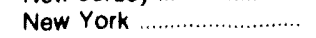 & 33,611 & 32,273 & 40,100 & 38,858 & 25,461 & 26,439 & 10,121 & 9,872 & 109,293 & 107,442 \\
\hline 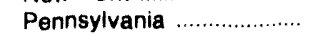 & 34,180 & 32,695 & 26,972 & 26,119 & 38,466 & 37,438 & 1,079 & 1.175 & 100,696 & 97,427 \\
\hline East North Central ........... & 121,192 & 111,457 & 101,478 & 95,370 & 172,761 & 170,723 & 13,105 & 12,727 & 408,536 & 390,276 \\
\hline Illinois & 29,474 & 26,527 & 29,240 & 26,505 & 33,251 & 35,310 & 6,829 & 6,917 & 98.793 & 95,258 \\
\hline 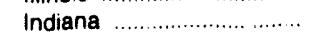 & 20,541 & 18,543 & 14,162 & 13,282 & 33,579 & 31,104 & 391 & 522 & 68,674 & 63,451 \\
\hline 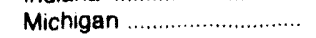 & 22,318 & 20,920 & 18,580 & 17,756 & 31,541 & 29,656 & 1,085 & 1,031 & 73,524 & 69,362 \\
\hline Ohio & 34,573 & 31,714 & 28,053 & 26,775 & $56,5,37$ & 57,684 & 3,787 & 3,726 & 122,950 & 119,899 \\
\hline Wisconsin & 14,286 & 13,753 & 11,442 & 11,052 & 17,853 & 16,969 & 1,013 & 531 & 44,595 & 42,306 \\
\hline West North Central .......... & 62,252 & 55,894 & 46,015 & 43,273 & 57,852 & 55,280 & 4,687 & 4,165 & 170,807 & 158,623 \\
\hline lowa & 9,388 & 8,283 & 6,159 & 5,819 & 11,264 & 10,186 & 1,215 & 650 & 28,026 & 24,938 \\
\hline 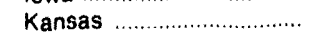 & 8,867 & 7,467 & 8,653 & 7,877 & 7,546 & 6,823 & 325 & 305 & 25,392 & 22,472 \\
\hline Minnesota & 12,648 & 12,342 & 7,109 & 7,055 & 20,028 & 19,415 & 533 & 716 & 40,319 & 39,527 \\
\hline 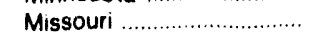 & 20,355 & 17,639 & 16,733 & 15,480 & 11,745 & 12,134 & 787 & 750 & 49,620 & 46,002 \\
\hline Nebraska & 5,906 & 5,522 & 4,526 & 4,417 & 4,208 & 3,642 & 1,077 & 1,042 & 15,717 & 14,624 \\
\hline North Dakota & 2,557 & 2,428 & 1,500 & 1,392 & 1,551 & 1,618 & 426 & 397 & 6,034 & 5,835 \\
\hline South Dakota & 2,531 & 2,214 & 1,335 & 1,232 & 1,510 & 1,473 & 323 & 306 & 5,700 & 5,225 \\
\hline South Atlantic & 198,349 & 182,854 & 140,880 & 133,634 & 132,947 & 129,878 & 15,484 & 14,817 & 487,661 & 461,184 \\
\hline Delaware & 2,587 & 2,364 & 2,208 & 2,093 & 2,808 & 2,698 & 48 & 48 & 7,651 & 7,203 \\
\hline District of Columbia ............. & 1,372 & 1,250 & 4,580 & 4,376 & 2,561 & 2,516 & 286 & 282 & 8,799 & 8,424 \\
\hline Florida & 64.837 & 62,262 & 45,900 & 43,652 & 13,159 & 13,362 & 3,949 & 3,899 & 127,845 & 123,176 \\
\hline 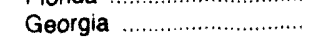 & 29,185 & 25,461 & 21,406 & 20,125 & 24,280 & 22,738 & 833 & 732 & 75,703 & 69,056 \\
\hline 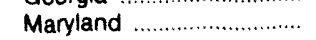 & 17,947 & 16,258 & 9,500 & 8,920 & 16,893 & 16,784 & 678 & 563 & 45,018 & 42,526 \\
\hline 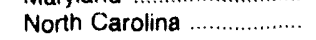 & 31,550 & 29,022 & 22,612 & 21,592 & 28,329 & 27,120 & 1,587 & 1,530 & 84,077 & 79,264 \\
\hline South Carolina & 17,484 & 15,125 & 11,180 & 10,308 & 22.156 & 22,039 & 703 & 694 & 51,522 & 48,167 \\
\hline Virginia & 26,301 & 24,361 & 18,945 & 18,159 & 14,176 & 13,795 & 7,323 & 6,983 & 66,745 & 63,297 \\
\hline West Virginia & 7,088 & 6,751 & 4.551 & 4,408 & 8,585 & 8,825 & 77 & 86 & 20,300 & 20,071 \\
\hline East South Central ........... & 73,537 & 66,379 & 34,450 & 31,669 & 100,483 & 96,121 & 4,243 & 4,082 & 212,713 & 198,251 \\
\hline Alabama & 19,475 & 17,678 & 10,058 & 9,388 & 25,031 & 23,328 & 537 & 556 & 55,100 & 50,950 \\
\hline Kentucky & 15,731 & 14,202 & 8,324 & 7,571 & 29,883 & 31,184 & 2,342 & 2,185 & 56,280 & 55,142 \\
\hline Mississippi ............................ & 11,595 & 10,487 & 6,182 & 5,517 & 11,741 & 11,049 & 498 & 500 & 30,016 & 27,552 \\
\hline Tennessee & 26,736 & 24,013 & 9,887 & 9,193 & 33,827 & 30,560 & 866 & 842 & 71,317 & 64,607 \\
\hline West South Central .......... & 118,274 & 109,168 & 80,813 & 78,023 & 115,945 & 110,686 & 13,807 & 13,398 & 328,839 & 311,275 \\
\hline 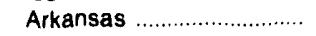 & 10,138 & 8,802 & 5,723 & 5,337 & 9,561 & 9,095 & 476 & 538 & 25,899 & 23,772 \\
\hline 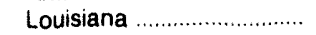 & 19,424 & 18,389 & 12,062 & 11,756 & 23,684 & 22,721 & 2,078 & 2,268 & 57,249 & 55,134 \\
\hline Oklahoma & 13,610 & 11.783 & 9,183 & 8,470 & 9,831 & 9,458 & 1,808 & 1,728 & 34,432 & 31,438 \\
\hline 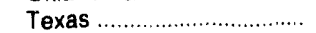 & 75,101 & 70,194 & 53,845 & 52,460 & 72,869 & 69,412 & 9,445 & 8,865 & 211,260 & 200,931 \\
\hline 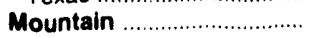 & 45,018 & 42,694 & 45,748 & 45,460 & 47,474 & 49,418 & 6,079 & 5,430 & 144,319 & 143,002 \\
\hline Arizona & 14,276 & 13,622 & 12,522 & 12,175 & 9,342 & 8,507 & 1,356 & 1,418 & 37,496 & 35,722 \\
\hline 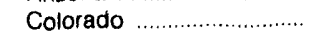 & 9,169 & 8,279 & 12,408 & 12,385 & 5,885 & 5,209 & 796 & 672 & 28.257 & 26,545 \\
\hline Idaho & 4,882 & 5,029 & 4,296 & 4,926 & 6,164 & 9,244 & 278 & 385 & 15,619 & 19,583 \\
\hline 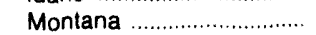 & 2,822 & 2,508 & 2,438 & 2,279 & 4,864 & 5,428 & 376 & 388 & 10,500 & 10,604 \\
\hline Nevada & 5,314 & 5.125 & 3,639 & 3,530 & 6,008 & 6,121 & 1,217 & 810 & 16,177 & 15,588 \\
\hline New Mexico ......................... & 3,259 & 3,100 & 4,087 & $4,0: 4$ & 3,926 & 3,550 & 1.183 & 1,044 & 12,454 & 11,707 \\
\hline 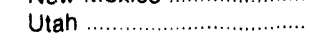 & 3,777 & 3,595 & 4,269 & 4,314 & 5,097 & 5,087 & 770 & 570 & 13,914 & 13,566 \\
\hline 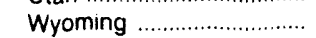 & 1,519 & 1,435 & 2,090 & 1,836 & 6,188 & 6,273 & 104 & 142 & 9,901 & 9,687 \\
\hline Pacific Contiguous .......... & 94,324 & 91,882 & 88,352 & 89,898 & 91,449 & 93,959 & 10,367 & 10,910 & 284,492 & 286,649 \\
\hline California & 56,270 & 57,002 & 62,292 & 65,094 & 50,716 & 49,986 & 6,652 & 6,886 & 175,930 & 178,969 \\
\hline Oregon & 13,240 & 11,946 & 10,123 & 9,936 & 12,136 & 12,779 & 498 & 465 & 35,997 & 35,125 \\
\hline 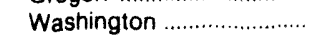 & 24,813 & 22,934 & 15,938 & 14,868 & 28,596 & 31,195 & 3,218 & 3,558 & 72,565 & 72,555 \\
\hline Paciflc Noncontiguous .... & 3,344 & 3,389 & 3,675 & 3,689 & 3,602 & 3,540 & 232 & 187 & 10,853 & 10,784 \\
\hline Alaska & 1,293 & 1,365 & 1,688 & 1,704 & 410 & 355 & 184 & 139 & 3,574 & 3,563 \\
\hline 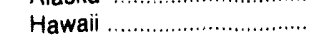 & 2,051 & 2,024 & 1,987 & 1,965 & 3,192 & 3,185 & 48 & 48 & 7,279 & 7,221 \\
\hline U.S. Total & 834,174 & 776,696 & 664,909 & 640,451 & 620,476 & 807,577 & 81,335 & 78,816 & $2,400,894$ & $2,303,539$ \\
\hline
\end{tabular}

' Includes public street and highway lighting, other sales to public authorities, sales to railroads and railways, and interdepartmental sales.

Notes: Estimates for 1992 are final and 1993 are preliminary. - Totals may not equal sum of components because of independent rounding. • Estimated sales and associated revenue are based on sales by the utilities in the sample. See technical notes for an explanation of the modification to the sample design as of January 1993 estimates.

Source: Energy Information Administration, Form EIA-826, "Monthly Electric Utility Sales and Revenue Report with State Distributions." 
Table 55. Revenue From U.S. Electric Utility Sales of Electricity to Uitimate Consumers by Sector, 1983 Through October 1993 (Million Dollars)

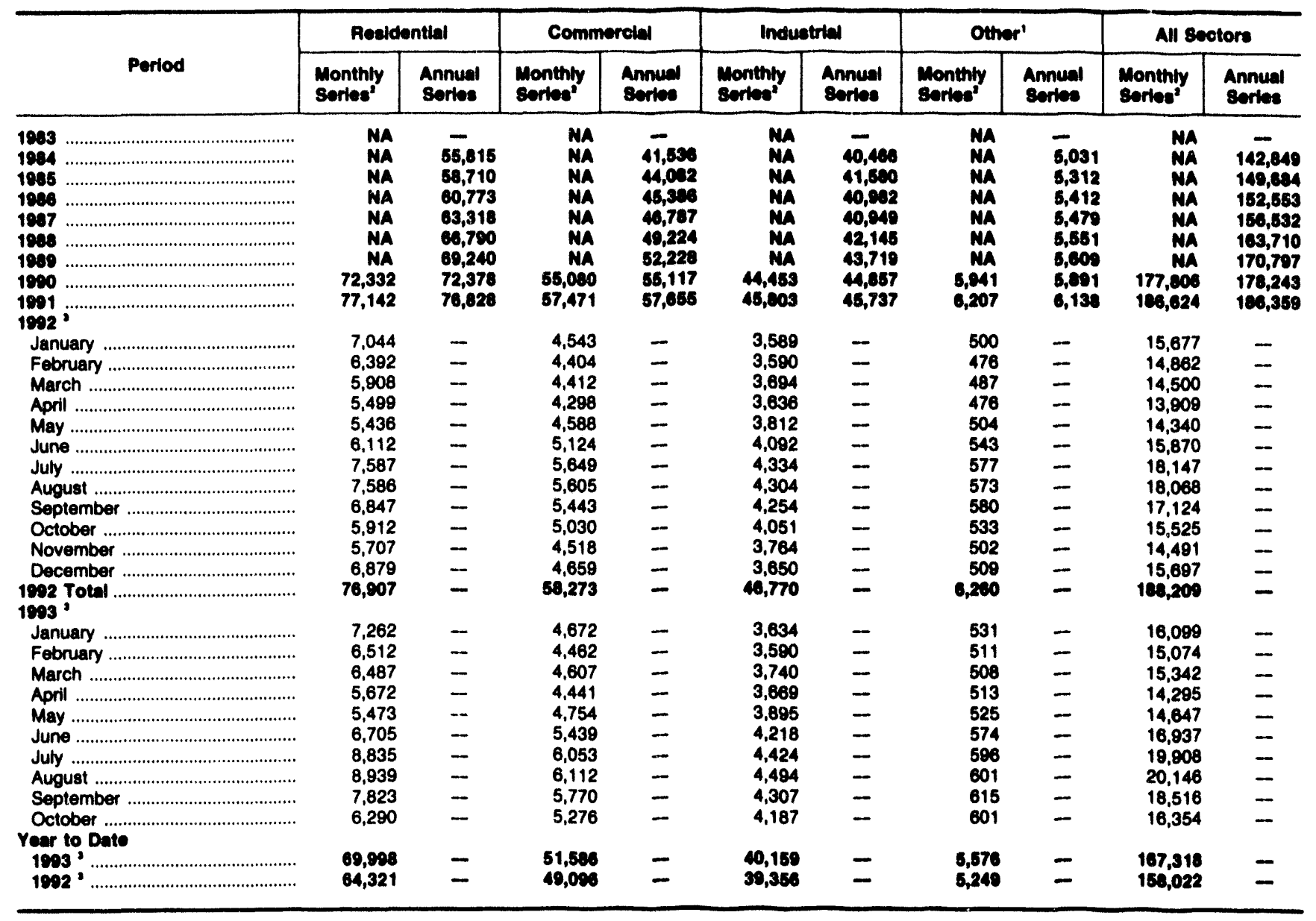

I Includes public street and highway lighting, other sales to public authorities, sales to railroads and ralhways, and interdepartmental sales.

2 See technical notes for an explanation of the modification to the sample design as of January 1993 estimates.

- Estimates for 1992 and prior years are final, and 1993 estimates are preliminary. For further information, $80 e$ the technical notes. NA $=$ Data not available.

Notes: - Totals may not equal sum of components because of independent rounding. - Monetary values are expressed in nominal terms. Revenue does not include sales and excise taxes. Estimated sales and associated revenue are based on sales by the utilities in the sarnple.

Sources: - Monthly Serlea: Energy Information Administration, Form ElA-826, "Monthly Electric Utility Sales and Revenue Report with State Distribu. tions," formerly the "Electric Utility Company Monthly Statement," and predecessor forms. •Annusl serles: Energy Information Adminiatration, Form EIA861. "Annual Electric Utility Report." 
Table 56. Electric Utility Revenue from Sales of Electricity to Uitimate Consumers by Sector, Census Division and State, October 1992 and 1993

(Million Dollars)

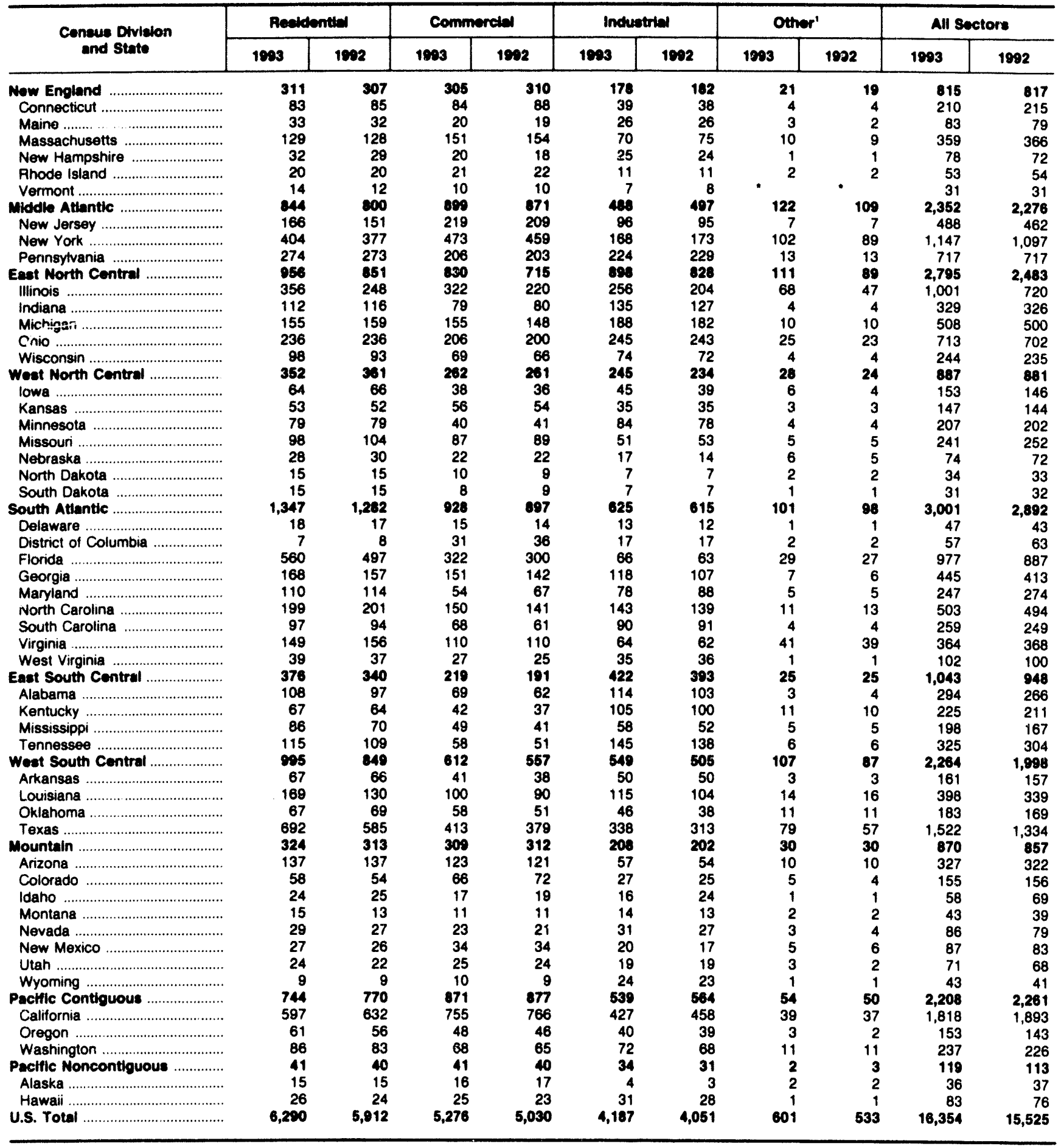

1 Includes public street and highway lighting, other sales to public authorities, sales to railroads and railways, and interdepartmental sales.

- Less than 0.5

Notes: Estimates for 1992 are final; 1993 estimates are preliminary. - Totals may not equal sum of components because of independent rounding. - Monetary values are expressed in nominal terms. Revenue does not include sales and excise taxes. •Estimated sales and associated revenue are based on sales by the utilities in the sample. -See technical notes for an explanation of the modification to the sample design as of January 1993 estimates. Source: Energy Information Administration, Form ElA-826, "Monthly Electric Utility Sales and Revenue Report with State Distributions." 
Table 57. Estimated Coefficients of Variation of Revenue from Electric Utility Sales of Electricity by Census Division and State, October 1993

(Percent)

\begin{tabular}{|c|c|c|c|c|c|}
\hline $\begin{array}{c}\text { Census Division } \\
\text { and State }\end{array}$ & Residential & Commercial & Induetrial & Other' & All Sectors \\
\hline Now England ...................................... & 0.6 & 1.1 & 0.6 & 2.9 & 0.7 \\
\hline Connecticut & .2 & .4 & .4 & 1.2 & .1 \\
\hline Maine & .1 & .1 & .6 & 8.3 & .7 \\
\hline 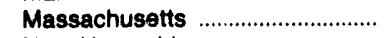 & 1.5 & 2.2 & 1.5 & 5.5 & 1.7 \\
\hline New Hampshire ............................. & .9 & .9 & 1.7 & 1.3 & .6 \\
\hline 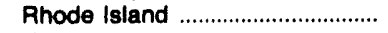 & .4 & .0 & .4 & .2 & .2 \\
\hline Vermont .............................................. & 2.0 & .3 & 1.3 & 1.6 & .7 \\
\hline Middle Atlantic ................................... & .8 & .8 & 1.5 & .9 & .8 \\
\hline 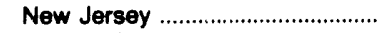 & 1.2 & .7 & 1.0 & .1 & .7 \\
\hline 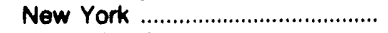 & .8 & 1.4 & .8 & .5 & .9 \\
\hline 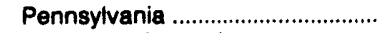 & 1.9 & 1.1 & 3.1 & 8.1 & 2.0 \\
\hline East North Central .......................... & 2.8 & 1.3 & 1.6 & 2.4 & 1.8 \\
\hline 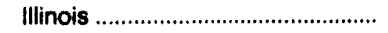 & 7.3 & 2.8 & 4.8 & 1.4 & 4.9 \\
\hline 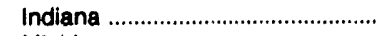 & 1.3 & 1.4 & .8 & 8.5 & .7 \\
\hline Michigan ........................................ & 2.2 & .6 & 2.0 & .4 & 1.5 \\
\hline Ohio & 2.1 & 2.5 & 2.6 & 9.8 & 2.1 \\
\hline 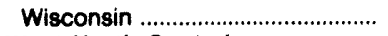 & 1.5 & 1.3 & 1.6 & .8 & .5 \\
\hline West North Central ......................... & 1.9 & 1.4 & 1.9 & 5.0 & 1.5 \\
\hline lowa & 3.5 & 3.3 & 5.0 & 11.8 & 3.5 \\
\hline 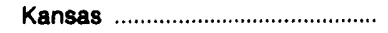 & 3.4 & 3.4 & 9.6 & 10.0 & 4.0 \\
\hline Minnesota & 3.7 & 3.3 & 1.4 & 1.7 & .7 \\
\hline 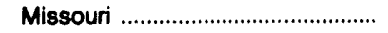 & 5.3 & 2.8 & 3.6 & .9 & 4.2 \\
\hline 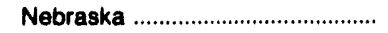 & 5.3 & 1.5 & 3.1 & 17.1 & 2.9 \\
\hline 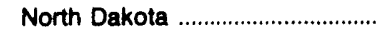 & 2.6 & 2.2 & 1.6 & 2.3 & 1.0 \\
\hline 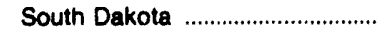 & 1.4 & 2.1 & .4 & 8.0 & 1.5 \\
\hline 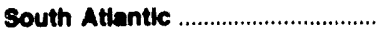 & 1.1 & .7 & 8 & 1.3 & .6 \\
\hline 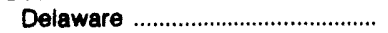 & 1.6 & 1.6 & 2.9 & .2 & 1.4 \\
\hline District of Columbia ......................... & .0 & .0 & .0 & .0 & .0 \\
\hline 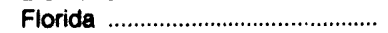 & 1.3 & 1.7 & 47 & 4.4 & 1.5 \\
\hline 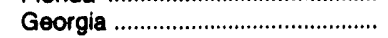 & 6.5 & .9 & 1.4 & 3.3 & 2.1 \\
\hline 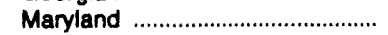 & 1.6 & 1.6 & 1.6 & 1.9 & 1.6 \\
\hline 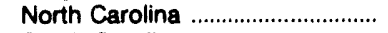 & 1.4 & 1.7 & 1.9 & 3.2 & .4 \\
\hline South Carolina ................................... & 2.4 & .7 & .8 & .3 & .5 \\
\hline 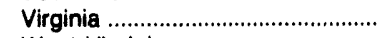 & 2.7 & 6 & .7 & .1 & 1.4 \\
\hline 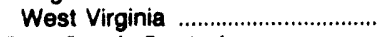 & 1.6 & .7 & 2.2 & .7 & .3 \\
\hline East South Central ......................... & 1.2 & 2.3 & 1.4 & 2.8 & 1.2 \\
\hline Alabama & 1.8 & 4.2 & .9 & 5.8 & 1.6 \\
\hline 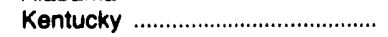 & 4.5 & 2.1 & 3.4 & 2.9 & 3.4 \\
\hline 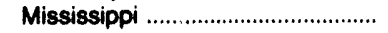 & 3.2 & 3.3 & 7.1 & 12.4 & 4.2 \\
\hline 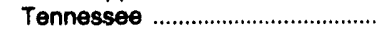 & 1.1 & 6.3 & 1.2 & 1.7 & 1.2 \\
\hline Weat South Central .......................... & 1.4 & .7 & .7 & 3.6 & .6 \\
\hline 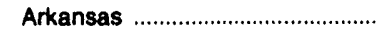 & 1.2 & .9 & .6 & 4.1 & .8 \\
\hline 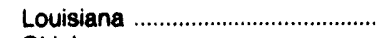 & 2.4 & 1.4 & 1.1 & 2.6 & 1.7 \\
\hline 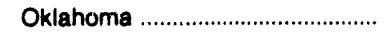 & 5.1 & 2.6 & 2.4 & 1.9 & .6 \\
\hline 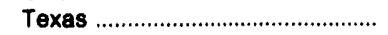 & 1.8 & .8 & 1.0 & 4.9 & .8 \\
\hline 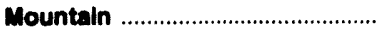 & 1.4 & 1.7 & 1.0 & 10.7 & 1.3 \\
\hline 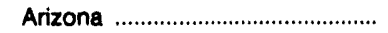 & .6 & 1.4 & 1.6 & 2.7 & .8 \\
\hline 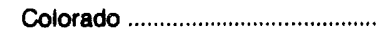 & 7.2 & 7.4 & 5.7 & 18.5 & 7.1 \\
\hline Idaho & 2.0 & 3.8 & 3.9 & 4.3 & 2.5 \\
\hline 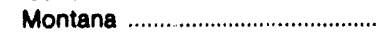 & 4.2 & 1.5 & 2.1 & 2.5 & 1.0 \\
\hline 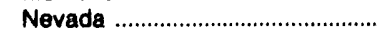 & .6 & .4 & 1.1 & 1.4 & .1 \\
\hline 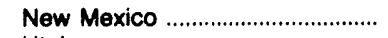 & 1.1 & 1.5 & 2.3 & 31.4 & 1.2 \\
\hline 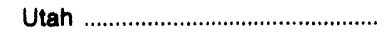 & 1.9 & .6 & 2.4 & 77.4 & .8 \\
\hline 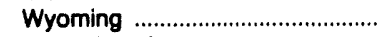 & 2.8 & 5.0 & .8 & 13.0 & .7 \\
\hline Pacific Contiguous .......................... & .8 & 1.5 & 3.4 & 7.0 & .7 \\
\hline 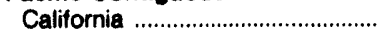 & .9 & 1.7 & 4.0 & 9.4 & .8 \\
\hline Oregon & 4.7 & .3 & 4.7 & 13.7 & 1.8 \\
\hline 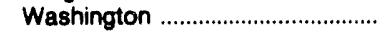 & 1.6 & .5 & 7.3 & 1.9 & 1.7 \\
\hline Pactfic Noncontiguous .................. & 1.0 & 1.5 & .5 & 6.0 & .9 \\
\hline 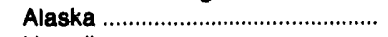 & 2.2 & 3.8 & 3.5 & 8.0 & 2.7 \\
\hline 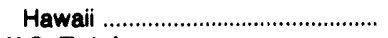 & 1.0 & .7 & .3 & 1.4 & .5 \\
\hline 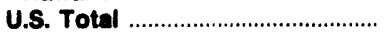 & .6 & .4 & .6 & 1.2 & .4 \\
\hline
\end{tabular}

1 Includes public street and highway lighting, other sales to public authorities, sales to railroads and railways, and interdepartmental sales. Notes: Estimates for 1993 are preliminary. $\bullet$ For an explanation of coefficient of variation, 80 the technical notes.

Source: Energy Information Administration, Form EIA-826, "Monthly Electric Utility Sales and Revenue Report with State Distributions." 
Table 58. Electric Utility Revenue from Sales to Uitimate Consumers by Sector, Census Division, and State, January Through October 1992 and 1993 (Million Dollars)

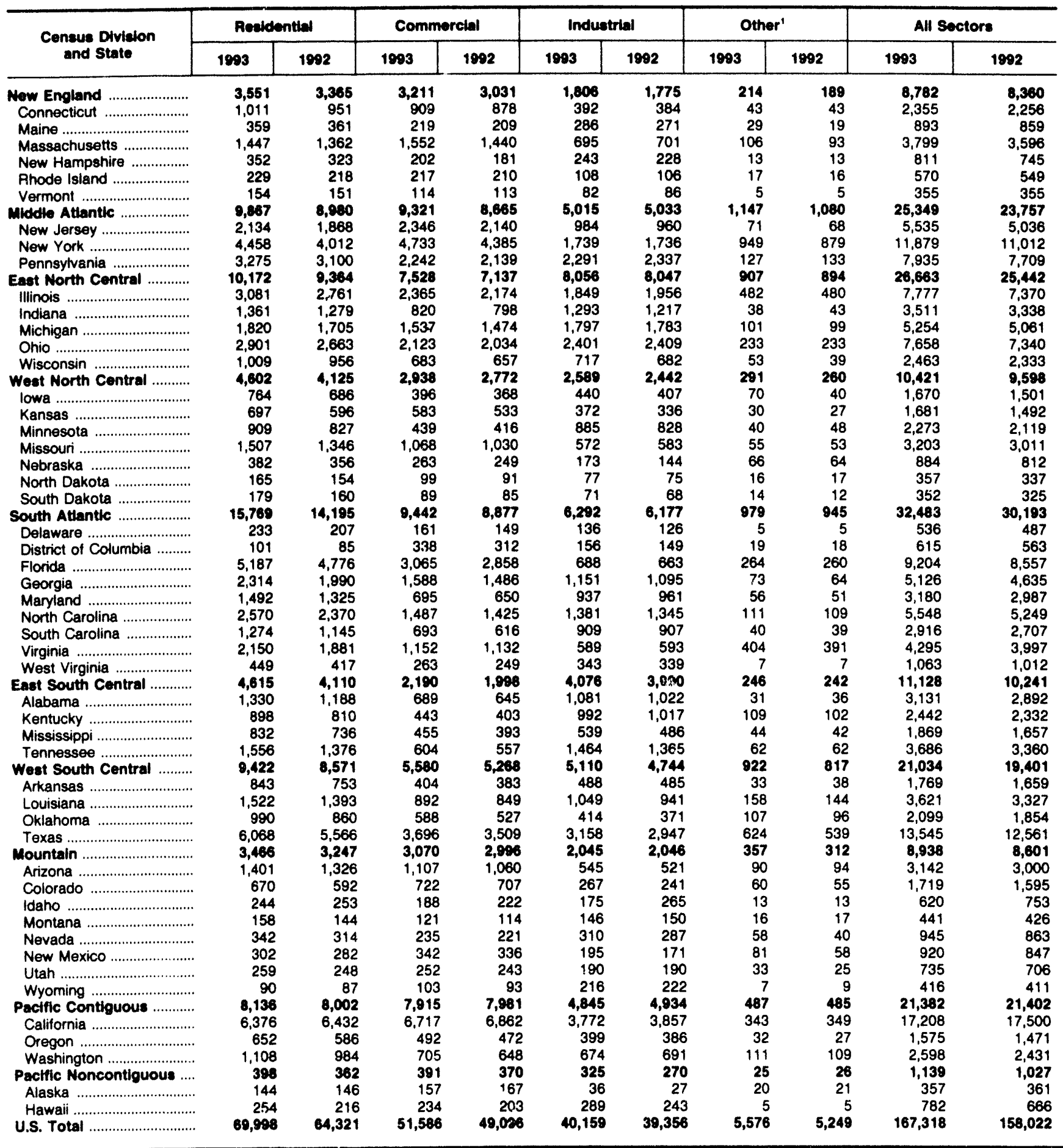

1 Includes public street and highway lighting, other sales to public authorities, sales to railroads and raitways, and interdepartmental sales.

Notes: Estimates for 1992 are final and 1993 are preliminary. - Totals may not equal sum of components because of independent rounding

- Monetary values are expressed in nominal terms. Revenue does not include sales and excise taxes. estimated sales and associated revenue are based on sales by the utilities in the sample. - See technical notes for an explanation of the modification to the sample design as of January 1993 estimates.

Source: Energy Information Administration, Form ElA-826, "Monthly Electric Utility Sales and Revenue Report with State Distributions." 
Table 59. U.S. Electric Utility Average Revenue per KIlowatthour by Sector, 1983 Through Ontober 1993

(Cents)

\begin{tabular}{|c|c|c|c|c|c|c|c|c|c|c|}
\hline \multirow[b]{2}{*}{ Period } & \multicolumn{2}{|c|}{ Residential } & \multicolumn{2}{|c|}{ Commercial } & \multicolumn{2}{|c|}{ Industrial } & \multicolumn{2}{|c|}{ Other' } & \multicolumn{2}{|c|}{ All Sectors } \\
\hline & $\begin{array}{l}\text { Monthly } \\
\text { Serlese }^{2}\end{array}$ & $\begin{array}{l}\text { Annual } \\
\text { Serles }\end{array}$ & $\begin{array}{l}\text { Monthly } \\
\text { Serles }^{2}\end{array}$ & $\begin{array}{c}\text { Annual } \\
\text { Serles }\end{array}$ & $\begin{array}{l}\text { Monthly } \\
\text { Series }^{2}\end{array}$ & $\begin{array}{l}\text { Annual } \\
\text { Series }\end{array}$ & $\begin{array}{l}\text { Monthly } \\
\text { Series }^{2}\end{array}$ & $\begin{array}{l}\text { Annual } \\
\text { Series }\end{array}$ & $\begin{array}{l}\text { Monthly } \\
\text { Serles }^{2}\end{array}$ & $\begin{array}{c}\text { Annual } \\
\text { Serles }\end{array}$ \\
\hline 1983 & 7.2 & - & 7.0 & - & 5.0 & - & 6.4 & - & 6.3 & $\ldots$ \\
\hline 1984 & 7.5 & 7.2 & 7.3 & 7.1 & 5.0 & 4.8 & 6.8 & 5.9 & 6.5 & 6.3 \\
\hline 1985 & 7.8 & 7.4 & 7.5 & 7.3 & 5.2 & 5.0 & 7.0 & 6.1 & 6.7 & 6.4 \\
\hline 1986 & 7.4 & 7.4 & 7.1 & 7.2 & 4.9 & 4.9 & 6.6 & 6.1 & 6.4 & 6.4 \\
\hline 1987 & 7.4 & 7.4 & 7.0 & 7.1 & 4.7 & 4.8 & 6.6 & 6.2 & 6.3 & 6.4 \\
\hline 1988 & 7.5 & 7.5 & 7.1 & 7.0 & 4.6 & 4.7 & 6.0 & 6.2 & 6.3 & 6.4 \\
\hline 1989 & 7.6 & 7.6 & 7.2 & 7.2 & 4.7 & 4.7 & 6.2 & 6.2 & 6.4 & 6.5 \\
\hline 1990 & 7.8 & 7.8 & 7.3 & 7.3 & 4.7 & 4.7 & 6.2 & 6.4 & 6.6 & 6.6 \\
\hline 1991 & 8.1 & 8.0 & 7.5 & 7.5 & 4.8 & 4.8 & 6.4 & 6.5 & 6.8 & 6.7 \\
\hline \\
\hline 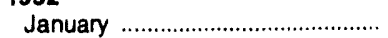 & 7.7 & - & 7.3 & - & 4.7 & -- & 6.5 & - & 6.6 & - \\
\hline 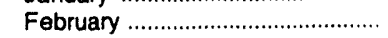 & 7.8 & -- & 7.4 & - & 4.7 & -- & 6.3 & -- & 6.6 & -- \\
\hline 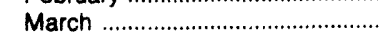 & 8.0 & -- & 7.4 & - & 4.7 & - & 6.5 & - & 6.6 & -- \\
\hline April & 8.0 & - & 7.4 & - & 4.7 & - & 6.4 & - & 6.6 & - \\
\hline 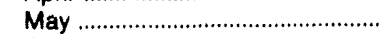 & 8.4 & -- & 7.6 & - & 4.8 & - & 6.5 & -- & 6.7 & -- \\
\hline 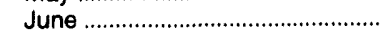 & 8.6 & -- & 7.9 & - & 4.9 & - & 6.9 & -- & 7.0 & - \\
\hline 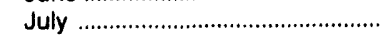 & 8.6 & - & 7.9 & - & 5.1 & - & 6.9 & -- & 7.2 & - \\
\hline 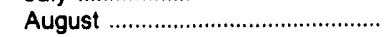 & 8.6 & - & 7.9 & - & 5.1 & - & 6.9 & - & 7.2 & -- \\
\hline 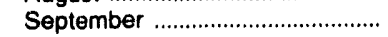 & 8.6 & - & 8.0 & - & 5.1 & - & 6.9 & -- & 7.2 & -- \\
\hline 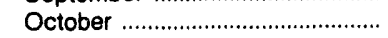 & 8.5 & - & 7.9 & -- & 4.9 & - & 6.9 & - & 6.9 & -- \\
\hline 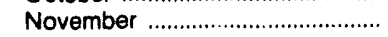 & 8.2 & - & 7.5 & - & 4.7 & -- & 6.7 & - & 6.6 & -- \\
\hline 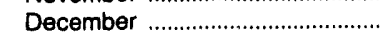 & 7.9 & - & 7.4 & - & 4.7 & - & 6.6 & -- & 6.7 & -- \\
\hline & 8.2 & - & 7.6 & - & 4.8 & - & 6.7 & - & 6.8 & - \\
\hline $1993^{3}$ & 7.7 & -- & 7.3 & - & 4.7 & - & 6.5 & - & 6.6 & - \\
\hline 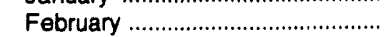 & 7.8 & -- & 7.4 & - & 4.7 & -- & 6.4 & - & 6.6 & - \\
\hline March & 7.8 &.- & 7.4 & - & 4.7 & - & 6.4 & - & 6.6 & - \\
\hline April & 8.1 & -- & 7.5 & - & 4.6 & - & 6.8 & - & 6.6 & -- \\
\hline May & 8.6 & -- & 7.7 & - & 4.7 & -- & 6.9 & - & 6.8 & - \\
\hline June & 8.8 & -- & 8.0 & - & 5.0 & -- & 7.1 & - & 7.1 & -- \\
\hline July & 8.7 & -- & 8.0 & - & 5.2 & - & 7.0 & - & 7.4 & -- \\
\hline August & 8.7 & - & 8.0 & - & 5.2 & - & 7.0 & - & 7.3 & - \\
\hline September & 8.8 & - & 8.0 & - & 5.1 & - & 7.1 & - & 7.3 & $\ldots$ \\
\hline October & 8.8 & - & 8.1 & - & 5.0 & - & 7.3 & - & 7.2 & - \\
\hline \multicolumn{11}{|l|}{ Year-to-Date Average } \\
\hline 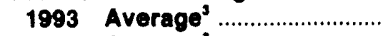 & 8.4 & - & 7.8 & - & 4.9 & - & 6.8 & - & 7.0 & - \\
\hline 1992 Average & 8.3 & - & 7.7 & - & 4.9 & - & 6.7 & - & 6.9 & - \\
\hline
\end{tabular}

- Includes public street and highway lighting, other sales to public authorities, sales to railroads and railways, and interdepartmental sales.

2 See the technical notes for an explanation of the modification to the sample design as of January 1993 estimates.

3 Estimates for 1992 and prior years are final, and 1993 are preliminary.

Notes: - Monetary values are expressed in nominal terms. Revenue and average revenue per kilowatthour do not include sales and excise taxes. - These estimates are calculated by dividing revenue by sales. Revenue may not correspond to sales for a particular month because of utility billing and accounting procedures. This could result in uncharacteristic increases or decreases in the monthly average revenue per kilowatthour.

Sources: -Monthly Serles: Energy Information Administration, Form EIA-826, "Monthly Electric Utility Sales and Revenue Report with State Distributions," formerly the "Electric Utility Company Monthly Statement," and predecessor forms. •Annual Serles: Energy Information Administration, Form ElA. 861. "Annual Electric Utility Report." 
Table 60. Electric UtIlity Average Revenue per Kilowatthour by Sector, Census Division, and State, October 1992 and 1993 (Cents)

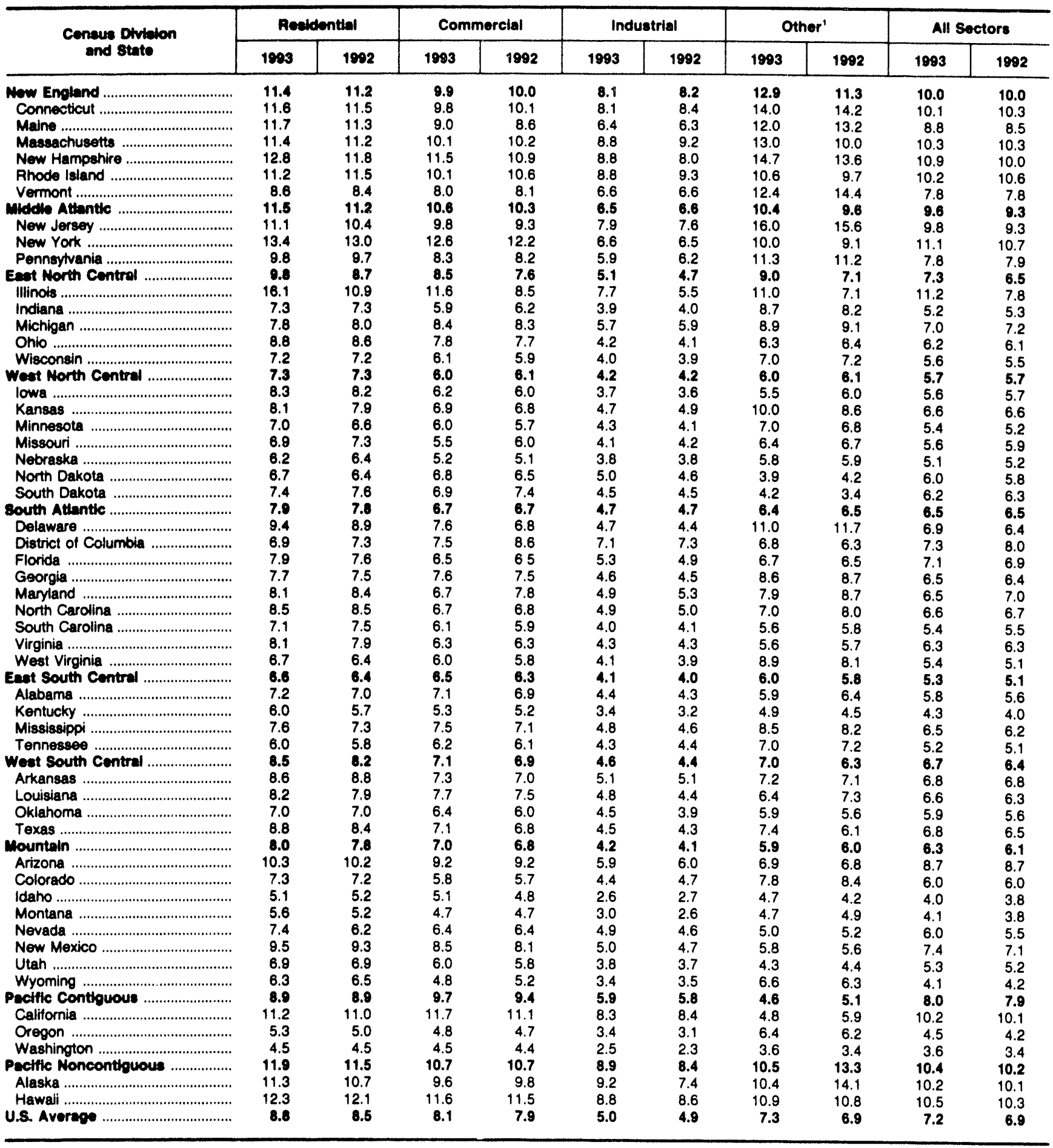

' Includes public street and highway lighting, other sales to public authorities, sales to railroads and railways, and interdepartmental sales.

Notes: - Estimates for 1992 are final, and 1993 are preliminary. - Monetary values are expressed in nominal terms. Revenue and average revenue per kilowatthour do not include sales and excise taxes. These estimates are calculated by dividing revenue by sales. Revenue may not correspond to sales for a particular month because of utility billing and accounting procedures. This could result in uncharacteristic increases or decreases in the monthly average revenue per kilowatthour. -See technical notes for an explanation of the modification to the sample design as of January 1993 estimates. Source: Energy Information Administration, Form EIA-826, "Monthly Electric Utility Sales and Revenue Report with State Distributions." 
Table 61. Estimated Coefficients of Variation for Electric Utility Average Revenue per Kilowatthour by Sector, Census Division, and State, October 1993

(Percent)

\begin{tabular}{|c|c|c|c|c|c|}
\hline $\begin{array}{l}\text { Census Division } \\
\text { and State }\end{array}$ & Residential & Commerclal & Industrial & Other' & All Sectors \\
\hline 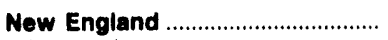 & 0.6 & 1.1 & 0.8 & 3.7 & 0.7 \\
\hline 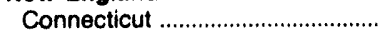 & .3 & .4 & .3 & .6 & .3 \\
\hline Maine & .7 & .2 & .5 & .8 & .1 \\
\hline Massachusetts ............................... & 1.1 & 2.3 & 2.0 & 8.0 & 1.5 \\
\hline New Harnpshire ............................... & 1.5 & 1.3 & .5 & 1.7 & .9 \\
\hline 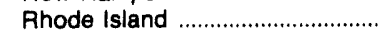 & .2 & .1 & .4 & .3 & .2 \\
\hline 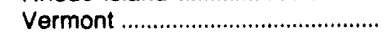 & 1.8 & .2 & 3.1 & 15.8 & 1.3 \\
\hline Middle Atlantic ............................... & .8 & .4 & .7 & 2.1 & .5 \\
\hline 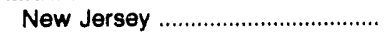 & .5 & .5 & .7 & .5 & .5 \\
\hline 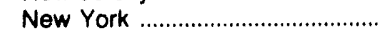 & 1.8 & .4 & 1.0 & 2.3 & .8 \\
\hline 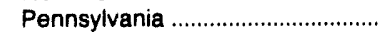 & 1.2 & 1.2 & 1.3 & 7.3 & 1.2 \\
\hline East North Central ......................... & 2.6 & 2.0 & 1.7 & 1.1 & 2.1 \\
\hline 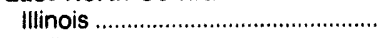 & 6.3 & 5.9 & 5.6 & 1.6 & 6.0 \\
\hline 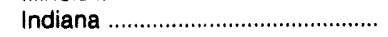 & 1.2 & .6 & .8 & 7.9 & .8 \\
\hline 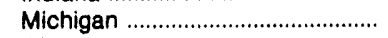 & .2 & .9 & .8 & .7 & .7 \\
\hline Ohio & 2.4 & 1.6 & 2.3 & 4.0 & 1.9 \\
\hline 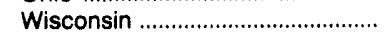 & .5 & .7 & 1.3 & 1.0 & 1.1 \\
\hline West North Central ....................... & 1.1 & 1.1 & 1.0 & 3.0 & 1.0 \\
\hline lowa & .8 & .4 & 1.8 & 6.3 & .9 \\
\hline Kansas & 2.1 & 1.4 & 2.3 & 15.2 & .9 \\
\hline 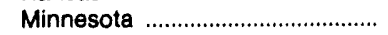 & 6 & .9 & .4 & 2.9 & .8 \\
\hline 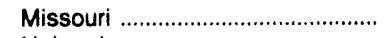 & 3.8 & 3.0 & 3.9 & 1.4 & 3.6 \\
\hline 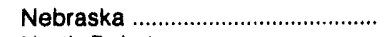 & 3.8 & 2.5 & 1.3 & 8.3 & 2.0 \\
\hline 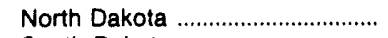 & 1.4 & 1.2 & 1.7 & 4.1 & 1.3 \\
\hline 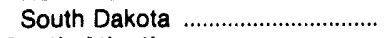 & .5 & .4 & 1.1 & 4.7 & .9 \\
\hline South Atlantic & .8 & .6 & .5 & .7 & .6 \\
\hline 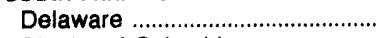 & .3 & 1.0 & 3.3 & 3.2 & 1.4 \\
\hline District of Columbia ........................ & .0 & .0 & .0 & .0 & .0 \\
\hline 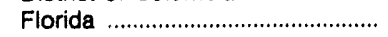 & 1.9 & 1.7 & 3.1 & 2.1 & 2.0 \\
\hline Georgia & 1.1 & .6 & .1 & .6 & .2 \\
\hline 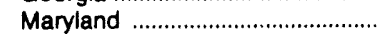 & 1.4 & 1.0 & 1.1 & 6.5 & 1.4 \\
\hline 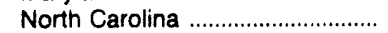 & .8 & .6 & 1.3 & 1.0 & .5 \\
\hline 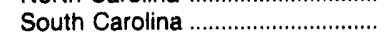 & .5 & 1.1 & 6 & .3 & .3 \\
\hline 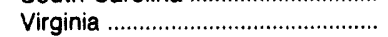 & 3 & .1 & .2 & .1 & .1 \\
\hline 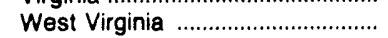 & .4 & 3 & 1.2 & 2.9 & .6 \\
\hline East South Central ......................... & .6 & .5 & .7 & .9 & .6 \\
\hline 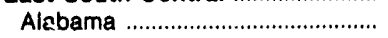 & 1.0 & .3 & .9 & 1.2 & .8 \\
\hline 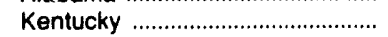 & 2.1 & .9 & 1.8 & 1.9 & 1.2 \\
\hline 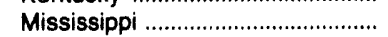 & 1.7 & 1.9 & 2.8 & 5.0 & 1.8 \\
\hline 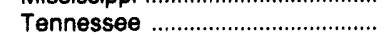 & .2 & .2 & 1.1 & 1.4 & .7 \\
\hline West South Central ....................... & 1.5 & 1.0 & .9 & 2.2 & 1.1 \\
\hline 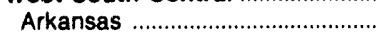 & .7 & .3 & .7 & .9 & .4 \\
\hline 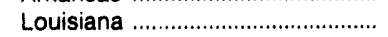 & .7 & .2 & .8 & 2.1 & .9 \\
\hline Oklahoma & 2.1 & 3.3 & 5.9 & .6 & .2 \\
\hline 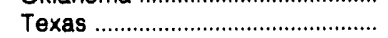 & 2.3 & 1.5 & 1.2 & 2.9 & 1.7 \\
\hline 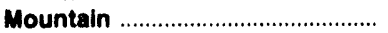 & .4 & .5 & .4 & 5.9 & .4 \\
\hline Arizona . . . . . . & .1 & .7 & 6 & 6.7 & .3 \\
\hline 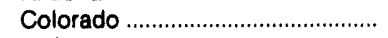 & 1.7 & 1.2 & 2.5 & 9.3 & 1.4 \\
\hline 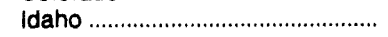 & 2.0 & 1.3 & 1.4 & 1.6 & .4 \\
\hline 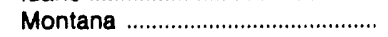 & 3.9 & 2.2 & .4 & 9.6 & 2.4 \\
\hline 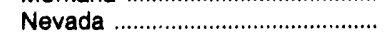 & 1.8 & 1.8 & 6 & .3 & $\begin{array}{r}2.4 \\
.5\end{array}$ \\
\hline 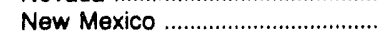 & 1.0 & .8 & .9 & 9.8 & 1.1 \\
\hline 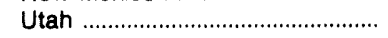 & 1.9 & .7 & 3 & 21.1 & 1.1 \\
\hline 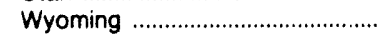 & 2.2 & 1.4 & .5 & 5.3 & .6 \\
\hline Pacific Contiguous ......................... & .8 & 1.6 & 1.7 & 5.1 & 1.2 \\
\hline 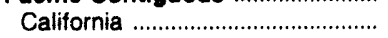 & 1.1 & 2.1 & 1.3 & 7.3 & $\begin{array}{l}1.2 \\
1.5\end{array}$ \\
\hline Oregon & .8 & .6 & 2.4 & 12.5 & 1.8 \\
\hline 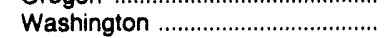 & 1.3 & 1.6 & 2.4 & 3.5 & 1.1 \\
\hline Pacific Noncontiguous ................. & .8 & 1.2 & .7 & 5.3 & .9 \\
\hline Alaska & 1.8 & 2.9 & 4.6 & 6.9 & 2.3 \\
\hline 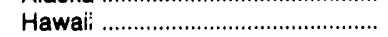 & 1.1 & .7 & .5 & 1.3 & .8 \\
\hline 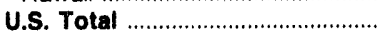 & .5 & .4 & .5 & 1.1 & .4 \\
\hline
\end{tabular}

' Includes public street and highway lighting, other sales to public authorities, sales to railroads and railways, and interdepartmental sales. Notes: - Estimates for 1993 are preliminary. -For an explanation of coefficient of variation, see the technical notes.

Source: Energy Information Administration, Form EIA-826, "Monthly Electric Utility Sales and Revenue Report with State Distributions." 
Monthly Plant Aggregates:

U.S. Electric Utility Net Generation,

Fuel Consumption, and Fuel Stocks 
Table 62. U.S. Electric Utility Net Generation, Fuel Consumption, and Fuel Stocks by Company and Plant, September 1893

\begin{tabular}{|c|c|c|c|c|c|c|c|c|c|c|c|}
\hline \multirow{2}{*}{$\begin{array}{c}\text { Company (Holding Company) } \\
\text { Plant (State) }\end{array}$} & \multicolumn{6}{|c|}{$\begin{array}{l}\text { Coneration } \\
\text { (thousend kllowatthours) }\end{array}$} & \multicolumn{3}{|c|}{$\begin{array}{l}\text { Coneumption } \\
\text { (thoueand) }\end{array}$} & \multicolumn{2}{|c|}{$\begin{array}{l}\text { Stocks } \\
\text { (thousand) }\end{array}$} \\
\hline & Cosl & Potrotoum & ans & Hydro & Nuclear & Other' & $\begin{array}{c}\text { Coal } \\
\text { (chort } \\
\text { tono) }\end{array}$ & $\begin{array}{l}\text { Potro- } \\
\text { loum } \\
\text { (bble) }\end{array}$ & $\underset{(M C f)}{\text { ans }}$ & $\begin{array}{l}\text { Coal } \\
\text { (chort } \\
\text { tons) }\end{array}$ & $\begin{array}{l}\text { Potro- } \\
\text { leum } \\
\text { (bble) }\end{array}$ \\
\hline
\end{tabular}

\begin{tabular}{|c|c|c|c|c|c|c|c|c|c|c|c|}
\hline $\begin{array}{l}\text { Abbeville Water Eloc Plant ........ } \\
\text { Abbeville (SC) }\end{array}$ & $\overline{-}$ & $\begin{array}{l}2 \\
2\end{array}$ & $\overline{-}$ & $\begin{array}{l}366 \\
366\end{array}$ & $\overline{-}$ & $\overline{-}$ & $\overline{-}$ & : & $\overline{-}$ & $\overline{-}$ & : \\
\hline \multicolumn{12}{|l|}{ Accomack-Northampton Electric } \\
\hline Smith (VA) & $\overline{-}$ & $\begin{array}{l}5 \\
3\end{array}$ & $\overline{-}$ & $\overline{-}$ & $\overline{-}$ & - & $\overline{-}$ & • & $\overline{-}$ & $\bar{z}$ & : \\
\hline 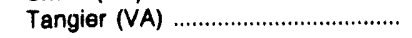 & - & 2 & - & - & -- & -- & - & • & - & -- & - \\
\hline Adrian Lt \& Water Comm ................ & - & - & - & - & - & - & - & - & - & - & - \\
\hline 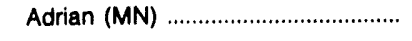 & -- & - & - & - & - & - & - & - & - & -- & - \\
\hline Altkin Publlc Uttlities Comm .......... & - & -8 & - & - & - & - & - & - & - & - & : \\
\hline 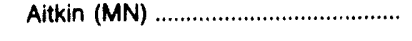 & - & -8 & - & - & - & - & - & - & - & - & • \\
\hline Alabama Elec Coop, Inc .................... & 288,953 & -214 & - & 1,025 & - & - & 125 & - & - & 349 & 4 \\
\hline Gantt (AL) & -- & - & - & 235 & - & - & - & - & - & - & -- \\
\hline 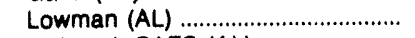 & 289,012 & - & - & - & - & - & 125 & - & - & 345 & -- \\
\hline 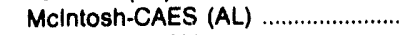 & - & -208 & $\cdots$ & - & - & - & - & - & - & - & 3 \\
\hline 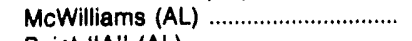 & -59 & - & - & -700 & $\overline{-}$ & - & - & - & - & 4 & -- \\
\hline 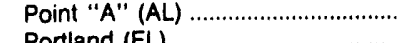 & - & - & $\overline{-}$ & $\ldots$ & $\overline{-}$ & $\overline{-}$ & - & $\because$ & $\overline{-}$ & $\overline{-}$ & - \\
\hline 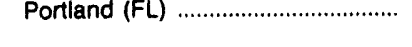 & - & $\rightarrow$ & & - & - & - & - & & - & - & 1 \\
\hline & $4,561,901$ & 1,654 & 20,045 & $\begin{array}{r}114,632 \\
4,634\end{array}$ & $1,016,513$ & - & 1,832 & 3 & 213 & 2,141 & 54 \\
\hline $\begin{array}{l}\text { Bankhead Dam (AL) } \\
\text { Barry (AL) }\end{array}$ & $1, \overline{011,522}$ & $=$ & & $\stackrel{4,634}{-}$ & & $\overline{-}$ & - & $\overline{-}$ & $-{ }_{24}$ & $\overline{5}_{561}$ & \\
\hline Chickasaw (AL) ................................... & - & $\overline{-}$ & -50 & - & - & $\overline{-}$ & - & $\bar{z}$ & -24 & - & $\begin{array}{r}16 \\
3\end{array}$ \\
\hline 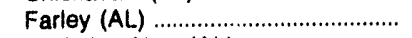 & - & - & - & - & $1,016,513$ & - & - & - & - & - & - \\
\hline 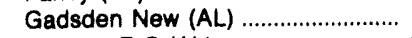 & 45,510 & 3 & 210 & - & - & - & 21 & • & 2 & 28 & 1 \\
\hline 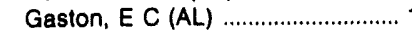 & $1,033,471$ & 714 & - & - & - & - & 422 & 1 & - & 462 & 16 \\
\hline 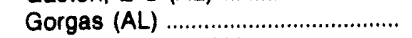 & 719,220 & 753 & - & - & - & - & 294 & 1 & - & 452 & 10 \\
\hline 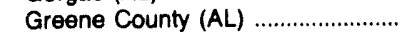 & 319,270 & 184 & - & - & - & - & 127 & • & - & 116 & \\
\hline H Neely Henry Dam (AL) .................. & - & - & - & 4,700 & - & - & - & - & -- & - & - \\
\hline Harris (AL) & - & - & - & 4,221 & - & - & - & - & - & - & - \\
\hline 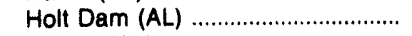 & - & - & - & 5,056 & - & - & - & - & - & - & - \\
\hline 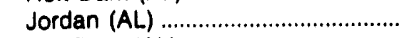 & - & - & - & 9,660 & - & - & - & - & - & - & - \\
\hline 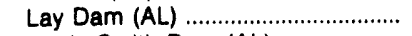 & - & - & - & 11,723 & - & - & - & - & - & - & - \\
\hline 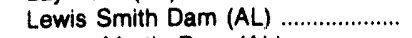 & - & - & - & 17,350 & - & - & - & - & - & - & - \\
\hline Logan Martin Dam (AL) .................... & - & - & - & 6,611 & - & - & - & - & - & - & - \\
\hline 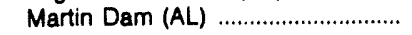 & -- & - & - & 13,043 & - & - & - & - & - & - & -- \\
\hline Miller (AL) & $1,432,998$ & - & 19,325 & - & - & - & 549 & - & 187 & 523 & 7 \\
\hline 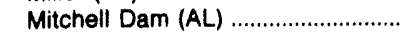 & - & - & - & 9,798 & - & - & - & - & - & - & - \\
\hline Thurlow Dam (AL) ............................. & - & -- & - & 9,508 & - & - & - & - & - & - & - \\
\hline Walter Bouldin Dam (AL) ................ & - & - & - & 7,271 & - & - & - & - & - & - & - \\
\hline 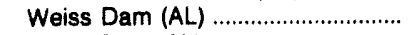 & - & - & - & 5,425 & - & - & - & - & - & - & - \\
\hline 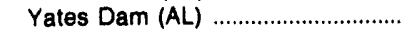 & - & - & - & 5,632 & - & - & - & - & -- & - & - \\
\hline Alaska Electric Lt \& Pwr Co ......... & - & 41 & - & 4,135 & - & - & - & - & - & - & \\
\hline Annex Creek (AK) & - & - & - & 2,262 & - & - & - & - & - & - & - \\
\hline Gold Creek (AK) ................................. & - & - & - & 323 & - & - & - & • & - & - & - \\
\hline Lemon Creek (AK) ............................. & - & 41 & - & - & - & - & - & " & - & - & \\
\hline Salmon Creek (AK) ........................... & - & - & - & 1,550 & - & - & - & - & - & - & - \\
\hline 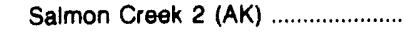 & - & - & - & - & - & - & - & - & - & - & - \\
\hline Alaska Power Administration ........ & - & - & - & 39,234 & - & - & - & - & - & - & - \\
\hline 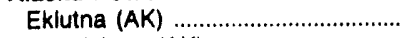 & - & - & - & 21,930 & - & - & - & - & - & - & - \\
\hline 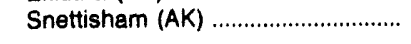 & - & - & - & 17,304 & - & - & - & - & - & - & - \\
\hline $\begin{array}{l}\text { Company } \\
\text { Chistochina (AK) }\end{array}$ & - & 16 & - & -200 & - & - & $\overline{-}$ & - & $\overline{-}$ & $\overline{-}$ & $\cdot^{2}$ \\
\hline Cottman Cove (AK) ............................ & - & 50 & - & - & - & - & - & - & - & - & - \\
\hline 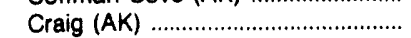 & - & 1,006 & -. & - & - & - & - & 2 & - & - & $\cdot$ \\
\hline 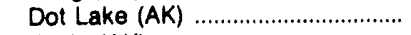 & - & - & - & - & - & - & - & - & - & - & - \\
\hline Eagle (AK) & - & 51 & - & - & - & - & - & • & - & - & ( \\
\hline Hollis (AK) . & - & 18 & - & - & - & - & - & : & - & - & $:$ \\
\hline 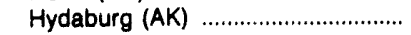 & - & 111 & - & - & - & - & - & - & - & - & - \\
\hline 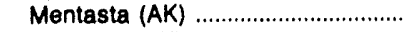 & - & 17 & - & - & - & - & - & • & - & - & : \\
\hline Skagway (AK) & - & 475 & - & 288 & - & - & - & 1 & - & - & " \\
\hline
\end{tabular}

See footnotes at end of table. 
Table 62. U.S. Electric Utility Net Generation, Fuel Consumption, and Fuel Stocks by Company and Plant, September 1993 (Continued)

\begin{tabular}{|c|c|c|c|c|c|c|c|c|c|c|c|}
\hline \multirow{2}{*}{$\begin{array}{c}\text { Company (Holding Company) } \\
\text { Plant (State) }\end{array}$} & \multicolumn{6}{|c|}{$\begin{array}{c}\text { Ceneration } \\
\text { (thoueand kllowatthours) }\end{array}$} & \multicolumn{3}{|c|}{$\begin{array}{l}\text { Consumption } \\
\text { (thousand) }\end{array}$} & \multicolumn{2}{|c|}{$\begin{array}{c}\text { Stocke } \\
\text { (thousand) }\end{array}$} \\
\hline & Cual & Potroleum & ans & Hydro & Nuclear & Other' & $\begin{array}{l}\text { Coal } \\
\text { (chort } \\
\text { tons) }\end{array}$ & $\begin{array}{l}\text { Potro- } \\
\text { loum } \\
\text { (bble) }\end{array}$ & $\begin{array}{c}\text { Gas } \\
\text { (Mef) }\end{array}$ & $\begin{array}{l}\text { Coal } \\
\text { (ahort } \\
\text { tons) }\end{array}$ & $\begin{array}{l}\text { Potro- } \\
\text { loum } \\
\text { (bblo) }\end{array}$ \\
\hline
\end{tabular}

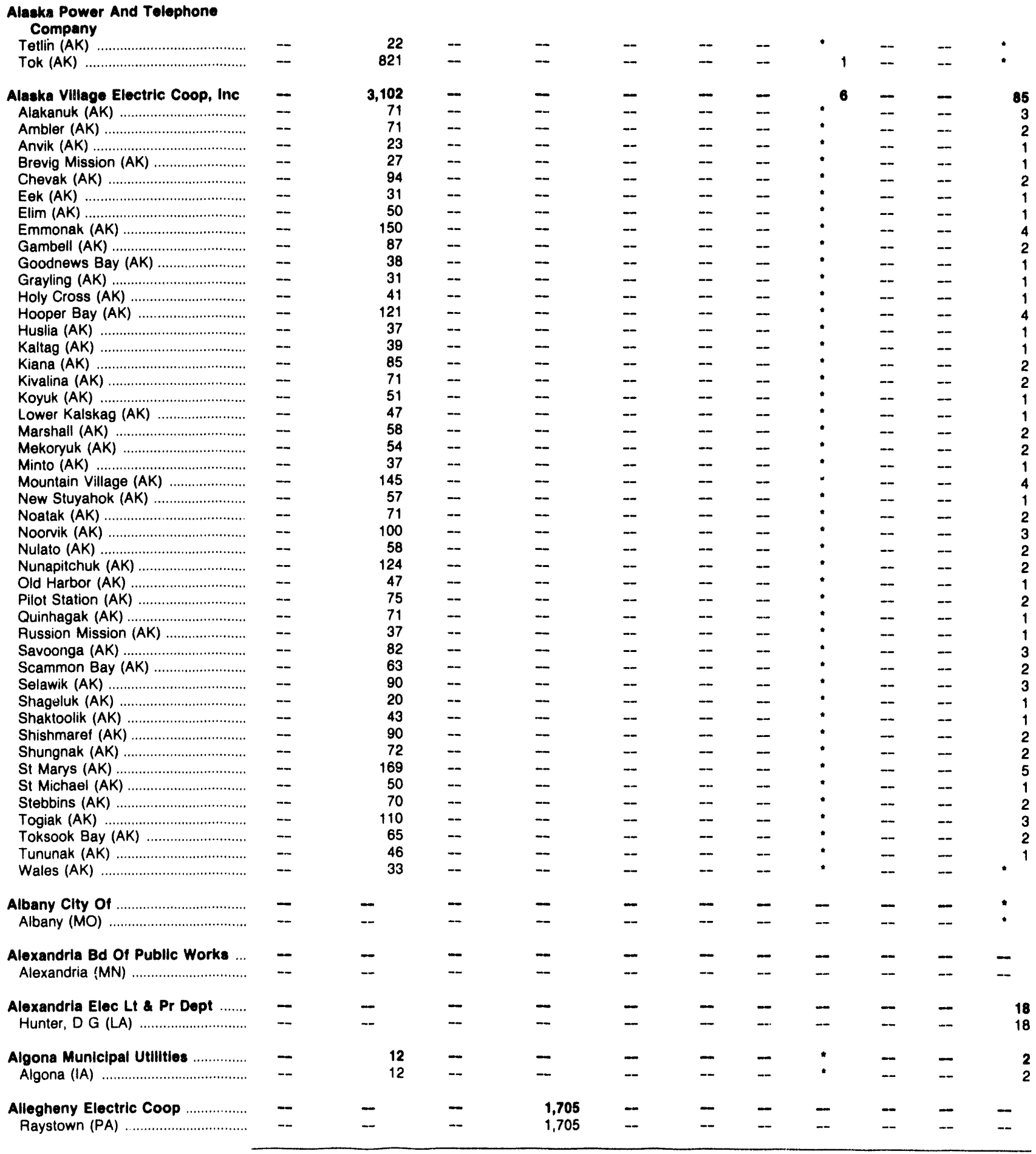

See footnotes at end of table. 
Table 62. U.S. Electric Utility Net Generation, Fuel Consumption, and Fuel Stocks by Company and Plant, September 1993 (Continued)

\begin{tabular}{|c|c|c|c|c|c|c|c|c|c|c|c|}
\hline \multirow{2}{*}{$\begin{array}{c}\text { Company (Holding Company) } \\
\text { Plant (State) }\end{array}$} & \multicolumn{6}{|c|}{$\begin{array}{c}\text { Ceneration } \\
\text { (thousand kllowatthours) }\end{array}$} & \multicolumn{3}{|c|}{$\begin{array}{l}\text { Consumption } \\
\text { (thousand) }\end{array}$} & \multicolumn{2}{|c|}{$\begin{array}{c}\text { Stocke } \\
\text { (thousand) }\end{array}$} \\
\hline & Coal & Petroleum & Gas & Hydro & Nuclear & Other' & $\begin{array}{c}\text { Coal } \\
\text { (ahort } \\
\text { tons) }\end{array}$ & $\begin{array}{l}\text { Petro- } \\
\text { leum } \\
\text { (bols) }\end{array}$ & $\begin{array}{c}\text { Gas } \\
\text { (Mcf) }\end{array}$ & $\begin{array}{c}\text { Coal } \\
\text { (short } \\
\text { tons) }\end{array}$ & $\begin{array}{l}\text { Potro- } \\
\text { loum } \\
\text { (bbls) }\end{array}$ \\
\hline Alta Mun Elec Li \& Pwr PIt Sys ... & - & -2 & - & - & - & - & - & - & - & -- & * \\
\hline Alta (IA) & -- & -2 & -- & - & - & - & -- & - & -- & -- & - \\
\hline \multicolumn{12}{|l|}{ American Municipal Power-Ohio } \\
\hline $\begin{array}{l}\text { Inc } \\
\text { Richard Gorsuch }(\mathrm{OH})\end{array}$ & $\begin{array}{l}87,750 \\
87,750\end{array}$ & - & $\begin{array}{l}569 \\
569\end{array}$ & - & - & - & $\begin{array}{l}60 \\
60\end{array}$ & - & $\begin{array}{l}9 \\
9\end{array}$ & $\begin{array}{l}108 \\
108\end{array}$ & - \\
\hline 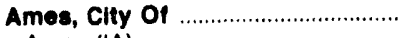 & 20,446 & 52 & - & - & - & - & 14 & * & - & 23 & 3 \\
\hline 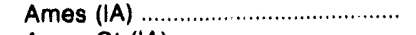 & 20,446 & 62 & -- & -- & -- & -- & 14 & $\bullet$ & -- & 23 & 1 \\
\hline 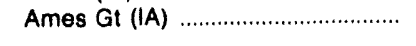 & -- & -10 & - & -- & -- & -- & - & • & -- & - & 2 \\
\hline Anchorage Mun Lt \& Pwr Dept .... & - & 2 & 34,366 & - & - & - & - & : & 478 & - & 36 \\
\hline 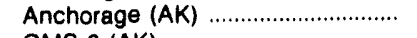 & -- & 2 & 570 & -- & -- & - & - & $\cdot$ & 11 & - & \\
\hline GMS 2 (AK) & -- & -- & 33,796 & - & - & -- & - & - & 467 & -- & 36 \\
\hline \multicolumn{12}{|l|}{ Aniak Light Power Company, } \\
\hline Aniak (AK) & - & 199 & - & - & - & -- & -- & " & -- & -- & 3 \\
\hline Anita Municipal Utilities .................. & - & - & - & - & - & - & - & - & - & - & - \\
\hline 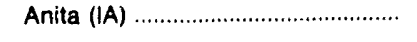 & -- & -- & - & - & - & - & -- & - & -- & - & - \\
\hline Ansley Municipal Light Plant ......... & - & - & - & - & - & - & - & -- & -- & - & - \\
\hline 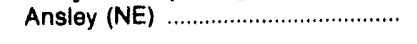 & - & -- & -- & - & -- & - & - & -- & - & -- & - \\
\hline 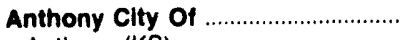 & - & 117 & 876 & - & - & -- & - & $\because$ & 10 & - & * \\
\hline 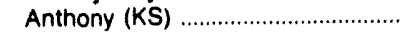 & - & 117 & 876 & - & - & - & -- & • & 10 & -- & - \\
\hline Appalachlan Power Co (AEP) ....... & $1,518,144$ & 2,845 & - & 19,719 & - & - & 599 & 5 & - & 2,301 & 50 \\
\hline 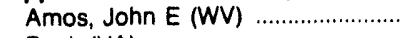 & 571,741 & 911 & -- & - & -- & -- & 236 & 2 & -- & 1,177 & 17 \\
\hline Buck (VA) & -- & - & -- & 2,107 & -- & -- & - & - & - & - & - \\
\hline Byllesby 2 (VA) & -- & -- & -- & 2,384 & - & - & -- & -- & -- & -- & -- \\
\hline 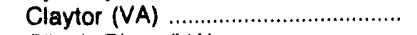 & - & + & -- & 9,154 & - & - & -- & $m$ & -- & - & - \\
\hline 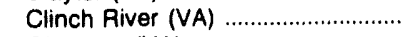 & 306,534 & 191 & -- & - & - & - & 112 & $\cdot$ & - & 318 & 1 \\
\hline 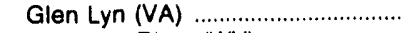 & 93,327 & 670 & -- & - & - & - & 37 & 1 & -- & 117 & 3 \\
\hline 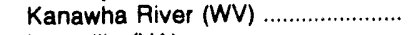 & 25,013 & 111 & -- & $-\bar{a}$ & -- & - & 11 & • & -- & 189 & -- \\
\hline Leesville (VA) ..................................... & - & - & -- & 2,470 & -- & -- & -- & -- & -- & -- & -- \\
\hline 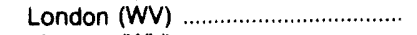 & -- & -- & -- & 2,573 & -- & - & -- & -- & -- & -- & - \\
\hline 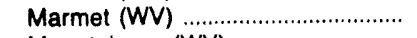 & - & - & -- & 2,074 & -- & -- & - & -- & - & - & -- \\
\hline 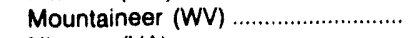 & 521,529 & 962 & - & - & -- & - & 204 & 2 & -- & 499 & 29 \\
\hline 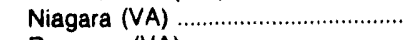 & -- & - & -- & 558 & -- & - & -- & - & - & - & -- \\
\hline 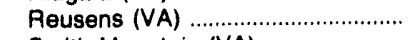 & -- & - & - & 1,233 & -- & -- & - & - & - & - & -- \\
\hline Smith Mountain (VA) ........................ & -- & $-\infty$ & - & $-7,019$ & - & - & -- & - & -- & $-\rightarrow$ & -- \\
\hline 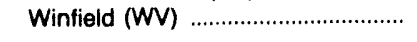 & - & -- & - & 4,185 & - & - & - & - & -- & -- & - \\
\hline Arcadia Mun Electric Utility ........... & - & 20 & 31 & - & - & - & - & * & " & - & 1 \\
\hline 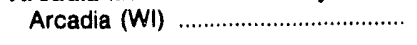 & - & 20 & 31 & - & - & - & -- & • & • & -- & 1 \\
\hline Arcanum Water \& Light Plant ...... & - & - & - & - & -- & - & - & - & -- & - & $:$ \\
\hline Arcanum (OH) & - & - & -- & - & - & -- & -- & - & - & - & * \\
\hline \multicolumn{12}{|l|}{ Argyle Municipal Elec \& Water } \\
\hline 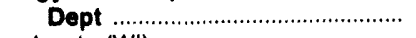 & - & - & - & - & - & - & - & - & - & - & - \\
\hline Argyle (WI) & - & - & - & -- & - & -- & -- & -- & - & -- & - \\
\hline Arizona Electric Pwr Coop, Inc .... & 215,682 & - & 1,863 & - & - & - & 112 & - & 20 & 158 & - \\
\hline Apache Station (AZ) .......................... & 215,682 & - & 1,863 & - & - & - & 112 & - & 20 & 158 & -- \\
\hline Arizona Public Service Company & $1,975,499$ & 391 & 128,298 & 2,631 & $1,631,661$ & - & 1,124 & 1 & 1,378 & 1,642 & 175 \\
\hline 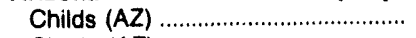 & - & - & - & 1,696 & - & - & - & - & - & - & -- \\
\hline 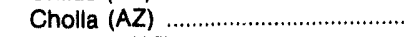 & 606,197 & 378 & 292 & - & -- & -- & 329 & 1 & 4 & 1,033 & 7 \\
\hline 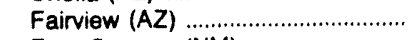 & -- & 13 & $-\bar{n}$ & - & - & -- & - & $\star$ & - & - & 8 \\
\hline 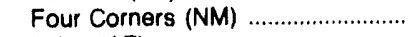 & $1,369,302$ & - & 2,790 & - & -- & -- & 795 & -- & 28 & 609 & - \\
\hline 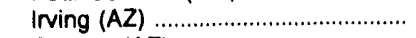 & - & -- & $\overline{0}$ & 935 & -- & - & - & - & - & -- & -- \\
\hline 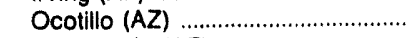 & -- & - & 37,770 & -- & -- & -- & - & -- & 432 & -- & 36 \\
\hline 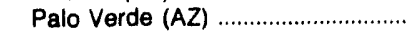 & -- & - & $\overline{-i}$ & -- & $1,631,661$ & -- & - & -- & - & -- & - \\
\hline 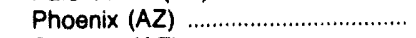 & - & - & 63,256 & - & - & - & -- & -- & 623 & -- & 26 \\
\hline Saguaro (AZ) & -- & -- & 6,242 & -- & - & - & -- & - & 78 & - & 41 \\
\hline
\end{tabular}

See footnotes at end of table. 
Table 62. U.S. Electric Utillty Net Generation, Fuel Consumption, and Fuel Stocks by Company and Plant, September 1993 (Continued)

\begin{tabular}{|c|c|c|c|c|c|c|c|c|c|c|c|}
\hline \multirow{2}{*}{$\begin{array}{c}\text { Company (Holding Company) } \\
\text { Plant (State) }\end{array}$} & \multicolumn{6}{|c|}{$\begin{array}{c}\text { Generation } \\
\text { (thousand kllowatthours) }\end{array}$} & \multicolumn{3}{|c|}{$\begin{array}{l}\text { Coneumption } \\
\text { (thousand) }\end{array}$} & \multicolumn{2}{|c|}{$\begin{array}{l}\text { Stooks } \\
\text { (thouseand) }\end{array}$} \\
\hline & Coal & Potroleum & Gas & Hydro & Nuclear & Other' & $\begin{array}{c}\text { Coal } \\
\text { (chort } \\
\text { tons) }\end{array}$ & $\begin{array}{l}\text { Potro- } \\
\text { leum } \\
\text { (bbla) }\end{array}$ & $\begin{array}{l}\text { Qas } \\
\text { (Mct) }\end{array}$ & $\begin{array}{l}\text { Cosl } \\
\text { (ehort } \\
\text { tons) }\end{array}$ & $\begin{array}{l}\text { Potro- } \\
\text { loum } \\
\text { (bble) }\end{array}$ \\
\hline \multicolumn{12}{|l|}{ Arizona Public Service Company } \\
\hline $\begin{array}{l}\text { Yucca (AZ) } \\
\text { Yuma Axis (AZ) }\end{array}$ & -- & -- & 17,800 & -- & -- & -- & -- & -- & 211 & -- & - \\
\hline Yuma Axis (AZ) & -- & -- & 148 & - & -- & -- & -- & -- & 3 & -- & 57 \\
\hline Arkansas Electric Coop Corp ....... & - & - & 7,603 & 19,838 & - & - & - & - & 82 & - & - \\
\hline Bailey (AR) & -- & -- & 7,603 & -- & -- & -- & -- & -- & 82 & -- & -- \\
\hline Clyde Ellis (AR) & - & -- & -- & 11,798 & -- & -- & - & -- & $\ldots$ & -- & -- \\
\hline Dam 9 (AR) & -- & -- & -- & 8,040 & - & -- & - & -- & -- & -- & -- \\
\hline 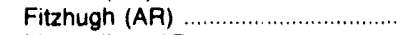 & -- & -- & -- & -- & -- & -- & -- & -- & -- & -- & -- \\
\hline Mc Clellan (AR) & -- & -- & - & -- & -- & -- & -- & - & -- & -- & -- \\
\hline \multirow{2}{*}{\multicolumn{12}{|c|}{$\begin{array}{l}\text { Arkansas Power L Light Co } \\
\text { (MSU) }\end{array}$}} \\
\hline & $1,365,443$ & 3,375 & 301,316 & 3,040 & 670,738 & - & 832 & 7 & 3,267 & 772 & 245 \\
\hline Arkansas Nuclear One(AR) ............ & -- & -- & -- & $-\infty$ & 670,738 & -- & - & -- & - & -- & -- \\
\hline Blytheville (AR) & -- & 987 & -- & - & -- & -- & - & 2 & - & -- & 18 \\
\hline 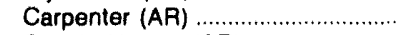 & -- & -- & -- & 1,888 & - & -- & -- & -- & - & -- & -- \\
\hline Couch, Harvey (AR) & -- & -- & 33,263 & - & - & - & - & -- & 361 & -- & 5 \\
\hline 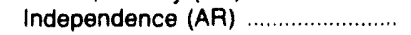 & 446,251 & 1,299 & -- & - & -- & -- & 267 & 2 & $\ldots$ & 337 & 28 \\
\hline 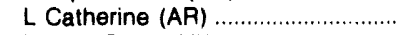 & -- & -- & 156,176 & - & -- & -- & -- & -- & 1,661 & -- & 39 \\
\hline 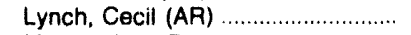 & -- & -- & -- & -- & - & -- & -- & -- & - & - & 2 \\
\hline Mablevale (AR) & -- & -- & 2 & -- & -- & -- & -- & - & - & -- & 9 \\
\hline 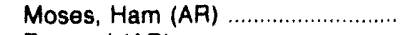 & - & -- & -- & - & -- & - & -- & -- & - & -- & 5 \\
\hline Remmel (AR) & -- & -- & - & 1,152 & -- & -- & - & -- & -- & -- & -_ \\
\hline Ritchie, RE (AR) & -- & - & 111,875 & -- & -- & -- & - & -- & 1,245 & -- & 103 \\
\hline 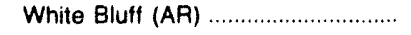 & 919,192 & 1,089 & -- & -- & -- & -- & 565 & 2 & - & 435 & 36 \\
\hline Arnold Municipal Plant ........................ & - & - & - & - & - & - & - & - & - & - & - \\
\hline 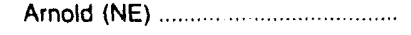 & -- & -- & -- & -- & -- & -- & - & -- & - & -- & • \\
\hline Ashland, City of & - & - & -- & - & - & - & - & - & - & - & - \\
\hline Ashland (KS) & -- & -- & -- & -- & -- & -- & -- & -- & - & - & * \\
\hline Associated Electric Coop, Inc ...... & 276,230 & 1,551 & - & - & - & - & 155 & 3 & - & 1,076 & 21 \\
\hline 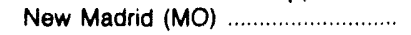 & 178,257 & 62 & -- & -- & -- & -- & 96 & - & -- & 296 & 1 \\
\hline Thomas Hill $(\mathrm{MO})$ & 97,973 & 1,475 & - & - & - & - & 59 & 3 & - & 781 & 5 \\
\hline 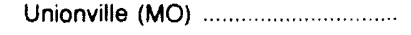 & -- & 14 & - & -- & - & -- & - & - & - & -- & 15 \\
\hline Atlantic Clty Electric Co (ACE) .... & 157,092 & 19,670 & 22,021 & - & - & - & 67 & 36 & 275 & 96 & 310 \\
\hline - Central Storage * & -- & -- & -- & - & -- & -- & - & -- & -- & -. & 68 \\
\hline Carlls Corner $(\mathrm{N} J)$ & -- & -- & 813 & -- & - & -- & -- & -- & 14 & -- & 8 \\
\hline Cedar $(N J)$ & -- & -166 & -- & -- & -- & -- & -- & • & - & -_ & 6 \\
\hline Cumberland St $(\mathrm{NJ})$ & -- & -- & 6,604 & $-\infty$ & -- & - & -- & -- & 84 & -- & 11 \\
\hline 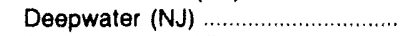 & 27.149 & 39 & 7,956 & - & -- & -- & 12 & - & 85 & 20 & 77 \\
\hline England, $\mathrm{B} L(\mathrm{NJ})$ & 129,943 & 19,607 & -- & - & -- & - & 56 & 34 & - & 76 & 98 \\
\hline Mickleton Street (NJ) ........................ & -- & -- & 1,477 & -- & -- & -- & -- & -- & 23 & -- & 1 \\
\hline 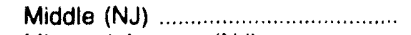 & -- & -275 & -- & -- & -- & -- & -- & $\cdot$ & - & - & 13 \\
\hline 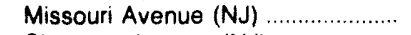 & -- & 465 & - & - & - & -- & -- & 1 & - & -- & 7 \\
\hline Sherman Avenue $(\mathrm{NJ})$ & -- & -- & 5,171 & -- & - & -- & -- & -- & 69 & -- & 23 \\
\hline Atlantic Mun Utilities & -- & -- & -- & - & - & - & -- & - & - & -- & - \\
\hline Atlantic (IA) & -- & -- & -- & -- & -- & -- & -- & -- & -- & -- & • \\
\hline Attica Clty Of & - & - & - & - & - & - & - & - & - & - & - \\
\hline Attica (KS) & -- & - & -- & -- & -- & -- & - & -- & - & -- & -- \\
\hline Auburn Clty Of & - & -58 & - & - & - & - & - & - & - & - & 1 \\
\hline Auburn (NE) & -- & -58 & -- & -- & - & -- & -- & - & - & - & 1 \\
\hline Augusta City of .. & -- & 42 & 1,164 & - & - & - & -- & • & 11 & - & • \\
\hline Plant No 1 (KS) & -- & -25 & -- & -- & -- & -- & -- & - & - & -. & . \\
\hline 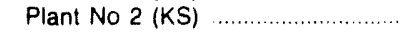 & -- & 67 & 1,164 & -- & -- & -- & -- & * & 11 & -- & • \\
\hline Augusta Light And Water Plant ... & -- & - & - & - & -- & - & - & -- & - & -- & -- \\
\hline Fairbanks (AR) & -- & -- & -- & -- & -- & -- & -- & -- & -- & -- & -- \\
\hline Austin Electric Dept & - & $\cdots$ & 344,233 & -- & - & 35 & - & -- & 3,623 & - & 252 \\
\hline Decker Creek $(T X)$ & -- & -- & 231,249 & -- & - & 35 & -- & -- & 2,375 & -- & 132 \\
\hline Holly Street $(T X)$ & -- & -- & 113,184 & - & -- & -- & -- & -- & 1,248 & -- & 116 \\
\hline Seaholm (TX) & - & -. & -200 & -- & -- & -- & -- & -- & -- & -- & 4 \\
\hline
\end{tabular}

See footnotes at end of table. 
Table 62. U.S. Electric Utility Net Generation, Fuel Consumption, and Fuel Stocks by Company and Plant, September 1993 (Continued)

\begin{tabular}{|c|c|c|c|c|c|c|c|c|c|c|c|}
\hline \multirow{2}{*}{$\begin{array}{c}\text { Company (Holding Company) } \\
\text { Plant (State) }\end{array}$} & \multicolumn{6}{|c|}{$\begin{array}{c}\text { Coneration } \\
\text { (thousand kHowatthours) }\end{array}$} & \multicolumn{3}{|c|}{$\begin{array}{l}\text { Consumption } \\
\text { (thousand) }\end{array}$} & \multicolumn{2}{|c|}{$\begin{array}{l}\text { Stocke } \\
\text { (thoucand) }\end{array}$} \\
\hline & Coal & Petroleum & Gas & Hydro & Nuclear & Other' & $\begin{array}{l}\text { Cont } \\
\text { (Chort } \\
\text { tone) }\end{array}$ & $\begin{array}{l}\text { Potro- } \\
\text { (Dum } \\
\text { (bole) }\end{array}$ & $\begin{array}{l}\text { Cas } \\
\text { (Mof) }\end{array}$ & $\begin{array}{l}\text { Cont } \\
\text { (chort } \\
\text { tons) }\end{array}$ & $\begin{array}{l}\text { Potro- } \\
\text { loum } \\
\text { (bblo) }\end{array}$ \\
\hline $\begin{array}{l}\text { Austin Utillties } \\
\text { Northeast Station (MN) }\end{array}$ & $\begin{array}{l}2,138 \\
2,138\end{array}$ & -- & $\begin{array}{l}499 \\
499\end{array}$ & - & - & - & $\begin{array}{l}1 \\
1\end{array}$ & - & $\begin{array}{l}3 \\
3\end{array}$ & $\begin{array}{l}20 \\
20\end{array}$ & - \\
\hline $\begin{array}{l}\text { Baldwin City of } \\
\text { Attica (KS) }\end{array}$ & - & $\overline{-}$ & 4 & - & $\overline{--}$ & - & - & $\stackrel{-}{\bullet}$ & $\begin{array}{l}1 \\
1\end{array}$ & - & $\stackrel{.}{\bullet}$ \\
\hline 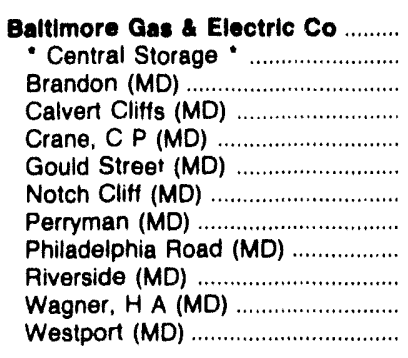 & $\begin{array}{l}994,058 \\
-\overline{-} \\
650,151 \\
-- \\
18 \overline{7.218} \\
-- \\
-- \\
-- \\
-- \\
\overline{156,689} \\
--\end{array}$ & $\begin{array}{r}68,810 \\
-- \\
4,234 \\
-- \\
200 \\
8,342 \\
-- \\
1,429 \\
205 \\
529 \\
53,865 \\
6\end{array}$ & $\begin{array}{l}17,936 \\
-- \\
-- \\
-- \\
-- \\
-\overline{1,943} \\
-- \\
-- \\
5,620 \\
9,216 \\
1,157\end{array}$ & $\begin{array}{l}- \\
-- \\
-- \\
-- \\
- \\
- \\
- \\
- \\
-\end{array}$ & $\begin{array}{l}1,210,197 \\
\overline{-} \\
1,210,197 \\
- \\
- \\
- \\
- \\
- \\
- \\
-\end{array}$ & $\begin{array}{l}- \\
-- \\
- \\
- \\
- \\
- \\
- \\
- \\
-\end{array}$ & $\begin{array}{l}390 \\
- \\
254 \\
-74 \\
- \\
- \\
- \\
- \\
-63\end{array}$ & $\begin{array}{c}120 \\
-\quad 7 \\
-\quad 17 \\
-\quad 4 \\
1 \\
2 \\
80\end{array}$ & $\begin{array}{l}228 \\
- \\
- \\
- \\
- \\
- \\
- \\
- \\
83 \\
93 \\
19\end{array}$ & $\begin{array}{l}793 \\
- \\
-474 \\
- \\
- \\
- \\
- \\
- \\
- \\
-\end{array}$ & $\begin{array}{r}485 \\
-\quad 2 \\
-\quad \\
-\quad 4 \\
26 \\
-\quad 47 \\
4 \\
81 \\
267 \\
20\end{array}$ \\
\hline 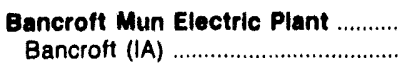 & - & - & - & - & - & - & - & - & - & - & - \\
\hline $\begin{array}{l}\text { Bangor Hydro Electric Co } \\
\text { Bar Harbor (ME) } \\
\text { Eastport (ME) } \\
\text { Ellsworth (ME) } \\
\text { Graham Station (ME) } \\
\text { Howland (ME) } \\
\text { Medway (ME) } \\
\text { Milford (ME) } \\
\text { Orono (ME) } \\
\text { Stiliwater (ME) } \\
\text { Veazie (ME) } \\
\text { Veazie A (ME) }\end{array}$ & $\begin{array}{l}- \\
- \\
- \\
- \\
- \\
- \\
- \\
- \\
- \\
-\end{array}$ & $\begin{array}{r}415 \\
\quad 39 \\
-\quad 12 \\
- \\
- \\
- \\
- \\
-- \\
-- \\
-\end{array}$ & $\begin{array}{l}- \\
-- \\
-- \\
-- \\
-- \\
-- \\
- \\
- \\
-\end{array}$ & $\begin{array}{r}12,843 \\
-- \\
-- \\
- \\
- \\
2509 \\
2,701 \\
3,517 \\
796 \\
940 \\
-4,140\end{array}$ & $\begin{array}{l}- \\
- \\
- \\
- \\
- \\
- \\
- \\
- \\
-\end{array}$ & $\begin{array}{l}- \\
- \\
- \\
- \\
- \\
- \\
- \\
- \\
-\end{array}$ & $\begin{array}{l}- \\
- \\
- \\
- \\
- \\
- \\
- \\
-\end{array}$ & $\begin{array}{l}1 \\
: \\
- \\
- \\
-1 \\
- \\
- \\
-\end{array}$ & $\begin{array}{l}- \\
\overline{-} \\
\overline{-} \\
\overline{-} \\
\overline{-} \\
-\end{array}$ & $\begin{array}{l}- \\
\overline{-} \\
\overline{-} \\
\overline{-} \\
- \\
- \\
-\end{array}$ & $\begin{array}{ll} & 1 \\
& 1 \\
- & 1 \\
- & \\
- & \\
- & \\
- & \\
- & \\
- & \end{array}$ \\
\hline 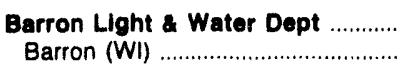 & - & - & - & $\begin{array}{l}27 \\
27\end{array}$ & - & - & - & - & - & - & 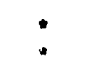 \\
\hline $\begin{array}{c}\text { Barrow Utils Elec Coop Inc } \\
\text { Barrow (AK) }\end{array}$ & - & $\overline{--}$ & $\begin{array}{l}2,936 \\
2,936\end{array}$ & - & - & - & - & : & $\begin{array}{l}54 \\
54\end{array}$ & - & . \\
\hline $\begin{array}{l}\text { Barton Village Electric Dept } \ldots \ldots \ldots . . . . \\
\text { W. Charleston (VT) } \ldots \ldots \ldots \ldots \ldots \ldots \ldots \ldots \ldots\end{array}$ & - & - & - & $\begin{array}{l}377 \\
377\end{array}$ & - & - & - & - & - & - & $\because$ \\
\hline $\begin{array}{l}\text { Basin Electric Pwr Coop, Inc ........ } \\
\text { Antelope Valley (ND) } \\
\text { Laramie River (WY) } \\
\text { Leland Olds (ND) } \\
\text { Sprit Mound (SD) }\end{array}$ & $\begin{array}{c}1,407,704 \\
564,362 \\
689,275 \\
154,067 \\
--\end{array}$ & $\begin{array}{r}1,244 \\
72 \\
1,080 \\
92 \\
-\end{array}$ & $\begin{array}{l}-- \\
-- \\
-- \\
-\end{array}$ & $\begin{array}{l}- \\
- \\
-- \\
--\end{array}$ & $\begin{array}{l}- \\
-- \\
-\end{array}$ & $\begin{array}{l}- \\
- \\
-\end{array}$ & $\begin{array}{r}1,037 \\
462 \\
444 \\
131 \\
-\end{array}$ & $\begin{array}{l}2 \\
. \\
+-\end{array}$ & $\begin{array}{l}- \\
- \\
-\end{array}$ & $\begin{array}{r}1,230 \\
172 \\
741 \\
317 \\
-\end{array}$ & $\begin{array}{r}39 \\
7 \\
20 \\
3 \\
9\end{array}$ \\
\hline $\begin{array}{l}\text { Baudette LIght Water Dept } \ldots \ldots . . . . \\
\text { Baudette (MN) }\end{array}$ & - & - & - & - & - & - & - & - & - & - & - \\
\hline 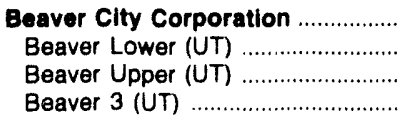 & - & $\begin{array}{l}- \\
-- \\
--\end{array}$ & $\begin{array}{l}- \\
-- \\
-- \\
--\end{array}$ & $\begin{array}{r}584 \\
64 \\
252 \\
268\end{array}$ & $\begin{array}{l}- \\
-- \\
-\end{array}$ & $\overline{-}$ & $\overline{-}$ & $\overline{-}$ & $\overline{-}$ & $\overline{-}$ & $\overline{-}$ \\
\hline $\begin{array}{l}\text { Beaver Clty Of } \\
\text { Beaver City (NE) }\end{array}$ & -- & - & - & - & - & - & - & - & - & - & 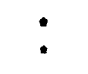 \\
\hline $\begin{array}{l}\text { Bedford Electric Dept } \\
\text { Snowden (VA) }\end{array}$ & - & - & - & $\begin{array}{l}480 \\
480\end{array}$ & - & - & - & - & - & - & $\overline{-}$ \\
\hline 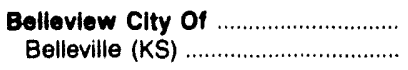 & - & $\begin{array}{l}40 \\
40\end{array}$ & $\begin{array}{l}341 \\
341\end{array}$ & - & - & - & - & • & 4 & - & \\
\hline 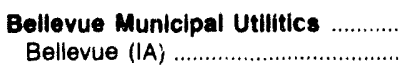 & - & $\begin{array}{l}6 \\
6\end{array}$ & - & - & - & - & - & 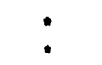 & $\overline{-}$ & - & : \\
\hline
\end{tabular}

See footnotes at end of table. 
Table 62. U.S. Electric Utility Net Generation, Fuel Consumption, and Fuel Stocks by Company and Plant, September 1993 (Continued)

\begin{tabular}{|c|c|c|c|c|c|c|c|c|c|c|c|}
\hline \multirow{2}{*}{$\begin{array}{c}\text { Company (Holding Company) } \\
\text { Plant (Stale) }\end{array}$} & \multicolumn{6}{|c|}{$\begin{array}{c}\text { Coneration } \\
\text { (thousand kllowatthours) }\end{array}$} & \multicolumn{3}{|c|}{$\begin{array}{l}\text { Coneumption } \\
\text { (thousand) }\end{array}$} & \multicolumn{2}{|c|}{$\begin{array}{l}\text { Stocks } \\
\text { (thousand) }\end{array}$} \\
\hline & Conl & Petroleum & Gas & Hydro & Nuctear & Othor' & $\begin{array}{c}\text { Cod } \\
\text { (ohort } \\
\text { tone) }\end{array}$ & $\begin{array}{l}\text { Potro- } \\
\text { loum } \\
\text { (bble) }\end{array}$ & (Mas) & $\begin{array}{c}\text { Coal } \\
\text { (ohort } \\
\text { tone) }\end{array}$ & $\begin{array}{l}\text { Potro- } \\
\text { loum } \\
\text { (bbls) }\end{array}$ \\
\hline
\end{tabular}

Belolt City of

Beloit (KS)

Benkelman City of

Benkeiman (NE)

Benson Water Llght Heat a Pwr Pit

Benson (MN)

Beriln Electric Light Plant Berlin (MD)

Bethany Clty Bethany $(\mathrm{MO})$

Bethel Utilities Commiseion ...........

Bethel (AK)

Bettles Lught \& Power Inc Bettles (AK)

Big Rivers Electric Corporation .. Coleman (KY)

Green $(K Y)$

Henderson li (KY)

Reid, Robert (KY)

Wilson (KY)

Black Hills Corp

French, Ben (SD)

Kirk (SD)

Osage (WY)

Simpson, Neil (WY)

Black River Falls, City of

Black River Falls (WI)

Block Island Power Co

Block island (RI)

Bloomfield Mun Utilities

Bloomfieid (IA)

Blooming Pralrie Pub Utils Comm Blooming Prairie (MN)

Blue Earth Lt \& Wtr Dept Blue Earth (MN)

Blue Ridge Electric Membership Corp Sharp Falls (NC)

Blutton UtIIIties Bluffton (IN)

Bonners Ferry City Of (Lt \& Wtr Dpt)

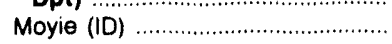

Boston Edison Company

Edgar (MA)

Framingham (MA)

$L$ Street (MA)

Mystic (MA)

New Boston (MA)

Pilgrim (MA)

West Medway (MA)

See footnotes at end of table.

\begin{tabular}{|c|c|c|c|c|c|c|c|c|c|c|}
\hline- & - & - & - & - & - & - & - & 5 & - & - \\
\hline- & -- & - & - & - & - & - & - & 5 & -- & • \\
\hline- & - & - & - & - & - & - & - & 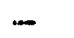 & - & - \\
\hline-- & - & -- & - & - & - & - & - & -- & -- & -- \\
\hline- & - & - & - & - & $-\infty$ & - & - & - & - & -- \\
\hline-- & -- & - & - & - & - & -- & -- & -- & - & -- \\
\hline- & 169 & - & - & - & - & - & - & - & - & 1 \\
\hline-- & 169 & -- & - & - & -- & - & - & -- & - & 1 \\
\hline- & - & - & - & - & - & - & - & - & - & 1 \\
\hline-- & -- & - & - & -- & -- & - & -- & -- & - & 1 \\
\hline- & 2,388 & - & - & - & - & - & 4 & - & - & 1 \\
\hline- & 2,388 & -- & -- & -- & - & - & 4 & -- & -- & 1 \\
\hline- & 65 & - & - & - & - & - & - & - & - & 1 \\
\hline- & 65 & - & - & - & - & - & • & - & -- & 1 \\
\hline 888,161 & 1,301 & 398 & - & - & - & 406 & 2 & 4 & 653 & 21 \\
\hline 204,495 & -- & 398 & -- & - & -- & 95 & -- & 4 & 148 & 2 \\
\hline 257,941 & 370 & - & - & - & -- & 129 & 1 & -- & 175 & 1 \\
\hline 173,648 & 138 & - & -- & - & -- & 72 & • & -- & - & 1 \\
\hline 31,610 & 40 & -- & - & - & - & 15 & - & -- & 85 & 6 \\
\hline 220,467 & 753 & -- & -- & - & - & 95 & 1 & -- & 245 & 11 \\
\hline 48,029 & 413 & 142 & - & - & - & 43 & 1 & 4 & 15 & 17 \\
\hline 4,295 & 345 & 142 & - & - & - & 3 & 1 & 4 & 3 & 17 \\
\hline 8,484 & - & -- & - & - & - & 8 & - & -- & 4 & - \\
\hline 21,507 & - & - & - & - & - & 21 & - & -- & 7 & -- \\
\hline 13,743 & 68 & - & -- & - & $m$ & 12 & • & -- & -- & * \\
\hline- & - & - & 240 & - & - & - & - & - & - & - \\
\hline-- & - & -- & 240 & - & - & - & -- & -- & -- & -- \\
\hline- & 935 & - & - & - & - & - & 1 & - & - & 1 \\
\hline- & 935 & -- & -- & -- & -- & - & 1 & -- & -- & 1 \\
\hline- & 7 & - & - & - & - & - & " & - & - & $\cdot$ \\
\hline- & 7 & - & - & - & -- & - & • & -- & - & • \\
\hline- & - & -- & - & - & - & - & • & - & - & $\bullet$ \\
\hline-- & - & -- & -- & - & -- & -- & • & -- & -- & • \\
\hline - & - & - & - & - & - & - & - & - & - & - \\
\hline- & -- & -- & - & - & - & - & - & - & - & - \\
\hline- & - & - & - & - & - & - & - & - & - & - \\
\hline$\cdots$ & - & -- & - & -- & - & - & -- & - & - & -- \\
\hline- & 1 & 44 & - & - & - & - & * & - & - & 1 \\
\hline- & 1 & 44 & - & - & - & -- & $\bullet$ & $\cdot$ & -- & 1 \\
\hline- & - & - & 2,337 & - & - & - & - & - & - & - \\
\hline- & - & - & 2,337 & - & -- & -- & -- & - & - & -- \\
\hline- & 262,431 & 214,269 & - & 409,080 & - & - & 437 & 2,087 & - & 402 \\
\hline-- & 185 & - & - & - & -- & -- & 1 & - & -- & 1 \\
\hline-- & 76 & - & -- & - & -- & - & $*$ & -- & -- & 2 \\
\hline- & 181 & -- & - & - & - & -- & • & -- & -- & 1 \\
\hline-- & 261,977 & 33,678 & -- & - & - & -- & 436 & 328 & - & 373 \\
\hline-- & -- & 180,187 & -- & -- & - & -- & - & 1.753 & - & 19 \\
\hline-- & -- & -- & - & 409,060 & - & - & -- & -- & -- & - \\
\hline- & 12 & 404 & - & - & -- & -- & • & 6 & -- & 7 \\
\hline
\end{tabular}


Table 62. U.S. Electric Utility Net Generation, Fuel Consumption, and Fuel Stocks by Company and Plant, September 1993 (Continued)

\begin{tabular}{|c|c|c|c|c|c|c|c|c|c|c|c|}
\hline \multirow{2}{*}{$\begin{array}{c}\text { Company (Holding Company) } \\
\text { Plant (State) }\end{array}$} & \multicolumn{6}{|c|}{$\begin{array}{c}\text { Generation } \\
\text { (thousand kllowatthours) }\end{array}$} & \multicolumn{3}{|c|}{$\begin{array}{l}\text { Consumption } \\
\text { (thousand) }\end{array}$} & \multicolumn{2}{|c|}{$\begin{array}{l}\text { Stocks } \\
\text { (thousand) }\end{array}$} \\
\hline & Coal & Petroloum & Cas & Hydro & Nuciear & Other' & $\begin{array}{c}\text { Coal } \\
\text { (short } \\
\text { tons) }\end{array}$ & $\begin{array}{l}\text { Petro- } \\
\text { loum } \\
\text { (bbla) }\end{array}$ & $\begin{array}{l}\text { Gas } \\
\text { (MC1) }\end{array}$ & $\begin{array}{c}\text { Coal } \\
\text { (short } \\
\text { tons) }\end{array}$ & $\begin{array}{l}\text { Potro- } \\
\text { leum } \\
\text { (bbla) }\end{array}$ \\
\hline 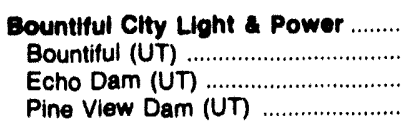 & $\begin{array}{l}\overline{-} \\
\overline{-}\end{array}$ & $\overline{-}$ & $\begin{array}{l}138 \\
138 \\
- \\
-\end{array}$ & $\begin{array}{r}2,707 \\
1,984 \\
723\end{array}$ & $\bar{z}$ & $\bar{z}$ & $\overline{-}$ & $\begin{array}{l}\ddot{*} \\
\cdots \\
-\end{array}$ & $\begin{array}{r}1 \\
- \\
--\end{array}$ & $\begin{array}{l}- \\
- \\
-\end{array}$ & $\begin{array}{r}1 \\
1 \\
-- \\
--\end{array}$ \\
\hline $\begin{array}{l}\text { Brady Water a Lught Worke } \\
\text { Brady }(T X) . . . . . .\end{array}$ & $\overline{-}$ & $\overline{-}$ & $\overline{-}$ & $\overline{-}$ & $\overline{-}$ & $\overline{-}$ & $\overline{-}$ & $\overline{-}$ & $\overline{-}$ & $\overline{-}$ & $\begin{array}{l}1 \\
1\end{array}$ \\
\hline 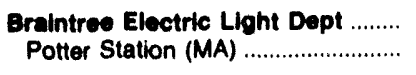 & $\overline{-}$ & $\begin{array}{l}8 \\
8\end{array}$ & $\begin{array}{l}5,790 \\
5,790\end{array}$ & $\overline{-}$ & $\overline{-}$ & $\overline{-}$ & $\overline{-}$ & : & $\begin{array}{l}60 \\
60\end{array}$ & $\overline{-}$ & $\begin{array}{l}18 \\
18\end{array}$ \\
\hline $\begin{array}{l}\text { Brazos Elec Pwr Coop, Inc } \\
\text { Miller, R W (TX) } \\
\text { North Texas (TX) }\end{array}$ & $\bar{z}$ & $\bar{z}$ & $\begin{array}{r}99,636 \\
90,009 \\
9,627\end{array}$ & $\bar{z}$ & $\overline{-}$ & $\bar{z}$ & $\bar{z}$ & $\overline{-}$ & $\begin{array}{r}1,112 \\
992 \\
120\end{array}$ & $\overline{-}$ & $\begin{array}{r}110 \\
100 \\
10\end{array}$ \\
\hline $\begin{array}{l}\text { Brazos River Authortty } \\
\text { M Shepppard (TX) }\end{array}$ & $\overline{-}$ & $\overline{-}$ & $\overline{-}$ & $\begin{array}{l}1,162 \\
1,162\end{array}$ & $\overline{-}$ & $\overline{-}$ & $\overline{-}$ & $\overline{-}$ & $\overline{-}$ & $\overline{-}$ & $\overline{-}$ \\
\hline $\begin{array}{l}\text { Breese CIty Or } \\
\text { Breese (IL) }\end{array}$ & $\overline{-}$ & $\begin{array}{l}18 \\
18\end{array}$ & $\overline{-}$ & $\overline{-}$ & $\overline{-}$ & $\overline{-}$ & $\ddot{-}$ & $:$ & $\overline{-}$ & $\overline{-}$ & $\begin{array}{l}1 \\
1\end{array}$ \\
\hline 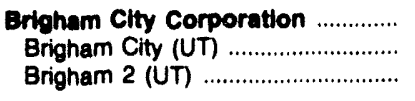 & $\overline{-}$ & $\bar{z}$ & $\overline{-}$ & $\begin{array}{l}720 \\
369 \\
351\end{array}$ & $\bar{z}$ & $\overline{-}$ & $\overline{-}$ & $\overline{-}$ & $\begin{array}{l}\overline{-} \\
--\end{array}$ & $\bar{z}$ & $\begin{array}{l}- \\
--\end{array}$ \\
\hline $\begin{array}{l}\text { Broken Bow Mun Utilities } \\
\text { Broken Bow (NE) }\end{array}$ & $\overline{-}$ & $\begin{array}{l}3 \\
3\end{array}$ & $\begin{array}{l}23 \\
23\end{array}$ & $\overline{-}$ & $\overline{-}$ & $\overline{-}$ & $\overline{-}$ & : & : & $\overline{-}$ & : \\
\hline $\begin{array}{l}\text { Brooklyn Mun Utilities } \\
\text { Brooklyn (IA) }\end{array}$ & $\overline{-}$ & $\begin{array}{l}2 \\
2\end{array}$ & $\overline{-}$ & $\overline{-}$ & $\overline{-}$ & $\overline{-}$ & $\overline{-}$ & $\dot{0}$ & $\overline{-}$ & $\overline{-}$ & : \\
\hline $\begin{array}{l}\text { Brownfleld Mun Pr \& Lt Plant ....... } \\
\text { Brownfield }(T X)\end{array}$ & $\overline{-}$ & $\overline{-}$ & $\begin{array}{l}-55 \\
-55\end{array}$ & $\overline{-}$ & $\overline{-}$ & $\overline{-}$ & $\overline{-}$ & $\overline{-}$ & $\overline{-}$ & $\overline{-}$ & : \\
\hline 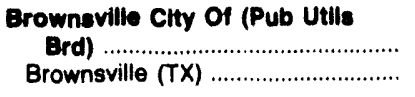 & $\overline{-}$ & $\overline{-}$ & $\begin{array}{l}18,664 \\
18,664\end{array}$ & $\overline{-}$ & - & $\overline{-}$ & $\overline{-}$ & $\overline{-}$ & $\begin{array}{l}271 \\
271\end{array}$ & $\overline{-}$ & $\begin{array}{l}28 \\
28\end{array}$ \\
\hline $\begin{array}{l}\text { Bryan City or } \\
\text { Bryan (TX) } \\
\text { Dansby (TX) }\end{array}$ & $\overline{-}$ & $\overline{-}$ & $\begin{array}{l}61,068 \\
16,563 \\
44,505\end{array}$ & $\bar{z}$ & $\overline{-}$ & $\overline{-}$ & $\overline{-}$ & $\bar{z}$ & $\begin{array}{l}675 \\
208 \\
467\end{array}$ & $\overline{-}$ & $\begin{array}{l}77 \\
40 \\
37\end{array}$ \\
\hline 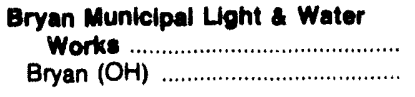 & $\overline{-}$ & $\begin{array}{l}9 \\
9\end{array}$ & $\begin{array}{l}64 \\
64\end{array}$ & $\overline{-}$ & $\overline{-}$ & $\overline{-}$ & $\overline{-}$ & : & $\begin{array}{l}1 \\
1\end{array}$ & $\overline{-}$ & $\begin{array}{l}8 \\
8\end{array}$ \\
\hline $\begin{array}{l}\text { Bryant Loht a Power Plant } \\
\text { Bryant (SD) }\end{array}$ & $\overline{-}$ & $\overline{-}$ & $\overline{-}$ & $\overline{-}$ & $\overline{-}$ & $\overline{-}$ & $\overline{-}$ & $\overline{-}$ & $=$ & $\overline{-}$ & $\overline{-}$ \\
\hline $\begin{array}{l}\text { Buhl Publle Utiltites Dept } \\
\text { Buhl (MN) }\end{array}$ & $\overline{-}$ & $\underline{-}$ & $\overline{-}$ & $\overline{-}$ & $\overline{-}$ & $\overline{-}$ & $\overline{-}$ & $\overline{-}$ & $\overline{-}$ & $\overline{-}$ & $\overline{-}$ \\
\hline 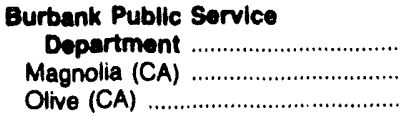 & $\overline{-}$ & $\overline{-}$ & $\begin{array}{r}15,832 \\
7,671 \\
8,161\end{array}$ & $\bar{z}$ & $\bar{z}$ & $\bar{z}$ & $\overline{-}$ & $\overline{-}$ & $\begin{array}{l}233 \\
118 \\
114\end{array}$ & $\begin{array}{l}- \\
\overline{-}\end{array}$ & $\begin{array}{r}41 \\
39 \\
2\end{array}$ \\
\hline $\begin{array}{l}\text { Burlingame City or } \\
\text { Burtingame (KS) }\end{array}$ & $\overline{-}$ & $\overline{-}$ & $\begin{array}{l}2 \\
2\end{array}$ & $\overline{-}$ & $\overline{-}$ & $\overline{-}$ & $\overline{-}$ & $\overline{-}$ & $:$ & $\overline{-}$ & $\overline{-}$ \\
\hline $\begin{array}{l}\text { Burlington City or } \\
\text { Burlington (KS) }\end{array}$ & $\overline{-}$ & $\begin{array}{l}39 \\
39\end{array}$ & $\begin{array}{l}279 \\
279\end{array}$ & $\overline{-}$ & $\overline{-}$ & $\overline{-}$ & $\overline{-}$ & 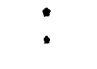 & $\begin{array}{l}3 \\
3\end{array}$ & $\overline{-}$ & $\begin{array}{l}1 \\
1\end{array}$ \\
\hline $\begin{array}{l}\text { Burlington Clty of } \\
\text { Burlington (CO) }\end{array}$ & $\overline{-}$ & $\overline{-}$ & $\overline{-}$ & $\overline{-}$ & $\overline{-}$ & $\overline{-}$ & $\overline{-}$ & $\overline{-}$ & $\overline{-}$ & $\overline{-}$ & $\overline{-}$ \\
\hline $\begin{array}{l}\text { Burlington Electrlc Loht Dept ...... } \\
\text { Burlington }(V T) \\
\text { J C McNeil (VT) }\end{array}$ & $\overline{-}$ & $\overline{-}$ & $\bar{z}$ & $\bar{z}$ & $\overline{-}$ & $\frac{4,019}{4,019}$ & $\bar{z}$ & $\ddot{\square}$ & $\overline{-}$ & $\overline{-}$ & $\begin{array}{l}3 \\
1 \\
2\end{array}$ \\
\hline
\end{tabular}

See footnotes at end of table. 
Table 62. U.S. Electric Utility Net Generation, Fuel Consumption, and Fuel Stocks by Company and Plant, September 1993 (Continued)

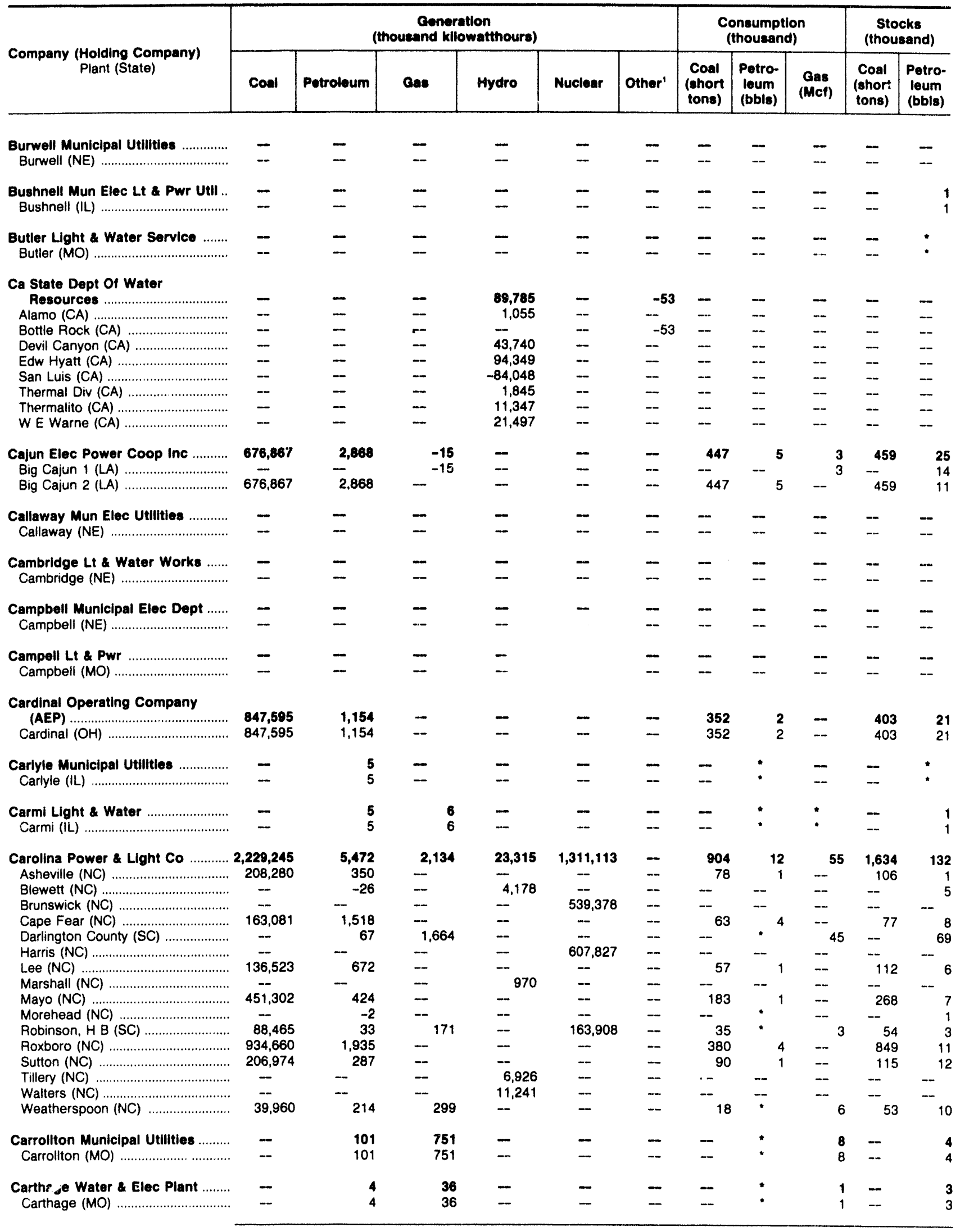

See footnotes at end of table 
Table 62. U.S. Electric Utility Net Generation, Fuel Consumption, and Fuel Stocks by Company and Plant, September 1993 (Continued)

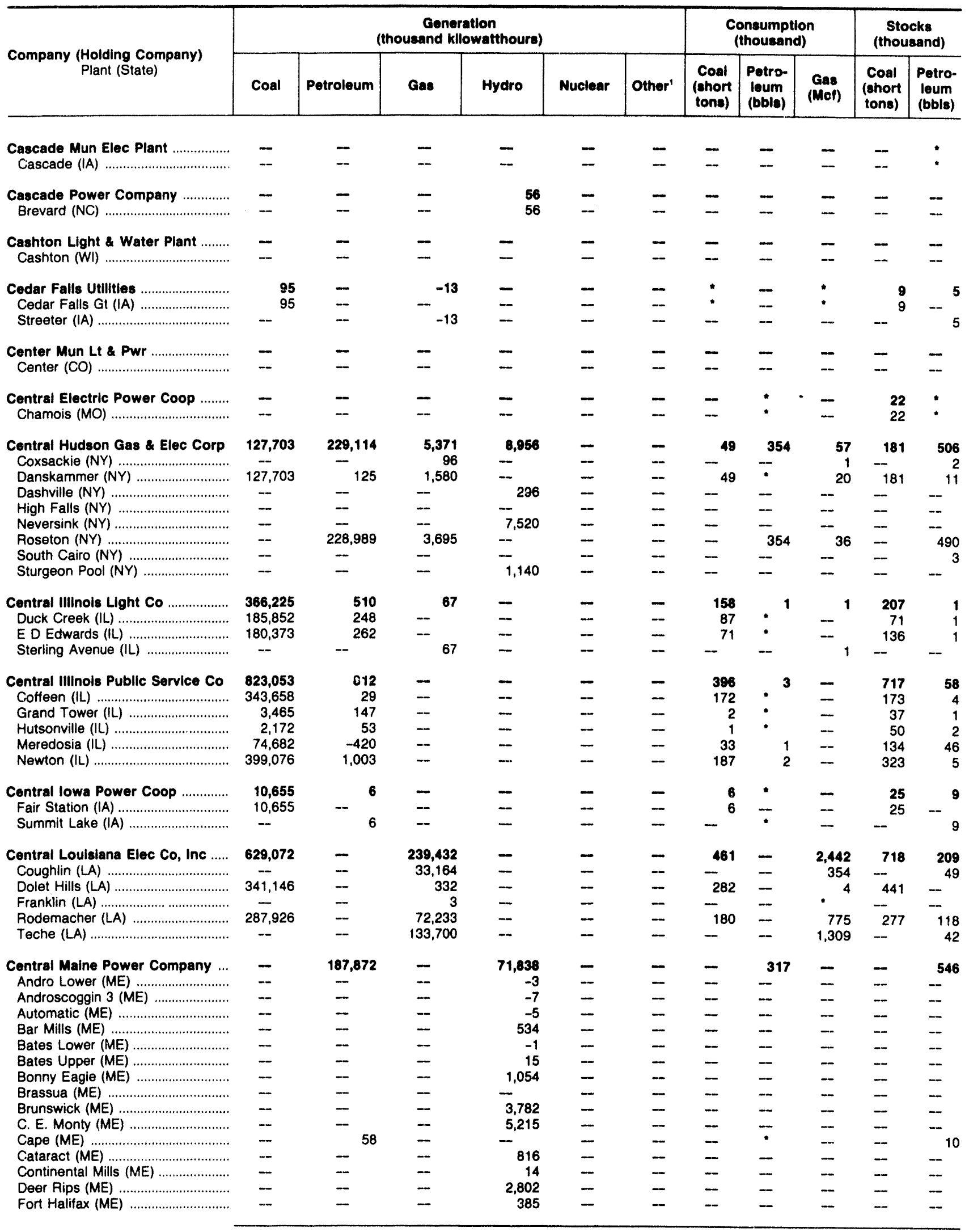

See footnotes at end of table. 
Table 62. U.S. Electric Utility Net Generation, Fuel Consumption, and Fuel Stocks by Company and Plant, September 1993 (Continued)

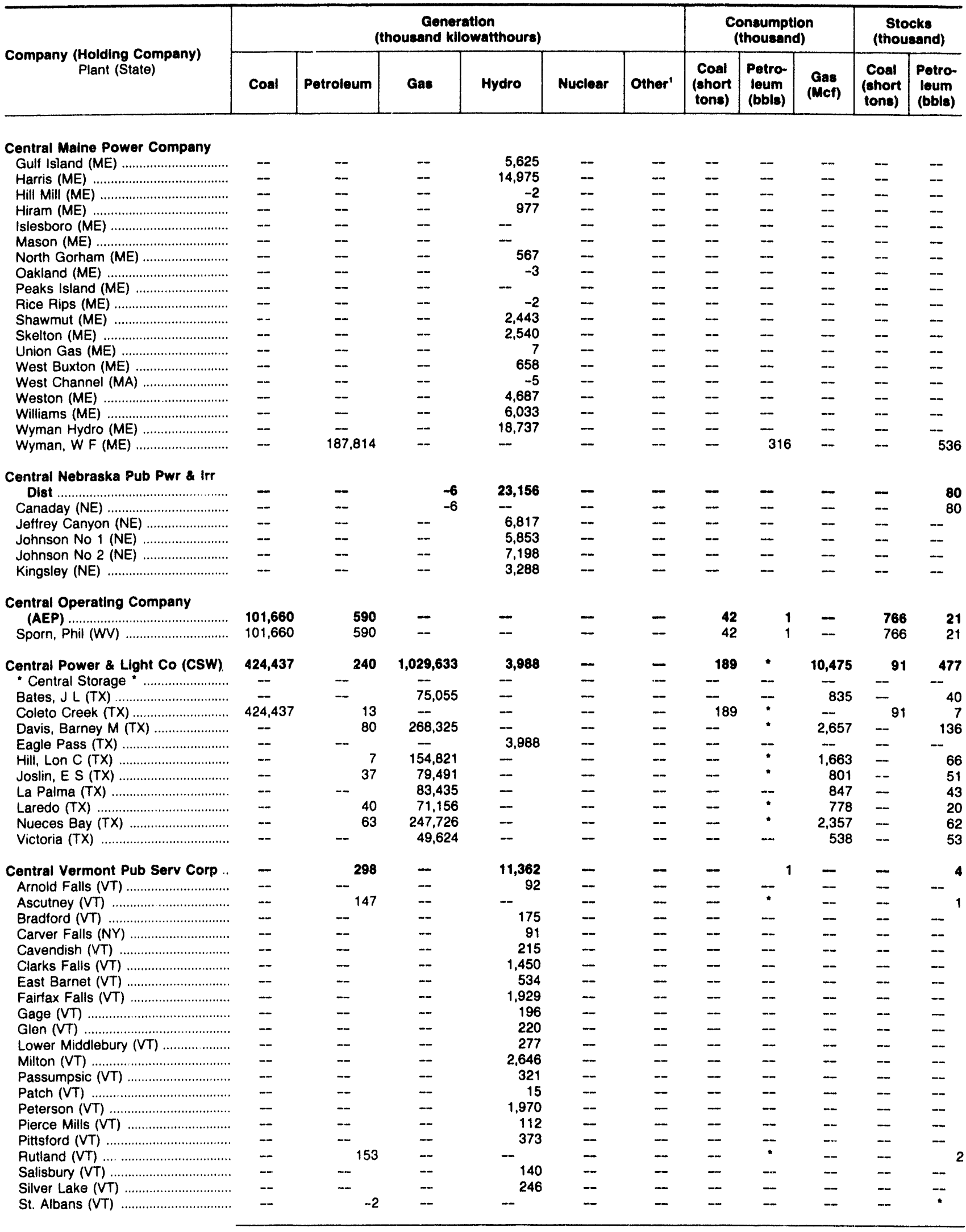

See footnotes at end of table. 
Table 62. U.S. Electric Utility Net Generation, Fuel Consumption, and Fuel Stocks by Company and Plant, September 1993 (Continued)

\begin{tabular}{|c|c|c|c|c|c|c|c|c|c|c|c|}
\hline \multirow{2}{*}{$\begin{array}{c}\text { Company (Holding Company) } \\
\text { Plant (State) }\end{array}$} & \multicolumn{6}{|c|}{$\begin{array}{c}\text { Generation } \\
\text { (thousend kllowatthours) }\end{array}$} & \multicolumn{3}{|c|}{$\begin{array}{l}\text { Consumption } \\
\text { (thousand) }\end{array}$} & \multicolumn{2}{|c|}{$\begin{array}{c}\text { Stocks } \\
\text { (thousand) }\end{array}$} \\
\hline & Coal & Petroleum & Gas & Hydro & Nuclear & Other' & $\begin{array}{l}\text { Coal } \\
\text { (short } \\
\text { tons) }\end{array}$ & $\begin{array}{c}\text { Petro- } \\
\text { leum } \\
\text { (bols) }\end{array}$ & $\begin{array}{c}\text { Gas } \\
\text { (Mcf) }\end{array}$ & $\begin{array}{c}\text { Coal } \\
\text { (short } \\
\text { tons) }\end{array}$ & $\begin{array}{l}\text { Petro- } \\
\text { leum } \\
\text { (bbls) }\end{array}$ \\
\hline
\end{tabular}

\begin{tabular}{|c|c|c|c|c|c|c|c|c|c|c|c|}
\hline $\begin{array}{l}\text { Central Vermont Pub serv Corp } \\
\text { Taftsville (VT) }\end{array}$ & -- & - & - & 3 & - & - & -- & -- & -- & -- & - \\
\hline 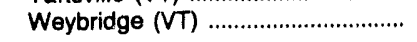 &.- & - & -- & 357 & $m$ & - & - & - & $-\cdots$ & -- & - \\
\hline Centralla Mun Hydro-Elec Plant ... & - & - & - & 4,084 & - & - & - & - & -- & -- & -- \\
\hline 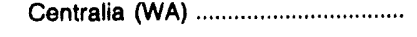 & -- & -- & - & 4,084 & -- & -- & - & - & -- & -- & - \\
\hline Champion International ................... & - & - & - & 807 & - & 5,669 & - & - & - & - & -- \\
\hline 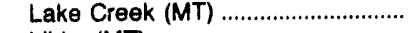 & - & - & - & 807 & -- & $\overline{-}-$ & - & -- & - & -- & -- \\
\hline 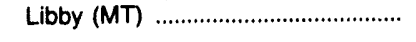 & - & - & - & - & -- & 5,669 & -- & $-\infty$ & - & -- & - \\
\hline Chanute City of ................................. & - & 21 & 268 & -- & - & - & - & • & 4 & -- & 1 \\
\hline 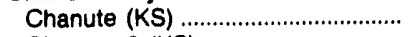 & -- & - & $-\infty$ & -- & - & -- & -- & -- & -- & -- & -- \\
\hline 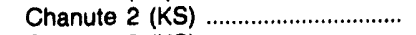 & - & -- & -28 & - & -- & -- & -- & - & - & -- & • \\
\hline 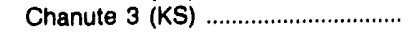 & -- & 21 & 296 & - & -- & -- & -- & • & 4 & -- & 1 \\
\hline 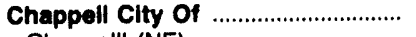 & -- & - & - & - & - & - & - & - & - & - & -- \\
\hline 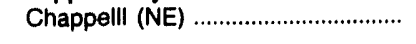 & -- & -- & - & - & - & - & -- & - & - & - & - \\
\hline Chelan County P U D No 1 ............ & - & - & - & 507,623 & - & - & - & - & - & - & -- \\
\hline 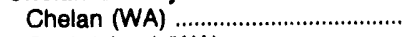 & - & - & -- & 14,586 & - & - & - & - & -- & -- & -- \\
\hline Rock Island (WA) & -- & - & - & 149,198 & -- & - & -- & - & -- & - & -- \\
\hline 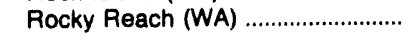 & - & - & - & 343,839 & - & - & - & -- & -- & - & -- \\
\hline Cheyenne Lt, Fuel \& Power Co ... & - & - & - & - & - & - & - & - & - & - & - \\
\hline Snyder (WY) & - & - & - & - & - & - & - & - & -- & - & - \\
\hline Chillicothe Municipal Utilities ........ & 2,105 & 7 & 5 & - & - & - & 2 & " & $"$ & 1 & 7 \\
\hline Beardmoie (MO) & 2,105 & 7 & 5 & - & -- & -- & 2 & * & * & 1 & 7 \\
\hline Chugach Electric Asen, Inc ............ & - & - & 129,201 & 42,936 & - & - & - & - & 1,485 & - & 10 \\
\hline 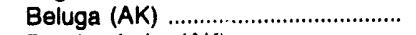 & - & - & 127,691 & -- & - & - & -- & -- & 1,457 & -- & -- \\
\hline Bernice Lake (AK) .............................. & - & -- & 838 & - & -- & -- & - & -- & 16 & - & 3 \\
\hline Bradley Lake (AK) ............................. & - & - & - & 42,901 & - & -- & -- & -- & -- & -- & $\cdots$ \\
\hline 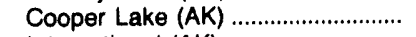 & -- & -- & - & 35 & - & -- & - & -- & -- & -- & -- \\
\hline International (AK) ............................. & - & - & - & - & - & - & -- & -- & - & -- & 7 \\
\hline 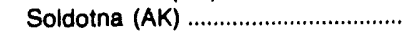 & -- & -- & 672 & - & - & -- & - & - & 12 & -- & -- \\
\hline $\begin{array}{l}\text { Cincinnatl Gas \& Electric Co } \ldots \ldots \ldots \\
\text { Beckiord, Walter } \mathrm{C}(\mathrm{OH})\end{array}$ & $\begin{array}{r}1,894,684 \\
325,700\end{array}$ & $\begin{array}{l}6,252 \\
1,083\end{array}$ & $-{ }^{462}$ & - & - & - & $\begin{array}{l}803 \\
147\end{array}$ & $\begin{array}{r}18 \\
2\end{array}$ & $-{ }^{19}$ & $\begin{array}{c}1,394^{\circ} \\
276\end{array}$ & $\begin{array}{r}185 \\
31\end{array}$ \\
\hline 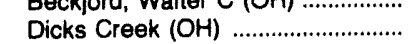 & - & 17 & -87 & - & - & -- & -- & $\cdot$ & 1 & - & 6 \\
\hline 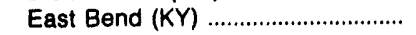 & 215,202 & 331 & $-m$ & - & - & -- & 99 & 1 & - & 207 & 5 \\
\hline Miami Fort $(\mathrm{OH})$ & 490,135 & 2,690 & - & - & - & - & 213 & 5 & -- & 381 & 24 \\
\hline 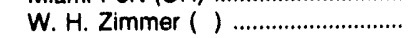 & 863,647 & 646 & - & - & - & - & 345 & 1 & -- & 530 & 25 \\
\hline Woodsdale $(\mathrm{OH})$ & - & 1,485 & 549 & - & - & -- & -- & 9 & 19 & - & 93 \\
\hline Citizens Utilities Co ......................... & - & - & - & 1,508 & - & - & - & - & - & -- & $*$ \\
\hline 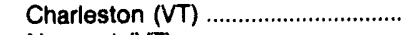 & - & - & - & 346 & -- & - & -- & -- & -- & -- & -- \\
\hline 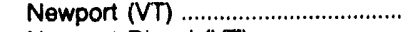 & - & - & -- & 1,100 & - & -- & - & - & -- & - & -- \\
\hline Newport Diesel (VT) ......................... & - & - & - & - & - & -- & - & - & -- & -- & 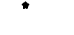 \\
\hline 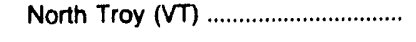 & - & - & - & 63 & - & - & - & -- & - & -- & -- \\
\hline Cltizens Utilities Company ............. & - & -23 & - & - & - & - & - & - & - & - & 1 \\
\hline 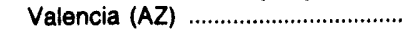 & -- & -23 & -- & - & - & - & -- & - & -- & - & 1 \\
\hline Clarkedale Water Light Dept .... & - & - & 6,289 & - & - & - & - & - & 72 & - & 13 \\
\hline South (MS) & -- & - & 6,289 & - & -- & - & - & - & 72 & - & 13 \\
\hline 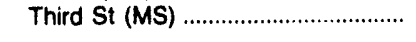 & -- & - & - & - & -- & - & - & - & -- & -- & - \\
\hline \multicolumn{12}{|l|}{ Clay Genter Mun Lt \& Water } \\
\hline 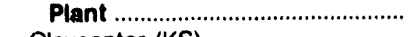 & - & 7 & 813 & - & - & - & - & $\star$ & 14 & - & 3 \\
\hline 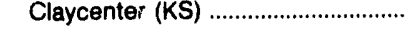 & - & 7 & 813 & - & -- & - & - & " & 14 & - & 3 \\
\hline Cleveland Elec Illum Co, The ....... & $1,043,130$ & 2,463 & - & - & 837,663 & - & 416 & 6 & - & 417 & 75 \\
\hline Ashtabula $(\mathrm{OH})$ & 117,283 & 539 & -- & - & -- & - & 52 & 1 & -- & 121 & 1 \\
\hline Avon Lake $(\mathrm{OH})$ & 338,584 & 102 & -- & - & -- & - & 132 & • & -- & 110 & 13 \\
\hline Eastlake $(\mathrm{OH})$ & 532,892 & 1,211 & - & - & - & - & 209 & 3 & -- & 165 & 11 \\
\hline 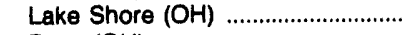 & 54,371 & 611 & - & - & $\overline{-}$ & - & 23 & 1 & -- & 22 & 50 \\
\hline Perry $(\mathrm{OH})$ & - & - & -- & - & 837,663 & - & -- & -- & -- & -- & - \\
\hline
\end{tabular}

See footnotes at end of table. 
Table 62. U.S. Electric Utillty Net Generation, Fuel Consumption, and Fuel Stocks by Company and Plant, September 1993 (Continued)

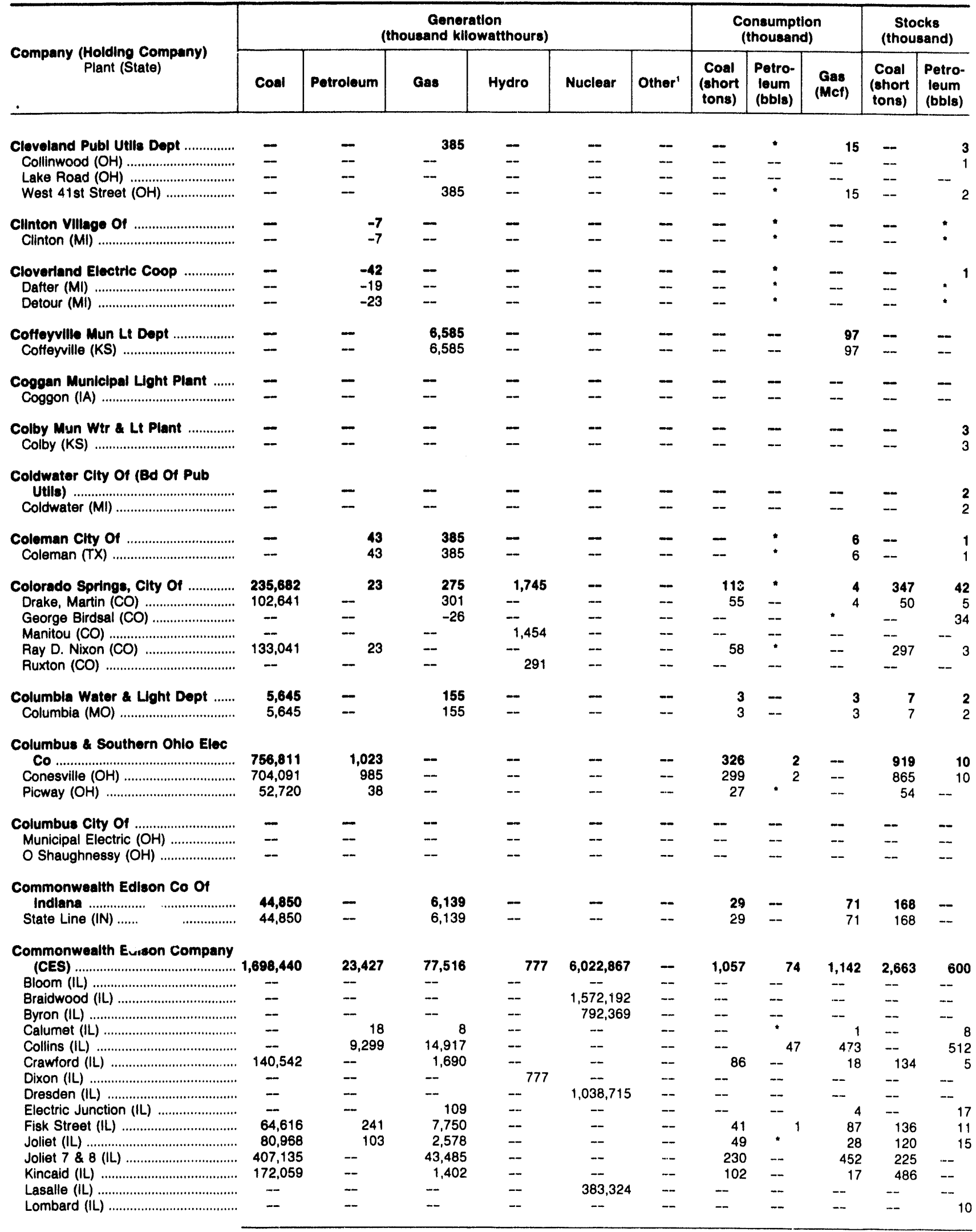

See footnotes at end of table. 
Table 62. U.S. Electric Utility Net Generation, Fuel Consumption, and Fuel Stocks by Company and Plant, September 1993 (Continued)

\begin{tabular}{|c|c|c|c|c|c|c|c|c|c|c|c|}
\hline \multirow{2}{*}{$\begin{array}{c}\text { Company (Holding Company) } \\
\text { Plant (State) }\end{array}$} & \multicolumn{6}{|c|}{$\begin{array}{c}\text { Generation } \\
\text { (thousand kllowatthours) }\end{array}$} & \multicolumn{3}{|c|}{$\begin{array}{l}\text { Consumption } \\
\text { (thousand) }\end{array}$} & \multicolumn{2}{|c|}{$\begin{array}{c}\text { Stocks } \\
\text { (thousand) }\end{array}$} \\
\hline & Coal & Petroleum & Gas & Hydro & Nuclear & Other' & $\begin{array}{c}\text { Coal } \\
\text { (short } \\
\text { tons) }\end{array}$ & $\begin{array}{l}\text { Petro- } \\
\text { leum } \\
\text { (bbls) }\end{array}$ & $\begin{array}{l}\text { Gas } \\
\text { (Mcf) }\end{array}$ & $\begin{array}{l}\text { Coal } \\
\text { (short } \\
\text { tons) }\end{array}$ & $\begin{array}{l}\text { Petro- } \\
\text { leum } \\
\text { (bb/s) }\end{array}$ \\
\hline
\end{tabular}

\section{Commonwealth Edison Company

$$
\text { (CES) }
$$

Powerton (IL)

Quad-cities (IL)

Sabrooke (IL)

Waukegan (IL)

Will County (IL) ........................... 319,042

Zion (IL)

Commonwealth Energy System (CES)

Airport Diesel (MA)

Blackstone Street (MA)

Canal (MA)

Cannon Street (MA)

Kendall Square (MA)

Oak Bluffs (MA)

West Tisbury (MA)

Connecticut Light \& Power Co (NU)

Bantam (CT)

Branford (CT)

Bulls Bridge (CT)

Cos Cob (CT)

Devon (CT)

Falls Village (CT)

Franklin (CT)

Middletown (CT)

Montville (CT)

Norwalk Harbor (CT)

Robertsville (CT)

Rocky River (CT)

Scotland (CT)

Shepaug (CT)

South Meadow (CT)

Stevenson (CT)

Taftville (CT)

Torrington (CT)

Tunnel (CT)

Connecticut Yankee Atomlc Pwr Co Haddam Neck (CT)

Consolidated Edison Co Of N $Y$,

Inc

- Central Storage

Arthur Kill (NY)

Astoria (NY) ....

Buchanan (NY)

East River (NY)

Gowanus (NY) .

Hudson Avenue (NY)

Indian Point (NY)

Narrows (NY)

Ravenswood (NY)

Waterside (NY)

59Th Street (NY)

74Th Street (NY)

Consolldated Water Power

\section{Company}

Biron (WI) ......

Du Bay (WI)

Stevens Point (WI)

Wisconsin Rapids (WI)

Wisconsin River Di (WI)

See tootnotes at end of table. $\begin{array}{lllllllllrr}299,236 & -- & -1,898 & -- & - & -- & 224 & -- & 25 & 1,103 & -- \\ -- & -- & -- & -- & 823,574 & -- & -- & -- & -- & -- & -- \\ -- & 182 & - & -- & - & -- & - & 1 & -- & - \\ 214,842 & 2,330 & 3,679 & -- & - & -- & 126 & 5 & 38 & 247 & 11 \\ 319,042 & 11,254 & -- & -- & -- & -- & 200 & 21 & -- & 212 & 2\end{array}$

430,822

422,60

7, 958

7,958

13

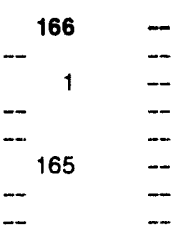

$\begin{array}{lll}-- & - & - \\ -- & - & - \\ -- & - & - \\ -- & - & - \\ -- & - & - \\ -- & - & - \\ -- & - & - \\ -- & - & -\end{array}$

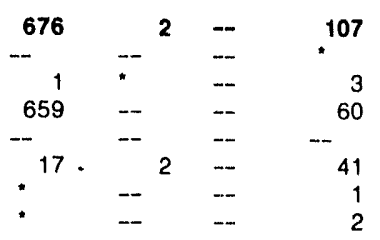

211,791

1,660

5,042

$\begin{array}{rrr}- \\ 149 & -- & \\ - & -- & 723\end{array}$

84

30,390

-

68,874

28,726

82,989

--

--

$-$

596

--

$-3$

$-$

$-$

$-$

1,660

$--$

$-$

$\cdots$

$-$

$-$

$-$

$-$

--

523

$-$

$--$

43

-36
10

1,900

1,564

198

109

--
--
--
--
--
--
--
--
--
--
--
--
--
--
--
--
--
--

35,051
-
--
--
--
-
-
--
--
--
--
--
--
--
-
35,051
--
--
--
-

$412 \quad 22 \quad-\quad 932$

$\begin{array}{llll}412 & 22 & - & 932 \\ - & - & -- & -\end{array}$

96 -- -

-

$\begin{array}{rrrr}60 & 22 & -- & 433 \\ 133 & - & 160\end{array}$

-.. $\quad$-.. $\quad--$

$\begin{array}{llll}- & -. & - & -\end{array}$

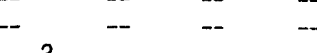

$\begin{array}{lllll}- & - & - & - & \\ - & -- & -- & -\end{array}$

$-$

1

$-\infty$

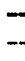

401,563

401,563

-
-
-
-
-
-
-
-
-
-
-
-
-

$\begin{array}{lll}664,584 & - & -- \\ -- & -- & -- \\ -- & -- & -- \\ -- & -- & -- \\ -- & -- & -- \\ -- & -- & -- \\ -- & -- & -- \\ -- & -- & -- \\ 664,584 & -- & - \\ -- & -- & -- \\ -- & -- & - \\ -- & -- & -- \\ -- & -- & -- \\ -- & - & -\end{array}$

$\begin{array}{rccr}348 & 7,640 & - & 2,809 \\ - & -- & -- & 1,800 \\ * & 747 & -- & 20 \\ 64 & 3,431 & -- & 240 \\ & - & -- & 4 \\ 100 & 406 & - & 294 \\ 18 & -- & -- & 59 \\ 20 & -- & -- & 199 \\ & -- & -- & 2 \\ 1 & 180 & -- & 71 \\ 123 & 2,398 & -- & 67 \\ & 479 & -- & 1 \\ 9 & -- & -- & 18 \\ 13 & -- & -- & 34\end{array}$

$\overline{-}$

\begin{tabular}{|c|c|c|c|c|c|c|c|}
\hline 11,161 & - & - & - & - & - & - & - \\
\hline 1,725 & -- & - & -- & - & - & -- & -- \\
\hline 3,643 & -- & -- & - & -- & -- & -- & -- \\
\hline 2,633 & - & -- & - & -- & -- & - & -- \\
\hline 2,820 & -- & -- & -- & -- & - & - & - \\
\hline 340 & -- & -- & -- & - & & 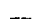 & \\
\hline
\end{tabular}


Table 62. U.S. Electric Utillty Net Generation, Fuel Consumption, and Fuel Stocks by Company and Plant, September 1993 (Continued)

\begin{tabular}{|c|c|c|c|c|c|c|c|c|c|c|c|}
\hline \multirow{2}{*}{$\begin{array}{c}\text { Company (Holding Company) } \\
\text { Plant (State) }\end{array}$} & \multicolumn{6}{|c|}{$\begin{array}{c}\text { Generation } \\
\text { (thousand kllowatthours) }\end{array}$} & \multicolumn{3}{|c|}{$\begin{array}{l}\text { Consumption } \\
\text { (thousand) }\end{array}$} & \multicolumn{2}{|c|}{$\begin{array}{l}\text { Stocks } \\
\text { (thousand) }\end{array}$} \\
\hline & Coal & Potroloum & Gas & Hydro & Nuclear & Other' & $\begin{array}{c}\text { Coal } \\
\text { (short } \\
\text { tons) }\end{array}$ & $\begin{array}{l}\text { Petro- } \\
\text { leum } \\
\text { (bbla) }\end{array}$ & $\begin{array}{c}\text { Gas } \\
\text { (Mcf) }\end{array}$ & $\begin{array}{c}\text { Coal } \\
\text { (short } \\
\text { tons) }\end{array}$ & $\begin{array}{l}\text { Petro- } \\
\text { leum } \\
\text { (bbls) }\end{array}$ \\
\hline Consumers Power Company ........ & $1,350,905$ & 7,357 & 2,359 & $-25,174$ & 26,931 & - & 553 & 21 & 44 & 688 & 130 \\
\hline 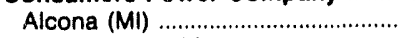 & - & - & -- & 2,812 & - & -- & - & - & - & - & $-\infty$ \\
\hline Allegan Dam (MI) ................................ & - & -- & -- & 1,296 & - & -- & -- & -- & - & -- & - \\
\hline Big Rock Point (MI) ......................... & - & - & - & - & 32,126 & -- & -- & -- & -- & - & -- \\
\hline 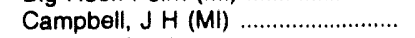 & 682,862 & 1,439 & - & -- & - & - & 262 & 2 & -- & 268 & 5 \\
\hline Cobb, B C (MI) & 164,753 & 234 & 784 & -- & -- & -- & 79 & $*$ & 8 & 154 & $\ldots$ \\
\hline Cooke (MI) & - & - & -- & 2,595 & - & -- & -- & -- & $\ldots$ & - & - \\
\hline Croton (MI) & - & -- & -- & 3,513 & -- & - & -- & -- & - & - & $\ldots$ \\
\hline 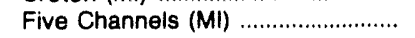 & - & - & -- & 2,389 & -- & -- & - & -- & -- & -- & -- \\
\hline 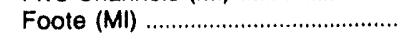 & - & - & -- & 2,975 & -- & -- & -- & -- & -- & - & - \\
\hline 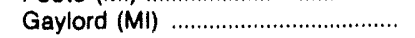 & - & - & 204 & - & -- & -- & -- & - & 2 & - & $\ldots$ \\
\hline Hardy (MI) & -- & -- & -- & 7,872 & -- & -- & -- & -- & - & -- & -- \\
\hline Karn, DE (MI) & 145,764 & 5,379 & 1,081 & -- & -- & -- & 62 & 18 & 25 & 152 & 123 \\
\hline 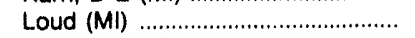 & - & - & -- & 1,836 & - & - & -- & -- & - & $-\infty$ & $\ldots$ \\
\hline 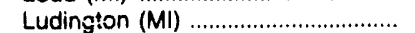 & - & - & - & $-64,039$ & - & -- & -- & $-\cdots$ & -- & -- & -- \\
\hline Mio (MI) & - & - & -- & 1,555 & - & -- & - & -- & - & -- & -- \\
\hline Morrow, B E (Mi) & -- & - & 48 & - & -- & -- & -- & - & 1 & $\ldots$ & $\ldots$ \\
\hline Palisades (MI) & - & - & - & - & $-5,195$ & -- & - & -- & - & -- & - \\
\hline 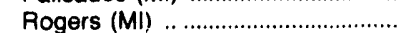 & -- & - & - & 2,679 & -- & -- & $\sim$ & -- & - & $\ldots$ & - \\
\hline 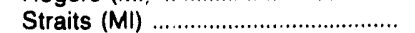 & -- & -- & -- & - & - & $-m$ & -- & - & -- & $\ldots$ & $\ldots$ \\
\hline 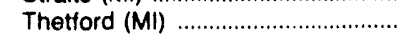 & -- & -- & 217 & -- & -- & -- & -- & - & 7 & -- & -- \\
\hline 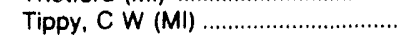 & - & - & -- & 4,815 & - & -- & - & -- & - & -- & -- \\
\hline 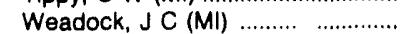 & 175,497 & 84 & 25 & $-\infty$ & -- & -- & 76 & $\cdot$ & 1 & 65 & - \\
\hline Webber $(\mathrm{MI})$ & - & - & - & 1,134 & -- & - & - & - & -- & -- & -- \\
\hline Whiting, J R (MI) ….......................... & 182,029 & 221 & -- & -- & -- & -- & 74 & 1 & -- & 49 & 2 \\
\hline Coon Rapids Mun Utilities .............. & - & - & - & - & - & - & - & - & - & -- & 1 \\
\hline 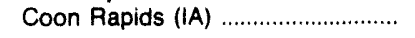 & -- & -- & -- & -- & - & - & -- & -- & - & -- & 1 \\
\hline Cooperative Power Association .. & 708,717 & - & - & - & - & - & 619 & - & -- & 1,027 & 30 \\
\hline Bonifacius (MN) & - & - & -- & - & - & -- & -- & -- & - & - & 6 \\
\hline Copper Valley Electric Assn, Inc & - & -123 & - & 6,096 & - & - & - & - & - & - & 1 \\
\hline 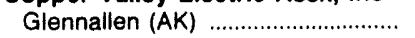 & -- & -51 & - & -- & -- & - & -- & • & -- & -- & 1 \\
\hline Valdez (AK) & -- & -- & - & 6,096 & -- & -- & -- & -- & - & -- & $\ldots$ \\
\hline Valdez (AK) & -- & -72 & -- & $-\infty$ & - & -- & -- & $\cdot$ & - & - & 1 \\
\hline Cordova City Ot & - & 1,483 & - & 369 & - & - & - & 3 & - & - & 1 \\
\hline 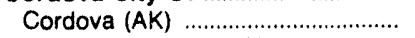 & - & 120 & -- & -- & - & -- & -- & * & -- & - & * \\
\hline Humpback Creek (AK) .................... & -- & -- & -- & 369 & -- & - & - & - & -- & -- & -- \\
\hline Ocean Dock (AK) ............................ & - & 1,363 & -- & - & - & - & -- & 3 & -- & -- & * \\
\hline Corn Belt Power Coop ...................... & 10,855 & - & 22 & - & - & - & 6 & - & * & 8 & - \\
\hline Humboldt (IA) & -36 & - & -- & - & -- & - & -- & - & -- & -- & -- \\
\hline Wisdom, Earl F (IA) ......................... & 10,891 & -- & 22 & -- & -- & - & 6 & -- & • & 8 & -- \\
\hline Corning Municipal Utilities ............. & - & - & - & - & - & - & - & - & - & -- & $-m$ \\
\hline 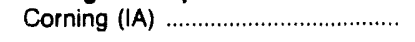 & -- & - & -- & -- & -- & -- & -- & -- & -- & -- & -- \\
\hline Craig-Botetourt Electric Coop ....... & - & - & - & - & - & - & - & - & - & -- & -- \\
\hline New Castle (VA) ................................. & -- & -- & -- & - & -- & -- & -- & -- & - & -- & -- \\
\hline Crawfordsville Elec Lt \& Pwr UtII & - & - & - & - & - & - & • & - & -- & 3 & -- \\
\hline 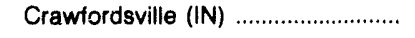 & -- & - & -- & -- & -- & -- & • & - & -- & 3 & -- \\
\hline 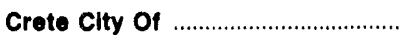 & - & 11 & 9 & - & - & - & - & $\bullet$ & * & - & 1 \\
\hline Crete (NE) & -- & 11 & 9 & - & -- & - & -- & * & * & -- & 1 \\
\hline Crisp County Power Commission & -112 & - & 4 & 1,519 & - & - & $*$ & - & 1 & 3 & - \\
\hline Crisp (GA) & -112 & -- & 4 & -- & - & - & • & -- & 1 & 3 & - \\
\hline 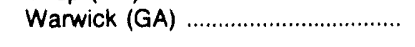 & -- & -- & - & 1,519 & -- & - & - & -- & - & -- & -- \\
\hline Crystal Falls Clty of (Lt \& Wtr) .... & - & - & - & 317 & - & - & - & - & - & - & - \\
\hline 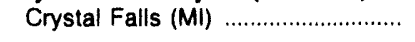 & - & -- & - & 317 & -- & -- & -- & - & -- & -- & -- \\
\hline 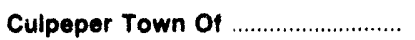 & -- & 39 & 36 & - & - & - & - & $*$ & 1 & - & 1 \\
\hline 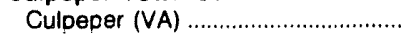 & - & 39 & 36 & - & -- & - & -- & - & 1 & - & 1 \\
\hline
\end{tabular}

See footnotes at end of table. 
Table 62. U.S. Electric Utility Net Generation, Fuel Consumption, and Fuel Stocks by Company and Plant, September 1993 (Continued)

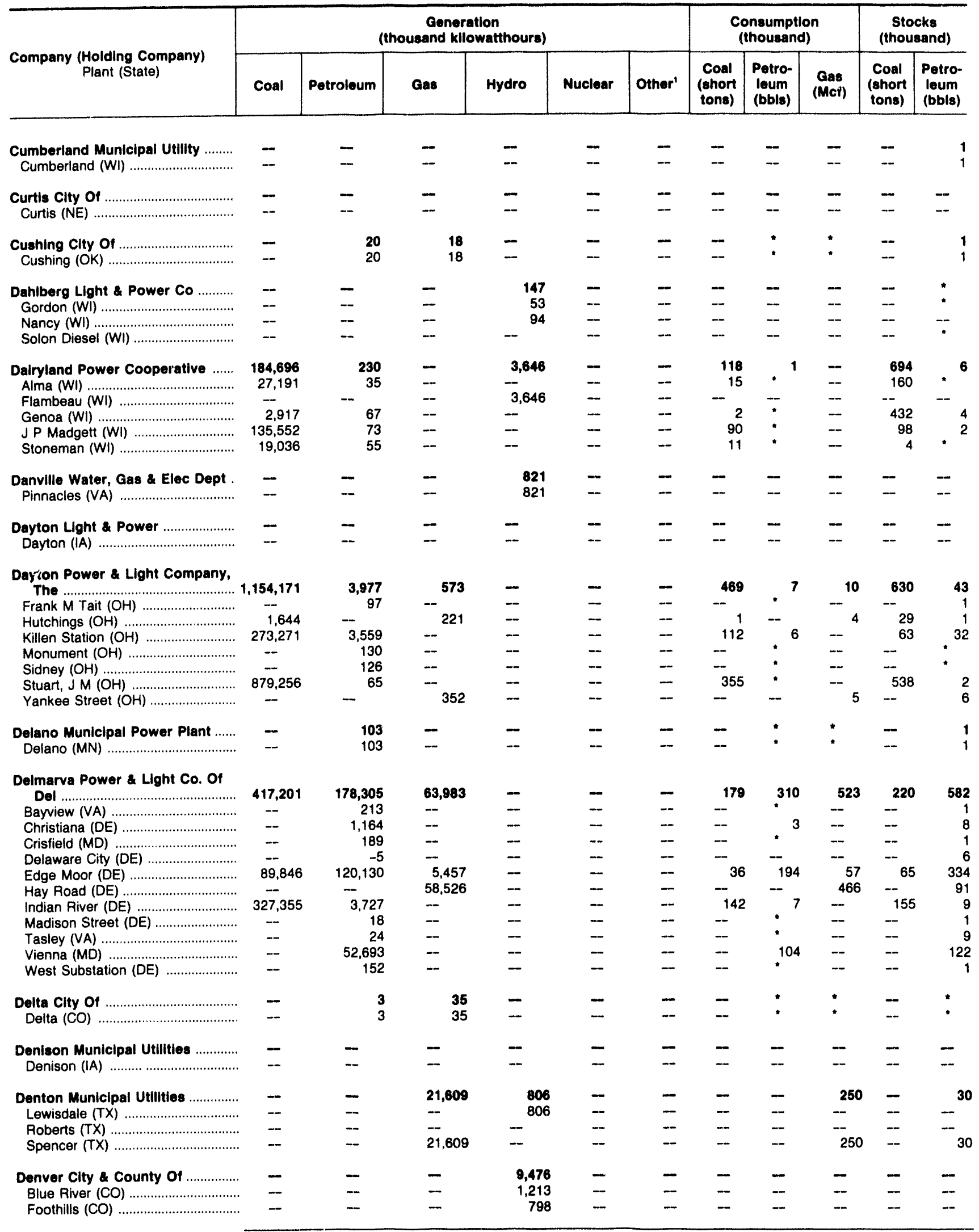

See footnotes at end of table. 
Table 62. U.S. Electric Utility Net Generation, Fuel Consumption, and Fuel Stocks by Company and Plant, September 1993 (Continued)

\begin{tabular}{|c|c|c|c|c|c|c|c|c|c|c|c|}
\hline \multirow{2}{*}{$\begin{array}{c}\text { Company (Holding Company) } \\
\text { Plant (State) }\end{array}$} & \multicolumn{6}{|c|}{$\begin{array}{c}\text { Generation } \\
\text { (thousand kllowatthours) }\end{array}$} & \multicolumn{3}{|c|}{$\begin{array}{l}\text { Consumption } \\
\text { (thousand) }\end{array}$} & \multicolumn{2}{|c|}{$\begin{array}{c}\text { Stocks } \\
\text { (thoueand) }\end{array}$} \\
\hline & Coal & Petroleum & Gas & Hydro & Nuclear & Other' & $\begin{array}{c}\text { Coal } \\
\text { (short } \\
\text { tons) }\end{array}$ & $\begin{array}{l}\text { Petro- } \\
\text { leum } \\
\text { (bbles) }\end{array}$ & $\begin{array}{l}\text { Gas } \\
\text { (Mcf) }\end{array}$ & $\begin{array}{l}\text { Coal } \\
\text { (short } \\
\text { tons) }\end{array}$ & $\begin{array}{l}\text { Petro- } \\
\text { leum } \\
\text { (bble) }\end{array}$ \\
\hline \multicolumn{12}{|l|}{ Denver City \& County of } \\
\hline 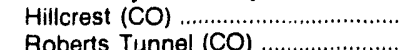 & - & - & -- & 1,088 & -- & -- & - & -- & -- & -. & - \\
\hline $\begin{array}{l}\text { Roberts Tunnel (CO) .. } \\
\text { Strontia Spros (CO) }\end{array}$ & -- & - & -- & 3,918 & -- & -- & - & -- & -- & -- & -- \\
\hline & -- & -- & -- & 765 & -- & -- & -- & -- & -- & -- & -- \\
\hline Williams Fork $(\mathrm{CO})$ & - & -- & -- & 1,694 & -- & -- & -- & -- & -- & -- & -- \\
\hline \multicolumn{12}{|l|}{ Deseret Generating \& } \\
\hline 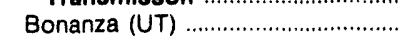 & 300,110 & 51 & -- & -- & -- & -- & 130 & • & - & 469 & $\begin{array}{l}2 \\
2\end{array}$ \\
\hline Deshler Munlcipal Utilities ................ & - & - & - & - & - & - & - & - & - & - & • \\
\hline Deshler (NE) & - & -- & - & -- & -- & -- & - & •" & -- & -- & - \\
\hline \multicolumn{12}{|l|}{ Detroit City Of (Pub Lighting } \\
\hline 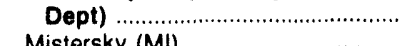 & - & 6,682 & -175 & - & - & - & - & 34 & - & - & 54 \\
\hline 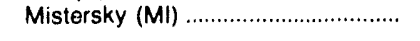 & - & 6,682 & -175 & -- & -- & -- & -- & 34 & - & -- & 54 \\
\hline \multicolumn{2}{|c|}{ Detrolt Edison Company, The ...... 3,381,038 } & 9,396 & 22,240 & - & 592,392 & - & 1,631 & 19 & 881 & 4,100 & 331 \\
\hline - Central Storage " & - & -- & & -- & -- & -- & - & - & -- & 689 & - \\
\hline Beacon Heating (MI) ........................... & -- & - & -486 & -- & -- & -- & -- & -- & -- & -- & 7 \\
\hline Belle River (MI) & 654,301 & 794 & -- & -- & -- & -- & 354 & 1 & -- & -- & 13 \\
\hline 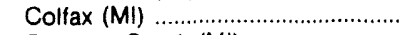 & -- & 17 & -- & -- & -- & - & -- & • & -- & -- & 1 \\
\hline Conners Creek (MI) ……..................... & - & 4 & -- & -- & - & -- & -- & • & - & -- & • \\
\hline 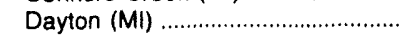 & - & -4 & -- & -- & -- & -- & $\cdots$ & • & -- & -- & - \\
\hline 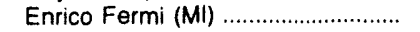 & -- & -9 & -- & - & 592,392 & -- & -- & * & - & -- & 5 \\
\hline 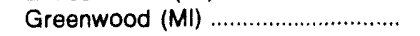 & -- & 369 & 1,663 & - & -- & -- & -- & 2 & 43 & -- & 225 \\
\hline 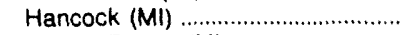 & - & - & 223 & -- & -- & -- & - & -- & 4 & -- & -- \\
\hline Harbor Beach (Mil) ............................... & 10,294 & 140 & -- & -- & -- & -- & 5 & • & -_ & 32 & - \\
\hline 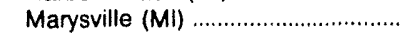 & 13,395 & -- & 748 & - & -- & -- & 8 & -- & 10 & 11 & -- \\
\hline Monroe (MI) & $1,633,357$ & 3,415 & -- & -- & - & - & 724 & 6 & $\ldots$ & 545 & 10 \\
\hline 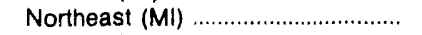 & -- & -44 & 17 & - & -- & - & -- & • & * & -- & 2 \\
\hline 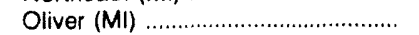 & -- & 81 & -- & -- & -- & -- & -- & - & -- & -- & •" \\
\hline 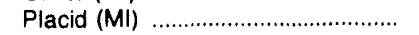 & - & 24 & -- & -- & -- & -- & -- & • & -- & -- & - \\
\hline Putnam (MI) & -- & 39 & -- & - & -- & -- & -- & • & -- & -_ & 1 \\
\hline 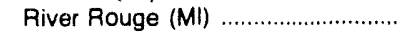 & 256,435 & 9 & 17,041 & - & -- & -- & 108 & • & 792 & 25 & • \\
\hline 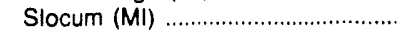 & -- & -2 & - & -- & -- & -- & - & • & - & -- & • \\
\hline St. Clair (MI) & 561,124 & 2,640 & 3,034 & -- & -- & -- & 315 & 5 & 32 & 2,571 & 55 \\
\hline Superior (MI) & -- & 129 & -- & - & -- & - & -- & 1 & -- & - & 2 \\
\hline Trenton Channel (MI) ......................... & 252,132 & 1.758 & -- & -- & - & -- & 117 & 3 & -- & 228 & 8 \\
\hline 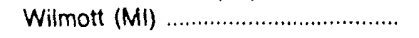 & -- & 36 & -- & - & -- & -- & -- & • & -- & -- & - \\
\hline \multirow{2}{*}{$\begin{array}{l}\text { Detroit Lakes Clty Of } \\
\text { Detroit Lakes (MN) }\end{array}$} & - & - & - & - & - & - & - & - & - & - & - \\
\hline & -- & - & -- & - & -- & -- & -- & - & -- & -- & - \\
\hline \multirow{2}{*}{$\begin{array}{l}\text { Douglas County Pud No } 1 \\
\text { Wells (WA) }\end{array}$} & - & - & - & 258,350 & - & - & - & - & - & - & - \\
\hline & -- & - & -- & 258,350 & - & -- & -- & -- & -- & -- & -- \\
\hline \multirow{2}{*}{ 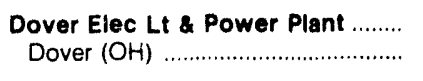 } & 6,627 & - & 7 & - & - & - & 4 & - & 1 & - & " \\
\hline & 6,627 & -- & 7 & -- & -- & -- & 4 & - & 1 & - & • \\
\hline \multirow{3}{*}{$\begin{array}{l}\text { Dover Electric Department } \\
\text { Mckee Run (DE) } \\
\text { Van Sant (DE) }\end{array}$} & - & 19,092 & 687 & -- & - & - & - & 37 & 8 & - & 14 \\
\hline & -- & 18,628 & 450 & -- & - & - & - & 36 & 5 & -- & 10 \\
\hline & -- & 464 & 237 & -- & -- & -- & - & 1 & 3 & -- & 4 \\
\hline \multirow{2}{*}{$\begin{array}{l}\text { Dowagiac Dept Of Public Service } \\
\text { Dowagiac (MI) }\end{array}$} & - & 70 & 10 & - & - & - & - & - & •" & - & • \\
\hline & - & 70 & 10 & -- & -- & - & -- & •" & " & - & •" \\
\hline \multirow{2}{*}{$\begin{array}{l}\text { Duke Power Company } \\
\text { Allen (NC) }\end{array}$} & $3,452,215$ & 3,484 & 5,654 & 10,825 & $3,594,430$ & - & 1,316 & 8 & 74 & 1,099 & 116 \\
\hline & 443,214 & 1,412 & -- & -- & -- & -- & 177 & 2 & -- & 128 & \\
\hline 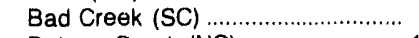 & -- & -- & -- & $-57,64$ & -- & - & -- & $\because$ & - & - & -- \\
\hline Belews Creek (NC) ....................... 1 & $1,437,239$ & 273 & -- & -- & -- & - & 539 & • & -- & 346 & \\
\hline 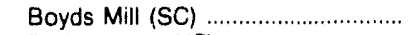 & -- & -- & -- & 215 & -- & -- & -- & -- & -- & -- & -- \\
\hline Bridgewater (NC) ................................... & -- & -- & -- & 1,432 & -- & -- & -- & - & - & -- & -- \\
\hline 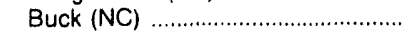 & 62,076 & 143 & 2,265 & -- & -- & -- & 27 & • & 24 & 63 & 16 \\
\hline Buzzard Roost (SC) ........................... & -- & -- & 1,187 & 2,301 & -- & -- & -- & -- & 23 & -- & 36 \\
\hline 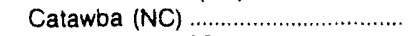 & -- & -- & -- & -- & $1,517,264$ & -- & -- & -- & -- & -- & -- \\
\hline Cedar Creek (SC) ................................. & - & -- & -- & 3,889 & -- & -- & - & -- & - & -- & -- \\
\hline 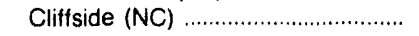 & 318,595 & 485 & - & -- & -- & -- & 125 & 1 & -- & 61 & 2 \\
\hline
\end{tabular}

See footnotes at end of table. 
Table 62. U.S. Electric Utility Net Generation, Fuel Consumption, and Fuel Stocks by Company and Plant, September 1993 (Continued)

\begin{tabular}{|c|c|c|c|c|c|c|c|c|c|c|c|}
\hline \multirow{2}{*}{$\begin{array}{c}\text { Company (Holding Company) } \\
\text { Plant (State) }\end{array}$} & \multicolumn{6}{|c|}{$\begin{array}{c}\text { Generation } \\
\text { (thousand kilowatthours) }\end{array}$} & \multicolumn{3}{|c|}{$\begin{array}{l}\text { Consumptlon } \\
\text { (thousand) }\end{array}$} & \multicolumn{2}{|c|}{$\begin{array}{l}\text { Stocks } \\
\text { (thousand) }\end{array}$} \\
\hline & Coal & Potroleum & Gas & Hydro & Nuclear & Other' & $\begin{array}{c}\text { Coal } \\
\text { (short } \\
\text { tons) }\end{array}$ & $\begin{array}{c}\text { Petro- } \\
\text { leum } \\
\text { (bble) }\end{array}$ & $\begin{array}{l}\text { Gas } \\
\text { (Mcf) }\end{array}$ & $\begin{array}{c}\text { Coal } \\
\text { (short } \\
\text { tons) }\end{array}$ & $\begin{array}{l}\text { Petro- } \\
\text { leum } \\
\text { (bbls) }\end{array}$ \\
\hline Duke Power Company & -- & -- & -- & 5,821 & -- & - & - & -- & -- & -- & - \\
\hline 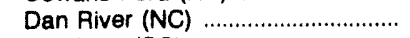 & 66,932 & 384 & 894 & - & -- & -- & 30 & 1 & 10 & 74 & 16 \\
\hline 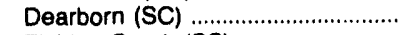 & -- & -- & -- & 4,566 & -- & - & -- & -- & -- & -- & - \\
\hline 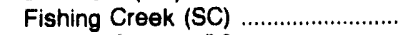 & -- & - & -- & 4,496 & -- & -- & -- & -- & -- & - & - \\
\hline Gaston Shoals (SC) & -- & -- & -- & 1,531 & - & -- & -- & - & - & -- & -- \\
\hline 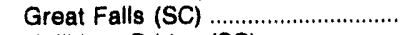 & - & -- & -- & 258 & -- & -- & -- & - & - & -- & - \\
\hline Hollidays Bridge (SC) ....................... & -- & -- & -- & 368 & -- & -- & -- & -- & - & -- & - \\
\hline Idols (NC) & -- & -- & -- & 232 & -- & -- & -- & -- & -- & - & -- \\
\hline 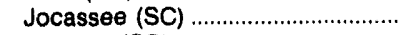 & - & -- & -- & 10,908 & - & -- & - & -- & -- & -- & -- \\
\hline 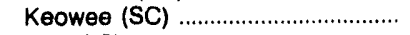 & - & -- & - & 6,757 & - & -- & -- & - & -- & - & -- \\
\hline Le日 (SC) & 62,845 & 267 & 1,028 & -- & - & -- & 28 & 1 & 11 & 85 & 14 \\
\hline Lookout Shoals (NC) ......................... & -- & - & -- & 3,199 & -- & -- & - & -- & - & -- & -- \\
\hline 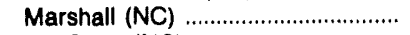 & 909,496 & 430 & -- & -- & $-\overline{-}$ & -- & 331 & 1 & - & 238 & 6 \\
\hline 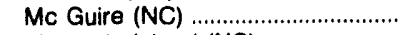 & -- & -- & - & -- & 268,267 & - & - & -- & -- & -- & - \\
\hline 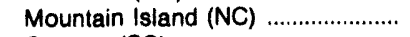 & - & - & -- & 3,639 & -- & -- & - & -- & -- & -- & - \\
\hline 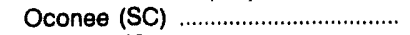 & - & - & -- & - & $1,808,899$ & - & -- & - & -- & -- & -- \\
\hline Oxford $(N C)$ & - & - & - & 3,391 & -- & - & -- & -- & -- & -- & -- \\
\hline 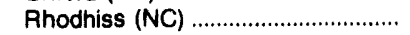 & -- & -- & - & 2,055 & -- & -- & - & - & -- & - & -- \\
\hline 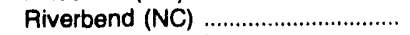 & 151,818 & 90 & 210 & - & - & - & 59 & 2 & 5 & 105 & 16 \\
\hline 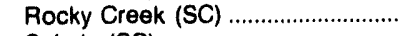 & - & -- & -- & 30 & - & - & -- & - & - & -- & -- \\
\hline 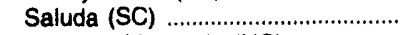 & - & - & -- & 225 & - & -- & - & -- & - & -- & -- \\
\hline Spencer Mountain (NC) .................. & - & - & - & 82 & - & - & - & - & -- & - & - \\
\hline Stice Shoals (NC) & -- & -- & - & 104 & - & - & -- & -- & -- & - & -- \\
\hline Turner Shoals (NC) ............................. & - & - & -- & 459 & - & -- & - & -- & -- & -- & -- \\
\hline 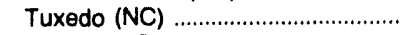 & -- & - & -- & 617 & - & -- & - & -- & -- & -- & - \\
\hline 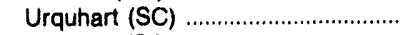 & - & -- & 70 & $=$ & - & -- & - & -- & 1 & -- & 3 \\
\hline 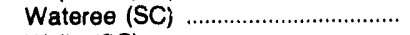 & - & - & -- & 5,289 & - & - & - & -- & -- & -- & - \\
\hline 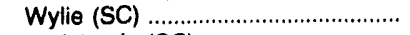 & -- & -- & $-\cdots$ & 3,875 & - & -- & -- & - & -- & -- & -- \\
\hline 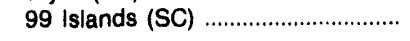 & - & -- & - & 2,770 & - & -- & - & -- & - & - & -- \\
\hline Duquesne Light Company .............. & 416,767 & -223 & 1,403 & - & 816,911 & - & 177 & 1 & 13 & 459 & 52 \\
\hline 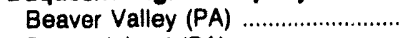 & -- & -- & -- & - & 816,911 & -- & - & -- & -- & -- & -- \\
\hline 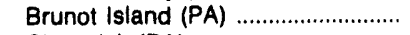 & -- & -915 & - & -- & -- & -- & - & -- & - & - & 49 \\
\hline 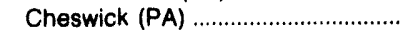 & 279,128 & -- & 1.403 & -- & - & -- & 108 & -- & 13 & 270 & - \\
\hline 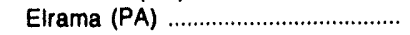 & 137,639 & 692 & - & - & - & -- & 69 & 1 & - & 189 & 3 \\
\hline Phillips, $F$ (PA) & -- & -- & -- & - & -- & -- & - & -- & - & - & -- \\
\hline Durant Municlpal Electric Plant .... & - & - & - & - & - & - & - & - & - & -- & " \\
\hline 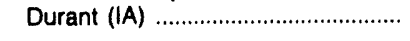 & - & - & -- & - & - & - & - & -- & -- & -- & " \\
\hline East Bay Mun Utility District .......... & - & - & - & 4,832 & - & - & - & - & - & - & - \\
\hline Camanche $(C A)$ & -- & -- & - & 745 & -- & - & -- & -- & - & -- & -- \\
\hline 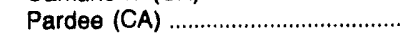 & -- & -- & - & 4,087 & - & -- & - & -- & - & -- & - \\
\hline $\begin{array}{l}\text { East Kentucky Power } \\
\text { Cooperative }\end{array}$ & 586,358 & 245 & & & & & & - & - & & \\
\hline Cooper (KY) & 178,564 & 113 & - & - & - & - & 74 & - & $\ldots$ & 77 & 1 \\
\hline Dale (KY) & 36,625 & 67 & -- & -- & -- & - & 19 & * & - & 47 & . \\
\hline 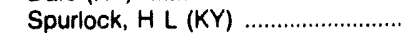 & 371,169 & 65 & -- & - & -- & - & 150 & * & - & 286 & 3 \\
\hline Eastern Maine Elec Coop, Inc ....... & - & - & - & - & - & - & - & • & - & - & • \\
\hline 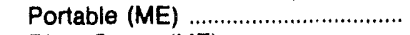 & -- & -- & -- & -- & -- & -- & -- & -- & - & -- & -- \\
\hline 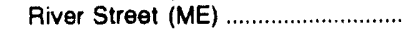 & -- & - & -- & -- & -- & - & -- & " & - & -- & " \\
\hline Easton Utilities Commlesion, The & - & 1,060 & 518 & - & - & - & - & 2 & 5 & - & 13 \\
\hline Easton (MD) & -- & 317 & 489 & -- & -- & -- & - & 1 & 5 & - & 9 \\
\hline 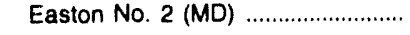 & -- & 743 & 29 & - & -- & - & - & 1 & * & $\cdots$ & 4 \\
\hline Edison Sault Electric Co ................. & - & 74 & - & 17,990 & - & - & - & • & - & -- & ' \\
\hline 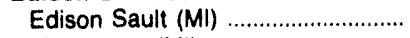 & - & -- & -- & 17,990 & - & -- & - & -- & -- & -- & - \\
\hline Manistique (MI) ...................................... & - & 74 & -- & - & -- & - & -- & " & -- & - & " \\
\hline Egeglk Light \& Power Company .. & - & 55 & - & - & - & - & - & : & - & -- & - \\
\hline 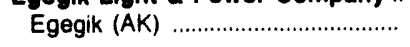 & - & 55 & -- & - & - & -- & - & $\cdot$ & - & - & -- \\
\hline El Paso Electric Company .............. & - & - & 260,383 & - & - & - & - & - & 2,889 & - & 78 \\
\hline Copper (TX) & -- & -- & 6,283 & - & - & - & - & - & 91 & -- & 7 \\
\hline
\end{tabular}

See footnotes at end of table. 
Table 62. U.S. Electric Utility Net Generation, Fuel Consumption, and Fuel Stocks by Company and Plant, September 1993 (Continued)

\begin{tabular}{|c|c|c|c|c|c|c|c|c|c|c|c|}
\hline \multirow{2}{*}{$\begin{array}{c}\text { Cornpany (Holding Company) } \\
\text { Plant (State) }\end{array}$} & \multicolumn{6}{|c|}{$\begin{array}{c}\text { Gonoration } \\
\text { (thousand kilowatthours) }\end{array}$} & \multicolumn{3}{|c|}{$\begin{array}{l}\text { Consumption } \\
\text { (thousand) }\end{array}$} & \multicolumn{2}{|c|}{$\begin{array}{c}\text { Stocks } \\
\text { (thousand) }\end{array}$} \\
\hline & Coal & Petroloum & Gas & Hydro & Nuclear & Other' & $\begin{array}{c}\text { Coal } \\
\text { (short } \\
\text { tons) }\end{array}$ & $\begin{array}{l}\text { Potro- } \\
\text { loum } \\
\text { (bbls) }\end{array}$ & $\begin{array}{l}\text { Gas } \\
\text { (Mcf) }\end{array}$ & $\begin{array}{c}\text { Coal } \\
\text { (short } \\
\text { tons) }\end{array}$ & $\begin{array}{l}\text { Petro- } \\
\text { leum } \\
\text { (bbls) }\end{array}$ \\
\hline \multicolumn{12}{|l|}{$\begin{array}{l}\text { El Paso Electric Company } \\
\text { Newman }(\mathrm{T})\end{array}$} \\
\hline 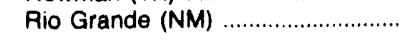 & - & $\cdots$ & 82,172 & - & -- & - & - & - & 987 & $\overline{--}$ & $\begin{array}{l}11 \\
61\end{array}$ \\
\hline \multirow{2}{*}{$\begin{array}{l}\text { Electra City Of } \\
\text { Electra (TX) }\end{array}$} & - & - & - & - & - & - & - & - & - & - & - \\
\hline & - & -- & -- & - & - & - & - & - & -- & -- & -- \\
\hline \multirow{2}{*}{$\begin{array}{l}\text { Eloctric Energy, Inc } \\
\text { Joppa Steam (IL) }\end{array}$} & 521,737 & 443 & - & - & - & - & 284 & 1 & - & 270 & 1 \\
\hline & 521,737 & 443 & $\cdots$ & $\cdots$ & - & -- & 284 & 1 & $\cdots$ & 270 & 1 \\
\hline \multirow{2}{*}{ 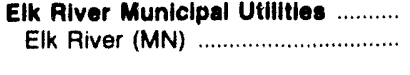 } & - & 16 & 5 & - & - & - & - & " & " & - & • \\
\hline & - & 16 & 5 & - & - & - & - & " & $\cdot$ & - & • \\
\hline \multirow{2}{*}{$\begin{array}{l}\text { Ellinwood Lt \& Water } \\
\text { Ellinwood (KS) }\end{array}$} & - & 2 & 1 & - & - & - & - & • & • & - & - \\
\hline & -- & 2 & 1 & - & -- & - & - & $\cdot$ & " & -- & -- \\
\hline \multirow{2}{*}{$\begin{array}{l}\text { Elroy City Of } \\
\text { Elroy }(W \mid)\end{array}$} & - & -2 & - & - & - & - & - & - & - & - & • \\
\hline & -- & -2 & - & - & -- & - & -- & $\cdots$ & - & -- & • \\
\hline \multirow{2}{*}{$\begin{array}{l}\text { Emerson Light \& Power Plant ....... } \\
\text { Emerson (NE) }\end{array}$} & - & 1 & - & - & - & - & - & • & - & -- & " \\
\hline & -- & 1 & - & - & -- & - & - & $\cdot$ & - & -- & $\star$ \\
\hline \multirow{5}{*}{$\begin{array}{l}\text { Empire District Electric Co } \\
\text { Asbury (MO) } \\
\text { Energy Center (MO) } \\
\text { Ozark Beach (MO) } \\
\text { Riverton (KS) }\end{array}$} & 160,491 & 25 & 238 & 5,440 & - & - & 100 & : & 6 & 149 & 33 \\
\hline & 128,061 & 131 & -- & - & -- & -- & 81 & • & -- & 112 & 1 \\
\hline & -- & -106 & -- & - & - & - & - & -- & -- & -- & 28 \\
\hline & $\overrightarrow{30}$ & -- & - & 5,440 & -- & - & -10 & - & - & -- & $\cdots$ \\
\hline & 32,430 & -- & 238 & - & -- & - & 19 & - & 6 & 38 & 4 \\
\hline \multirow{4}{*}{$\begin{array}{l}\text { Enosburg Falls Inc Village Of ........ } \\
\text { Diesel Plt (VT) } \\
\text { Kendall (VT) } \\
\text { Village Plt (VT) }\end{array}$} & - & - & - & 401 & - & - & - & - & - & - & : \\
\hline & - & -- & -- & - & - & - & $\cdots$ & -- & - & -- & * \\
\hline & -- & - & - & 128 & -- & - & $\cdots$ & -- & -- & -- & - \\
\hline & -- & - & - & 273 & -- & - & -- & - & -- & -- & -- \\
\hline \multirow{4}{*}{ 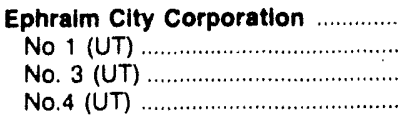 } & - & - & - & 382 & - & - & - & - & -- & -- & - \\
\hline & - & - & - & 144 & - & - & -- & -- & - & -- & - \\
\hline & - & -- & - & 218 & - & -- & - & - & -- & -- & -- \\
\hline & -- & -- & -- & 20 & -- & -- & -- & -- & -- & - & -- \\
\hline \multirow{2}{*}{$\begin{array}{l}\text { Erie Water \& Light } \\
\text { Erie (KS) }\end{array}$} & - & - & - & - & - & - & - & - & - & - & - \\
\hline & $\cdots$ & -- & -- & - & - & -- & - & -- & -- & -- & -- \\
\hline \multirow{3}{*}{$\begin{array}{l}\text { Escondido Mutual Wtr Co } \\
\text { Bear Valley (CA) } \\
\text { Rincon Pwr (CA) }\end{array}$} & - & - & - & 1,057 & - & - & - & - & -- & -- & - \\
\hline & - & - & -- & 1,043 & -- & -- & -- & -- & -- & -- & -- \\
\hline & -- & -- & - & 14 & - & - & -- & - & -- & -- & -- \\
\hline \multirow{2}{*}{$\begin{array}{l}\text { Estherville City of } \\
\text { Esterville (IA) }\end{array}$} & - & - & - & - & - & - & - & - & - & - & 2 \\
\hline & - & -- & - & - & -- & -- & - & " & - & -- & 2 \\
\hline \multirow{5}{*}{ 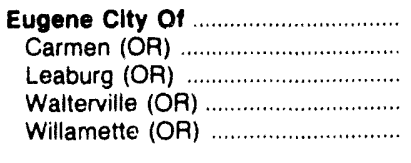 } & - & - & - & 25,004 & - & - & - & - & - & - & - \\
\hline & - & - & -- & 13,559 & - & -- & - & -- & -- & - & -- \\
\hline & -- & - & - & 7,107 & - & -- & -- & - & - & -- & - \\
\hline & - & - & -- & 4,338 & - & -- & -- & -- & -- & -- & - \\
\hline & - & - & - & - & - & - & - & - & -- & - & -- \\
\hline Fairbanks Mun Utilities System .... & 5,434 & 19 & - & - & - & - & 6 & - & - & 5 & 1 \\
\hline Chena (AK) & 5,434 & 19 & -- & - & - & -- & 6 & • & - & 5 & 1 \\
\hline Fairbury Light \& Water Dept ........ & - & - & -- & - & - & - & - & - & - & - & - \\
\hline 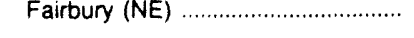 & - & -- & - & - & - & -- & $\cdots$ & - & -- & - & $\cdot$ \\
\hline Fairtax Mun Pwr Plant .......................... & - & - & - & - & - & - & - & - & -- & - & - \\
\hline Fairfax (MN) . & - & - & -- & - & - & -- & - & - & -- & - & $\cdots$ \\
\hline Fairfield Municipal Light Plant ...... & -- & - & - & - & - & - & - & - & - & - & - \\
\hline Fairfield (IL) ....................................... & -- & - & - & -- & - & - & -- & - & - & -- & -- \\
\hline Fairmont Pub Util Comm ............... & -24 & -24 & -48 & - & - & - & - & - & - & 2 & 1 \\
\hline 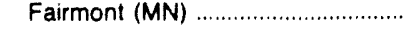 & -24 & -24 & -48 & - & -- & -- & $\cdots$ & -- & -- & 2 & 1 \\
\hline
\end{tabular}

See footnotes at end of table. 
Table 62. U.S. Electric Utility Net Generation, Fuel Consumption, and Fuel Stocks by Company and Plant, September 1993 (Continued)

\begin{tabular}{|c|c|c|c|c|c|c|c|c|c|c|c|}
\hline \multirow{2}{*}{$\begin{array}{c}\text { Company (Holding Company) } \\
\text { Plant (State) }\end{array}$} & \multicolumn{6}{|c|}{$\begin{array}{c}\text { Generation } \\
\text { (thousand kllowatthours) }\end{array}$} & \multicolumn{3}{|c|}{$\begin{array}{l}\text { Consumption } \\
\text { (thousand) }\end{array}$} & \multicolumn{2}{|c|}{$\begin{array}{c}\text { Stocks } \\
\text { (thousand) }\end{array}$} \\
\hline & Coal & Petroleum & Gas & Hydro & Nuclear & Other' & $\begin{array}{c}\text { Conl } \\
\text { (ohort } \\
\text { tons) }\end{array}$ & $\begin{array}{l}\text { Petro- } \\
\text { leum } \\
\text { (bble) }\end{array}$ & $\begin{array}{c}\text { Cas } \\
\text { (Mcf) }\end{array}$ & $\begin{array}{c}\text { Coal } \\
\text { (ohort } \\
\text { tons) }\end{array}$ & $\begin{array}{l}\text { Potro- } \\
\text { leum } \\
\text { (bbls) }\end{array}$ \\
\hline
\end{tabular}

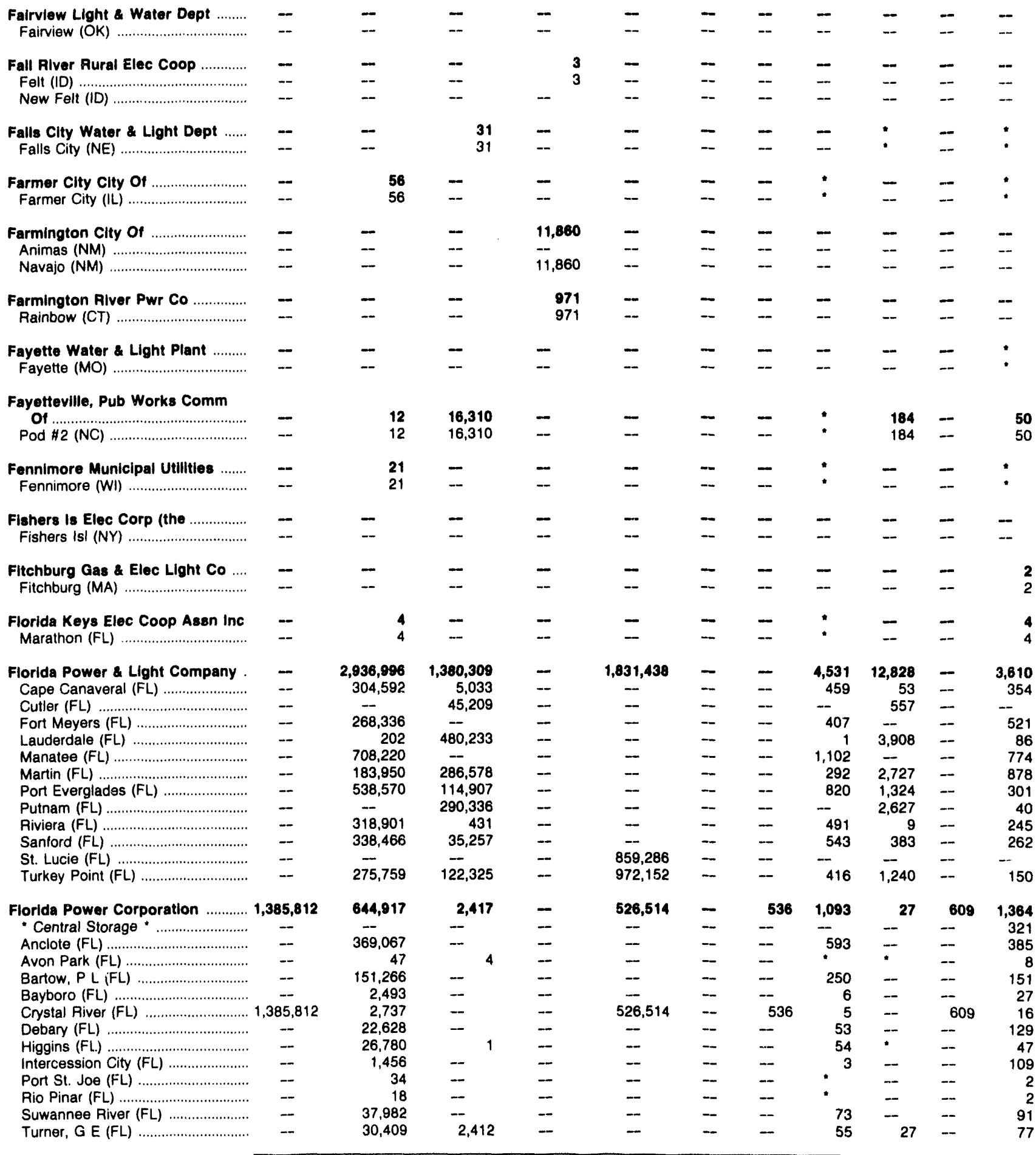

See footnotes at end of table. 
Table 62. U.S. Electric Utillty Net Generation, Fuel Consumption, and Fuel Stocks by Company and Plant, September 1993 (Continued)

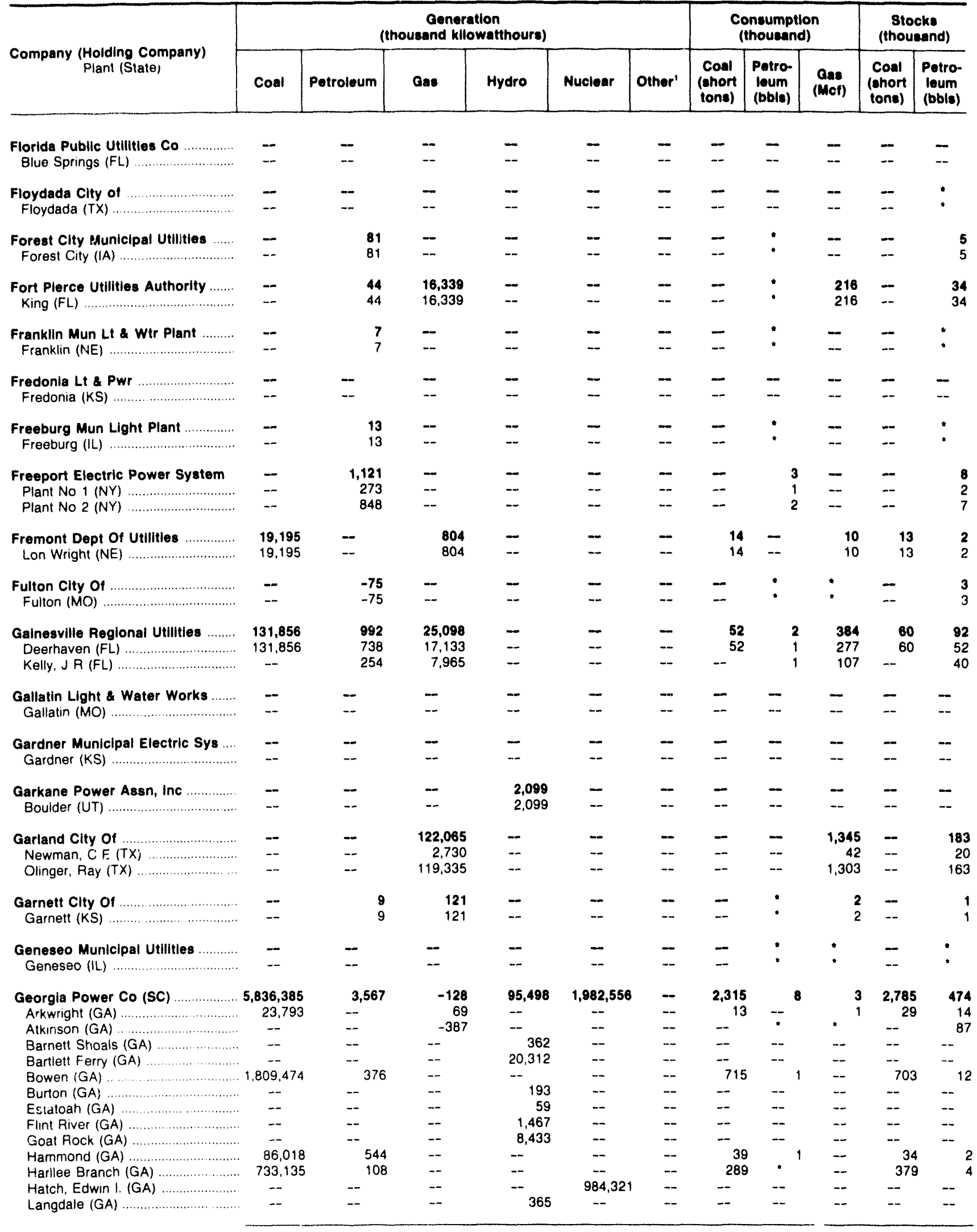

See footnotes at end of table. 
Table 62. U.S. Electric Utility Net Generation, Fuel Consumption, and Fuel Stocks by Company and Plant, September 1993 (Continued)

\begin{tabular}{|c|c|c|c|c|c|c|c|c|c|c|c|}
\hline \multirow{2}{*}{$\begin{array}{c}\text { Company (Holding Company) } \\
\text { Plant (State) }\end{array}$} & \multicolumn{6}{|c|}{$\begin{array}{c}\text { Generation } \\
\text { (thousand kllowatthours) }\end{array}$} & \multicolumn{3}{|c|}{$\begin{array}{l}\text { Consumption } \\
\text { (thousand) }\end{array}$} & \multicolumn{2}{|c|}{$\begin{array}{l}\text { Stocke } \\
\text { (thousand) }\end{array}$} \\
\hline & Coal & Potroleum & Cas & Hydro & Nuclear & Othor' & $\begin{array}{c}\text { Coal } \\
\text { (short } \\
\text { tons) }\end{array}$ & $\begin{array}{l}\text { Potro- } \\
\text { loum } \\
\text { (bbla) }\end{array}$ & $\underset{(\operatorname{Man})}{\operatorname{ans}}$ & $\begin{array}{c}\text { Coal } \\
\text { (chort } \\
\text { tone) }\end{array}$ & $\begin{array}{l}\text { Petro- } \\
\text { leum } \\
\text { (bbls) }\end{array}$ \\
\hline
\end{tabular}

\begin{tabular}{|c|c|c|c|c|c|c|c|c|c|c|c|}
\hline $\begin{array}{l}\text { Georgla Power Co (SC) } \\
\text { Lloyd Shoals (GA) }\end{array}$ & - & -- & -- & 356 & -- & - & - & - & -- & -- & - \\
\hline Mcdonough, $\mathrm{J}(\mathrm{GA})$ & 304,541 & 327 & 190 & -- & - & - & 116 & 1 & 2 & 110 & -- \\
\hline 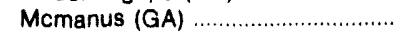 & - & -585 & -- & -- & -- & 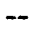 & - & $\cdot$ & -- & - & 145 \\
\hline 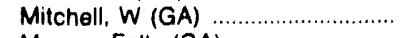 & 66,993 & 1,352 & - & - & -- & - & 28 & 2 & - & 47 & 76 \\
\hline Morgan Falls $(G A)$ & -- & -- & -- & 4,018 & -- & - & $-\infty$ & -- & - & - & -- \\
\hline 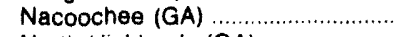 & -- & -- & -- & 119 & -- & -- & - & -- & -- & -- & -- \\
\hline North Highlands (GA) ..................... & -- & -- & -- & 6,438 & - & -- & - & - & -- & -- & -- \\
\hline 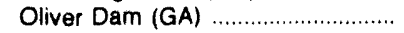 & -- & -- & -- & 10,980 & -- & - & - & -- & - & -- & -- \\
\hline Riverview (GA) & -- & -- & -- & 68 & -- & - & -- & - & - & $-\infty$ & $\ldots$ \\
\hline 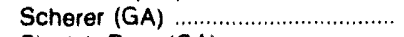 & $1,519,568$ & 350 & - & $-\overline{-}$ & -- & - & 583 & 1 & - & 456 & 18 \\
\hline 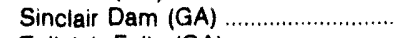 & -- & -- & -- & 3,822 & - & -- & - & $m$ & -- & $-\infty$ & -- \\
\hline 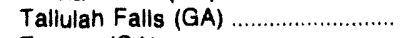 & -- & -- & - & 466 & - & - & -- & -- & - & - & -- \\
\hline 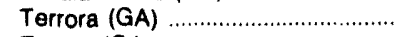 & - & - & -- & 395 & -- & -- & - & - & - & -- & - \\
\hline 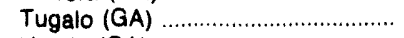 & -- & $-\infty$ & -- & 1,668 & -- & -- & - & - & - & - & - \\
\hline Vogtle $(G A)$ & -- & $-\cdots$ & -- & - & 998,235 & -- & $-\cdots$ & - & - & - & - \\
\hline 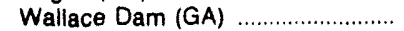 & -- & -- & -- & 35,069 & -- & -- & -- & - & - & $-\infty$ & - \\
\hline 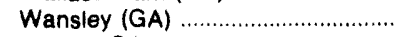 & 905,118 & 394 & -- & -- & -- & -- & 361 & 1 & - & 577 & 31 \\
\hline 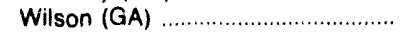 & - & -124 & -- & -- & -- & - & - & • & - & - & 83 \\
\hline 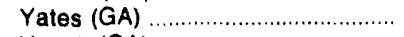 & 387.745 & 825 & - & -- & -- & - & 172 & 2 & -- & 449 & 3 \\
\hline 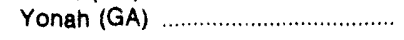 & -- & -- & -- & 908 & -- & - & -- & -- & - & $-\infty$ & -- \\
\hline Gilman Brothers Co .......................... & - & - & - & 1 & - & - & - & - & - & - & - \\
\hline 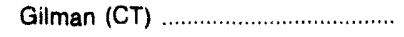 & -- & -- & - & 1 & - & - & - & -- & - & -- & - \\
\hline Girard Clty of & - & - & -- & - & -- & - & - & - & - & - & - \\
\hline 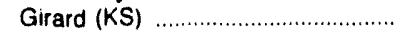 & -- & -- & -- & -- & - & -- & -- & - & - & - & -- \\
\hline Glencoe Mun Electric Plant ............ & -- & 279 & 55 & - & - & - & - & - & 1 & - & 2 \\
\hline Glencoe $(\mathrm{MN})$ & -- & 279 & 55 & -- & - & -- & - & - & 1 & -- & 2 \\
\hline Glendale Public Service Dept ........ & - & 21 & 18,053 & - & - & - & - & * & 250 & - & 51 \\
\hline 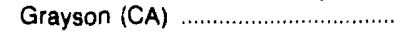 & -- & 21 & 18,053 & - & -- & - & - & • & 259 & -- & 51 \\
\hline Golden Valley Elec Aasn, Inc ....... & 17,436 & 22,726 & - & - & - & - & 15 & 43 & - & - & 32 \\
\hline Fairbanks (ÁK) & - & -34 & -- & -- & -- & - & -- & - & - & - & 5 \\
\hline Healy $(A K)$ & 17,436 & 33 & - & - & -- & - & 15 & $\cdot$ & -- & $\cdot$ & 1 \\
\hline 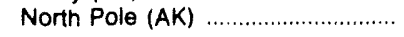 & -- & 22,727 & -- & -- & -- & - & -- & 43 & - & - & 27 \\
\hline Goodland Municipal Electric Dep & - & 75 & 491 & - & - & - & - & - & 9 & - & 2 \\
\hline 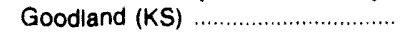 & - & 75 & 491 & -- & -- & - & - & • & 9 & - & 2 \\
\hline Gouverneur Elec PIt ........................... & - & - & - & 72 & - & - & - & - & - & - & - \\
\hline 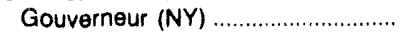 & -- & -- & -- & 72 & -- & - & - & -- & - & -- & - \\
\hline Gowrie Light \& Water Plant .......... & - & - & - & - & - & - & - & - & - & - & - \\
\hline 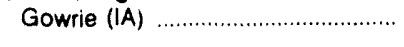 & - & -- & - & -- & -- & -- & -- & - & - & -- & $\cdot$ \\
\hline Graeftinger Mun Light Plant ......... & - & -- & - & - & - & - & - & - & - & - & - \\
\hline Graettinger (IA) & -- & - & -- & - & -- & - & -- & - & -- & -- & - \\
\hline Grafton Light \&ater Dept ......... & - & - & - & - & - & - & - & - & - & - & - \\
\hline Grafton (ND) & - & - & -- & - & - & -- & -- & -- & - & -- & -- \\
\hline Grand Haven Board of Lt a Pwr. & 30,351 & 3 & - & - & - & - & 16 & - & - & 86 & 10 \\
\hline 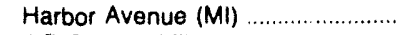 & $\overline{-i n}$ & 3 & -- & -- & $-\cdot$ & - & - & -- & - & $-\cdots$ & 10 \\
\hline J B Simms (MI) & 30,351 & - & - & - & - & - & 16 & -- & - & 96 & - \\
\hline Grand Island Water \& LIght Dept & 41,213 & - & -119 & -- & - & - & 29 & - & : & 59 & 56 \\
\hline Burdick, C W (NE) & $-\infty$ & -- & -119 & -- & - & - & -- & -- & • & -- & 56 \\
\hline 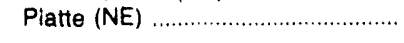 & 41,213 & - & -- & -- & - & -- & 29 & -- & -- & 59 & - \\
\hline Grand Junction Mun Lt Plant ....... & - & - & - & - & - & - & - & - & - & - & - \\
\hline 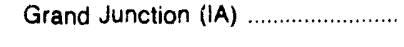 & -- & -- & - & -- & - & - & - & -- & - & - & - \\
\hline Grand Marals, Vlliage of ................. & - & -1 & - & - & - & - & - & $\cdot$ & - & - & $\bullet$ \\
\hline 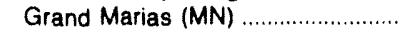 & -- & -1 & -- & $\cdots$ & - & -- & - & " & -- & - & • \\
\hline
\end{tabular}

See footnotes at end of table. 
Table 62. U.S. Electric Utillty Net Generation, Fuel Consumption, and Fuel Stocks by Company and Plant, September 1993 (Continued)

\begin{tabular}{|c|c|c|c|c|c|c|c|c|c|c|c|}
\hline \multirow{2}{*}{$\begin{array}{c}\text { Company (Holding Company) } \\
\text { Plant (State) }\end{array}$} & \multicolumn{6}{|c|}{$\begin{array}{l}\text { Generation } \\
\text { (thousand kllowatthours) }\end{array}$} & \multicolumn{3}{|c|}{$\begin{array}{l}\text { Consumption } \\
\text { (thousand) }\end{array}$} & \multicolumn{2}{|c|}{$\begin{array}{c}\text { Stocks } \\
\text { (thousand) }\end{array}$} \\
\hline & Coal & Potroleum & Gas & Hydro & Nuclear & Other' & $\begin{array}{c}\text { Coal } \\
\text { (short } \\
\text { tons) }\end{array}$ & $\begin{array}{l}\text { Potro- } \\
\text { leum } \\
\text { (bble) }\end{array}$ & $\begin{array}{l}\text { Gas } \\
\text { (Mcf) }\end{array}$ & $\begin{array}{c}\text { Coal } \\
\text { (short } \\
\text { tons) }\end{array}$ & $\begin{array}{l}\text { Potro- } \\
\text { loum } \\
\text { (bbls) }\end{array}$ \\
\hline 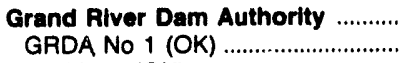 & $\begin{array}{l}510,243 \\
510,243\end{array}$ & $\overline{-}$ & $\begin{array}{l}2,377 \\
2,377\end{array}$ & 77,953 & $\overline{-}$ & $\overline{-}$ & $\begin{array}{l}322 \\
322\end{array}$ & : & $\begin{array}{l}25 \\
25\end{array}$ & $\begin{array}{l}232 \\
232\end{array}$ & $\begin{array}{l}1 \\
1\end{array}$ \\
\hline 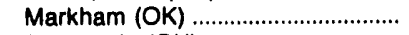 & - & - & - & 29,848 & -- & - & - & - & - & - & - \\
\hline 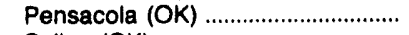 & -- & - & - & 51,343 & - & - & - & -- & -- & -- & -- \\
\hline Salina (OK) & - & - & - & $-3,238$ & - & - & -- & - & -- & - & - \\
\hline Granite Falis City Of ............................ & - & - & - & 363 & - & - & - & - & - & - & - \\
\hline Granite Falls (MN) & - & - & - & 363 & -- & - & -- & -- & -- & - & -- \\
\hline Grant County P U D No $2 \ldots \ldots \ldots \ldots$ & - & - & - & 610,819 & - & - & - & - & - & - & - \\
\hline Pec Hdwks (WA) & - & - & - & 2,113 & -- & -- & -- & - & -. & -- & -- \\
\hline 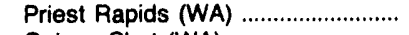 & - & - & -- & 305,838 & - & - & - & -- & -- & - & -- \\
\hline 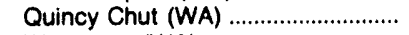 & - & - & -- & 2,694 & -- & - & - & -- & - & -- & -- \\
\hline 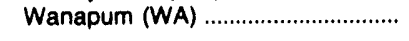 & - & - & - & 300,174 & - & - & - & - & - & -- & - \\
\hline Green Mountain Power & & & & & & & & & & & \\
\hline Corporation & - & 161 & - & 6,181 & - & - & - & 1 & - & - & 11 \\
\hline Berlin (VT) & - & 146 & - & -- & -- & - & -- & 1 & - & - & 8 \\
\hline Bolton Falls (VT) & -- & - & - & - & -- & - & - & -- & -- & - & -- \\
\hline Carthusians (VT) & -- & -- & - & - & -- & - & - & - & -- & -- & -- \\
\hline Colchester (VT) & - & -- & - & $-\bar{n}$ & - & - & - & - & -- & -- & 1 \\
\hline 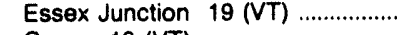 & - & - & -- & 2,693 & - & - & - & - & -- & - & 1 \\
\hline Gorge $18(\mathrm{VT})$ & -- & - & -- & 228 & -- & - & -- & -- & -- & -- & -- \\
\hline 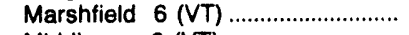 & -- & - & $-\infty$ & 490 & - & -- & - & -- & -- & -- & - \\
\hline 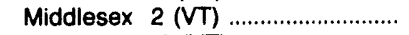 & -- & - & -- & 666 & - & - & - & - & -- & -- & -- \\
\hline 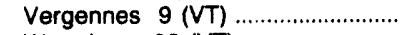 & -- & 15 & -- & 612 & - & - & - & • & -- & -- & * \\
\hline 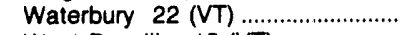 & -- & -- & - & 1,164 & - & - & - & -- & -- & - & -- \\
\hline 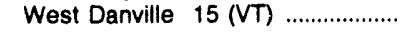 & -- & - & -- & 328 & - & - & -- & - & - & - & -- \\
\hline Greenfield Municipal Utilities ......... & - & 17 & - & - & - & - & - & * & - & - & - \\
\hline 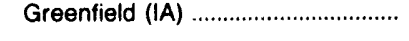 & - & 17 & - & - & - & - & - & * & -- & - & * \\
\hline Greenport Village of ............................. & -- & -22 & - & - & - & - & - & - & - & - & * \\
\hline 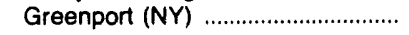 & - & -22 & -- & - & -- & - & - & - & - & -- & • \\
\hline Greensburg Mun Lt \& Pwr ............ & - & 21 & - & - & - & - & - & - & * & - & 1 \\
\hline 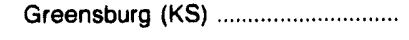 & -- & 21 & $\cdots$ & - & - & - & - & - & - & -- & 1 \\
\hline Greenville Electric Dept ................... & - & - & 7,912 & - & - & - & - & - & 100 & - & 11 \\
\hline 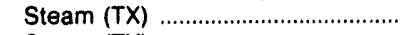 & - & - & -22 & - & -- & - & - & - & & - & 1 \\
\hline 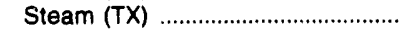 & -- & -- & 7,934 & - & - & - & - & - & 100 & -- & 11 \\
\hline Greenwood Utilities Commission & - & - & 637 & - & - & - & - & - & 12 & 11 & 6 \\
\hline 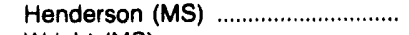 & - & - & 637 & - & - & - & -- & -- & 12 & 10 & 5 \\
\hline 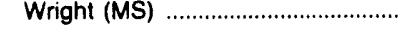 & - & - & - & - & - & - & -- & -- & - & 1 & 2 \\
\hline Gresham Mun Light Plant ............... & - & - & - & 417 & - & - & - & - & - & - & - \\
\hline 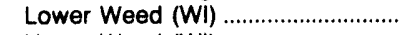 & - & - & -- & 203 & - & - & - & - & -- & -- & -_ \\
\hline 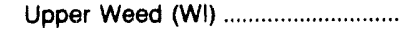 & -- & -- & - & 214 & - & - & -- & - & -- & -- & - \\
\hline Grundy Center Mun Lt \& Pwr ........ & - & 11 & 6 & - & - & - & - & - & - & - & - \\
\hline Grundy Center (IA) & - & 11 & 6 & - & - & -- & -- & * & - & - & * \\
\hline Guadalupe-Blanco River Auth ...... & - & - & - & 5,426 & - & - & - & - & - & - & - \\
\hline Abbott Tp $3(T X)$ & - & - & - & 758 & -- & - & - & - & - & -- & -- \\
\hline 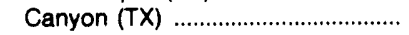 & -- & -- & - & 920 & - & -- & - & -- & -- & -- & -- \\
\hline 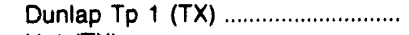 & - & - & - & 1,181 & - & - & -- & -- & - & - & -- \\
\hline $\mathrm{H}-4$ (TX) & -- & -- & - & 506 & - & - & - & -- & -- & -- & -- \\
\hline $\mathrm{H}-5(\mathrm{TX})$ & -- & -- & -- & 699 & -- & - & -- & - & - & -- & -. \\
\hline Nolte $(T X)$ & - & -- & - & 649 & -- & -- & -- & -- & -- & -- & -- \\
\hline 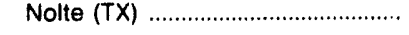 & -- & - & - & 713 & - & - & -- & -- & -- & -- & -- \\
\hline 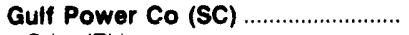 & 696,779 & 1,115 & 542 & - & - & - & 310 & 2 & 6 & 176 & 5 \\
\hline Crist (FL) & 444,223 & 872 & 542 & - & - & -- & 188 & 2 & 6 & 125 & 1 \\
\hline 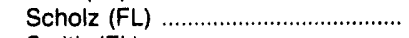 & 26,731 & 27 & - & - & - & - & 14 & $:$ & -- & 5 & • \\
\hline Smith (FL) . . & 225,825 & 216 & -- & - & -- & -- & 97 & * & - & 46 & 4 \\
\hline Gulf States Utilities Company ...... & 370,890 & 111,846 & $1,871,074$ & 22,880 & 586,021 & - & 234 & * & 18,806 & 139 & 455 \\
\hline
\end{tabular}

See footnotes at end of table. 
Table 62. U.S. Electric Utility Net Generation, Fuel Consumption, and Fuel Stocks by Company and Plant, September 1993 (Continued)

\begin{tabular}{|c|c|c|c|c|c|c|c|c|c|c|c|}
\hline \multirow{2}{*}{$\begin{array}{c}\text { Company (Holding Company) } \\
\text { Plant (State) }\end{array}$} & \multicolumn{6}{|c|}{$\begin{array}{l}\text { Ceneration } \\
\text { (thousand kllowatthours) }\end{array}$} & \multicolumn{3}{|c|}{$\begin{array}{l}\text { Consumption } \\
\text { (thousand) }\end{array}$} & \multicolumn{2}{|c|}{$\begin{array}{c}\text { Stocks } \\
\text { (thousand) }\end{array}$} \\
\hline & Coal & Petroloum & Gas & Hydro & Nuclear & Other' & $\begin{array}{c}\text { Coal } \\
\text { (short } \\
\text { tons) }\end{array}$ & $\begin{array}{l}\text { Potro- } \\
\text { leum } \\
\text { (bbls) }\end{array}$ & $\begin{array}{l}\text { Gas } \\
\text { (Mct) }\end{array}$ & $\begin{array}{c}\text { Coal } \\
\text { (short } \\
\text { tons) }\end{array}$ & $\begin{array}{l}\text { Petro- } \\
\text { leum } \\
\text { (bbls) }\end{array}$ \\
\hline \multicolumn{12}{|l|}{ Culf States Utillitios Company } \\
\hline $\begin{array}{l}\text { Lewis Creek (TX) } \\
\text { Lovisiana } 1 \text { (LA) }\end{array}$ & $\overline{-}$ & $\overline{-}$ & $\begin{array}{l}235,896 \\
120,895\end{array}$ & $\overline{-}$ & $\overline{-}$ & & - & - & 2,414 & -- & 34 \\
\hline 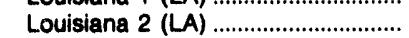 & - & -- & 120,090 & $\overline{-}$ & $\overline{-}$ & $\overline{-}$ & $\overline{-}$ & - & 1,059 & - & $\overline{-}$ \\
\hline Neches $(T X)$ & -- & -- & -- & -_ & $=$ & $\overline{-}$ & $\overline{-}$ & $\overline{-}$ & $\overline{--}$ & $\overline{-}$ & -- \\
\hline Nelson, R S (LA) ................................ & 370,890 & 111,842 & 249,166 & - & - & -- & 234 & 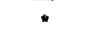 & 2,534 & 133 & 112 \\
\hline 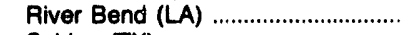 & -- & - & - & - & 586,021 & - & - & - & -- & -- & -- \\
\hline Sabine (TX) & -- & 4 & 846,841 & $\overline{0}$ & - & - & -- & • & 8,503 & -- & 103 \\
\hline 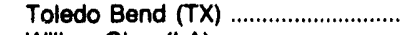 & -- & - & $-\overline{-}$ & 22,880 & - & -- & -- & - & - & -- & - \\
\hline 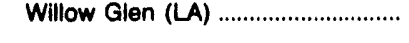 & - & - & 418,276 & - & - & - & -- & - & 4,296 & - & 205 \\
\hline Gwitchyaa Zhee Utility Company & - & 202 & - & - & - & - & - & * & - & - & * \\
\hline 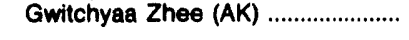 & -- & 202 & -- & - & - & - & - & • & -- & - & * \\
\hline GPU Nuclear Corporation ................ & - & - & - & - & 541,580 & - & - & - & - & - & - \\
\hline Oyster Creek (NJ) & -- & - & -- & - & 437,183 & - & - & - & - & - & -- \\
\hline 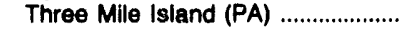 & - & - & - & - & 104,397 & - & - & - & - & - & - \\
\hline Hageratown Mun Elec Lt Plant .... & - & - & - & - & - & - & - & - & - & - & - \\
\hline 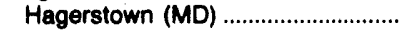 & - & - & -- & - & - & - & - & -- & -- & -- & -- \\
\hline \multicolumn{12}{|l|}{ Haines Llght And Power } \\
\hline Company ................................. & - & 895 & - & - & - & - & - & 2 & - & - & - \\
\hline Haines (AK) & - & 895 & - & - & - & - & -- & 2 & - & -- & -- \\
\hline Halatad Municipal Utilitios ............... & - & - & - & - & - & - & - & - & - & -- & * \\
\hline Halstad (MN) & - & - & -- & - & - & - & - & -- & - & -- & • \\
\hline 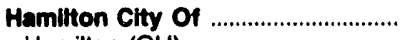 & 30,598 & 23 & 1,056 & 41,598 & - & - & 16 & * & 16 & 8 & 3 \\
\hline Hamilton $(\mathrm{OH})$ & 30,598 & 23 & 1,056 & - & - & - & 16 & * & 16 & 8 & \\
\hline 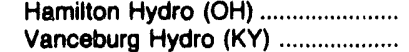 & $\overline{-}$ & $\overline{-}$ & -- & $\overline{41,598}$ & $\overline{-}$ & $\overline{-}$ & $\overline{-}$ & $\overline{-}$ & $\overline{-}$ & -- & -- \\
\hline Hardwlck Eloctric Department ..... & - & - & - & & - & - & - & - & & & * \\
\hline 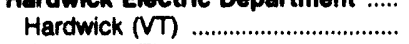 & - & $=$ & - & -20 & $=$ & $=$ & $\overline{-}$ & $\overline{-}$ & $\overline{-}$ & $=$ & . \\
\hline 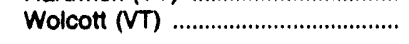 & - & - & - & 257 & - & - & - & - & - & - & -- \\
\hline Hart Hydro Electric Dept ................. & - & - & - & 2 & - & - & - & • & - & - & - \\
\hline Hart (MI) & - & - & - & - & - & - & - & - & -- & -- & • \\
\hline 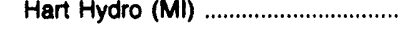 & -- & - & - & 2 & - & -- & - & -- & - & - & -- \\
\hline 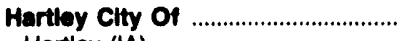 & - & - & - & - & - & - & - & - & - & - & - \\
\hline 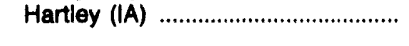 & - & - & -- & - & - & -- & -- & -- & -- & -- & -- \\
\hline 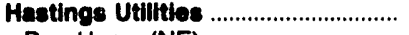 & 30,704 & 34 & - & - & - & - & 21 & " & - & 76 & 7 \\
\hline 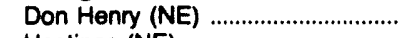 & $-\overline{0}$ & - & - & - & - & - & - & -- & -- & & 2 \\
\hline 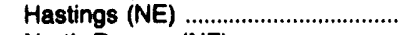 & 30,704 & 34 & - & - & - & -- & 21 & " & -- & 76 & 2 \\
\hline North Denver (NE) .................................... & - & - & -- & - & - & - & - & - & - & -- & 4 \\
\hline Hawall Eloctric Lught Co, Inc ......... & - & 45,327 & - & 738 & - & - & - & 99 & - & - & 71 \\
\hline Kanoelehua $(\mathrm{HI})$ & - & 2,829 & - & - & -- & - & - & 6 & -- & - & 3 \\
\hline 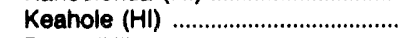 & - & 9,669 & -- & - & - & -- & - & 21 & -- & -- & 5 \\
\hline 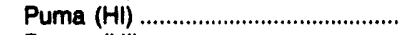 & - & 16,233 & -- & - & -- & -- & - & 38 & -- & -- & 16 \\
\hline 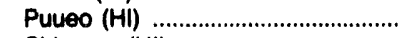 & - & - & - & 700 & - & - & -- & - & -- & -- & \\
\hline 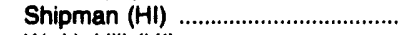 & - & 1,383 & - & - & - & -- & - & 4 & -- & -- & 6 \\
\hline 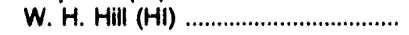 & - & 13,856 & -- & - & - & - & -- & 28 & -- & - & 39 \\
\hline 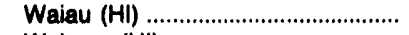 & - & - & - & 38 & - & - & - & - & -- & -- & -- \\
\hline 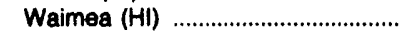 & - & 1,357 & - & - & - & -- & - & 3 & -- & -- & 1 \\
\hline Hawalian Electric Company, Inc .. & - & 388,812 & - & - & - & - & - & 665 & - & - & 667 \\
\hline - Central Storage * & - & $\bar{x} z$ & - & -- & - & - & - & & -- & -- & 167 \\
\hline 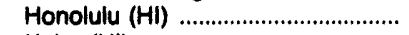 & -- & 15,740 & - & - & - & - & - & 33 & -- & -- & 37 \\
\hline Kahe $(H I)$ & - & 284,368 & - & - & - & - & - & 459 & -- & - & 323 \\
\hline 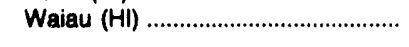 & -- & 98,704 & - & - & - & - & -- & 173 & - & - & 140 \\
\hline 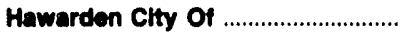 & - & - & - & - & - & - & - & - & - & - & - \\
\hline Hawarden $(\mid A)$ & - & - & -- & - & - & - & - & -- & - & - & -- \\
\hline
\end{tabular}

See footnotes at end of table. 
Table 62. U.S. Electric Utllity Net Generation, Fuel Consumption, and Fuel Stocks by Company and Plant, September 1993 (Continued)

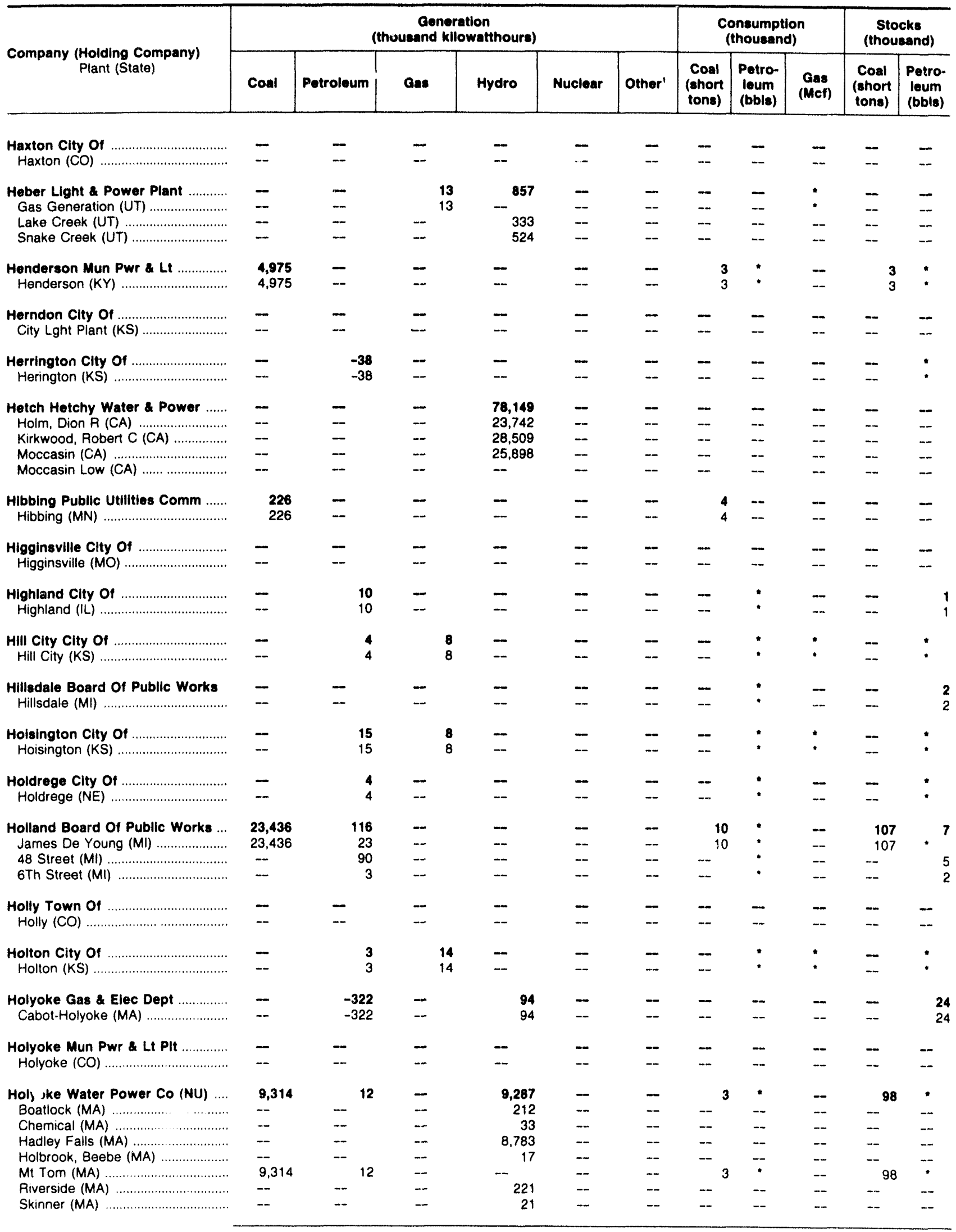

See footnotes at end of table. 
Table 62. U.S. Electric Utility Net Generation, Fuel Consumption, and Fuel Stocks by Company and Plant, September 1993 (Continued)

\begin{tabular}{|c|c|c|c|c|c|c|c|c|c|c|c|}
\hline \multirow{2}{*}{$\begin{array}{c}\text { Company (Holding Company) } \\
\text { Plant (State) }\end{array}$} & \multicolumn{6}{|c|}{$\begin{array}{l}\text { Generation } \\
\text { (thousand kllowatthours) }\end{array}$} & \multicolumn{3}{|c|}{$\begin{array}{l}\text { Consumption } \\
\text { (thousand) }\end{array}$} & \multicolumn{2}{|c|}{$\begin{array}{c}\text { Stocks } \\
\text { (thousand) }\end{array}$} \\
\hline & Coal & Petroleum & Gas & Hydro & Nuclear & Other' & $\begin{array}{l}\text { Coal } \\
\text { (short } \\
\text { tons) }\end{array}$ & $\begin{array}{l}\text { Petro- } \\
\text { leum } \\
\text { (bbls) }\end{array}$ & $\begin{array}{l}\text { Gas } \\
\text { (Mct) }\end{array}$ & $\begin{array}{l}\text { Coal } \\
\text { (short } \\
\text { tons) }\end{array}$ & $\begin{array}{l}\text { Potro- } \\
\text { leum } \\
\text { (bbls) }\end{array}$ \\
\hline 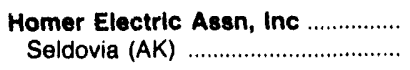 & $\overline{-}$ & $\begin{array}{l}31 \\
31\end{array}$ & $\overline{-}$ & $\overline{-}$ & - & $\overline{-}$ & $\overline{-}$ & : & $\overline{-}$ & - & : \\
\hline $\begin{array}{c}\text { Homestead City of } \\
\text { G W Ivey (FL) }\end{array}$ & $\overline{-}$ & $\begin{array}{l}494 \\
494\end{array}$ & $\begin{array}{l}4,595 \\
4,595\end{array}$ & $\overline{-}$ & $\overline{-}$ & $\overline{-}$ & $\overline{-}$ & $\begin{array}{l}1 \\
1\end{array}$ & $\begin{array}{l}56 \\
56\end{array}$ & $\overline{-}$ & $\begin{array}{l}3 \\
3\end{array}$ \\
\hline $\begin{array}{l}\text { Hoosler Energy, Ind Statewide } \\
\text { Rec } \\
\text { Merom (IN) } \\
\text { Ratts (IN) }\end{array}$ & $\begin{array}{r}506,562 \\
436,934 \\
69,628\end{array}$ & $\begin{array}{r}319 \\
289 \\
30\end{array}$ & $\begin{array}{l}- \\
-\end{array}$ & $\overline{-}$ & $\begin{array}{l}-- \\
--\end{array}$ & $\bar{z}$ & $\begin{array}{r}250 \\
219 \\
31\end{array}$ & $\begin{array}{r}1 \\
+1\end{array}$ & $\overline{-}$ & $\begin{array}{r}493 \\
418 \\
76\end{array}$ & - \\
\hline $\begin{array}{l}\text { Hopkinton Municipal Utllity } \\
\text { Hopkinton (IA) }\end{array}$ & $\ddot{-}$ & $\overline{-}$ & $\overline{-}$ & $\overline{-}$ & $\overline{-}$ & $\overline{-}$ & $\overline{-}$ & $\overline{-}$ & $\overline{-}$ & $\overline{-}$ & : \\
\hline $\begin{array}{l}\text { Houma Light \& Water Plant } \\
\text { Houma (LA) }\end{array}$ & $\overline{-}$ & $\begin{array}{l}-6 \\
-6\end{array}$ & $\begin{array}{l}8,849 \\
8,849\end{array}$ & $\overline{-}$ & $\overline{-}$ & $\overline{-}$ & - & : & $\begin{array}{r}114 \\
114\end{array}$ & $\overline{-}$ & $\begin{array}{l}6 \\
6\end{array}$ \\
\hline $\begin{array}{l}\text { Houston Lighting Power Co } \\
\text { Central Storage } \\
\text { Bertron, Sam (TX) } \\
\text { Cedar Bayou (TX) } \\
\text { Clarke, Hiram (TX) } \\
\text { Deepwater (TX) } \\
\text { Greens Bayou (TX) } \\
\text { Limestone (TX) } \\
\text { Parish, W A (TX) } \\
\text { Robinson, P H (TX) } \\
\text { South Texas (TX) } \\
\text { Webster (TX) } \\
\text { Wharton, TH (TX) }\end{array}$ & $\begin{array}{c}2,015,522 \\
-- \\
-- \\
-- \\
-- \\
- \\
- \\
635,403 \\
1,380,119 \\
-- \\
-- \\
-- \\
--\end{array}$ & 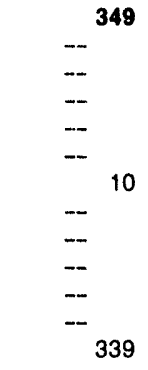 & $\begin{array}{r}3,036,727 \\
158,198 \\
986,817 \\
2,639 \\
25,490 \\
190,013 \\
8,220 \\
494,817 \\
778,933 \\
-- \\
145,370 \\
246,230\end{array}$ & $\begin{array}{l}- \\
\overline{-} \\
= \\
= \\
\overline{-} \\
\overline{-} \\
\overline{-} \\
\overline{-} \\
-\end{array}$ & $\begin{array}{l}-19,382 \\
- \\
- \\
- \\
- \\
- \\
- \\
- \\
- \\
-19,382 \\
--\end{array}$ & $\begin{array}{l}- \\
\overline{-} \\
\overline{-} \\
\overline{-} \\
\overline{-} \\
\overline{-} \\
\overline{-} \\
\overline{-}\end{array}$ & $\begin{array}{l}1,386 \\
-- \\
- \\
- \\
- \\
- \\
- \\
527 \\
859 \\
-- \\
= \\
-\end{array}$ & 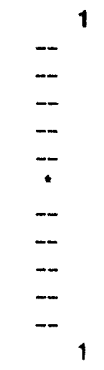 & $\begin{array}{r}30,440 \\
- \\
1,778 \\
9,841 \\
47 \\
287 \\
1,954 \\
85 \\
4,819 \\
7,634 \\
\overline{1,491} \\
2,503\end{array}$ & $\begin{array}{l}1,521 \\
-- \\
-- \\
-- \\
-- \\
- \\
556 \\
965 \\
-- \\
- \\
-\end{array}$ & $\begin{array}{r}688 \\
21 \\
64 \\
277 \\
-- \\
-- \\
128 \\
32 \\
7 \\
116 \\
-- \\
-- \\
\end{array}$ \\
\hline $\begin{array}{l}\text { Hudson Light \& Power Dept ........... } \\
\text { Cherry Street (MA) }\end{array}$ & $\overline{-}$ & $\begin{array}{l}44 \\
44\end{array}$ & $\begin{array}{l}199 \\
199\end{array}$ & $\overline{-}$ & $\overline{-}$ & $\overline{-}$ & $\overline{-}$ & 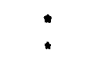 & $\begin{array}{l}2 \\
2\end{array}$ & $\overline{-}$ & \\
\hline $\begin{array}{l}\text { Hughes Power Light Company } \\
\text { Hughes (AK) }\end{array}$ & $\begin{array}{l}-- \\
--\end{array}$ & $\begin{array}{l}20 \\
20\end{array}$ & $\begin{array}{l}-- \\
--\end{array}$ & $\overline{-}$ & $\overline{-}$ & $\overline{-}$ & $\overline{-}$ & : & - & - & : \\
\hline $\begin{array}{l}\text { Hugoton City of } \\
\text { Hugoton (KS) } \\
\text { Hugoton } \$ 2 \text { (KS) }\end{array}$ & $\begin{array}{l}-- \\
-\end{array}$ & $\begin{array}{r}184 \\
19 \\
165\end{array}$ & $\begin{array}{r}2,062 \\
210 \\
1,852\end{array}$ & $\begin{array}{l}\overline{-} \\
\overline{-}\end{array}$ & $\overline{-}$ & $\bar{m}$ & $\overline{-}$ & : & $\begin{array}{r}21 \\
2 \\
18\end{array}$ & $\overline{-}$ & . 1 \\
\hline $\begin{array}{l}\text { Hutchinson Utillties Comm } \\
\text { Plant No. } 1 \text { (MN) } \\
\text { Plant No. } 2 \text { (MN) }\end{array}$ & $\begin{array}{l}-- \\
--\end{array}$ & $\begin{array}{l}3 \\
3\end{array}$ & $\begin{array}{r}17 \\
-\quad 17 \\
-\quad\end{array}$ & $=$ & $=$ & $\begin{array}{l}- \\
\overline{-}\end{array}$ & $\overline{-}$ & $:$ & $:$ & $\overline{-}$ & $\begin{array}{l}4 \\
1 \\
3\end{array}$ \\
\hline $\begin{array}{l}\text { Hydro Dev Group Inc } \\
\# 3 \text { Mill (NY) } \\
\# 6 \text { Mill (NY) } \\
\text { Copenhagen (NY) } \\
\text { Dexter (NY) } \\
\text { Diamond Island (NY) } \\
\text { Fowler (NY) } \\
\text { Goodyear Lake (NY) } \\
\text { Hailesboro (NY) } \\
\text { Pyrites (NY) } \\
\text { Pyrites } \# 2 \text { (NY) } \\
\text { Theresa (NY) }\end{array}$ & $\begin{array}{l}- \\
\overline{-} \\
-- \\
- \\
\overline{-} \\
- \\
- \\
- \\
- \\
-\end{array}$ & $\begin{array}{l}\overline{-} \\
\overline{-} \\
\overline{-} \\
\overline{-} \\
\overline{-} \\
- \\
\overline{-} \\
-\end{array}$ & $\begin{array}{l}- \\
-- \\
- \\
- \\
- \\
\overline{-} \\
\overline{-} \\
- \\
- \\
-\end{array}$ & $\begin{array}{r}5,460 \\
330 \\
350 \\
644 \\
1,116 \\
395 \\
425 \\
75 \\
677 \\
129 \\
1,155 \\
164\end{array}$ & $\begin{array}{l}- \\
- \\
- \\
- \\
- \\
- \\
- \\
- \\
- \\
- \\
- \\
-\end{array}$ & $\begin{array}{c}- \\
- \\
- \\
- \\
- \\
\overline{-} \\
\overline{-} \\
- \\
\overline{-} \\
\overline{-} \\
-\end{array}$ & $\begin{array}{l}- \\
-- \\
-- \\
-- \\
-- \\
-- \\
-- \\
-- \\
-- \\
-\end{array}$ & $\begin{array}{l}- \\
- \\
-- \\
- \\
- \\
- \\
- \\
- \\
- \\
- \\
- \\
-\end{array}$ & $\begin{array}{c}- \\
- \\
- \\
- \\
- \\
-- \\
- \\
- \\
- \\
\overline{-} \\
\overline{-}\end{array}$ & $\begin{array}{l}- \\
- \\
-- \\
-- \\
- \\
- \\
- \\
- \\
- \\
- \\
- \\
-\end{array}$ & $\begin{array}{l}-- \\
- \\
- \\
- \\
- \\
- \\
- \\
\overline{-} \\
- \\
- \\
- \\
-\end{array}$ \\
\hline $\begin{array}{l}\text { Hyrum Clty Corporation } \\
\text { Hyrum (UT) }\end{array}$ & $\overline{-}$ & $\overline{-}$ & $\overline{-}$ & $\begin{array}{l}273 \\
273\end{array}$ & $\overline{-}$ & $\overline{-}$ & $\overline{-}$ & $\overline{-}$ & $\overline{-}$ & $\overline{-}$ & 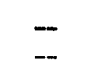 \\
\hline 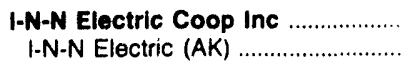 & $\overline{-}$ & $\begin{array}{l}200 \\
200\end{array}$ & $\overline{-}$ & - & $\overline{-}$ & $\overline{-}$ & $\overline{-}$ & 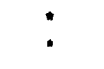 & $\overline{-}$ & - & 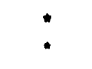 \\
\hline 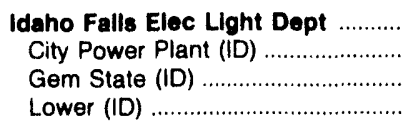 & $\begin{array}{l}- \\
\overline{-} \\
-\end{array}$ & $\begin{array}{l}- \\
\bar{z} \\
\overline{-}\end{array}$ & $\begin{array}{l}-- \\
-- \\
--\end{array}$ & $\begin{array}{r}22,429 \\
4,103 \\
9,837 \\
-1\end{array}$ & $\begin{array}{l}\overline{-} \\
\bar{z}\end{array}$ & $\begin{array}{l}- \\
\overline{-} \\
-\end{array}$ & $\begin{array}{l}\overline{-} \\
\overline{-} \\
\overline{-}\end{array}$ & $\begin{array}{l}- \\
- \\
-\end{array}$ & $\begin{array}{l}- \\
- \\
--\end{array}$ & $\begin{array}{l}- \\
-- \\
-\end{array}$ & $\begin{array}{l}- \\
\overline{-} \\
-\end{array}$ \\
\hline
\end{tabular}

See footnotes at end of table. 
Table 62. U.S. Electric Utility Net Generation, Fuel Consumption, and Fuel Stocks by Company and Plant, September 1993 (Continued)

\begin{tabular}{|c|c|c|c|c|c|c|c|c|c|c|c|}
\hline \multirow{2}{*}{$\begin{array}{c}\text { Company (Holding Company) } \\
\text { Plant (State) }\end{array}$} & \multicolumn{6}{|c|}{$\begin{array}{l}\text { Generation } \\
\text { (thousand kllowatthours) }\end{array}$} & \multicolumn{3}{|c|}{$\begin{array}{l}\text { Consumption } \\
\text { (thousand) }\end{array}$} & \multicolumn{2}{|c|}{$\begin{array}{c}\text { Stocks } \\
\text { (thousand) }\end{array}$} \\
\hline & Coal & Petroleum & Cas & Hydro & Nuclear & Other' & $\begin{array}{l}\text { Coal } \\
\text { (short } \\
\text { tons) }\end{array}$ & $\begin{array}{l}\text { Potro- } \\
\text { leum } \\
\text { (bbls) }\end{array}$ & $\begin{array}{c}\text { Gas } \\
\text { (Mcf) }\end{array}$ & $\begin{array}{c}\text { Coal } \\
\text { (short } \\
\text { tons) }\end{array}$ & $\begin{array}{l}\text { Potro- } \\
\text { loum } \\
\text { (bble) }\end{array}$ \\
\hline
\end{tabular}

\section{Idaho Falls Elec LIght Dept}

Lower \#1 (ID)

Upper Power Plant (ID)

Idaho Power Company

American Falls (ID)

Bliss (ID)

Brownlee (ID)

Cascade (ID)

Clear Lake (ID)

Hells Canyon (OR)

Lower Malad (ID)

Lower Salmon (ID)

Milner (ID)

Oxbow (OR)

Salmon (ID)

Shoshone Falls (ID)

Strike, C J (ID)

Swan Falls (ID)

Thousand Springs (ID)

Twin Falls (ID)

Upper Malad (ID)

Upper Salmon (ID)

Upper Salmon (ID)

Wood River (ID)

IIIinols Power Company ............... $\quad 876,884$

Baldwin (IL)

Clinton (IL)

Havana (IL)

Hennepin (IL)

Oglesby (IL)

Stallings (IL)

Vermilion (IL)

Wood River (IL)

Imperial City of

Imperial (NE)

Imperial Irrigation District

Brawley (CA)

Coachella (CA)

Double Weir (CA)

Drop No 1 (CA)

Drop No. 5 (CA)

Drop 2 (CA)

Drop 3 (CA)

Drop 4 (CA)

E Highline (CA)

El Centro (CA)

Pilot Knob (CA)

Rockwood (CA)

Turnip (CA) ..

Independence City of

Independence (IA)

Independence Clty Of

Blue Valley (MO)

Jackson Square (MO)

Missouri City (MO)

Station $\mathrm{H}(\mathrm{MO})$

Station I (MO)

Indiana Michigan Power Co

(AEP) .......... 1,793,379

Berrien Springs (MI)

Breed (IN)

2,521

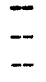

2,135

$-\cdots$

$-$

$\begin{array}{rr}103 & - \\ 20 & 1,613\end{array}$

$-\quad-$

- $\quad 85,348$

$-$

$+$

$-$

\begin{tabular}{rrrrr}
4,015 & - & - & - & - \\
4,475 & - & - & - & - \\
563,365 & - & - & - & - \\
36,445 & - & - & - & - \\
28,552 & - & - & - & - \\
164,639 & - & - & - & - \\
6,684 & - & - & - & - \\
1,367 & - & - & - & - \\
144,038 & - & - & - & - \\
9,969 & - & - & - & - \\
19,327 & - & - & - & - \\
510 & - & - & - & - \\
74,268 & - & - & - & - \\
\hline & - & - & - & - \\
7,585 & - & - & - & - \\
31,791 & - & - & - & - \\
6,901 & - & - & - & - \\
4,700 & - & - & - & - \\
-4 & - & - & - & - \\
5,418 & - & - & - & - \\
11,015 & - & - & - & - \\
10,160 & - & - & - & - \\
-- & - & - & - & -
\end{tabular}

$\begin{array}{lll}-- & -- & - \\ -- & -- & - \\ - & - & \end{array}$

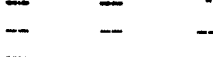

$-\infty \quad-\infty$

- $\quad-$

-- $\quad-$

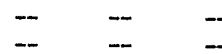

$-\quad-$

$+\quad--$

-- - -

-- $\quad--$

$-$

$-$

$-$

$-$

$-$

$$
\begin{array}{ccccc}
443,566 & - & 451 & & 6 \\
-- & -- & 252 & & 1 \\
443,566 & -- & - & - & \\
-- & - & 32 & & 4 \\
- & - & 57 & - & \\
- & -- & - & \vdots \\
- & - & - & - \\
- & - & 43 & \vdots \\
- & -- & 69
\end{array}
$$

$-$

$-$

$-$

511

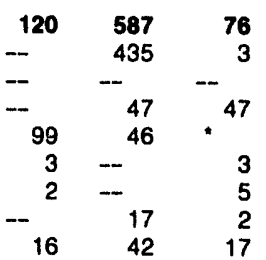

See footnotes at ena of table. 
Table 62. U.S. Electric Utility Net Generation, Fuel Consumption, and Fuel Stocks by Company and Plant, September 1993 (Continued)

\begin{tabular}{|c|c|c|c|c|c|c|c|c|c|c|c|}
\hline \multirow{2}{*}{$\begin{array}{c}\text { Company (Holding Company) } \\
\text { Plant (State) }\end{array}$} & \multicolumn{6}{|c|}{$\begin{array}{c}\text { Generation } \\
\text { (thousand kllowatthours) }\end{array}$} & \multicolumn{3}{|c|}{$\begin{array}{l}\text { Consumption } \\
\text { (thousand) }\end{array}$} & \multicolumn{2}{|c|}{$\begin{array}{l}\text { Stocks } \\
\text { (thousand) }\end{array}$} \\
\hline & Coal & Potroloum & Gas & Hydro & Nuclear & Other' & $\begin{array}{c}\text { Coal } \\
\text { (short } \\
\text { tons) }\end{array}$ & $\begin{array}{l}\text { Potro- } \\
\text { loum } \\
\text { (bbls) }\end{array}$ & $\begin{array}{l}\text { Gas } \\
\text { (Mcf) }\end{array}$ & $\begin{array}{l}\text { Coal } \\
\text { (short } \\
\text { tons) }\end{array}$ & $\begin{array}{l}\text { Petro- } \\
\text { leum } \\
\text { (bbls) }\end{array}$ \\
\hline \multicolumn{12}{|l|}{$\begin{array}{l}\text { Indiana \&ichigan Power Co } \\
\text { (AEP) }\end{array}$} \\
\hline 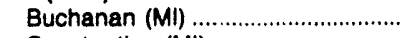 & - & -- & - & 1,873 & - & -- & -- & - & - & -- & -- \\
\hline 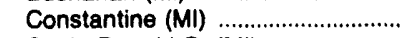 & -- & -- & - & 496 & - & -- & - & - & -- & - & -- \\
\hline Cook, Donald C. (MI) ......................... & -- & - & - & -- & $1,183,846$ & - & - & -- & -- & -- & -- \\
\hline 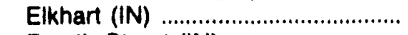 & - & - & -- & 1,853 & -- & -- & - & - & -- & -- & \multirow{2}{*}{$-{ }_{1}$} \\
\hline 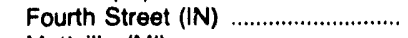 & - & 6 & - & - & - & - & -- & $\cdot$ & -- & -- & \\
\hline 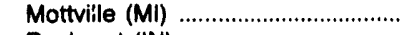 & -- & - & -- & 757 & -- & - & -- & - & -- & -- & \multirow{3}{*}{$\begin{array}{r}-- \\
46 \\
15\end{array}$} \\
\hline 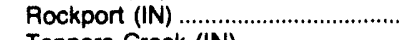 & $1,560,003$ & 2,254 & -- & -- & - & - & 946 & \multirow[t]{2}{*}{4} & -- & 660 & \\
\hline 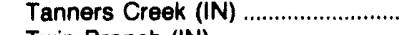 & 233,376 & 261 & $-\infty$ & $-\overline{0}$ & -- & -- & 101 & & -- & 547 & \\
\hline 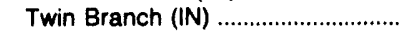 & - & -- & - & 3,021 & - & - & -- & -- & -- & -- & -- \\
\hline Indlana Municipal Power Agency & - & 5 & 22 & - & - & - & - & - & - & - & 7 \\
\hline Anderson (IN) & -- & 5 & 22 & - & - & - & - & • & • & - & 7 \\
\hline Indiana-Kentucky Electric Corp ... & 689,301 & 84 & - & - & - & - & 310 & : & - & 398 & 4 \\
\hline Clifty Creek (IN) & 689,301 & 84 & - & - & - & - & 310 & $\cdot$ & -- & 398 & 4 \\
\hline Indianapolis Pwr \& Lt Co .................. & 969,735 & 2,374 & 3,001 & - & - & - & 460 & 5 & - & 1,624 & 29 \\
\hline 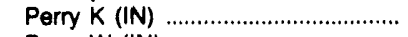 & -612 & - & 3,001 & - & - & - & - & - & - & 66 & 4 \\
\hline 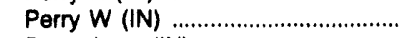 & - & -57 & - & - & - & - & - & -- & -- & -- & $i$ \\
\hline 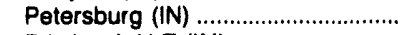 & 657,893 & 1,711 & -- & - & - & -- & 310 & 3 & -- & 1,235 & 3 \\
\hline Pritchard, $\mathrm{H} T(\mathrm{IN}) \ldots \ldots \ldots \ldots \ldots \ldots \ldots \ldots$ & 42,721 & 373 & - & - & - & - & 22 & 1 & -- & 116 & 10 \\
\hline 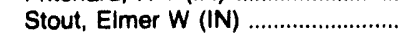 & 269,733 & 347 & -- & - & -- & - & 128 & 1 & - & 207 & 12 \\
\hline Indianola Mun Light \& Pwr ............ & - & -19 & -8 & - & - & - & - & - & - & - & 11 \\
\hline 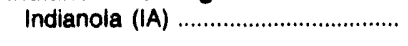 & - & -19 & -8 & -- & - & - & - & • & * & - & 11 \\
\hline Interatate Power Company ............ & 87,333 & 227 & 32,856 & - & - & - & 54 & 1 & 373 & 334 & 31 \\
\hline Dubuque (IA) & 1,121 & -6 & 142 & -- & - & -- & 1 & - & 2 & 30 & $\cdot$ \\
\hline 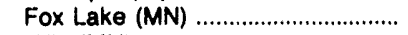 & -- & -10 & 31,085 & - & - & - & -- & - & 352 & 19 & 22 \\
\hline Hills (MN) & - & -6 & - & - & - & - & - & - & -- & - &. \\
\hline Kapp, $M L(I A)$ & 54,543 & - & 1,629 & -- & - & - & 28 & -- & 19 & 122 & -- \\
\hline 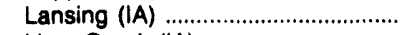 & 31,669 & 320 & - & - & -- & - & 26 & 1 & -- & 163 & 2 \\
\hline Lime Creek (IA) ................................ & - & -54 & -- & - & - & -- & - & - & -- & -- & 4 \\
\hline 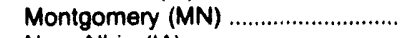 & - & -8 & -- & - & - & - & $-\cdot$ & - & -- & -- & 2 \\
\hline 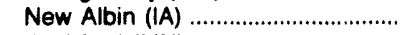 & - & -3 & - & - & - & - & - & - & -- & -- & - \\
\hline Rushford (MN) ................................... & -- & -6 & -- & - & - & - & - & -- & -- & -- & $\cdot$ \\
\hline Iola Electric System .............................. & - & 14 & - & - & - & - & - & * & 6 & - & 1 \\
\hline 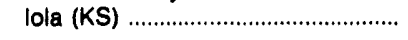 & - & 14 & -- & - & - & - & -- & - & 6 & -- & 1 \\
\hline lowa Eloctric Lt \& Pwr Co .............. & 112,493 & 608 & 2,438 & 755 & $-3,579$ & 2,270 & 82 & 2 & 51 & 351 & 51 \\
\hline Ames (IA) & - & - & - & - & - & - & - & -- & - & -- & 1 \\
\hline Anamosa (IA) & -- & -- & -- & 53 & - & - & -- & - & -- & -- & -- \\
\hline 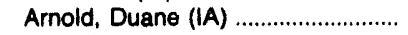 & -- & - & -- & - & $-3,579$ & - & -- & - & -- & -_ & -- \\
\hline 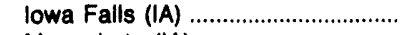 & - & - & - & 318 & - & - & - & -- & -. & -- & - \\
\hline 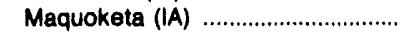 & -- & - & - & 384 & - & -- & -- & -- & -- & -- & -- \\
\hline 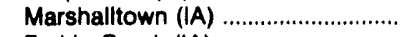 & - & 556 & - & - & - & -- & - & 1 & -- & -- & 47 \\
\hline 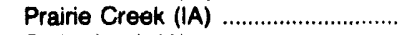 & 73,353 & 52 & 215 & - & - & - & 52 & - & 3 & 133 & 2 \\
\hline Sutherland $(\mathrm{IA})$ & 30,129 & - & -- & - & - & -- & 22 & - & -. & 218 & -- \\
\hline 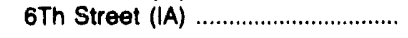 & 9,011 & - & 2,223 & - & - & 2,270 & 8 & - & 48 & 1 & 2 \\
\hline lowa Southern Utilities Co ............. & 384,155 & 78 & -20 & - & - & - & 235 & - & - & 460 & 17 \\
\hline 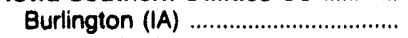 & 21,277 & 90 & - & - & - & - & 14 & - & -. & 72 & \\
\hline 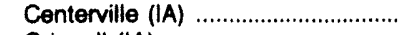 & - & -16 & - & - & - & - & - & -- & -- & & \\
\hline 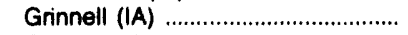 & - & - & -20 & - & - & -- & - & -- & -- & -. & - \\
\hline 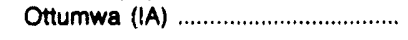 & 362,878 & 4 & - & - & - & - & 221 & - & -- & 388 & 17 \\
\hline lowa-lilinols Gas \& Eloctric Co .... & 301,336 & 8 & 1,849 & - & - & - & 193 & - & 23 & 243 & 13 \\
\hline 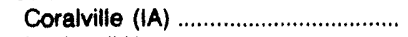 & - & - & -51 & - & - & - & - & -- & - & -- & \\
\hline 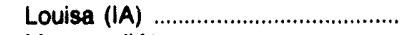 & 288,971 & 8 & 621 & - & - & - & 186 & - & 6 & 223 & 10 \\
\hline 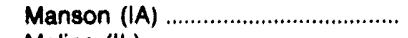 & - & - & $-\infty$ & - & - & - & - & - & -- & -- & - \\
\hline 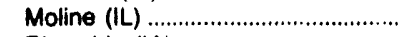 & - & - & -60 & - & - & - & - & -- & -- & -- & 2 \\
\hline 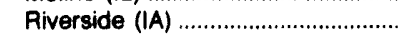 & 12,365 & - & 1,339 & - & - & - & 7 & - & 17 & 20 & $-{ }^{2}$ \\
\hline Ipawich Mun Lt Dept ....................... & - & 48 & 59 & - & - & - & - & - & 1 & - & 1 \\
\hline 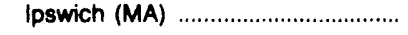 & - & 48 & 59 & - & - & - & -- & - & 1 & -- & 1 \\
\hline
\end{tabular}

See footnotes at end of tabie. 
Table 62. U.S. Electric UtIllty Net Generation, Fuel Consumption, and Fuel Stocks by Company and Plant, September 1993 (Continued)

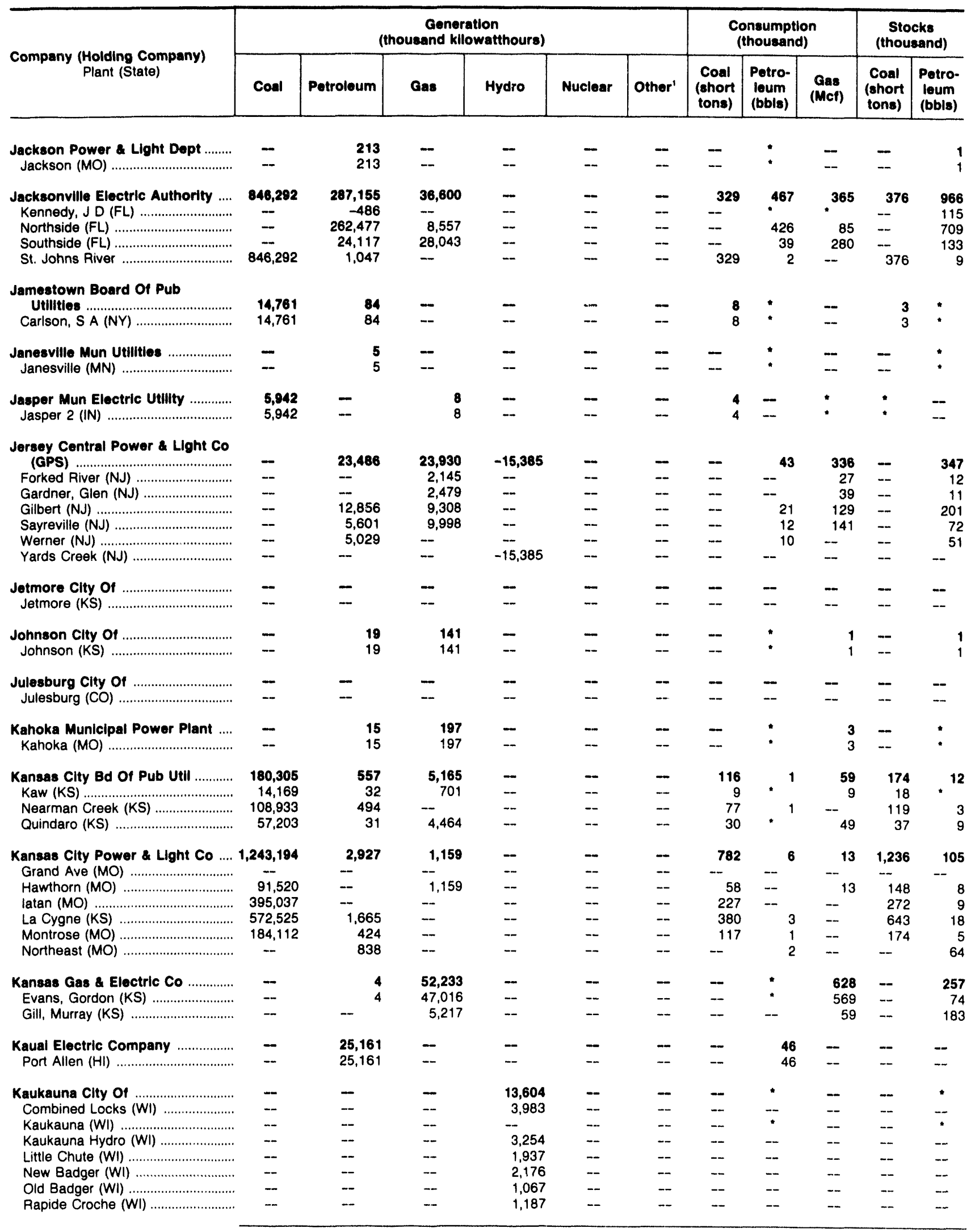

See footnotes at end of table. 
Table 62. U.S. Electric Utllity Net Generation, Fuel Consumption, and Fuel Stocks by Company and Plant, September 1993 (Continued)

\begin{tabular}{|c|c|c|c|c|c|c|c|c|c|c|c|}
\hline \multirow[b]{2}{*}{$\begin{array}{c}\text { Company (Holding Company) } \\
\text { Plarit (State) }\end{array}$} & \multicolumn{6}{|c|}{$\begin{array}{c}\text { Generation } \\
\text { (thouseand kllowatthours) }\end{array}$} & \multicolumn{3}{|c|}{$\begin{array}{l}\text { Consumption } \\
\text { (thousand) }\end{array}$} & \multicolumn{2}{|c|}{$\begin{array}{c}\text { Stocks } \\
\text { (thousand) }\end{array}$} \\
\hline & Coal & Potroleum & Gas & Hydro & Nuclear & Other' & $\begin{array}{c}\text { Coal } \\
\text { (short } \\
\text { tons) }\end{array}$ & $\begin{array}{l}\text { Petro- } \\
\text { leum } \\
\text { (bbls) }\end{array}$ & $\begin{array}{l}\text { Gas } \\
\text { (Mct) }\end{array}$ & $\begin{array}{l}\text { Coal } \\
\text { (short } \\
\text { tons) }\end{array}$ & $\begin{array}{l}\text { Petro- } \\
\text { leum } \\
\text { (bbls) }\end{array}$ \\
\hline $\begin{array}{l}\text { Kennett Board Of Public Works... } \\
\text { Kennett (MO) }\end{array}$ & $\overline{-}$ & $\overline{-}$ & $\overline{-}$ & $\overline{-}$ & $\overline{-}$ & $\overline{-}$ & $\overline{-}$ & * & : & -- & $\begin{array}{l}6 \\
6\end{array}$ \\
\hline $\begin{array}{l}\text { Kentucky Power Co (AEP) } \\
\text { Big Sandy (KY) }\end{array}$ & $\begin{array}{l}\mathbf{5 3 5 , 5 5 8} \\
\mathbf{5 3 5 , 5 5 8}\end{array}$ & $\begin{array}{l}2,002 \\
2,002\end{array}$ & $\overline{-}$ & $\overline{-}$ & $\overline{-}$ & $\overline{-}$ & $\begin{array}{l}217 \\
217\end{array}$ & $\begin{array}{l}3 \\
3\end{array}$ & $\overline{-}$ & $\begin{array}{l}381 \\
381\end{array}$ & $\begin{array}{l}8 \\
8\end{array}$ \\
\hline $\begin{array}{l}\text { Kentucky Utillties Company } \\
\text { Brown, E W (KY) } \\
\text { Dix Dam (KY) } \\
\text { Ghent (KY) } \\
\text { Green River (KY) } \\
\text { Haefling (KY) } \\
\text { Lock } 7(\mathrm{KY}) \\
\text { Pineville (KY) } \\
\text { Tyrone (KY) }\end{array}$ & $\begin{array}{r}1,100,978 \\
172,487 \\
-- \\
855,531 \\
68,534 \\
-- \\
\overline{2,076} \\
2,350\end{array}$ & $\begin{array}{r}401 \\
35 \\
-\quad 447 \\
-\quad 31 \\
-\quad \\
-\quad 2 \\
-114\end{array}$ & $\begin{array}{l}-12 \\
= \\
= \\
- \\
- \\
- \\
--\end{array}$ & 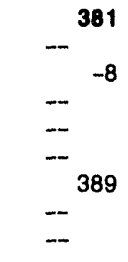 & $\begin{array}{l}\overline{-} \\
\overline{-} \\
\overline{-} \\
\overline{-} \\
\overline{-} \\
-\end{array}$ & $\begin{array}{l}-- \\
- \\
- \\
- \\
- \\
-- \\
--\end{array}$ & $\begin{array}{r}465 \\
-75 \\
- \\
355 \\
-33 \\
- \\
-\quad 1 \\
1\end{array}$ & $\begin{array}{l}4 \\
-- \\
: \\
\vdots \\
\vdots\end{array}$ & $\begin{array}{l}\overline{-} \\
\overline{-} \\
\overline{-} \\
\overline{-} \\
\overline{-} \\
-\end{array}$ & $\begin{array}{r}1,184 \\
222 \\
-- \\
880 \\
-54 \\
-- \\
-- \\
\quad 6 \\
22\end{array}$ & $\begin{array}{r}35 \\
-\quad 5 \\
-\quad 11 \\
1 \\
-\quad 6 \\
-11\end{array}$ \\
\hline $\begin{array}{l}\text { Kenyon Municipal Utilities } \\
\text { Kenyon (MN) }\end{array}$ & $\overline{-}$ & $\overline{-}$ & $\overline{-}$ & $\overline{-}$ & $\overline{--}$ & $\overline{--}$ & $\overline{-}$ & $\overline{--}$ & $\overline{-}$ & $\begin{array}{l}-- \\
--\end{array}$ & $\begin{array}{l}-- \\
-\end{array}$ \\
\hline $\begin{array}{l}\text { Ketchlkan Public Utilities } \\
\text { Beaver Falls (AK) } \\
\text { Ketchikan (AK) } \\
\text { Ketchikan (AK) } \\
\text { Silvis (AK) } \\
\text { Swan Lake (AK) } \\
\text { Totem Bight (AK) }\end{array}$ & $\begin{array}{l}\overline{-} \\
\overline{-} \\
\overline{-} \\
\overline{-}\end{array}$ & $\begin{array}{l}4,586 \\
\overline{-} \\
\overline{4,603} \\
- \\
- \\
-17\end{array}$ & $\begin{array}{l}-- \\
-- \\
= \\
- \\
-\end{array}$ & $\begin{array}{r}5,818 \\
2,113 \\
4119 \\
-\quad 582 \\
2,704 \\
--\end{array}$ & $\begin{array}{l}- \\
\overline{-} \\
\overline{-} \\
\overline{-} \\
-\end{array}$ & $\begin{array}{l}-- \\
-- \\
-- \\
-- \\
--\end{array}$ & $\begin{array}{l}- \\
-- \\
-- \\
-- \\
--\end{array}$ & $\begin{array}{l}-- \\
-- \\
-- \\
--\end{array}$ & $\begin{array}{l}- \\
- \\
-- \\
- \\
- \\
-\end{array}$ & $\begin{array}{l}- \\
\overline{-} \\
= \\
= \\
-\end{array}$ & $\begin{array}{l}{ }^{4} \\
- \\
- \\
- \\
=\end{array}$ \\
\hline $\begin{array}{l}\text { Key West, Clty Of } \\
\text { Big Pine (FL) } \\
\text { Cudjoe (FL) } \\
\text { Key West (FL) } \\
\text { Stock Island (FL) } \\
\text { Stock Island D I (FL) }\end{array}$ & $\begin{array}{l}\overline{-} \\
\overline{-} \\
\overline{-} \\
\overline{-}\end{array}$ & $\begin{array}{r}7,279 \\
18 \\
67 \\
14 \\
5,930 \\
1,250\end{array}$ & $\begin{array}{l}- \\
\overline{-} \\
- \\
\overline{-} \\
--\end{array}$ & $\begin{array}{l}\overline{-} \\
\bar{z} \\
\overline{-} \\
\overline{-}\end{array}$ & $\begin{array}{l}\overline{-} \\
\overline{-} \\
\bar{z} \\
\overline{-}\end{array}$ & $\begin{array}{l}- \\
-- \\
-- \\
- \\
--\end{array}$ & $\begin{array}{l}-- \\
-- \\
-- \\
-- \\
--\end{array}$ & $:^{17}$ & $\begin{array}{l}- \\
\overline{-} \\
\overline{-} \\
-- \\
-\end{array}$ & $\begin{array}{l}- \\
=- \\
= \\
= \\
-\end{array}$ & $\begin{array}{r}64 \\
1 \\
1 \\
16 \\
-\quad 46\end{array}$ \\
\hline $\begin{array}{l}\text { Kimball City of } \\
\text { Kimball (NE) }\end{array}$ & $\overline{-}$ & $\overline{-}$ & $\overline{-}$ & $\overline{-}$ & $\overline{-}$ & $\overline{-}$ & $\overline{-}$ & 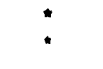 & : & $\overline{-}$ & : \\
\hline $\begin{array}{c}\text { Kimballton Mun Light Plant } \ldots \ldots \ldots \ldots . . . . . \\
\text { Kimballton (IA) }\end{array}$ & $\overline{-}$ & $\overline{-}$ & $\overline{-}$ & $\overline{-}$ & $\overline{-}$ & $\overline{-}$ & $\overline{-}$ & $\overline{--}$ & $\overline{-}$ & $\overline{-}$ & $\ddot{--}$ \\
\hline $\begin{array}{l}\text { Kingfisher Clty Of } \\
\text { Kingtisher (OK) }\end{array}$ & $\overline{-}$ & $\begin{array}{l}5 \\
5\end{array}$ & $\overline{-}$ & $\overline{-}$ & $\overline{-}$ & $\overline{-}$ & - & : & $\overline{-}$ & - & : \\
\hline $\begin{array}{l}\text { KIngman City of } \\
\text { Kingman (KS) }\end{array}$ & $\overline{-}$ & $\begin{array}{l}139 \\
139\end{array}$ & $\begin{array}{l}3,073 \\
3,073\end{array}$ & $\overline{-}$ & $\overline{-}$ & $\overline{-}$ & $\overline{-}$ & $\dot{2}$ & $\begin{array}{l}34 \\
34\end{array}$ & $\overline{-}$ & 1 \\
\hline $\begin{array}{l}\text { Kings River Conservation Dist ...... } \\
\text { Pine Flat (CA) }\end{array}$ & $\overline{-}$ & $\overline{-}$ & $\overline{-}$ & $\begin{array}{l}42,707 \\
42,707\end{array}$ & $\overline{-}$ & $\overline{-}$ & $\overline{-}$ & $\overline{-}$ & 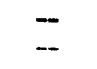 & $\overline{-}$ & $\overline{-}$ \\
\hline $\begin{array}{l}\text { Kissimmoe City Of } \\
\text { Kissimmee (FL) }\end{array}$ & $\overline{-}$ & $\begin{array}{l}1 \\
1\end{array}$ & $\begin{array}{l}25,000 \\
25,000\end{array}$ & $\overline{-}$ & $\overline{-}$ & $\overline{-}$ & $\overline{-}$ & * & $\begin{array}{l}299 \\
299\end{array}$ & $\overline{-}$ & $\begin{array}{l}22 \\
22\end{array}$ \\
\hline $\begin{array}{l}\text { Kodlak Electric Assn, Inc } \\
\text { Kodiac A (AK) } \\
\text { Port Lions (AK) } \\
\text { Terror Lake AK) }\end{array}$ & $\begin{array}{l}- \\
-- \\
--\end{array}$ & $\begin{array}{r}37 \\
43 \\
-\quad 6 \\
-\quad\end{array}$ & $\begin{array}{l}- \\
\overline{-} \\
-\end{array}$ & $\begin{array}{l}9,070 \\
\overline{-} \\
\overline{9,070}\end{array}$ & $\begin{array}{l}- \\
\bar{z} \\
\overline{-}\end{array}$ & $\begin{array}{l}- \\
\overline{-} \\
\overline{-}\end{array}$ & $\begin{array}{l}- \\
-- \\
--\end{array}$ & $\begin{array}{l}: \\
- \\
--\end{array}$ & $\begin{array}{l}- \\
-- \\
--\end{array}$ & $\begin{array}{l}- \\
-- \\
-\end{array}$ & $\begin{array}{r}1 \\
- \\
-\end{array}$ \\
\hline 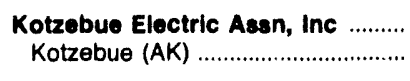 & $\overline{-}$ & $\begin{array}{l}1,481 \\
1,481\end{array}$ & $\overline{-}$ & $\overline{-}$ & $\overline{-}$ & $\overline{-}$ & $\overline{--}$ & $\begin{array}{l}3 \\
3\end{array}$ & $\overline{-}$ & - & $\begin{array}{l}38 \\
38\end{array}$ \\
\hline $\begin{array}{l}\text { La Crosse City of } \\
\text { Larned (KS) }\end{array}$ & $\overline{-}$ & $\overline{-}$ & $\overline{-}$ & $\overline{-}$ & $\overline{-}$ & $\overline{-}$ & $\overline{-}$ & $\overline{-}$ & $\overline{-}$ & - & - \\
\hline $\begin{array}{l}\text { La Junta Municipal Utillties } \\
\text { La Junta }(\mathrm{CO})\end{array}$ & $\overline{-}$ & $\begin{array}{l}-134 \\
-134\end{array}$ & $\overline{-}$ & $\overline{-}$ & $\overline{-}$ & $\overline{-}$ & $\overline{--}$ & : & " & - & 4 \\
\hline $\begin{array}{l}\text { La Plata Electric Assn, The } \\
\text { La Plata }(M O)\end{array}$ & $\overline{-}$ & $\overline{-}$ & $\overline{-}$ & $\overline{-}$ & $\overline{-}$ & $\overline{-}$ & $\overline{-}$ & $\overline{-}$ & $\overline{-}$ & $\overline{-}$ & " \\
\hline
\end{tabular}

See footnotes at end of table. 
Table 62. U.S. Electric Utility Net Generation, Fuel Consumption, and Fuel Stocks by Company and Plant, September 1993 (Continued)

\begin{tabular}{|c|c|c|c|c|c|c|c|c|c|c|c|}
\hline \multirow{2}{*}{$\begin{array}{c}\text { Company (Holding Company) } \\
\text { Plant (State) }\end{array}$} & \multicolumn{6}{|c|}{$\begin{array}{c}\text { Genoration } \\
\text { (thousand kllowatthours) }\end{array}$} & \multicolumn{3}{|c|}{$\begin{array}{c}\text { Consumption } \\
\text { (thousand) }\end{array}$} & \multicolumn{2}{|c|}{$\begin{array}{c}\text { Stocks } \\
\text { (thousand) }\end{array}$} \\
\hline & Coal & Petroloum & Gas & Hydro & Nuclear & Other' & $\begin{array}{c}\text { Coal } \\
\text { (short } \\
\text { tons) }\end{array}$ & $\begin{array}{l}\text { Potro- } \\
\text { loum } \\
\text { (bbls) }\end{array}$ & $\begin{array}{c}\text { Gas } \\
\text { (Mcf) }\end{array}$ & $\begin{array}{l}\text { Coal } \\
\text { (short } \\
\text { tons) }\end{array}$ & $\begin{array}{l}\text { Petro- } \\
\text { leum } \\
\text { (bbls) }\end{array}$ \\
\hline $\begin{array}{l}\text { La Porte Clty Municipal Utilities .. } \\
\text { La Porte (IA) .................................. }\end{array}$ & $\overline{-}$ & $\overline{-}$ & $\overline{-}$ & $\overline{-}$ & $\overline{-}$ & $\overline{-}$ & $\overline{-}$ & $\overline{-}$ & $\overline{-}$ & $\overline{-}$ & : \\
\hline 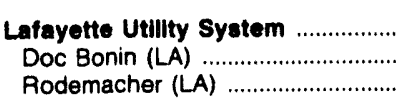 & $\overline{-}$ & $\overline{-}$ & $\begin{array}{r}33,772 \\
33,814 \\
-42\end{array}$ & $\overline{-}$ & $\overline{-}$ & $\overline{-}$ & $\bar{m}$ & $\bar{z}$ & $\begin{array}{r}416 \\
416 \\
--\end{array}$ & $\begin{array}{l}-- \\
-- \\
-\end{array}$ & $\begin{aligned} 120 \\
120 \\
--\end{aligned}$ \\
\hline 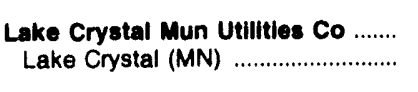 & $\overline{-}$ & $\begin{array}{l}31 \\
31\end{array}$ & $\begin{array}{l}10 \\
10\end{array}$ & $\overline{-}$ & $\overline{-}$ & $\overline{-}$ & $\overline{-}$ & : & $:$ & $\overline{-}$ & : \\
\hline $\begin{array}{l}\text { Lake Lure Elec Pwr Facility ............ } \\
\text { Lake Lure (NC) }\end{array}$ & $\overline{-}$ & - & $\overline{-}$ & $\begin{array}{l}281 \\
281\end{array}$ & $\overline{-}$ & $\overline{-}$ & $\overline{-}$ & $\overline{-}$ & $\overline{-}$ & $\overline{-}$ & $\overline{--}$ \\
\hline 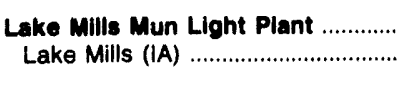 & $\overline{-}$ & $\begin{array}{l}-21 \\
-21\end{array}$ & $\overline{-}$ & $\overline{-}$ & $\overline{-}$ & $\overline{-}$ & $\overline{-}$ & $\overline{-}$ & $\overline{-}$ & $\overline{-}$ & $\begin{array}{l}1 \\
1\end{array}$ \\
\hline 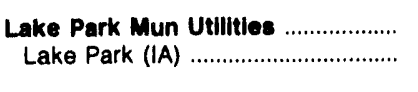 & $\overline{-}$ & $\overline{-}$ & - & $\overline{-}$ & $\overline{-}$ & $\overline{-}$ & $\overline{-}$ & - & - & - & - \\
\hline 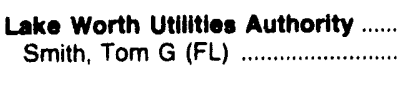 & - & $\begin{array}{l}100 \\
100\end{array}$ & $\begin{array}{l}14,645 \\
14,645\end{array}$ & $\overline{-}$ & $\overline{-}$ & $\overline{-}$ & - & : & $\begin{array}{l}168 \\
168\end{array}$ & $\overline{-}$ & $\begin{array}{l}13 \\
13\end{array}$ \\
\hline 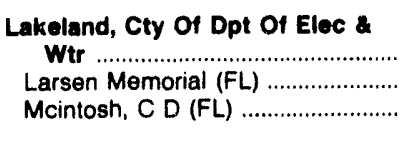 & $\begin{array}{c}199,837 \\
\overline{-} \\
199,837\end{array}$ & $\begin{array}{r}30,494 \\
473 \\
30,021\end{array}$ & $\begin{array}{l}46,637 \\
33,403 \\
13,234\end{array}$ & $\overline{-}$ & $\overline{-}$ & $\overline{-}$ & $\begin{array}{r}79 \\
-- \\
79\end{array}$ & $\begin{array}{r}56 \\
1 \\
55\end{array}$ & $\begin{array}{l}477 \\
322 \\
156\end{array}$ & $\begin{array}{r}92 \\
-92\end{array}$ & $\begin{array}{r}183 \\
76 \\
107\end{array}$ \\
\hline $\begin{array}{l}\text { Lamar Utilities Brd of } \\
\text { Lamar (CO) }\end{array}$ & - & $\overline{-}$ & $\begin{array}{l}6,573 \\
6,573\end{array}$ & $\overline{-}$ & $\overline{-}$ & - & - & $\overline{-}$ & $\begin{array}{l}92 \\
92\end{array}$ & - & $\begin{array}{l}6 \\
6\end{array}$ \\
\hline 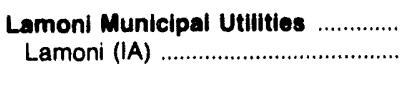 & $\overline{-}$ & - & $\overline{-}$ & $\overline{-}$ & $\overline{-}$ & $\overline{-}$ & - & $\overline{-}$ & $\overline{-}$ & $=$ & $\begin{array}{l}1 \\
1\end{array}$ \\
\hline $\begin{array}{l}\text { Lanesboro Public Utilities } \\
\text { Lansboro (MN) }\end{array}$ & $\overline{-}$ & - & $\overline{-}$ & - & - & $\overline{-}$ & $\overline{-}$ & - & - & $\overline{-}$ & - \\
\hline 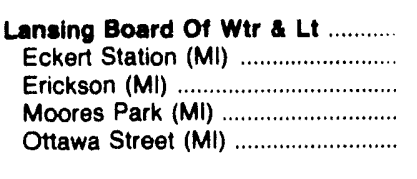 & $\begin{array}{c}119,883 \\
38,897 \\
80,986 \\
-- \\
-\end{array}$ & $\begin{array}{r}293 \\
252 \\
-- \\
--\end{array}$ & $\begin{array}{l}- \\
- \\
- \\
-\end{array}$ & $\begin{array}{l}256 \\
-- \\
--\end{array}$ & $\begin{array}{l}- \\
\overline{-} \\
-\end{array}$ & $\begin{array}{l}- \\
\overline{-} \\
\overline{-}\end{array}$ & $\begin{array}{r}50 \\
19 \\
31 \\
-- \\
--\end{array}$ & $\begin{array}{r}1 \\
. \\
-- \\
--\end{array}$ & $\begin{array}{l}- \\
\overline{-} \\
\overline{-}\end{array}$ & $\begin{array}{r}145 \\
31 \\
114 \\
-- \\
--\end{array}$ & $\begin{array}{r}1 \\
. \\
--\end{array}$ \\
\hline 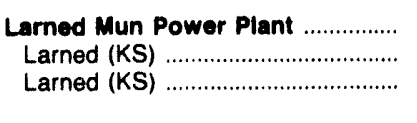 & $\overline{-}$ & $\overline{-}$ & $\begin{array}{r}1,500 \\
-- \\
1,500\end{array}$ & $\overline{-}$ & $\overline{-}$ & $\overline{-}$ & $\begin{array}{l}-- \\
--\end{array}$ & $\overline{-}$ & $\begin{array}{r}23 \\
-- \\
23\end{array}$ & $\overline{--}$ & -4 \\
\hline $\begin{array}{l}\text { Larsen Bay City Of } \\
\text { Larsen (AK) }\end{array}$ & $\overline{-}$ & - & $\overline{-}$ & $\begin{array}{l}24 \\
24\end{array}$ & $\overline{-}$ & $\overline{-}$ & $\overline{-}$ & $\overline{-}$ & $\overline{-}$ & $\overline{--}$ & -- \\
\hline 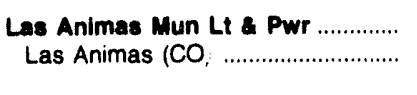 & $\overline{-}$ & $\begin{array}{l}-16 \\
-16\end{array}$ & - & - & $\overline{-}$ & - & $\overline{-}$ & 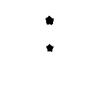 & $:$ & $\overline{-}$ & 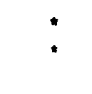 \\
\hline 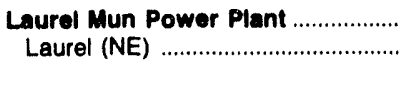 & $\overline{-}$ & $\overline{-}$ & $\overline{-}$ & $\overline{-}$ & $\overline{-}$ & $\overline{-}$ & $\overline{-}$ & $\overline{-}$ & $\overline{-}$ & $\overline{-}$ & : \\
\hline 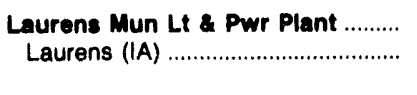 & - & $\overline{-}$ & $\overline{-}$ & $\overline{-}$ & - & $\overline{-}$ & $\overline{-}$ & - & $\overline{-}$ & $\overline{--}$ & -- \\
\hline 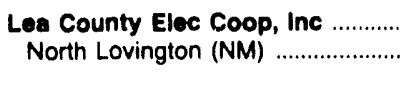 & - & - & $\overline{-}$ & - & - & - & - & $\overline{--}$ & -- & $\overline{-}$ & $\begin{array}{l}-- \\
--\end{array}$ \\
\hline $\begin{array}{c}\text { Lebanon Clty Of } \\
\text { Lebanon }(\mathrm{OH})\end{array}$ & - & $\overline{-}$ & $\overline{-}$ & $\overline{-}$ & $\overline{-}$ & $\overline{-}$ & $\overline{-}$ & - & $\overline{--}$ & $\overline{--}$ & \\
\hline 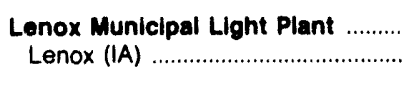 & - & - & -- & - & - & - & - & - & -- & -- & - \\
\hline $\begin{array}{l}\text { Lewiston Public Works } \\
\text { Andro Upper (ME) }\end{array}$ & $\overline{-}$ & $\overline{-}$ & $\overline{-}$ & $\begin{array}{l}264 \\
264\end{array}$ & $\overline{-}$ & $\overline{-}$ & $\overline{-}$ & $\overline{--}$ & $\overline{-}$ & $\overline{-}$ & $\overline{-}$ \\
\hline
\end{tabular}

See footnotes at end of table. 
Table 62. U.S. Electric Utility Net Generation, Fuel Consumption, and Fuel Stocks by Company and Plant, September 1993 (Continued)

\begin{tabular}{|c|c|c|c|c|c|c|c|c|c|c|c|}
\hline \multirow{2}{*}{$\begin{array}{c}\text { Company (Holding Company) } \\
\text { Plant (State) }\end{array}$} & \multicolumn{6}{|c|}{$\begin{array}{c}\text { Generation } \\
\text { (thousand kllowatthours) }\end{array}$} & \multicolumn{3}{|c|}{$\begin{array}{l}\text { Consumption } \\
\text { (thousand) }\end{array}$} & \multicolumn{2}{|c|}{$\begin{array}{c}\text { Stocks } \\
\text { (thousand) }\end{array}$} \\
\hline & Coal & Petroloum & Gas & Hydro & Nuclear & Other' & $\begin{array}{l}\text { Cosal } \\
\text { (short } \\
\text { tona) }\end{array}$ & $\begin{array}{l}\text { Petro- } \\
\text { Ioum } \\
\text { (bble) }\end{array}$ & $\begin{array}{c}\text { Gas } \\
\text { (Mct) }\end{array}$ & $\begin{array}{c}\text { Coal } \\
\text { (ehort } \\
\text { tons) }\end{array}$ & $\begin{array}{l}\text { Petro- } \\
\text { loum } \\
\text { (bbls) }\end{array}$ \\
\hline 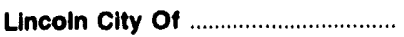 & - & 8 & - & - & - & - & - & * & - & - & $\bullet$ \\
\hline 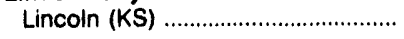 & -- & 8 & - & - & -- & - & -- & • & - & - & * \\
\hline LIncoln Electric Sys ......................... & - & - & 1 & - & - & - & - & - & * & - & 7 \\
\hline 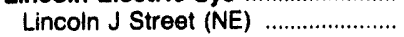 & -- & -- & 1 & -- & - & - & -- & -- & $\cdot$ & - & 3 \\
\hline 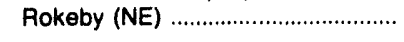 & - & -- & - & - & - & -- & - & -- & - & - & 5 \\
\hline Lindsay Public Works Authority .. & - & - & - & - & - & - & - & - & - & - & - \\
\hline Lindsay $(\mathrm{OK})$ & -- & - & - & - & - & - & - & -- & -- & - & - \\
\hline 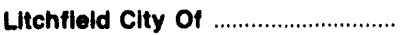 & - & 1 & 3 & - & - & - & - & • & * & -- & * \\
\hline 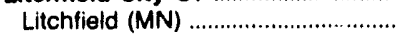 & -- & 1 & 3 & - & - & - & -- & " & • & -- & * \\
\hline Lockhart Power Company .............. & - & - & - & 3,234 & - & - & - & - & - & - & - \\
\hline 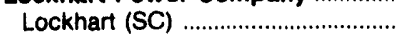 & - & -- & -- & 3,234 & - & - & - & -- & -- & - & -- \\
\hline Lodgepole City Of ............................. & - & - & - & - & - & - & - & - & - & - & - \\
\hline 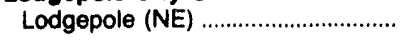 & - & - & - & - & - & -- & -- & - & - & -- & - \\
\hline \multicolumn{12}{|l|}{ Logan Clty Municlpal Elect Lght } \\
\hline 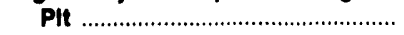 & - & -32 & - & 2,077 & - & - & - & $\bullet$ & - & - & 1 \\
\hline 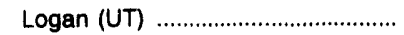 & - & - & -- & 496 & - & -- & $\rightarrow$ & -- & - & -- & - \\
\hline 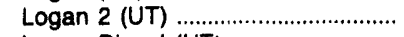 & -- & -- & - & 1,581 & - & -- & -- & -- & -- & -- & - \\
\hline 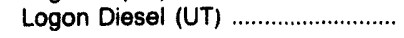 & -- & -32 & -- & - & -- & - & -- & $\bullet$ & -- & -- & 1 \\
\hline Logansport Municipal Utilities ....... & 12,434 & - & - & - & - & - & 7 & - & -- & 8 & 2 \\
\hline 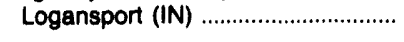 & 12,434 & -- & - & - & - & - & 7 & - & -- & 8 & 2 \\
\hline Long Island Lighting Company .... & - & 410,011 & 401,490 & - & - & - & - & 683 & 4,254 & - & 2,076 \\
\hline 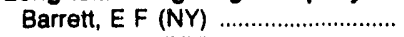 & -- & 48 & 187,458 & - & - & -- & - & $\cdot$ & 1,983 & - & 352 \\
\hline Brookhaven (NY) ............................. & -- & 4,177 & - & - & - & - & -- & 9 & - & - & 36 \\
\hline 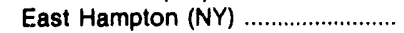 & - & 611 & - & - & -- & -- & - & 1 & - & -- & 1 \\
\hline 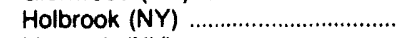 & - & 5,886 & - & - & - & - & - & 13 & - & -- & 103 \\
\hline 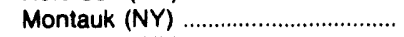 & -- & 1 & - & - & - & - & - & $\cdot$ & -- & -- & 1 \\
\hline 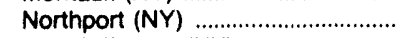 & -- & 249,804 & 174,410 & - & - & - & - & 414 & 1,798 & -- & 1,122 \\
\hline Port Jefferson (NY) ......................... & - & 149,024 & -- & - & - & - & -- & 244 & - & -- & 364 \\
\hline 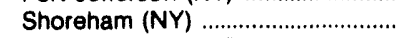 & - & -2 & - & - & - & - & - & 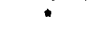 & - & -- & 20 \\
\hline 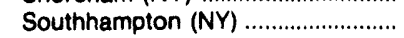 & - & 7 & - & -- & - & -- & -- & • & -- & - & 3 \\
\hline 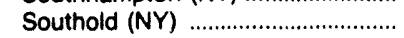 & -- & -11 & - & - & -- & - & - & -- & - & -- & 2 \\
\hline 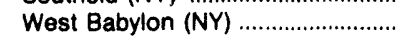 & -- & -9 & - & - & - & - & - & - & - & -- & 10 \\
\hline Longmont Clty 01 & - & - & - & 278 & - & - & - & - & - & - & - \\
\hline Longmont $(\mathrm{CO})$ & - & - & -- & 278 & - & -- & - & -- & - & - & - \\
\hline \multicolumn{12}{|c|}{ Los Angeles Dept Of Water And } \\
\hline 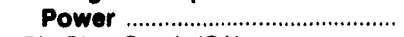 & $1,110,312$ & 1,106 & 499,350 & 56,334 & - & 182 & 449 & 2 & 5,187 & 1,443 & 3,221 \\
\hline 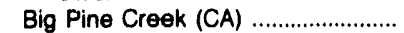 & -. & - & - & 1,010 & - & - & - & - & - & - & - \\
\hline Castaic (CA) & - & - & -- & $-10,357$ & - & - & -- & - & -- & - & -- \\
\hline 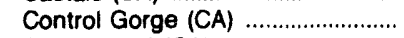 & -- & - & - & 11,270 & - & - & - & - & - & - & -- \\
\hline 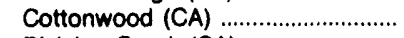 & - & -- & - & 297 & - & - & - & - & - & -- & -- \\
\hline Division Creek (CA) ............................. & - & - & - & 321 & - & - & - & - & -- & -- & - \\
\hline Foothill (CA) & -- & - & - & 1,658 & -- & -- & - & -- & - & - & $\rightarrow$ \\
\hline 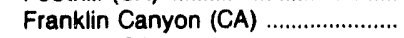 & -- & - & - & 311 & - & - & - & - & -- & - & - \\
\hline 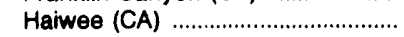 & -- & -- & - & 1,628 & -- & -- & - & - & - & - & - \\
\hline Harbor (CA) & - & - & -129 & - & - & - & - & • & 4 & -- & 15 \\
\hline 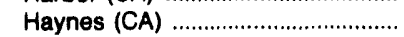 & - & - & 319,451 & - & - & - & - & - & 3,260 & - & 2,361 \\
\hline 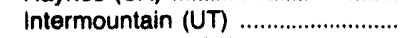 & $1,110,312$ & 1,106 & - & - & - & - & 449 & 2 & - & 1,443 & 8 \\
\hline 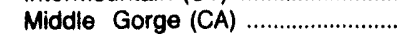 & - & - & - & 11,076 & -- & - & -- & -- & -- & -2 & - \\
\hline 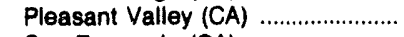 & - & -- & - & 937 & - & - & - & - & - & - & - \\
\hline 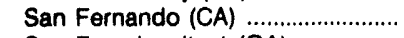 & -- & -- & -- & 2,119 & - & - & -- & -- & - & - & -- \\
\hline San Francisquito 1 (CA) ................... & - & -- & - & 17,484 & - & - & -- & - & - & - & - \\
\hline San Francisquito 2 (CA) ................... & - & - & - & 7,518 & -- & -- & - & -- & - & -- & - \\
\hline 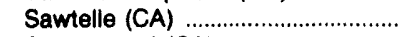 & -- & -- & - & 251 & - & -- & -- & - & -- & - & - \\
\hline 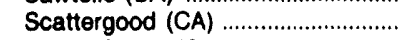 & - & - & 154,836 & - & - & 182 & - & - & 1,605 & - & 391 \\
\hline 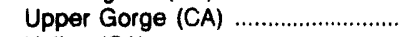 & - & -- & -- & 10,811 & - & - & - & - & - & - & $-\infty$ \\
\hline 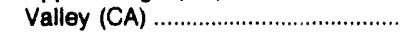 & - & -- & 25,192 & - & - & - & - & -- & 317 & - & 446 \\
\hline
\end{tabular}

See footnotes at end of table. 
Table 62. U.S. Electric Utility Net Generation, Fuel Consumption, and Fuel Stocks by Company and Plant, September 1993 (Continued)

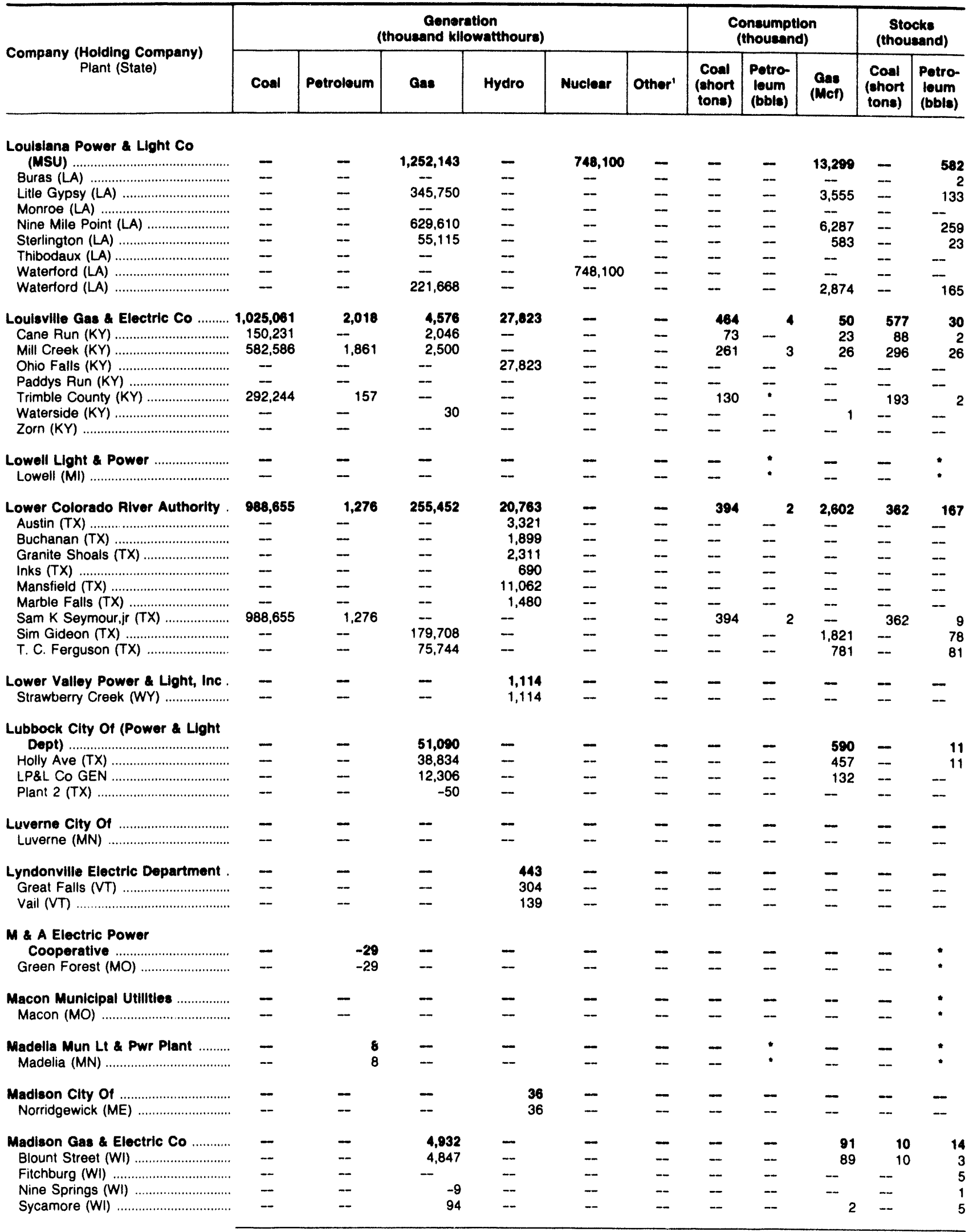

See footnotes at end of table. 
Table 62. U.S. Electric Utility Net Generation, Fuel Consumption, and Fuel Stocks by Company and Plant, September 1993 (Continued)

\begin{tabular}{|c|c|c|c|c|c|c|c|c|c|c|c|}
\hline \multirow{2}{*}{$\begin{array}{c}\text { Company (Holding Company) } \\
\text { Plant (State) }\end{array}$} & \multicolumn{6}{|c|}{$\begin{array}{c}\text { Ceneration } \\
\text { (thousand kllowatthours) }\end{array}$} & \multicolumn{3}{|c|}{$\begin{array}{l}\text { Consumption } \\
\text { (thousand) }\end{array}$} & \multicolumn{2}{|c|}{$\begin{array}{l}\text { Stocks } \\
\text { (thousand) }\end{array}$} \\
\hline & Coal & Petroloum & Gas & Hydro & Nuclear & Other' & $\begin{array}{c}\text { Coal } \\
\text { (short } \\
\text { tons) }\end{array}$ & $\begin{array}{l}\text { Potro- } \\
\text { loum } \\
\text { (bble) }\end{array}$ & $\begin{array}{l}\text { Gas } \\
\text { (Mcf) }\end{array}$ & $\begin{array}{l}\text { Coal } \\
\text { (short } \\
\text { tons) }\end{array}$ & $\begin{array}{l}\text { Potro- } \\
\text { leum } \\
\text { (bbls) }\end{array}$ \\
\hline $\begin{array}{l}\text { Madison Municipal Uttities } \\
\text { Madison (MN) }\end{array}$ & $\overline{-}$ & $\overline{-}$ & $\overline{-}$ & $\overline{-}$ & $\overline{-}$ & $\overline{-}$ & $\overline{-}$ & $\overline{-}$ & $\overline{-}$ & $\overline{-}$ & $\overline{--}$ \\
\hline $\begin{array}{l}\text { Maine Public Service Co } \\
\text { Caribou (ME) } \\
\text { Flos Inn (ME) } \\
\text { Houlton (ME) } \\
\text { Squa Pan (ME) }\end{array}$ & $\begin{array}{l}\overline{-} \\
\bar{z} \\
\overline{-}\end{array}$ & $\begin{array}{r}383 \\
355 \\
22 \\
-\quad 6\end{array}$ & $\begin{array}{l}- \\
-- \\
-- \\
-\end{array}$ & $\begin{array}{r}494 \\
\quad 498 \\
- \\
-\quad \\
-\end{array}$ & $\begin{array}{l}\overline{-} \\
\overline{-} \\
\overline{-}\end{array}$ & $\begin{array}{l}\overline{-} \\
\bar{z} \\
\overline{-}\end{array}$ & $\begin{array}{l}- \\
\overline{-} \\
\overline{-} \\
-\end{array}$ & $\begin{array}{r}1 \\
: \\
-\end{array}$ & $\begin{array}{l}-- \\
-- \\
-- \\
-\end{array}$ & $\begin{array}{l}- \\
-- \\
-- \\
--\end{array}$ & $\dot{ }^{11}+11$ \\
\hline 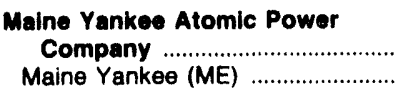 & $\overline{-}$ & $\overline{-}$ & $\overline{-}$ & $\overline{-}$ & $\overline{-}$ & $\overline{-}$ & $\overline{-}$ & $\overline{-}$ & $\ddot{-}$ & $\overline{-}$ & $\ddot{-}$ \\
\hline $\begin{array}{l}\text { Malden City Of } \\
\text { Malden (MO) }\end{array}$ & $\overline{-}$ & $\begin{array}{l}15 \\
15\end{array}$ & $\begin{array}{l}9 \\
9\end{array}$ & $\overline{-}$ & $\overline{-}$ & 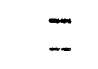 & $\overline{-}$ & : & : & $\overline{-}$ & $\begin{array}{l}1 \\
1\end{array}$ \\
\hline $\begin{array}{l}\text { Mangum City of } \\
\text { Mangum (OK) }\end{array}$ & $\overline{-}$ & $\begin{array}{l}17 \\
17\end{array}$ & $\begin{array}{l}213 \\
213\end{array}$ & $\overline{-}$ & $\overline{-}$ & $\ddot{-}$ & $\overline{-}$ & : & $\begin{array}{l}3 \\
3\end{array}$ & $\overline{-}$ & $\begin{array}{l}1 \\
1\end{array}$ \\
\hline $\begin{array}{l}\text { Manilla Mun Service Dept } \\
\text { Manilla (IA) }\end{array}$ & $\overline{-}$ & $\overline{-}$ & $\overline{-}$ & $\overline{-}$ & $\overline{-}$ & $\overline{-}$ & $\overline{-}$ & $\overline{-}$ & $\overline{-}$ & - & $\ddot{-}$ \\
\hline $\begin{array}{l}\text { Manitowoc Public Utilitios } \\
\text { Manitowoc (WI) }\end{array}$ & $\begin{array}{l}13,435 \\
13,435\end{array}$ & $\begin{array}{l}6,345 \\
6,345\end{array}$ & $\begin{array}{l}36 \\
36\end{array}$ & $\overline{-}$ & $\overline{-}$ & $\overline{-}$ & $\begin{array}{l}7 \\
7\end{array}$ & : & : & $\begin{array}{l}25 \\
25\end{array}$ & $\begin{array}{l}1 \\
1\end{array}$ \\
\hline 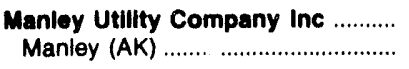 & $\overline{-}$ & $\begin{array}{l}23 \\
23\end{array}$ & $\overline{-}$ & $\overline{-}$ & $\overline{-}$ & $\overline{-}$ & $\overline{-}$ & : & $\overline{-}$ & - & : \\
\hline $\begin{array}{l}\text { Manning Municlpal Llght Plant ..... } \\
\text { Manning (IA) . . }\end{array}$ & $\overline{-}$ & $\overline{-}$ & $\overline{-}$ & $\overline{-}$ & $\overline{-}$ & $\overline{-}$ & $\overline{-}$ & $\overline{-}$ & $\overline{-}$ & $\overline{-}$ & $\overline{-}$ \\
\hline $\begin{array}{l}\text { Manti City Corp Light a Power } \\
\text { Dept ..T. } \\
\text { Lower (UT) } \\
\text { Manti (UT) }\end{array}$ & $\bar{z}$ & $\overline{-}$ & $\overline{-}$ & $\begin{array}{r}862 \\
95 \\
767\end{array}$ & $\overline{-}$ & $\overline{-}$ & $\overline{-}$ & $\overline{-}$ & $\overline{-}$ & $\begin{array}{l}-- \\
- \\
-\end{array}$ & $\begin{array}{l}- \\
-\end{array}$ \\
\hline $\begin{array}{c}\text { Maquoketa Mun Lt Pwr } \\
\text { Maquoketa (IA) }\end{array}$ & $\overline{-}$ & 4 & $\begin{array}{l}173 \\
173\end{array}$ & $\overline{-}$ & $\overline{-}$ & $\overline{-}$ & $\overline{-}$ & : & $\begin{array}{l}2 \\
2\end{array}$ & - & $\begin{array}{l}1 \\
1\end{array}$ \\
\hline 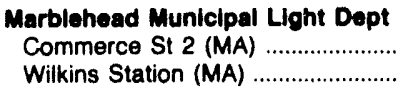 & $\begin{array}{l}- \\
- \\
-\end{array}$ & $\begin{array}{r}12 \\
--\quad 12\end{array}$ & $\begin{array}{l}\overline{-} \\
\overline{-}\end{array}$ & $\overline{-}$ & $\begin{array}{l}- \\
- \\
-\end{array}$ & $\bar{z}$ & $\begin{array}{l}- \\
-\end{array}$ & $\ddot{-}$ & $\begin{array}{l}- \\
- \\
-\end{array}$ & $\bar{z}$ & ${ }^{*} 1$ \\
\hline $\begin{array}{l}\text { Marquatte Bd Of Lt P Pwr } \\
\text { Plant Four (MI) } \\
\text { Plant Two (MI) } \\
\text { Russell, Frank J (MI) } \\
\text { Shiras (MI) }\end{array}$ & $\begin{array}{l}17,854 \\
\overline{--} \\
\overline{17,854}\end{array}$ & $\begin{array}{l}- \\
- \\
- \\
-\end{array}$ & $\begin{array}{l}\overline{-} \\
\overline{-} \\
\overline{-}\end{array}$ & $\begin{array}{r}330 \\
-\quad 260 \\
-\end{array}$ & $\begin{array}{l}\overline{-} \\
\bar{z} \\
\overline{-}\end{array}$ & $\begin{array}{l}\overline{-} \\
\bar{z} \\
-\end{array}$ & $\begin{array}{l}-{ }^{13} \\
-- \\
-{ }_{13}\end{array}$ & $\begin{array}{l}\ddot{-} \\
\ddot{-}\end{array}$ & $\begin{array}{l}- \\
\overline{-} \\
\overline{-} \\
-\end{array}$ & $\begin{array}{l}65 \\
-- \\
-- \\
-65\end{array}$ & $\begin{array}{r}5 \\
-\quad 4 \\
-\quad 1\end{array}$ \\
\hline $\begin{array}{l}\text { Marshall City Wir Elec Works .. } \\
\text { Marshall (MI) }\end{array}$ & $\overline{-}$ & $\overline{-}$ & $\overline{-}$ & $\overline{-}$ & $\overline{-}$ & $\overline{-}$ & $\overline{-}$ & $\overline{-}$ & $\overline{-}$ & $\overline{-}$ & $\begin{array}{l}1 \\
1\end{array}$ \\
\hline 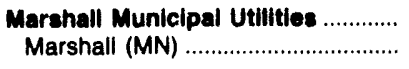 & $\overline{-}$ & $\overline{-}$ & $\overline{-}$ & $\overline{-}$ & $\overline{-}$ & $\overline{-}$ & $\overline{-}$ & $\overline{-}$ & $\overline{-}$ & - & $\overline{-}$ \\
\hline 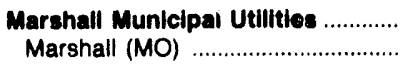 & $\begin{array}{l}5,784 \\
5,784\end{array}$ & $\overline{-}$ & $\begin{array}{l}442 \\
442\end{array}$ & $\overline{-}$ & - & $\overline{-}$ & $\begin{array}{l}3 \\
3\end{array}$ & $\overline{-}$ & $\begin{array}{l}9 \\
9\end{array}$ & $\overline{-}$ & $\begin{array}{l}2 \\
2\end{array}$ \\
\hline $\begin{array}{l}\text { Marshfield Electric \& Water Dept } \\
\text { Wildwood (WI) }\end{array}$ & $\overline{-}$ & $\overline{-}$ & $\overline{-}$ & $\overline{-}$ & $\overline{-}$ & $\overline{-}$ & $\overline{-}$ & $\overline{-}$ & $\overline{-}$ & - & $\overline{-}$ \\
\hline $\begin{array}{l}\text { Martinsville Electric Department .. } \\
\text { Martinsville (VA) .............................. }\end{array}$ & $\overline{-}$ & $\overline{-}$ & $\overline{-}$ & $\begin{array}{l}321 \\
321\end{array}$ & $\overline{-}$ & $\overline{-}$ & $\overline{-}$ & $\overline{-}$ & $\overline{-}$ & $\overline{-}$ & $\overline{-}$ \\
\hline 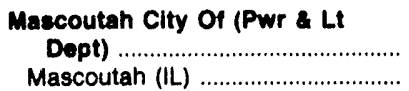 & $\overline{-}$ & $\begin{array}{l}1 \\
1\end{array}$ & $\overline{-}$ & $\overline{-}$ & $\overline{-}$ & $\overline{-}$ & $\overline{-}$ & : & $\overline{-}$ & $\overline{-}$ & $\begin{array}{l}1 \\
1\end{array}$ \\
\hline
\end{tabular}

See footnotes at end of table. 
Table 62. U.S. Electric Utility Net Generation, Fuel Consumption, and Fuel Stocks by Company and Plant, September 1993 (Continued)

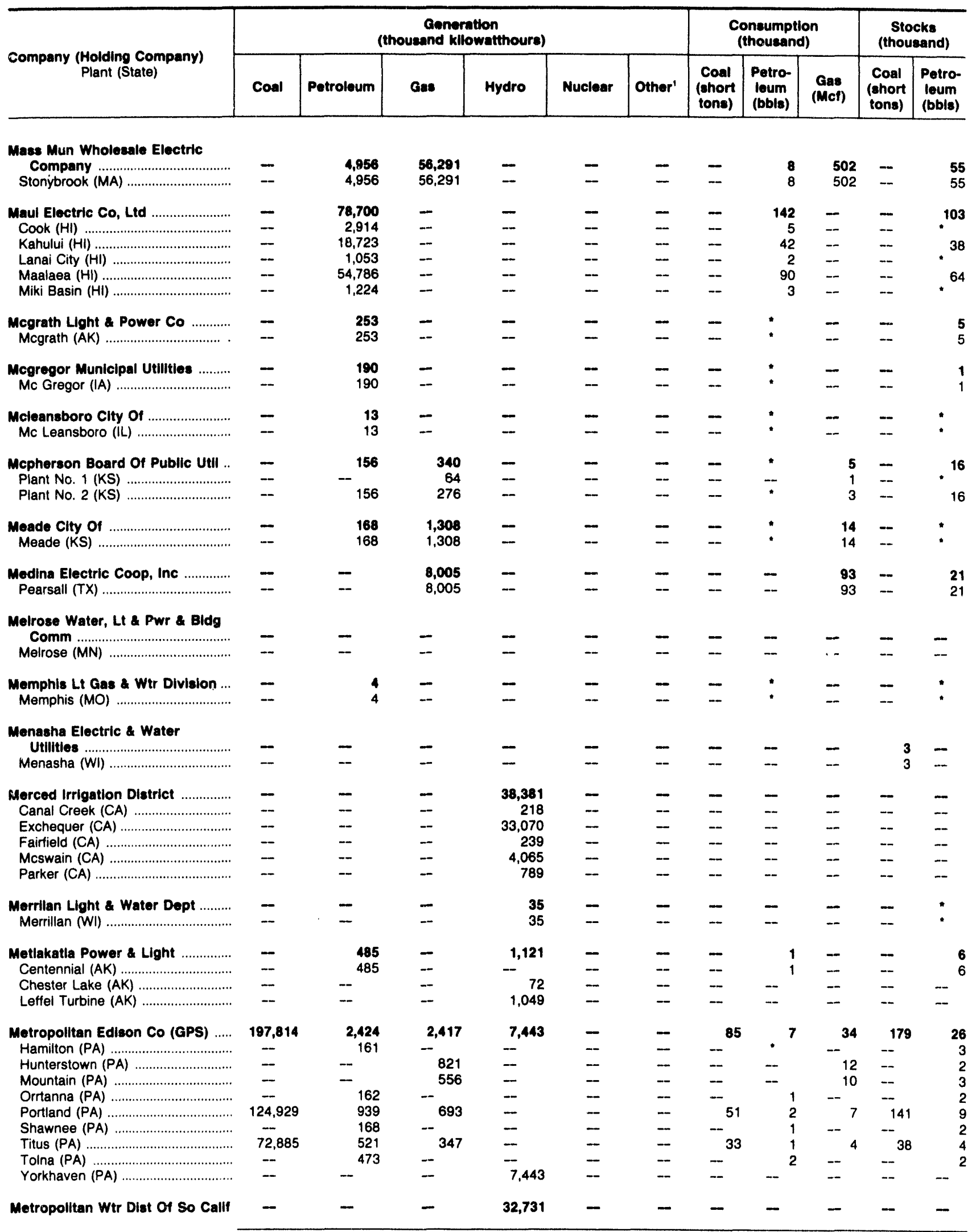

See footnotes at end of table. 
Table 62. U.S. Electric Utility Net Generation, Fuel Consumption, and Fuel Stocks by Company and Plant, September 1993 (Continued)

\begin{tabular}{|c|c|c|c|c|c|c|c|c|c|c|c|}
\hline \multirow{2}{*}{$\begin{array}{c}\text { Company (Holding Company) } \\
\text { Plant (State) }\end{array}$} & \multicolumn{6}{|c|}{$\begin{array}{c}\text { Generation } \\
\text { (thousand kilowatthours) }\end{array}$} & \multicolumn{3}{|c|}{$\begin{array}{l}\text { Consumption } \\
\text { (thousand) }\end{array}$} & \multicolumn{2}{|c|}{$\begin{array}{c}\text { Stocks } \\
\text { (thousand) }\end{array}$} \\
\hline & Coal & Potroleum & Gas & Hydro & Nuclear & Other' & $\begin{array}{c}\text { Coal } \\
\text { (short } \\
\text { tons) }\end{array}$ & $\begin{array}{l}\text { Petro- } \\
\text { leum } \\
\text { (bole) }\end{array}$ & $\begin{array}{c}\text { Gas } \\
\text { (Mcf) }\end{array}$ & $\begin{array}{c}\text { Coal } \\
\text { (ehort } \\
\text { tons) }\end{array}$ & $\begin{array}{l}\text { Petro- } \\
\text { loum } \\
\text { (bbls) }\end{array}$ \\
\hline
\end{tabular}

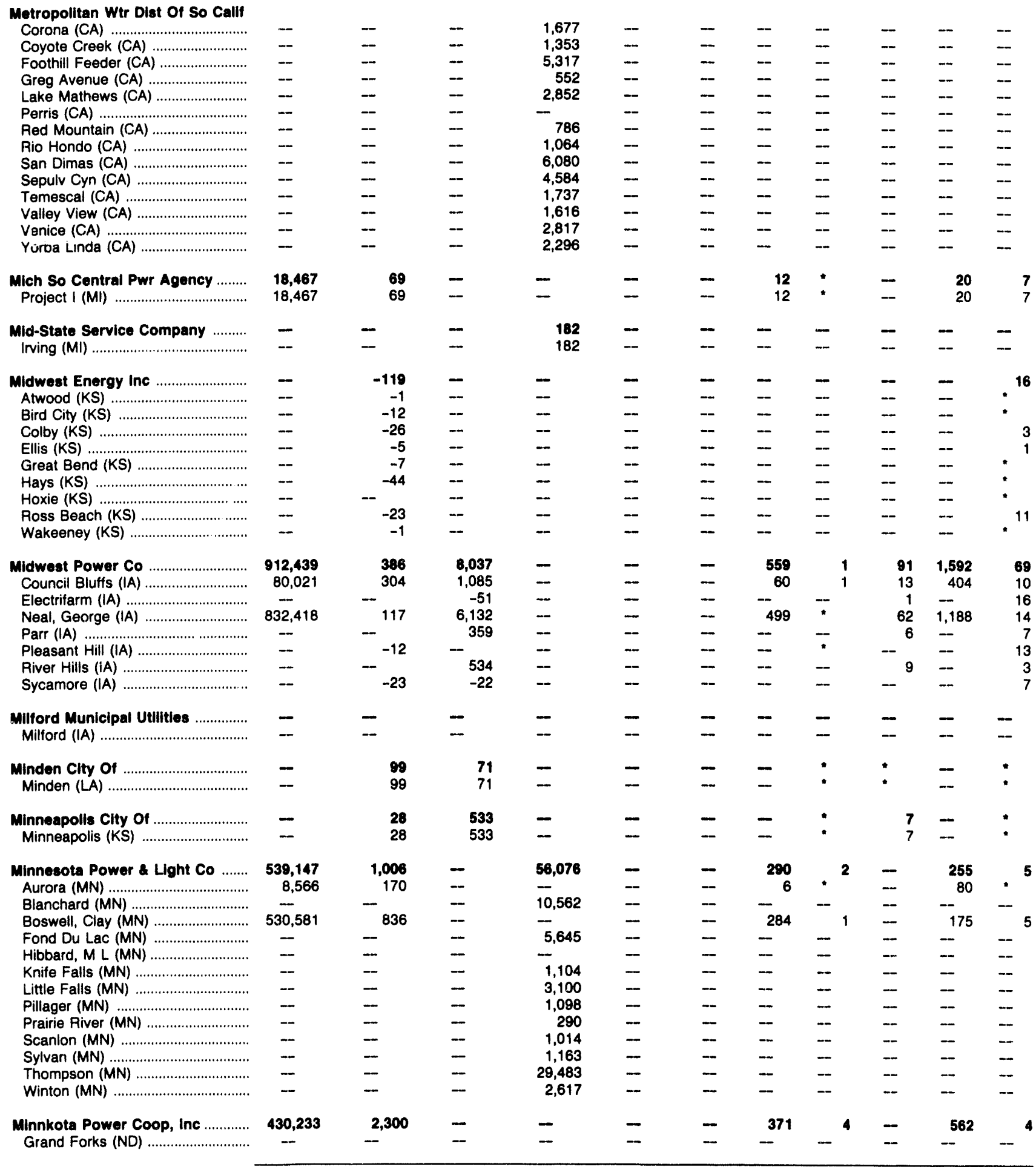

See footnotes at end of table. 
Table 62. U.S. Electric Utility Net Generation, Fuel Consumption, and Fuel Stocks by Company and Plant, September 1993 (Continued)

\begin{tabular}{|c|c|c|c|c|c|c|c|c|c|c|c|}
\hline \multirow{2}{*}{$\begin{array}{c}\text { Company (Holding Company) } \\
\text { Plant (State) }\end{array}$} & \multicolumn{6}{|c|}{$\begin{array}{c}\text { Generation } \\
\text { (thousand kilowatthours) }\end{array}$} & \multicolumn{3}{|c|}{$\begin{array}{l}\text { Consumption } \\
\text { (thoueand) }\end{array}$} & \multicolumn{2}{|c|}{$\begin{array}{c}\text { Stocks } \\
\text { (thousand) }\end{array}$} \\
\hline & Coal & Petroleum & Gas & Hydro & Nuclear & Other' & $\begin{array}{c}\text { Coal } \\
\text { (short } \\
\text { tona) }\end{array}$ & $\begin{array}{l}\text { Potro- } \\
\text { Ioum } \\
\text { (bble) }\end{array}$ & $\begin{array}{c}\text { Gas } \\
\text { (Mcf) }\end{array}$ & $\begin{array}{c}\text { Coal } \\
\text { (ahort } \\
\text { tons) }\end{array}$ & $\begin{array}{l}\text { Petro } \\
\text { leum } \\
\text { (bbls) }\end{array}$ \\
\hline
\end{tabular}

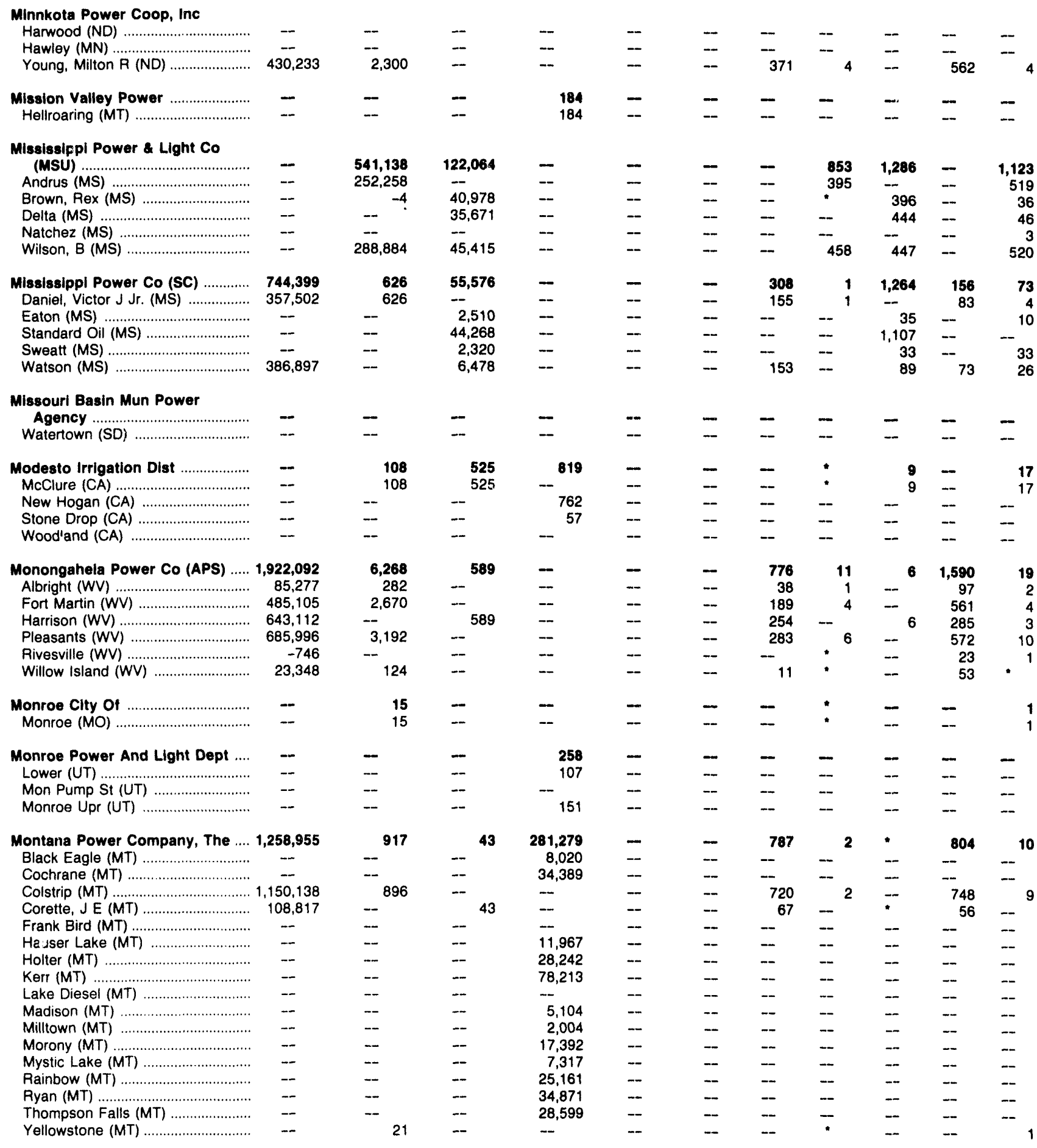

See footnotes at end of table. 
Table 62. U.S. Electric Utility Net Generation, Fuel Consumption, and Fuel Stocks by Company and Plant, September 1993 (Continued)

\begin{tabular}{|c|c|c|c|c|c|c|c|c|c|c|c|}
\hline \multirow{2}{*}{$\begin{array}{c}\text { Company (Holding Company) } \\
\text { Plant (State) }\end{array}$} & \multicolumn{6}{|c|}{$\begin{array}{c}\text { Generation } \\
\text { (thousand kllowatthours) }\end{array}$} & \multicolumn{3}{|c|}{$\begin{array}{l}\text { Conoumption } \\
\text { (thousand) }\end{array}$} & \multicolumn{2}{|c|}{$\begin{array}{l}\text { Stocks } \\
\text { (thousand) }\end{array}$} \\
\hline & Coal & Petroleum & Gas & Hydro & Nuclear & Other' & $\begin{array}{l}\text { Coal } \\
\text { (ehort } \\
\text { tons) }\end{array}$ & $\begin{array}{l}\text { Petro- } \\
\text { loum } \\
\text { (bble) }\end{array}$ & $\begin{array}{l}\text { Gas } \\
\text { (Mcf) }\end{array}$ & $\begin{array}{l}\text { Coal } \\
\text { (short } \\
\text { tons) }\end{array}$ & $\begin{array}{l}\text { Petro- } \\
\text { loum } \\
\text { (bbls) }\end{array}$ \\
\hline $\begin{array}{c}\text { Montana-Dakota Utilities Co } \\
\text { Coyote (ND) }\end{array}$ & $\begin{array}{l}257,107 \\
202,767\end{array}$ & $\begin{array}{l}948 \\
948\end{array}$ & {$\left[\begin{array}{l}61 \\
--\end{array}\right.$} & $\overline{-}$ & $\overline{-}$ & $\overline{-}$ & $\begin{array}{l}216 \\
165\end{array}$ & 2 & $\ldots 1$ & 287 & 7 \\
\hline 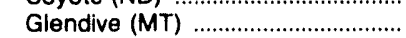 & -- & -- & 20 & -- & - & - & - & $\cdot^{2}$ & - & -238 & $\begin{array}{l}4 \\
2\end{array}$ \\
\hline 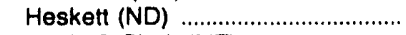 & 36,222 & -- & -- & -- & -- & -- & 33 & -- & • & 36 & -. \\
\hline 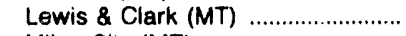 & 18,118 & -- & 53 & -- & - & - & 18 & -- & 1 & 12 & -- \\
\hline 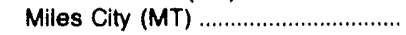 & - & -- & -5 & -- & -- & -- & - & • & - & -- & 1 \\
\hline Williston (ND) & -- & -- & -7 & -- & - & - & - & - & -- & -- & - \\
\hline Montaup Electric Co (EU) ............... & 12,873 & 1,473 & - & - & - & - & 7 & 4 & - & 83 & 88 \\
\hline Somerset (MA) & 12,873 & 1,473 & -- & -- & - & -- & 7 & 4 & - & 83 & 88 \\
\hline Montezuma Mun Elec Lt \& Pwr .... & -- & - & - & - & - & - & - & - & - & -- & - \\
\hline 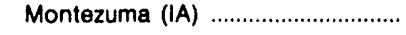 & - & -- & -- & -- & - & -- & -- & -- & -- & -- & • \\
\hline Moon Lake Electric Assn, Inc ....... & - & - & - & 1,261 & - & - & - & - & - & - & - \\
\hline 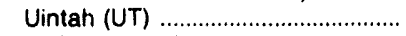 & - & -- & -- & 736 & -- & - & -- & - & -. & -- & -. \\
\hline 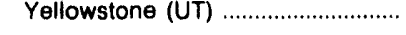 & - & -- & -- & 525 & - & - & - & -- & - & - & -- \\
\hline Moorhead Public Service Dept .... & - & 1 & - & - & - & - & -- & • & - & 2 & - \\
\hline 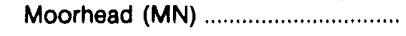 & - & 1 & -- & -- & -- & - & -- & - & -- & 2 & • \\
\hline Moose Lake Publlc UtIl Comm ..... & - & 48 & - & - & - & - & - & - & - & - & - \\
\hline Moose Lake (MN) & -- & 48 & -- & -- & -- & - & - & • & - & - & - \\
\hline Mora Municipal Electric Utllity ..... & - & - & - & - & - & - & - & - & - & - & - \\
\hline Mora (MN) & - & -- & - & - & -- & - & - & - & - & - & -- \\
\hline 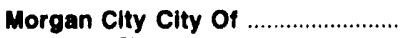 & - & - & 6,651 & - & - & - & - & - & 95 & - & - \\
\hline Morgan City (LA) & -- & - & 6,651 & -- & -- & -- & - & -- & 95 & -- & -- \\
\hline Morrisville Water \& LIght Dept .... & - & - & - & 929 & - & - & - & - & - & - & - \\
\hline Cadys Falls (VT) & -- & - & -- & 383 & - & - & - & - & -- & -- & -- \\
\hline 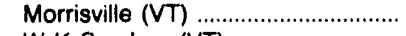 & - & - & - & 496 & -- & -- & - & - & - & -- & -- \\
\hline 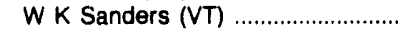 & -- & -- & -- & 50 & -- & - & - & - & -- & -- & -- \\
\hline Mount Pleasant Mun Utils ............... & - & - & -- & - & - & - & - & - & - & - & - \\
\hline 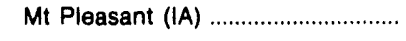 & -- & - & -- & -- & -- & - & - & -- & - & -- & • \\
\hline Mount Pleasant Municipal ............... & - & - & - & 243 & - & - & - & - & - & - & - \\
\hline 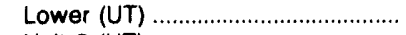 & -- & -- & - & 36 & -- & - & - & - & -- & - & -- \\
\hline 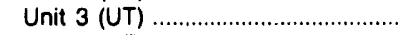 & -- & - & - & 78 & -- & - & - & -- & -- & -_ & -. \\
\hline 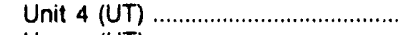 & -- & - & - & 29 & -- & - & -- & - & -- & - & -- \\
\hline 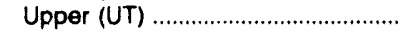 & -- & -- & -- & 100 & - & - & - & - & - & -- & - \\
\hline Mountain Lake Munlcipal Util ....... & - & - & - & - & - & - & - & - & - & - & - \\
\hline Mountain Lake (MN) ............................ & - & -- & - & - & -- & - & - & - & - & -- & - \\
\hline 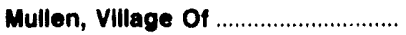 & - & - & - & - & - & - & - & - & - & - & • \\
\hline Mullen (NE) & -- & - & -- & - & - & -- & - & - & - & -- & • \\
\hline 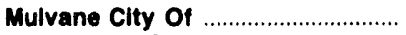 & - & 3 & 13 & - & - & - & - & • & - & - & - \\
\hline 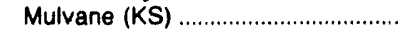 & -- & 3 & 13 & - & -- & - & -- & - & - & -. & • \\
\hline Murray Clty Corporation ..................... & - & 7 & 20 & 1,078 & - & - & - & - & * & - & . \\
\hline Diesel (UT) & -- & 7 & 20 & -- & - & -- & - & - & - & -- & - \\
\hline 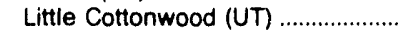 & -- & -- & -- & 1,078 & -- & - & - & - & - & -. & - \\
\hline Muscatine City of & 78,471 & - & 131 & - & - & - & 52 & - & 1 & 182 & 2 \\
\hline 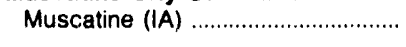 & 78,471 & -- & 131 & - & - & - & 52 & - & 1 & 182 & 2 \\
\hline Muscoda Light \& Water Comm .... & - & -- & - & - & - & - & - & - & - & - & - \\
\hline Muscoda (WI) & -- & - & - & -- & -- & - & - & - & - & -- & - \\
\hline Naknek Electric Assn, Inc .............. & - & 1,507 & - & - & - & - & - & 2 & - & - & 34 \\
\hline Naknek (AK) ......................................... & - & 1,507 & - & -- & - & - & -- & 2 & - & - & 34 \\
\hline Nantahala Power Light Co ......... & - & - & - & 30,561 & - & - & - & - & - & - & - \\
\hline Bear Creek (NC) & -- & -- & -- & 568 & -- & - & - & -- & - & - & - \\
\hline
\end{tabular}

See footnotes at end of table. 
Table 62. U.S. Electric Utility Net Generation, Fuel Consumption, and Fuel Stocks by Company and Plant, September 1993 (Continued)

\begin{tabular}{|c|c|c|c|c|c|c|c|c|c|c|c|}
\hline \multirow{2}{*}{$\begin{array}{c}\text { Company (Holding Company) } \\
\text { Plant (State) }\end{array}$} & \multicolumn{6}{|c|}{$\begin{array}{l}\text { Ceneration } \\
\text { (thousand kllowatthours) }\end{array}$} & \multicolumn{3}{|c|}{$\begin{array}{l}\text { Consumption } \\
\text { (thousand) }\end{array}$} & \multicolumn{2}{|c|}{$\begin{array}{l}\text { Stocks } \\
\text { (thousend) }\end{array}$} \\
\hline & Coal & Petroleum & Gas & Hydro & Nuclear & Other' & $\begin{array}{c}\text { Coal } \\
\text { (short } \\
\text { tons) }\end{array}$ & $\begin{array}{l}\text { Petro- } \\
\text { loum } \\
\text { (bols) }\end{array}$ & (Mef) & $\begin{array}{c}\text { Coal } \\
\text { (hort } \\
\text { tone) }\end{array}$ & $\begin{array}{l}\text { Potro- } \\
\text { loum } \\
\text { (bble) }\end{array}$ \\
\hline
\end{tabular}

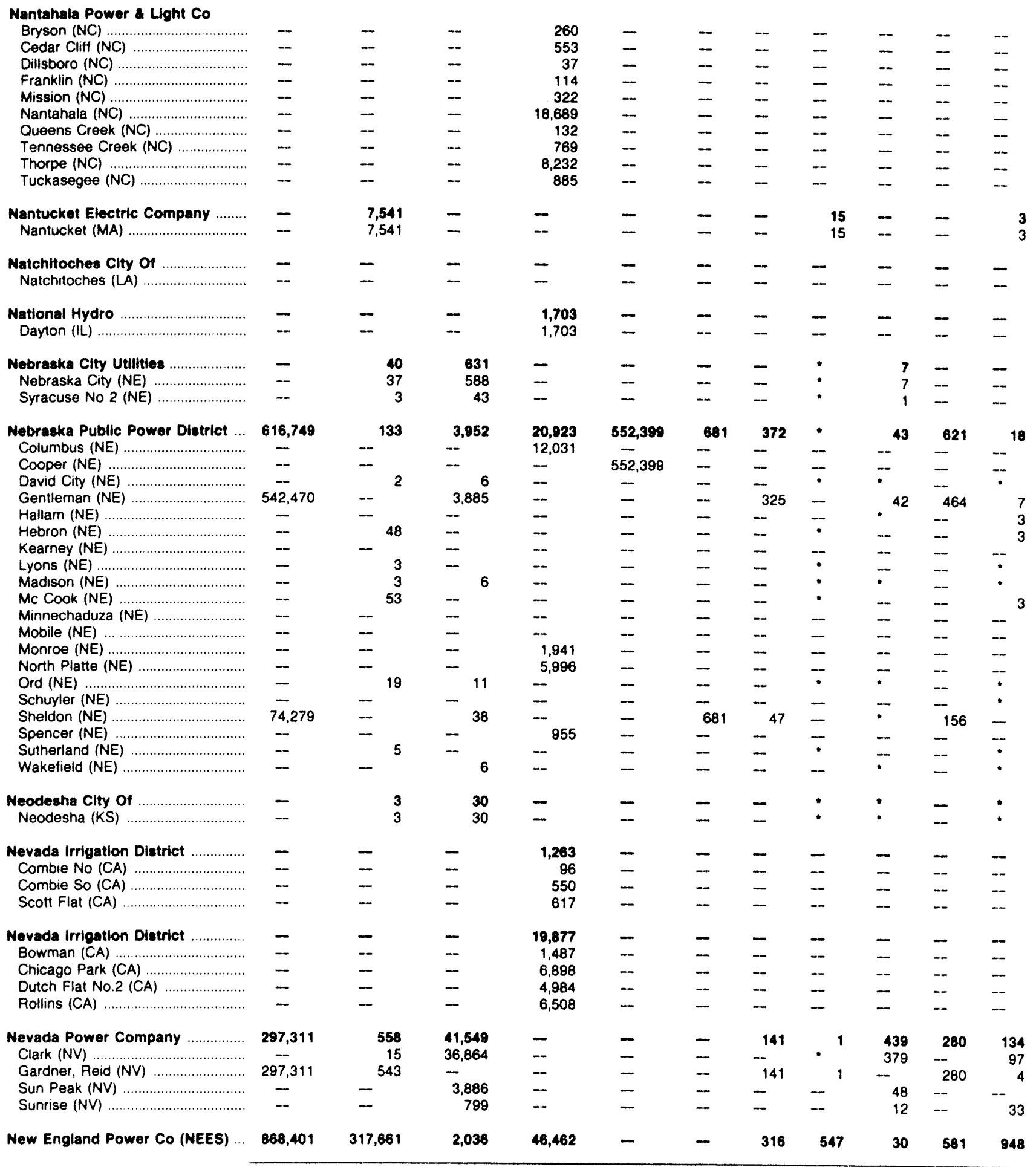

See footnotes at end of table. 
Table 62. U.S. Electric Utility Net Generation, Fuel Consumption, and Fuel Stocks by Company and Plant, September 1993 (Continued)

\begin{tabular}{|c|c|c|c|c|c|c|c|c|c|c|c|}
\hline \multirow{2}{*}{$\begin{array}{c}\text { Company (Holding Company) } \\
\text { Plant (State) }\end{array}$} & \multicolumn{6}{|c|}{$\begin{array}{c}\text { Ceneration } \\
\text { (thousand kllowatthours) }\end{array}$} & \multicolumn{3}{|c|}{$\begin{array}{l}\text { Consumption } \\
\text { (thousand) }\end{array}$} & \multicolumn{2}{|c|}{$\begin{array}{c}\text { Stocks } \\
\text { (thousand) }\end{array}$} \\
\hline & Coal & Petroleum & ass & Hydro & Nuchar & Other' & $\begin{array}{c}\text { Coal } \\
\text { (short } \\
\text { tons) }\end{array}$ & $\begin{array}{l}\text { Petro- } \\
\text { loum } \\
\text { (bbles) }\end{array}$ & $\begin{array}{l}\text { Gas } \\
\text { (Mct) }\end{array}$ & $\begin{array}{c}\text { Coal } \\
\text { (short } \\
\text { tons) }\end{array}$ & $\begin{array}{l}\text { Petro- } \\
\text { leum } \\
\text { (bbls) }\end{array}$ \\
\hline
\end{tabular}

\begin{tabular}{|c|c|c|c|c|c|c|c|c|c|c|c|}
\hline $\begin{array}{l}\text { Now England Power Co (NEES) } \\
\text { Bear Swamp (MA) }\end{array}$ & -- & -- & - & $-15,464$ & - & -- & -- & -- & $\ldots$ & -- & -. \\
\hline Bellows Falls (NT) & -- & - & - & $\begin{array}{r}-10,404 \\
9,357\end{array}$ & - & -- & -- & .. & .. & -- & $\overline{-}$ \\
\hline 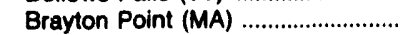 & 705,199 & 117,521 & - & - & - & -- & 251 & 213 & -- & 461 & 705 \\
\hline 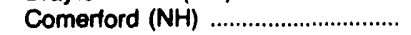 & -- & - & - & 12,205 & - & - & - & - & -- & -- & -- \\
\hline 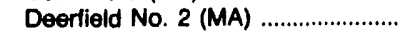 & - & - & - & 681 & - & -- & - & -- & -- & -- & -- \\
\hline 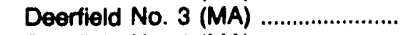 & -- & $\cdots$ & - & 1,142 & -- & - & - & - & -- & -- & -- \\
\hline 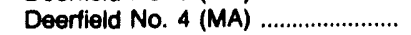 & - & - & - & 1,049 & - & - & -- & -- & - & -- & -- \\
\hline 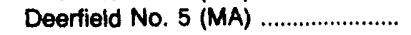 & -- & - & -- & 2,390 & - & -- & - & -- & - & -- & -. \\
\hline 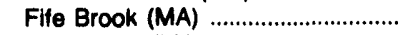 & - & - & - & 1,274 & - & - & -- & -- & -- & -- & -- \\
\hline 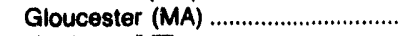 & -- & 76 & - & - & - & - & - & * & - & -- & 2 \\
\hline 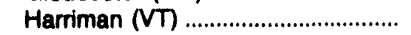 & - & - & - & 4,972 & -- & - & - & - & -- & -- & -- \\
\hline Manchester Street (RI) ................... & - & - & 2,036 & - & - & - & -- & -- & 30 & -- & -- \\
\hline 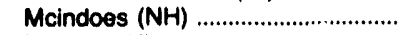 & - & - & - & 2,975 & - & - & -- & -- & - & -- & - \\
\hline 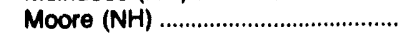 & -- & - & - & 11,119 & - & - & -- & -- & -- & -- & - \\
\hline Newburyport (MA) ............................... & - & 27 & -- & - & - & - & - & • & -- & -- & 1 \\
\hline 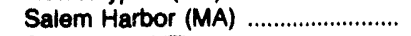 & 163,202 & 200,037 & - & - & - & -- & 65 & 333 & -- & 121 & 240 \\
\hline Searsburg (VT) ......................... & - & - & -- & 1,361 & - & - & -- & - & -- & - & -- \\
\hline 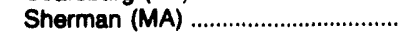 & -- & -- & - & 1,292 & -- & - & - & - & -- & -- & -- \\
\hline South Street (RI) ................................. & -- & - & - & - & - & -- & -- & - & -- & - & -- \\
\hline 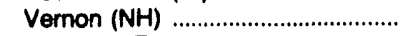 & - & - & - & 1,456 & - & - & - & - & -- & -- & - \\
\hline 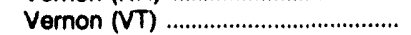 & - & -- & - & 3,189 & - & - & - & - & -- & -- & - \\
\hline Wilder $(\mathrm{NH})$ & - & - & - & 7,454 & - & -- & - & -- & -- & -- & -_- \\
\hline 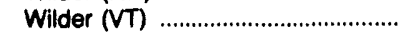 & -- & - & - & - & - & - & - & - & -- & -- & - \\
\hline Now Hampton Mun Light Plant .... & - & - & - & - & - & - & - & - & - & - & • \\
\hline 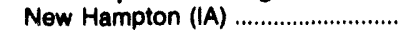 & - & - & -- & - & - & - & - & -. & -- & - & - \\
\hline Now Luabon Mun Electrlc Dept .... & - & - & 4 & - & - & - & - & - & - & - & 1 \\
\hline 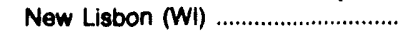 & -- & -- & 4 & - & - & - & -- & - & • & -- & 1 \\
\hline Now Orbans Publlc Service & & & & & & & & & & & \\
\hline 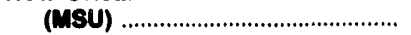 & - & -10 & 236,400 & - & - & - & - & - & 2,637 & - & 141 \\
\hline 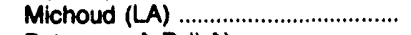 & - & - & 236,400 & - & - & - & -- & $\cdots$ & 2,637 & - & 139 \\
\hline 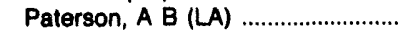 & - & -10 & - & - & -- & -- & - & • & - & -_ & 3 \\
\hline Now Prague Mun Utils Comm ........ & - & 25 & 91 & - & - & - & - & • & 1 & - & - \\
\hline New Prague (MN) ................................ & - & 25 & 91 & - & -- & - & - & • & 1 & -- & . \\
\hline Now Roads Llght \& Water Plant .. & - & - & - & - & - & - & - & * & - & - & 1 \\
\hline 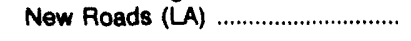 & - & - & - & - & - & - & -- & * & -- & -- & 1 \\
\hline New Smyrna Beach City of ........... & - & 74 & - & - & - & - & - & " & - & - & 2 \\
\hline 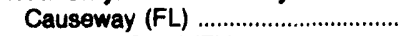 & -- & - & - & - & - & -. & -- & - & - & -- & -- \\
\hline 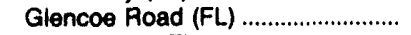 & -- & -- & - & - & - & -- & -- & - & -- & -- & -- \\
\hline New Smyra (FL) ................................ & - & 33 & - & - & - & - & - & • & -- & -- & 1 \\
\hline 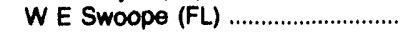 & -- & 41 & - & - & - & -- & - & • & - & - & 1 \\
\hline Now Ulm Pub Utils Comm .............. & - & - & 4,401 & - & - & - & - & - & 87 & 3 & 4 \\
\hline 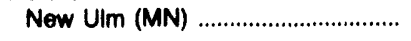 & $-\infty$ & -- & 4,401 & - & -- & - & - & - & 87 & 3 & 4 \\
\hline Now York State Elec \& Gas Corp & 681,697 & 1,097 & - & 21,330 & - & 1,267 & 280 & 2 & - & 655 & 6 \\
\hline 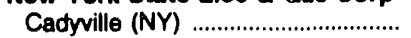 & - & - & - & 1,400 & - & - & -- & - & - & -- & - \\
\hline 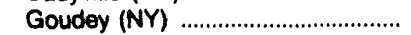 & 37,276 & 236 & - & - & -- & - & 13 & - & - & 61 & 1 \\
\hline 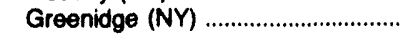 & 63,554 & 45 & - & - & -- & -- & 21 & - & -- & 110 & 2 \\
\hline 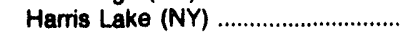 & - & 6 & - & - & - & -- & -- & • & -- & -- & - \\
\hline 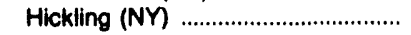 & 28,019 & -- & - & - & - & -- & 10 & -- & -- & 57 & -- \\
\hline 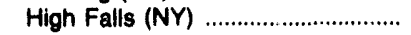 & - & -- & - & 6,745 & - & -- & - & -- & - & -. & -- \\
\hline 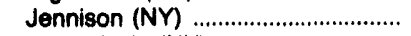 & 16,411 & - & - & - & - & 1,267 & 13 & -- & -- & 44 & -- \\
\hline 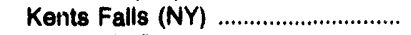 & - & -- & - & 6,336 & - & - & - & -- & - & - & - \\
\hline 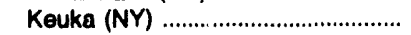 & - & -- & - & - & - & - & - & - & -- & - & $\ldots$ \\
\hline 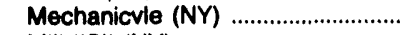 & - & - & - & $\mathbf{3 , 3 3 9}$ & - & - & - & -- & -- & -.- & - \\
\hline 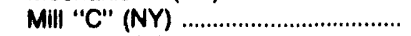 & - & -- & - & 2,057 & - & - & -- & -- & - & - & -- \\
\hline 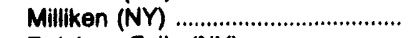 & 195,645 & 567 & - & - & - & - & 68 & 1 & - & 85 & 2 \\
\hline 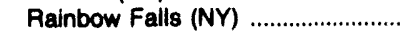 & - & - & -- & 1,408 & - & - & - & - & -- & -- & -- \\
\hline 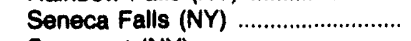 & - & - & - & 32 & - & - & - & -- & -- & - & -- \\
\hline 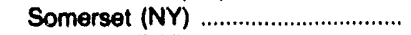 & 320,792 & 243 & - & - & - & - & 127 & . & - & 297 & 1 \\
\hline 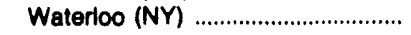 & - & - & - & 22 & - & - & - & - & -- & -- & -- \\
\hline
\end{tabular}

See footnotes at end of table: 
Table 62. U.S. Electric Utility Net Generation, Fuel Consumption, and Fuel Stocks by Company and Plant, September 1993 (Continued)

\begin{tabular}{|c|c|c|c|c|c|c|c|c|c|c|c|}
\hline \multirow{2}{*}{$\begin{array}{c}\text { Company (Holding Company) } \\
\text { Plant (State) }\end{array}$} & \multicolumn{6}{|c|}{$\begin{array}{c}\text { Generation } \\
\text { (thousand kllowatthours) }\end{array}$} & \multicolumn{3}{|c|}{$\begin{array}{l}\text { Consumption } \\
\text { (thousand) }\end{array}$} & \multicolumn{2}{|c|}{$\begin{array}{c}\text { Stocks } \\
\text { (thousand) }\end{array}$} \\
\hline & Cool & Petroloum & Gas & Hydro & Nuclear & Other' & $\begin{array}{l}\text { Coal } \\
\text { (short } \\
\text { tons) }\end{array}$ & $\begin{array}{l}\text { Petro- } \\
\text { leum } \\
\text { (bbla) }\end{array}$ & $\begin{array}{l}\text { Gas } \\
\text { (Mef) }\end{array}$ & $\begin{array}{c}\text { Coal } \\
\text { (short } \\
\text { tons) }\end{array}$ & $\begin{array}{l}\text { Petro- } \\
\text { loum } \\
\text { (bbls) }\end{array}$ \\
\hline
\end{tabular}

\begin{tabular}{|c|c|c|c|c|c|c|c|c|c|c|c|}
\hline $\begin{array}{l}\text { Newberry Wtr \& Lt Board } \\
\text { Newbery (MI) }\end{array}$ & $\overline{-}$ & $\overline{-}$ & $\overline{-}$ & - & - & - & $\overline{-}$ & $\overline{-}$ & $\overline{-}$ & $\overline{--}$ & : \\
\hline Newport Electric Corporation ...... & - & 209 & - & - & - & - & - & • & -- & - & 2 \\
\hline Eldred (RI) & -- & 123 & - & -- & -. & -- & -- & - & -- & -_. & 1 \\
\hline 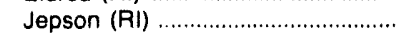 & - & 86 & -- & -- & - & - & -- & • & -- & -. & 1 \\
\hline Niagara Mohawk Power Corp ....... & 487,762 & 73,243 & 3,036 & 156,567 & $1,145,431$ & -- & 240 & 150 & 39 & 189 & 1,168 \\
\hline Albany (NY) & -- & 67,334 & 3,036 & - & - & -- & -- & 137 & 39 & $-\infty$ & 344 \\
\hline 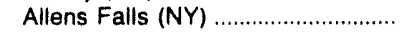 & -- & - & -- & 1,969 & -- & -- & -- & -- & -. & -- & -- \\
\hline Bakers Falls (NY) ………………...... & -- & -- & -- & - & -- & -- & -- & -- & -. & -- & ... \\
\hline 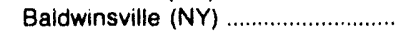 & -- & -- & -- & -3 & - & -- & -- & -- & -- & -. & - \\
\hline 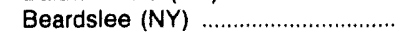 & -- & -- & -- & 2,351 & -- & -- & -- & -- & -- & -. & -- \\
\hline Beebee Island (NY) ................... & - & -- & -- & 2,609 & -- & -- & -- & -- & -- & -- & -- \\
\hline 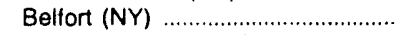 & -- & -- & -- & 816 & - & -- & -- & -- & -- & -. & -- \\
\hline Bennetts Bridge (NY) ....................... & -- & - & - & 5,576 & - & -- & -- & -- & -- & -. & -- \\
\hline Black River (NY) . . & -- & -- & -- & 2,501 & - & -- & - & -- & - & - & - \\
\hline 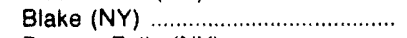 & - & -- & - & 3,009 & - & -- & -- & -- & -- & -- & -- \\
\hline Browns Falls (NY) . & -- & - & -- & 2,271 & -- & -- & -- & -- & -- & -- & $\ldots$ \\
\hline Chasm (NY) . & -- & - &.- & 1,441 & -- & -- & -- & -- & -- & -.. & -- \\
\hline 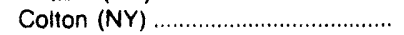 & - & -- & -- & 12,984 & -- & -- & -- & -- & -- & -- & -. \\
\hline Deferiet $(N Y)$ & -- & - & -- & 3,514 & -- & -- & -- & -- & $\ldots$ & -- & -.. \\
\hline 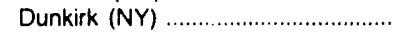 & 230,902 & 665 & -- & - & -- & -- & 125 & 2 & -- & 96 & 1 \\
\hline Eagle (NY) & -- & - & - & 2,169 & -- & -- & -- & -- & -- & $\ldots$ & -- \\
\hline 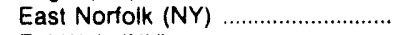 & -- & - & -- & -13 & -- & -- & -- & -- & -- & -- & -- \\
\hline 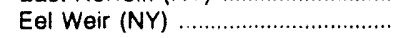 & -- & -- & -- & 236 & -- & -- & -- & -- & -- & -- & -- \\
\hline Effley (NY) & - & -- & -- & 1,089 & -- & -- & -- & - & - & - & - \\
\hline Elmer (NY) & -- & - & -- & 700 & -- & -- & -- & -- & -- & $\ldots$ & -- \\
\hline 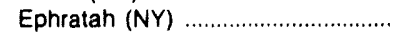 & -- & -- & - & 450 & - & -- & -- & -. & -- & $\ldots$ & -- \\
\hline 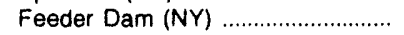 & -- & - & -- & 1,502 & - & -- & -- & -- & -- & $\ldots$ & -- \\
\hline Five Falls $(N Y)$ & -- & -- & -- & 4,696 & -- & -- & .. & -- & - & -- & -- \\
\hline Flat Rock (NY) & -- & -- & -- & 464 & -- & -- & -- & -- & -- & -- & -- \\
\hline 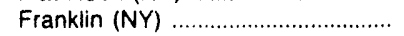 & -- & - & -- & 473 & -- & -- & _. & -- & -_ & -. & -. \\
\hline Fulton (NY) & - & -- & -- & 687 & - & -- & -- & - & - & $\ldots$ & - \\
\hline 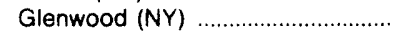 & -- & -- & - & 428 & -- & -- & -- & -- & -- & -- & -- \\
\hline 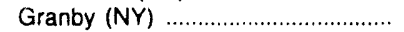 & -- & -- & -- & -55 & -- & -- & -- & -- & -- & -. & -- \\
\hline 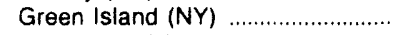 & -- & -- & -- & 2,434 & -- & -- & -- & -- & -- & .- & -- \\
\hline 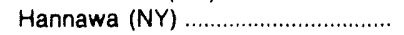 & - & -- & -- & 3,182 & -- & -- & -- & -- & -- & -- & $\ldots$ \\
\hline 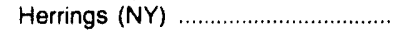 & -- & -- & -- & 1,179 & -- & -- & -_ & -- & -- & -- & -- \\
\hline 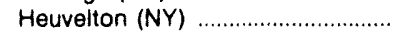 & - & -- & -- & 320 & -- & -- & -- & -- & -_- & -- & -- \\
\hline High Dam (NY) & -- & -- & -- & 1,667 & -- & -- & -- & -- & -- & -- & $\ldots$ \\
\hline 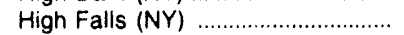 & -- & -- & -- & 2,320 & -- & -- & -- & -_ & -_. & -- & $\ldots$ \\
\hline 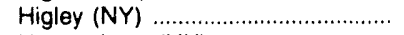 & -- & -- & -- & 1,901 & -- & -- & -- & - & -- & -- & -. \\
\hline 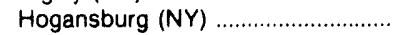 & -- & -- & -- & 207 & -- & -- & -- & -- & -- & .. & -- \\
\hline Huntley, C R (NY) & 256,860 & 4 & -- & -- & -- & -- & 116 & 2 & -- & 93 & 3 \\
\hline 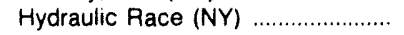 & -- & -- & -- & 1,516 & -- & -- & -_ & -- & -. & -_ & -- \\
\hline 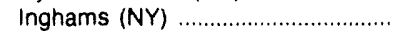 & -- & -- & - & 1,733 & -- & -- & -- & -- & -_ & -- & -- \\
\hline Johnsonville (NY) ............................. & - & -- & -- & 94 & -- & -- & -.- & -- & -- & -- & -- \\
\hline 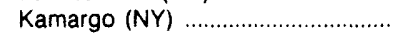 & -- & -- & -.. & 1,239 & -- & -- & -- & -- & -.. & -_- & - \\
\hline Lighthouse Hill (NY) .......................... & - & - & -- & 1,070 & -- & -- & -- & -- & -- & -- & $\ldots$ \\
\hline Macomb (NY) & -- & -- & -- & 434 & -- & -- & -- & -- & -- & $\ldots$ & -. \\
\hline Minetto (NY) & -- & - & - & 1,057 & -. & -- & -- & -_ & -- & -- & -. \\
\hline 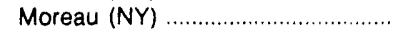 & -- & -- & -- & -4 & -- & -- & -.. & -. & -. & $\ldots$ & $\ldots$ \\
\hline 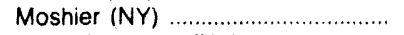 & -- & -- & -- & 1,912 & -- & -- & -- & -- & -.- & -- & -. \\
\hline 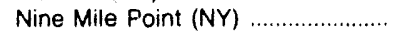 & -- & 8 & -- & -- & $1,145,431$ & -- & -- & - & -. & -_- & 2 \\
\hline Norfolk (NY) & -- & -- & -- & 1,596 & - & -- & -- & $\sim$ & - & - & $-{ }^{2}$ \\
\hline Norwood (NY) & -- & -- & -- & 912 & -- & -- & -- & -- & -- & $\ldots$ & - \\
\hline 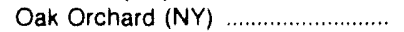 & -- & -- & -- & 180 & -- & -- & -- & -- & -- & -.. & -- \\
\hline 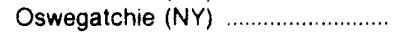 & -- & -- & - & -- & -- & -- & -- & -- & .- & $\ldots$ & $\ldots$ \\
\hline 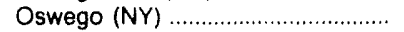 & -- & 5,232 & -- & -- & -- & -. & -- & 9 & * & -- & 819 \\
\hline Oswego Falls Es (NY) & -- & - & -- & 1,405 & -- & - & -- & -- & -- & & \\
\hline Oswego Falls Ws (NY) .......................... & -. & -- & -- & 230 & - & -- & -- & -- & -- & -.. & -- \\
\hline 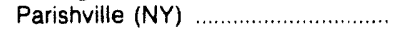 & -- & -- & -- & 1,224 & - & -- & - & -- & -- & & -. \\
\hline Piercefield (NY) & -- & -- & -- & 870 & -- & -- & -- & -- & .. & -.. & -.. \\
\hline Prospect (NY) & -- & -- & -- & 3,609 & -- & -- & -- & -- & -- & -. & -_ \\
\hline Rainbow (NY) & - & - & -- & 4,672 & -- & -- & -- & -- & -- & -- & -_ \\
\hline 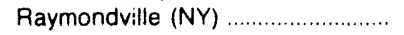 & -- & -- & -- & 900 & -. & -- & -- & -. & -. & -- & -- \\
\hline 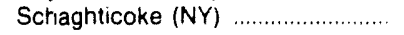 & -- & -- & -- & 1.593 & -- & -- & -- & -- & -. & -- & -.. \\
\hline
\end{tabular}

See footnotes at end of table. 
Table 62. U.S. Electric Utility Net Generation, Fuel Consumption, and Fuel Stocks by Company and Plant, September 1993 (Continued)

\begin{tabular}{|c|c|c|c|c|c|c|c|c|c|c|c|}
\hline \multirow{2}{*}{$\begin{array}{c}\text { Company (Holding Company) } \\
\text { Plant (State) }\end{array}$} & \multicolumn{6}{|c|}{$\begin{array}{c}\text { Generation } \\
\text { (thousand kllowatthours) }\end{array}$} & \multicolumn{3}{|c|}{$\begin{array}{l}\text { Consumption } \\
\text { (thousand) }\end{array}$} & \multicolumn{2}{|c|}{$\begin{array}{c}\text { Stocks } \\
\text { (thousand) }\end{array}$} \\
\hline & Coal & Petroleum & Gas & Hydro & Nuclear & Other' & $\begin{array}{c}\text { Coal } \\
\text { (short } \\
\text { tons) }\end{array}$ & $\begin{array}{l}\text { Petro- } \\
\text { leum } \\
\text { (bbla) }\end{array}$ & $\begin{array}{c}\text { Gas } \\
\text { (Mcf) }\end{array}$ & $\begin{array}{c}\text { Coal } \\
\text { (short } \\
\text { tons) }\end{array}$ & $\begin{array}{l}\text { Petro- } \\
\text { leum } \\
\text { (bbls) }\end{array}$ \\
\hline
\end{tabular}

Niagara Mohawk Power Corp

School Street (NY)

Schuylerville (NY)

Sewalls (NY)

Sherman island (NY)

So Glens Falis (NY)

Soft Maple (NY)

South Colton (NY)

South Edwards (NY)

Spier Falls (NY)

Stark (NY)

Stewarts Bridge (NY)

Stuyvesant Falls (NY)

Sugar Island (NY)

Taylorville (NY)

Trenton (NY)

Varick (NY) ......

Waterport (NY)

West, E J (NY)

Yaleville (NY)

Niles Board Of Public Works

Niles (MI)

Nodak Rural Elec Coop Inc Mobile (ND)

Nome Light And Power Utillties

Snake River (AK)

North American Hydro Inc

Wautoma (WI)

North Branch Lt \& Pwr Comm .... North Branch (MN)

North Central Power Co Inc ......... Arpin (WI)

Radisson (WI)

Winter (WI)

North Little Rock Clty of

Murray (AR)

Northeast Mlasourl Elec Pwr

$$
\text { Coop . }
$$

South River Station (MO)

Northeast Nuclear Energy Co ...

Millstone (CT)

Northern Indiana Public Serv Co . 1,126,613

Bailly (IN)

264,424

Mitchell, Dean H (IN) ................... 132,628

Norway (IN)

Oakdale (IN)

Schahter, R. M. (IN) ..................... 438,874

Northern States Power Co ........... 1,509,853

Apple River (WI)

Bay Front (WI)

Big Falls (WI)

Black Dog (MN)

Blue Lake (MN)

Cedar Fails (WI)

Chippewa Falls (WI)

Cornell (WI)

\begin{tabular}{clr}
$\mathbf{5 0 9 , 8 5 3}$ & \multicolumn{2}{l}{$\mathbf{5 7 , 9 2 3}$} \\
-- & -- & \\
224 & -- & \\
-- & -- & \\
61,145 & \multicolumn{2}{|r}{36} \\
-- & \multicolumn{2}{r}{-31} \\
-- & -- & \\
-- & -- & \\
-- & --
\end{tabular}

$-$
8,513

-3
970
6,910
-
1,740
3,806
972
10,914
1,046
10,495
-3
2,472
1,640
7,941
1,279
654
5,619
286

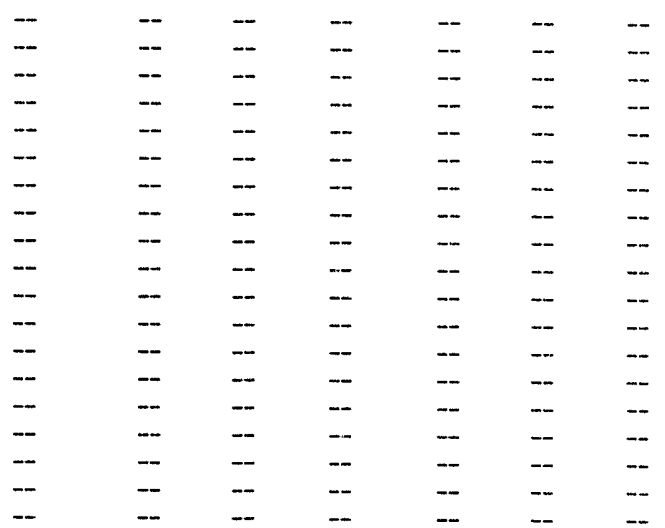

$-$

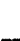

$-$

$\overline{-}$

$=$

-

\section{1}

$$
\begin{aligned}
& 626 \\
& 109
\end{aligned}
$$$$
146
$$

14,109

14,109

$-$

$-\quad-$

$-$

$-$

$12,401 \quad 7,897$

$\begin{array}{rr}- & 12,401 \\ - & 2,218\end{array}$

$\begin{array}{lr}- & 2,218 \\ - & 783\end{array}$

-- 919

-- $\quad$--

8,48

$-$

$-$

3,055

4,842

4,239

$-$

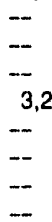

58,660

1,213

$-\overline{2}, 673$

$-$

$-$

2,579

3,647

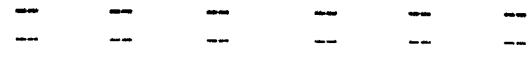

$-\quad-$

$=-$

$=-$

$\overline{-}$

$-$

$-\overline{-}$

$\overline{--}$

$--$

$=$
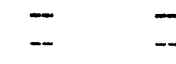

732,390

732,390

$-$

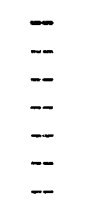

$\overline{-}$

$1,149,394$
--
-
--
--
-
-
-
- \begin{tabular}{ccc}
39,249 & 974 & \multicolumn{2}{c}{2} \\
-- & - & -- \\
13,214 & - & - \\
- & - & -- \\
- & 43 & -- \\
-- & - & - \\
-- & - & - \\
- & - & --
\end{tabular}

See footnotes at end of table. 
Table 62. U.S. Electric Utllity Net Generation, Fuel Consumption, and Fuel Stocks by Company and Plant, September 1993 (Continued)

\begin{tabular}{|c|c|c|c|c|c|c|c|c|c|c|c|}
\hline \multirow{2}{*}{$\begin{array}{c}\text { Company (Holding Company) } \\
\text { Plant (State) }\end{array}$} & \multicolumn{6}{|c|}{$\begin{array}{c}\text { Ceneration } \\
\text { (thousand kllowatthours) }\end{array}$} & \multicolumn{3}{|c|}{$\begin{array}{l}\text { Concumption } \\
\text { (thoueand) }\end{array}$} & \multicolumn{2}{|c|}{$\begin{array}{c}\text { Stocks } \\
\text { (thoueand) }\end{array}$} \\
\hline & Coal & Potroleum & Cas & Hydro & Nuclear & Other' & $\begin{array}{c}\text { Coal } \\
\text { (chort } \\
\text { tons) }\end{array}$ & $\begin{array}{l}\text { Petro- } \\
\text { leum } \\
\text { (bble) }\end{array}$ & $\begin{array}{l}\text { Qes } \\
\text { (Mct) }\end{array}$ & $\begin{array}{c}\text { Coal } \\
\text { (short } \\
\text { tona) }\end{array}$ & $\begin{array}{l}\text { Potro- } \\
\text { leum } \\
\text { (bbls) }\end{array}$ \\
\hline
\end{tabular}

\section{Northern States Power Co}

Dells $(W I)$

Flambeau (WI)

French Island (WI)

Granite City (MN)

Hayward (WI)

Hennepin Island (MN)

High Bridge (MN)

Holcombe (WI)

Holland (MN)

Inver Hills (MN)

Jim Falls $(W I)$

Key City (MN)

King (MN) ........

Menomonie (WI)

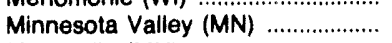

Monticello (MN)

Pathtinder (SD)

Prairie Island (MN)

Redwing (MN)

Riverdale (WI)

Riverside (MN)

Saxon Falls (MI)

St Croix Falls (WI)

Superior Falls (MI)

Thornapple (WI)

Trego (WI)

West Faribault (MN)

Wheaton (WI)

White River (WI)

Wilmarth (MN)

Wissota (WI)

Northway Power \& Light Inc

Northway (AK).

\section{Northwestern Public Service}

$$
\text { Company }
$$

Aberdeen (SD)

Clark (SD)

Faulkton (SD)

Highmore (SD)

Huron (SD)

Mobile (SD)

Redfield (SD)

Webster (SD)

Yankton New (SD)

Northwestern Wisconsin Elec Co

Black Brook (WI)

Clam Falls (WI)

Clam River Dam (WI)

Danbury (WI)

Frederic (WI)

Grantsburg (WI)

Northwood Clty Of Mun Lt \& Pwr Northwood (ND)

Norton City of

Norton (KS)

Norway Electric Light Dept

Norway (MI)

Norwich Dept Of Pub Utils

See footnotes at end of table.
3,313

-
-
-
-
-
$\overline{17,628}$
-
-
$\overline{-}$
-

$-$

4,850

-

$-$

$--$

$\overline{132,732}$

132,732

$1,004,316$

--

$-$

$-$

$-$

$-$

$-$

$-$

-

$\begin{array}{lll}- & & \\ -51 & - & 4 \\ - & & -23\end{array}$

$-$

--

$-6$

$\begin{array}{lll}- & -6 & - \\ - & -\end{array}$

46,113

$-$

$-1$

$-$

$-$

$-$

11,155

881

$-$

$-$

$-$

$-$

$--189$

$-$

$-$

$-$

$\begin{array}{rrrr}-48 & & -20 & - \\ -2 & -- & - \\ -12 & - & & - \\ -8 & -- & & - \\ -10 & -- & & - \\ - & & -28 & - \\ -2 & -- & & - \\ -4 & & -7 & - \\ -12 & - & & - \\ 2 & & 6 & -\end{array}$

-
-
-
-
-
-
-

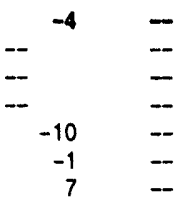

708
142
29
306
231
$-\quad$

$\begin{array}{llll}- & - & - & - \\ - & \overline{1} & - & - \\ - & 5,612 & - & - \\ - & - & - & -\end{array}$

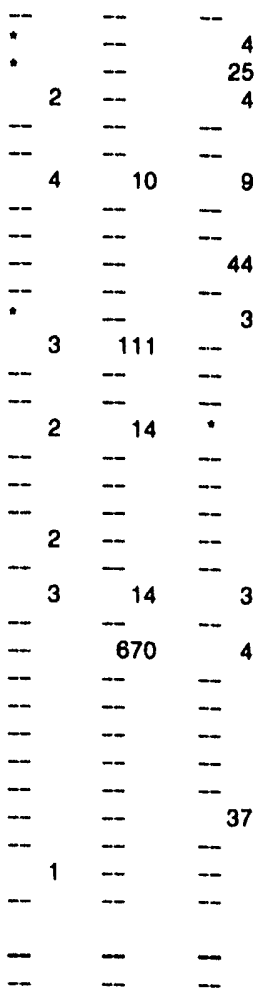

$\overline{-}$

$-$

5,728

5,183

$\overline{7}, 071$

$-$

834

$-$

$-$

$\overline{-}$

$-$

- 928

7,896

1,004

784
552

-

311

$-\quad 48,087$

- $-\overline{-}$

$-{ }{ }^{13}-$

$\begin{array}{lll}- & - & - \\ -- & - & -\end{array}$

2,362 $158 \quad$

- $-\quad$ -

$-\quad-^{4}$

- $\quad--\quad=$

$\overline{8,227}-\overline{-}$

$-$

$-6$

$-$

$-$

$-$

$-$

$-$

$\overline{9,825}$

$\begin{array}{lll}- & - \\ - & - & - \\ - & - & - \\ - & - & - \\ - & - & - \\ - & -\end{array}$

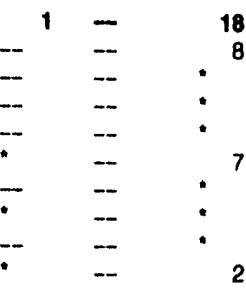

-
-
-
-
-

$-$

$-$

$--$

$=$

-

$-\quad-$

--

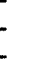

284 
Table 62. U.S. Electric Utility Net Generation, Fuel Consuniption, and Fuel Stocks by Company and Plant, September 1993 (Continued)

\begin{tabular}{|c|c|c|c|c|c|c|c|c|c|c|c|}
\hline \multirow{2}{*}{$\begin{array}{c}\text { Company (Holding Company) } \\
\text { Plant (State) }\end{array}$} & \multicolumn{6}{|c|}{$\begin{array}{l}\text { Ceneratlon } \\
\text { (thousand kllowatthours) }\end{array}$} & \multicolumn{3}{|c|}{$\begin{array}{l}\text { Consumption } \\
\text { (thousand) }\end{array}$} & \multicolumn{2}{|c|}{$\begin{array}{c}\text { Stocks } \\
\text { (thousand) }\end{array}$} \\
\hline & Coal & Petroleum & Cas & Hydro & Nuclear & Other' & $\begin{array}{c}\text { Coal } \\
\text { (short } \\
\text { tons) }\end{array}$ & $\begin{array}{l}\text { Petro- } \\
\text { Ieum } \\
\text { (blole) }\end{array}$ & $\begin{array}{c}\text { Gas } \\
\text { (Mcf) }\end{array}$ & $\begin{array}{c}\text { Coal } \\
\text { (ahort } \\
\text { tons) }\end{array}$ & $\begin{array}{l}\text { Petro- } \\
\text { leum } \\
\text { (bbls) }\end{array}$ \\
\hline
\end{tabular}

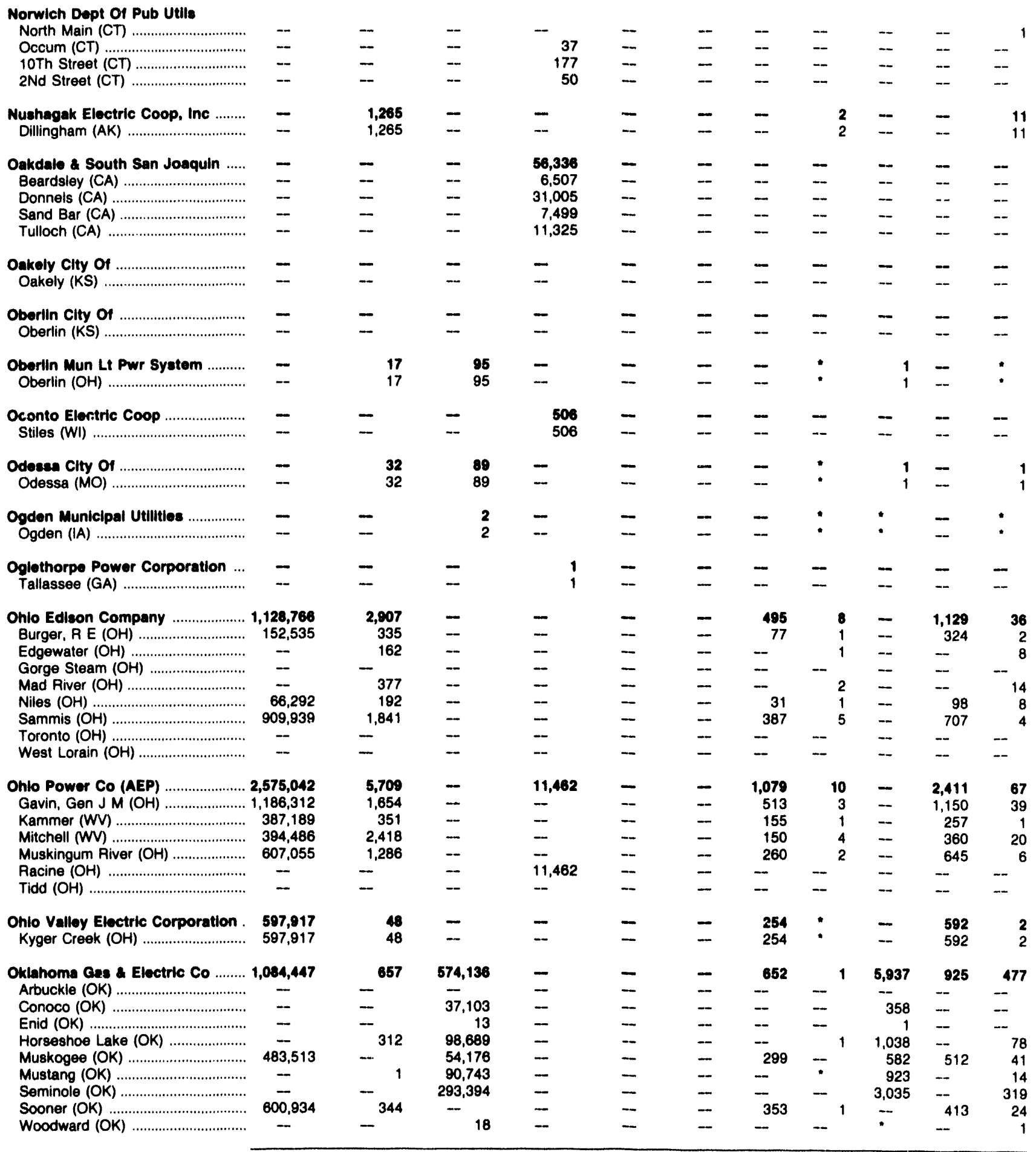

See footnotes at end of table. 
Table 62. U.S. Electric Utility Net Generation, Fuel Consumption, and Fuel Stocks by Company and Plant, September 1993 (Continued)

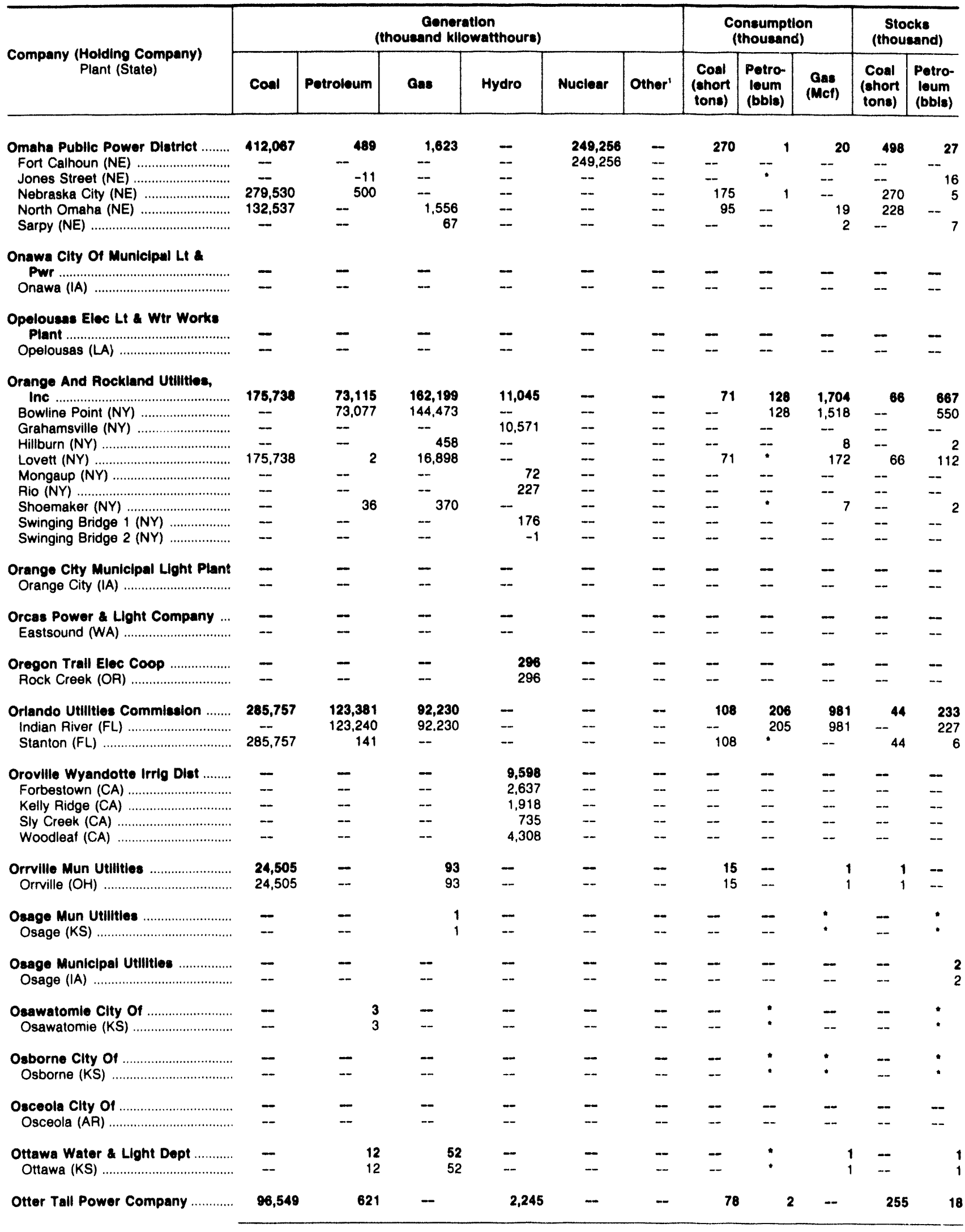

See footnotes at end of table. 
Table 62. U.S. Electric Utility Net Generation, Fuel Consumption, and Fuel Stocks by Company and Plant, September 1993 (Continued)

\begin{tabular}{|c|c|c|c|c|c|c|c|c|c|c|c|}
\hline \multirow{2}{*}{$\begin{array}{c}\text { Company (Holding Company) } \\
\text { Plant (State) }\end{array}$} & \multicolumn{6}{|c|}{$\begin{array}{c}\text { Generation } \\
\text { (thousand kilowatthours) }\end{array}$} & \multicolumn{3}{|c|}{$\begin{array}{l}\text { Consumption } \\
\text { (thousand) }\end{array}$} & \multicolumn{2}{|c|}{$\begin{array}{c}\text { Stocks } \\
\text { (thousand) }\end{array}$} \\
\hline & Coal & Potroloum & Gas & Hydro & Nuclear & Other ${ }^{1}$ & $\begin{array}{c}\text { Coal } \\
\text { (short } \\
\text { tona) }\end{array}$ & $\begin{array}{l}\text { Petro- } \\
\text { loum } \\
\text { (bbls) }\end{array}$ & $\begin{array}{c}\text { Gas } \\
\text { (Mcf) }\end{array}$ & $\begin{array}{c}\text { Coal } \\
\text { (short } \\
\text { tons) }\end{array}$ & $\begin{array}{l}\text { Petro- } \\
\text { leum } \\
\text { (bbls) }\end{array}$ \\
\hline
\end{tabular}

\section{Otter Tall Power Company}

Bemidji (MN)

Big Stone (SD)

Dayton Hollow (MN)

Hoot Lake (MN) .

Jamestown (ND)

Lake Preston (SD) ........

Pisgah (MN)

Port 148 (MN)

Taplin Gorge (MN)

Wright (MN)

Ottumwa Wtr Wrke Hydro EI PI

Ottumwa (IA)

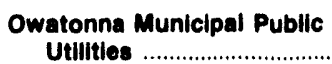

Owatonna (MN)

Owensboro Municlpal Utillities ...

Elmer Smith (KY)

Owensville Municipal Utilities

Owensville (MO)

Oxford Mun Lt \& Pwr Plant

Oxford (NE)

Pacific Gas \& Eloctric Company.

- Central Storage *

Alta (CA)

Angels (CA)

Balch 1 (CA)

Baich 2 (CA)

Belden (CA)

Black, James B (CA)

Bucks Creek (CA)

Butt Valley (CA)

Caribou 1 (CA)

Caribou 2 (CA)

Centerville (CA)

Chili Bar (CA)

Coal Canyon (CA)

Coleman (CA)

Contra Costa (CA)

Cow Creek (CA) ...

Crane Valley (CA)

Cresta (CA) .

De Sabla (CA)

Deer Creek (CA)

Diablo Canyon (CA)

Downieville (CA)

Drum 1 (CA)

Drum 2 (CA)

Dutch Flat (CA)

El Dorado (CA)

Electra (CA)

Haas (CA)

Halsey (CA)

Hamilton Branch

Hamillon Branch (CA)

Hat Creek 1 (CA)

Hat Creok 2 (CA)

Helms (CA)

Humbolt Bay (CA)

unters Point (CA)

Inskip (CA)

Kerckhot (CA)

Kerckhot 2 (CA)

See footnotes at end of table.

\begin{tabular}{|c|c|c|c|c|c|c|c|}
\hline 244 & - & -- & -- & - & -- & -- & -- \\
\hline-- & - & -- & 54 & " & - & 255 & \\
\hline 663 & - & -- & -- & -- & -- & - & - \\
\hline 313 & - & - & 24 & • & - & 1 & $\star$ \\
\hline- & - & -- & -- & & - & - & \\
\hline-- & - & - & - & - & - & $-\infty$ & \\
\hline 388 & -- & -- & - & -- & - & - & -- \\
\hline-- & - & -- & $\cdots$ & -- & -- & -- & -- \\
\hline 340 & - & -- & -- & -- & - & -- & -- \\
\hline 297 & -- & - & - & -- & -- & -- & - \\
\hline - & - & - & - & - & - & - & - \\
\hline- & -- & - & - & -- & - & - & \\
\hline
\end{tabular}

$\begin{array}{rrr}- & & 451 \\ - & & 451 \\ 188 & - & - \\ 188 & - & - \\ 175 & - & - \\ 175 & - & - \\ - & - & -\end{array}$

$8951,676,613 \quad 1,053,709$

$\begin{array}{lll}- & -- \\ -- & -- \\ -- & -- \\ - & - \\ -- & --\end{array}$

470
636

18,436

66,902

60,056

42,931

3,261

24,430

23,327

70,362

1,568

2,327

372

5,150

- 425

425
190

27,402

4,337

--

$-$

7,644
10,469

3,007

-6
34,998

83,986

6,378

2,537

1,962

3,167

43,510

--

3,392

-23
48,055
$1,561,057 \quad 538,750$

--

$--$

--

$-$

-

$-$

$-$

-

--

--

$-$

$-$

$-$

$-$

$-$

$-$

-

--

-

-

$-\quad \quad-$

$+$

-

$\begin{array}{ll}- & - \\ - & --\end{array}$

-

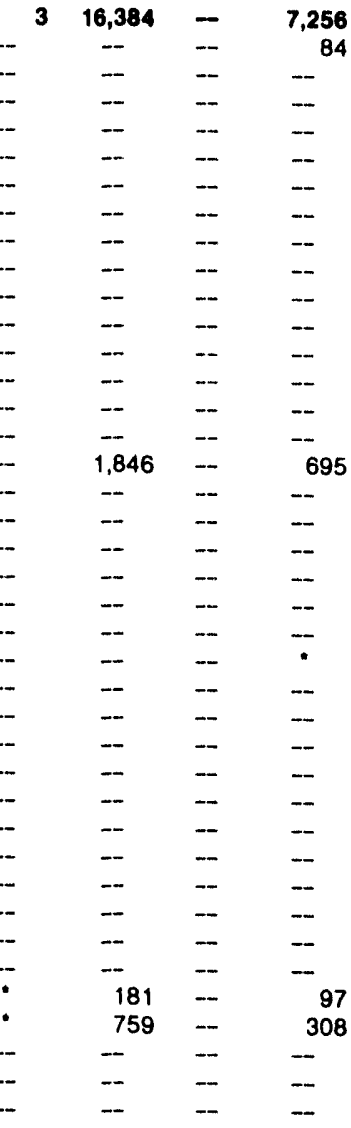

$-$

$-$

$=$ 
Table 62. U.S. Electric Utility Net Generation, Fuel Consumption, and Fuel Stocks by Company and Plant, September 1993 (Continued)

\begin{tabular}{|c|c|c|c|c|c|c|c|c|c|c|c|}
\hline \multirow{2}{*}{$\begin{array}{c}\text { Company (Holding Company) } \\
\text { Plant (State) }\end{array}$} & \multicolumn{6}{|c|}{$\begin{array}{c}\text { Gonoration } \\
\text { (thousand kilowatthours) }\end{array}$} & \multicolumn{3}{|c|}{$\begin{array}{l}\text { Consumption } \\
\text { (thousand) }\end{array}$} & \multicolumn{2}{|c|}{$\begin{array}{l}\text { Stocks } \\
\text { (thousand) }\end{array}$} \\
\hline & Coal & Petroleum & Cas & Hydro & Nuclear & Other' & $\begin{array}{l}\text { Coal } \\
\text { (short } \\
\text { tons) }\end{array}$ & $\begin{array}{l}\text { Petro- } \\
\text { loum } \\
\text { (bbls) }\end{array}$ & $\begin{array}{l}\text { Gas } \\
(M c f)\end{array}$ & $\begin{array}{c}\text { Coal } \\
\text { (short } \\
\text { tons) }\end{array}$ & $\begin{array}{l}\text { Petro- } \\
\text { leum } \\
\text { (bb/s) }\end{array}$ \\
\hline
\end{tabular}

Pacific Gas Electric Company

Kern (CA)....

Kern Canyon (CA)

Kilarc (CA)

Kings Aiver (CA)

Lime Saddle (CA)

Merced Falls (CA)

Mobile Turbine (CA)

Morro Bay (CA)

Moss Landing (CA)

Murphys (CA)

Narrows (CA) .

Newcastle (CA)

Oak Flat (CA)

Oakland (CA)

Phoenix (CA)

Pit 1 (CA)

Pit 3 (CA)

Pit 4 (CA)

Pit 5 (CA)

Pit 6 (CA)

Pit 7 (CA)

Pittsburg (CA)

Poe (CA)

Potrero (CA)

Potter Valley (CA)

PVUSA 1 (CA)

PVUSA 3 (CA)

Rock Creek (CA)

Salt Springs (CA)

San Joaquin No. 1a (CA)

San Joaquin No. 2 (CA)

San Joaquin 3 (CA)

South (CA)

Spaulding No. 1 (CA)

Spaulding No. 2 (CA)

Spaulding No. 3 (CA)

Spring Gap (CA)

Stanislaus (CA)

The Geysers (CA)

Tiger Creek (CA)

Toadtown (CA)

Tule River (CA)

Volta (CA)

Volta 2 (CA)

West Point (CA)

Wise (CA)

Wise 2 (CA)

Wishon, A G (CA)

Paciflcorp

American Fork (UT)

Ashton (ID)

Beaver Upper (UT)

Bend (OR)

Big Fork (MT)

Blundell (UT)

Bridger, Jim (WY)

Carbon (UT)

Centralia (WA)

Clearwater 1 (OR)

Clearwater 2 (OR)

Cline Falls (OR)

Condit (WA)

Copco 1 (CA)

Copco 2 (CA)

Cove (ID)

Cutler (UT)

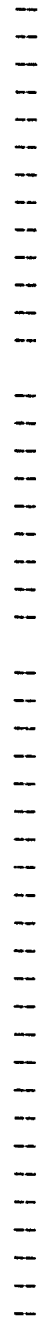

$4,947,906$

$-$

--

$-$

$34 \overline{9,633}$

118,490

941,539

$--$

$-$

$-$

$-$

$-$

$-$

See footnotes at end of table. 
Table 62. U.S. Electric Utility Net Generation, Fuel Consumption, and Fuel Stocks by Company and Plant, September 1993 (Continued)

\begin{tabular}{|c|c|c|c|c|c|c|c|c|c|c|c|}
\hline \multirow{2}{*}{$\begin{array}{c}\text { Company (Holding Company) } \\
\text { Plant (State) }\end{array}$} & \multicolumn{6}{|c|}{$\begin{array}{l}\text { Coneration } \\
\text { (thoueand kllowatthoure) }\end{array}$} & \multicolumn{3}{|c|}{$\begin{array}{l}\text { Consumption } \\
\text { (thouasnd) }\end{array}$} & \multicolumn{2}{|c|}{$\begin{array}{l}\text { Stocke } \\
\text { (thousand) }\end{array}$} \\
\hline & Coal & Potroleum & Cas & Hydro & Nuctear & Other' & $\begin{array}{l}\text { Coel } \\
\text { (ehort } \\
\text { tons) }\end{array}$ & $\begin{array}{l}\text { Potro- } \\
\text { leum } \\
\text { (bbls) }\end{array}$ & Gas & $\begin{array}{c}\text { Coal } \\
\text { (short } \\
\text { tons) }\end{array}$ & $\begin{array}{l}\text { Potro- } \\
\text { loum } \\
\text { (bbla) }\end{array}$ \\
\hline
\end{tabular}

\section{Pacticorp}

Eagle Point (OA)

East Side (OR)

Fall Creek (CA)

Fish Creok (OR)

Ftn Green (UT)

Gadsby (UT)

Grace (ID)

Granite (UT)

Hunter (emery) (UT)

Huntington Canyon (UT

Hydro No. 1 (UT)

Hydro No. 2 (UT)

Hydro No. 3 (UT)

Iron Gate (CA)

John C Boyle (OR)

Johnston, Dave (WY)

Last Chance (UT)

Lemolo 1 (OR)

Lemolo 2 (OR)

Little Mountain (UT)

Menwin (WA)

Naches (WA)

Naches Drop (WA)

Naughton (WY

Olmstead (UT)

Onoida (ID)

Paris (ID)

Pioneer (UT)

Powerdale (OR)

Prospect 1 (OR)

Prospect 2 (OR)

Prospect 3 (OR)

Plospect 4 (OR)

Skookumchuck (WA)

Slide Creek (OR)

Snake Creek (UT)

Soda (ID)

Soda Springs (OR)

St Anthony (ID)

Stairs (UT)

Stayton (OR)

Swift No. 2 (WA)

Swift 1 (WA) ...

Toketee (OR)

Viva (WY)

Wallowa Falls (OR)

Weber (UT)

West Side (OR)

Wyodak (WY

Yale (WA)

Palneeville Mun Lt Plant ................

Painesville (OH)

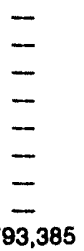

566,837

$-$

$-$

-

$5 \overline{57}, 421$

$-$

-

$-$

$-$

-

$-$

$\overline{384,774}$

$-$

$-$

$-$

$-$

$-$

$-$

$-$

-

-

$-$

$-$

$-$

$-$

$-$

$-$

$-$

$-$

-

$\overline{-}$

$-$

235,827

12,505

12,505

Palmyra Clty of Bd of Pub

Worke

Palmyra (MO)

Paimyra 2 (MO)

Paragould Lught Plant Comm .......

Paragould (AR)

Paria Lught \& Power Dept .............. Paris (KY)

Parowan Clty Corporation ............. Center Creok (UT)

Paragonah (UT)

See footnotes at end of table. 
Table 62. U.S. Electric Utility Net Generation, Fuel Consumption, and Fuel Stocks by Company and Plant, September 1993 (Continued)

\begin{tabular}{|c|c|c|c|c|c|c|c|c|c|c|c|}
\hline \multirow{2}{*}{$\begin{array}{c}\text { Company (Holding Company) } \\
\text { Plant (State) }\end{array}$} & \multicolumn{6}{|c|}{$\begin{array}{c}\text { Generation } \\
\text { (thousand kllowatthours) }\end{array}$} & \multicolumn{3}{|c|}{$\begin{array}{l}\text { Consumption } \\
\text { (thousend) }\end{array}$} & \multicolumn{2}{|c|}{$\begin{array}{c}\text { Stocks } \\
\text { (thousand) }\end{array}$} \\
\hline & Coal & Petroleum & Gas & Hydro & Nuclear & Other' & $\begin{array}{l}\text { Coal } \\
\text { (stort } \\
\text { tons) }\end{array}$ & $\begin{array}{l}\text { Potro- } \\
\text { loum } \\
\text { (bbla) }\end{array}$ & $\begin{array}{l}\text { ans } \\
\text { (Mct) }\end{array}$ & $\begin{array}{c}\text { Coal } \\
\text { (chort } \\
\text { tons) }\end{array}$ & $\begin{array}{l}\text { Potro- } \\
\text { loum } \\
\text { (bbla) }\end{array}$ \\
\hline
\end{tabular}

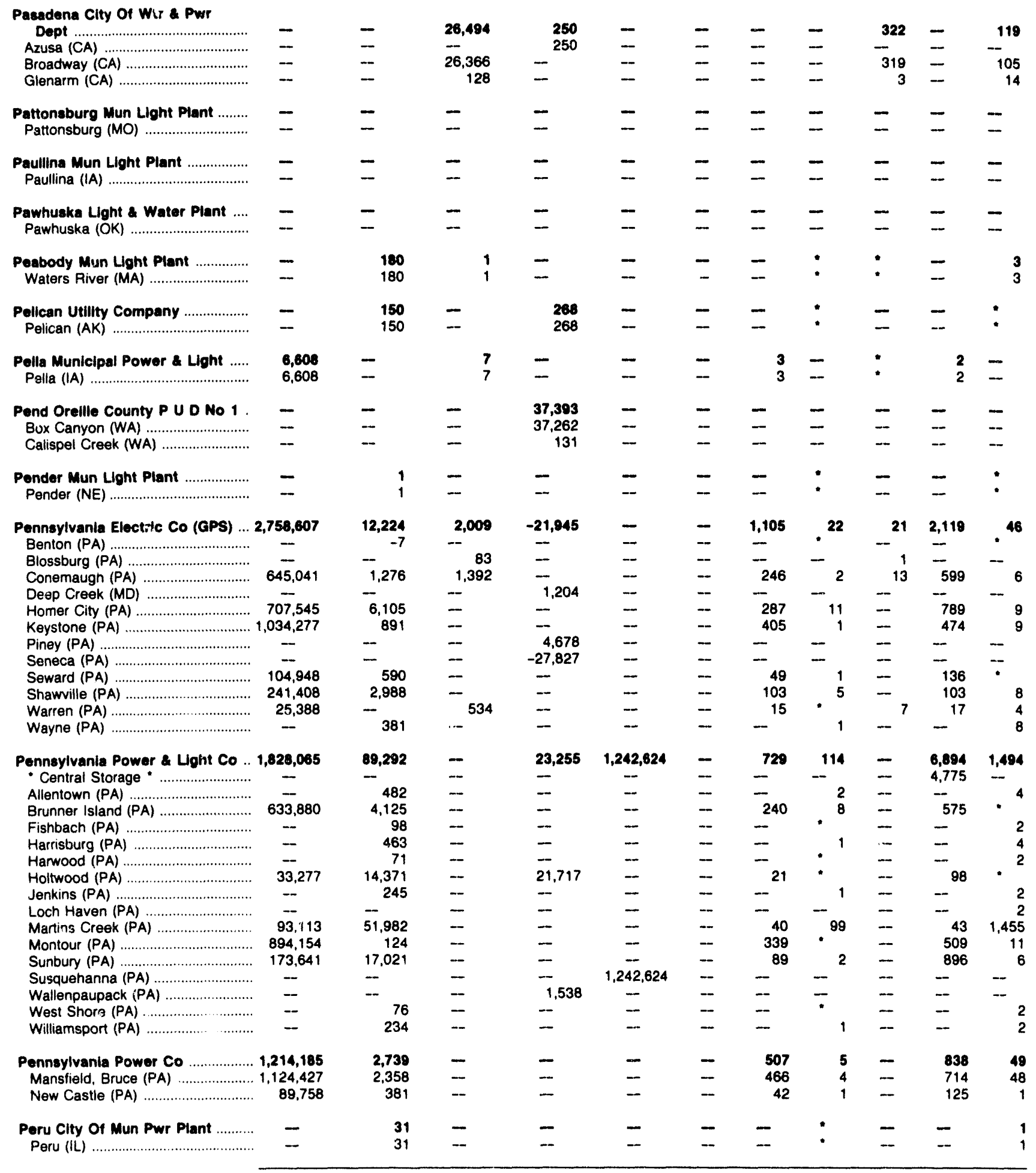

See footnotes at end of table. 
Table 62. U.S. Electric Utility Net Generation, Fuel Consumption, and Fuel Stocks by Company and Plant, September 1993 (Continued)

\begin{tabular}{|c|c|c|c|c|c|c|c|c|c|c|c|}
\hline \multirow{2}{*}{$\begin{array}{c}\text { Company (Holding Company) } \\
\text { Plant (State) }\end{array}$} & \multicolumn{6}{|c|}{$\begin{array}{c}\text { Ceneration } \\
\text { (thoueand kllowatthours) }\end{array}$} & \multicolumn{3}{|c|}{$\begin{array}{l}\text { Consumption } \\
\text { (thousand) }\end{array}$} & \multicolumn{2}{|c|}{$\begin{array}{c}\text { Stocks } \\
\text { (thousand) }\end{array}$} \\
\hline & Coal & Potroloum & Oas & Hydro & Nuclear & Other' & $\begin{array}{c}\text { Coal } \\
\text { (chort } \\
\text { tons) }\end{array}$ & $\begin{array}{l}\text { Petro- } \\
\text { loum } \\
\text { (bole) }\end{array}$ & $\begin{array}{c}\text { Gas } \\
\text { (Mcf) }\end{array}$ & $\begin{array}{c}\text { Coal } \\
\text { (short } \\
\text { tons) }\end{array}$ & $\begin{array}{l}\text { Potro- } \\
\text { leum } \\
\text { (bble) }\end{array}$ \\
\hline 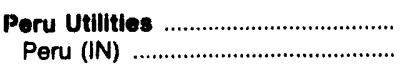 & $\begin{array}{l}1,401 \\
1,401\end{array}$ & $\begin{array}{l}27 \\
27\end{array}$ & - & $\overline{-}$ & $\overline{-}$ & - & $\begin{array}{l}1 \\
1\end{array}$ & : & $\overline{--}$ & . & 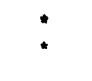 \\
\hline 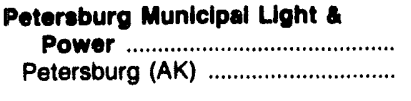 & $\overline{-}$ & $\begin{array}{l}1,172 \\
1,172\end{array}$ & $\overline{-}$ & $\begin{array}{l}410 \\
410\end{array}$ & $\overline{-}$ & - & - & $\begin{array}{l}2 \\
2\end{array}$ & $\overline{-}$ & $\overline{--}$ & $:$ \\
\hline 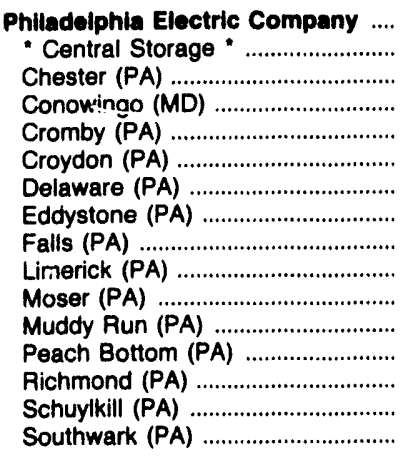 & $\begin{array}{l}341,855 \\
- \\
- \\
58,986 \\
- \\
\overline{-} \\
282,869 \\
- \\
- \\
- \\
- \\
- \\
-\end{array}$ & $\begin{array}{r}225,901 \\
-- \\
-\quad 1 \\
46,952 \\
3,021 \\
53,698 \\
89,824 \\
-\quad 76 \\
-\quad 172 \\
-\quad \\
-\quad 756 \\
31,490 \\
11\end{array}$ & $\begin{array}{l}28,743 \\
-- \\
-- \\
\overline{25,439} \\
- \\
- \\
3,304 \\
- \\
- \\
-- \\
- \\
- \\
- \\
-\end{array}$ & $\begin{array}{l}-20,329 \\
-- \\
- \\
38,398 \\
-- \\
- \\
- \\
- \\
- \\
- \\
- \\
-58,727 \\
- \\
- \\
- \\
-\end{array}$ & $\begin{array}{c}2,406,437 \\
- \\
- \\
- \\
- \\
- \\
- \\
- \\
- \\
1,356,151 \\
- \\
- \\
1,050,286 \\
- \\
- \\
-\end{array}$ & $\begin{array}{l}- \\
- \\
- \\
- \\
- \\
-- \\
- \\
- \\
- \\
- \\
- \\
-\end{array}$ & $\begin{array}{l}142 \\
- \\
-- \\
- \\
- \\
- \\
- \\
119 \\
- \\
-- \\
- \\
-- \\
- \\
-- \\
--\end{array}$ & $\begin{array}{r}391 \\
- \\
- \\
- \\
- \\
78 \\
6 \\
92 \\
156 \\
-\quad \\
-\quad 1 \\
-\quad 2 \\
-56\end{array}$ & $\begin{array}{l}299 \\
- \\
- \\
264 \\
- \\
- \\
-35 \\
- \\
-- \\
- \\
-- \\
- \\
-- \\
-- \\
--\end{array}$ & $\begin{array}{l}222 \\
-- \\
-- \\
-- \\
-- \\
- \\
- \\
172 \\
-- \\
-- \\
- \\
- \\
-- \\
-- \\
- \\
--\end{array}$ & $\begin{array}{r}304 \\
15 \\
5 \\
-- \\
33 \\
41 \\
34 \\
137 \\
-\quad 5 \\
-5 \\
-\quad \\
- \\
- \\
- \\
20 \\
3 \\
6\end{array}$ \\
\hline $\begin{array}{l}\text { Piggott Public Improvement Piat } \\
1 \text { Piggott (AR) }\end{array}$ & - & $\begin{array}{l}30 \\
30\end{array}$ & - & - & $\overline{-}$ & - & - & 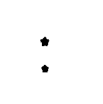 & - & - & : \\
\hline $\begin{array}{l}\text { Piqua Municipal Power System .... } \\
\text { Piqua (OH) }\end{array}$ & $\begin{array}{l}4,486 \\
4,486\end{array}$ & -4 & - & - & - & - & 4 & $\cdot$ & - & 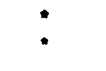 & $\begin{array}{l}3 \\
3\end{array}$ \\
\hline 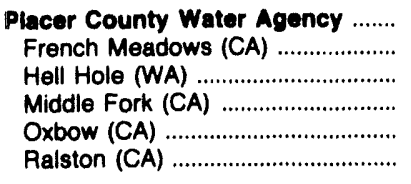 & $\begin{array}{l}- \\
- \\
- \\
-\end{array}$ & $\begin{array}{l}- \\
- \\
- \\
-\end{array}$ & $\begin{array}{l}- \\
- \\
- \\
-\end{array}$ & $\begin{array}{r}90,543 \\
11,041 \\
341 \\
46,217 \\
2,156 \\
30,788\end{array}$ & $\begin{array}{l}- \\
- \\
- \\
- \\
-\end{array}$ & $\begin{array}{l}- \\
- \\
- \\
--\end{array}$ & $\begin{array}{l}- \\
- \\
- \\
-\end{array}$ & $\begin{array}{l}- \\
- \\
- \\
- \\
-\end{array}$ & $\begin{array}{l}- \\
-- \\
- \\
-\end{array}$ & $\begin{array}{l}- \\
-- \\
-- \\
--\end{array}$ & $\begin{array}{l}- \\
- \\
- \\
- \\
-\end{array}$ \\
\hline 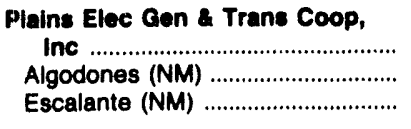 & $\begin{array}{l}144,668 \\
144,668\end{array}$ & - & $\begin{array}{r}19 \\
--\quad 19\end{array}$ & - & - & $\overline{-}$ & $\begin{array}{r}82 \\
-82\end{array}$ & - & 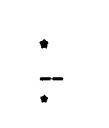 & $\frac{133}{133}$ & $\begin{array}{r}9 \\
-\quad 9\end{array}$ \\
\hline 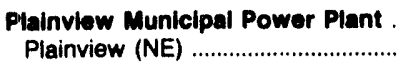 & - & - & - & - & - & - & - & - & $\overline{-}$ & - & - \\
\hline 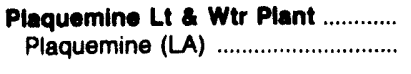 & - & - & - & - & - & - & - & $\overline{-}$ & : & $\overline{-}$ & - \\
\hline 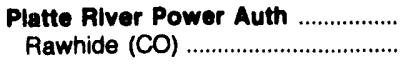 & $\begin{array}{l}170,292 \\
170,292\end{array}$ & - & - & - & - & - & $\begin{array}{l}102 \\
102\end{array}$ & - & - & $\begin{array}{l}163 \\
163\end{array}$ & $\begin{array}{l}4 \\
4\end{array}$ \\
\hline $\begin{array}{l}\text { Ponca Clty Mun Wtr Lt Dept .... } \\
\text { Ponca Steam (OK) } \\
\text { Ponca Steam (OK) }\end{array}$ & - & $\begin{array}{r}32 \\
-\quad 32\end{array}$ & $\begin{array}{r}293 \\
-- \\
293\end{array}$ & - & - & - & - & 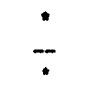 & --4 & - & $\begin{array}{r}1 \\
-\quad 1\end{array}$ \\
\hline 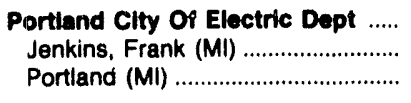 & $\overline{-}$ & $\overline{-}$ & - & $\begin{array}{r}153 \\
-\quad 153\end{array}$ & - & $\overline{-}$ & - & - & - & - & $\begin{array}{l}- \\
-- \\
--\end{array}$ \\
\hline $\begin{array}{l}\text { Portland General Electric } \\
\text { Company } \\
\text { Beaver (OR) } \\
\text { Bethel (OR) } \\
\text { Boardman (OR) } \\
\text { Bull Run (OR) } \\
\text { Faraday (OR) } \\
\text { North Fork (OR) }\end{array}$ & $\begin{array}{c}359,886 \\
- \\
-- \\
359,886 \\
- \\
- \\
-\end{array}$ & $\begin{array}{r}310 \\
-\quad 1 \\
-\quad 318 \\
- \\
-\end{array}$ & $\begin{array}{c}167,748 \\
167,748 \\
- \\
- \\
- \\
- \\
-\end{array}$ & $\begin{array}{l}142,977 \\
- \\
- \\
- \\
2,407 \\
3,788 \\
4,129\end{array}$ & $\begin{array}{l}- \\
- \\
- \\
- \\
-\end{array}$ & $\begin{array}{l}- \\
- \\
- \\
- \\
- \\
-\end{array}$ & $\begin{array}{l}212 \\
- \\
- \\
- \\
-\end{array}$ & $\begin{array}{l}1 \\
-\quad 1 \\
- \\
- \\
-\end{array}$ & $\begin{array}{l}1,638 \\
1,638 \\
-- \\
-- \\
-- \\
-- \\
--\end{array}$ & $\begin{array}{l}586 \\
-- \\
- \\
586 \\
-- \\
-- \\
--\end{array}$ & $\begin{array}{r}232 \\
211 \\
16 \\
\\
- \\
- \\
- \\
-\end{array}$ \\
\hline
\end{tabular}

See iootnotes at end of table. 
Table 62. U.S. Electric Utility Net Generation, Fuel Consumption, and Fuel Stocks by Company and Plant, September 1993 (Continued)

\begin{tabular}{|c|c|c|c|c|c|c|c|c|c|c|c|}
\hline \multirow{2}{*}{$\begin{array}{c}\text { Company (Holding Company) } \\
\text { Plant (State) }\end{array}$} & \multicolumn{6}{|c|}{$\begin{array}{c}\text { Generation } \\
\text { (thousand kllowatthours) }\end{array}$} & \multicolumn{3}{|c|}{$\begin{array}{l}\text { Consumption } \\
\text { (thousand) }\end{array}$} & \multicolumn{2}{|c|}{$\begin{array}{c}\text { Stocks } \\
\text { (thousand) }\end{array}$} \\
\hline & Coal & Petroleum & Gas & Hydro & Nuclear & Other' & $\begin{array}{l}\text { Coal } \\
\text { (short } \\
\text { tons) }\end{array}$ & $\begin{array}{l}\text { Potro- } \\
\text { loum } \\
\text { (bbls) }\end{array}$ & $\begin{array}{l}\text { Gas } \\
\text { (Mcf) }\end{array}$ & $\begin{array}{l}\text { Coal } \\
\text { (short } \\
\text { tons) }\end{array}$ & $\begin{array}{l}\text { Petro- } \\
\text { leum } \\
\text { (bbls) }\end{array}$ \\
\hline
\end{tabular}

\begin{tabular}{|c|c|c|c|c|c|c|c|c|c|c|c|}
\hline $\begin{array}{l}\text { Portiand General Electric } \\
\text { Company }\end{array}$ & & & & & & & & & & & \\
\hline Oak Grove (OR) & -- & - & - & 13,466 & -- & -- & -- & -- & -- & -- & -- \\
\hline Pelton (OR) & - & - & - & 29,571 & - & -- & -- & -- & - & - & - \\
\hline Pelton Re Regulation (OR) .............. & -- & -- & - & 5,552 & -- & - & -- & -- & -- & -- & -- \\
\hline Portland Hydro Proj 1 (OR) ............ & - & - & - & 2,310 & - & - & -- & -- & -- & -- & - \\
\hline Portland Hydro Proj 2 (OR) ............. & -- & -- & - & - & -- & -- & - & -- & -- & -- & -- \\
\hline 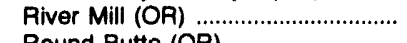 & - & -- & -- & 2,667 & - & -- & -- & -- & -- & -- & - \\
\hline 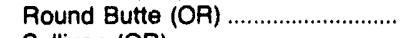 & - & - & -- & 67,539 & - & -- & -- & -- & -- & -- & $-\cdots$ \\
\hline Sullivan (OR) .................................. & - & -- & -- & 11,548 & - & -- & - & -- & -- & -- & -- \\
\hline 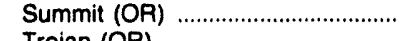 & - & - & -- & -- & - & - & -- & -- & -- & - & " \\
\hline 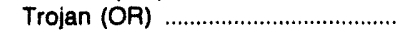 & - & -- & -- & - & -- & - & -- & -- & - & -- & -- \\
\hline Potomac Edison Co (APS) ............. & 21,210 & 291 & - & 1,709 & - & - & 10 & 1 & -- & 54 & * \\
\hline Dam 4 (WV) & -- & -- & -- & 560 & - & -- & -- & -- & -- & $-\infty$ & -- \\
\hline Dam 5 (WV) & -- & -- & - & 393 & -- & - & -- & -- & -- & - & -- \\
\hline 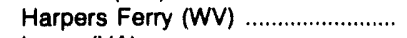 & -- & -- & -- & - & - & - & -- & -- & -- & -- & -- \\
\hline 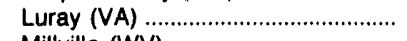 & - & -- & - & - & - & - & -- & -- & -- & -- & -- \\
\hline 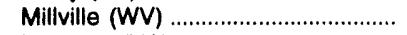 & - & -- & -- & 364 & - & - & - & - & -- & -- & -- \\
\hline 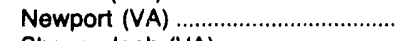 & -- & - & -- & 152 & -- & -- & -- & -- & -- & -- & -- \\
\hline 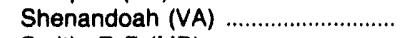 & -- & -- & - & 65 & -- & -- & - & -- & - & -- & -- \\
\hline 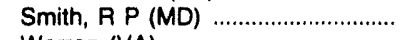 & 21,210 & 291 & - & - & - & - & 10 & 1 & -- & 54 & • \\
\hline 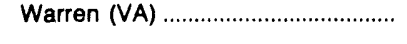 & -- & - & -- & 175 & - & -- & - & -- & - & -- & -- \\
\hline Potomac Electric Power Co ........... & 965,918 & 188,006 & 71,754 & - & - & - & 372 & 403 & 961 & 966 & 1,123 \\
\hline 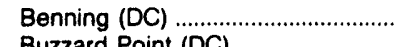 & -- & 27,685 & -- & -- & - & -- & - & 62 & - & - & 101 \\
\hline Buzzard Point (DC) ......................... & -- & 1,267 & -- & - & - & -- & -- & 4 & -- & -- & 12 \\
\hline 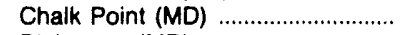 & 76,221 & 110,428 & 58,100 & -- & -- & -- & 31 & 259 & 798 & 253 & 748 \\
\hline 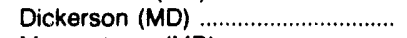 & 301,769 & 2,700 & 13,654 & -- & - & -- & 114 & 5 & 163 & 238 & 41 \\
\hline Morgantown (MD) ................................ & 420,667 & 44,579 & -- & -- & -- & -- & 155 & 70 & - & 403 & 220 \\
\hline Potomac River (VA) ................................. & 167,261 & 1,347 & -- & - & -- & -- & 71 & 3 & -- & 71 & 1 \\
\hline Power Authority of The State of & & & & & & & & & & & \\
\hline 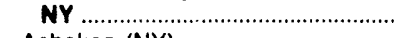 & - & 17,687 & 138,613 & $1,728,191$ & 480,930 & - & - & 35 & 1,714 & - & 223 \\
\hline 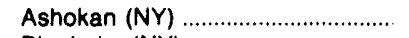 & -- & - & - & 1,079 & -- & -- & -- & -- & - & -- & -- \\
\hline 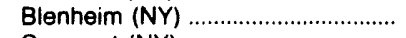 & -- & -- & -- & $-70,903$ & - & -- & -- & -- & -- & -- & - \\
\hline 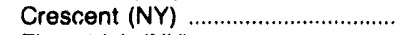 & -- & -- & -- & 2,346 & - & -- & -- & -- & -- & - & - \\
\hline 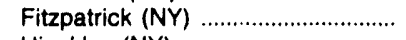 & -- & - & - & - & 480,930 & -- & -- & -- & - & -- & -- \\
\hline 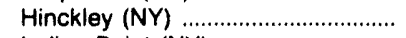 & -- & - & - & 1,661 & -- & -- & - & -- & -- & -- & -- \\
\hline Indian Point (NY) . & -- & -- & -- & - & -- & -- & - & -- & -- & -- & -- \\
\hline 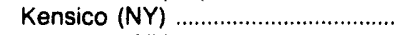 & - & - & -- & 1,055 & -- & -- & -- & -- & -- & -- & -- \\
\hline 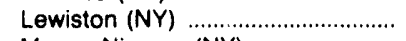 & -- & -- & - & $-32,274$ & -- & -- & - & -- & - & -- & - \\
\hline Moses Niagara (NY) ........................ & -- & -- & -- & $1,227,824$ & -- & -- & -- & - & -- & -- & -- \\
\hline Moses Power Dam (NY) ................. & -- & - & - & 595,228 & - & -- & -- & - & -- & -- & -- \\
\hline 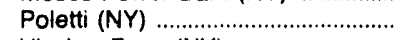 & -- & 17,687 & 138,613 & - & -- & -- & -- & 35 & 1,714 & -- & 223 \\
\hline 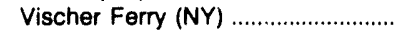 & -- & -- & - & 2,175 & -- & -- & - & -- & - & -- & -- \\
\hline Pratt Clty of & - & 395 & 4,671 & - & - & - & - & 2 & 59 & - & 1 \\
\hline 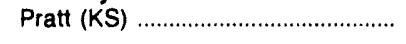 & - & 395 & 4,671 & - & - & - & - & 2 & 59 & -- & 1 \\
\hline Preston Publlc Utilities Comm ..... & - & -7 & - & - & - & - & - & - & - & - & * \\
\hline Preston (MN) .......................................... & -- & -7 & -- & -- & - & - & -- & -- & - & -- & - \\
\hline Preston, Town of Mun Lt \& Pwr . & - & - & - & - & - & - & - & - & - & - & - \\
\hline 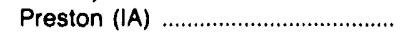 & -- & -- & -- & - & - & - & -- & -- & -- & -- & -- \\
\hline Primghar Municipal Lt Plant ........... & - & - & - & - & - & - & - & - & -- & -- & -- \\
\hline 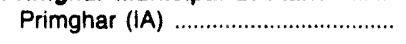 & -- & -- & -- & -- & - & - & -- & -- & -- & - & -- \\
\hline Princeton Mun Pwr \& Lt Plant ...... & - & - & - & - & - & - & - & - & -- & - & - \\
\hline 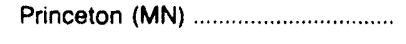 & - & -- & -- & -- & - & - & -- & -- & -- & -- & •" \\
\hline Princeton Municipal Utilities .......... & - & 19 & 32 & - & - & - & - & • & • & - & 1 \\
\hline 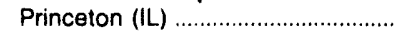 & - & 19 & 32 & - & - & - & -- & $\cdot$ & • & -- & 1 \\
\hline Providence Lt \& Wtr ........................... & - & - & - & - & - & - & - & - & - & - & -- \\
\hline Providence (RI) . & -- & - & - & - & - & - & -- & -- & -- & $\ldots$ & - \\
\hline
\end{tabular}

See footnotes at end of table. 
Table 62. U.S. Electric Utility Net Generation, Fuel Consumption, and Fuel Stocks by Company and Plant, September 1993 (Continued)

\begin{tabular}{|c|c|c|c|c|c|c|c|c|c|c|c|}
\hline \multirow{2}{*}{$\begin{array}{c}\text { Company (Holding Company) } \\
\text { Plant (State) }\end{array}$} & \multicolumn{6}{|c|}{$\begin{array}{l}\text { Generation } \\
\text { (thousand kllowatthours) }\end{array}$} & \multicolumn{3}{|c|}{$\begin{array}{l}\text { Consumption } \\
\text { (thousand) }\end{array}$} & \multicolumn{2}{|c|}{$\begin{array}{l}\text { Stocks } \\
\text { (thousand) }\end{array}$} \\
\hline & Coal & Petroleum & Gas & Hydro & Nuctear & Other' & $\begin{array}{l}\text { Coal } \\
\text { (chort } \\
\text { tons) }\end{array}$ & $\begin{array}{l}\text { Petro- } \\
\text { loum } \\
\text { (bble) }\end{array}$ & $\begin{array}{l}\text { Gas } \\
\text { (Mct) }\end{array}$ & $\begin{array}{c}\text { Coal } \\
\text { (short } \\
\text { tons) }\end{array}$ & $\begin{array}{l}\text { Potro- } \\
\text { loum } \\
\text { (bble) }\end{array}$ \\
\hline \multicolumn{12}{|l|}{$\begin{array}{l}\text { Provo City Corporation Dept Of } \\
\text { Util }\end{array}$} \\
\hline 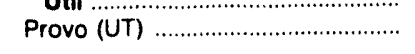 & - & - & 3 & $\overline{-}$ & - & $\overline{-}$ & - & - & $\cdot$ & $\overline{-}$ & 1 \\
\hline \multicolumn{3}{|l|}{$\begin{array}{l}\text { Public Serv Co of Oklahoma } \\
\text { (CSW) }\end{array}$} & 620,287 & - & - & - & 264 & - & 6,209 & 421 & 98 \\
\hline 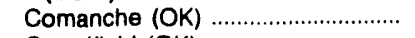 & -- & 15 & 110,017 & - & - & -- & $-\cdots$ & : & 910 & -- & $:$ \\
\hline 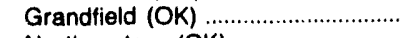 & $-\infty$ & 2 & $\overline{-100} \cdot 157$ & - & - & - & - & : & $-\overline{100}$ & -- & : \\
\hline 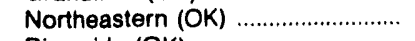 & 428,206 & 14 & 190.157 & - & - & -- & 264 & • & 1,956 & 421 & - \\
\hline 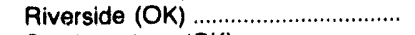 & -- & $-\infty$ & 278,347 & - & - & -- & -- & $\because$ & 2,831 & $-\infty$ & 46 \\
\hline 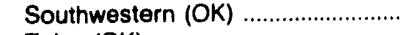 & -- & 10 & 41,301 & - & - & - & - & : & 505 & - & .50 \\
\hline Tulsa (OK) & -- & 6 & - & -- & - & - & - & * & - & -- & : \\
\hline 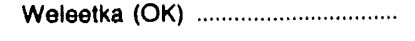 & - & - & 465 & - & - & - & -- & - & 7 & -- & " \\
\hline \multirow{2}{*}{\multicolumn{2}{|c|}{$\begin{array}{l}\text { Publlc Service Co Of Colorado } \ldots . .1,246,801 \\
\text { Alamosa (CO) }\end{array}$}} & 10 & 20,658 & 15,281 & - & - & 649 & - & 203 & 1,971 & 97 \\
\hline & & -- & 5 & -- & -- & - & -- & - & 1 & - & 6 \\
\hline Ames (CO) & $=-$ & - & - & 1,131 & - & -- & -- & -- & - & - & -- \\
\hline Arapahoe (CO) & 67.990 & - & 436 & $-\bar{n}$ & -- & - & 34 & - & 5 & 120 & -- \\
\hline 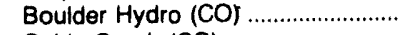 & -- & - & -- & 2,098 & - & - & - & - & -- & - & - \\
\hline Cabin Creek $(\mathrm{CO})$ & $\ddot{-\pi}$ & - & -- & $-2,879$ & - & - & - & - & - & - & 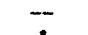 \\
\hline Cameo (CO) & 37,568 & - & 193 & - & - & -- & 19 & - & 2 & 45 & - \\
\hline 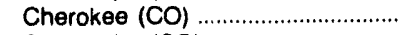 & 384,796 & - & 11,198 & -- & -- & - & 176 & -- & 92 & 343 & - \\
\hline Comanche (CO) . & 260,413 & - & 1,290 & - & -- & - & 162 & - & 14 & 405 & 2 \\
\hline 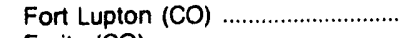 & -- & -- & 62 & - & - & - & - & $\because$ & 1 & -- & .14 \\
\hline Fruita $(\mathrm{CO})$ & - & 1 & 17 & -- & - & $\overline{-}$ & - & " & 1 & - & " \\
\hline Georgetown Hydro (CO) ................. & $-\overline{-}$ & - & -- & 538 & - & - & $-\overline{10}$ & $\because$ & - & - & - \\
\hline 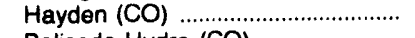 & 301,587 & 3 & 229 & -500 & -- & - & 148 & • & 2 & 555 & 4 \\
\hline 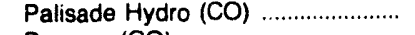 & -- & -- & $-\overline{c o s}(10)$ & 1,596 & -- & - & - & $\because$ & -- & - & - \\
\hline 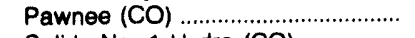 & 89,148 & 6 & 6,649 & - & - & - & 64 & " & 74 & 404 & 8 \\
\hline Salida No. 1 Hydro $(\mathrm{CO})$................... & -- & -- & - & 409 & - & - & -- & - & -- & -- & - \\
\hline Salida No. 2 Hydro (CO) ................ & - & -- & -- & $\begin{array}{r}328 \\
-950\end{array}$ & -- & - & - & -- & -- & -- & - \\
\hline 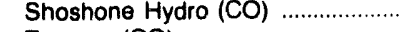 & - & - & - & 10,650 & - & - & - & - & - & - & -- \\
\hline 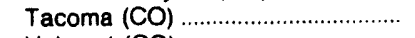 & $-\overline{0}$ & - & -- & 1,410 & - & - & - & - & -- & - & - \\
\hline Valmont (CO) ........................................ & 105,299 & - & 730 & - & -- & -- & 47 & - & 10 & 99 & 9 \\
\hline Zuni $(\mathrm{CO})$ & - & - & -151 & - & - & -- & - & -- & -- & - & 53 \\
\hline \multicolumn{12}{|l|}{$\begin{array}{l}\text { Public Service Co Of New } \\
\text { Hampshire }\end{array}$} \\
\hline 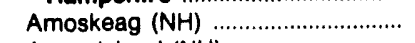 & - & - & - & 2,665 & - & - & - & -- & -- & -- & -- \\
\hline 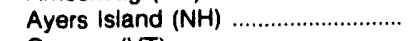 & - & - & -- & 2,130 & - & - & -- & - & -- & - & -- \\
\hline Canaan $(V T)$ & - & - & - & 738 & - & - & - & -- & -- & -- & - \\
\hline Eastman Falls (NH) & -- & -- & - & 1,124 & - & -- & -- & - & -- & -- & -- \\
\hline 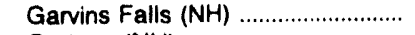 & -- & -- & -- & 1,451 & - & -- & - & - & - & - & - \\
\hline 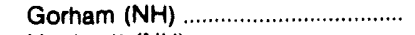 & -- & - & - & 1,061 & - & - & - & -- & - & -- & -- \\
\hline 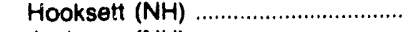 & - & - & - & 189 & - & - & - & - & -- & - & -- \\
\hline Jackman $(\mathrm{NH})$ & -- & - & - & -1 & - & - & - & - & - & - & - \\
\hline Lost Nation $(\mathrm{NH})$ & $-\overline{0}-1000$ & -9 & - & - & - & - & - & - & -- & - & 2 \\
\hline Merrimack (NH) & 234,673 & -12 & - & - & - & - & 91 & 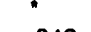 & - & 295 & 3 \\
\hline 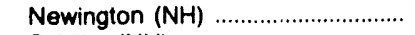 & -- & 193,870 & -- & - & -- & - & - & 342 & -- & - & 213 \\
\hline Schiller $(\mathrm{NH})$ & 53,323 & 13,091 & -- & - & $-\overline{c o s}$ & - & 26 & 26 & - & 72 & 91 \\
\hline 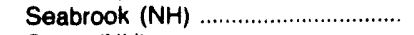 & - & - & - & -700 & 586,020 & - & - & - & - & - & -- \\
\hline 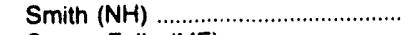 & - & - & - & 7,636 & - & - & - & -- & -- & -- & $\because$ \\
\hline Swans Falls (ME) & - & -2 & - & -- & - & - & - & $\because$ & -- & - & * \\
\hline White Lake (NH) & - & -2 & - & - & - & - & -- & • & - & -- & 2 \\
\hline \multicolumn{12}{|c|}{$\begin{array}{l}\text { Publlic Service Co Of New } \\
\text { Mexico }\end{array}$} \\
\hline Mexico & $\begin{array}{l}799,154 \\
-\ldots\end{array}$ & $\begin{array}{r}2,686 \\
-9\end{array}$ & --240 & $\overline{-}$ & $\overline{-}$ & $\overline{-}$ & - & $-{ }^{5}$ & $\overline{-}$ & $-{ }_{-}^{625}$ & $\begin{array}{r}29 \\
4\end{array}$ \\
\hline 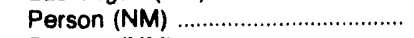 & -- & -- & -- & - & - & - & - & -- & -- & -- & - \\
\hline 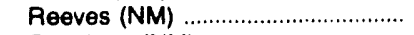 & -- & -- & -240 & - & - & - & - & -- & -- & -- & -- \\
\hline 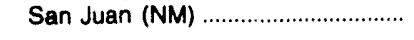 & 799.154 & 2,694 & -- & - & - & - & 486 & 5 & - & 625 & 25 \\
\hline Public Service Elec And Gas Co. & 148,151 & 30,801 & 249,100 & - & $2,273,698$ & - & 54 & 70 & 2,868 & 445 & 469 \\
\hline Bayonne (NJ) & -- & 149 & $-\overline{c o s e}(0)$ & $-\infty$ & - & - & -- & • & -- & -- & 4 \\
\hline 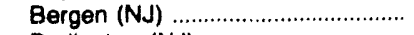 & -- & - & 46,534 & - & - & - & - & - & 634 & - & -- \\
\hline Burlington (NJ) ..................................... & -- & 2,221 & 30,923 & - & - & -- & -- & 3 & 288 & - & 52 \\
\hline Edison (NJ) & -- & 373 & $\vec{a} \operatorname{sen}$ & - & - & -- & -- & 1 & - & -- & * \\
\hline Essex (NJ) ......................................... & -- & 545 & 16,660 & - & - & - & - & 1 & 206 & -- & 36 \\
\hline
\end{tabular}

See footnotes at end of table. 
Table 62. U.S. Electric Utllity Net Generation, Fuel Consumption, and Fuel Stocks by Company and Plant, September 1993 (Continued)

\begin{tabular}{|c|c|c|c|c|c|c|c|c|c|c|c|}
\hline \multirow{2}{*}{$\begin{array}{c}\text { Company (Holding Company) } \\
\text { Plant (State) }\end{array}$} & \multicolumn{6}{|c|}{$\begin{array}{c}\text { Generation } \\
\text { (thousand kilowatthours) }\end{array}$} & \multicolumn{3}{|c|}{$\begin{array}{l}\text { Consumption } \\
\text { (thousand) }\end{array}$} & \multicolumn{2}{|c|}{$\begin{array}{l}\text { Stocks } \\
\text { (thousand) }\end{array}$} \\
\hline & Coal & Petroloum & Gas & Hydro & Nuclear & Other' & $\begin{array}{c}\text { Coal } \\
\text { (short } \\
\text { tons) }\end{array}$ & $\begin{array}{l}\text { Petro- } \\
\text { Ioum } \\
\text { (bble) }\end{array}$ & $\begin{array}{c}\text { Gas } \\
\text { (Mct) }\end{array}$ & $\begin{array}{c}\text { Coal } \\
\text { (enort } \\
\text { tons) }\end{array}$ & $\begin{array}{l}\text { Potro- } \\
\text { leum } \\
\text { (bole) }\end{array}$ \\
\hline \multicolumn{12}{|l|}{ Public Service Elec And Gas Co } \\
\hline 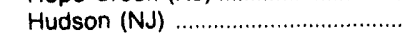 & - & 61 & 80,987 & - & - & - & - & $\cdot$ & 875 & 227 & 113 \\
\hline 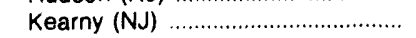 & -- & 7,806 & 4,701 & - & - & -- & -- & 18 & 68 & - & 40 \\
\hline 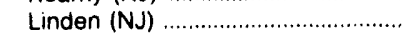 & - & 19,326 & 1,271 & -- & -- & - & -- & 44 & 25 & - & 126 \\
\hline 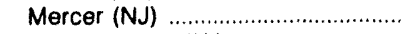 & 148,151 & -45 & 40,179 & $-\infty$ & -- & -- & 54 & -- & 409 & 218 & - \\
\hline National Park (NJ) ............................ & -- & -6 & -- & -- & $-\infty$ & - & -- & -- & -- & -- & 1 \\
\hline 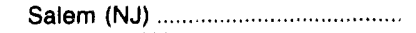 & - & 27 & $-\infty$ & -- & $1,534,108$ & -- & -- & $\cdot$ & -- & -- & 16 \\
\hline 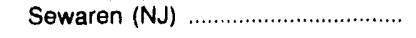 & -- & 344 & 27,845 & - & -- & $\rightarrow$ & -- & 1 & 362 & -- & 81 \\
\hline \multicolumn{12}{|l|}{ Puget Sound Power Light } \\
\hline Electron (WA) .................................. & -- & $-\infty$ & $-\infty$ & 11,663 & -- & $\sim$ & - & -- & - & - & -- \\
\hline 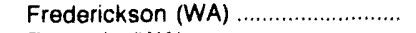 & - & 60 & 51 & - & -- & -- & -- & $\bullet$ & 1 & -- & 93 \\
\hline 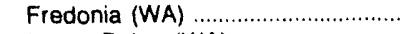 & - & -- & 174 & -ingon & -- & - & -- & -- & 2 & -- & 100 \\
\hline 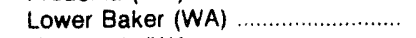 & - & - & -- & 22,809 & -- & - & -- & -- & - & -- & -- \\
\hline 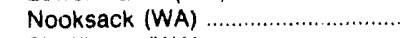 & -- & $-\infty$ & -- & 782 & - & - & -- & -- & -- & -- & -- \\
\hline Shuffleton (WA) & -- & -41 & -- & -2 & - & - & - & -- & -- & -- & • \\
\hline 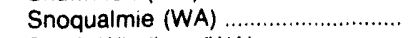 & -- & -- & -- & 5,803 & - & - & -- & -- & - & -- & - \\
\hline 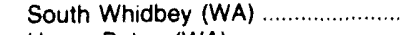 & -- & 16 & -- & - & -- & - & -- & $"$ & -- & - & 10 \\
\hline 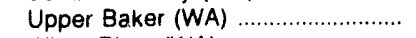 & -- & - & -- & 21,876 & - & - & -- & -- & -- & -- & -- \\
\hline White River (WA) & - & - & -- & 3,387 & -- & -- & -- & - & -- & - & - \\
\hline Whitehorn (WA) & -- & 57 & -- & - & $\cdots$ & - & -- & $\cdot$ & -- & - & 141 \\
\hline 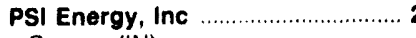 & $2,203,722$ & 9,173 & 2,281 & 25,231 & - & - & 1,022 & 18 & 24 & 1,497 & 41 \\
\hline Cayuga (IN) & 271,920 & 3,335 & 2,281 & - & - & -- & 129 & 6 & 24 & 254 & 10 \\
\hline Connersville $(\mathrm{IN})$ & -- & 130 & - & - & - & - & - & 1 & -- & - & 8 \\
\hline 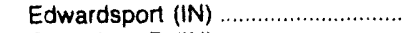 & 4,466 & 145 & - & -- & - & -- & 3 & $\bullet$ & - & 23 & 3 \\
\hline Gallagher, $R($ IN) & 177,190 & 1,087 & -- & - & - & -- & 77 & 2 & $-\infty$ & 118 & 2 \\
\hline Gibson $(\mathrm{IN})$ & $1,525,901$ & 1,930 & -- & $\overline{-}$ & -- & -- & 702 & 3 & -- & 984 & 5 \\
\hline Markland $(\mathrm{IN})$ & -- & -- & -- & 25,231 & -- & -- & -- & -- & -- & -- & - \\
\hline 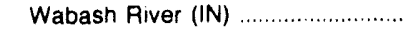 & 222,628 & 2,484 & -- & -- & - & -- & 109 & 5 & -- & 91 & 2 \\
\hline R V Light \& Power ........................... & - & -- & $-\infty$ & 63 & - & - & - & - & - & - & - \\
\hline 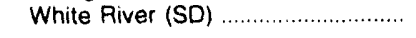 & -- & -- & -- & 63 & -- & - & - & -- & -- & -- & -- \\
\hline Radford Mun Power Plant .............. & -- & -- & -- & 274 & - & - & - & - & - & - & - \\
\hline Radford (VA) & -- & -- & -- & 274 & - & -- & -- & - & -- & -- & -- \\
\hline \multicolumn{12}{|l|}{ Rantoul, Village Of, Lt \& Pwr } \\
\hline Dept & - & - & - & - & - & - & - & - & - & - & * \\
\hline Rantoul (IL) & -- & -- & -- & - & -- & - & $-\cdots$ & -- & -- & -- & * \\
\hline Raton Public Service Company ... & 1,953 & - & -- & -- & - & $-\infty$ & 1 & - & - & 2 & - \\
\hline 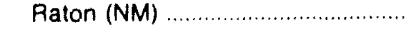 & 1,953 & -- & -- & -- & - & -- & 1 & -- & -- & 2 & -- \\
\hline Rayne Elec Lt \& Pwr ........................ & - & - & -- & - & - & - & - & - & - & -- & -- \\
\hline Rayne (LA) & -- & -- & -- & -- & -- & - & - & -- & - & -- & -- \\
\hline Ped Bud Clty Of & - & - & - & - & - & - & - & - & - & - & 1 \\
\hline 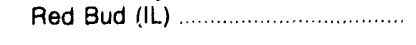 & -- & -- & - & -- & -- & - & +- & -- & - & -- & 1 \\
\hline Red Cloud Clty Of Mun Lt \& Pwr & -- & 2 & - & - & - & - & -- & - & - & - & * \\
\hline Red Cloud (NE) & -- & 2 & - & - & - & -- & -- & • & -- & -- & * \\
\hline Redding City of & -- & $-\infty$ & -- & 606 & - & - & -- & -- & -- & -- & - \\
\hline 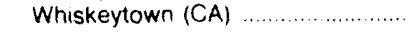 & - & - & -- & 606 & -- & - & - & -- & -- & -- & -- \\
\hline Redlands Water \& Power Co ......... & - & - & -- & 920 & - & - & - & - & - & -- & - \\
\hline Redlands (CO) & -- & - & -- & 920 & -- & -- & -- & -- & -- & -- & -- \\
\hline Redwood Falls Pub Util Comm .... & - & 79 & - & 152 & - & $\cdots$ & - & " & - & - & 2 \\
\hline Redwood Falls (MN) ........................ & - & 79 & - & 152 & -- & - & -- & * & -- & -- & 2 \\
\hline
\end{tabular}

See footnotes at end of table. 
Table 62. U.S. Electric Utility Net Generation, Fuel Consumption, and Fuel Stocks by Company and Plant, September 1993 (Continued)

\begin{tabular}{|c|c|c|c|c|c|c|c|c|c|c|c|}
\hline \multirow{2}{*}{$\begin{array}{c}\text { Company (Holding Company) } \\
\text { Plant (State) }\end{array}$} & \multicolumn{6}{|c|}{$\begin{array}{c}\text { Generation } \\
\text { (thousand kllowatthours) }\end{array}$} & \multicolumn{3}{|c|}{$\begin{array}{l}\text { Consumption } \\
\text { (thousand) }\end{array}$} & \multicolumn{2}{|c|}{$\begin{array}{l}\text { Stocks } \\
\text { (thousand) }\end{array}$} \\
\hline & Coal & Petroloum & Gas & Hydro & Nuclear & Other' & $\begin{array}{l}\text { Coal } \\
\text { (short } \\
\text { tons) }\end{array}$ & $\begin{array}{l}\text { Petro- } \\
\text { loum } \\
\text { (bble) }\end{array}$ & $\begin{array}{c}\text { Gas } \\
\text { (Mcf) }\end{array}$ & $\begin{array}{c}\text { Coal } \\
\text { (short } \\
\text { tons) }\end{array}$ & $\begin{array}{l}\text { Petro- } \\
\text { leum } \\
\text { (bbls) }\end{array}$ \\
\hline
\end{tabular}

\section{Rensaelaer City of Mun Power} Plant .......

Rensselaer (IN)

Renwick LIght \& Power Plant Renwick (IA)

Pich HIII City of Rich Hill (MO)

Richmond Power \& Light

Whitewater Valley (IN)

River Falls Municipal Utillties ..... Junction (WI)

Powell Falls (WI)

Robstown Clty of

Robstown (TX)

Rochelle Municipal Utilities

Rochelle No. 1 (IL)

Rochelle No. 2 (IL)

Rochester Clty Of Elec Dept ...

Cascade Creek (MN)

Rochester (MN)

Silver Lake (MN)

Rochester Gas \& Elec Corp

Ginna (NY)

Station 160 (NY)

Station 170 (NY)

Station 172 (NY)

Station 2 (NY)

Station 26 (NY)

Station 3 (NY)

Station 5 (NY)

Station 7 (NY)

Station 9 (NY)

Rock Port Bd Of Public Works Rockport (MO)

Rock Rapids City of (Util Div)..

Rock Rapids (IA)

Rockford Mun Light Plant

Rocklord (IA)

Rockville Center El Lt Dept .......... Rockville (NY)

Roseau, Village of Roseau (MN)

Russell Clty of Russell (KS)

Ruston Water \& Light Plant Ruston (LA)

Sabetha Clty of

Sabetha (KS)

Sacramento Municipal Utility Dist Camino (CA)

Camp Far W (CA)

See footnotes at end of table.

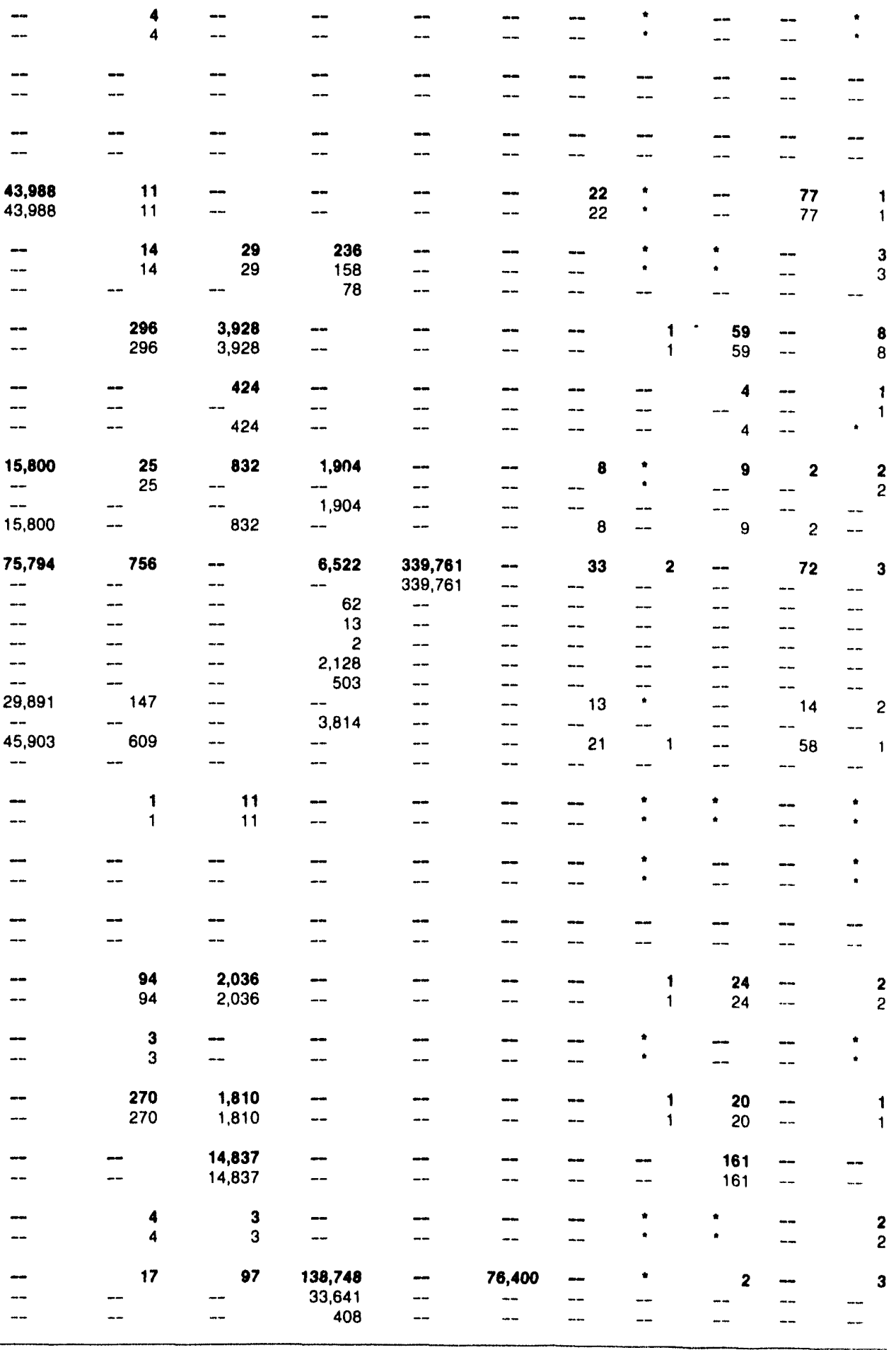


Table 62. U.S. Electric Utility Net Generation, Fuel Consumption, and Fuel Stocks by Company and Plant, September 1993 (Continued)

\begin{tabular}{|c|c|c|c|c|c|c|c|c|c|c|c|}
\hline \multirow[b]{2}{*}{$\begin{array}{c}\text { Company (Holding Company) } \\
\text { Plant (State) }\end{array}$} & \multicolumn{6}{|c|}{$\begin{array}{c}\text { Ceneration } \\
\text { (thousand kllowatthours) }\end{array}$} & \multicolumn{3}{|c|}{$\begin{array}{l}\text { Consumption } \\
\text { (thousend) }\end{array}$} & \multicolumn{2}{|c|}{$\begin{array}{l}\text { Stocks } \\
\text { (thousand) }\end{array}$} \\
\hline & Coal & Petroloum & Gas & Hydro & Nuclear & Other' & $\begin{array}{l}\text { Coal } \\
\text { (short } \\
\text { tons) }\end{array}$ & $\begin{array}{l}\text { Potro- } \\
\text { Deum } \\
\text { (bbta) }\end{array}$ & $\begin{array}{c}\text { Gass } \\
\text { (Mcf) }\end{array}$ & $\begin{array}{l}\text { Coal } \\
\text { (chort } \\
\text { tons) }\end{array}$ & $\begin{array}{l}\text { Petro- } \\
\text { loum } \\
\text { (bbla) }\end{array}$ \\
\hline \multicolumn{12}{|l|}{ Sacramento Municipal Utility Diat } \\
\hline Coldwater Creek (CA) ....................... & -- & - & -- & - & - & 35,532 & - & -- & - & -- & -- \\
\hline 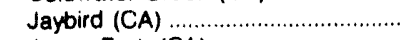 & -- & - & - & 54,145 & - & - & - & - & -- & -- & -- \\
\hline Jones Fork (CA) & -- & - & - & -6 & -- & - & - & - & -- & -- & - \\
\hline Loon Lake (CA) & -- & -- & -- & 5,847 & - & -- & -- & - & -- & - & - \\
\hline 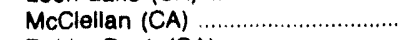 & - & 17 & 97 & - & - & - & -- & $\cdot$ & 2 & -- & 3 \\
\hline 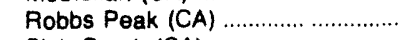 & - & - & - & 1,686 & - & - & - & -- & -- & -- & - \\
\hline 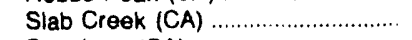 & - & - & -- & 279 & -- & - & -- & - & -- & -- & - \\
\hline 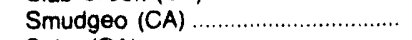 & -- & - & - & - & - & 40,510 & - & -- & $\rightarrow$ & - & -- \\
\hline 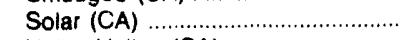 & - & - & -- & - & - & 358 & - & - & -- & -- & - \\
\hline 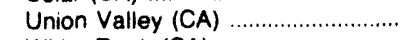 & - & - & - & 11,665 & - & - & -- & -- & - & -- & -- \\
\hline 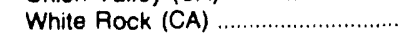 & - & - & - & 31,083 & - & - & - & - & - & - & -- \\
\hline Safe Harbor Water Power Corp ... & - & - & - & 20,723 & - & - & - & - & - & - & - \\
\hline 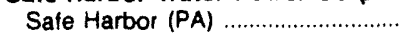 & -- & - & - & 20,723 & - & - & -- & - & - & -- & -- \\
\hline Salt River Prol Agri Imp Pwr Dist & $1,995,185$ & 731 & 36,154 & 49,523 & - & - & 929 & 1 & 359 & 2,260 & 461 \\
\hline - Central Storago & - & - & - & - & - & - & - & - & - & - & 23 \\
\hline Agua Fria $(A Z)$ & - & - & -241 & - & - & -- & - & - & 10 & - & 211 \\
\hline Coronado $(A Z)$ & 470,343 & 247 & - & - & - & - & 243 & $\cdot$ & - & 818 & 23 \\
\hline 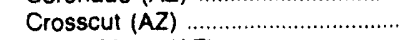 & - & - & - & 38 & -- & - & - & -- & - & - & - \\
\hline 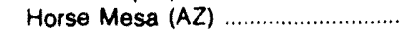 & - & - & - & 27,909 & - & - & - & -- & - & - & - \\
\hline Kyrene $(A Z)$ & - & -1 & -313 & - & - & - & - & $\cdot$ & 2 & - & 64 \\
\hline 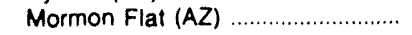 & -- & - & - & 15,207 & - & - & - & - & -- & - & - \\
\hline Navajo $(A Z)$ & $1,524,852$ & 458 & - & - & - & - & 686 & 1 & -- & 1,441 & 35 \\
\hline 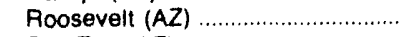 & - & $-\infty$ & $\bar{a}$ & - & - & - & - & -- & - & - & -- \\
\hline $\operatorname{San} \operatorname{Tan}(A Z)$ & - & 27 & 36,708 & -- & -- & - & -- & $\cdot$ & 347 & - & 105 \\
\hline South Con $(A Z)$ & - & - & - & 62 & - & - & - & - & - & - & - \\
\hline 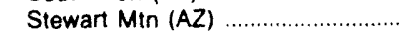 & -- & - & - & 6,307 & - & - & - & -- & -- & -- & - \\
\hline San Antonio Public Service Bd .... & 887,309 & 293 & 450,249 & - & - & - & 537 & 1 & 4,662 & 595 & 430 \\
\hline Braunig. $\vee H(T X)$ & -- & - & 154,936 & - & - & - & - & - & 1,664 & - & 203 \\
\hline 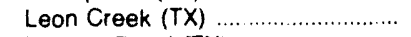 & - & - & -134 & - & - & - & - & - & - & - & 2 \\
\hline 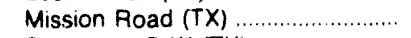 & - & - & -180 & - & - & - & - & -- & - & -- & - \\
\hline 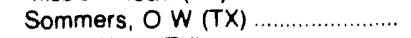 & - & 24 & 248,599 & -- & $m$ & - & - & $\cdot$ & 2,488 & - & -- \\
\hline 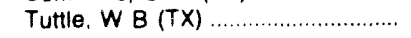 & - & - & 47,025 & - & - & - & -- & - & 510 & -- & 52 \\
\hline \multicolumn{12}{|l|}{ San Dlego Gas Electric } \\
\hline Company & - & 994 & 357,168 & - & - & - & - & 2 & 3,849 & - & 1,128 \\
\hline - Central Storage * ......................... & -- & - & - & - & - & - & - & - & - & -- & $\because$ \\
\hline 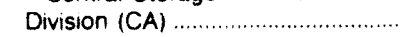 & -- & 12 & -- & - & - & -- & - & $\cdot$ & - & - & - \\
\hline 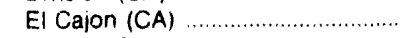 & - & 18 & 29 & -- & -- & - & - & - & 1 & -- & 1 \\
\hline Encina (CA) & -- & 590 & 166,470 & - & - & - & - & 1 & 1,899 & - & 716 \\
\hline Kearny (CA) & - & 45 & 407 & - & -- & -- & - & - & 8 & -- & 23 \\
\hline 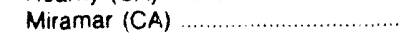 & - & 27 & 695 & - & -- & - & - & • & 12 & -- & 4 \\
\hline 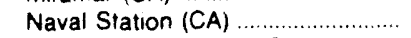 & - & 12 & 561 & - & - & - & - & - & 6 & - & 15 \\
\hline 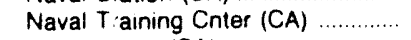 & - & - & -- & - & - & - & - & - & - & - & 1 \\
\hline 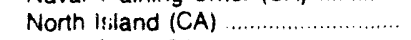 & - & 20 & 51 & - & - & - & - & $\cdot$ & 1 & -- & 4 \\
\hline 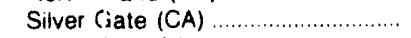 & - & - & - & - & -- & -- & - & - & - & - & - \\
\hline 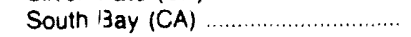 & -- & 270 & 188,955 & - & - & - & - & $\bullet$ & 1,923 & -- & 364 \\
\hline 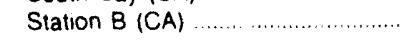 & -- & - & - & - & - & - & -- & - & - & -- & $-\cdots$ \\
\hline San Miguwl Eloctric Coop .............. & 273,242 & 443 & - & - & - & - & 305 & 1 & - & 203 & 21 \\
\hline 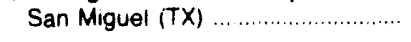 & 273,242 & 443 & - & - & - & - & 305 & 1 & - & 203 & 21 \\
\hline Sanborn Municipal Light Plant ..... & - & - & - & - & - & - & - & - & - & - & - \\
\hline Sanborn (IA) & -- & -- & - & - & - & $\rightarrow$ & -- & - & -- & -- & - \\
\hline Santa Clara City Of & - & - & 1,726 & 292 & - & - & - & - & 26 & - & 2 \\
\hline 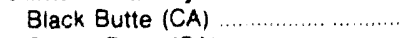 & - & -- & - & + & - & -- & -- & -- & - & - & -- \\
\hline Cogen Plant (CA) & -- & -- & 1,726 & - & - & - & -- & - & 26 & -- & -- \\
\hline Gianera (CA) & -- & - & - & - & - & - & -- & - & - & -- & 2 \\
\hline 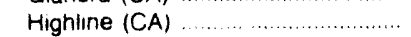 & - & -- & -- & 178 & - & - & - & - & - & - & - \\
\hline Stony Gorge $(C A)$ & $-\cdots$ & -- & -- & 114 & -- & -- & -- & - & -- & -- & -- \\
\hline Sargent City Of & - & - & - & - & - & - & - & - & - & - & • \\
\hline Sargent (NE) & -- & -- & - & - & - & -- & - & -- & - & - & • \\
\hline
\end{tabular}

See footnotes at and of table. 
Table 62. U.S. Electric Utility Net Generation, Fuel Consumption, and Fuel Stocks by Company and Plant, September 1993 (Continued)

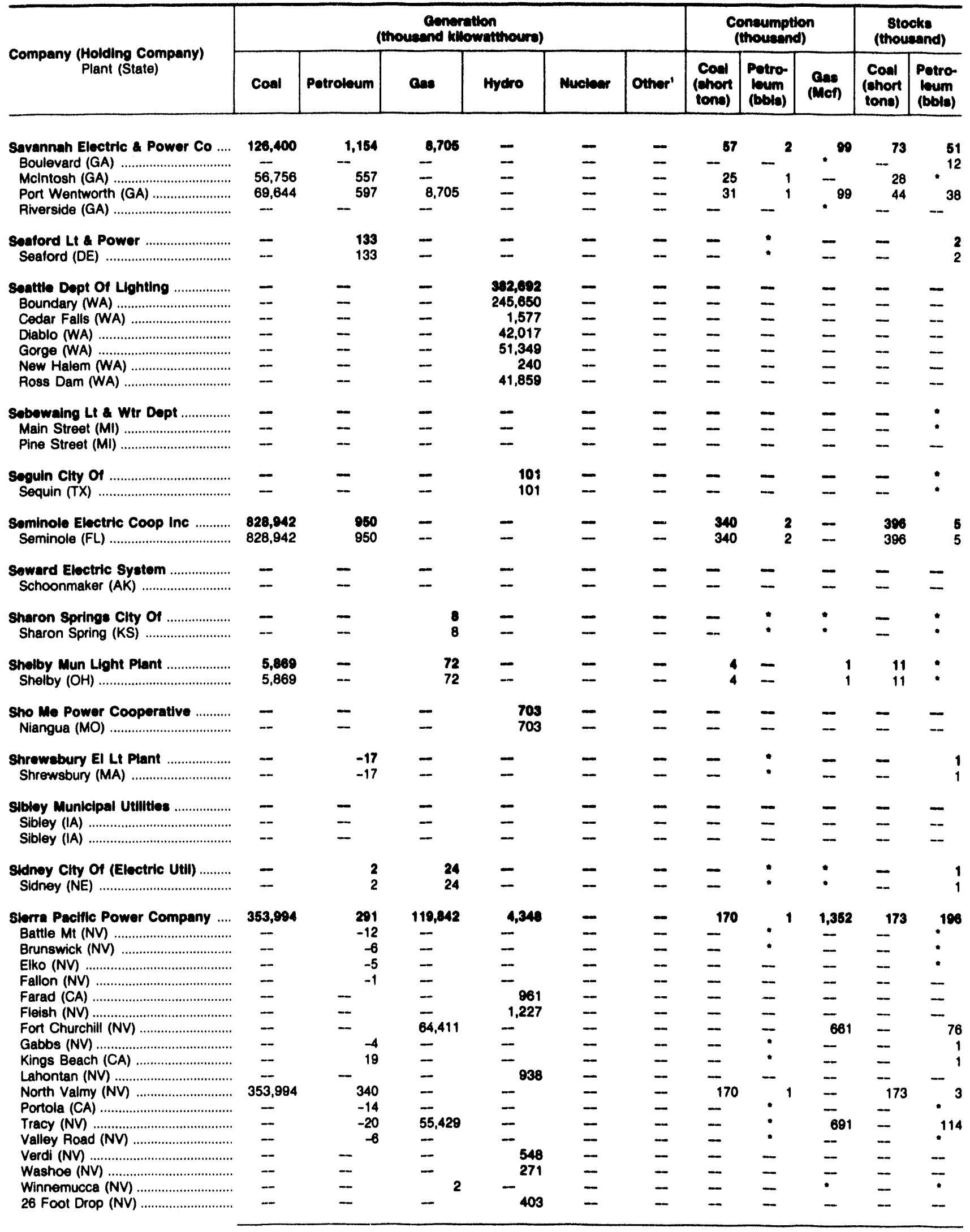

See footnotes at end of table. 
Table 62. U.S. Electric Utility Net Generation, Fuel Consumptlon, and Fuel Stocks by Company and Plant, September 1993 (Continued)

\begin{tabular}{|c|c|c|c|c|c|c|c|c|c|c|c|}
\hline \multirow{2}{*}{$\begin{array}{c}\text { Company (Holding Company) } \\
\text { Plant (State) }\end{array}$} & \multicolumn{6}{|c|}{$\begin{array}{c}\text { Generation } \\
\text { (thousand kllowatthours) }\end{array}$} & \multicolumn{3}{|c|}{$\begin{array}{l}\text { Consumption } \\
\text { (thousand) }\end{array}$} & \multicolumn{2}{|c|}{$\begin{array}{c}\text { Stocks } \\
\text { (thousand) }\end{array}$} \\
\hline & Coal & Potroloum & Gas & Hydro & Nuclear & Other' & $\begin{array}{c}\text { Coal } \\
\text { (short } \\
\text { tons) }\end{array}$ & $\begin{array}{l}\text { Potro- } \\
\text { loum } \\
\text { (bbla) }\end{array}$ & $\begin{array}{c}\text { Gas } \\
\text { (Mct) }\end{array}$ & $\begin{array}{c}\text { Coal } \\
\text { (ehort } \\
\text { tons) }\end{array}$ & $\begin{array}{l}\text { Potro- } \\
\text { leum } \\
\text { (bbls) }\end{array}$ \\
\hline Sikeston Bd Of Mun Utilitles ........ & 110,071 & 48,579 & - & - & - & - & 45 & - & - & 108 & 3 \\
\hline Coleman, E. P. (MO) & -- & $-\overline{10}=70$ & -- & -- & -- & -- & - & $\because$ & $\cdots$ & -- & \\
\hline Sikeston (MO) & 110,071 & 48,579 & -- & -- & -- & -- & 45 & • & -- & 108 & 2 \\
\hline Sloux Center Mun Lt \& Pwr ........... & - & - & - & - & - & - & - & - & - & - & - \\
\hline Sioux Center (IA) & -- & - & -- & - & -- & -- & -- & -- & -- & - & -- \\
\hline Sitka City of (Electric Dept) .......... & - & 7 & - & 6,727 & - & - & - & • & - & -- & 4 \\
\hline 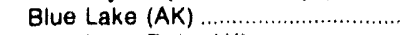 & -- & - & -- & 2,526 & -- & - & -- & -- & -- & -- & -- \\
\hline 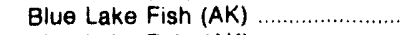 & -- & -- & -- & 55 & -- & -- & - & -- & -- & -- & -- \\
\hline Blue Lake Pulp (AK) ........................ & -- & -- & -- & 8 & -- & -- & -- & -- & -- & -. & -- \\
\hline 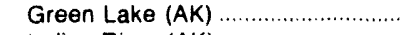 & -- & -- & - & 4,138 & - & - & -- & -- & -- & -- & -- \\
\hline 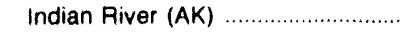 & -- & 7 & -- & -- & -- & -- & -- & • & -- & -- & 4 \\
\hline Skaneateles City of ............................. & - & - & - & - & - & - & - & -- & - & -- & - \\
\hline Skaneateles (NY) & -- & -- & -- & -- & -- & -- & -- & -- & -- & - & -- \\
\hline Sleepy Eye Public Util Comm ....... & - & 14 & 14 & - & - & - & - & - & • & -- & - \\
\hline Sleepy Éye (MN) & - & 14 & 14 & - & -- & - & -- & $\cdot$ & $\cdot$ & -- & - \\
\hline Soda Springs City of ........................... & - & - & - & 187 & - & - & - & - & - & -- & - \\
\hline 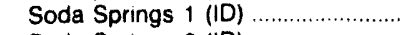 & - & - & -- & 91 & -- & -- & -- & -- & -- & -- & -- \\
\hline Soda Springs 2 (ID) ........................... & - & - & -- & 96 & - & -- & -- & -- & -- & -- & -- \\
\hline South Belolt Wtr Gas \& Elec Co.. & - & - & - & 629 & - & - & -- & - & -- & -- & - \\
\hline 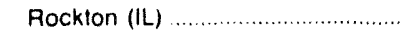 & -- & - & -- & 629 & - & - & -- & -- & - & -- & - \\
\hline \multicolumn{12}{|c|}{ South Carolina Public Service } \\
\hline Auth & $1,040,738$ & 1,072 & - & 20,560 & - & - & 423 & 2 & - & 516 & 118 \\
\hline Cross (SC) & 311,567 & 537 & -- & -- & -- & -- & 122 & 1 & -- & 183 & 4 \\
\hline Grainger, Dolphus M (SC) ............ & 69,838 & 45 & -- & -- & -- & -- & 28 & $\cdot$ & -- & 27 & - \\
\hline Hilton Head (SC) & -- & -44 & -- & -- & -- & -- & -- & - & -- & -- & 27 \\
\hline 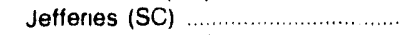 & 149,961 & 116 & -- & 15,995 & -- & $\cdots$ & 62 & • & -. & 42 & 47 \\
\hline Myrtle Beach (SC) & - & 5 & -- & -- & -- & -- & $\cdots$ & • & • & -- & 32 \\
\hline Spillway (SC) & - & -- & -- & 1,075 & -- & -- & -- & -- & -- & -- & -- \\
\hline St. Stephen (SC) & - & -- & -- & 3,490 & -- & -- & -- & -- & -- & -- & -- \\
\hline Winyah (SC) & 509,372 & 413 & - & -- & -- & -- & 211 & 1 & -- & 264 & 7 \\
\hline South Mississippl Elec Pwr Assn & 210,208 & 108 & 50,039 & - & -- & - & 91 & - & 603 & 247 & 47 \\
\hline Benndale (MS) & $\cdots$ & -- & 159 & -- & -- & -- & - & -- & 2 & -- & -- \\
\hline Morrow (MS) & 210,208 & 108 & -- & -- & -- & -- & 91 & • & -- & 247 & 11 \\
\hline Moselle (MS) & -- & -- & 49,880 & -- & -- & -- & - & -- & 601 & - & 35 \\
\hline Paulding (MS) & -- & $\cdots$ & -- & -- & -- & -- & -- & -- & -- & -- & 1 \\
\hline South Norwalk Elec Wks, The & - & 101 & - & - & - & - & - & - & - & - & 2 \\
\hline South Norwalk (CT) & -- & 101 & -- & - & -- & -- & -- & • & -- & -- & 2 \\
\hline South Texas Elec Coop, Inc ......... & - & - & 417 & - & - & - & - & - & 7 & -- & - \\
\hline Playburn, Sam (TX) & -- & - & 417 & -- & -- & -- & - & -- & 7 & -- & -- \\
\hline \multicolumn{12}{|l|}{ Southern Callfornia Edison } \\
\hline & 815,585 & 2,533 & $1,744,945$ & 455,091 & $1,532,169$ & - & 377 & 5 & 16,892 & 673 & 4,555 \\
\hline - Central Storage ${ }^{\circ}$ & -- & -- & -- & -- & -- & -- & $\cdots$ & -- & - & - & 771 \\
\hline Alamitos (CA) & -- & 4 & 350,780 & -- & -- & -- & - & • & 3,480 & -- & 533 \\
\hline Baker Dam (CA) & -- & -- & -- & -- & $\cdots$ & -- & -- & -- & -- & -- & $\ldots$ \\
\hline Big Creek 1 (CA). & -- & -- & - & 51,166 & -- & -- & -- & -- & -- & -- & -- \\
\hline Big Creek 2 (CA) & -- & -- & -- & 46,135 & -- & -- & -- & -- & -- & -. & -. \\
\hline Big Creek 2a (CA) & -- & -- & - & 65,172 & -- & -- & -- & -- & -- & -- & -.. \\
\hline Big Creek 3 (CA) & -- & -- & -- & 78,545 & -- & -- & -- & -- & -. & -- & -. \\
\hline Big Creek 4 (CA) & -- & -- & -- & 42,298 & -- & -. & -- & - & -- & -- & -- \\
\hline Big Creek 8 (CA) $\ldots . .$. & - & -- & -- & 39,300 & - & -- & -- & -- & -- & - & -.. \\
\hline Bishop Creek 2 (CA) & -. & -- & - & 4,022 & -- & -- & -- & -- & -- & -- & -. \\
\hline Bishop Creek 3 (CA). & -- & -- & - & 3,564 & -- & -- & -- & -. & -. & -- & -.. \\
\hline Bishop Creek 4 (CA). & -- & -- & -- & 4,632 & -- & -- & -- & -- & -- & -- & -. \\
\hline Bishop Creek 5 (CA) & -- & -- & -- & 1,869 & -- & -- & -- & -. & -- & -. & -- \\
\hline Bishop Creek 6 (CA) $\ldots \ldots \ldots \ldots \ldots$ & -- & -- & -- & 1,040 & -- & -- & -- & -- & -- & -. & -- \\
\hline Borel $(C A)$ & -- & -- & -- & 7,410 & -- & -- & -- & -- & -- & -. & .. \\
\hline Cool Water (CA) & -- & -- & 108.611 & -- & -- & -- & -- & -- & 1.055 & -- & 426 \\
\hline
\end{tabular}

See footnotes at end of table 
Table 62. U.S. Electric Utility Net Generation, Fuel Consumption, and Fuel Stocks by Company and Plant, September 1993 (Continued)

\begin{tabular}{|c|c|c|c|c|c|c|c|c|c|c|c|}
\hline \multirow{2}{*}{$\begin{array}{c}\text { Company (Holding Company) } \\
\text { Plant (State) }\end{array}$} & \multicolumn{6}{|c|}{$\begin{array}{c}\text { Generation } \\
\text { (thousand kllowatthoure) }\end{array}$} & \multicolumn{3}{|c|}{$\begin{array}{c}\text { Consumptlon } \\
\text { (thousand) }\end{array}$} & \multicolumn{2}{|c|}{$\begin{array}{l}\text { Stocks } \\
\text { (thousand) }\end{array}$} \\
\hline & Coal & Petroleum & Gas & Hydro & Nuclear & Other' & $\begin{array}{l}\text { Coal } \\
\text { (short } \\
\text { tons) }\end{array}$ & $\begin{array}{l}\text { Petro- } \\
\text { leum } \\
\text { (bble) }\end{array}$ & $\begin{array}{l}\text { Qass } \\
\text { (Mct) }\end{array}$ & $\begin{array}{c}\text { Coal } \\
\text { (ahort } \\
\text { tons) }\end{array}$ & $\begin{array}{l}\text { Potro- } \\
\text { loum } \\
\text { (bbis) }\end{array}$ \\
\hline
\end{tabular}

\section{Southern Callfornia Edison}

$$
\text { Company }
$$

Eastwood (CA) .

El Segundo (CA)

Ellwood (CA)

Etiwanda (CA)

Fontana (CA)

Highgrove (CA)

Huntington Beach (CA)

Kaweah 1 (CA)

Kaweah 2 (CA)

Kaweah 3 (CA)

Kern River 1 (CA)

Kern River 3 (CA)

Long Berch (CA)

Lundy (CA)

Lytle Creek (CA)

Mammoth Pool (CA)

Mendalay (CA)

Mill Creek 1 (CA)

Mill Creek 283 (CA)

Mill Creek 3 (CA)

Mohave (NV)

Ontario 1 (CA)

Ontario 2 (CA)

Ormond Beach (CA)

Pebbly Beach (CA)

Poole (CA)

Portal (CA)

Redondo Beach (CA)

Rush Creek (CA)

San Bernardino (CA)

San Gorgonio (CA)

San Gorgonio (CA)

San Onofre (CA)

Santa Ana 1 (CA)

Santa Ana 2 (CA)

Santa Ana 3 (CA)

Sierra (CA)

Tule River (CA)

Southern Illinols Power Coop ..... $\quad 65,629$

Marion (IL) ............................... 65,629

Southern Indiana Gas a Electrlc

$$
\text { Co }
$$

A. B. Brown (IN)

Broadway (IN)

Culley (IN) .

Northeast (IN)

Warrick (IN)

Southwest Public Power District .

Palisade (NE)

Southwestern Elec Power Co

$$
\text { (CSW) }
$$

Arsenal Hill (LA)

Flint Creek (AR)

Knox Lee (TX)

Lieberman (LA)

Lone Star (TX)

Pirkey (TX) .

Welsh (TX)

Wilkes (TX)

Southwestern Public Service Co . 1,298,604 Carlsbad (NM)

81

--
--
-
--
-
-
--
-
-
--
-
--
-
--
-
--
-
--
-

$--$

-- $\quad 171,249$

215
$-\quad 187,241$

$-$

$-$

$+$

$-\infty$

$-$

$--$

$--$

$-$

430

$-$

$-$

$-$

$-$

$-$

2,099

$--$

$-$

$-$

$-$

$-$

$-$

$-$

$-$

--

$-$

$$
63
$$

121,647

$\rightarrow$

$-$

$-$

$\overline{19} 618$

19,618

$-$

-

140,143

--

18,845

$$
--
$$

367,157

367,157

$-$

$--$

259,547

$--17$

$--$

$-$

$-$

$-$

$-$

$-$

17.608

$-$

-.

966

-..

1,190

195
651

17,396

6,230

- 705

329
44,050

44,050

500

1,399

$-534$

534
182

$\overline{--}$

6,133

5

5,982

311

$-$

1,752

\begin{tabular}{|c|c|c|}
\hline \multirow[t]{2}{*}{72} & 808 & - \\
\hline & 425 & - \\
\hline \multirow{4}{*}{72} & 52 & $\ldots$ \\
\hline & 40 & 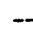 \\
\hline & 62 & \\
\hline & 327 & \\
\hline
\end{tabular}

799

694

349
549

$63 \quad--\quad-$

-
-
-
-
--

$-$

$\begin{array}{lrl}1,866 & 321,626 & - \\ -- & 2,552 & -- \\ -844 & -- & -- \\ - & 94,468 & -- \\ - & 26,526 & -- \\ -- & -12 & -- \\ -1,022 & 179 & -- \\ -- & 197,913 & --\end{array}$

$76 \quad 422,252$

$-$

$\begin{array}{lllllll}-- & - & - & - & - & -- & - \\ -- & -- & -- & -- & 1,629 & -- & -\end{array}$

$-$

$-$

$\overline{-}$

$\overline{--}$

$\overline{-}$

$-\overline{-}$

$\overline{-} \quad-$

$-\overline{-} \quad-$

$=\quad-$

$\overline{-}=$

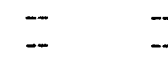

$-$

--

$-$

$-$

$--$

$-$

$-$

$-$

$1,532,169$

--

$-$

$--$

$\begin{array}{lll}- & - & 39 \\ - & -- & 39\end{array}$

$\begin{array}{rrr}1,629 & -- & 292 \\ 1,865 & -- & --\end{array}$

$\begin{array}{lll}1,865 \quad-- & 601 \\ -- & -\cdots & -0\end{array}$

$\begin{array}{rrr}3 & - & 43 \\ 1,154 & - & 180\end{array}$

$-+\quad-$

$--\quad--\quad-$

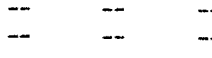

$223 \quad-\quad 124$

$\begin{array}{lllll}- & - & -- & -- & - \\ -- & -- & -- & -- & -\end{array}$

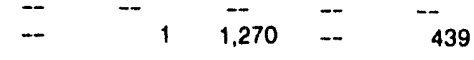

$\begin{array}{lllll}-- & -- & -- & - & - \\ -- & -- & -- & - & -\end{array}$

$\begin{array}{lllll}-- & - & - & - & - \\ -- & - & - & -- & -\end{array}$

$\begin{array}{ccccc}377 & -- & 186 & 673 \\ -- & -- & -- & -- & -\end{array}$

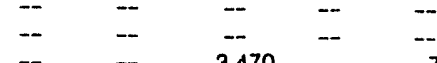

$\begin{array}{rrrrrr}- & - & & 3,470 & - & 780 \\ - & & 4 & - & - & 5\end{array}$

-

$\overline{--}$

$-+$

$--$

$-$

$-$

$-$

$\begin{array}{ll}- & = \\ -- & = \\ = & = \\ - & =\end{array}$

$\begin{array}{rrrrrr}191 & - & 16 & 432 & 3 \\ 58 & -- & 5 & 205 & 2 \\ -- & - & 1 & - & 1 \\ 109 & - & & & 151 & \cdot \\ - & -- & 6 & - & -- \\ 24 & -- & & 3 & 75 & --\end{array}$

$\begin{array}{lllllll}- & - & - & - & - & - & -\end{array}$

$\begin{array}{llllllll}- & - & 915 & & 3 & 3,406 & 1,591 & 117 \\ - & - & - & - & 48 & - & - \\ - & - & 156 & & 2 & - & 209 & 10 \\ - & - & - & -- & 927 & - & 66 \\ - & - & - & - & 292 & -- & 20 \\ - & - & - & - & - & - & 14 \\ - & -- & 294 & - & & 2 & 280 & - \\ - & - & 465 & & 2 & -- & 1,101 & 7 \\ - & -- & - & - & 2,137 & -- & \\ - & 25,964 & 737 & - & 4,426 & 578 & 87 \\ - & -- & - & -- & 18 & - & -\end{array}$

See footnotes at end of table. 
Table 62. U.S. Electric Utility Not Generation, Fuel Consumption, and Fuel Stocks by Company and Plant, September 1993 (Continued)

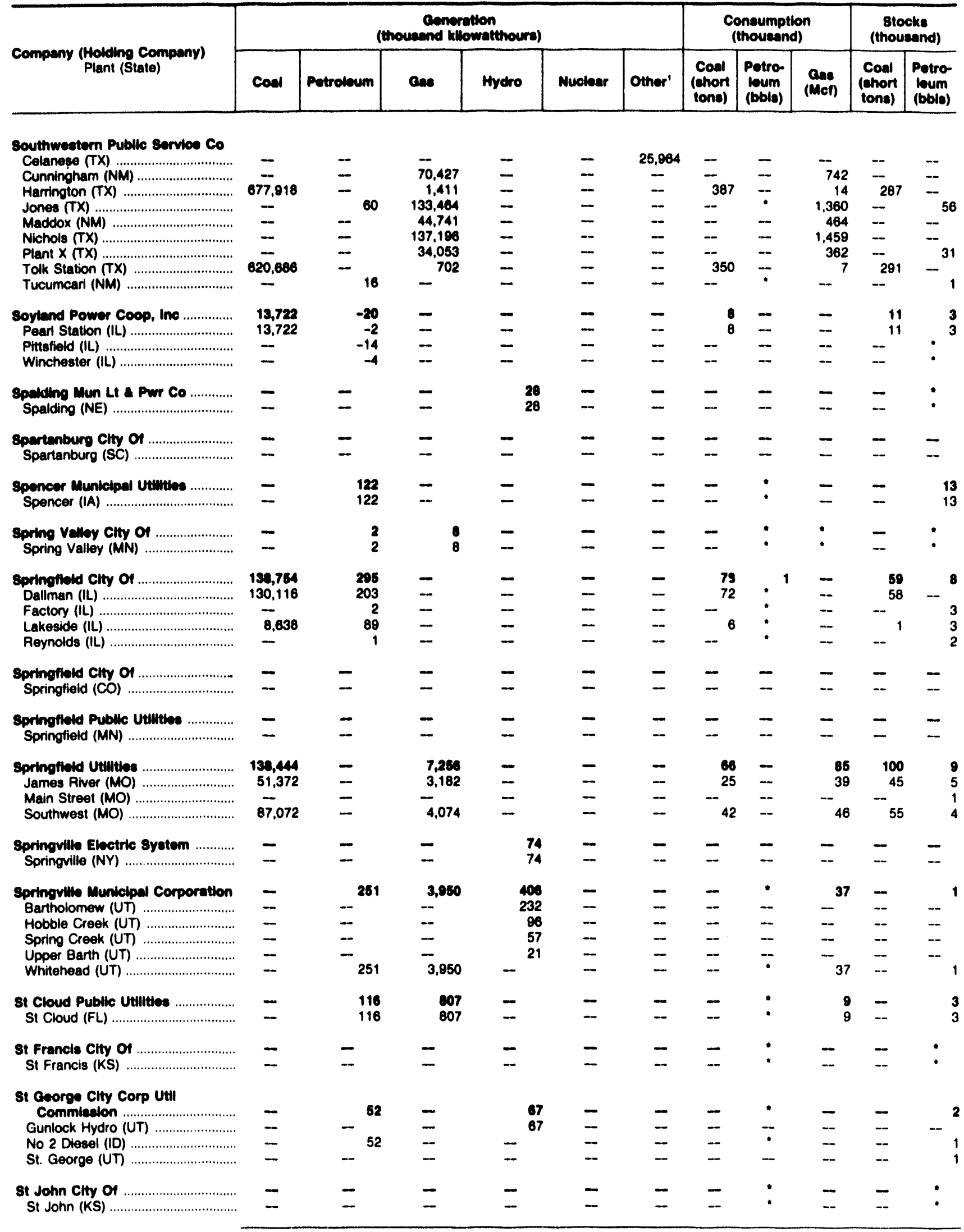

See footnotes at end of table. 
Table 62. U.S. Electric Utility Net Generation, Fuel Consumption, and Fuel Stocks by Company and Plant, September 1993 (Continued)

\begin{tabular}{|c|c|c|c|c|c|c|c|c|c|c|c|}
\hline \multirow[b]{2}{*}{$\begin{array}{c}\text { Company (Holding Company) } \\
\text { Plant (State) }\end{array}$} & \multicolumn{6}{|c|}{$\begin{array}{c}\text { Ceneration } \\
\text { (thousand kllowatthours) }\end{array}$} & \multicolumn{3}{|c|}{$\begin{array}{l}\text { Consumption } \\
\text { (thousand) }\end{array}$} & \multicolumn{2}{|c|}{$\begin{array}{l}\text { Stocks } \\
\text { (thousand) }\end{array}$} \\
\hline & Coal & Potroloum & ans & Hydro & Nuclear & Other' & $\begin{array}{l}\text { Coal } \\
\text { (chort } \\
\text { tons) }\end{array}$ & $\begin{array}{l}\text { Potro- } \\
\text { loum } \\
\text { (bblo) }\end{array}$ & $\begin{array}{l}\text { Gas } \\
\text { (Mof) }\end{array}$ & $\begin{array}{c}\text { Coal } \\
\text { (ahort } \\
\text { tona) }\end{array}$ & $\begin{array}{l}\text { Petro- } \\
\text { loum } \\
\text { (bbla) }\end{array}$ \\
\hline 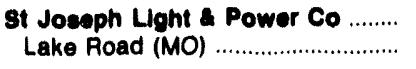 & $\begin{array}{l}4,467 \\
4,467\end{array}$ & $\begin{array}{l}1,240 \\
1,240\end{array}$ & $\begin{array}{l}331 \\
331\end{array}$ & $\overline{-}$ & $\overline{-}$ & $\overline{-}$ & $\begin{array}{l}3 \\
3\end{array}$ & $\begin{array}{l}3 \\
3\end{array}$ & $\stackrel{9}{9}$ & $\begin{array}{l}36 \\
36\end{array}$ & $\begin{array}{l}41 \\
41\end{array}$ \\
\hline $\begin{array}{l}\text { st Louls Electric UtIIty } \\
\text { Saint Louis (MI) }\end{array}$ & $\overline{-}$ & $\overline{-}$ & $\overline{-}$ & $\overline{-}$ & $\overline{-}$ & $\overline{-}$ & $\overline{-}$ & $\overline{-}$ & - & $\overline{-}$ & $\overline{-}$ \\
\hline $\begin{array}{l}\text { St Marys Mun Lt \& Pwr Plant ........ } \\
\text { Saint Marys (OH) }\end{array}$ & $\begin{array}{l}2,600 \\
2,600\end{array}$ & $\begin{array}{l}110 \\
110\end{array}$ & $\overline{-}$ & $\overline{-}$ & $\overline{-}$ & $\overline{-}$ & $\begin{array}{l}2 \\
2\end{array}$ & : & $\overline{-}$ & : & : \\
\hline $\begin{array}{l}\text { Staftord Clty of } \\
\text { Stafford (KS) }\end{array}$ & $\overline{-}$ & $\overline{-}$ & $\overline{-}$ & $\overline{-}$ & $\overline{-}$ & $\overline{-}$ & $\overline{-}$ & $\overline{-}$ & $\overline{-}$ & $\overline{-}$ & : \\
\hline $\begin{array}{l}\text { Stanberry Powor Co } \\
\text { Stanberry (MO) }\end{array}$ & $\overline{-}$ & $\overline{-}$ & $\overline{-}$ & $\overline{-}$ & $\overline{-}$ & $\overline{-}$ & $\overline{-}$ & $\overline{-}$ & $\overline{-}$ & $\overline{-}$ & $\overline{-}$ \\
\hline $\begin{array}{l}\text { Starke Mun Lt a Wtr Plant } \\
\text { Stark (FL) }\end{array}$ & $\overline{-}$ & $\begin{array}{l}43 \\
43\end{array}$ & $\begin{array}{l}341 \\
341\end{array}$ & $\overline{-}$ & $\overline{-}$ & $\overline{-}$ & $\overline{-}$ & : & $\begin{array}{l}7 \\
7\end{array}$ & - & $\begin{array}{l}1 \\
1\end{array}$ \\
\hline $\begin{array}{l}\text { State Conter Mun LI Plant } \\
\text { State Center (IA) }\end{array}$ & $\overline{-}$ & $\overline{-}$ & $\overline{-}$ & $\overline{-}$ & $\overline{-}$ & $\overline{-}$ & $\overline{-}$ & $\overline{-}$ & $\overline{-}$ & $\overline{-}$ & : \\
\hline $\begin{array}{l}\text { storling City of } \\
\text { Sterling (KS) }\end{array}$ & $\overline{-}$ & $\begin{array}{l}11 \\
11\end{array}$ & $\begin{array}{l}72 \\
72\end{array}$ & $\overline{-}$ & $\overline{-}$ & $\overline{-}$ & $\overline{-}$ & : & $\begin{array}{l}1 \\
1\end{array}$ & $\overline{-}$ & $\begin{array}{l}2 \\
2\end{array}$ \\
\hline $\begin{array}{l}\text { stullwater Clty of } \\
\text { Boomer Lake (OK) }\end{array}$ & $\overline{-}$ & $\overline{-}$ & $\overline{-}$ & $\overline{-}$ & $\overline{-}$ & $\overline{-}$ & $\overline{-}$ & $\overline{-}$ & $\overline{-}$ & $\overline{-}$ & $\begin{array}{l}1 \\
1\end{array}$ \\
\hline $\begin{array}{l}\text { Stockton City of } \\
\text { Stockton (KS) }\end{array}$ & $\overline{-}$ & $\begin{array}{l}5 \\
5\end{array}$ & $\begin{array}{l}74 \\
74\end{array}$ & $\overline{-}$ & $\overline{-}$ & $\overline{-}$ & $\overline{-}$ & : & $\begin{array}{l}1 \\
1\end{array}$ & $\overline{-}$ & 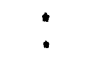 \\
\hline 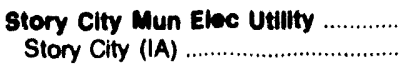 & $\overline{-}$ & $\overline{-}$ & $\overline{-}$ & $\underline{-}$ & $\overline{-}$ & $\overline{-}$ & $\overline{-}$ & $\overline{-}$ & $\overline{-}$ & $\overline{-}$ & $\overline{-}$ \\
\hline $\begin{array}{l}\text { Strawberry Point Lt a Wir Dopt .. } \\
\text { Strawberry Point (IA) }\end{array}$ & $\overline{-}$ & $\overline{-}$ & $\overline{-}$ & $\overline{-}$ & $\overline{-}$ & $\overline{-}$ & $\overline{-}$ & $\overline{-}$ & $\overline{-}$ & $\overline{-}$ & : \\
\hline $\begin{array}{l}\text { Strawberry Water Users Aseoc ... } \\
\text { Payson (UT) } \\
\text { Spanish Fork (UT) }\end{array}$ & $\bar{z}$ & $\bar{z}$ & $\bar{z}$ & $\begin{array}{l}274 \\
103 \\
171\end{array}$ & $\bar{z}$ & $\bar{z}$ & $\bar{z}$ & $\begin{array}{l}- \\
-\end{array}$ & $\bar{z}$ & $\bar{z}$ & $\overline{-}$ \\
\hline $\begin{array}{l}\text { Stuart Light } 2 \text { Power Plant } \\
\text { Stuart (NE) }\end{array}$ & $\overline{-}$ & $\overline{-}$ & $\overline{-}$ & $\overline{-}$ & $\overline{-}$ & $\overline{-}$ & $\overline{-}$ & - & $\overline{-}$ & $\overline{-}$ & 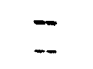 \\
\hline $\begin{array}{l}\text { Stuart Lught a Power System } \\
\text { Stuart (IA) ..... }\end{array}$ & $\overline{-}$ & 1 & $\overline{-}$ & $\overline{-}$ & $\overline{-}$ & $\overline{-}$ & $\overline{-}$ & : & $\overline{-}$ & $\overline{-}$ & : \\
\hline $\begin{array}{l}\text { Sturgle City Ot } \\
\text { Centerville (MI) } \\
\text { Sturgis (MI) }\end{array}$ & $\overline{-}$ & $-\quad 29$ & $\begin{array}{r}91 \\
-\quad 91\end{array}$ & $\begin{array}{r}916 \\
916 \\
-\quad\end{array}$ & $\overline{-}$ & $\overline{-}$ & $\overline{-}$ & $\ddot{-}$ & $-{ }^{1}$ & $\overline{-}$ & -1 \\
\hline $\begin{array}{l}\text { Sullivan Crty Of } \\
\text { Sullivan (IL) }\end{array}$ & $\overline{-}$ & $\begin{array}{l}14 \\
14\end{array}$ & $\begin{array}{l}50 \\
50\end{array}$ & $\overline{-}$ & $\overline{-}$ & $\overline{-}$ & $\overline{-}$ & $:$ & $\begin{array}{l}1 \\
1\end{array}$ & - & 2 \\
\hline $\begin{array}{l}\text { Summer Municipal Light Dopt ...... } \\
\text { Summer (IA) ... }\end{array}$ & $\overline{-}$ & $\overline{-}$ & - & $\overline{-}$ & $\overline{-}$ & $\overline{-}$ & $\overline{-}$ & - & $\overline{-}$ & $=$ & $\begin{array}{l}1 \\
1\end{array}$ \\
\hline $\begin{array}{l}\text { Sunflower Eloctrlc Coop } \\
\text { Garden City (KS) } \\
\text { Holcomb (KS) }\end{array}$ & $\frac{93,151}{93,151}$ & $\overline{-}$ & $\begin{array}{r}1,588 \\
1.168 \\
420\end{array}$ & $\bar{z}$ & $=$ & $\overline{-}$ & $-{ }^{53}$ & $\overline{-}$ & $\begin{array}{r}23 \\
19 \\
5\end{array}$ & $\begin{array}{c}104 \\
-104\end{array}$ & $=$ \\
\hline $\begin{array}{l}\text { Superior Water LI \& Pwr Co. } \\
\text { Winslow (WI) }\end{array}$ & $\overline{-}$ & $\overline{-}$ & $\overline{-}$ & $\overline{-}$ & $\overline{-}$ & $\overline{-}$ & $\overline{-}$ & - & $\overline{-}$ & $\overline{-}$ & - \\
\hline $\begin{array}{l}\text { Swans Ieland Eloctric Coop, Inc.. } \\
\text { Minturn (ME) }\end{array}$ & $\overline{-}$ & $\overline{-}$ & $\overline{-}$ & $\underline{-}$ & $\overline{-}$ & $\overline{-}$ & $\overline{-}$ & $=$ & $\overline{-}$ & $\overline{-}$ & $=$ \\
\hline $\begin{array}{l}\text { 8wanton Eloctric Department } \\
\text { Higate Falls (VT) }\end{array}$ & $\overline{-}$ & $\overline{-}$ & $=$ & $\begin{array}{l}2,205 \\
2,205\end{array}$ & $\overline{-}$ & $\overline{-}$ & $\overline{-}$ & $\overline{-}$ & $\overline{-}$ & $\overline{-}$ & $\overline{-}$ \\
\hline
\end{tabular}

See footnotes at end of table. 
Table 62. U.S. Electric Utility Net Generation, Fuel Consumption, and Fuel Stocks by Company and Plant, September 1993 (Continued)

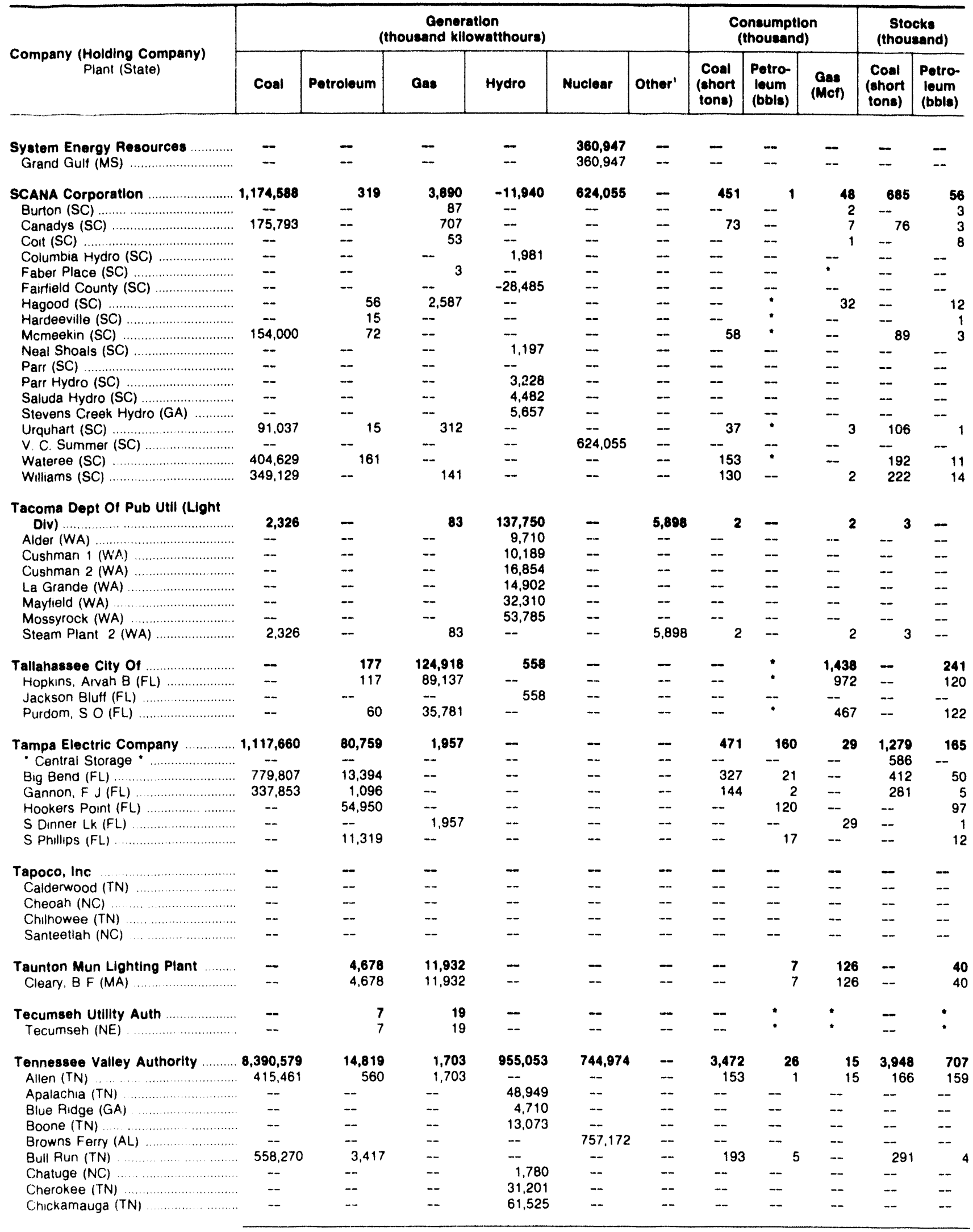

See footnotes at end of table. 
Table 62. U.S. Electric Utility Net Generation, Fuel Consumption, and Fuel Stocks by Company and Plant, September 1993 (Continued)

\begin{tabular}{|c|c|c|c|c|c|c|c|c|c|c|c|}
\hline \multirow{2}{*}{$\begin{array}{c}\text { Company (Holding Company) } \\
\text { Plant (State) }\end{array}$} & \multicolumn{6}{|c|}{$\begin{array}{c}\text { Generation } \\
\text { (thousand kllowatthours) }\end{array}$} & \multicolumn{3}{|c|}{$\begin{array}{l}\text { Consumption } \\
\text { (thouseand) }\end{array}$} & \multicolumn{2}{|c|}{$\begin{array}{l}\text { Stocks } \\
\text { (thousand) }\end{array}$} \\
\hline & Coal & Potroleum & Cas & Hydro & Nuclear & Other' & $\begin{array}{c}\text { Coal } \\
\text { (short } \\
\text { tons) }\end{array}$ & $\begin{array}{l}\text { Potro- } \\
\text { leum } \\
\text { (bble) }\end{array}$ & $\begin{array}{c}\text { Gas } \\
\text { (Mct) }\end{array}$ & $\begin{array}{c}\text { Coal } \\
\text { (ehort } \\
\text { tons) }\end{array}$ & $\begin{array}{l}\text { Petro- } \\
\text { leum } \\
\text { (bbls) }\end{array}$ \\
\hline
\end{tabular}

Tennessee Valley Authority

Colbert (AL)

Cumberland (TN) ............................ 1,422,582

Douglas (TN)

Fontana (NC)

Fort Loudoun (TN)

Fort Patrick Henry (TN)

Gallatin (TN)

Great Falls (TN)

Guntersville (AL)

Hiwassee (NC)

Johnsonville (TN)

Kentucky (KY)

Kingston (TN)

Melton Hill (TN)

Nickajack (TN)

Norris (TN) .

Nottely (GA)

OCOOe 1 (TN)

Ocoee 2 (TN)

Ocoes 3 (TN)

Paradise (KY)

Pickwick (TN)

Raccoon Mountair

Sequoyah (TN)
Sevier, John (TN)

Shawnee (KY)

South Holston (TN)

Tims Ford (TN)

Watauga (TN)

Watts Bar (TN)

Watts Bar (TN)

Wheeler (AL)

Wivows Creek (AL)

Wilbur (TN)

Wilson (AL)

Texas Municipal Power Agency

Gibbons Creek (TX)

Texas Utila Electric Co (TU) ........ 3,022,293

Big Brown (TX) ........................... 506,337

Collin (TX)

Comanche Peak (TX)

Dallas (TX)

De Cordova (TX)

Eagle Mountain (TX)

Graham (TX)

Handiey (TX)

Lake Creek (TX)

Lake Hubbard (TX)

Martin Lake (TX)

Monticello (TX) ........

Morgan Creek (TX)

Mountain Creek (TX)

North Lake (TX)

North Main (TX)

Parkdale (TX)

Permian Basin (TX)

River Crest (TX)

Sandow (TX) ........

Stryker Creok (TX)

Tradinghouse Creak (TX)

Trinidad (TX)

valley $(\mathrm{TX})$

Texas-Now Mexico Power Co ..... 133,195 Lordsburg (NM)

TNP One (TX)

133,195
2438

$-$

$-$

598

626,663

$-$

617,252

881,650

$-$

$-$

$-$

$-$

$, 295,846$

--

461,362

737,876

$-$

$-120$

$-120$

$84 \overline{9}, 329$

$-$

244,420

244,420

506,337

$-$

-

$-$

$-$

$-$

$, 066,844$

$1,058,707$

-

-

$-$

$-$

$-$

390,405

-

$-$

$\overline{-}$

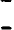

3,109

254

--

$-$

$-$

$-$

$-$

--

$-$

74

$-$

$-$

$-$

$-$

-

$-$

$-$

-

4,525

--

$-$

$-$

$=$

$-$

$-$

--

1,696

2,578

--

$-$

$-$

$+$

$-$

--

238

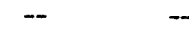

-

78,646

78,646
$-$

31,462

88,818

65,912

7,595

1,005

51,250

25,913

73,687

$\overline{12,679}$

47,266

41,824

2,433

6,160

10,227

16,948

81,055

$-57,587$

$-$

$-$

15,746

1,224

4,386

$\overline{67,259}$

66,466

- 653

131,434

29,599
29,599

$-$

$, 794,513$

13,975

$-217$

250,053

59,793

170,529

133,520

75,718

250,555

--

292,708

124,083

152,641

86
1,295

291,835

$\begin{array}{r}-93 \\ \hline\end{array}$

-

277,173

443,140
-242

257,863

$\begin{array}{rr}22 & - \\ - & -\end{array}$

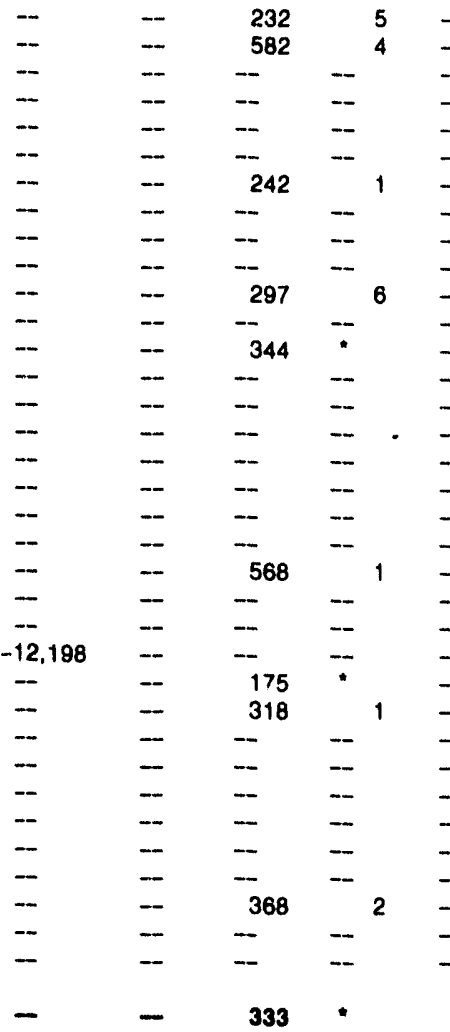

$\begin{array}{rrr}- & 416 & 121 \\ - & 496 & 8\end{array}$

$-$

$--$

$137 \quad 141$

-. -

$356 \quad 261$

$400 \quad 3$

-

$-$

$-$

814

365

$-$

$-$

-

325

$107-$

- 2,582

- 1,193,

$1, \overline{193,521}$

$-$

\begin{tabular}{|c|c|c|c|c|c|}
\hline $\begin{array}{r}2,592 \\
431\end{array}$ & -- & 8 & $\begin{array}{r}28,211 \\
154\end{array}$ & $\begin{array}{r}1,708 \\
296\end{array}$ & $\begin{array}{l}2,396 \\
--\end{array}$ \\
\hline- & -- & & 5 & - & 65 \\
\hline - & - & & -- & - & -- \\
\hline-- & $\cdots$ & & • & -- & 44 \\
\hline- & - & & 2,545 & - & 195 \\
\hline- & - & & 735 & - & 71 \\
\hline- & - & & 1,759 & - & 58 \\
\hline- & - & & 1,129 & - & 252 \\
\hline- & - & & 754 & -- & 117 \\
\hline- & - & & 2,637 & - & 216 \\
\hline $\begin{array}{l}881 \\
947\end{array}$ & & $\begin{array}{l}3 \\
5\end{array}$ & 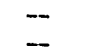 & $\begin{array}{l}547 \\
362\end{array}$ & 61 \\
\hline- & -- & & 3,031 & -- & 250 \\
\hline- & - & & 1,284 & - & 148 \\
\hline- & - & & 1,635 & - & 167 \\
\hline- & - & & 5 & - & 14 \\
\hline- & - & & 23 & - & 50 \\
\hline- & - & & 2,855 & - & 234 \\
\hline- & - & & - & - & 3 \\
\hline 333 & 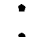 & & - & 503 & -- \\
\hline-- & • & & 2,701 & - & \\
\hline- & - & & 4,311 & -- & 181 \\
\hline- & -- & & - & -- & 35 \\
\hline- & -- & & 2,649 & - & 103 \\
\hline 104 & - & & " & 116 & - \\
\hline-- & - & & - & $-\infty$ & - \\
\hline 104 & - & & • & 116 & - \\
\hline
\end{tabular}

See footnotes at end of table. 
Table 62. U.S. Electric Utility Not Generation, Fuel Coneumption, and Fuel Stocks by Company and Plant, September 1993 (Continued)

\begin{tabular}{|c|c|c|c|c|c|c|c|c|c|c|c|}
\hline \multirow{2}{*}{$\begin{array}{l}\text { Company (Holding Company) } \\
\text { Plant (State) }\end{array}$} & \multicolumn{6}{|c|}{$\begin{array}{l}\text { Ceneration } \\
\text { (thousand kllowatthours) }\end{array}$} & \multicolumn{3}{|c|}{$\begin{array}{l}\text { Contumption } \\
\text { (thoueand) }\end{array}$} & \multicolumn{2}{|c|}{$\begin{array}{c}\text { Stocks } \\
\text { (thousand) }\end{array}$} \\
\hline & Cool & Potroteum & aes & Hydro & Nuolew & Other' & $\begin{array}{l}\text { Coal } \\
\text { (chort } \\
\text { tons') }\end{array}$ & $\begin{array}{l}\text { Potro- } \\
\text { bum } \\
\text { (bole) }\end{array}$ & (Mct) & $\begin{array}{c}\text { Coal } \\
\text { (ahort } \\
\text { tons) }\end{array}$ & $\begin{array}{l}\text { Petro- } \\
\text { leum } \\
\text { (bble) }\end{array}$ \\
\hline
\end{tabular}

Thief River Falls Wtr a Lt Dept ...

Thief River Falls (MN)

Thumb Elec Coop or Michloen ....

Caro (M!)

Ubly (MI)

Tipton Mun Utilities

Tipton (IA)

Toledo Edison Company

Acme (OH)

Bay Shore (OH)

Davis-Besse (OH)

Richland $(\mathrm{OH})$

Stryker $(\mathrm{OH})$

Traer Municlpal Utilities

Traer (IA)

Traveres Clty 01

Bayside (MI)

Boardman (MI)

Brown Bridge (MI)

Elk Rapids (MI) .

Sabin (MI)

Trenton Light a Power Plant .......

Trenton (NE)

Trenton Municipal Utaltibe

Trenton (MO) .

Trenton PKG (MO)

Tri State Gen a Trans Asen, Inc.. Burtington (CO)

Craig (CO)

Nucla (CO)

Ouray (CO)

Trinidad Mun Purr a te Dapt Trinidad (CO) .

Truman Public Uthl Comm Truman (MN)

Tucson Electric Power Company De Moss Petrie (AZ)

Irvington (AZ)

North Loop (AZ)

Springerville (AZ)

Tulla Light a Power Plant ............. Tulia (TX)

Turlock Irrigation Diat Hickman (CA)

Lagrange (CA)

New Don Pedro (CA)

Turlock Lake (CA)

Uppr Dawson (CA)

Walnut (CA)

Two Harbore Wtr L Lept .........

Two Harbors (MN)

Unalaklest Viliage Elec Asece

Unalakleet (AK)

Unalakleet (AK)

Seo footnotes at end of table.

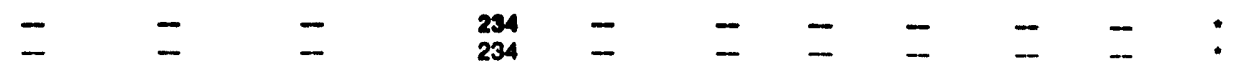

$=\quad$

$-$

214,115

$2 \overline{4}, 115$

$-$

$-$

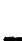

-

$-$

-

-

$-$

$-$

$-$

-

$-$

$-$

49,258

708,011

41,245

$-$

\section{$-21$}

$-21$

- $\quad-$

$-$

$-$

$-$

$-$

$24 \quad-$
$-\quad-$

$437 \quad 1,272$

$\begin{array}{ll}- & 1,272 \\ - & - \\ 437 & -272\end{array}$

$-$

$\begin{array}{ll}-2 & - \\ -2 & -\end{array}$

-

503,103

4085,200

-

$-$

54,318

448,875

$-486$

4,787
81

$\bar{z}$
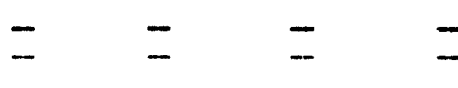

$-$

$-$

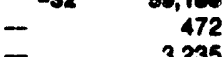

- $\quad 3,235$

$\begin{array}{rrr}- & - & 53,380 \\ - & - & 987\end{array}$

$\begin{array}{ll}- & - \\ - & -32\end{array}$

$-$

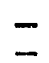

- 290

$\bar{z} \equiv \bar{z} \equiv \bar{z}$

629,977 - $81 \quad 1 \quad 183$

\begin{tabular}{lllllr}
- & - & - & - & - & 19 \\
\hline & 81 & 1 & - & 164
\end{tabular}

628,977

$-\quad-$
$-$

8,10

1,104

eng

132

221

-

$=$

$\bar{z}$

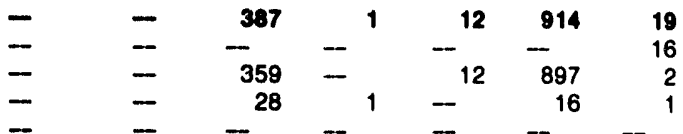

$\overline{-}=\overline{-}=11$

$= \pm= \pm=$ :

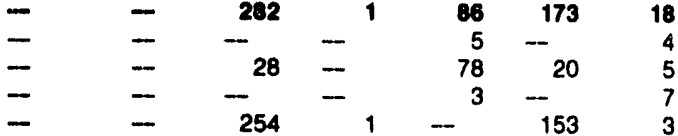

$=\overline{-}=\overline{-}=$

$\bar{z}=\overline{-}-\overline{-}{ }^{3}$

$\bar{z}=\bar{z}=\overline{-}$

$=\bar{z}=\bar{z}$

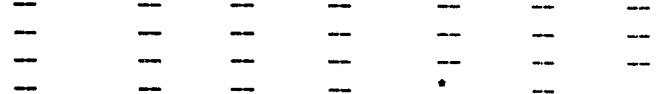

$\overline{-}=\overline{-} \overline{-} \overline{-}$

$=-\overline{-}-1 \pm-7$

$\begin{array}{rrr}290 & - & - \\ 290 & - & -\end{array}$


Table 62. U.S. Electric Utility Net Generation, Fuel Consumption, and Fuel Stocks by Company and Plant, September 1993 (Continued)

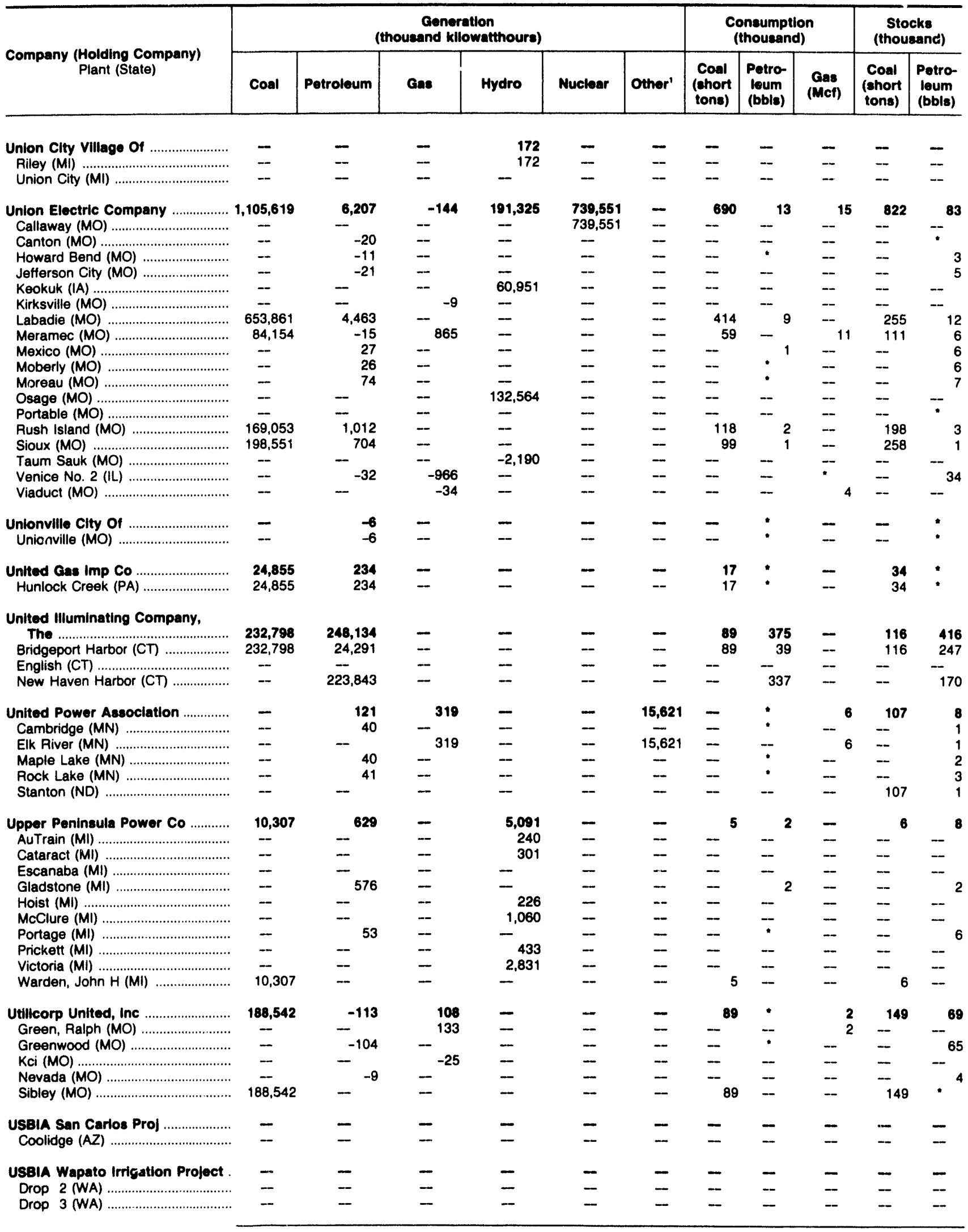

See footnotes at end of table. 
Table 62. U.S. Electric Utility Net Generation, Fuel Consumption, and Fuel Stocks by Company and Plant, September 1993 (Continued)

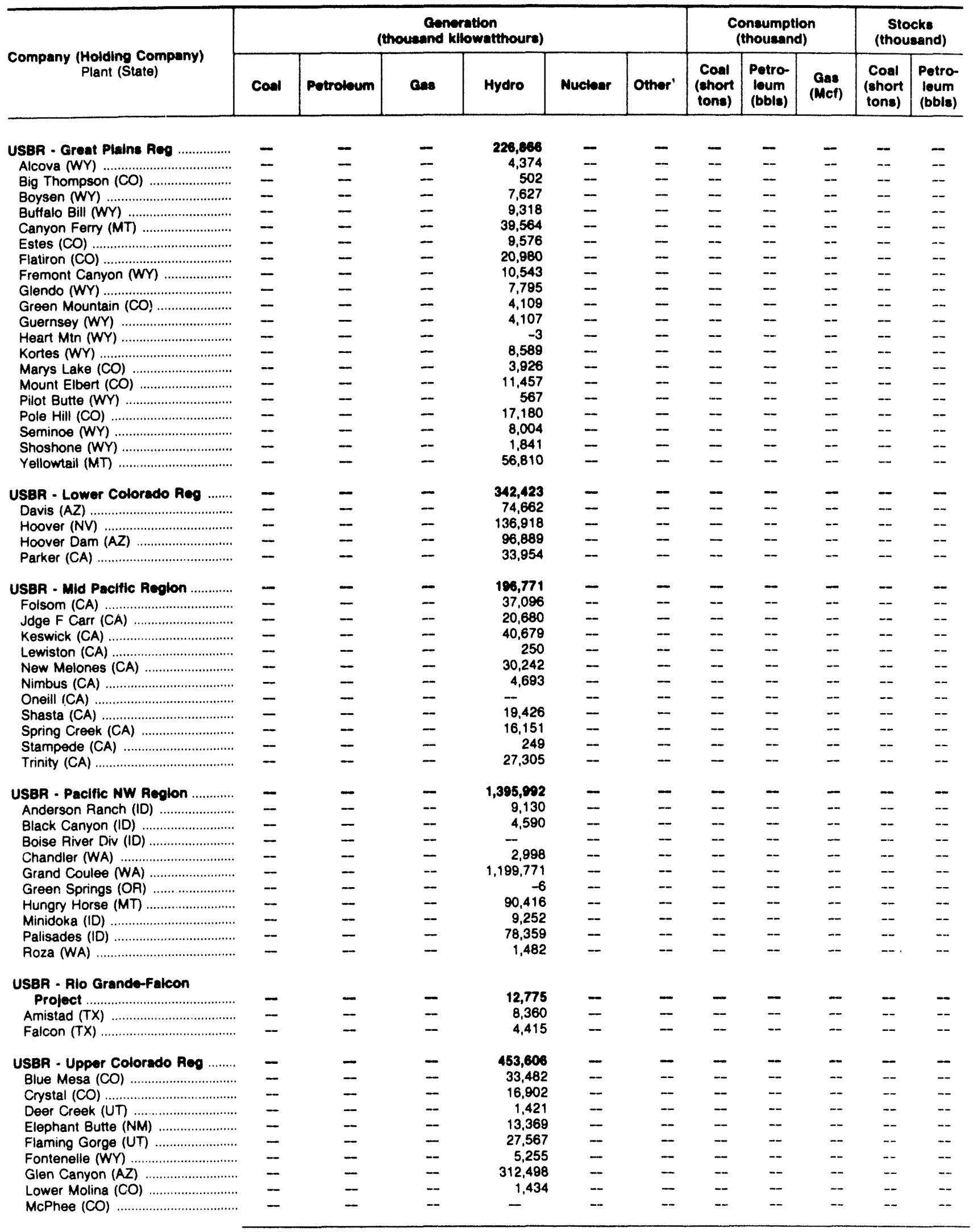

See footnotes at end of table. 
Table 62. U.S. Electric Utility Net Generation, Fuel Consumption, and Fuel Stocks by Company and Plant, September 1993 (Continued)

\begin{tabular}{|c|c|c|c|c|c|c|c|c|c|c|c|}
\hline \multirow{2}{*}{$\begin{array}{c}\text { Company (Holding Company) } \\
\text { Plant (State) }\end{array}$} & \multicolumn{6}{|c|}{$\begin{array}{c}\text { Ceneration } \\
\text { (thoueand kllowatthours) }\end{array}$} & \multicolumn{3}{|c|}{$\begin{array}{l}\text { Consumption } \\
\text { (thousend) }\end{array}$} & \multicolumn{2}{|c|}{$\begin{array}{c}\text { Stocks } \\
\text { (thousennd) }\end{array}$} \\
\hline & Coal & Potroleum & Ges & Hydro & Nuclear & Other' & $\begin{array}{l}\text { Coal } \\
\text { (chort } \\
\text { tons) }\end{array}$ & $\begin{array}{l}\text { Potro- } \\
\text { loum } \\
\text { (bols) }\end{array}$ & $\begin{array}{l}\text { Cas } \\
\text { (Mct) }\end{array}$ & $\begin{array}{l}\text { Coal } \\
\text { (chort } \\
\text { tons) }\end{array}$ & $\begin{array}{l}\text { Potro- } \\
\text { loum } \\
\text { (bble) }\end{array}$ \\
\hline
\end{tabular}

USBR - Upper Colorado Reg Morrow Point (CO)

Towaoc (CO)

Upper Molina (CO)

USCE - Blakely Mtn .

Blakely Mountain (AR)

Degray (AR)

Narrows (AR)

USCE - Fort Worth District

R. D. Willis (TX)

Rayburn, Sam (TX)

Whitney (TX)

USCE - Hartwell Power Plant Hartwell Lake (GA)

USCE - J Strom Thur Power

Plant

$\mathrm{J}$ Strom Thur............

USCE - Kansas City Dist

Harry Truman (MO)

Stockton (MO)

Wilson (KS).

USCE - Little Rock

Beaver (AR)

Bull Shoals (AR)

Dardanelle (AR)

Greers Ferry Lake (AR)

Norfork (AR)

Ozark (AP)

Table Rock (MO)

USCE - Mobile District

Allatoona (GA)

Buford (GA)

Carters (GA)

George, Walter $F$ (GA)

Jones Bluff (AL)

Millers Ferry (AL)

West Point (GA)

Woodruff, $J(F L)$

USCE - Nashville

Barkley (KY)

Center Hill (TN)

Cheatham (TN)

Cordell Hull (TN

Dale Hollow (TN)

Laurel (KY)

Old Hickory (TN)

Priest, JP (TN)

Wolf Creek (KY)

USCE - North Pacific Div ............... Albeni Falls (ID)

Big Clitf (OR)

Bonneville (OR)

Chief Joseph (WA)

Cougar (OR)

Dalles (WA)

Day, John (OR)

Detroit (OR)

Dexter (OR)

Dworshak (ID)

\begin{tabular}{|c|c|c|c|c|c|c|c|c|c|c|}
\hline- & -- & - & 39,227 & - & - & - & - & - & - & -- \\
\hline -- & - & - & - & - & - & - & - & - & - & -- \\
\hline-- & -- & -- & 2,451 & - & - & - & - & - & - & - \\
\hline- & - & - & 4,839 & - & - & - & - & - & - & - \\
\hline- & - & -- & 3,210 & - & - & - & - & - & -- & -- \\
\hline- & - & - & 1,370 & - & - & - & - & - & -- & - \\
\hline- & - & - & 259 & - & - & - & - & - & - & -- \\
\hline- & - & - & 18,060 & - & - & - & - & - & - & - \\
\hline- & - & - & 4,427 & - & - & - & - & - & - & - \\
\hline$\cdots$ & - & - & 10,475 & - & - & - & -- & - & - & -- \\
\hline-- & - & -- & 3,987 & - & - & - & - & -- & - & -- \\
\hline- & - & - & 28,605 & - & - & - & - & - & - & - \\
\hline-- & - & - & 28,605 & - & - & - & $-s$ & - & - & -- \\
\hline- & - & - & 34,906 & - & - & - & - & - & - & - \\
\hline-- & - & - & 34,966 & - & - & - & - & - & - & - \\
\hline- & - & - & 21,817 & - & 2 & - & - & - & - & - \\
\hline-- & - & -- & 16,935 & - & - & - & - & - & - & -- \\
\hline- & - & - & 4,882 & - & - & - & - & - & - & -- \\
\hline- & - & -- & - & - & 2 & - & $-\infty$ & - & -- & - \\
\hline- & - & - & 204,312 & - & - & - & - & - & - & - \\
\hline-- & - & -- & 2,289 & - & - & - & - & - & - & - \\
\hline- & - & -- & 37,118 & - & - & - & - & - & - & - \\
\hline-- & - & -- & 70,500 & - & - & - & - & - & - & - \\
\hline-- & - & -- & 3,187 & - & - & - & - & - & - & - \\
\hline-+ & -- & - & 3,860 & - & - & - & - & - & -- & - \\
\hline- & -- & -- & 50,319 & - & - & - & - & - & - & -- \\
\hline-- & - & -- & 36,939 & - & - & - & - & - & - & - \\
\hline- & - & - & 77,947 & - & - & - & - & - & - & - \\
\hline-- & - & - & 6,309 & - & - & - & - & - & -- & - \\
\hline-- & - & - & 14,493 & - & - & - & -- & - & - & - \\
\hline- & - & - & $-8,589$ & - & - & - & - & - & -- & - \\
\hline-- & - & - & 18,906 & - & - & - & - & - & - & - \\
\hline- & - & - & 11,071 & - & - & - & - & - & - & - \\
\hline-- & - & -- & 15,528 & - & - & - & - & -- & - & - \\
\hline- & - & - & 8,329 & - & - & - & - & - & - & - \\
\hline- & - & -- & 11,900 & - & - & - & - & - & - & - \\
\hline- & - & - & 147,237 & - & - & - & - & - & - & - \\
\hline-- & - & - & 36,327 & - & - & - & - & - & - & - \\
\hline- & - & - & 10,463 & - & - & - & - & - & -- & - \\
\hline- & - & - & 9,915 & - & - & - & - & - & - & - \\
\hline- & - & -- & 19,686 & - & - & - & - & - & - & - \\
\hline- & - & - & 5,650 & - & - & - & - & - & - & - \\
\hline-- & -- & - & 1,041 & - & - & - & - & - & - & - \\
\hline-- & - & - & 22,365 & - & - & - & - & - & -- & -- \\
\hline- & - & - & -86 & - & - & - & - & - & - & - \\
\hline- & - & - & 41,896 & - & - & - & - & - & - & - \\
\hline- & - & - & $2,984,229$ & - & - & - & - & - & - & - \\
\hline-- & - & - & 23,483 & - & - & - & - & - & - & -- \\
\hline- & - & - & 7,929 & - & - & - & - & - & - & - \\
\hline- & - & - & 313,259 & - & - & - & - & - & - & - \\
\hline- & - & - & 636,123 & - & - & - & - & - & - & - \\
\hline-- & - & - & 16,663 & - & - & - & - & - & -- & - \\
\hline- & -- & - & 405,554 & - & - & - & - & -- & -- & - \\
\hline- & - & - & 510,984 & - & - & - & - & - & - & - \\
\hline-- & - & - & 33,051 & - & - & - & - & - & - & - \\
\hline-- & - & - & 6,964 & - & - & - & - & - & - & - \\
\hline- & - & - & 35,742 & - & -- & - & - & - & - & - \\
\hline
\end{tabular}

See footnotes at end of table. 
Table 62. U.S. Electric Utility Net Generation, Fuel Consumption, and Fuel Stocks by Company and Plant, September 1993 (Continued)

\begin{tabular}{|c|c|c|c|c|c|c|c|c|c|c|c|}
\hline \multirow{2}{*}{$\begin{array}{c}\text { Company (Holding Company) } \\
\text { Plant (State) }\end{array}$} & \multicolumn{6}{|c|}{$\begin{array}{c}\text { Ceneration } \\
\text { (thousand kllowatthoura) }\end{array}$} & \multicolumn{3}{|c|}{$\begin{array}{l}\text { Consumption } \\
\text { (thousand) }\end{array}$} & \multicolumn{2}{|c|}{$\begin{array}{l}\text { Stocks } \\
\text { (thousand) }\end{array}$} \\
\hline & Coal & Potroleum & Cas & Hydro & Nuclear & Other' & $\begin{array}{c}\text { Coal } \\
\text { (short } \\
\text { tons) }\end{array}$ & $\begin{array}{l}\text { Petro- } \\
\text { leum } \\
\text { (bbis) }\end{array}$ & $\begin{array}{c}\text { Gas } \\
(\mathrm{Mct})\end{array}$ & $\begin{array}{c}\text { Coal } \\
\text { (short } \\
\text { tons) }\end{array}$ & $\begin{array}{l}\text { Petro- } \\
\text { loum } \\
\text { (bbls) }\end{array}$ \\
\hline
\end{tabular}

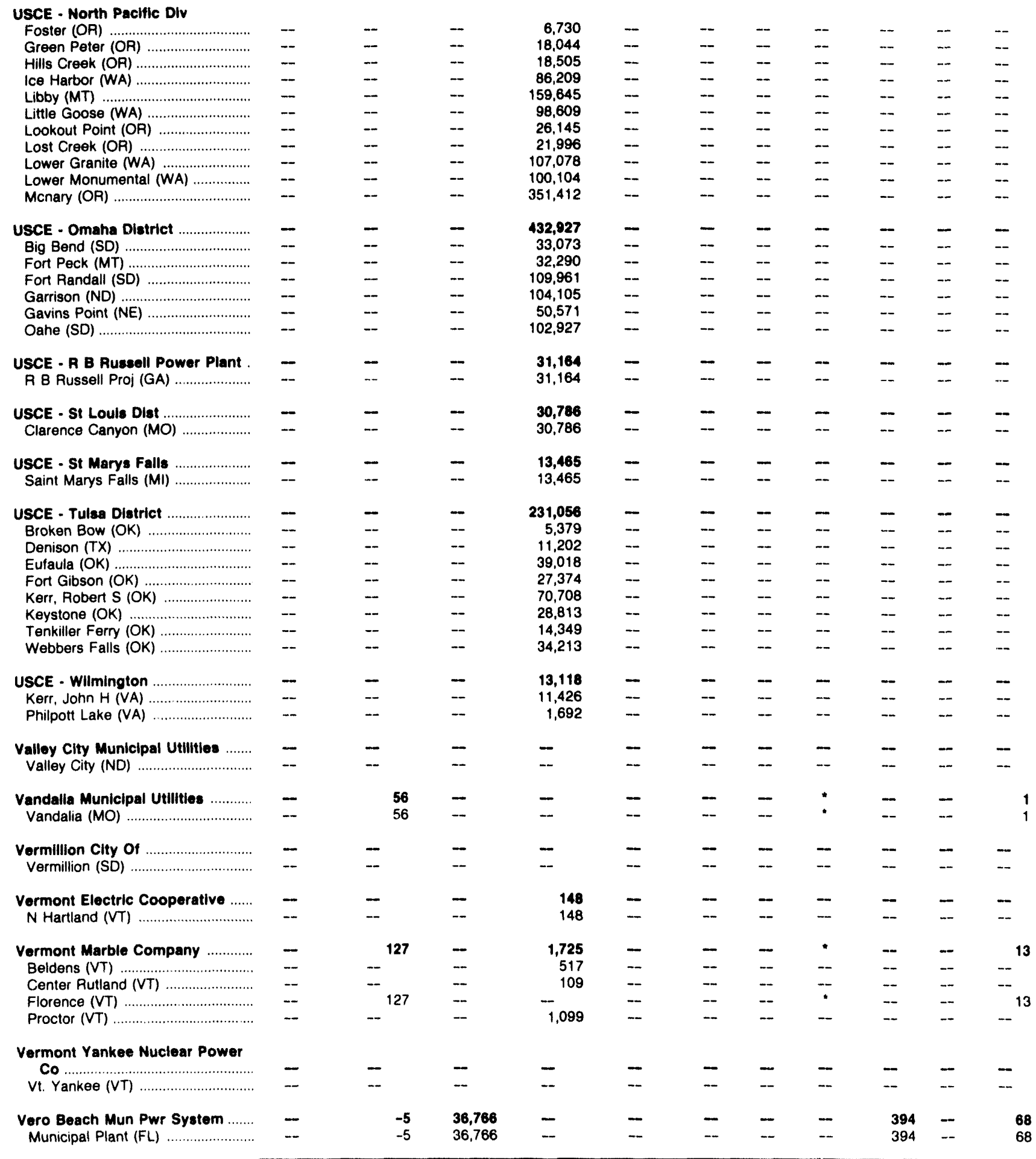

See footnotes at end of table. 
Table 62. U.S. Electric Utility Net Generation, Fuel Consumption, and Fuel Stocks by Company and Plant, September 1993 (Continued)

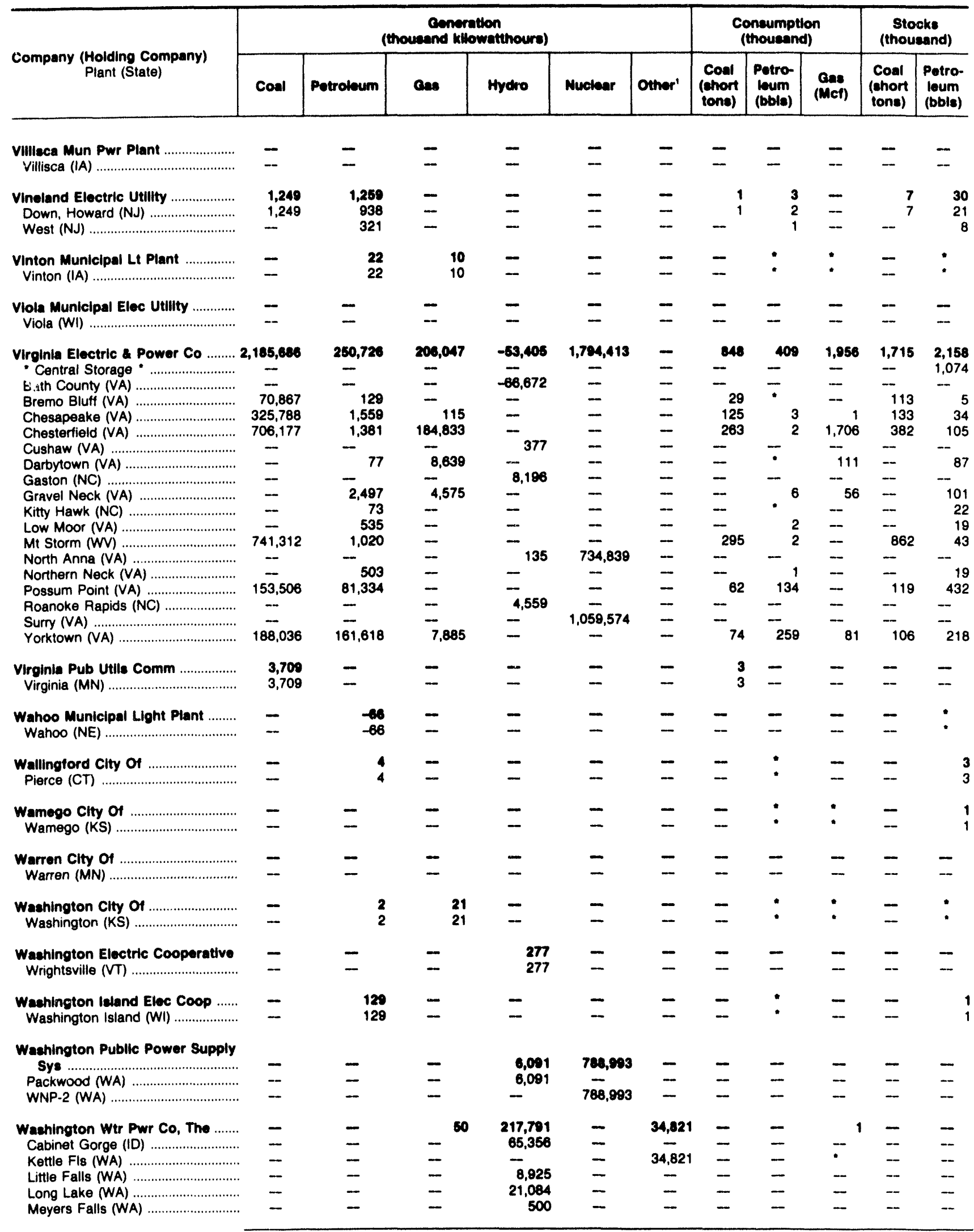

See footnotes at end of table. 
Table 62. U.S. Electric Utility Net Generation, Fuel Consumption, andi Fuel Stocks by Company and Plant, September 1993 (Continued)

\begin{tabular}{|c|c|c|c|c|c|c|c|c|c|c|c|}
\hline \multirow{2}{*}{$\begin{array}{c}\text { Company (Holding Company) } \\
\text { Plant (State) }\end{array}$} & \multicolumn{6}{|c|}{$\begin{array}{c}\text { Generation } \\
\text { (thousand kilowatthours) }\end{array}$} & \multicolumn{3}{|c|}{$\begin{array}{l}\text { Consumption } \\
\text { (thousand) }\end{array}$} & \multicolumn{2}{|c|}{$\begin{array}{c}\text { Stocks } \\
\text { (thousand) }\end{array}$} \\
\hline & Coal & Patroleum & Gas & Mydro & Nuclear & Other' & $\begin{array}{l}\text { Coal } \\
\text { (ahort } \\
\text { tons) }\end{array}$ & $\begin{array}{l}\text { Potro- } \\
\text { leum } \\
\text { (bble) }\end{array}$ & $\begin{array}{l}\text { Gas } \\
\text { (Mct) }\end{array}$ & $\begin{array}{l}\text { Coal } \\
\text { (short } \\
\text { tons) }\end{array}$ & $\begin{array}{l}\text { Potro- } \\
\text { loum } \\
\text { (bbla) }\end{array}$ \\
\hline
\end{tabular}

\begin{tabular}{|c|c|c|c|c|c|c|c|c|c|c|c|}
\hline Washington Wtr Pwr Co, The & & & & & & & & & & & \\
\hline 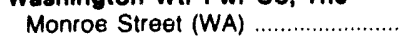 & - & - & -- & 6,096 & -- & -- & -- & -- & - & -- & -- \\
\hline Nine Mile (WA) & - & -- & -- & 5,094 & -. & -- & - & - & -- & -- & -. \\
\hline 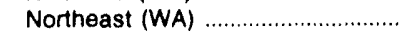 & $\ldots$ & -- & 50 & - & -- & - & -- & -- & 1 & -- & -- \\
\hline 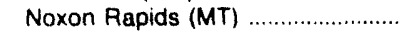 & - & -- & - & 100,605 & -- & -- & -- & -- & -- & -- & -- \\
\hline 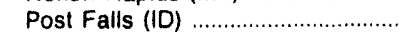 & - & -- & -- & 4,328 & -- & - & -- & -- & -- & -- & -- \\
\hline Upper Falls (WA) ..................................... & -- & -- & -- & 5,803 & - & -- & -- & -- & - & -- & -- \\
\hline Waterloo City Light \& Power ........ & - & - & - & - & - & - & - & - & - & - & 1 \\
\hline Waterloo (IL) . . . . . . & -- & - & -- & -- & -- & -- & -- & -- & -- & - & $i$ \\
\hline Watertown Clty of & - & - & - & 1,514 & - & - & - & - & - & - & - \\
\hline Watertown (NY) & -- & -- & -- & 1,514 & -- & - & - & -- & - & -- & -- \\
\hline Wauchula City Of & - & - & - & - & - & - & -- & - & - & - & 2 \\
\hline Wauchula $(F L)$ & -- & -- & -- & -- & -- & - & - & -- & -- & -- & 2 \\
\hline Waverly City of & - & - & - & 208 & - & - & - & - & - & - & 1 \\
\hline 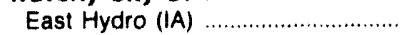 & -- & -- & -- & 208 & -- & - & -- & -- & -- & -- & -- \\
\hline East Plant (IA) & -- & - & -- & -- & -- & - & -- & -- & -- & -- & • \\
\hline 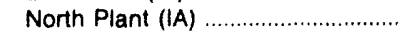 & -- & -- & -- & -- & $\cdots$ & -- & - & - & -- & -- & 1 \\
\hline Wayne Clty of & - & 29 & - & - & -- & - & - & - & - & - & 1 \\
\hline Wayne (NE) & -- & 29 & -- & - & -- & -- & - & • & -- & -- & 1 \\
\hline Weatherford Clty of & - & - & - & - & - & - & - & - & - & - & -- \\
\hline Weathertord (TX) & - & -- & -- & -- & - & - & -- & -- & -- & -- & - \\
\hline Weber Basin Water Conservancy & & & & & & & & & & & \\
\hline 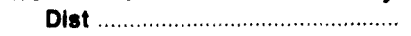 & - & - & - & 3,631 & - & - & - & - & - & -- & -- \\
\hline 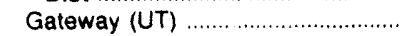 & -- & -- & -- & 2,257 & - & -- & -- & -- & -- & -- & -. \\
\hline 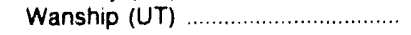 & -- & -- & -- & 1,374 & -- & -- & - & -- & -- & -- & -- \\
\hline Webster Clty Mun Lt \& Pwr ........... & -- & - & - & - & - & - & - & - & -- & -- & -- \\
\hline Webster City (IA) & -- & -- & -- & - & -- & -- & -- & -- & -- & -- & -- \\
\hline Wellington Clty of & - & - & 430 & -- & - & - & - & - & 5 & - & 3 \\
\hline 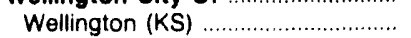 & -- & - & 125 & -- & -- & -- & -- & -- & 1 & -- & 2 \\
\hline 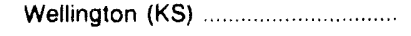 & -- & -- & 305 & $\cdots$ & -- & - & - & -- & 5 & -. & 1 \\
\hline Wells Electric Assoclation ............. & -- & - & - & - & - & - & - & - & - & - & • \\
\hline Wells (MN) & -- & -- & -- & - & -- & -- & -- & -- & -- & -- & - \\
\hline West Bend Mun Power Plant ......... & - & 1 & 15 & - & - & - & - & - & - & - & • \\
\hline West Bend (IA) . & -- & 1 & 15 & -- & - & -- & -- & $\cdot$ & " & -- & . \\
\hline West Liberty Municipal Elec Util .. & - & - & - & - & - & - & - & -. & -- & - & • \\
\hline West Liberty (IA) & -- & -- & -- & -- & -- & -- & -- & -- & -- & -- & • \\
\hline West Penn Power Co (APS) .......... & $\mathbf{9 8 7 , 7 1 1}$ & 672 & 212 & 3,825 & - & - & 401 & 1 & 2 & 871 & 72 \\
\hline Armstrong (PA) & 195,399 & 100 & -- & $-\ldots$ & -- & -- & 83 & * & -- & 124 & $\cdot$ \\
\hline Hatfields Ferry (PA) ........................... & 692,008 & 572 & -- & -- & -- & -- & 275 & 1 & -- & 676 & 4 \\
\hline Lake Lynn $(W V)$ & - & -- & -- & 3,825 & -- & -- & - & -- & -- & -- & -- \\
\hline 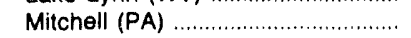 & 100,304 & -- & 212 & -- & -- & - & 43 & -- & 2 & 71 & 68 \\
\hline Springdale (PA) & -. & -- & -- & -- & -- & -. & -- & -- & $\ldots$ & $\ldots$ & -. \\
\hline West Point Light \& Water Works & -- & 2 & 14 & - & -- & - & - & * & - & - & * \\
\hline West Point (NE) & -- & 2 & 14 & -- & -- & -- & -- & • & - & -- & . \\
\hline West Texas Utilities Co (CSW) ..... & 466,431 & 364 & 372,011 & -- & - & - & 278 & 1 & 3,765 & 274 & 289 \\
\hline Abilene $(T X)$ & -- & - & -34 & - & -- & - & -- & -- & 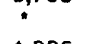 & -- & 4 \\
\hline 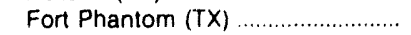 & -- & 207 & 147,020 & -- & -- & -- & -- & - & 1,325 & -- & 101 \\
\hline 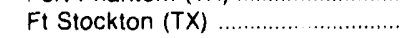 & -- & -- & - & -- & -- & - & -- & -- & - & -- & -- \\
\hline Lake Pauline (TX) & -- & -- & -109 & -- & -- & -- & -- & -- & 2 & -- & 18 \\
\hline Oak Creek $(T X)$ & -- & -- & 38,064 & -- & -- & -- & -- & $-\ldots$ & 406 & -- & 28 \\
\hline Oklaunion $(T X)$ & 466,431 & 157 & -- & -- & -- & -- & 278 & • & -- & 274 & 4 \\
\hline 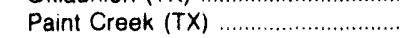 & - & -- & 38,977 & -- & -- & -- & -- & - & 505 & $\ldots$ & 80 \\
\hline Presidio (TX) & -- & -- & -- & $-\sim$ & -- & -- & -- & - & -- & -- & 1 \\
\hline
\end{tabular}

See footnotes at end of table 
Table 62. U.S. Electric Utility Net Generation, Fuel Consumption, and Fuel Stocks by Company and Plant, September 1993 (Continued)

\begin{tabular}{|c|c|c|c|c|c|c|c|c|c|c|c|}
\hline \multirow{2}{*}{$\begin{array}{c}\text { Company (Holding Company) } \\
\text { Plant (State) }\end{array}$} & \multicolumn{6}{|c|}{$\begin{array}{c}\text { Generation } \\
\text { (thousand kllowatthours) }\end{array}$} & \multicolumn{3}{|c|}{$\begin{array}{l}\text { Consumption } \\
\text { (thousand) }\end{array}$} & \multicolumn{2}{|c|}{$\begin{array}{l}\text { Stocks } \\
\text { (thoueand) }\end{array}$} \\
\hline & Coal & Petroleum & Gas & Hydro & Nuclear & Other' & $\begin{array}{c}\text { Coal } \\
\text { (ehort } \\
\text { tons) }\end{array}$ & $\begin{array}{l}\text { Potro- } \\
\text { loum } \\
\text { (bbla) }\end{array}$ & $\begin{array}{l}\text { Gas } \\
\text { (Mct) }\end{array}$ & $\begin{array}{l}\text { Coal } \\
\text { (ehort } \\
\text { tons) }\end{array}$ & $\begin{array}{l}\text { Potro- } \\
\text { leum } \\
\text { (bbia) }\end{array}$ \\
\hline \multicolumn{12}{|l|}{ West Texas Utillties Co (CSW) } \\
\hline Rio $P \theta \cos (T X)$ & -- & -- & 73,628 & -- & - & -- & -- & - & 797 & -- & 24 \\
\hline San Angelo (TX) & -- & -- & 74,468 & -- & -- & -- & -- & - & 730 & -- & 28 \\
\hline Vernon $(T X)$ & -- & -- & -3 & -- & - & -- & -- & - & -- & -- & 1 \\
\hline \multirow{2}{*}{$\begin{array}{l}\text { Westbrook Mun Lt \& Pwr Plant ... } \\
\text { Westbrook (MN) }\end{array}$} & - & -- & - & - & - & - & - & - & - & - & - \\
\hline & -- & -- & -- & -- & -- & -- & - & - & -- & -- & -- \\
\hline Western Farmers Elec Coop ........ & 204,395 & 129 & 100,673 & - & - & - & 129 & - & 877 & 193 & 43 \\
\hline Anadarko (OK) & -- & -- & 94,116 & -- & -- & -- & -- & - & 810 & - & 40 \\
\hline 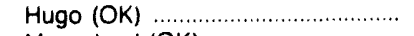 & 204,395 & 129 & $\cdots$ & -- & -- & -- & 129 & • & -- & 193 & 3 \\
\hline Mooreland $(O K)$ & -- & -- & 6,557 & -- & - & - & -- & -- & 67 & $-\infty$ & -- \\
\hline Western Mass Electric Co (NU) ... & -- & 2,308 & 6,173 & $-23,478$ & - & - & - & 5 & 74 & - & 145 \\
\hline Cabot (MA) & -- & -- & -- & 10.730 & - & -- & - & - & - & -- & -- \\
\hline Cobble Mountain (MA) ...................... & -- & -- & -- & 1,366 & -- & $\rightarrow$ & - & - & -- & -- & - \\
\hline Doreen (MA) & -- & -13 & - & -- & -- & - & -- & - & -- & - & 1 \\
\hline 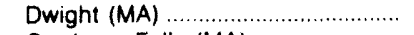 & - & -- & -- & 265 & -- & $-\cdot$ & - & - & - & - & - \\
\hline Gardners Falls (MA) .......................... & -- & -- & -- & 457 & -- & - & -- & - & -- & - & - \\
\hline 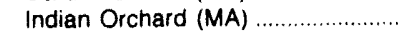 & -- & -- & -- & 122 & -- & -- & - & - & - & - & -- \\
\hline Northfield Mountain (MA) .............. & -- & -- & -- & -37.019 & -- & -- & -- & - & -- & -- & -- \\
\hline Putts Bridge (MA) & - & -- & - & 119 & -- & -- & - & - & -- & -- & - \\
\hline 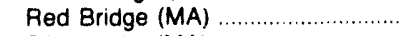 & -- & -- & -- & 485 & - & -- & -- & -- & - & - & - \\
\hline Silver Lake (MA) & -- & - & -- & -- & -- & -- & - & - & - & -- & -- \\
\hline Turners Falls (MA) . & -- & -- & -- & -3 & -- & -- & - & - & -- & - & - \\
\hline West Springfield (MA) ......................... & -- & 2,330 & 6,173 & -- & - & -- & -- & 4 & 74 & -- & 143 \\
\hline Woodland Road (MA) ....................... & -- & -9 & -- & -- & -- & -- & - & $\cdot$ & - & -- & 1 \\
\hline Western Resources, Inc .................. & $1,096,657$ & 325 & 5,787 & - & - & - & 705 & 1 & 81 & 1,114 & 161 \\
\hline Abilene (KS) & -- & -- & 1.177 & $\cdots$ & -- & -- & -- & - & 17 & -- & 16 \\
\hline Hutchinson (KS) & - & 318 & 1,200 & - & - & -- & - & 1 & 22 & - & 99 \\
\hline Jeffrey (KS) & 878,897 & 7 & -- & - & - & -- & 583 & $\cdot$ & - & 814 & 37 \\
\hline Lawrence (KS) & 169,977 & -- & 2,742 & -- & -- & -- & 93 & - & 34 & 264 & 3 \\
\hline 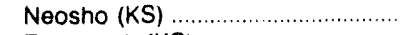 & - & -- & -- & -- & - & -- & -- & - & - & - & - \\
\hline 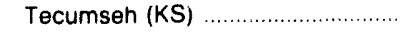 & 47,783 & -- & 668 & -- & - & -- & 29 & - & 9 & 36 & 7 \\
\hline WestPlains Energy ..... & 22,692 & -36 & 45,592 & - & - & - & 13 & - & 612 & 7 & 79 \\
\hline 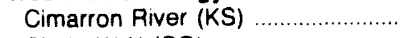 & -- & -- & 11,914 & -- & -- & - & -- & -- & 172 & - & - \\
\hline Clark, W N (CO) & 22,692 & -- & -- & -- & -- & - & 13 & - & - & 7 & - \\
\hline Clifton (KS) & -- & -- & -7 & -- & - & -- & -- & -- & 4 & - & 6 \\
\hline Judson Large (KS) ............................. & -- & -- & 29,622 & -- & - & - & -- & - & 370 & -- & 43 \\
\hline Mullergren, Arthur (KS) .................. & -- & -- & -147 & -- & -- & - & -- & - & - & - & 22 \\
\hline Puebio $(\mathrm{CO})$ & -- & -- & 4,210 & -- & -- & -- & - & - & 66 & - & 5 \\
\hline Rocky Ford $(\mathrm{CO})$ & -- & -36 & -- & -- & -- & -- & - & - & - & - & 4 \\
\hline Whitesboro Electric Utillty ............ & - & - & -- & - & - & - & - & - & - & - & - \\
\hline Whitesboro (TX) & -- & $\cdots$ & - & - & - & - & - & - & - & -- & -- \\
\hline Whittemore Mun Lt \& Pwr Co ...... & - & -- & -- & - & - & - & - & - & - & - & - \\
\hline Whittemore (IA) & -- & -- & -- & - & -- & -- & -- & - & -- & -- & - \\
\hline Wilber Clty Of & - & - & - & - & - & - & - & - & - & - & - \\
\hline Wilber (NE) & -- & -- & -- & -- & -- & -- & -- & - & - & -- & - \\
\hline Willmar Municipal Util Comm ......... & 3,148 & - & 18 & - & - & - & 4 & - & " & 6 & - \\
\hline Wilmar (MN) & 3,148 & -- & 18 & -- & -- & -- & 4 & - & - & 6 & - \\
\hline Wilton Mun Lt \& Pwr System ....... & - & - & - & - & - & - & - & - & - & - & - \\
\hline Wilton Junction (IA) ................................ & -- & -- & -- & - & -- & -- & -- & - & -- & -- & -- \\
\hline Windom Municipal Utilities .............. & - & - & - & - & - & - & - & - & - & - & - \\
\hline Windom (MN) & -- & -- & - & -- & - & -- & - & - & - & - & - \\
\hline Winfield Mun Light \& Power PIt ... & - & 362 & 400 & - & - & - & - & 1 & 4 & - & 4 \\
\hline Winfield (KS) & -- & -- & -- & - & - & -- & - & - & - & - & - \\
\hline 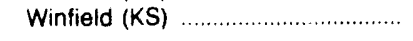 & -- & 362 & 400 & -- & - & -- & -- & 1 & 4 & - & 4 \\
\hline Winnetka, Village of .......................... & - & 7 & 47 & - & - & - & - & - & 3 & - & 2 \\
\hline 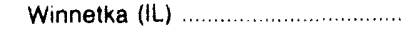 & -.. & 7 & 47 & - & - & - & -- & - & 3 & - & 2 \\
\hline
\end{tabular}

See footnotes at end of table. 
Table 62. U.S. Electric Utility Net Generation, Fuel Consumption, and Fuel Stocks by Company and Plant, September 1993 (Continued)

\begin{tabular}{|c|c|c|c|c|c|c|c|c|c|c|c|}
\hline \multirow{2}{*}{$\begin{array}{c}\text { Company (Holding Company) } \\
\text { Plant (State) }\end{array}$} & \multicolumn{6}{|c|}{$\begin{array}{l}\text { Cenoration } \\
\text { (thousand kllowatthoura) }\end{array}$} & \multicolumn{3}{|c|}{$\begin{array}{l}\text { Consumption } \\
\text { (thousend) }\end{array}$} & \multicolumn{2}{|c|}{$\begin{array}{l}\text { Stocks } \\
\text { (thousand) }\end{array}$} \\
\hline & Coal & Potroloum & Qas & Hydro & Nuclear & Other' & $\begin{array}{c}\text { Coal } \\
\text { (ehort } \\
\text { tons) }\end{array}$ & $\begin{array}{l}\text { Potro- } \\
\text { loum } \\
\text { (bbla) }\end{array}$ & $\begin{array}{l}\text { Gas } \\
\text { (Mef) }\end{array}$ & $\begin{array}{c}\text { Coal } \\
\text { (ahort } \\
\text { tons) }\end{array}$ & $\begin{array}{l}\text { Potro- } \\
\text { loum } \\
\text { (bble) }\end{array}$ \\
\hline $\begin{array}{l}\text { Winterset Clty of } \\
\text { Winterset (IA) }\end{array}$ & - & $\overline{-}$ & $\overline{-}$ & $\overline{-}$ & $\overline{-}$ & $\overline{-}$ & $\overline{-}$ & - & - & $\overline{-}$ & 1 \\
\hline Wisconsin Electric Power Co ........ & $1,213,346$ & 1,887 & 7,387 & 19,302 & 636,507 & - & 645 & 3 & 76 & 1,783 & 40 \\
\hline " Central Storage • & -- & -- & -- & - & -- & -- & - & - & -- & - & 5 \\
\hline Appleton (WI) & -- & -- & - & 1,245 & -- & -- & - & -- & -- & -- & - \\
\hline 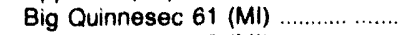 & -- & -- & - & -- & - & -- & -- & -- & -- & - & -- \\
\hline 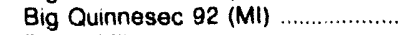 & - & -- & -- & -- & -- & -- & -- & -- & -. & -- & -- \\
\hline Brule (MI) & -- & - & - & 966 & -- & -- & - & -- & - & - & - \\
\hline 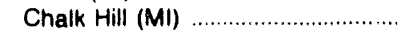 & -- & -- & - & 2,234 & - & -- & -- & -- & -- & -- & - \\
\hline Concord $(w 1)$ & -- & -- & -- & -- & -- & -- & -- & -- & -- & -- & - \\
\hline 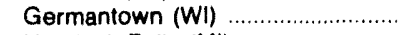 & -- & -60 & - & -- & -- & - & - & - & - & - & 12 \\
\hline Hemlock Falls (MI) & $\cdots$ & -- & -- & -1 & -- & -- & - & - & -- & -- & -. \\
\hline Kingstord $(M I)$ & -- & -- & -- & 2,231 & -- & -- & -- & - & -- & -- & -- \\
\hline Lower Paint (MI) & -- & -- & -- & 42 & -- & -- & -- & - & - & -- & - \\
\hline Michigamme Falls (MI) ..................... & -- & -- & -- & 2,241 & - & - &.- & - & - & -- & -- \\
\hline Oconto Falls (WI) & - & - & -- & 647 & - & $\cdots$ & -- & -- & -- & $\ldots$ & -- \\
\hline 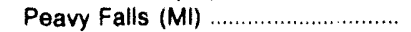 & - & - & - & 3,804 & -- & -- & -- & -- & -- & -. & -- \\
\hline Pine $(W I)$ & -- & -- & - & 183 & -- & -- & - & -- & - & -- & -- \\
\hline Pleasant Prairie (WI) ......................... & 587.533 & 20 & 2,349 & -- & - & -- & 373 & • & 25 & 655 & 8 \\
\hline Point Beach (WI) . & - & 31 & -- & -- & 636,507 & - & - & - & -- & -- & 3 \\
\hline 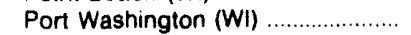 & 17,003 & 1 & 274 & -- & -- & - & 11 & - & 4 & 112 & 3 \\
\hline Presque Isle (MI) & 158,588 & 453 & - & -- & -- & -- & 82 & 1 & -- & 854 & 5 \\
\hline South Oak Creek (WI) & 413,194 & 1,439 & 4,602 & -- & - & -- & 159 & 2 & 44 & 106 & 4 \\
\hline Sturgeon (MI) & -- & -- & -- & 388 & -- & -- & -- & - & -- & $-\infty$ & -- \\
\hline Twin Falls (MI) & $\cdots$ & -- & $-\infty$ & 2,217 & - & -- & - & -- & - & - & -- \\
\hline Valley $(W I)$ & $\$ 7,028$ & 3 & 162 & -- & -- & - & 20 & - & 3 & 57 & - \\
\hline 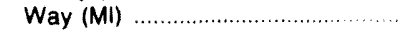 & -- & -- & -- & 236 & -- & -- & - & -- & -- & -- & -- \\
\hline 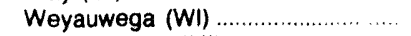 & -- & - & - & 28 & -- & -- & -- & -- & -- & -- & -- \\
\hline White Rapids (MI) & $\cdots$ & -- & - & 2,841 & - & - & -- & - & -. & -- & - \\
\hline Wisconsin Power \& Light Co ....... & 875,929 & 1,070 & 288 & 21,322 & - & 2,288 & 507 & 2 & 6 & 804 & 12 \\
\hline Blackhawk (WI) & -- & -- & 22 & 285 & -- & -- & - & - & 1 & -- & - \\
\hline Columbia $(W \mid)$ & 383,458 & 538 & -- & -- & - & - & 238 & 1 & - & 470 & 5 \\
\hline 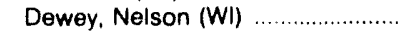 & 75,903 & 54 & -- & -- & - & 1,463 & 43 & • & -- & 194 & - \\
\hline 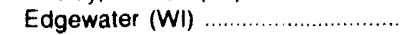 & 396,105 & 397 & -- & - & -- & - & 215 & 1 & -- & 185 & 2 \\
\hline 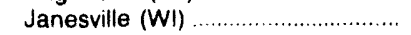 & -- & - & - & 236 & -- & -- & -- & -- & - & -- & -- \\
\hline Kilbourn $(W I)$ & -- & - & - & 6,093 & - & -- & -- & - & -- & - & -- \\
\hline 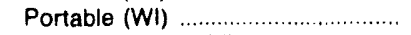 & -- & -- & - & -- & -- & -- & -- & -- & - & -- & -- \\
\hline 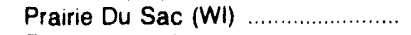 & -- & -- & -- & 14,278 & -- & - & -- & - & -- & - & -- \\
\hline 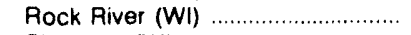 & 20,463 & 81 & 252 & -- & -- & 825 & 11 & • & 5 & 55 & 1 \\
\hline Shawano $(W \mid)$ & -- & -- & -- & 430 & -- & -- & -- & -- & -- & -- & -- \\
\hline Sheepskin (WI) & -- & - & 14 & -- & -- & -- & -- & -- & • & -- & 4 \\
\hline Wisconsin Public Service Corp. & 346,652 & 275 & 2,633 & 25,613 & 376,951 & - & 220 & 1 & 33 & 106 & 33 \\
\hline$r(W I)$ & -. & -- & -- & 1,989 & -- & - & -- & -- & $-\infty$ & -- & -- \\
\hline 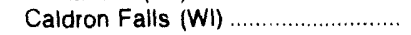 & -- & -- & -- & 1,898 & -- & -- & - & -- & -- & -- & -- \\
\hline iver $(W \mid)$ & - & 12 & -- & - & - & -- & -- & • & -- & -- & 1 \\
\hline 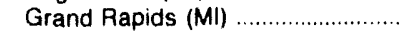 & -- & -- & -- & 3,357 & - & -- & -- & - & -- & -- & -. \\
\hline Grandfather Falls (WI) .......................... & - & -- & -- & 7,948 & -- & - & -- & -- & -- & -- & -- \\
\hline Hat Rapids (WI) & -- & -- & -- & 627 & -- & -- & -- & -- & -- & -- & -- \\
\hline High Falis (WI) & -- & -- & -- & 1,940 & -- & -- & -- & -- & -- & -- & -- \\
\hline Jersey $(W I)$ & -- & -- & - & 219 & -- & - & - & -- & -- & - & - \\
\hline 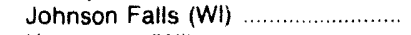 & $\cdots$ & -- & -- & 1,223 & -- & -- & - & - & -- & - & -- \\
\hline 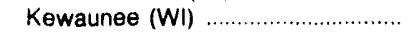 & - & -- & -- & -- & - & -- & -- & -- & -- & -- & -- \\
\hline 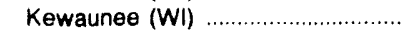 & - & -- & - & - & 376,951 & -- & -- & - & - & -- & -- \\
\hline 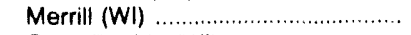 & - & $\cdots$ & -- & 485 & - & -- & -- & -- & -- & -- & -- \\
\hline 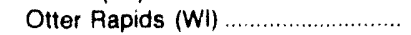 & -- & -- & -- & 117 & -- & -- & -- & -. & -- & -- & -- \\
\hline 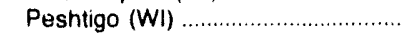 & -- & -- & -- & 370 & -- & -- & - & -. & -- & -- & -- \\
\hline Potato Rapids (WI) & -- & -- & -- & 557 & -- & -- & - & - & -- & - & -- \\
\hline Pulliam (WI) & 127,489 & -- & 1,584 & $-\infty$ & - & -- & 82 & -- & 19 & 18 & 1 \\
\hline 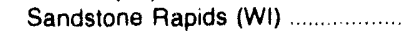 & -- & - & -- & 1,340 & -- & -- & -- & -- & -- & -- & -- \\
\hline 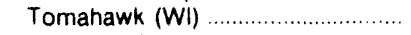 & -- & -- & -- & 783 & -- & -- & - & -- & -- & $\ldots$ & -- \\
\hline Wausau (WI) & -- & -- & - & 2,760 & - & -- & - & -- & -- & -- & -- \\
\hline 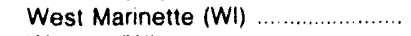 & -- & -- & 303 & -- & -- & - & -- & -- & 5 & - & 16 \\
\hline Weston (WI) & 219,163 & 263 & 746 & - & -- & -- & 138 & 1 & 8 & 89 & 15 \\
\hline Wisconsin River Power Company & - & - & -- & 18,897 & - & - & - & - & - & - & - \\
\hline
\end{tabular}

See footnotes at end of table. 
Table 62. U.S. Electric Utility Net Generation, Fuel Consumption, and Fuel Stocke by Company and Plant, September 1993 (Continued)

\begin{tabular}{|c|c|c|c|c|c|c|c|c|c|c|c|}
\hline \multirow{2}{*}{$\begin{array}{c}\text { Company (Holding Company) } \\
\text { Plant (State) }\end{array}$} & \multicolumn{6}{|c|}{$\begin{array}{l}\text { Ceneration } \\
\text { (thouaend kllowatthours) }\end{array}$} & \multicolumn{3}{|c|}{$\begin{array}{l}\text { Consumption } \\
\text { (thousand) }\end{array}$} & \multicolumn{2}{|c|}{$\begin{array}{l}\text { 8tocks } \\
\text { (thousand) }\end{array}$} \\
\hline & Coal & Petroloum & ase & Hydro & Muctear & Other' & $\begin{array}{c}\text { Coal } \\
\text { (ehort } \\
\text { tons) }\end{array}$ & $\begin{array}{l}\text { Potro- } \\
\text { leum } \\
\text { (bbls) }\end{array}$ & (Mas & $\begin{array}{l}\text { Coal } \\
\text { (Chort } \\
\text { tona) }\end{array}$ & $\begin{array}{l}\text { Potro- } \\
\text { loum } \\
\text { (boto) }\end{array}$ \\
\hline $\begin{array}{l}\text { Wheconaln Alver Power Company } \\
\text { Castie Rock (WI) } \\
\text { Petenwell (WI) }\end{array}$ & $\overline{-}$ & $\overline{-}$ & $\overline{-}$ & $\begin{array}{l}9,421 \\
9,476\end{array}$ & $\ddot{-}$ & $\overline{--}$ & $\overline{-}$ & $\overline{-}$ & 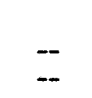 & - & $=$ \\
\hline $\begin{array}{l}\text { Whener Eloc Lt \& Wir Dept } \\
\text { Wisner (NE) }\end{array}$ & - & $\overline{-}$ & $\overline{-}$ & $\overline{-}$ & $\overline{-}$ & $\overline{-}$ & $\overline{-}$ & $\overline{-}$ & $\overline{-}$ & $\overline{-}$ & $\overline{-}$ \\
\hline $\begin{array}{l}\text { Woff Crook Nuclear Corporation. } \\
\text { Wolf Croek (KS) ... }\end{array}$ & $\overline{-}$ & $\overline{-}$ & $\overline{-}$ & $\overline{-}$ & $\begin{array}{l}820,905 \\
820,805\end{array}$ & $\overline{-}$ & $\overline{-}$ & $\overline{-}$ & $\overline{-}$ & $\overline{-}$ & $\overline{-}$ \\
\hline 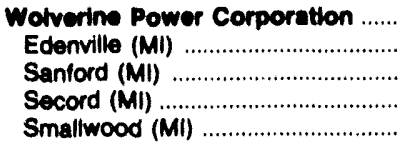 & $\bar{m}=$ & $\begin{array}{l}\bar{z} \\
\bar{z}\end{array}$ & $\begin{array}{l}\bar{z} \\
\bar{z}\end{array}$ & $\begin{array}{r}1,783 \\
804 \\
486 \\
233 \\
140\end{array}$ & $\begin{array}{l}\overline{-} \\
\bar{z}\end{array}$ & $\begin{array}{l}\bar{z} \\
\bar{z}\end{array}$ & $\begin{array}{l}\overline{-} \\
\overline{-} \\
\overline{-}\end{array}$ & $\begin{array}{l}- \\
\overline{-} \\
\overline{-}\end{array}$ & $\begin{array}{l}- \\
\overline{-} \\
\overline{-}\end{array}$ & $\begin{array}{l}- \\
\overline{-} \\
\overline{-}\end{array}$ & $\bar{z}$ \\
\hline 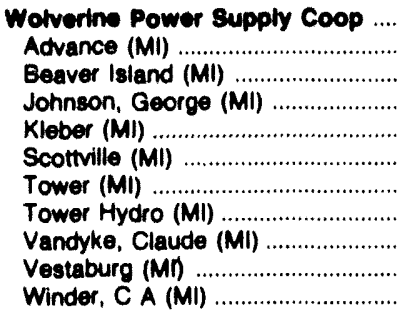 & $\begin{array}{l}13,516 \\
13,516 \\
= \\
= \\
= \\
= \\
= \\
=\end{array}$ & $\begin{array}{r}245 \\
232 \\
-3 \\
3 \\
-\quad 13 \\
-13 \\
21 \\
-\quad-3 \\
-3 \\
5\end{array}$ & $\begin{array}{l}- \\
= \\
- \\
= \\
= \\
= \\
=\end{array}$ & $\begin{array}{l}{ }^{642} \\
= \\
- \\
= \\
= \\
=\end{array}$ & $\begin{array}{l}\bar{z} \\
= \\
= \\
= \\
=\end{array}$ & $\begin{array}{l}\bar{z} \\
\bar{z} \\
\bar{z} \\
\bar{z} \\
\bar{z}\end{array}$ & $\begin{array}{l} \\
\quad 7 \\
= \\
= \\
= \\
= \\
= \\
=\end{array}$ & $\begin{array}{l}\dot{ }^{1} \\
\ddot{-} \\
\ddot{\vdots} \\
\dot{-}\end{array}$ & 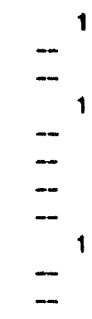 & $\begin{array}{l}42 \\
42 \\
- \\
- \\
- \\
= \\
= \\
= \\
- \\
-\end{array}$ & $\begin{array}{l}{ }^{7} \\
{ }^{2} \\
\div \\
\div \\
{ }^{2}\end{array}$ \\
\hline $\begin{array}{l}\text { Woodofield Elec Loht Plant ......... } \\
\text { Anadarko }(\mathrm{OH})\end{array}$ & $\overline{-}$ & $\overline{-}$ & $\overline{-}$ & $\overline{-}$ & $\overline{-}$ & $\overline{-}$ & $\overline{-}$ & $\overline{-}$ & $\overline{-}$ & $\overline{-}$ & $\overline{-}$ \\
\hline $\begin{array}{l}\text { Wrangell Loght Plant } \\
\text { Wrangell (AK) }\end{array}$ & $\overline{-}$ & $\begin{array}{l}618 \\
618\end{array}$ & $\overline{-}$ & $\overline{-}$ & $\overline{-}$ & $\overline{-}$ & $\overline{-}$ & $\begin{array}{l}1 \\
1\end{array}$ & $\overline{-}$ & $\overline{-}$ & : \\
\hline $\begin{array}{l}\text { Wyandotte Dept of Mun Service. } \\
\text { Wyandotte (MI) . }\end{array}$ & $\begin{array}{l}15,711 \\
15,711\end{array}$ & $\overline{-}$ & $\overline{-}$ & $\overline{-}$ & - & $\overline{-}$ & 8 & $\overline{-}$ & $\overline{-}$ & $\begin{array}{l}28 \\
28\end{array}$ & $\overline{-}$ \\
\hline $\begin{array}{l}\text { Yadkin, Inc } \\
\text { Falls (NC) } \\
\text { High Rock (NC) } \\
\text { Narrows (NC) } \\
\text { Tuckertown (NC) }\end{array}$ & $\begin{array}{l}\bar{z} \\
\bar{z} \\
\bar{z}\end{array}$ & $\begin{array}{l}\bar{z} \\
\bar{z}\end{array}$ & $\begin{array}{l}\bar{z} \\
\bar{z}\end{array}$ & $\begin{array}{r}34,710 \\
5,193 \\
5,650 \\
18,396 \\
5,471\end{array}$ & $\begin{array}{l}\bar{z} \\
\bar{z}\end{array}$ & $\begin{array}{l}- \\
\overline{-} \\
\overline{-}\end{array}$ & $\begin{array}{l}\bar{z} \\
\bar{z} \\
\overline{-}\end{array}$ & $\begin{array}{l}\overline{-} \\
\overline{-}\end{array}$ & $\begin{array}{l}- \\
\overline{-} \\
\overline{-}\end{array}$ & $\begin{array}{l}\overline{-} \\
\overline{-} \\
\overline{-}\end{array}$ & $\begin{array}{l}\overline{-} \\
\bar{z}\end{array}$ \\
\hline $\begin{array}{l}\text { Yakutat Power Ine } \\
\text { Yakutat (AK) }\end{array}$ & $\overline{-}$ & $\begin{array}{l}831 \\
831\end{array}$ & $\overline{-}$ & $\overline{-}$ & $\overline{-}$ & $\overline{-}$ & $\overline{-}$ & $\begin{array}{l}1 \\
1\end{array}$ & $\overline{-}$ & - & : \\
\hline $\begin{array}{l}\text { Yazoo Clty Publlc Sorvice Comm } \\
\text { Yazoo (MS) }\end{array}$ & $\overline{-}$ & $\overline{-}$ & $\overline{-}$ & $\overline{-}$ & - & $\overline{-}$ & - & $\overline{-}$ & $\overline{-}$ & - & $\underline{-}$ \\
\hline 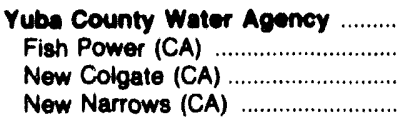 & $\bar{z}$ & $\begin{array}{l}\overline{-} \\
\overline{-}\end{array}$ & $\bar{z}$ & $\begin{array}{r}81,981 \\
101 \\
72,683 \\
9,197\end{array}$ & $\begin{array}{l}- \\
m\end{array}$ & $\overline{-}$ & $\bar{z}$ & $\begin{array}{l}\overline{-} \\
\overline{-} \\
-\end{array}$ & $\begin{array}{l}- \\
\bar{z}\end{array}$ & $\begin{array}{l}- \\
\overline{-}\end{array}$ & $\overline{-}$ \\
\hline $\begin{array}{l}\text { Yuma Clty Of } \\
\text { Yuma (CO) }\end{array}$ & $\overline{-}$ & $\overline{-}$ & $\overline{-}$ & $\overline{-}$ & $=$ & $\overline{-}$ & $\overline{-}$ & $\overline{-}$ & $\overline{-}$ & $\overline{-}$ & $\overline{-}$ \\
\hline $\begin{array}{l}\text { Zeoland Board of Publle Worke .. } \\
\text { Zooland (MI) }\end{array}$ & $\overline{-}$ & $\begin{array}{l}11 \\
11\end{array}$ & $\begin{array}{l}210 \\
210\end{array}$ & $\overline{-}$ & $\overline{-}$ & $\overline{-}$ & $\overline{-}$ & : & $\begin{array}{l}3 \\
3\end{array}$ & $\overline{-}$ & : \\
\hline
\end{tabular}

U. 8. Total ........................................133,855,840

$9,758,691 \quad 26,020,206 \quad 17,000,200$

$40,968,643 \quad 803,531 \quad 66,504 \quad 15,760 \quad 260,812 \quad 112,773 \quad 60,673$

- Other energy sources include geothermal, solar, wood, wind, and waste.

Less than 0.5

Notes: - Totals may not equal sum of components because of independent rounding. -Net generation for jointly owned units is reported by the operator. Negative generation denotes that electric power consumed for plant use exceeds gross generation. - Station losses include energy used for pumped storage. -Generation is included for plants in test status. -Nuclear generation is included for those plants with an operating license issued authorizing fuel loading/low power testing prior to receipt of full power amendment. - Central storage is a common area for fuel stocks not assigned to specific plants. -Mcf = thousand cubic feet and bbls = barrels. Data for 1992 are final. $\bullet$ Holding Companies are: AEP is American Electric Power, APs is Allegheny Power System, ACE is Atlantic City Electric, CSW is Central \& South West Corporation, CES is Commonwealth Energy System, DijV is Delmarva, EU is Eastern Utilities Associates Company, QPS is General Public Utilities, MsU is Middle South Utilities, NEEs is New England Electric Systern, NU is Northeast Utilities, SC is Southern Company, TU is Texas Utilities.

Source: Energy Information Administration, Form EIA-759, "Monthly Power Plant Report." 


\section{Monthly Plant Aggregates: \\ U.S. Electric Utility \\ Recelpts, Cost, and \\ Quality of Fossil Fuels}


Table 63. Recelpts, Average Cost, and Quality of Foasll Fuels Dellvered to U.S. Electric Utilities by Company and Plant, September 1993

\begin{tabular}{|c|c|c|c|c|c|c|c|c|c|c|c|c|c|c|}
\hline \multirow{3}{*}{$\begin{array}{l}\text { Utiltiy (Holding Company) } \\
\text { Plant (State) }\end{array}$} & \multicolumn{4}{|c|}{ Coal } & \multicolumn{4}{|c|}{ Potroloum' } & \multicolumn{3}{|c|}{ Gas } & \multicolumn{3}{|c|}{$\%$ of Total Btu } \\
\hline & \multirow{2}{*}{\begin{tabular}{|c|} 
Recolpte \\
$(1,000$ \\
tons)
\end{tabular}} & \multicolumn{2}{|c|}{$\begin{array}{l}\text { Averape } \\
\text { Coot' }\end{array}$} & \multirow{2}{*}{$\mid \begin{array}{c}\text { Avg. } \\
\text { sut } \\
\text { fur } \\
\%\end{array}$} & \multirow{2}{*}{\begin{tabular}{|c|} 
Recolpte \\
$\begin{array}{c}1,000 \\
\text { bble })\end{array}$
\end{tabular}} & \multicolumn{2}{|c|}{$\begin{array}{l}\text { Average } \\
\text { Cost }\end{array}$} & \multirow{2}{*}{$\begin{array}{l}\text { Avg. } \\
\text { sur } \\
\text { fur } \\
*\end{array}$} & \multirow{2}{*}{$\begin{array}{c}\text { Aecelpta } \\
\begin{array}{c}(1,000 \\
M 01)\end{array}\end{array}$} & \multicolumn{2}{|c|}{$\begin{array}{l}\text { Average } \\
\text { Cost? }^{3}\end{array}$} & \multirow[b]{2}{*}{ Coal } & \multirow[b]{2}{*}{$\begin{array}{l}\text { Pe- } \\
\text { tro- } \\
\text { leum }\end{array}$} & \multirow[b]{2}{*}{ Cas } \\
\hline & & $\begin{array}{l}\text { (Conto } \\
\text { per } \\
10^{\circ} \\
\text { Btu) }\end{array}$ & $\begin{array}{l}\text { (S por } \\
\text { chort } \\
\text { tori) }\end{array}$ & & & $\begin{array}{l}\text { (Cente } \\
\text { per } \\
10^{\circ} \\
\text { Btu) }\end{array}$ & bor & & & $\begin{array}{l}\text { (Conte } \\
\text { per } \\
10^{\circ} \\
\text { Btu) }\end{array}$ & $\begin{array}{l}8 \text { per } \\
\text { Mor }\end{array}$ & & & \\
\hline $\begin{array}{l}\text { Alabama Eleotric Coop Inc } \\
\text { Lowman (AL) }\end{array}$ & 69 & $\begin{array}{l}144.0 \\
144.0\end{array}$ & $\begin{array}{l}34.90 \\
34.90\end{array}$ & $\begin{array}{l}1.52 \\
1.52\end{array}$ & $\bullet$ & $\begin{array}{l}491.6 \\
491.6\end{array}$ & $\begin{array}{l}26.94 \\
26.94\end{array}$ & $\begin{array}{r}0.10 \\
.10\end{array}$ & - & - & - & $\begin{array}{l}100 \\
100\end{array}$ & 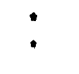 & - \\
\hline 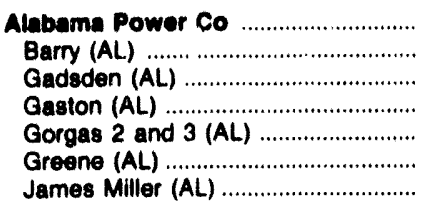 & $\begin{array}{r}1,384 \\
89 \\
20 \\
334 \\
440 \\
103 \\
399\end{array}$ & $\begin{array}{l}102.0 \\
217.5 \\
182.2 \\
175.3 \\
166.1 \\
140.2 \\
240.3\end{array}$ & $\begin{array}{l}46.83 \\
53.50 \\
45.89 \\
42.25 \\
39.47 \\
34.54 \\
60.53\end{array}$ & $\begin{array}{r}1.13 \\
.91 \\
1.81 \\
1.63 \\
1.21 \\
1.47 \\
.56\end{array}$ & 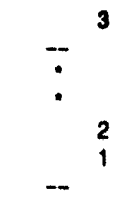 & $\begin{array}{l}410.0 \\
- \\
408.3 \\
392.9 \\
415.3 \\
404.6 \\
-\end{array}$ & $\begin{array}{c}23.99 \\
23.94 \\
23.00 \\
24.30 \\
23.64 \\
-\end{array}$ & $\begin{array}{l}.00 \\
.00 \\
.00 \\
.00 \\
.00 \\
\cdots\end{array}$ & $\begin{array}{r}213 \\
24 \\
-\quad 2 \\
-\quad \\
-\quad \\
-\quad 187\end{array}$ & $\begin{array}{l}283.8 \\
270.0 \\
274.7 \\
-- \\
-- \\
-- \\
285.9\end{array}$ & $\begin{array}{l}2.87 \\
2.92 \\
2.75 \\
-- \\
-- \\
-- \\
2.86\end{array}$ & $\begin{array}{r}99 \\
99 \\
99 \\
100 \\
100 \\
100 \\
98\end{array}$ & $\begin{array}{l}\dot{*} \\
\dot{-} \\
\dot{-} \\
--\end{array}$ & $\begin{array}{r}1 \\
1 \\
-- \\
-- \\
- \\
-\end{array}$ \\
\hline 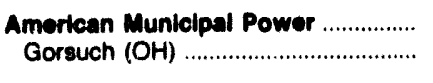 & $\begin{array}{l}63 \\
63\end{array}$ & $\begin{array}{l}91.5 \\
91.5\end{array}$ & $\begin{array}{l}21.01 \\
21.01\end{array}$ & $\begin{array}{l}4.67 \\
4.67\end{array}$ & - & $\overline{-}$ & - & - & 9 & & $\begin{array}{l}3.85 \\
3.85\end{array}$ & $\begin{array}{l}99 \\
99\end{array}$ & - & $\begin{array}{l}1 \\
1\end{array}$ \\
\hline Ames City of & $\begin{array}{l}18 \\
18\end{array}$ & $\begin{array}{l}135.4 \\
135.4\end{array}$ & $\begin{array}{l}23.81 \\
23.81\end{array}$ & $\begin{array}{l}.28 \\
.26\end{array}$ & $\begin{array}{l}1 \\
1\end{array}$ & $\begin{array}{l}392.0 \\
392.0\end{array}$ & $\begin{array}{l}23.31 \\
23.31\end{array}$ & $\begin{array}{l}.30 \\
.30\end{array}$ & - & - & - & $\begin{array}{l}98 \\
98\end{array}$ & $\begin{array}{l}2 \\
2\end{array}$ & $\overline{-}$ \\
\hline 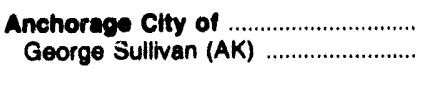 & - & - & - & - & - & - & - & - & $\begin{array}{l}268 \\
268\end{array}$ & & $\begin{array}{l}2.53 \\
2.53\end{array}$ & - & - & $\begin{array}{l}100 \\
100\end{array}$ \\
\hline 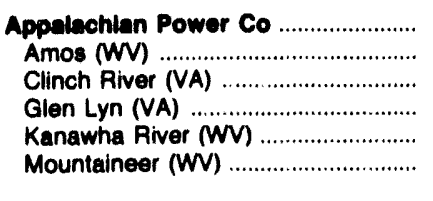 & $\begin{array}{r}561 \\
196 \\
120 \\
38 \\
36 \\
171\end{array}$ & $\begin{array}{l}167.5 \\
193.4 \\
131.4 \\
141.7 \\
176.9 \\
167.9\end{array}$ & $\begin{array}{l}41.57 \\
47.48 \\
32.85 \\
36.73 \\
44.38 \\
41.43\end{array}$ & $\begin{array}{l}.72 \\
.73 \\
.69 \\
.80 \\
.76 \\
.68\end{array}$ & $\begin{array}{r}22 \\
10 \\
-\quad 2 \\
-\quad 2 \\
10\end{array}$ & $\begin{array}{l}452.5 \\
407.2 \\
686.0 \\
443.1 \\
425.3 \\
495.8\end{array}$ & $\begin{array}{l}28.28 \\
23.86 \\
40.46 \\
25.83 \\
24.90 \\
28.50\end{array}$ & $\begin{array}{l}.00 \\
.00 \\
.00 \\
.00 \\
.00 \\
.00\end{array}$ & $\begin{array}{l}- \\
- \\
- \\
-\end{array}$ & $\begin{array}{l}- \\
-- \\
-- \\
-- \\
--\end{array}$ & $\begin{array}{l}- \\
- \\
- \\
-- \\
-\end{array}$ & $\begin{array}{r}99 \\
99 \\
100 \\
99 \\
100 \\
99\end{array}$ & $\begin{array}{r}1 \\
1 \\
+1 \\
1 \\
1\end{array}$ & $\begin{array}{l}- \\
- \\
-- \\
- \\
-\end{array}$ \\
\hline 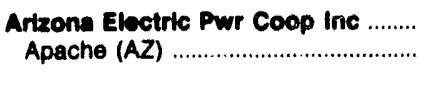 & $\begin{array}{l}105 \\
105\end{array}$ & $\begin{array}{l}128.6 \\
126.6\end{array}$ & $\begin{array}{l}25.54 \\
25.54\end{array}$ & $\begin{array}{l}.43 \\
.43\end{array}$ & - & - & - & - & $\begin{array}{l}20 \\
20\end{array}$ & $\begin{array}{l}215.6 \\
215.6\end{array}$ & $\begin{array}{l}2.22 \\
2.22\end{array}$ & $\begin{array}{l}99 \\
99\end{array}$ & - & $\begin{array}{l}1 \\
1\end{array}$ \\
\hline 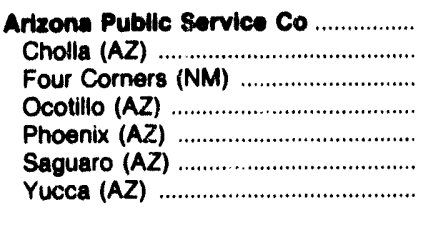 & $\begin{array}{r}1,137 \\
345 \\
792 \\
-- \\
- \\
-\end{array}$ & $\begin{array}{l}129.1 \\
172.0 \\
107.5 \\
- \\
- \\
-\end{array}$ & $\begin{array}{l}23.60 \\
34.65 \\
18.79 \\
- \\
- \\
- \\
-\end{array}$ & $\begin{array}{l}.63 \\
.42 \\
.72 \\
-- \\
-- \\
-- \\
--\end{array}$ & $\begin{array}{l}- \\
- \\
- \\
-\end{array}$ & $\begin{array}{l}- \\
\overline{-} \\
\overline{-} \\
-\end{array}$ & $\begin{array}{l}- \\
- \\
- \\
-\end{array}$ & $\begin{array}{l}- \\
-- \\
- \\
- \\
-\end{array}$ & $\begin{array}{r}1,355 \\
3 \\
35 \\
420 \\
613 \\
69 \\
215\end{array}$ & $\begin{array}{l}325.7 \\
363.6 \\
376.2 \\
337.0 \\
338.0 \\
335.0 \\
257.0\end{array}$ & $\begin{array}{l}3.33 \\
3.72 \\
3.80 \\
3.51 \\
3.41 \\
3.46 \\
2.63\end{array}$ & $\begin{array}{r}94 \\
100 \\
100 \\
-- \\
-- \\
--\end{array}$ & $\begin{array}{l}- \\
- \\
- \\
- \\
-- \\
-\end{array}$ & $\begin{array}{l}: 6 \\
100 \\
100 \\
100 \\
100\end{array}$ \\
\hline 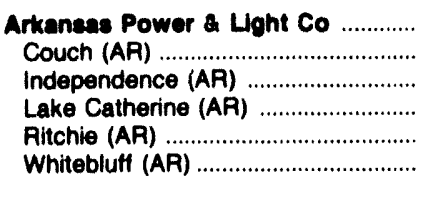 & $\begin{array}{l}840 \\
- \\
- \\
- \\
- \\
560\end{array}$ & $\begin{array}{l}\frac{178.5}{177.4} \\
\overline{179.1}\end{array}$ & $\begin{array}{l}31.10 \\
31.30 \\
-- \\
-- \\
31.00\end{array}$ & $\begin{array}{l}.34 \\
-- \\
.21 \\
-- \\
-.40\end{array}$ & $\begin{array}{ll} & 4 \\
- & \\
- & \\
- & \end{array}$ & $\begin{array}{l}435.8 \\
441.6 \\
- \\
- \\
429.2\end{array}$ & $\begin{array}{c}25.03 \\
\overline{25.36} \\
- \\
\overline{24.65}\end{array}$ & $\begin{array}{c}.30 \\
-30 \\
- \\
- \\
-30\end{array}$ & $\begin{array}{r}3,223 \\
402 \\
-\quad \\
1,661 \\
1,160 \\
-\end{array}$ & $\begin{array}{l}242.2 \\
174.5 \\
-- \\
254.7 \\
249.8 \\
--\end{array}$ & $\begin{array}{l}2.47 \\
1.92 \\
-- \\
2.57 \\
2.53 \\
--\end{array}$ & $\begin{array}{c}82 \\
-- \\
100 \\
-- \\
--\end{array}$ & $\begin{array}{l}\cdot \\
-- \\
-- \\
--\end{array}$ & $\begin{array}{c}18 \\
100 \\
-- \\
100 \\
100 \\
--\end{array}$ \\
\hline $\begin{array}{l}\text { Associated Electric Coop lnc } \\
\text { Madrid (MO) }\end{array}$ & $\begin{array}{l}286 \\
266\end{array}$ & $\begin{array}{l}112.6 \\
112.6\end{array}$ & $\begin{array}{l}20.03 \\
20.03\end{array}$ & $\begin{array}{l}.43 \\
.43\end{array}$ & - & - & - & - & - & - & - & $\begin{array}{l}100 \\
100\end{array}$ & $\overline{-}$ & - \\
\hline 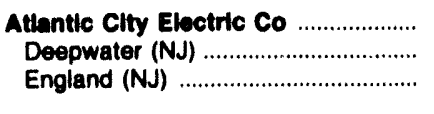 & $\begin{array}{r}46 \\
-\quad 46\end{array}$ & $\frac{164.4}{164.4}$ & $\frac{42.85}{42.85}$ & $\frac{2.42}{2.42}$ & $-\begin{array}{r}50 \\
50\end{array}$ & $\frac{281.7}{261.7}$ & $\overline{16.54}$ & $\begin{array}{c}.75 \\
-75\end{array}$ & $\begin{array}{r}85 \\
85 \\
-\quad\end{array}$ & $\begin{array}{l}236.5 \\
236.5 \\
-\end{array}$ & $\begin{array}{l}2.46 \\
2.46 \\
--\end{array}$ & $\begin{array}{c}75 \\
--\end{array}$ & $\begin{array}{c}10 \\
-- \\
21\end{array}$ & $\begin{array}{r}5 \\
100 \\
--\end{array}$ \\
\hline 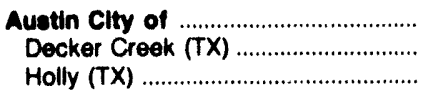 & $\overline{-}$ & $\overline{-}$ & $\overline{-}$ & $\overline{-}$ & - & $\overline{-}$ & $\overline{-}$ & $\overline{-}$ & $\begin{array}{l}3,789 \\
2,443 \\
1,347\end{array}$ & $\begin{array}{l}265.9 \\
263.5 \\
270.3\end{array}$ & $\begin{array}{l}2.65 \\
2.63 \\
2.69\end{array}$ & - & - & $\begin{array}{l}100 \\
100 \\
100\end{array}$ \\
\hline 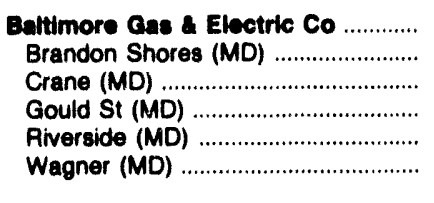 & $\begin{array}{r}368 \\
227 \\
-\quad 81 \\
- \\
-\quad 58\end{array}$ & $\begin{array}{l}155.7 \\
154.9 \\
153.7 \\
- \\
- \\
162.1\end{array}$ & $\begin{array}{l}39.97 \\
39.59 \\
40.85 \\
-- \\
-- \\
40.23\end{array}$ & $\begin{array}{r}1.02 \\
.68 \\
2.09 \\
- \\
- \\
.89\end{array}$ & $\begin{array}{r}124 \\
-\quad 3 \\
-\quad 23 \\
-\quad 98\end{array}$ & $\begin{array}{l}237.3 \\
387.5 \\
-- \\
\overline{233.8} \\
\overline{234.0}\end{array}$ & $\begin{array}{c}14.96 \\
22.42 \\
\overline{14.77} \\
\overline{14.77}\end{array}$ & $\begin{array}{c}.97 \\
.18 \\
- \\
.99 \\
-.99\end{array}$ & $\begin{array}{l}171 \\
-- \\
-\end{array}$ & $\begin{array}{l}306.4 \\
-- \\
-- \\
-- \\
288.6 \\
321.4\end{array}$ & $\begin{array}{l}3.18 \\
- \\
- \\
- \\
3.00 \\
3.35\end{array}$ & $\begin{array}{c}91 \\
100 \\
100 \\
- \\
-- \\
67\end{array}$ & $\begin{array}{c}8 \\
\overline{100} \\
-- \\
29\end{array}$ & $\begin{array}{r}2 \\
-- \\
-- \\
- \\
100 \\
4\end{array}$ \\
\hline $\begin{array}{l}\text { Bein Electric Power Coop } \ldots . . . . . . . . . . . \\
\text { Antelope Valley (ND) }\end{array}$ & $\begin{array}{r}1,172 \\
463\end{array}$ & $\begin{array}{l}67.1 \\
70.1\end{array}$ & $\begin{array}{l}9.69 \\
9.30\end{array}$ & $\begin{array}{l}.50 \\
.54\end{array}$ & - & $\begin{array}{l}527.0 \\
-\end{array}$ & $\begin{array}{c}30.52 \\
--\end{array}$ & -34 & - & - & - & $\begin{array}{l}100 \\
100\end{array}$ & $\stackrel{-}{--}$ & - \\
\hline
\end{tabular}

See notes and footnotes at end of table. 
Table 63. Recelpte, Average Cost, and Quality of Fossll Fuels Dellvered to U.S. Electric Utilities by Company and Plant, September 1993 (Continued)

\begin{tabular}{|c|c|c|c|c|c|c|c|c|c|c|c|c|c|c|}
\hline \multirow{3}{*}{$\begin{array}{l}\text { Uthlty (Holding Company) } \\
\text { Plant (State) }\end{array}$} & \multicolumn{4}{|c|}{ Coal } & \multicolumn{4}{|c|}{ Petroleum' } & \multicolumn{3}{|c|}{ Gas } & \multicolumn{3}{|c|}{$\%$ of Total Btu } \\
\hline & \multirow{2}{*}{\begin{tabular}{|c|} 
Recolpts \\
$(1,000$ \\
tons)
\end{tabular}} & \multicolumn{2}{|c|}{$\begin{array}{c}\text { Average } \\
\text { Coot }^{3}\end{array}$} & \multirow{2}{*}{$\begin{array}{l}\text { Avg. } \\
\text { Sul- } \\
\text { fur } \\
\%\end{array}$} & \multirow{2}{*}{\begin{tabular}{|c|} 
Rocelpts \\
$\begin{array}{c}(1,000 \\
\text { bols })\end{array}$
\end{tabular}} & \multicolumn{2}{|c|}{$\begin{array}{l}\text { Average } \\
\text { Cost? }^{3}\end{array}$} & \multirow{2}{*}{$\begin{array}{l}\text { Avg. } \\
\text { Sul- } \\
\text { fur } \\
\%\end{array}$} & \multirow{2}{*}{$\begin{array}{c}\text { Recelpts } \\
(1,000 \\
\text { Mcf })\end{array}$} & \multicolumn{2}{|c|}{$\begin{array}{c}\text { Average } \\
\text { Cost }^{3}\end{array}$} & \multirow[b]{2}{*}{ Coal } & \multirow[b]{2}{*}{$\begin{array}{c}\text { Pe- } \\
\text { tro- } \\
\text { leum }\end{array}$} & \multirow[b]{2}{*}{ Gas } \\
\hline & & $\begin{array}{l}\text { (Conte } \\
\text { per } \\
10^{\circ} \\
\text { Btu) }\end{array}$ & $\begin{array}{l}\text { (s por } \\
\text { ehort } \\
\text { ton) }\end{array}$ & & & $\begin{array}{l}\text { (Conts } \\
\text { per } \\
10^{\circ} \\
\text { Btu) }\end{array}$ & por & & & $\begin{array}{l}\text { (Cents } \\
\text { per } \\
10^{\circ} \\
\text { Btu) }\end{array}$ & per & & & \\
\hline 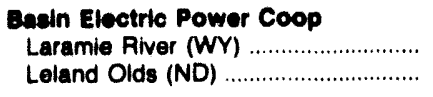 & $\begin{array}{l}447 \\
262\end{array}$ & $\begin{array}{l}60.2 \\
76.5\end{array}$ & $\begin{array}{r}9.88 \\
10.03\end{array}$ & $\begin{array}{r}0.40 \\
.61\end{array}$ & -- & $\begin{array}{l}527.0 \\
--\end{array}$ & $\begin{array}{c}30.52 \\
--\end{array}$ & $\begin{array}{l}0.34 \\
--\end{array}$ & -- & -- & -- & $\begin{array}{l}100 \\
100\end{array}$ & $\dot{-}$ & -- \\
\hline 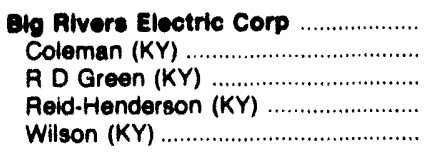 & $\begin{array}{r}426 \\
115 \\
133 \\
72 \\
106\end{array}$ & $\begin{array}{l}123.1 \\
106.1 \\
120.4 \\
123.3 \\
143.3\end{array}$ & $\begin{array}{l}28.01 \\
23.93 \\
25.52 \\
30.31 \\
33.98\end{array}$ & $\begin{array}{l}3.23 \\
2.53 \\
3.96 \\
2.71 \\
3.43\end{array}$ & $\begin{array}{l}-- \\
-- \\
-- \\
--\end{array}$ & $\begin{array}{l}- \\
-- \\
-- \\
--\end{array}$ & $\begin{array}{l}-- \\
-- \\
-- \\
--\end{array}$ & $\begin{array}{l}-- \\
-- \\
-- \\
--\end{array}$ & $\begin{array}{l}-- \\
-- \\
--\end{array}$ & $\begin{array}{l}358.2 \\
358.2 \\
-- \\
-- \\
--\end{array}$ & $\begin{array}{l}3.58 \\
3.58 \\
- \\
\cdots \\
-\end{array}$ & $\begin{array}{l}100 \\
100 \\
100 \\
100 \\
100\end{array}$ & $\begin{array}{l}-- \\
-- \\
-- \\
--\end{array}$ & $\begin{array}{l}\cdot \\
-- \\
-- \\
--\end{array}$ \\
\hline 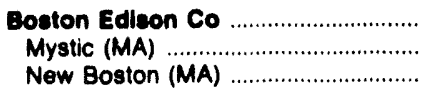 & - & $\overline{-}$ & - & -- & $\begin{array}{r}616 \\
616 \\
--\end{array}$ & $\begin{array}{l}224.5 \\
224.5 \\
--\end{array}$ & $\begin{array}{c}14.37 \\
14.37 \\
--\end{array}$ & $\begin{array}{r}.96 \\
.96 \\
--\end{array}$ & $\begin{array}{r}2,030 \\
303 \\
1,727\end{array}$ & $\begin{array}{l}278.9 \\
263.6 \\
281.7\end{array}$ & $\begin{array}{l}2.91 \\
2.87 \\
2.91\end{array}$ & +- & $\begin{array}{c}65 \\
92 \\
--\end{array}$ & $\begin{array}{r}35 \\
8 \\
100\end{array}$ \\
\hline 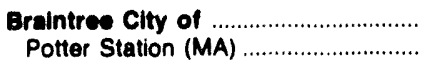 & - & - & $\overline{-}$ & - & - & - & - & - & $\begin{array}{l}60 \\
60\end{array}$ & $\begin{array}{l}275.0 \\
275.0\end{array}$ & $\begin{array}{l}2.84 \\
2.84\end{array}$ & $\cdots$ & -- & $\begin{array}{l}100 \\
100\end{array}$ \\
\hline 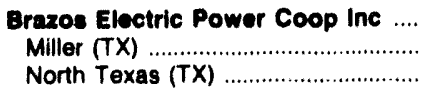 & - & - & -- & - & - & - & - & -- & $\begin{array}{r}1,135 \\
1,010 \\
125\end{array}$ & $\begin{array}{l}234.4 \\
232.7 \\
247.8\end{array}$ & $\begin{array}{l}2.38 \\
2.36 \\
2.60\end{array}$ & -- & -- & $\begin{array}{l}100 \\
100 \\
100\end{array}$ \\
\hline $\begin{array}{l}\text { Bryan Clty of } \\
\text { Bryan (TX) } \\
\text { Dansby (TX) }\end{array}$ & - & - & - & - & - & -- & -- & $\overline{--}$ & $\begin{array}{l}654 \\
205 \\
449\end{array}$ & $\begin{array}{l}220.1 \\
215.1 \\
222.5\end{array}$ & $\begin{array}{l}2.33 \\
2.30 \\
2.35\end{array}$ & $\begin{array}{l}-- \\
--\end{array}$ & $\begin{array}{l}-- \\
--\end{array}$ & $\begin{array}{l}100 \\
100 \\
100\end{array}$ \\
\hline 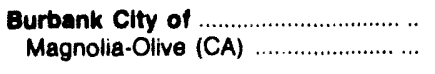 & - & - & -- & - & - & -- & - & - & $\begin{array}{l}233 \\
233\end{array}$ & $\begin{array}{l}293.0 \\
293.0\end{array}$ & $\begin{array}{l}3.00 \\
3.00\end{array}$ & -- & -- & $\begin{array}{l}100 \\
100\end{array}$ \\
\hline 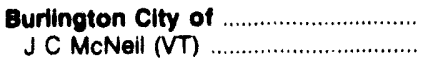 & - & - & - & - & 1 & $\begin{array}{l}465.2 \\
465.2\end{array}$ & $\begin{array}{l}25.31 \\
25.31\end{array}$ & $\begin{array}{l}.27 \\
.27\end{array}$ & - & -- & -- & -- & $\begin{array}{l}100 \\
100\end{array}$ & $\overline{-}$ \\
\hline 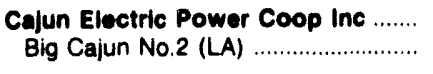 & $\begin{array}{l}510 \\
510\end{array}$ & $\begin{array}{l}154.6 \\
154.6\end{array}$ & $\begin{array}{l}25.78 \\
25.78\end{array}$ & $\begin{array}{l}.40 \\
.40\end{array}$ & $\begin{array}{l}6 \\
6\end{array}$ & $\begin{array}{l}392.0 \\
392.0\end{array}$ & $\begin{array}{l}23.05 \\
23.05\end{array}$ & $\begin{array}{l}.00 \\
.00\end{array}$ & - & -- & - & $\begin{array}{l}100 \\
100\end{array}$ & $\dot{*}$ & -- \\
\hline $\begin{array}{l}\text { Cambridge Electric Light Co } \\
\text { Kendall Square (MA) }\end{array}$ & - & - & - & - & $\begin{array}{l}28 \\
28\end{array}$ & $\begin{array}{l}256.7 \\
256.7\end{array}$ & $\begin{array}{l}\mathbf{1 6 . 2 0} \\
16.20\end{array}$ & $\begin{array}{l}.48 \\
.48\end{array}$ & $\begin{array}{l}2 \\
2\end{array}$ & $\begin{array}{l}257.0 \\
257.0\end{array}$ & $\begin{array}{l}2.57 \\
2.57\end{array}$ & -- & $\begin{array}{l}99 \\
99\end{array}$ & $\begin{array}{l}1 \\
1\end{array}$ \\
\hline $\begin{array}{c}\text { Canal Electric Co } \\
\text { Canal (MA) }\end{array}$ & - & - & $-\overline{-}$ & - & $\begin{array}{l}565 \\
565\end{array}$ & $\begin{array}{l}203.4 \\
203.4\end{array}$ & $\begin{array}{l}12.87 \\
12.87\end{array}$ & $\begin{array}{l}1.76 \\
1.76\end{array}$ & - & - & - & -- & $\begin{array}{l}100 \\
100\end{array}$ & -- \\
\hline $\begin{array}{l}\text { Cardinal Operating Co } \\
\text { Cardinal }(\mathrm{OH})\end{array}$ & $\begin{array}{l}215 \\
215\end{array}$ & $\begin{array}{l}151.7 \\
151.7\end{array}$ & $\begin{array}{l}36.47 \\
36.47\end{array}$ & $\begin{array}{l}2.51 \\
2.51\end{array}$ & - & -- & -- & $-\overline{-}$ & -- & - & -- & $\begin{array}{l}100 \\
100\end{array}$ & -- & - \\
\hline $\begin{array}{l}\text { Carolina Power LIght Co } \\
\text { Asheville (NC) } \\
\text { Cape Fear (NC) } \\
\text { Lee (NC) } \\
\text { Mayo (NC) } \\
\text { Robinson (SC) } \\
\text { Roxboro (NC) } \\
\text { Sutton (NC) } \\
\text { Weatherspoon (NC) }\end{array}$ & $\begin{array}{r}891 \\
82 \\
51 \\
59 \\
158 \\
22 \\
476 \\
23 \\
21\end{array}$ & $\begin{array}{l}177.0 \\
132.8 \\
208.9 \\
203.4 \\
192.7 \\
168.6 \\
174.3 \\
169.2 \\
163.5\end{array}$ & $\begin{array}{l}44.33 \\
34.42 \\
53.47 \\
52.36 \\
46.96 \\
42.41 \\
43.48 \\
42.36 \\
41.69\end{array}$ & $\begin{array}{r}1.01 \\
1.24 \\
.97 \\
.99 \\
.65 \\
.98 \\
1.07 \\
1.16 \\
1.21\end{array}$ & $\begin{array}{l}-- \\
-- \\
-- \\
--\end{array}$ & $\begin{array}{l}410.4 \\
417.3 \\
390.3 \\
-- \\
416.0 \\
-- \\
410.9 \\
-- \\
--\end{array}$ & $\begin{array}{c}23.79 \\
24.19 \\
22.62 \\
-- \\
24.11 \\
-- \\
23.82 \\
-- \\
--\end{array}$ & $\begin{array}{l}.20 \\
.20 \\
.20 \\
-- \\
.20 \\
-- \\
.20 \\
-- \\
--\end{array}$ & $\begin{array}{l}-- \\
-- \\
-- \\
-- \\
-- \\
-- \\
-- \\
--\end{array}$ & $\begin{array}{l}-- \\
-- \\
-- \\
-- \\
-- \\
-- \\
-- \\
--\end{array}$ & $\begin{array}{l}-- \\
-- \\
-- \\
-- \\
-- \\
-- \\
-- \\
-- \\
--\end{array}$ & $\begin{array}{l}100 \\
100 \\
100 \\
100 \\
100 \\
100 \\
100 \\
100 \\
100\end{array}$ & $\begin{array}{l}: \\
: \\
:- \\
-- \\
-- \\
--\end{array}$ & $\begin{array}{l}-- \\
-- \\
-- \\
-- \\
-- \\
-- \\
-- \\
--\end{array}$ \\
\hline $\begin{array}{l}\text { Cedar Falls Clty of } \\
\text { Streeter (IA) }\end{array}$ & - & - & - & - & - & - & - & - & . & $\begin{array}{l}458.0 \\
458.0\end{array}$ & $\begin{array}{l}4.58 \\
4.58\end{array}$ & -- & -- & $\begin{array}{l}100 \\
100\end{array}$ \\
\hline $\begin{array}{l}\text { Centel Corp } \\
\text { Cimarron River (KS) } \\
\text { Large (KS) }\end{array}$ & - & - & -- & $\begin{array}{l}- \\
--\end{array}$ & -- & +- & $\begin{array}{l}-- \\
--\end{array}$ & -- & $\begin{array}{l}436 \\
112 \\
324\end{array}$ & $\begin{array}{l}211.1 \\
213.7 \\
210.1\end{array}$ & $\begin{array}{l}2.12 \\
2.26 \\
2.07\end{array}$ & $\begin{array}{l}-- \\
--\end{array}$ & -- & $\begin{array}{l}100 \\
100 \\
100\end{array}$ \\
\hline $\begin{array}{l}\text { Central Hudeon Gas \& Elec Corp ... } \\
\text { Danskammer (NY) } \\
\text { Roseton (NY) }\end{array}$ & $\begin{array}{r}51 \\
51 \\
-\quad\end{array}$ & $\begin{array}{l}183.1 \\
183.1 \\
--\end{array}$ & $\begin{array}{l}48.02 \\
48.02 \\
-\cdots\end{array}$ & $\begin{array}{r}.59 \\
.59 \\
--\end{array}$ & $\begin{array}{r}495 \\
-- \\
495\end{array}$ & $\begin{array}{l}207.0 \\
207.0\end{array}$ & $\begin{array}{c}13.28 \\
-- \\
13.28\end{array}$ & $\begin{array}{l}1.22 \\
1.22\end{array}$ & $\begin{array}{l}55 \\
20 \\
36\end{array}$ & $\begin{array}{l}370.8 \\
561.6 \\
265.7\end{array}$ & $\begin{array}{l}3.77 \\
5.71 \\
2.70\end{array}$ & $\begin{array}{r}29 \\
99 \\
--\end{array}$ & $\begin{array}{c}70 \\
-- \\
99\end{array}$ & $\begin{array}{l}1 \\
1 \\
1\end{array}$ \\
\hline Central Illinols Llght Co & 212 & 175.2 & 42.62 & 1.77 & 1 & 431.4 & 24.97 & .23 & - & $\cdots$ & -- & 100 & - & - \\
\hline
\end{tabular}

See notes and footnotes at end of table. 
Table 63. Recelpts, Average Cost, and Quality of Fossil Fuels Delivered to U.S. Electric Utilities by Company and Plant, September 1993 (Continued)

\begin{tabular}{|c|c|c|c|c|c|c|c|c|c|c|c|c|c|c|}
\hline \multirow{3}{*}{$\begin{array}{l}\text { Utillty (Holding Company) } \\
\text { Plant (State) }\end{array}$} & \multicolumn{4}{|c|}{ Coal } & \multicolumn{4}{|c|}{ Potroleum' } & \multicolumn{3}{|c|}{ Gas } & \multicolumn{3}{|c|}{ \% of Total Btu } \\
\hline & \multirow{2}{*}{\begin{tabular}{|c|} 
Recelpts \\
$(1,000$ \\
tons $)$
\end{tabular}} & \multicolumn{2}{|c|}{$\begin{array}{l}\text { Average } \\
\text { Cost }^{3}\end{array}$} & \multirow{2}{*}{$\begin{array}{l}\text { Avg. } \\
\text { sut: } \\
\text { fur } \\
\times\end{array}$} & \multirow{2}{*}{\begin{tabular}{|c|} 
Recelpts \\
$(1,000$ \\
bble $)$
\end{tabular}} & \multicolumn{2}{|c|}{$\begin{array}{l}\text { Average } \\
\text { Cost }^{2}\end{array}$} & \multirow{2}{*}{$\begin{array}{l}\text { Avg. } \\
\text { sul: } \\
\text { fur } \\
\%\end{array}$} & \multirow{2}{*}{$\begin{array}{c}\text { Recelpte } \\
\left(\begin{array}{c}1,000 \\
\text { Mef })\end{array}\right.\end{array}$} & \multicolumn{2}{|c|}{$\begin{array}{l}\text { Average } \\
\text { Cost? }\end{array}$} & \multirow[b]{2}{*}{ Coal } & \multirow[b]{2}{*}{$\mid \begin{array}{l}\text { Po- } \\
\text { tro- } \\
\text { loum }\end{array}$} & \multirow[b]{2}{*}{ Gas } \\
\hline & & $\begin{array}{l}\text { (Cents } \\
\text { par } \\
10^{\circ} \\
\text { Btu) }\end{array}$ & $\begin{array}{l}\text { (\$ per } \\
\text { short } \\
\text { ton) }\end{array}$ & & & $\begin{array}{l}\text { (Conte } \\
\text { perr } \\
10^{8} \\
\text { Btu) }\end{array}$ & soor & & & $\begin{array}{c}\text { (Cents } \\
\text { por } \\
10^{\circ} \\
\text { Btu) }\end{array}$ & per & & & \\
\hline 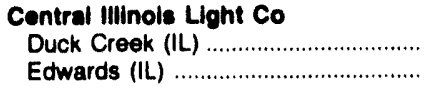 & $\begin{array}{r}91 \\
121\end{array}$ & $\begin{array}{l}183.5 \\
170.2\end{array}$ & $\begin{array}{l}39.64 \\
44.85\end{array}$ & $\begin{array}{r}2.95 \\
.88\end{array}$ & $\begin{array}{l}1 \\
1\end{array}$ & $\begin{array}{l}425.8 \\
436.6\end{array}$ & $\begin{array}{l}24.65 \\
25.27\end{array}$ & $\begin{array}{r}0.24 \\
.22\end{array}$ & $\begin{array}{l}-- \\
-\end{array}$ & $\overline{--}$ & -- & $\begin{array}{l}100 \\
100\end{array}$ & $\vdots$ & $\overline{--}$ \\
\hline 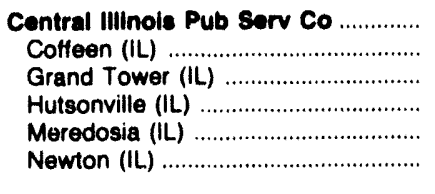 & $\begin{array}{r}396 \\
153 \\
8 \\
12 \\
35 \\
188\end{array}$ & $\begin{array}{l}159.3 \\
158.4 \\
183.1 \\
120.9 \\
154.0 \\
162.3\end{array}$ & $\begin{array}{l}35.71 \\
33.68 \\
42.50 \\
26.72 \\
34.84 \\
37.81\end{array}$ & $\begin{array}{l}2.46 \\
3.10 \\
2.76 \\
2.13 \\
3.00 \\
1.84\end{array}$ & $\dot{-}$ & $\begin{array}{l}435.2 \\
433.1 \\
435.2 \\
-- \\
-- \\
439.9\end{array}$ & $\begin{array}{l}25.10 \\
25.09 \\
25.12 \\
-- \\
-- \\
25.12\end{array}$ & $\begin{array}{l}.30 \\
.28 \\
.18 \\
-- \\
-- \\
.39\end{array}$ & $\begin{array}{l}- \\
-- \\
\overline{-} \\
-\end{array}$ & $\begin{array}{l}- \\
\overline{-} \\
\overline{-} \\
-\end{array}$ & $\begin{array}{l}- \\
\overline{-} \\
\overline{-} \\
\overline{-}\end{array}$ & $\begin{array}{r}100 \\
100 \\
99 \\
100 \\
100 \\
100\end{array}$ & $\begin{array}{l}\dot{1} \\
1 \\
--\end{array}$ & $\begin{array}{l}- \\
\ddot{-} \\
- \\
-\end{array}$ \\
\hline $\begin{array}{l}\text { Contral lowa Power Coop } \\
\text { Fair Station (IA) ................................................. }\end{array}$ & 4 & $\begin{array}{l}126.6 \\
126.6\end{array}$ & $\begin{array}{l}28.13 \\
28.13\end{array}$ & $\begin{array}{l}3.07 \\
3.07\end{array}$ & $\overline{-}$ & $\overline{-}$ & $\overline{-}$ & $\overline{-}$ & 1 & $\begin{array}{l}391.8 \\
391.8\end{array}$ & $\begin{array}{l}4.02 \\
4.02\end{array}$ & $\begin{array}{l}99 \\
99\end{array}$ & $\overline{-}$ & \\
\hline 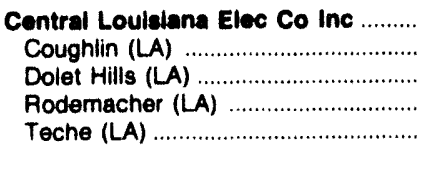 & $\begin{array}{r}-460 \\
-287 \\
- \\
-\end{array}$ & $\begin{array}{l}154.8 \\
\overline{133.5} \\
183.3 \\
--\end{array}$ & $\begin{array}{l}23.49 \\
-- \\
18.56 \\
31.68 \\
--\end{array}$ & $\begin{array}{l}.66 \\
-.78 \\
.46 \\
--\end{array}$ & $\begin{array}{l}\overline{-} \\
\overline{-} \\
-\end{array}$ & $\begin{array}{l}- \\
= \\
- \\
-\end{array}$ & $\begin{array}{l}- \\
- \\
- \\
-\end{array}$ & $\begin{array}{l}\overline{-} \\
\overline{-} \\
\overline{-}\end{array}$ & $\begin{array}{r}2,442 \\
354 \\
4 \\
776 \\
1,308\end{array}$ & $\begin{array}{l}262.1 \\
275.6 \\
275.6 \\
271.0 \\
253.2\end{array}$ & $\begin{array}{l}2.72 \\
2.91 \\
2.82 \\
2.73 \\
2.66\end{array}$ & $\begin{array}{c}73 \\
- \\
100 \\
-79\end{array}$ & $\begin{array}{l}\overline{-} \\
\overline{-} \\
--\end{array}$ & $\begin{array}{r}21 \\
100\end{array}$ \\
\hline 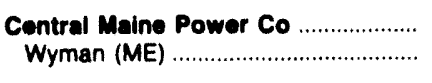 & $\overline{-}$ & $\overline{-}$ & $\overline{-}$ & $\overline{-}$ & $\begin{array}{l}445 \\
445\end{array}$ & $\begin{array}{l}206.9 \\
206.9\end{array}$ & $\begin{array}{l}13.11 \\
13.11\end{array}$ & $\begin{array}{l}1.32 \\
1.32\end{array}$ & $\overline{-}$ & $\overline{-}$ & $\overline{-}$ & $\overline{-}$ & $\begin{array}{l}100 \\
100\end{array}$ & $\overline{-}$ \\
\hline $\begin{array}{l}\text { Central Nebraska Pub Pal Dist ........ } \\
\text { Canaday (NE) }\end{array}$ & $\overline{-}$ & $\overline{-}$ & $\overline{-}$ & - & - & - & $\overline{-}$ & - & : & $\begin{array}{l}597.6 \\
597.6\end{array}$ & $\begin{array}{l}5.75 \\
5.75\end{array}$ & $\overline{-}$ & $\overline{-}$ & $\begin{array}{l}100 \\
100\end{array}$ \\
\hline 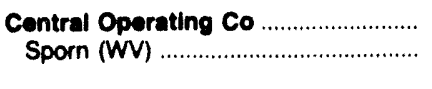 & $\begin{array}{l}51 \\
51\end{array}$ & $\begin{array}{l}161.0 \\
161.0\end{array}$ & $\begin{array}{l}40.20 \\
40.20\end{array}$ & $\begin{array}{l}1.19 \\
1.19\end{array}$ & 9 & $\begin{array}{l}487.1 \\
487.1\end{array}$ & $\begin{array}{l}28.10 \\
28.10\end{array}$ & $\begin{array}{l}.00 \\
.00\end{array}$ & - & - & $\ddot{-}$ & $\begin{array}{l}96 \\
96\end{array}$ & 4 & $\overline{-}$ \\
\hline $\begin{array}{l}\text { Central Power \& Lught Co } \\
\text { Bates (TX) . } \\
\text { Coleto Creek (TX) } \\
\text { Davis (TX) } \\
\text { Hill (TX) } \\
\text { Joslin (TX) } \\
\text { La Palma (TX) } \\
\text { Laredo (TX) } \\
\text { Nueces Bay (TX) } \\
\text { Victoria (TX) }\end{array}$ & $\begin{array}{l}227 \\
- \\
- \\
= \\
= \\
= \\
-\end{array}$ & $\begin{array}{l}181.7 \\
\overline{181.7} \\
- \\
- \\
- \\
\overline{-} \\
- \\
-\end{array}$ & $\begin{array}{l}39.61 \\
39.61 \\
- \\
= \\
= \\
= \\
- \\
-\end{array}$ & $\begin{array}{l}.45 \\
- \\
.45 \\
-- \\
-- \\
-- \\
-- \\
-\end{array}$ & $\begin{array}{l}\overline{-} \\
\overline{-} \\
\overline{-} \\
\overline{-} \\
\overline{-}\end{array}$ & $\begin{array}{l}- \\
- \\
- \\
- \\
- \\
- \\
-\end{array}$ & $\begin{array}{l}- \\
= \\
= \\
= \\
= \\
= \\
- \\
-\end{array}$ & $\begin{array}{l}- \\
\overline{-} \\
\overline{-} \\
= \\
= \\
= \\
-\end{array}$ & $\begin{array}{r}10,541 \\
831 \\
--\quad \\
2,694 \\
1,673 \\
770 \\
815 \\
803 \\
2,422 \\
533\end{array}$ & $\begin{array}{l}244.7 \\
233.6 \\
241.3 \\
241.9 \\
244.9 \\
233.3 \\
278.1 \\
244.9 \\
254.1\end{array}$ & $\begin{array}{l}2.53 \\
2.41 \\
2.48 \\
2.50 \\
2.54 \\
2.44 \\
2.89 \\
2.53 \\
2.66\end{array}$ & $\begin{array}{l}31 \\
-- \\
100 \\
-- \\
-- \\
-- \\
-- \\
-- \\
-- \\
--\end{array}$ & $\begin{array}{l}- \\
- \\
\overline{-} \\
-- \\
- \\
= \\
-\end{array}$ & $\begin{array}{c}69 \\
100 \\
-- \\
100 \\
100 \\
100 \\
100 \\
100 \\
100 \\
100\end{array}$ \\
\hline 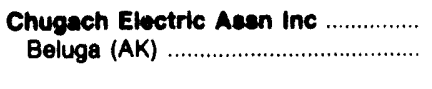 & $\overline{-}$ & $\overline{-}$ & $\overline{-}$ & $\overline{-}$ & $\overline{-}$ & $\overline{-}$ & $\overline{-}$ & $\overline{-}$ & $\begin{array}{l}609 \\
609\end{array}$ & $\begin{array}{l}63.3 \\
63.3\end{array}$ & $\begin{array}{l}.63 \\
.63\end{array}$ & - & $\overline{-}$ & $\begin{array}{l}100 \\
100\end{array}$ \\
\hline $\begin{array}{l}\text { Cincinnati Gas Electric Co } \\
\text { Beckjord (OH) } \\
\text { East Bend (KY) } \\
\text { Miami Fort (OH) } \\
\text { Zimmer (OH) }\end{array}$ & $\begin{array}{r}682 \\
133 \\
97 \\
159 \\
293\end{array}$ & $\begin{array}{l}150.9 \\
182.8 \\
182.4 \\
168.7 \\
116.8\end{array}$ & $\begin{array}{l}36.07 \\
42.72 \\
42.75 \\
41.11 \\
28.11\end{array}$ & $\begin{array}{l}1.92 \\
1.01 \\
1.31 \\
1.15 \\
2.94\end{array}$ & $x^{2}$ & $\begin{array}{l}425.1 \\
416.5 \\
434.4 \\
427.3 \\
426.2\end{array}$ & $\begin{array}{l}24.46 \\
24.41 \\
25.00 \\
24.43 \\
24.42\end{array}$ & $\begin{array}{l}.43 \\
.42 \\
.49 \\
.43 \\
.39\end{array}$ & $\begin{array}{l}\overline{-} \\
\overline{-} \\
-\end{array}$ & $\begin{array}{l}- \\
- \\
- \\
-\end{array}$ & $\begin{array}{l}- \\
\overline{-} \\
\overline{-} \\
-\end{array}$ & $\begin{array}{r}100 \\
100 \\
100 \\
99 \\
100\end{array}$ & $:$ & $\begin{array}{l}- \\
\overline{-} \\
--\end{array}$ \\
\hline $\begin{array}{l}\text { Cloveland Eloctric Illum Co } \\
\text { Ashtabula }(\mathrm{OH}) \\
\text { Avon Lake }(\mathrm{OH}) \\
\text { Eastlake }(\mathrm{OH}) \\
\text { Lake Shore }(\mathrm{OH})\end{array}$ & $\begin{array}{r}412 \\
40 \\
149 \\
193 \\
30\end{array}$ & $\begin{array}{l}136.1 \\
154.2 \\
122.7 \\
138.0 \\
167.6\end{array}$ & $\begin{array}{l}34.94 \\
39.36 \\
32.08 \\
34.83 \\
44.00\end{array}$ & $\begin{array}{r}2.09 \\
3.46 \\
1.15 \\
2.77 \\
.58\end{array}$ & - & $\begin{array}{l}408.4 \\
404.9 \\
- \\
410.6 \\
407.4\end{array}$ & $\begin{array}{l}23.60 \\
23.54 \\
-- \\
23.97 \\
23.71\end{array}$ & $\begin{array}{r}.28 \\
.27 \\
-. \\
.28 \\
.30\end{array}$ & $\begin{array}{l}\overline{-} \\
\overline{-} \\
\overline{-}\end{array}$ & $\begin{array}{l}- \\
\bar{z} \\
\overline{-}\end{array}$ & $\begin{array}{l}\overline{-} \\
\overline{-} \\
\overline{-}\end{array}$ & $\begin{array}{r}100 \\
99 \\
100 \\
100 \\
99\end{array}$ & $\begin{array}{c}\cdot 1 \\
-- \\
1\end{array}$ & $\begin{array}{l}- \\
\overline{-} \\
\overline{-}\end{array}$ \\
\hline $\begin{array}{l}\text { Cofteyville City of } \\
\text { Coffeyville (KS) }\end{array}$ & $\overline{-}$ & $\overline{-}$ & - & $\overline{-}$ & $\overline{-}$ & $\overline{-}$ & $\overline{-}$ & $=$ & $\begin{array}{l}97 \\
87\end{array}$ & $\begin{array}{l}223.9 \\
223.9\end{array}$ & $\begin{array}{l}2.24 \\
2.24\end{array}$ & $\overline{-}$ & $\overline{-}$ & $\begin{array}{l}100 \\
100\end{array}$ \\
\hline 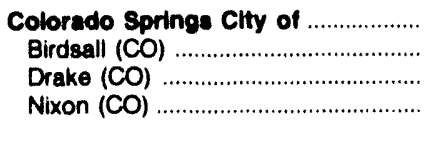 & $-\begin{array}{r}92 \\
62 \\
31\end{array}$ & $\begin{array}{l}163.5 \\
- \\
190.6 \\
113.7\end{array}$ & $\begin{array}{l}34.47 \\
-- \\
39.03 \\
25.34\end{array}$ & $\begin{array}{r}.38 \\
-- \\
.36 \\
.41\end{array}$ & $\begin{array}{l}- \\
\overline{-} \\
-\end{array}$ & $\overline{-}$ & $\begin{array}{l}- \\
- \\
-\end{array}$ & $\overline{-}$ & $\begin{array}{l}7 \\
4 \\
4\end{array}$ & $\begin{array}{l}333.0 \\
333.0 \\
333.0 \\
--\end{array}$ & $\begin{array}{l}3.26 \\
3.26 \\
3.26 \\
--\end{array}$ & $\begin{array}{l}100 \\
-- \\
100 \\
100\end{array}$ & $\begin{array}{l}- \\
\overline{-} \\
-\end{array}$ & i0 \\
\hline $\begin{array}{l}\text { Columbla Clty of } \\
\text { Columbia (MO) }\end{array}$ & $\begin{array}{l}5 \\
5\end{array}$ & $\begin{array}{l}210.1 \\
210.1\end{array}$ & $\begin{array}{l}57.29 \\
57.29\end{array}$ & $\begin{array}{l}.61 \\
.61\end{array}$ & $=$ & $=$ & $\overline{-}$ & $\overline{-}$ & $\begin{array}{l}5 \\
5\end{array}$ & $\begin{array}{l}291.0 \\
291.0\end{array}$ & $\begin{array}{l}2.91 \\
2.91\end{array}$ & $\begin{array}{l}97 \\
97\end{array}$ & $\overline{-}$ & \\
\hline
\end{tabular}

See notes and footnotes at end of table. 
Table 63. Recelpts, Average Cost, and Quality of Fossll Fuele Dellvered to U.S. Electric Utilities by Company and Plant, September 1993 (Continued)

\begin{tabular}{|c|c|c|c|c|c|c|c|c|c|c|c|c|c|c|}
\hline \multirow{3}{*}{$\begin{array}{l}\text { Utility (Holding Company) } \\
\text { Plant (State) }\end{array}$} & \multicolumn{4}{|c|}{ Coal } & \multicolumn{4}{|c|}{ Potroloum' } & \multicolumn{3}{|c|}{ Gas } & \multicolumn{3}{|c|}{$\%$ of Total Btu } \\
\hline & \multirow{2}{*}{\begin{tabular}{|c|} 
Recelpts \\
$(1,000$ \\
tons $)$
\end{tabular}} & \multicolumn{2}{|c|}{$\begin{array}{l}\text { Averege } \\
\text { Coot }\end{array}$} & \multirow{2}{*}{$\begin{array}{l}\text { Avg. } \\
\text { sut- } \\
\text { fur } \\
\times\end{array}$} & \multirow{2}{*}{\begin{tabular}{|c|} 
Recelpte \\
$(1,000$ \\
bbte $)$
\end{tabular}} & \multicolumn{2}{|c|}{$\begin{array}{l}\text { Average } \\
\text { Coot }\end{array}$} & \multirow{2}{*}{$\begin{array}{c}\text { Avg. } \\
\text { Sul- } \\
\text { fur } \\
\times\end{array}$} & \multirow{2}{*}{$\begin{array}{c}\text { Recelpts } \\
\begin{array}{c}(1,000 \\
\text { Mct }\end{array}\end{array}$} & \multicolumn{2}{|c|}{$\begin{array}{l}\text { Average } \\
\text { Cost }^{3}\end{array}$} & \multirow[b]{2}{*}{ Coal } & \multirow[b]{2}{*}{$\begin{array}{c}\text { Po- } \\
\text { tro- } \\
\text { leum }\end{array}$} & \multirow[b]{2}{*}{ Gas } \\
\hline & & $\begin{array}{l}\text { (Cents } \\
\text { per } \\
10^{\circ} \\
\text { Btu) }\end{array}$ & $\begin{array}{l}\text { (S per } \\
\text { chort } \\
\text { ton) }\end{array}$ & & & $\begin{array}{l}\text { (Cents } \\
\text { port } \\
10^{4} \\
\text { Btu) }\end{array}$ & por & & & $\begin{array}{l}\text { (Conts } \\
\text { per } \\
10^{8} \\
\text { Btu) }\end{array}$ & $\begin{array}{l}\text { s per } \\
\text { Mcf }\end{array}$ & & & \\
\hline 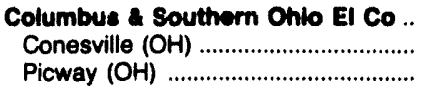 & $\begin{array}{r}302 \\
273 \\
29\end{array}$ & $\begin{array}{l}152.9 \\
158.1 \\
102.0\end{array}$ & $\begin{array}{l}36.27 \\
37.63 \\
23.49\end{array}$ & $\begin{array}{l}3.28 \\
3.30 \\
3.12\end{array}$ & $\begin{array}{r}1 \\
\cdot 1\end{array}$ & $\begin{array}{l}417.7 \\
419.9 \\
406.7\end{array}$ & $\begin{array}{l}24.60 \\
24.73 \\
23.96\end{array}$ & $\begin{array}{r}0.00 \\
.00 \\
.00\end{array}$ & $\bar{z}$ & $\overline{-}$ & $\overline{-}$ & $\begin{array}{l}100 \\
100 \\
100\end{array}$ & : & $\overline{-}$ \\
\hline 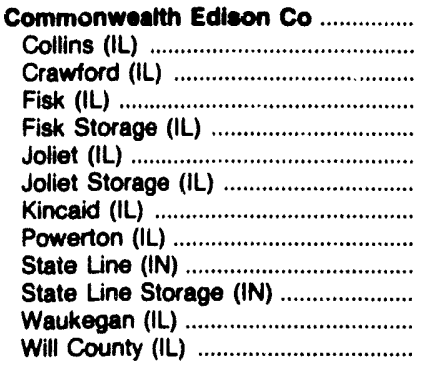 & $\begin{array}{r}1,027 \\
-\quad 97 \\
-\quad 37 \\
-\quad 194 \\
-\quad 223 \\
182 \\
-\quad 13 \\
-\quad 102 \\
179\end{array}$ & $\begin{array}{l}213.8 \\
272.3 \\
273.5 \\
\overline{254} \\
\overline{102.7} \\
259.4 \\
252.2 \\
\overline{271.4} \\
202.7\end{array}$ & $\begin{array}{l}40.24 \\
\overline{52.03} \\
52.25 \\
\overline{48.22} \\
\overline{21.58} \\
46.15 \\
\overline{48.19} \\
\overline{48.08} \\
34.69\end{array}$ & $\begin{array}{l}1.07 \\
.37 \\
.37 \\
- \\
.36 \\
- \\
3.76 \\
.24 \\
.37 \\
-.32 \\
.35\end{array}$ & 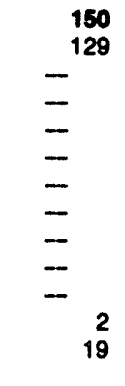 & $\begin{array}{l}282.8 \\
266.6 \\
\overline{ } \\
\overline{ } \\
\overline{ } \\
\overline{ } \\
\overline{ } \\
395.5 \\
391.1\end{array}$ & $\begin{array}{l}17.86 \\
17.04 \\
\overline{-} \\
\overline{=} \\
= \\
\overline{=} \\
\overline{23.15} \\
22.87\end{array}$ & $\begin{array}{l}.61 \\
66 \\
= \\
= \\
= \\
= \\
= \\
= \\
.29 \\
.27\end{array}$ & $\begin{array}{r}1,516 \\
924 \\
-\quad 4 \\
-\quad 147 \\
-\quad 311 \\
317 \\
17 \\
-\quad 25 \\
-\quad 88 \\
-\quad\end{array}$ & $\begin{array}{l}249.5 \\
246.4 \\
820.6 \\
\overline{250.0} \\
\overline{236.5} \\
315.6 \\
384.4 \\
\overline{250.8} \\
\overline{-}\end{array}$ & $\begin{array}{l}2.53 \\
2.50 \\
8.37 \\
- \\
2.55 \\
-- \\
2.40 \\
3.25 \\
3.87 \\
\overline{2.56} \\
- \\
--\end{array}$ & $\begin{array}{c}89 \\
-- \\
100 \\
100 \\
-- \\
100 \\
-- \\
100 \\
99 \\
100 \\
-- \\
99 \\
97\end{array}$ & $\begin{array}{c}47 \\
47 \\
-- \\
-- \\
-- \\
-- \\
-- \\
-- \\
- \\
-- \\
1 \\
3\end{array}$ & $\begin{array}{l}\overrightarrow{53} \\
\ddot{100} \\
\overline{100}\end{array}$ \\
\hline 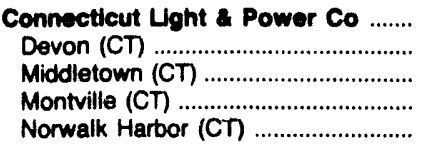 & $\bar{z}$ & $\begin{array}{l}\bar{z} \\
\bar{z}\end{array}$ & $\begin{array}{l}\bar{z} \\
\bar{z}\end{array}$ & $\begin{array}{l}\bar{z} \\
\bar{z}\end{array}$ & $\begin{array}{r}419 \\
41 \\
128 \\
94 \\
156\end{array}$ & $\begin{array}{l}244.9 \\
235.4 \\
267.2 \\
238.5 \\
233.4\end{array}$ & $\begin{array}{l}15.55 \\
15.13 \\
16.69 \\
15.16 \\
14.97\end{array}$ & $\begin{array}{l}.71 \\
.70 \\
.47 \\
.91 \\
.78\end{array}$ & $\begin{array}{l}-{ }^{21} \\
-{ }_{-}\end{array}$ & $\begin{array}{l}544.0 \\
\overline{544.0} \\
--\end{array}$ & $\begin{array}{l}5.60 \\
\overline{-} \\
5.60 \\
--\end{array}$ & $\begin{array}{l}- \\
\bar{z} \\
\overline{-}\end{array}$ & $\begin{array}{r}99 \\
100 \\
100 \\
97 \\
100\end{array}$ & $\begin{array}{c}1 \\
- \\
--\end{array}$ \\
\hline $\begin{array}{l}\text { Consolldated Edieon Co-NY Inc } \\
\text { Arthur Kill (NY) } \\
\text { Astoria (NY) } \\
\text { East River (NY) } \\
\text { Ravenswood (NY) } \\
\text { Storage Facility } \# 4+\ldots \ldots \ldots \ldots \ldots \\
\text { Storage Facility } \# 5 \\
\text { Storage Facility } \\
\text { Waterside (NY) }\end{array}$ & $\begin{array}{l}\bar{z} \\
\bar{z} \\
\bar{z}\end{array}$ & $\begin{array}{l}\bar{z} \\
\bar{z} \\
\bar{z} \\
\bar{z}\end{array}$ & $\begin{array}{l}\bar{z} \\
\bar{z} \\
\bar{z} \\
\bar{z}\end{array}$ & $\begin{array}{l}\bar{z} \\
\bar{z} \\
\bar{z} \\
\bar{z}\end{array}$ & $\begin{array}{r}982 \\
-\quad 73 \\
-\quad 86 \\
-\quad 194 \\
306 \\
-323\end{array}$ & $\begin{array}{l}250.7 \\
\overline{245.2} \\
246.4 \\
\overline{254.6} \\
249.5 \\
251.9 \\
--\end{array}$ & $\begin{array}{c}15.70 \\
\overline{15.41} \\
15.48 \\
\overline{15.98} \\
15.69 \\
15.67 \\
-\end{array}$ & $\begin{array}{l}.29 \\
-.29 \\
.29 \\
-.28 \\
.28 \\
.30 \\
-\end{array}$ & $\begin{array}{r}7,103 \\
747 \\
3,209 \\
406 \\
2,264 \\
-- \\
- \\
- \\
-477\end{array}$ & $\begin{array}{l}260.0 \\
259.2 \\
260.1 \\
260.1 \\
260.1 \\
\overline{-} \\
\overline{260.1}\end{array}$ & $\begin{array}{l}2.69 \\
2.68 \\
2.69 \\
2.69 \\
2.69 \\
-- \\
-- \\
-\overline{2.69}\end{array}$ & $\begin{array}{l}- \\
- \\
- \\
- \\
- \\
\overline{-} \\
-\end{array}$ & $\begin{array}{c}46 \\
\overline{12} \\
56 \\
-\overline{100} \\
100 \\
100 \\
--\end{array}$ & $\begin{array}{r}54 \\
100 \\
88 \\
44 \\
100 \\
-- \\
- \\
- \\
100\end{array}$ \\
\hline 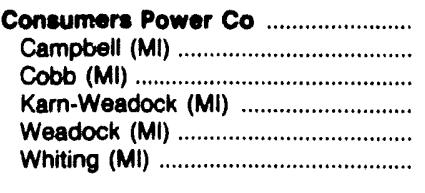 & $\begin{array}{r}794 \\
323 \\
86 \\
153 \\
144 \\
89\end{array}$ & $\begin{array}{l}165.1 \\
173.9 \\
156.4 \\
164.5 \\
152.3 \\
160.6\end{array}$ & $\begin{array}{l}40.39 \\
43.74 \\
35.83 \\
41.29 \\
34.34 \\
40.90\end{array}$ & $\begin{array}{l}.81 \\
.80 \\
.77 \\
.86 \\
.75 \\
.84\end{array}$ & $\begin{array}{r}75 \\
3 \\
-\quad 67 \\
5 \\
1\end{array}$ & $\begin{array}{l}284.7 \\
378.8 \\
- \\
273.3 \\
401.0 \\
390.1\end{array}$ & $\begin{array}{l}18.28 \\
21.96 \\
\overline{17.75} \\
23.24 \\
22.61\end{array}$ & $\begin{array}{l}.90 \\
.50 \\
-.95 \\
.50 \\
.50\end{array}$ & $\begin{array}{l}-^{150} \\
- \\
-\end{array}$ & $\begin{array}{l}294.0 \\
- \\
- \\
294.0 \\
--\end{array}$ & $\begin{array}{l}3.00 \\
-- \\
-- \\
3.00 \\
--\end{array}$ & $\begin{array}{r}97 \\
100 \\
100 \\
87 \\
99 \\
100\end{array}$ & $\begin{array}{c}2 \\
-{ }^{2} \\
10 \\
1\end{array}$ & $\begin{array}{c}1 \\
-- \\
- \\
-- \\
--\end{array}$ \\
\hline $\begin{array}{l}\text { Coop Power Acen } \\
\text { Coal Croek (ND) }\end{array}$ & $\begin{array}{l}771 \\
771\end{array}$ & $\begin{array}{l}63.7 \\
63.7\end{array}$ & $\begin{array}{l}8.09 \\
8.09\end{array}$ & $\begin{array}{l}.79 \\
.79\end{array}$ & $\overline{-}$ & $\overline{-}$ & $\overline{-}$ & $\overline{-}$ & $\overline{-}$ & $\overline{-}$ & $\overline{-}$ & $\begin{array}{l}100 \\
100\end{array}$ & $\overline{-}$ & $\overline{-}$ \\
\hline $\begin{array}{l}\text { Dairyland Power Coop } \\
\text { Alma-Madgett (WI) } \\
\text { Genoa No.3 (WI) } \\
\text { Stoneman (WI) }\end{array}$ & $\begin{array}{r}262 \\
178 \\
-83 \\
-\quad\end{array}$ & $\begin{array}{l}132.2 \\
136.6 \\
123.8 \\
-\end{array}$ & $\begin{array}{l}27.10 \\
27.06 \\
27.17 \\
-\end{array}$ & $\begin{array}{l}.71 \\
.68 \\
.78 \\
-\end{array}$ & $\dot{\div}$ & $\begin{array}{l}\frac{437.9}{-} \\
437.9\end{array}$ & $\overline{25.75}$ & $\begin{array}{l}.50 \\
- \\
-.50\end{array}$ & $\bar{z}$ & $\begin{array}{l}- \\
\overline{-} \\
-\end{array}$ & $\bar{z}$ & $\begin{array}{l}100 \\
100 \\
100 \\
-\end{array}$ & $\overline{1}$ & $\begin{array}{l}- \\
-- \\
--\end{array}$ \\
\hline 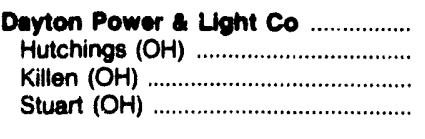 & $\begin{array}{r}769 \\
-- \\
138 \\
631\end{array}$ & $\begin{array}{l}135.7 \\
\overline{133.5} \\
136.2\end{array}$ & $\begin{array}{l}32.22 \\
\overline{32.65} \\
32.13\end{array}$ & $\begin{array}{l}.90 \\
-.64 \\
.96\end{array}$ & $\bar{z}$ & $\bar{z}$ & $\bar{z}$ & $\bar{z}$ & $\overline{-}$ & $\begin{array}{l}495.8 \\
495.8 \\
-- \\
--\end{array}$ & $\begin{array}{l}5.06 \\
5.06 \\
-- \\
--\end{array}$ & $\begin{array}{l}100 \\
-100 \\
100\end{array}$ & $\begin{array}{l}- \\
- \\
-\end{array}$ & $\begin{array}{l}\dot{100} \\
\dot{-}\end{array}$ \\
\hline 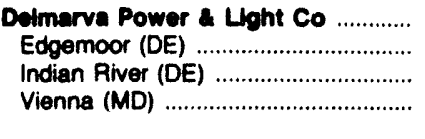 & $\begin{array}{r}108 \\
30 \\
-\quad 76\end{array}$ & $\begin{array}{l}167.3 \\
179.7 \\
162.4 \\
-\end{array}$ & $\begin{array}{l}43.39 \\
47.66 \\
41.73 \\
-\end{array}$ & $\begin{array}{l}.87 \\
.83 \\
.88 \\
--\end{array}$ & $\begin{array}{r}423 \\
312 \\
7 \\
105\end{array}$ & $\begin{array}{l}219.0 \\
221.7 \\
394.1 \\
200.4\end{array}$ & $\begin{array}{l}13.95 \\
14.16 \\
23.22 \\
12.71\end{array}$ & $\begin{array}{r}1.14 \\
.88 \\
.25 \\
1.98\end{array}$ & $\begin{array}{r}57 \\
-\quad 57 \\
-\quad \\
-\quad\end{array}$ & $\begin{array}{l}284.0 \\
284.0 \\
-- \\
--\end{array}$ & $\begin{array}{l}2.93 \\
2.93 \\
-- \\
--\end{array}$ & $\begin{array}{r}50 \\
28 \\
98 \\
-\end{array}$ & $\begin{array}{r}49 \\
70 \\
2 \\
100\end{array}$ & $\begin{array}{r}1 \\
2 \\
- \\
-\end{array}$ \\
\hline $\begin{array}{l}\text { Denton City of } \\
\text { Spencer (TX) }\end{array}$ & $\overline{-}$ & $\overline{-}$ & $\overline{-}$ & $\overline{-}$ & $\overline{-}$ & $\overline{-}$ & $\overline{-}$ & $\overline{-}$ & $\begin{array}{l}293 \\
293\end{array}$ & $\begin{array}{l}239.9 \\
239.9\end{array}$ & $\begin{array}{l}2.58 \\
2.58\end{array}$ & - & $\overline{-}$ & $\begin{array}{l}100 \\
100\end{array}$ \\
\hline $\begin{array}{l}\text { Deeoret Generation } \& \text { Tran Coop .. } \\
\text { Bonanza (UT) }\end{array}$ & $\begin{array}{l}124 \\
124\end{array}$ & $\begin{array}{l}223.0 \\
223.0\end{array}$ & $\begin{array}{l}47.54 \\
47.54\end{array}$ & $\begin{array}{l}.42 \\
.42\end{array}$ & $\overline{-}$ & - & $\overline{-}$ & $\overline{-}$ & $\overline{-}$ & $\overline{-}$ & $\overline{-}$ & $\begin{array}{l}100 \\
100\end{array}$ & - & $\overline{-}$ \\
\hline
\end{tabular}

See notes and footnotes at end of table. 
Table 63. Receipts, Average Cost, and Quality of Fossil Fuels Delivered to U.S. Electric Utilities by Company and Plant, September 1993 (Continued)

\begin{tabular}{|c|c|c|c|c|c|c|c|c|c|c|c|c|c|c|}
\hline \multirow{3}{*}{$\begin{array}{l}\text { Utility (Holding Company) } \\
\text { Plant (State) }\end{array}$} & \multicolumn{4}{|c|}{ Cosl } & \multicolumn{4}{|c|}{ Petroleum' } & \multicolumn{3}{|c|}{ Gas } & \multicolumn{3}{|c|}{$\%$ of Total Btu } \\
\hline & \multirow{2}{*}{\begin{tabular}{|c|} 
Recelpts \\
$\begin{array}{c}(1,000 \\
\text { tons })\end{array}$ \\
\end{tabular}} & \multicolumn{2}{|c|}{$\begin{array}{l}\text { Average } \\
\text { Cost }^{3}\end{array}$} & \multirow{2}{*}{\begin{tabular}{c|} 
Avg. \\
Sut- \\
fur \\
$\%$
\end{tabular}} & \multirow{2}{*}{$\begin{array}{c}\text { Recelpts } \\
\begin{array}{c}(1,000 \\
\text { bblo })\end{array}\end{array}$} & \multicolumn{2}{|c|}{$\begin{array}{l}\text { Average } \\
\text { Cost }^{2}\end{array}$} & \multirow{2}{*}{\begin{tabular}{l|} 
Avg. \\
Sul- \\
fur \\
$\%$
\end{tabular}} & \multirow{2}{*}{$\begin{array}{c}\text { Recelpts } \\
\begin{array}{c}(1,000 \\
\text { Mcf })\end{array}\end{array}$} & \multicolumn{2}{|c|}{$\begin{array}{l}\text { Average } \\
\text { Cost" }\end{array}$} & \multirow[b]{2}{*}{ Coal } & \multirow[b]{2}{*}{$\begin{array}{l}\text { Po- } \\
\text { tro- } \\
\text { loum }\end{array}$} & \multirow[b]{2}{*}{ Gas } \\
\hline & & $\begin{array}{l}\text { (Cents } \\
\text { per } \\
10^{\circ} \\
\text { Btu) }\end{array}$ & $\begin{array}{l}\text { (f por } \\
\text { ohort } \\
\text { ton) }\end{array}$ & & & $\begin{array}{l}\text { (Conts } \\
\text { per } \\
10^{\circ} \\
\text { Btu) }\end{array}$ & bol & & & $\begin{array}{l}\text { (Cents } \\
\text { per } \\
10^{\circ} \\
\text { Btu) }\end{array}$ & $\begin{array}{l}\text { S per } \\
\text { Mef }\end{array}$ & & & \\
\hline $\begin{array}{l}\text { Detrolt Clty of } \\
\text { Mistersky (MI) }\end{array}$ & - & - & $\overline{-}$ & $\overline{-}$ & 44 & $\begin{array}{l}288.4 \\
288.4\end{array}$ & $\begin{array}{l}17.62 \\
17.62\end{array}$ & $\begin{array}{r}0.78 \\
.78\end{array}$ & $\overline{-}$ & - & - & - & $\begin{array}{l}100 \\
100\end{array}$ & $\overline{-}$ \\
\hline 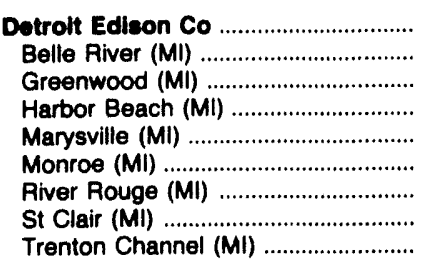 & $\begin{array}{r}1,869 \\
526 \\
-\quad \\
6 \\
11 \\
526 \\
93 \\
547 \\
160\end{array}$ & $\begin{array}{l}148.4 \\
152.1 \\
- \\
157.2 \\
159.1 \\
137.2 \\
148.6 \\
152.1 \\
162.2\end{array}$ & $\begin{array}{l}30.70 \\
28.96 \\
\overline{41.58} \\
41.02 \\
31.04 \\
34.94 \\
28.97 \\
37.66\end{array}$ & $\begin{array}{l}0.56 \\
.38 \\
- \\
.74 \\
.90 \\
.90 \\
.66 \\
.38 \\
.57\end{array}$ & $\begin{array}{r}15 \\
-\quad 4 \\
-\quad \\
-\quad 7 \\
-\quad 2 \\
1\end{array}$ & $\begin{array}{l}379.1 \\
385.9 \\
\overline{388.3} \\
\overline{379.2} \\
\overline{367.1} \\
376.0\end{array}$ & $\begin{array}{c}21.96 \\
22.35 \\
\overline{22.43} \\
\overline{21.97} \\
\overline{2} \\
21.25 \\
21.83\end{array}$ & $\begin{array}{l}.27 \\
.25 \\
-. \\
.29 \\
- \\
.30 \\
- \\
.25 \\
.26\end{array}$ & 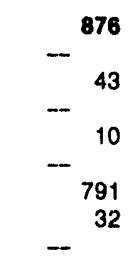 & $\begin{array}{l}235.5 \\
\overline{272.5} \\
\overline{3} \\
\overline{1} \\
\overline{185.0} \\
394.0 \\
-\end{array}$ & $\begin{array}{l}0.68 \\
-- \\
2.76 \\
-- \\
4.01 \\
-- \\
.39 \\
4.02 \\
--\end{array}$ & $\begin{array}{r}99 \\
100 \\
-- \\
99 \\
97 \\
100 \\
93 \\
100 \\
100\end{array}$ & $\begin{array}{l}: \\
- \\
- \\
- \\
- \\
-\end{array}$ & $\begin{array}{c}-1 \\
\overline{100} \\
- \\
-3 \\
-7 \\
-\end{array}$ \\
\hline 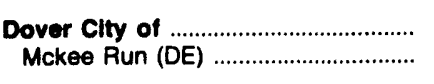 & - & - & - & - & $\begin{array}{l}42 \\
42\end{array}$ & $\begin{array}{l}271.0 \\
271.0\end{array}$ & $\begin{array}{l}17.05 \\
17.05\end{array}$ & $\begin{array}{l}1.01 \\
1.01\end{array}$ & $\begin{array}{l}8 \\
8\end{array}$ & $\begin{array}{l}392.0 \\
392.0\end{array}$ & $\begin{array}{l}4.05 \\
4.05\end{array}$ & - & $\begin{array}{l}97 \\
97\end{array}$ & $\begin{array}{l}3 \\
3\end{array}$ \\
\hline $\begin{array}{l}\text { Duke Power Co } \\
\text { Allen (NC) } \\
\text { Belews Creek (NC) } \\
\text { Buck (NC) } \\
\text { Cliftside (NC) } \\
\text { Dan River (NC) } \\
\text { Lee (SC) } \\
\text { Marshall (NC) } \\
\text { Riverbend (NC) }\end{array}$ & $\begin{array}{r}1,129 \\
154 \\
463 \\
27 \\
96 \\
17 \\
19 \\
245 \\
108\end{array}$ & $\begin{array}{l}168.3 \\
179.5 \\
162.2 \\
163.6 \\
179.6 \\
161.0 \\
162.1 \\
172.7 \\
161.2\end{array}$ & $\begin{array}{l}41.89 \\
45.10 \\
39.70 \\
41.18 \\
45.37 \\
40.98 \\
40.83 \\
43.22 \\
41.13\end{array}$ & $\begin{array}{r}1.00 \\
.95 \\
.97 \\
.90 \\
.96 \\
.84 \\
1.00 \\
1.02 \\
1.20\end{array}$ & $\begin{array}{l}- \\
- \\
- \\
-\end{array}$ & $\begin{array}{l}402.0 \\
405.8 \\
401.6 \\
\overline{407.6} \\
\overline{3} \\
-- \\
-\end{array}$ & $\begin{array}{c}23.40 \\
23.64 \\
23.30 \\
- \\
23.63 \\
\overline{2} \\
22.78 \\
-- \\
-\end{array}$ & $\begin{array}{c}.30 \\
.30 \\
.30 \\
- \\
.30 \\
\cdots \\
.30 \\
- \\
--\end{array}$ & $\begin{array}{l}- \\
- \\
- \\
- \\
- \\
-\end{array}$ & $\begin{array}{l}- \\
- \\
- \\
- \\
- \\
- \\
-\end{array}$ & $\begin{array}{l}- \\
- \\
- \\
- \\
- \\
-\end{array}$ & $\begin{array}{r}100 \\
100 \\
100 \\
100 \\
100 \\
100 \\
99 \\
100 \\
100\end{array}$ & $\begin{array}{l}: \\
: \\
- \\
-1 \\
--\end{array}$ & $\begin{array}{l}- \\
\overline{-} \\
\overline{-} \\
\overline{-} \\
-\end{array}$ \\
\hline $\begin{array}{l}\text { Duquesne Light Co } \\
\text { Cheswick (PA) } \\
\text { Elrama (PA) }\end{array}$ & $\begin{array}{r}221 \\
149 \\
72\end{array}$ & $\begin{array}{l}144.1 \\
134.1 \\
165.5\end{array}$ & $\begin{array}{l}36.38 \\
34.18 \\
40.92\end{array}$ & $\begin{array}{l}1.55 \\
1.49 \\
1.71\end{array}$ & 4 & $\frac{411.4}{411.4}$ & $\frac{23.94}{23.94}$ & -26 & $\begin{array}{r}13 \\
13 \\
-\quad\end{array}$ & $\begin{array}{l}287.5 \\
287.5 \\
--\end{array}$ & $\begin{array}{l}2.99 \\
2.99 \\
-\end{array}$ & $\begin{array}{r}99 \\
100 \\
99\end{array}$ & - & $\dot{-}$ \\
\hline 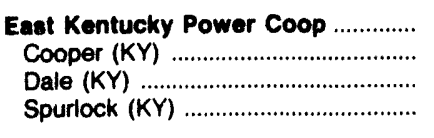 & $\begin{array}{r}256 \\
54 \\
38 \\
164\end{array}$ & $\begin{array}{l}115.0 \\
118.8 \\
112.8 \\
114.3\end{array}$ & $\begin{array}{l}28.44 \\
29.36 \\
28.09 \\
28.21\end{array}$ & $\begin{array}{r}1.30 \\
1.66 \\
.91 \\
1.28\end{array}$ & $\dot{-}$ & $\begin{array}{l}397.0 \\
397.0 \\
-- \\
-\end{array}$ & $\begin{array}{c}23.11 \\
23.11 \\
-\end{array}$ & $\begin{array}{l}.20 \\
.20 \\
-- \\
--\end{array}$ & $\overline{-}$ & $\begin{array}{l}- \\
- \\
-\end{array}$ & $\overline{-}$ & $\begin{array}{l}100 \\
100 \\
100 \\
100\end{array}$ & $\ddot{-}$ & $\overline{-}$ \\
\hline $\begin{array}{l}\text { El Paso Electric Co } \\
\text { Newman }(T X) \\
\text { Rio Grande }(\mathrm{TX})\end{array}$ & $\overline{-}$ & $\overline{-}$ & - & - & - & - & - & - & $\begin{array}{r}2,797 \\
1,811 \\
986\end{array}$ & $\begin{array}{l}228.2 \\
225.0 \\
234.0\end{array}$ & $\begin{array}{l}2.34 \\
2.31 \\
2.40\end{array}$ & - & - & $\begin{array}{l}100 \\
100 \\
100\end{array}$ \\
\hline 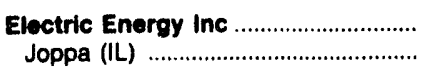 & $\begin{array}{l}326 \\
326\end{array}$ & $\begin{array}{l}99.1 \\
99.1\end{array}$ & $\begin{array}{l}19.05 \\
19.05\end{array}$ & $\begin{array}{l}.98 \\
.98\end{array}$ & $\begin{array}{l}2 \\
2\end{array}$ & $\begin{array}{l}442.0 \\
442.0\end{array}$ & $\begin{array}{l}25.62 \\
25.62\end{array}$ & $\begin{array}{l}.36 \\
.36\end{array}$ & - & - & - & $\begin{array}{l}100 \\
100\end{array}$ & 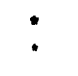 & $\overline{-}$ \\
\hline $\begin{array}{l}\text { Emplre District Electric Co } \\
\text { Asbury (MO) } \\
\text { Riverton (KS) }\end{array}$ & $\begin{array}{r}148 \\
129 \\
19\end{array}$ & $\begin{array}{l}103.2 \\
100.9 \\
117.1\end{array}$ & $\begin{array}{l}18.71 \\
17.97 \\
23.65\end{array}$ & $\begin{array}{r}.73 \\
.62 \\
1.47\end{array}$ & $\ddot{\bullet}$ & $\begin{array}{l}414.4 \\
414.4 \\
-\end{array}$ & $\begin{array}{c}24.27 \\
24.27 \\
-\end{array}$ & $\begin{array}{l}.00 \\
.00 \\
-\end{array}$ & - & $\frac{246.9}{246.9}$ & $\begin{array}{l}2.47 \\
2.47\end{array}$ & $\begin{array}{l}100 \\
100 \\
100\end{array}$ & - & $\ddot{-}$ \\
\hline $\begin{array}{l}\text { Fayetteville Public Works } \\
\text { Butler Warner (NC) }\end{array}$ & $\overline{-}$ & - & - & - & - & - & - & - & $\begin{array}{l}414 \\
414\end{array}$ & $\begin{array}{l}354.2 \\
354.2\end{array}$ & $\begin{array}{l}3.66 \\
3.66\end{array}$ & - & - & $\begin{array}{l}100 \\
100\end{array}$ \\
\hline 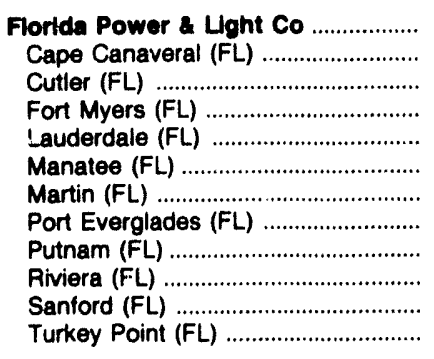 & $\begin{array}{l}- \\
- \\
- \\
- \\
- \\
- \\
- \\
-\end{array}$ & $\begin{array}{l}- \\
- \\
- \\
- \\
- \\
- \\
-\end{array}$ & $\begin{array}{l}- \\
- \\
- \\
- \\
- \\
- \\
- \\
-\end{array}$ & $\begin{array}{l}- \\
\bar{z} \\
\bar{z} \\
\bar{z} \\
\overline{-}\end{array}$ & $\begin{array}{r}4,083 \\
367 \\
-\quad 501 \\
-\quad \\
1,087 \\
226 \\
737 \\
-\quad \\
518 \\
358 \\
291\end{array}$ & $\begin{array}{l}211.8 \\
207.4 \\
\overline{199.8} \\
214.9 \\
248.4 \\
216.4 \\
\overline{183.7} \\
225.5 \\
219.7\end{array}$ & $\begin{array}{c}13.51 \\
13.09 \\
\overline{1} \\
12.63 \\
\overline{13.80} \\
15.79 \\
13.84 \\
\overline{11.73} \\
14.16 \\
14.20\end{array}$ & $\begin{array}{l}1.44 \\
2.00 \\
- \\
2.08 \\
- \\
.95 \\
.61 \\
.98 \\
\overline{2.33} \\
2.04 \\
.99\end{array}$ & $\begin{array}{r}12,273 \\
53 \\
557 \\
-- \\
3,601 \\
-\quad \\
2,727 \\
1,076 \\
2,627 \\
9 \\
383 \\
1,240\end{array}$ & $\begin{array}{l}211.9 \\
211.9 \\
211.9 \\
- \\
211.9 \\
- \\
211.9 \\
211.9 \\
211.9 \\
211.9 \\
211.9 \\
211.9\end{array}$ & $\begin{array}{l}2.12 \\
2.12 \\
2.12 \\
-- \\
2.12 \\
\overline{2.12} \\
2.12 \\
2.12 \\
2.12 \\
2.12 \\
2.12\end{array}$ & $\begin{array}{l}- \\
- \\
- \\
- \\
- \\
- \\
-\end{array}$ & $\begin{array}{c}68 \\
98 \\
\overline{100} \\
\overline{100} \\
35 \\
81 \\
\overline{100} \\
86 \\
60\end{array}$ & $\begin{array}{r}32 \\
2 \\
100 \\
- \\
100 \\
\overline{65} \\
18 \\
100 \\
15 \\
40\end{array}$ \\
\hline 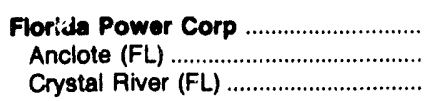 & $\begin{array}{r}451 \\
- \\
336\end{array}$ & $\begin{array}{l}175.1 \\
\overline{181.1}\end{array}$ & $\begin{array}{l}44.12 \\
\overline{45.66}\end{array}$ & $\frac{.81}{.83}$ & $\begin{array}{r}935 \\
5 \\
4\end{array}$ & $\begin{array}{l}213.1 \\
410.8 \\
418.7\end{array}$ & $\begin{array}{l}13.53 \\
24.11 \\
24.57\end{array}$ & $\begin{array}{r}1.57 \\
.15 \\
.15\end{array}$ & $\begin{array}{l}27 \\
-\end{array}$ & $\begin{array}{l}377.4 \\
- \\
-\end{array}$ & $\begin{array}{l}3.89 \\
- \\
-\end{array}$ & $\frac{88}{100}$ & $\begin{array}{r}34 \\
100\end{array}$ & $\begin{array}{l}- \\
-\end{array}$ \\
\hline
\end{tabular}

See notes and footnotes at end of table. 
Table 63. Recelpts, Average Cost, and Quality of Fossil Fuels Delivered to U.S. Electric Utilities by Company and Plant, September 1993 (Continued)

\begin{tabular}{|c|c|c|c|c|c|c|c|c|c|c|c|c|c|c|}
\hline \multirow{3}{*}{$\begin{array}{c}\text { Uttlity (Holding Compeny) } \\
\text { Plant (State) }\end{array}$} & \multicolumn{4}{|c|}{ Coal } & \multicolumn{4}{|c|}{ Patroleum' } & \multicolumn{3}{|c|}{ Gas } & \multicolumn{3}{|c|}{$\%$ of Total Btu } \\
\hline & \multirow{2}{*}{\begin{tabular}{|c|} 
Recelpts \\
$(1,000$ \\
tons)
\end{tabular}} & \multicolumn{2}{|c|}{$\begin{array}{c}\text { Average } \\
\text { Cost }^{3}\end{array}$} & \multirow{2}{*}{$\begin{array}{l}\text { Avg. } \\
\text { Sut } \\
\text { fur } \\
\%\end{array}$} & \multirow{2}{*}{\begin{tabular}{|c|} 
Recelpts \\
$(1,000$ \\
bbls)
\end{tabular}} & \multicolumn{2}{|c|}{$\begin{array}{c}\text { Average } \\
\text { Cost }^{3}\end{array}$} & \multirow{2}{*}{$\begin{array}{l}\text { Avg. } \\
\text { Sul- } \\
\text { fur } \\
\%\end{array}$} & \multirow{2}{*}{\begin{tabular}{|c|} 
Recelpts \\
$\begin{array}{c}(1,000 \\
\text { Mcf })\end{array}$
\end{tabular}} & \multicolumn{2}{|c|}{$\begin{array}{l}\text { Average } \\
\text { Cost }^{3}\end{array}$} & \multirow[b]{2}{*}{ Coal } & \multirow[b]{2}{*}{$\begin{array}{c}\text { Pe- } \\
\text { tro- } \\
\text { leum }\end{array}$} & \\
\hline & & $\begin{array}{l}\text { (Cents } \\
\text { per } \\
10^{\circ} \\
\text { Btu) }\end{array}$ & $\begin{array}{l}\text { (s por } \\
\text { chort } \\
\text { ton) }\end{array}$ & & & $\begin{array}{c}\text { (Cents } \\
\text { per } \\
10^{\circ} \\
\text { Btu) }\end{array}$ & bor & & & $\begin{array}{c}\text { (Cents } \\
\text { per } \\
10^{\circ} \\
\text { Btu) }\end{array}$ & $\begin{array}{l}5 \text { per } \\
\text { Mcf }\end{array}$ & & & Gas \\
\hline Florlda Power Corp & & & & & & & & & & & & & & \\
\hline 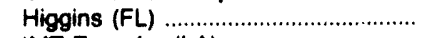 & - & -- & - & - & 39 & 227.9 & 14.33 & 0.95 & -- & - & -- & -- & 100 & $-\infty$ \\
\hline 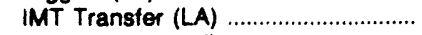 & 83 & 168.8 & 42.11 & 0.76 & -- & - & - & -- & -- & -- & - & 100 & - & $-\infty$ \\
\hline Storage Facility $\# 1$............................... & - & -- & - & -- & 779 & 206.6 & 13.13 & 1.65 & -- & -- & -- & -- & 100 & - \\
\hline Storage Facility $\# 2$ & - & - & - & - & 77 & 245.8 & 15.67 & 130 & - & -- & - & -- & 100 & -- \\
\hline 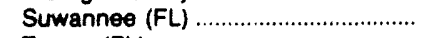 & - & - & - & -- & 27 & 222.2 & 14.12 & 2.41 & -- & $\cdots$ & - & -- & 100 & -- \\
\hline 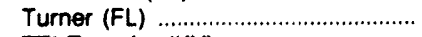 & - & - & - & - & 3 & 183.6 & 11.71 & 1.00 & 27 & 377.4 & 3.89 & - & 42 & 58 \\
\hline 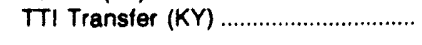 & 33 & 130.8 & 33.42 & .63 & -- & -- & - & -- & -- & - & -- & 100 & -- & -- \\
\hline Fort Pierce City of ........................... & - & - & - & - & * & 422.0 & 24.57 & .05 & 192 & 360.3 & 3.72 & - & 1 & 99 \\
\hline H O King (FL) & - & - & - & - & $\cdot$ & 422.0 & 24.57 & .05 & 192 & 360.3 & 3.72 & -- & 1 & 99 \\
\hline Fremont Clty of ................................... & 17 & 73.3 & 11.93 & .30 & - & - & -- & - & 10 & 251.5 & 2.51 & 97 & - & 3 \\
\hline Wright (NE) & 17 & 73.3 & 11.93 & .30 & - & - & - & - & 10 & 251.5 & 2.51 & 97 & -- & 3 \\
\hline 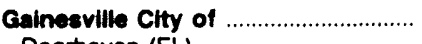 & 46 & 191.0 & 51.18 & .62 & 1 & 266.7 & 16.96 & 1.07 & 375 & 300.9 & 3.10 & 76 & 1 & 24 \\
\hline 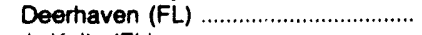 & 46 & 191.0 & 51.18 & .62 & 1 & 266.4 & 16.96 & .90 & 269 & 300.9 & 3.10 & 81 & $\cdot$ & 18 \\
\hline Jr Kelly (FL) & - & $\rightarrow$ & - & - & $\cdot$ & 267.3 & 16.96 & 1.46 & 106 & 300.9 & 3.11 & -- & 2 & 98 \\
\hline 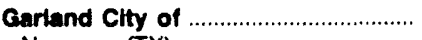 & - & - & - & - & - & - & - & - & 1,341 & 233.6 & 2.40 & - & - & 100 \\
\hline Newman $(T X)$ & - & -- & - & - & - & - & - & - & 42 & 239.2 & 2.46 & -- & - & 100 \\
\hline Olinger (TX) & - & - & - & - & - & -- & - & -- & 1,299 & 233.4 & 2.39 & -- & -- & 100 \\
\hline Georgla Power Co .............................. & 1,812 & 176.6 & 43.69 & 1.31 & 11 & 414.6 & 24.12 & .50 & 6 & 546.7 & 5.62 & 100 & • & - \\
\hline Arkwright (GA) & 27 & 179.7 & 45.23 & 1.53 & -- & -- & -- & -- & 3 & 431.1 & 4.43 & 100 & -- & - \\
\hline Atkinson-Mcdonough (GA) .................. & 94 & 164.4 & 42.15 & 1.31 & -- & - & - & - & 4 & 639.7 & 6.58 & 100 & -- & $\cdot$ \\
\hline Bowen (GA) & 631 & 164.6 & 40.36 & 1.47 & 2 & 410.8 & 23.90 & .50 & - & -- & - & 100 & $\cdot$ & -- \\
\hline 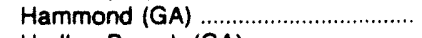 & 40 & 175.7 & 46.72 & 1.67 & 1 & 410.5 & 23.88 & .50 & -- & - & -- & 99 & 1 & - \\
\hline 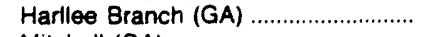 & 243 & 159.8 & 39.23 & 1.20 & $\cdot$ & 425.7 & 24.76 & .50 & -- & - & - & 100 & $\cdot$ & -- \\
\hline 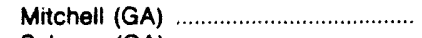 & 28 & 187.4 & 48.14 & 1.42 & -- & -- & -- & - & -- & -- & - & 100 & -- & -- \\
\hline Scherer (GA) & 335 & 205.3 & 51.64 & .68 & 4 & 418.3 & 24.33 & .50 & - & -- & - & 100 & $\cdot$ & - \\
\hline Wansley $(G A)$ & 262 & 182.8 & 44.52 & 1.54 & 2 & 407.1 & 23.68 & .50 & -- & - & -- & 100 & $\cdot$ & -- \\
\hline Yates $(G A)$ & 152 & 184.0 & 44.84 & 1.65 & 2 & 418.9 & 24.37 & .50 & - & - & -- & 100 & $\cdot$ & -- \\
\hline Glendale Clty of .................................... & - & - & - & - & - & - & - & - & 259 & 285.0 & 2.92 & - & - & 100 \\
\hline Glendale (CA) & - & - & -- & - & -- & - & - & -- & 259 & 285.0 & 2.92 & -- & -- & 100 \\
\hline Grand Haven Clty of .......................... & 23 & 155.8 & 34.38 & 3.10 & - & - & - & - & 1 & 443.5 & 4.43 & 100 & - & * \\
\hline J B Simms (MI) & 23 & 155.8 & 34.38 & 3.10 & - & -- & -- & -- & 1 & 443.5 & 4.43 & 100 & -- & • \\
\hline 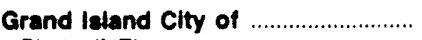 & 21 & 70.4 & 11.81 & .35 & - & - & $-\infty$ & - & - & - & - & 100 & - & - \\
\hline Platte (NE) & 21 & 70.4 & 11.81 & .35 & - & -- & -- & -- & -- & - & -- & 100 & -- & -- \\
\hline Grand Aiver Dam Authority ................ & 340 & 91.6 & 15.37 & .36 & - & - & - & - & 25 & 276.3 & 2.81 & 100 & - & - \\
\hline 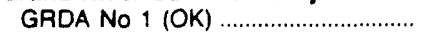 & 340 & 91.6 & 15.37 & .36 & - & -- & -- & -- & 25 & 276.3 & 2.81 & 100 & -- & $\cdot$ \\
\hline Greenvilie Clty of & - & - & - & - & - & - & - & - & 100 & 258.0 & 2.78 & - & - & 100 \\
\hline 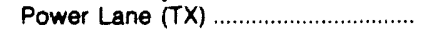 & - & - & - & - & - & - & -- & -- & 100 & 258.0 & 2.78 & - & -- & 100 \\
\hline Gulf Power Co ...................................... & 153 & 194.1 & 46.97 & 1.08 & 4 & 406.2 & 23.37 & .45 & 23 & 243.0 & 2.43 & 99 & 1 & 1 \\
\hline 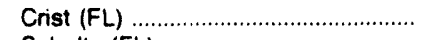 & 119 & 193.3 & 46.86 & .96 & 1 & 396.0 & 22.79 & .45 & 23 & 243.0 & 2.43 & 99 & $\cdot$ & 1 \\
\hline Scholtz (FL) & 9 & 163.8 & 39.71 & 1.18 & $\cdot$ & 393.9 & 22.67 & .45 & -- & -- & - & 99 & 1 & -- \\
\hline Smith (FL) & 25 & 209.5 & 50.24 & 1.59 & 3 & 411.1 & 23.65 & .45 & -- & -- & -- & 97 & 3 & - \\
\hline Gulf States Utilities Co ..................... & 256 & 171.4 & 29.50 & .44 & - & - & - & - & 19,571 & 254.1 & 2.62 & 18 & -- & 82 \\
\hline 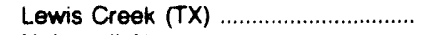 & - & - & $\bar{m}$ & - & -- & - & - & - & 2,405 & 252.0 & 2.59 & -- & - & 100 \\
\hline 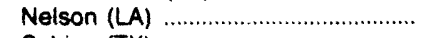 & 256 & 171.4 & 29.50 & .44 & - & - & -- & - & 2,984 & 246.9 & 2.59 & 58 & -- & 42 \\
\hline Sabine $(\mathrm{TX})$ & - & - & -- & - & -- & - & - & - & 8,313 & 255.5 & 2.63 & - & -- & 100 \\
\hline 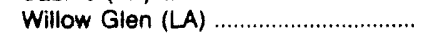 & - & - & - & - & -- & -- & - & - & 5,869 & 256.9 & 2.65 & -- & - & 100 \\
\hline 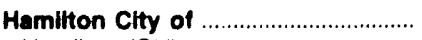 & 17 & 143.5 & 35.78 & .76 & " & 438.0 & 25.20 & .21 & 12 & 414.4 & 4.24 & 97 & • & 3 \\
\hline Hamilton $(\mathrm{OH})$ & 17 & 143.5 & 35.78 & .76 & • & 438.0 & 25.20 & .21 & 12 & 414.4 & 4.24 & 97 & " & 3 \\
\hline 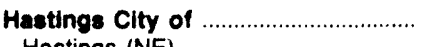 & 20 & 78.6 & 13.38 & .39 & - & - & - & - & - & - & - & 100 & - & $\cdots$ \\
\hline 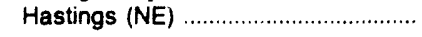 & 20 & 78.6 & 13.38 & .39 & -- & -- & -- & -- & - & -- & -- & 100 & -- & -- \\
\hline
\end{tabular}

See notes and footnotes at end of table. 
Table 63. Receipts, Average Cost, and Quality of Fossil Fuels Delivered to U.S. Electric Utilities by Company and Plant, September 1993 (Continued)

\begin{tabular}{|c|c|c|c|c|c|c|c|c|c|c|c|c|c|c|}
\hline \multirow{3}{*}{$\begin{array}{c}\text { Utility (Holding Company) } \\
\text { Plant (State) }\end{array}$} & \multicolumn{4}{|c|}{ Coal } & \multicolumn{4}{|c|}{ Potroleum' } & \multicolumn{3}{|c|}{ Gas } & \multicolumn{3}{|c|}{$\%$ of Total Btu } \\
\hline & \multirow{2}{*}{\begin{tabular}{|c|} 
Recoipts \\
$\begin{array}{l}(1,000 \\
\text { tons) }\end{array}$ \\
\end{tabular}} & \multicolumn{2}{|c|}{$\begin{array}{l}\text { Average } \\
\text { Cost }^{3}\end{array}$} & \multirow{2}{*}{$\begin{array}{l}\text { Avg. } \\
\text { Sul- } \\
\text { fur } \\
\%\end{array}$} & \multirow{2}{*}{\begin{tabular}{|c|} 
Recelpts \\
$\begin{array}{c}(1,000 \\
\text { bble })\end{array}$ \\
\end{tabular}} & \multicolumn{2}{|c|}{$\begin{array}{l}\text { Average } \\
\text { Costs }^{3}\end{array}$} & \multirow{2}{*}{$\begin{array}{l}\text { Avg. } \\
\text { Sul- } \\
\text { fur } \\
\%\end{array}$} & \multirow{2}{*}{\begin{tabular}{|c|} 
Rocelpts \\
$\begin{array}{c}(1,000 \\
\text { Mef) }\end{array}$
\end{tabular}} & \multicolumn{2}{|c|}{$\begin{array}{l}\text { Average } \\
\text { Cost }^{3}\end{array}$} & \multirow[b]{2}{*}{ Coal } & & \\
\hline & & $\begin{array}{l}\text { (Cents } \\
\text { per } \\
10^{\circ} \\
\text { Btu) }\end{array}$ & $\begin{array}{l}\text { (\$ per } \\
\text { short } \\
\text { ton) }\end{array}$ & & & $\begin{array}{c}\text { (Conts } \\
\text { per } \\
10^{\circ} \\
\text { Btu) }\end{array}$ & per & & & $\begin{array}{l}\text { (Cents } \\
\text { per } \\
10^{4} \\
\text { Btu) }\end{array}$ & $\begin{array}{l}\text { S per } \\
\text { Mct }\end{array}$ & & $\begin{array}{l}\text { tro- } \\
\text { loum }\end{array}$ & Gas \\
\hline Hawallan Electric Co Inc ..................... & -- & - & - & - & 497 & 283.9 & 17.85 & 0.41 & - & - & - & - & 100 & - \\
\hline 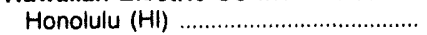 & -- & -- & -- & -- & 33 & 280.0 & 17.61 & .37 & -- & -- & -- & -- & 100 & -- \\
\hline Kahe $(H I)$ & -- & -- & -- & -- & 128 & 281.2 & 17.66 & .43 & -- & - & -_ & -- & 100 & -- \\
\hline Storage Facility $\sharp 1$ & -- & -- & - & -- & 172 & 293.5 & 18.49 & .44 & -- & -- & -- & -- & 100 & -- \\
\hline Waiau $(\mathrm{HI})$ & -- & -- & -- & -- & 164 & 276.7 & 17.36 & .37 & - & -- & - & - & 100 & -- \\
\hline Holland City of & 28 & 180.0 & 46.61 & 0.84 & - & - & - & - & - & - & - & 100 & - & -- \\
\hline James De Young (MI) ................................. & 28 & 180.0 & 46.61 & .84 & - & -- & - & -- & -- & - & -- & 100 & -- & -- \\
\hline Holyoke Water Power Co ................... & 14 & 159.4 & 42.35 & 1.49 & - & - & - & - & - & - & - & 100 & - & - \\
\hline Mount Tom (MA) & 14 & 159.4 & 42.35 & 1.49 & - & -- & - & - & - & -- & -- & 100 & -- & - \\
\hline Hoosier Energy R E C Inc $\ldots \ldots \ldots \ldots \ldots$ & 230 & 131.9 & 29.44 & 3.38 & - & - & - & - & - & - & - & 100 & - & - \\
\hline Frank E Ratts (IN) & 31 & 139.4 & 31.38 & 3.00 & - & -- & -- & -- & - & - & -- & 100 & -- & - \\
\hline 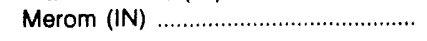 & 199 & 130.7 & 29.13 & 3.44 & -- & - & - & - & -- & - & - & 100 & - & - \\
\hline Houston Lighting \& Power Co ......... & 1,445 & 157.5 & 24.03 & .58 & - & - & - & - & 28,321 & 234.3 & 2.41 & 43 & - & 57 \\
\hline Bertron (TX) . & - & -- & -- & - & - & -- & - & - & 1,745 & 245.3 & 2.51 & -- & -- & 100 \\
\hline 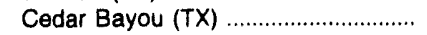 & - & -- & -- & -- & - & - & - & -- & 7,866 & 231.8 & 2.38 & - & -- & 100 \\
\hline 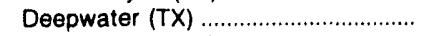 & -- & -- & - & - & - & - & -- & - & 288 & 237.7 & 2.44 & -- & -- & 100 \\
\hline 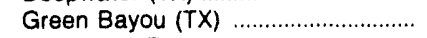 & -- & - & - & -- & -- & -- & - & - & 1,668 & 248.7 & 2.58 & - & .. & 100 \\
\hline 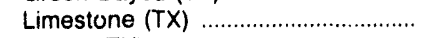 & 586 & 111.8 & 14.20 & .89 & -- & -- & - & - & 112 & 246.4 & 2.52 & 98 & - & 2 \\
\hline Parish (TX) & 859 & 180.8 & 30.74 & .37 & - & -- & -- & -- & 4,808 & & 2.2 & 75 & -- & 25 \\
\hline 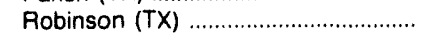 & $\ldots$ & -- & $\ldots$ & - & -- & - & - & -- & 7,615 & 234.5 & 2.41 & - & -- & 100 \\
\hline Storage Facility $\not 2$ & - & -- & - & -- & - & $\cdots$ & -- & -- & 684 & 25 & 2.58 & - & - & 100 \\
\hline 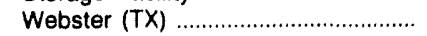 & -- & -- & - & -- & -- & -- & - & - & 1,483 & 240.2 & 2.47 & -- & -- & 100 \\
\hline Wharton $(T X)$ & -- & -- & -- & -- & - & -- & -- & -- & 2,052 & 247.1 & 2.52 & -- & -- & 100 \\
\hline Illinois Power Co & 534 & 142.2 & 29.73 & 1.43 & 1 & 412.3 & 23.80 & .30 & 115 & 333.3 & 3.39 & 99 & * & \\
\hline Baldwin (IL) .................. & 357 & 137.9 & 27.34 & 1.47 & $i$ & 412.3 & 23.90 & .30 & -- & - & - & 100 & * & - \\
\hline 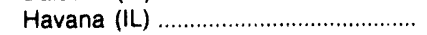 & 24 & 169.6 & 41.34 & .57 & -- & -- & - & - & - & - & -- & 100 & -- & - \\
\hline 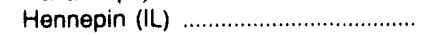 & 68 & 161.4 & 36.81 & 1.62 & - & - & - & - & 99 & 332.7 & 3.38 & 94 & - & 6 \\
\hline Vermilion (IL) ........................................ & 34 & 135.5 & 29.23 & 2.71 & - & -- & -- & -- & -- & - & - & 100 & -- & - \\
\hline 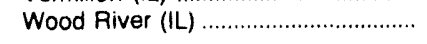 & 51 & 134.2 & 31.94 & .48 & -- & -- & - & - & 16 & 336.5 & 3.41 & 99 & - & 1 \\
\hline Imperial Irrigation District ................... & - & - & - & - & - & - & - & - & 809 & 280.6 & 2.88 & - & - & 100 \\
\hline El Centro (CA) & -- & -- & -- & -- & - & - & - & - & 809 & 280.6 & 2.88 & -- & -- & 100 \\
\hline Independence City of.. & 4 & 126.0 & 31.01 & 3.26 & - & - & - & - & 23 & 288.3 & 2.68 & 82 & - & 18 \\
\hline Blue Valley $(\mathrm{MO})$ & 4 & 126.0 & 31.01 & 3.26 & - & - & - & -- & 23 & 268.3 & 2.68 & 82 & - & 18 \\
\hline Indiana \& Michigan Electric Co ....... & 925 & 112.2 & 20.04 & .56 & 22 & 386.1 & 22.15 & .00 & - & - & - & 99 & 1 & -- \\
\hline Breed (IN) ....... & 5 & 158.6 & 33.28 & 3.65 & • & 458.6 & 26.33 & .00 & -- & -- & - & 99 & 1 & -- \\
\hline Rockport (IN) ............. & 806 & 105.9 & 18.15 & .33 & 22 & 385.4 & 22.11 & .00 & - & - & - & 99 & 1 & -- \\
\hline Tanners Creek (IN) ................................... & 113 & 144.4 & 32.89 & 2.07 & -- & -- & - & -- & - & -- & - & 100 & -- & - \\
\hline Indlana-Kentucky Electric Corp ...... & 302 & 103.0 & 23.47 & 3.48 & - & 514.9 & 29.79 & .28 & - & - & - & 100 & - & - \\
\hline Clitty Creek (IN) . & 302 & 103.0 & 23.47 & 3.48 & • & 514.9 & 29.79 & .28 & - & - & - & 100 & * & - \\
\hline Indianapolis Power \& Light Co ....... & 469 & 107.4 & 24.06 & 2.23 & 4 & 422.2 & 24.45 & .36 & - & - & - & 100 & • & - \\
\hline 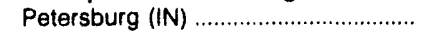 & & & & 2.43 & - & -- & - & -- & -- & - & - & & -- & -- \\
\hline 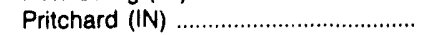 & 45 & 115.6 & 26.38 & 1.25 & 4 & 422.2 & 24.45 & .36 & -- & - & - & 98 & 2 & -- \\
\hline 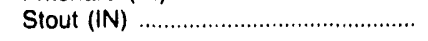 & 70 & 117.3 & 26.08 & 1.82 & -- & -- & -- & - & - & - & - & 100 & -- & - \\
\hline Interstate Power Co ... & 173 & 212.0 & 39. & 1.06 & - & - & - & - & 370 & 254.8 & 2.55 & 90 & - & 10 \\
\hline Dubuque $(\mid A)$ & 6 & 197.7 & 46.03 & 2.71 & - & - & - & -- & -- & - & - & 100 & -- & -- \\
\hline Fox Lake (MN) & -- & - & -- & - & - & - & - & - & 352 & 254.0 & 2.54 & - & -- & 100 \\
\hline Kapp (IA) & 41 & 137.3 & 30.74 & 2.03 & -- & -- & -- & - & 19 & 269.0 & 2.71 & 98 & -- & 2 \\
\hline 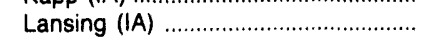 & 126 & 243.9 & 42.62 & .66 & - & -- & -- & - & -- & - & - & 100 & - & - \\
\hline lowa Electric Light \& Power Co ..... & 109 & 113.8 & 20.98 & .81 & 1 & 421.1 & 24.50 & .00 & 91 & 332.9 & 3.33 & 96 & : & \\
\hline Praire Creek (IA) & 64 & 123.5 & 22.96 & .90 & 1 & 421.1 & 24.50 & .00 & 3 & & 3.60 & 99 & •" & \\
\hline Sutherland $(\mid A)$ & 35 & 77.5 & 13.20 & .28 & -- & - & -- & - & 40 & 326.0 & 3.26 & 94 & -- & 6 \\
\hline 6th St (IA) & 10 & 158.8 & 35.56 & 2.13 & - & $\cdots$ & -- & - & 48 & 337.0 & 3.37 & 82 & - & 18 \\
\hline
\end{tabular}

See notes and footnotes at end of table. 
Table 63. Receipts, Average Cost, and Quality of Fossil Fuels Delivered to U.S. Electric Utilities by Company and Plant, September 1993 (Continued)

\begin{tabular}{|c|c|c|c|c|c|c|c|c|c|c|c|c|c|c|}
\hline \multirow{3}{*}{$\begin{array}{l}\text { Utility (Holding Company) } \\
\text { Plant (State) }\end{array}$} & \multicolumn{4}{|c|}{ Coal } & \multicolumn{4}{|c|}{ Petroleum' } & \multicolumn{3}{|c|}{ an } & \multicolumn{3}{|c|}{ \% of Total Btu } \\
\hline & \multirow{2}{*}{\begin{tabular}{|c|} 
Peceipts \\
$(1,000$ \\
tons $)$
\end{tabular}} & \multicolumn{2}{|c|}{$\begin{array}{l}\text { Average } \\
\text { Cost? }\end{array}$} & \multirow{2}{*}{$\begin{array}{l}\text { Avg. } \\
\text { Sut- } \\
\text { fur } \\
\%\end{array}$} & \multirow{2}{*}{\begin{tabular}{|c|} 
Recelpte \\
$(1,000$ \\
bbla $)$
\end{tabular}} & \multicolumn{2}{|c|}{$\begin{array}{l}\text { Average } \\
\text { Coot" }\end{array}$} & \multirow{2}{*}{$\begin{array}{l}\text { Avg. } \\
\text { sut } \\
\text { fur } \\
\%\end{array}$} & \multirow{2}{*}{\begin{tabular}{c|} 
Recelpts \\
$\left(\begin{array}{c}1,000 \\
\text { Mcf })\end{array}\right.$
\end{tabular}} & \multicolumn{2}{|c|}{$\begin{array}{l}\text { Average } \\
\text { Cost }^{3}\end{array}$} & \multirow[b]{2}{*}{ Coal } & \multirow[b]{2}{*}{$\begin{array}{l}\text { Po- } \\
\text { tro- } \\
\text { leum }\end{array}$} & \multirow[b]{2}{*}{ Cas } \\
\hline & & $\begin{array}{l}\text { (Cents } \\
\text { per } \\
10^{\circ} \\
\text { Btu) }\end{array}$ & $\begin{array}{l}\text { (s per } \\
\text { short } \\
\text { ton) }\end{array}$ & & & $\begin{array}{l}\text { (Cents } \\
\text { per } \\
10^{\circ} \\
\text { Btu) }\end{array}$ & bor & & & $\begin{array}{l}\text { (Cents } \\
\text { per } \\
10^{\circ} \\
\text { Btu) }\end{array}$ & $\begin{array}{l}5 \text { per } \\
\text { Mcf }\end{array}$ & & & \\
\hline 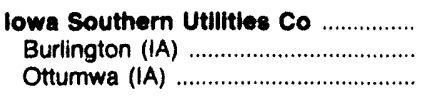 & $\begin{array}{r}306 \\
57 \\
249\end{array}$ & $\begin{array}{r}102.0 \\
87.8 \\
105.3\end{array}$ & $\begin{array}{l}17.17 \\
14.96 \\
17.68\end{array}$ & $\begin{array}{r}0.48 \\
1.05 \\
.35\end{array}$ & $\stackrel{\cdot}{-}$ & $\begin{array}{l}422.7 \\
422.7 \\
-\end{array}$ & $\begin{array}{c}24.48 \\
24.48 \\
-\end{array}$ & $\begin{array}{r}0.04 \\
.04 \\
-\end{array}$ & - & $\overline{-}$ & - & $\begin{array}{l}100 \\
100 \\
100\end{array}$ & $\stackrel{-}{-}$ & - \\
\hline 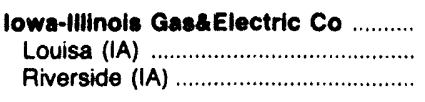 & $\begin{array}{r}235 \\
224 \\
11\end{array}$ & $\begin{array}{r}94.0 \\
92.5 \\
117.1\end{array}$ & $\begin{array}{l}15.95 \\
15.42 \\
26.73\end{array}$ & $\begin{array}{r}.42 \\
.32 \\
2.47\end{array}$ & - & - & $\overline{-}$ & - & $\begin{array}{r}29 \\
6 \\
23\end{array}$ & $\begin{array}{l}388.8 \\
310.4 \\
409.1\end{array}$ & $\begin{array}{l}3.98 \\
3.15 \\
4.19\end{array}$ & $\begin{array}{r}99 \\
100 \\
91\end{array}$ & - & $\begin{array}{r}1 \\
9\end{array}$ \\
\hline $\begin{array}{l}\text { Jeckeonvilie Electric Auth } \\
\text { Kennedy (FL) } \\
\text { Northside (FL) } \\
\text { Southside (FL) } \\
\text { St Johns River (FL) }\end{array}$ & $\begin{array}{l}308 \\
- \\
- \\
-\end{array}$ & $\begin{array}{l}150.5 \\
-- \\
- \\
150.5\end{array}$ & $\begin{array}{l}36.54 \\
- \\
- \\
36.54\end{array}$ & $\begin{array}{l}.88 \\
-- \\
- \\
- \\
.88\end{array}$ & $\begin{array}{r}297 \\
- \\
- \\
-\end{array}$ & $\begin{array}{l}211.2 \\
\overline{203.9} \\
225.5 \\
-\end{array}$ & $\begin{array}{c}13.43 \\
\overline{12.97} \\
14.32 \\
--\end{array}$ & $\begin{array}{l}1.42 \\
\overline{1.65} \\
.97 \\
-\end{array}$ & $\begin{array}{r}327 \\
31 \\
296 \\
-\quad\end{array}$ & $\begin{array}{l}263.8 \\
263.8 \\
263.8 \\
263.8 \\
--\end{array}$ & $\begin{array}{l}2.74 \\
2.74 \\
2.74 \\
2.74 \\
--\end{array}$ & $\begin{array}{l}77 \\
-- \\
- \\
100\end{array}$ & $\begin{array}{l}19 \\
-\overline{98} \\
67 \\
-\end{array}$ & $\begin{array}{r}3 \\
100 \\
2 \\
33 \\
--\end{array}$ \\
\hline 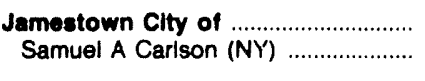 & $\begin{array}{l}9 \\
9\end{array}$ & $\begin{array}{l}128.9 \\
128.9\end{array}$ & $\begin{array}{l}32.92 \\
32.92\end{array}$ & $\begin{array}{l}1.83 \\
1.83\end{array}$ & - & - & - & - & - & - & - & $\begin{array}{l}100 \\
100\end{array}$ & - & - \\
\hline $\begin{array}{l}\text { Jersey Central Power\&Lught Co } \ldots . . . \\
\text { Gilbert (NJ) } \\
\text { Sayreville (NJ) }\end{array}$ & - & $\overline{-}$ & $\overline{-}$ & $\overline{-}$ & $\begin{array}{r}35 \\
-\quad 35 \\
-\end{array}$ & $\begin{array}{l}393.0 \\
393.0 \\
-\end{array}$ & $\begin{array}{c}22.66 \\
22.66 \\
-\end{array}$ & $\begin{array}{l}.16 \\
.16 \\
-\end{array}$ & $\begin{array}{r}194 \\
113 \\
81\end{array}$ & $\begin{array}{l}274.2 \\
280.8 \\
265.0\end{array}$ & $\begin{array}{l}2.83 \\
2.91 \\
2.73\end{array}$ & - & $\begin{array}{c}50 \\
63 \\
-\end{array}$ & $\begin{array}{r}50 \\
37 \\
100\end{array}$ \\
\hline 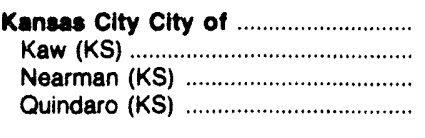 & $\begin{array}{r}160 \\
12 \\
110 \\
39\end{array}$ & $\begin{array}{r}148.0 \\
125.8 \\
93.5 \\
266.5\end{array}$ & $\begin{array}{l}27.40 \\
26.82 \\
15.58 \\
61.12\end{array}$ & $\begin{array}{r}.73 \\
.43 \\
.38 \\
1.84\end{array}$ & - & $\begin{array}{l}- \\
-- \\
-\end{array}$ & $\overline{-}$ & - & $\begin{array}{r}59 \\
9 \\
49\end{array}$ & $\begin{array}{l}318.0 \\
334.8 \\
- \\
314.7\end{array}$ & $\begin{array}{l}3.17 \\
3.39 \\
3.12\end{array}$ & $\begin{array}{r}98 \\
96 \\
100 \\
95\end{array}$ & - & $\begin{array}{r}2 \\
-4 \\
-5\end{array}$ \\
\hline $\begin{array}{l}\text { Kaneas Clty Power \& LIght Co } \ldots \ldots . . . \\
\text { Hawthorne (MO) } \\
\text { latan (MO) } \\
\text { La Cygne (KS) } \\
\text { Montrose (MO) }\end{array}$ & $\begin{array}{r}966 \\
23 \\
326 \\
455 \\
162\end{array}$ & $\begin{array}{l}84.1 \\
82.6 \\
84.9 \\
79.7 \\
94.9\end{array}$ & $\begin{array}{l}14.64 \\
14.57 \\
14.92 \\
13.75 \\
16.61\end{array}$ & $\begin{array}{l}.38 \\
.15 \\
.33 \\
.49 \\
.20\end{array}$ & $\begin{array}{l}- \\
- \\
- \\
-\end{array}$ & $\begin{array}{l}- \\
- \\
-\end{array}$ & $\begin{array}{l}- \\
- \\
- \\
-\end{array}$ & $\begin{array}{l}- \\
- \\
- \\
-\end{array}$ & - & $\begin{array}{l}260.2 \\
260.2 \\
- \\
- \\
-\end{array}$ & $\begin{array}{l}2.60 \\
2.60 \\
- \\
- \\
-\end{array}$ & $\begin{array}{r}100 \\
97 \\
100 \\
100 \\
100\end{array}$ & $\begin{array}{l}- \\
-- \\
-- \\
-\end{array}$ & $\begin{array}{l}-3 \\
-- \\
- \\
-\end{array}$ \\
\hline 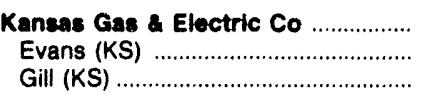 & - & - & - & - & - & - & - & - & $\begin{array}{r}624 \\
569 \\
55\end{array}$ & $\begin{array}{l}238.2 \\
236.4 \\
256.3\end{array}$ & $\begin{array}{l}2.30 \\
2.29 \\
2.43\end{array}$ & - & - & $\begin{array}{l}100 \\
100 \\
100\end{array}$ \\
\hline $\begin{array}{l}\text { Kansas Power \& Lht Co ............... } \\
\text { Jeffrey Energy Cnt (KS) } \\
\text { Lawrence (KS) } \\
\text { Tecumseh (KS) }\end{array}$ & $\begin{array}{r}756 \\
661 \\
61 \\
34\end{array}$ & $\begin{array}{l}107.5 \\
104.4 \\
125.7 \\
123.1\end{array}$ & $\begin{array}{l}18.36 \\
17.23 \\
26.16 \\
26.23\end{array}$ & $\begin{array}{l}.36 \\
.35 \\
.46 \\
.45\end{array}$ & - & 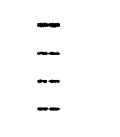 & $\begin{array}{l}- \\
- \\
-\end{array}$ & $\overline{-}$ & $\begin{array}{r}43 \\
34 \\
9\end{array}$ & $\begin{array}{l}266.1 \\
\overline{282.9} \\
206.6\end{array}$ & $\begin{array}{l}2.49 \\
\overline{2.61} \\
2.03\end{array}$ & $\begin{array}{r}100 \\
100 \\
98 \\
99\end{array}$ & $\overline{-}$ & $\begin{array}{r}- \\
- \\
2\end{array}$ \\
\hline 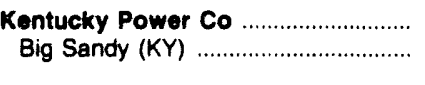 & $\begin{array}{l}180 \\
180\end{array}$ & $\begin{array}{l}107.8 \\
107.8\end{array}$ & $\begin{array}{l}26.11 \\
26.11\end{array}$ & $\begin{array}{l}1.11 \\
1.11\end{array}$ & 4 & $\begin{array}{l}435.9 \\
435.9\end{array}$ & $\begin{array}{l}25.24 \\
25.24\end{array}$ & $\begin{array}{l}.00 \\
.00\end{array}$ & - & - & - & $\begin{array}{l}99 \\
99\end{array}$ & $\begin{array}{l}1 \\
1\end{array}$ & - \\
\hline 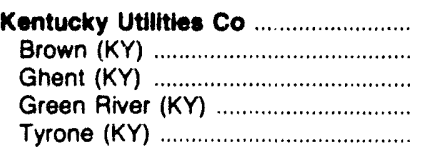 & $\begin{array}{r}488 \\
111 \\
321 \\
47 \\
9\end{array}$ & $\begin{array}{l}115.0 \\
114.8 \\
116.3 \\
105.6 \\
118.5\end{array}$ & $\begin{array}{l}27.98 \\
27.64 \\
28.48 \\
24.96 \\
29.80\end{array}$ & $\begin{array}{r}1.51 \\
1.43 \\
1.46 \\
2.17 \\
.94\end{array}$ & - & $\begin{array}{l}494.7 \\
493.2 \\
496.3 \\
- \\
--\end{array}$ & $\begin{array}{l}29.09 \\
29.00 \\
29.18 \\
- \\
-\end{array}$ & $\begin{array}{r}.40 \\
.40 \\
.40 \\
-- \\
--\end{array}$ & $\begin{array}{l}- \\
- \\
-\end{array}$ & $\begin{array}{l}- \\
- \\
-\end{array}$ & $\begin{array}{l}- \\
- \\
- \\
-\end{array}$ & $\begin{array}{r}100 \\
99 \\
100 \\
100 \\
100\end{array}$ & $\begin{array}{c}\cdot 1 \\
. \\
--\end{array}$ & $\overline{-}$ \\
\hline $\begin{array}{l}\text { Lafayette Clty of } \\
\text { Bonin (LA) }\end{array}$ & - & $\overline{-}$ & - & - & $\overline{-}$ & - & - & - & $\begin{array}{l}416 \\
416\end{array}$ & $\begin{array}{l}247.3 \\
247.3\end{array}$ & $\begin{array}{l}2.58 \\
2.58\end{array}$ & - & - & $\begin{array}{l}100 \\
100\end{array}$ \\
\hline 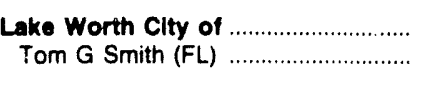 & - & - & - & - & $\begin{array}{l}1 \\
1\end{array}$ & $\begin{array}{l}437.0 \\
437.0\end{array}$ & $\begin{array}{l}25.63 \\
25.63\end{array}$ & $\begin{array}{l}.14 \\
.14\end{array}$ & $\begin{array}{l}168 \\
168\end{array}$ & $\begin{array}{l}294.0 \\
294.0\end{array}$ & $\begin{array}{l}3.03 \\
3.03\end{array}$ & - & $\begin{array}{l}3 \\
3\end{array}$ & $\begin{array}{l}97 \\
97\end{array}$ \\
\hline 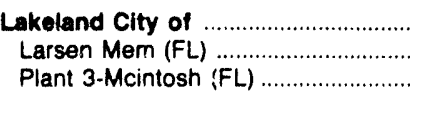 & -84 & $\begin{array}{l}165.8 \\
\overline{165.8}\end{array}$ & $\begin{array}{l}42.85 \\
\overline{42.85}\end{array}$ & $\frac{1.14}{1.14}$ & -86 & $\frac{239.1}{239.1}$ & $\frac{15.02}{15.02}$ & $\begin{array}{l}1.94 \\
-- \\
1.94\end{array}$ & $\begin{array}{l}509 \\
344 \\
165\end{array}$ & $\begin{array}{l}248.9 \\
250.0 \\
249.7\end{array}$ & $\begin{array}{l}2.58 \\
2.58 \\
2.57\end{array}$ & $\begin{array}{c}67 \\
-- \\
75\end{array}$ & $\frac{17}{19}$ & $\begin{array}{r}16 \\
100 \\
6\end{array}$ \\
\hline $\begin{array}{l}\text { Lansing Clty of } \ldots \ldots \ldots \ldots \ldots \ldots \ldots \ldots \ldots \ldots \ldots \ldots \ldots \ldots \ldots \ldots \ldots \ldots \ldots \ldots \ldots \ldots \ldots \ldots \ldots \ldots \ldots \ldots \ldots \ldots \ldots \ldots \ldots \\
\text { Eckert (MI) }\end{array}$ & $\begin{array}{l}34 \\
20 \\
15\end{array}$ & $\begin{array}{l}174.3 \\
175.8 \\
172.5\end{array}$ & $\begin{array}{l}44.05 \\
43.90 \\
44.24\end{array}$ & $\begin{array}{l}.88 \\
.86 \\
.91\end{array}$ & 1 & $\begin{array}{l}421.0 \\
421.0 \\
421.0\end{array}$ & $\begin{array}{l}24.40 \\
24.40 \\
24.40\end{array}$ & $\begin{array}{l}.30 \\
.30 \\
.30\end{array}$ & - & - & - & $\begin{array}{r}100 \\
99 \\
100\end{array}$ & . 1 & - \\
\hline Long Island Lighting Co ................... & - & - & - & - & 807 & 273.4 & 17.49 & .95 & 4,122 & 253.6 & 2.59 & - & 55 & 45 \\
\hline
\end{tabular}

See notes and footnotes at end of table. 
Table 63. Receipts, Average Cost, and Quality of Fossil Fuels Dellvered to U.S. Electric Utilities by Company and Plant, September 1993 (Continued)

\begin{tabular}{|c|c|c|c|c|c|c|c|c|c|c|c|c|c|c|}
\hline \multirow{3}{*}{$\begin{array}{l}\text { Utility (Holding Company) } \\
\text { Plant (State) }\end{array}$} & \multicolumn{4}{|c|}{ Coal } & \multicolumn{4}{|c|}{ Petroleum' } & \multicolumn{3}{|c|}{ Cas } & \multicolumn{3}{|c|}{ \% of Total Btu } \\
\hline & \multirow{2}{*}{\begin{tabular}{|c|} 
Recelpts \\
$(1,000$ \\
tons $)$
\end{tabular}} & \multicolumn{2}{|c|}{$\begin{array}{l}\text { Average } \\
\text { Cost }^{2}\end{array}$} & \multirow{2}{*}{$\begin{array}{l}\text { Avo. } \\
\text { Sut } \\
\text { fur } \\
\%\end{array}$} & \multirow{2}{*}{$\begin{array}{c}\text { Recelpts } \\
\begin{array}{c}(1,000 \\
\text { bble })\end{array}\end{array}$} & \multicolumn{2}{|c|}{$\begin{array}{l}\text { Average } \\
\text { Coot? }\end{array}$} & \multirow{2}{*}{$\begin{array}{l}\text { Avg. } \\
\text { sut } \\
\text { fur } \\
\%\end{array}$} & \multirow{2}{*}{$\begin{array}{c}\text { Recelpte } \\
\begin{array}{c}1,000 \\
\text { Mct }\end{array}\end{array}$} & \multicolumn{2}{|c|}{$\begin{array}{l}\text { Average } \\
\text { Cost }\end{array}$} & \multirow[b]{2}{*}{ Coal } & \multirow{2}{*}{$\begin{array}{l}\text { Po- } \\
\text { tro- } \\
\text { loum }\end{array}$} & \multirow[b]{2}{*}{ Cas } \\
\hline & & $\begin{array}{c}\text { (Cents } \\
\text { per } \\
10^{6} \\
\text { Btu) }\end{array}$ & $\begin{array}{l}\text { (6 per } \\
\text { chort } \\
\text { ton) }\end{array}$ & & & $\begin{array}{c}\text { (Cents } \\
\text { per } \\
10^{\circ} \\
\text { Btu) }\end{array}$ & bor & & & $\begin{array}{c}\text { (Cents } \\
\text { per } \\
10^{6} \\
\text { Btu) }\end{array}$ & $\begin{array}{l}\text { per } \\
\text { Mef }\end{array}$ & & & \\
\hline Long Island Lighting Co & & & & & & & & & & & & & & \\
\hline 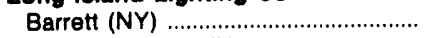 & - & - & - & - & - & -- & - & - & 1,852 & 254.8 & 2.62 & -- & - & 100 \\
\hline 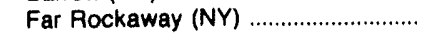 & -- & - & - & - & - & -- & - & - & 153 & 247.6 & 2.56 & - & -- & 100 \\
\hline 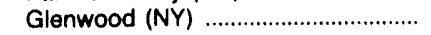 & -- & -- & - & - & - & - & - & - & 310 & 257.0 & 2.63 & - & - & 100 \\
\hline 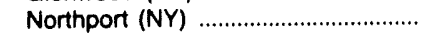 & -- & - & - & - & 615 & 273.1 & 17.49 & 0.99 & 1,798 & 252.3 & 2.56 & - & 68 & 32 \\
\hline 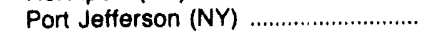 & - & - & - & - & 192 & 274.4 & 17.47 & .83 & - & - & - & -- & 100 & - \\
\hline 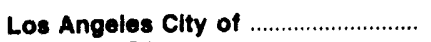 & 396 & 150.5 & $\mathbf{3 5 . 3 3}$ & 0.45 & - & - & - & - & 5,182 & 348.9 & $\mathbf{3 . 5 7}$ & 64 & - & 36 \\
\hline Haynes (CA) & -- & - & - & - & - & - & - & - & 3,260 & 348.9 & 3.56 & - & - & 100 \\
\hline 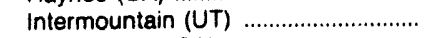 & 396 & 150.5 & 35.33 & .45 & - & $r$ & - & - & - & -- & - & 100 & - & - \\
\hline 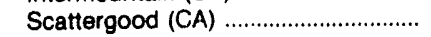 & - & - & - & - & - & - & - & - & 1,605 & 348.9 & 3.59 & - & - & 100 \\
\hline 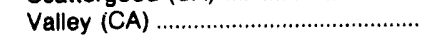 & - & - & - & - & - & - & - & - & 317 & 348.9 & 3.58 & - & - & 100 \\
\hline Louisiana Power \& Light Co ............ & - & - & - & - & - & - & - & - & 13,410 & 266.1 & 2.79 & - & - & 100 \\
\hline 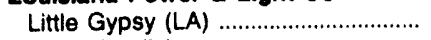 & - & - & - & - & - & - & - & - & 3,701 & 270.2 & 2.81 & - & - & 100 \\
\hline 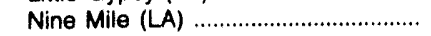 & -- & - & - & - & - & - & - & - & 6,267 & 269.6 & 2.82 & - & - & 100 \\
\hline 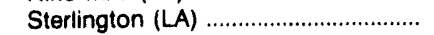 & -- & - & - & - & - & - & -- & -- & 1,007 & 253.0 & 2.67 & -- & - & 100 \\
\hline 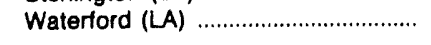 & - & - & - & - & - & - & - & - & 2,435 & 256.5 & 2.72 & - & - & 100 \\
\hline Louisville Gas \& Electric Co ............. & 514 & 113.9 & 26.79 & 2.02 & - & - & - & - & 24 & 297.1 & 3.05 & 100 & - & * \\
\hline 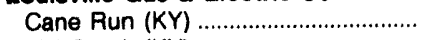 & 105 & 116.6 & 27.42 & 3.06 & - & - & - & - & 14 & 297.1 & 3.05 & 99 & - & 1 \\
\hline 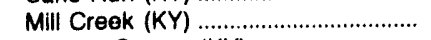 & 270 & 113.6 & 26.85 & 3.03 & - & - & - & - & 10 & 297.1 & 3.05 & 100 & - & $\cdot$ \\
\hline Trimble County (KY) ............................. & 139 & 112.5 & 26.19 & 2.61 & - & - & - & - & - & - & - & 100 & - & - \\
\hline Lower Colorado Rlver Authority .... & 490 & 125.8 & 21.66 & .40 & - & - & - & - & 2,604 & 227.4 & 2.37 & 76 & - & 24 \\
\hline Gideon (TX) & -- & - & - & - & - & - & - & - & 1,822 & 230.3 & 2.41 & - & - & 100 \\
\hline S Seymour-Fayette $(\mathrm{TX})$ & 490 & 125.8 & 21.66 & .40 & - & - & - & - & - & - & - & 100 & - & - \\
\hline 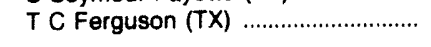 & - & - & - & - & - & - & - & - & 782 & 220.7 & 2.29 & - & - & 100 \\
\hline 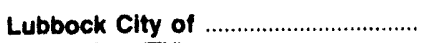 & - & - & - & - & - & - & - & - & 457 & 237.8 & 2.40 & - & - & 100 \\
\hline 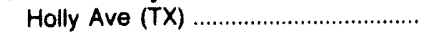 & -- & - & - & - & - & - & - & - & 457 & 237.8 & 2.40 & - & - & 100 \\
\hline Madison Gas E Electric Co ............... & 1 & 100.0 & 24.24 & .76 & - & - & - & - & 89 & 196.2 & 1.98 & 21 & - & 79 \\
\hline 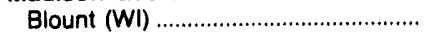 & 1 & 100.0 & 24.24 & .76 & - & - & - & - & 89 & 196.2 & 1.88 & 21 & - & 79 \\
\hline Manitowoc Public Utilities .................... & 14 & 167.9 & 42.63 & .97 & - & - & - & - & - & - & - & 100 & - & - \\
\hline 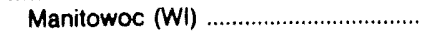 & 14 & 167.9 & 42.63 & .97 & - & - & - & - & - & - & - & 100 & - & - \\
\hline 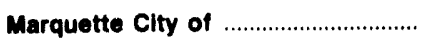 & 3 & 298.8 & 73.90 & .78 & - & - & - & - & - & - & - & 100 & - & - \\
\hline 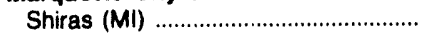 & 3 & 296.8 & 73.90 & .78 & - & - & - & - & - & - & - & 100 & - & - \\
\hline Massachusetts Mun Wholes El Co. & - & - & - & - & - & - & - & - & 502 & 239.6 & 2.44 & - & - & 100 \\
\hline Stonybrook (MA) & - & - & - & - & -- & - & - & - & 502 & 239.6 & 2.44 & - & - & 100 \\
\hline Medina Electric Coop Inc .................... & - & - & - & - & - & - & - & - & 93 & 235.0 & 2.57 & - & - & 100 \\
\hline 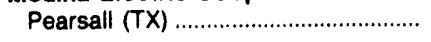 & - & - & - & - & - & - & - & - & 93 & 235.0 & 2.57 & - & - & 100 \\
\hline Metropolitan Edison Co ..................... & 98 & 175.1 & 44.91 & 1.79 & 1 & 388.7 & 22.60 & .13 & - & - & - & 100 & - & - \\
\hline Portland (PA) & 67 & 172.9 & 44.11 & 1.87 & - & - & - & - & - & - & -- & 100 & - & - \\
\hline 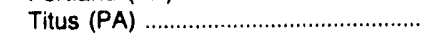 & 32 & 179.6 & 46.60 & 1.43 & 1 & 388.7 & 22.60 & .13 & - & - & - & 99 & 1 & - \\
\hline Michigan South Central Pwr Agy ... & 12 & 164.3 & 39.16 & 3.47 & - & - & - & - & - & - & - & 100 & - & - \\
\hline 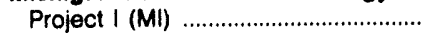 & 12 & 164.3 & 39.16 & 3.47 & - & - & - & - & - & - & - & 100 & - & - \\
\hline 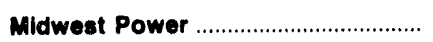 & 477 & 80.5 & 14.04 & .39 & - & - & - & - & 76 & 348.0 & 3.46 & 9 & - & 1 \\
\hline 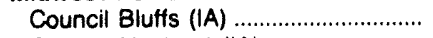 & - & - & - & - & - & - & - & - & 13 & 320.4 & 3.12 & - & - & 100 \\
\hline 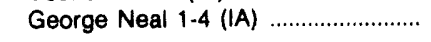 & 477 & 80.5 & 14.04 & .39 & - & - & - & - & 63 & 353.5 & 3.54 & 98 & - & 1 \\
\hline Minnesota Power \& Light Co .......... & 298 & 107.0 & 19.28 & .55 & 1 & 443.4 & 25.52 & .20 & - & - & - & 100 & : & - \\
\hline Boswell Energy Center (MN) ............... & 276 & 107.0 & 19.31 & .54 & 1 & 447.4 & 25.74 & .20 & - & - & - & 100 & * & - \\
\hline Laskin Energy Center (MN) ................. & 22 & 107.1 & 18.98 & .66 & 1 & 439.6 & 25.29 & .20 & - & - & - & 89 & 1 & - \\
\hline Minnkota Power Coop Inc ................. & 371 & 48.7 & 6.57 & .85 & 4 & 485.7 & 28.58 & .40 & - & - & - & 100 & " & - \\
\hline Young (ND) & 371 & 48.7 & 6.57 & .85 & 4 & 485.7 & 28.56 & .40 & - & - & - & 100 & • & - \\
\hline
\end{tabular}

See notes and footnotes at end of table. 
Table 63. Receipts, Average Cost, and Quality of Fossil Fuels Dellvered to U.S. Electric Utilities by Company and Plant, September 1993 (Continued)

\begin{tabular}{|c|c|c|c|c|c|c|c|c|c|c|c|c|c|c|}
\hline \multirow{3}{*}{$\begin{array}{l}\text { Utility (Holding Company) } \\
\text { Plant (State) }\end{array}$} & \multicolumn{4}{|c|}{ Coal } & \multicolumn{4}{|c|}{ Potroleum' } & \multicolumn{3}{|c|}{ Gas } & \multicolumn{3}{|c|}{$\%$ of Total Btu } \\
\hline & \multirow{2}{*}{\begin{tabular}{|c|} 
Recolpts \\
$\begin{array}{c}(1,000 \\
\text { tons })\end{array}$ \\
\end{tabular}} & \multicolumn{2}{|c|}{$\begin{array}{l}\text { Average } \\
\text { Cost }^{3}\end{array}$} & \multirow{2}{*}{$\begin{array}{l}\text { Avg. } \\
\text { Sul- } \\
\text { fur } \\
\%\end{array}$} & \multirow{2}{*}{$\begin{array}{c}\text { Recelpte } \\
\begin{array}{c}(1,000 \\
\text { bble })\end{array}\end{array}$} & \multicolumn{2}{|c|}{$\begin{array}{l}\text { Average } \\
\text { Cost? }\end{array}$} & \multirow{2}{*}{$\begin{array}{l}\text { Avg. } \\
\text { Sul- } \\
\text { fur } \\
\%\end{array}$} & \multirow{2}{*}{$\begin{array}{c}\text { Recelpts } \\
\begin{array}{c}1,000 \\
\text { Mef) }\end{array}\end{array}$} & \multicolumn{2}{|c|}{$\begin{array}{l}\text { Average } \\
\text { Cost' }\end{array}$} & \multirow[b]{2}{*}{ Coal } & \multirow[b]{2}{*}{$\begin{array}{c}\text { Po- } \\
\text { tro- } \\
\text { loum }\end{array}$} & \\
\hline & & $\begin{array}{c}\text { (Cents } \\
\text { per } \\
10^{\circ} \\
\text { Btu) }\end{array}$ & $\begin{array}{l}\text { (s per } \\
\text { short } \\
\text { ton) }\end{array}$ & & & $\begin{array}{c}\text { (Cents } \\
\text { per } \\
10^{\circ} \\
\text { Btu) }\end{array}$ & ber & & & $\begin{array}{c}\text { (Cente } \\
\text { per } \\
10^{\circ} \\
\text { Btu) }\end{array}$ & $\begin{array}{l}\text { per } \\
\text { Nat }\end{array}$ & & & Gas \\
\hline Mieslesippl Power Lught Co ......... & - & - & - & - & 853 & 176.1 & 11.35 & 2.80 & 1,444 & 268.1 & 2.78 & - & 79 & 21 \\
\hline 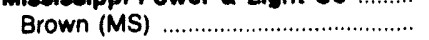 & - & - & - & - & $\cdot$ & 425.1 & 24.41 & .30 & 491 & 273.1 & 2.82 & -- & 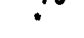 & 100 \\
\hline Delta (MS) & -- & -- & -- & - & - & - & - & - & 445 & 256.9 & 2.67 & -- & - & 100 \\
\hline Gerald Andrus (MS) & - & -- & - & - & 395 & 178.1 & 11.61 & 2.94 & - & - & - & - & 100 & - \\
\hline 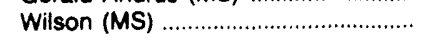 & - & - & -- & - & 458 & 174.3 & 11.12 & 2.87 & 507 & 273.1 & 2.82 & - & 85 & 15 \\
\hline Mieslestppl Power Co ........................... & 245 & 152.6 & 37.61 & 1.40 & 1 & 399.0 & 23.13 & .00 & 158 & 237.8 & 2.42 & 97 & - & 3 \\
\hline Daniel (MS) & 142 & 165.4 & 39.86 & .54 & - & 402.0 & 23.22 & .00 & - & - & - & 100 & . & - \\
\hline Eaton (MS) & - & - & -- & - & $\cdot$ & 392.7 & 22.93 & .00 & 35 & 224.3 & 2.28 & - & 3 & 97 \\
\hline 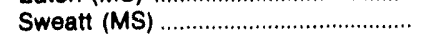 & -- & - & -- & $-\cdots$ & -- & -- & - & $-\infty$ & 33 & 238.5 & 2.44 & -- & $-\infty$ & 100 \\
\hline Watson (MS) & 103 & 135.9 & 34.51 & 2.58 & - & - & -- & - & 89 & 243.0 & 2.47 & 97 & - & 3 \\
\hline Missouri Public Service Comm ....... & 132 & 105.9 & 22.58 & .42 & - & - & - & - & - & - & - & 100 & - & - \\
\hline Sibley $(\mathrm{MO})$ & 132 & 105.9 & 22.58 & .42 & - & - & - & -- & - & - & -- & 100 & -- & - \\
\hline Monongahela Power Co ................... & 656 & 130.3 & 32.95 & 2.36 & 11 & 434.6 & 25.74 & .30 & 9 & 408.4 & 4.06 & 100 & - & • \\
\hline Albright (WV) & 26 & 101.1 & 25.15 & 1.54 & 1 & 448.1 & 26.54 & .30 & -- & - & $-\ldots$ & 100 & * & -- \\
\hline 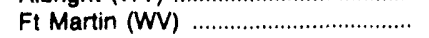 & 193 & 145.7 & 36.60 & 1.28 & 2 & 445.5 & 26.38 & .30 & -- & - & - & 100 & - & - \\
\hline 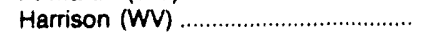 & 211 & 144.3 & 38.08 & 2.88 & $\cdot$ & 446.5 & 26.44 & .30 & 9 & 406.4 & 4.06 & 100 & $\cdot$ & $\cdot$ \\
\hline 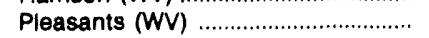 & 168 & 100.5 & 24.70 & 3.41 & 7 & 425.9 & 25.22 & .30 & -- & - & -- & 99 & 1 & - \\
\hline 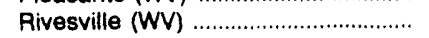 & 12 & 123.1 & 30.33 & .81 & -- & -- & - & - & - & - & -- & 100 & - & - \\
\hline 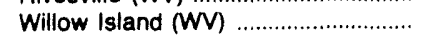 & 46 & 122.2 & 29.19 & 1.59 & $\cdot$ & 545.8 & 32.32 & .30 & - & -- & -- & 100 & $\cdot$ & - \\
\hline Montana Power Co ............................ & 801 & 66.8 & 11.48 & .65 & - & - & - & - & - & - & - & 100 & - & - \\
\hline Colstrip (MT) & 730 & 66.4 & $\$ 1.39$ & .66 & -- & -- & -- & - & - & - & - & 100 & - & -- \\
\hline Corette (MT) & 71 & 71.2 & 12.45 & .58 & - & - & - & - & - & -- & -- & 100 & -- & -- \\
\hline Montana-Dakota Utillties Co ............ & 211 & 84.2 & 11.70 & 1.27 & 2 & 471.6 & 27.05 & .30 & $\cdot$ & 442.1 & 4.94 & 100 & - & • \\
\hline Coyote (ND) & 160 & 77.9 & 10.87 & 1.41 & 2 & 471.6 & 27.05 & .30 & - & - & - & 100 & • & -- \\
\hline Heskett (ND) & 33 & 105.8 & 14.86 & .93 & -- & - & - & 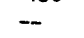 & • & 425.5 & 4.69 & 100 & - & 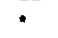 \\
\hline Lewis and Clark (MT) ......................... & 18 & 100.7 & 13.20 & .63 & -- & -- & -- & -- & $\cdot$ & 450.3 & 5.07 & 100 & - & - \\
\hline Montaup Electric Co ........................... & 14 & 177.8 & 45.18 & 1.41 & - & - & - & - & - & - & - & 100 & - & - \\
\hline Somerset (MA) & 14 & 177.8 & 45.18 & 1.41 & -- & -- & -- & - & -- & -- & - & 100 & -- & -- \\
\hline Morgan Clty City of ............................. & - & - & - & - & - & - & - & - & 95 & 254.0 & 2.71 & - & - & 100 \\
\hline 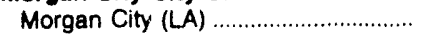 & - & -- & - & -- & -- & -- & -- & - & 95 & 254.0 & 2.71 & -- & -- & 100 \\
\hline Muscatine Clty of ................................ & 108 & 83.9 & 13.98 & .97 & - & - & - & - & 1 & 311.9 & 3.18 & 100 & - & - \\
\hline 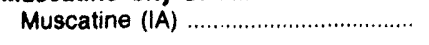 & 108 & 83.9 & 13.98 & .97 & -- & -- & -- & - & 1 & 311.9 & 3.18 & 100 & - & - \\
\hline Nobraska Public Power District ...... & 358 & 72.5 & 12.74 & .33 & - & - & - & - & 43 & 291.8 & 2.77 & 99 & - & 1 \\
\hline 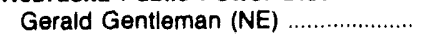 & 279 & 70.9 & 12.45 & .33 & -- & -- & -- & -- & 42 & 289.0 & 2.75 & 99 & - & 1 \\
\hline Sheldon (NE) & 79 & 78.2 & 13.78 & .32 & -- & - & -- & -- & $\cdot$ & 551.8 & 5.52 & 100 & - & $\cdot$ \\
\hline Novada Power Co .................................. & 92 & 196.1 & 46.66 & .49 & - & - & - & - & 374 & 245.5 & 2.52 & 85 & - & 15 \\
\hline Clark (NV) & $-\infty$ & -- & - & - & -- & - & -- & - & 374 & 245.5 & 2.52 & $-\infty$ & - & 100 \\
\hline Gardner (NV) & 92 & 196.1 & 46.66 & .49 & - & - & -- & -- & - & - & -- & 100 & -- & -- \\
\hline Now England Power Co .................... & 286 & 162.1 & 41.61 & .87 & 276 & 400.0 & 25.38 & 2.00 & 32 & 271.4 & 2.76 & 79 & 20 & • \\
\hline Brayton (MA) & 230 & 165.2 & 42.45 & .92 & - & -- & - & - & - & - & - & 100 & $-\infty$ & $\ldots$ \\
\hline 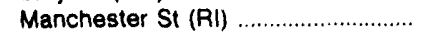 & -- & - & - & -- & - & - & -- & - & 32 & 271.4 & 2.76 & -- & -- & 100 \\
\hline 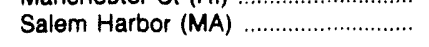 & 36 & 142.0 & 36.30 & .57 & 276 & 400.0 & 25.38 & 2.00 & - & -- & - & 35 & 65 & -- \\
\hline Now Orleans Public Service Inc ...... & - & - & - & - & - & - & - & - & 2,547 & 252.6 & 2.64 & - & - & 100 \\
\hline 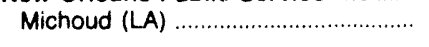 & -- & -- & -- & -- & -- & - & -- & - & 2,547 & 252.6 & 2.64 & -- & -- & 100 \\
\hline Now York State Elec Gas Corp ... & 205 & 132.6 & 33.32 & 1.39 & 1 & 532.4 & 30.63 & .14 & - & - & - & 100 & * & - \\
\hline Goudey (NY) & 17 & 126.2 & 32.40 & 1.80 & • & 516.7 & 29.73 & .14 & - & -- & -- & 100 & • & - \\
\hline 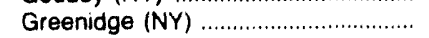 & 37 & 134.0 & 34.58 & 1.86 & -- & -- & - & - & - & - & -- & 100 & - & - \\
\hline 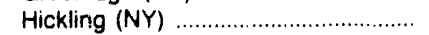 & 17 & 129.5 & 28.00 & .83 & - & - & - & - & -- & - & -- & 100 & - & $\ldots$ \\
\hline 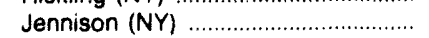 & 20 & 155.2 & 34.40 & .79 & -- & -- & - & - & - & - & - & 100 & - & - \\
\hline 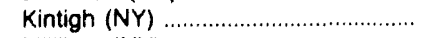 & 74 & 132.2 & 34.03 & 1.40 & $\cdot$ & 569.3 & 32.76 & .14 & - & - & - & 100 & • & -- \\
\hline 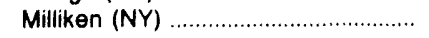 & 38 & 125.7 & 32.99 & 1.31 & 1 & 527.7 & 30.36 & .14 & - & -- & -- & 99 & 1 & - \\
\hline
\end{tabular}

See notes and footnotes at end of table. 
Table 63. Recelpts, Average Cost, and Quality of Fossill Fuels Dellvered to U.S. Electric Utilities by Company and Plant, September 1993 (Continued)

\begin{tabular}{|c|c|c|c|c|c|c|c|c|c|c|c|c|c|c|}
\hline \multirow{3}{*}{$\begin{array}{c}\text { Utllity (Holding Company) } \\
\text { Plant (State) }\end{array}$} & \multicolumn{4}{|c|}{ Coal } & \multicolumn{4}{|c|}{ Potroloum' } & \multicolumn{3}{|c|}{ ans } & \multicolumn{3}{|c|}{ \% of Total Btu } \\
\hline & \multirow{2}{*}{\begin{tabular}{|c|} 
Recelpts \\
$(1,000$ \\
tons $)$
\end{tabular}} & \multicolumn{2}{|c|}{$\begin{array}{l}\text { Average } \\
\text { Cost' }^{3}\end{array}$} & \multirow{2}{*}{$\begin{array}{l}\text { Avg. } \\
\text { Sul- } \\
\text { fur } \\
\%\end{array}$} & \multirow{2}{*}{\begin{tabular}{|c|} 
Recolpts \\
$\begin{array}{c}(1,000 \\
\text { bble })\end{array}$
\end{tabular}} & \multicolumn{2}{|c|}{$\begin{array}{l}\text { Avorage } \\
\text { Cost' }^{\circ}\end{array}$} & \multirow{2}{*}{$\begin{array}{c}\text { Avg. } \\
\text { sul- } \\
\text { fur } \\
\%\end{array}$} & \multirow{2}{*}{$\begin{array}{c}\text { Recolpte } \\
\begin{array}{c}1,000 \\
\text { Men }\end{array}\end{array}$} & \multicolumn{2}{|c|}{$\begin{array}{l}\text { Average } \\
\text { Coot }\end{array}$} & \multirow[b]{2}{*}{ Coal } & \multirow[b]{2}{*}{$\begin{array}{l}\text { Po- } \\
\text { tro- } \\
\text { loum }\end{array}$} & \\
\hline & & $\begin{array}{l}\text { (Cente } \\
\text { per } \\
10^{\circ} \\
\text { Btu) }\end{array}$ & $\begin{array}{l}\text { (5 per } \\
\text { short } \\
\text { ton) }\end{array}$ & & & $\begin{array}{c}\text { (Cente } \\
\text { per } \\
10^{4} \\
\text { Btu) }\end{array}$ & spor & & & $\begin{array}{l}\text { (Cente } \\
\text { por } \\
10^{4} \\
\text { Btu) }\end{array}$ & Sor & & & Oas \\
\hline $\begin{array}{l}\text { Niagara Mohawk Power Corp } \\
\text { Albany (NY) }\end{array}$ & $\begin{array}{r}179 \\
-\end{array}$ & $\begin{array}{l}145.4 \\
--\end{array}$ & 38.17 & 1.77 & $\begin{array}{l}244 \\
240\end{array}$ & $\begin{array}{l}204.3 \\
2012\end{array}$ & $\begin{array}{l}13.07 \\
12.89\end{array}$ & $\begin{array}{l}1.47 \\
1.49\end{array}$ & 6 & 282.6 & 2.88 & 75 & 25 & : \\
\hline 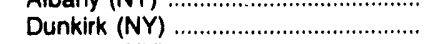 & 83 & 132.5 & 34.91 & 2.06 & 2 & 426.2 & 24.68 & .43 & - & $2 / 4.0$ & 2.78 & 99 & $\begin{array}{r}100 \\
1\end{array}$ & - \\
\hline Huntley (NY) .................................... & 95 & 156.8 & 41.01 & 1.53 & 2 & 437.8 & 25.32 & .47 & - & - & - & 100 & - & - \\
\hline Oswego (NY) & - & -- & -- & - & -- & - & -- & - & 2 & 299.1 & 3.08 & -- & -- & 100 \\
\hline Northern Indiana Pub Serv Co ......... & 524 & 141.8 & 29.49 & 1.42 & - & - & - & - & 123 & 282.7 & 2.88 & 99 & - & 1 \\
\hline 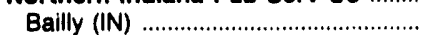 & 115 & 132.3 & 29.15 & 3.07 & -- & -- & -- & -- & 23 & 281.8 & 2.87 & $9 \theta$ & -. & 1 \\
\hline 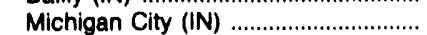 & 131 & 146.6 & 28.93 & .45 & - & -- & -- & - & 8 & 291.8 & 2.87 & 100 & - & - \\
\hline Mitchell (IN) & 83 & 101.6 & 17.13 & .35 & -- & - & - & -- & 11 & 290.5 & 2.96 & 99 & -- & 1 \\
\hline 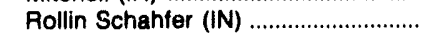 & 195 & 157.2 & 35.30 & 1.56 & -- & -- & - & - & 82 & 281.1 & 2.86 & 98 & - & 2 \\
\hline Northern States Power Co ................. & 1,062 & 117.2 & 20.53 & .46 & - & - & - & - & 50 & 278.5 & 2.82 & 100 & - & • \\
\hline 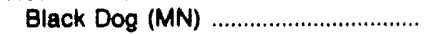 & 88 & 97.3 & 17.17 & .24 & -- & -- & - & - & 38 & 277.7 & 2.82 & 98 & - & 2 \\
\hline High Bridge (MN) & 24 & 95.2 & 16.72 & .20 & - & -- & - & - & 4 & 261.0 & 2.72 & 99 & -- & 1 \\
\hline 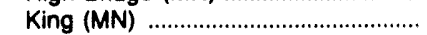 & 145 & 95.0 & 16.75 & .34 & - & -- & - & -- & 3 & 261.0 & 2.68 & 100 & - & - \\
\hline Riverside (MN) & 64 & 87.0 & 15.24 & .18 & -- & -- & - & -- & 6 & 286.0 & 2.91 & 99 & -- & 1 \\
\hline Sherburne County (MN) .......................... & 741 & 127.4 & 22.25 & .54 & - & - & -- & - & - & - & - & 100 & -- & - \\
\hline Ohio Edison Co & 526 & 119.8 & 29.10 & 1.87 & 4 & 422.0 & 24.50 & .32 & - & - & - & 100 & - & - \\
\hline Burger $(\mathrm{OH})$ & 86 & 103.5 & 25.25 & 3.45 & • & 440.0 & 25.56 & .36 & - & -- & -- & 100 & - & - \\
\hline Niles $(\mathrm{OH})$ & 32 & 112.2 & 26.87 & 2.65 & • & 415.0 & 24.09 & .30 & - & -- & -- & 100 & - & -- \\
\hline Sammis $(\mathrm{OH})$ & 408 & 123.9 & 30.09 & 1.47 & 4 & 420.5 & 24.41 & .32 & -- & - & -- & 100 & * & - \\
\hline Ohlo Power Co & 918 & 164.9 & 39.14 & 3.33 & 2 & 421.4 & 24.31 & .00 & - & - & - & 100 & - & - \\
\hline Gavin $(\mathrm{OH})$ & 240 & 209.8 & 48.14 & 3.26 & -- & - & - & - & - & - & -_ & 100 & -- & - \\
\hline Kammer $(W V)$ & 190 & 120.5 & 29.68 & 4.30 & • & 439.7 & 25.38 & .00 & -- & -- & - & 100 & - & -- \\
\hline Mitchell (WV) & 184 & 125.4 & 30.73 & 1.03 & -- & -- & - & - & - & - & - & 100 & -- & - \\
\hline 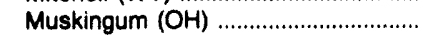 & 288 & 187.4 & 43.62 & 4.21 & 2 & 417.9 & 24.10 & .00 & - & - & - & 100 & • & -- \\
\hline Tidd $(\mathrm{OH})$ & 16 & 130.7 & 32.02 & 3.42 & $-\cdots$ & - & - & - & - & - & - & 100 & - & - \\
\hline Ohio Valley Electric Corp ................... & 97 & 100.5 & 23.02 & 3.59 & 1 & 575.6 & 33.60 & .37 & - & - & - & 100 & - & - \\
\hline Kyger Creok $(\mathrm{OH})$ & 97 & 100.5 & 23.02 & 3.59 & 1 & 575.6 & 33.60 & .37 & -- & - & -- & 100 & - & - \\
\hline Oklahoma Gas \& Electric Co ............ & 727 & 111.9 & 19.51 & .34 & - & - & - & - & 5,578 & 337.8 & 3.50 & 69 & - & 31 \\
\hline Horseshoe Lake (OK) ............................ & -- & -- & - & - & -- & -- & -- & - & 1,038 & 335 & 3.48 & -- & -- & 100 \\
\hline 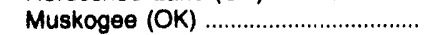 & 432 & 117.4 & 20.65 & .33 & -- & -- & -- & - & 582 & 339.0 & 3.52 & 93 & -- & 7 \\
\hline 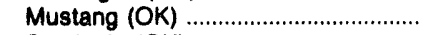 & - & - & -- & -- & -- & -- & - & - & 923 & 339.6 & 3.52 & $\ldots$ & - & 100 \\
\hline Seminole $(\mathrm{OK})$ & - & -- & -- & - & -- & -- & -- & - & 3,035 & 337.6 & 3.50 & - & - & 100 \\
\hline 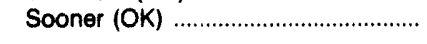 & 294 & 103.6 & 17.85 & .35 & -- & - & - & - & - & - & - & 100 & -- & - \\
\hline Omaha Publlc Power District ............ & 338 & 67.5 & 11.22 & .40 & 2 & 426.8 & 24.65 & .20 & 19 & 330.3 & 3.26 & 90 & - & • \\
\hline 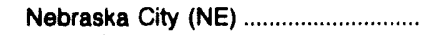 & 217 & 67.5 & 11.22 & .39 & 2 & 426.9 & 24.65 & .20 & - & - & - & 100 & - & -- \\
\hline 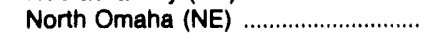 & 121 & 67.6 & 11.23 & .41 & - & -- & - & - & 10 & 330.3 & 3.26 & 99 & -- & 1 \\
\hline Orange \& Rockland Utils Inc ............ & 82 & 195.7 & 50.95 & .55 & 140 & 254.3 & 15.71 & .36 & 1,690 & 290.3 & 3.01 & 45 & 18 & 37 \\
\hline 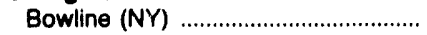 & - & & -- & -- & 140 & 254.3 & 15.71 & .36 & 1,518 & 290.3 & 3.01 & -- & 35 & 65 \\
\hline 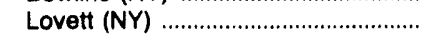 & 82 & 195.7 & 50.95 & .55 & -- & -- & - & - & 172 & 290.5 & 3.01 & 92 & - & 8 \\
\hline Orlando Utilitios Comm ......................... & 103 & 181.0 & 47.12 & .88 & 119 & 220.9 & 14.08 & 1.00 & 770 & 282.6 & 2.92 & 63 & 18 & 10 \\
\hline Indian River (FL) & & & - & - & 119 & 220.9 & 14.08 & 1.00 & & 282 & 2.92 & -- & 49 & 51 \\
\hline Stanton Energy $(F L)$ & 103 & 181.0 & 47.12 & .88 & - & - & -- & -- & - & - & - & 100 & - & - \\
\hline Orrville City o & 16 & 100.2 & 23.97 & 3.76 & - & - & - & - & - & - & - & 100 & - & - \\
\hline Orville $(\mathrm{OH})$ & 16 & 100.2 & 23.97 & 3.76 & -- & - & - & - & - & - & - & 100 & - & - \\
\hline Otter Tall Power Co ...... & 54 & 117.1 & 17.29 & .70 & • & 445.0 & 26.17 & .31 & - & - & - & 100 & . & - \\
\hline Big Stone (SD) & 34 & 115.3 & 14.37 & .91 & -- & -- & - & - & - & -- & -- & 100 & -_ & -- \\
\hline 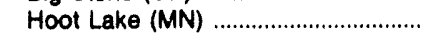 & 20 & 119.2 & 22.25 & .33 & $*$ & 445.0 & 26.17 & .31 & - & - & - & 100 & - & -- \\
\hline Owensboro Clty of & 87 & 95.2 & 21.16 & 2.72 & : & 404.9 & 23.47 & .38 & - & - & - & 100 & - & - \\
\hline 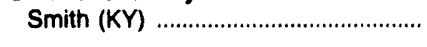 & 87 & 95.2 & 21.16 & 2.72 & • & 404.9 & 23.47 & .38 & - & - & - & 100 & • & - \\
\hline atric Co ....................... & - & - & - & - & - & - & - & - & 16,649 & 274.0 & 2.8 & - & - & 100 \\
\hline 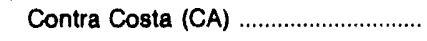 & - & -- & -- & -- & -- & -- & -- & -- & 1,876 & 274.0 & 2.82 & - & - & 100 \\
\hline
\end{tabular}

See notes and footnotes at end of table. 
Table 63. Recelpts, Average Cost, and Quality of Fossll Fuels Delivered to U.S. Electric Utilities by Company and Plant, September 1993 (Continued)

\begin{tabular}{|c|c|c|c|c|c|c|c|c|c|c|c|c|c|c|}
\hline \multirow{3}{*}{$\begin{array}{l}\text { Utility (Hoiding Company) } \\
\text { Plant (State) }\end{array}$} & \multicolumn{4}{|c|}{ Coal } & \multicolumn{4}{|c|}{ Potroleum' } & \multicolumn{3}{|c|}{ Ces } & \multicolumn{3}{|c|}{ \% of Total Btu } \\
\hline & \multirow{2}{*}{\begin{tabular}{|c|} 
Recolpts \\
$\begin{array}{c}(1,000 \\
\text { tons })\end{array}$ \\
\end{tabular}} & \multicolumn{2}{|c|}{$\begin{array}{l}\text { Average } \\
\text { Coot" }\end{array}$} & \multirow{2}{*}{$\begin{array}{l}\text { Avg. } \\
\text { sut } \\
\text { fur } \\
\%\end{array}$} & \multirow{2}{*}{\begin{tabular}{|c|} 
Recolpts \\
$(1,000$ \\
bble $)$
\end{tabular}} & \multicolumn{2}{|c|}{$\begin{array}{l}\text { Average } \\
\text { Cost }^{+}\end{array}$} & \multirow{2}{*}{$\begin{array}{l}\text { Avg. } \\
\text { sut- } \\
\text { fur } \\
\%\end{array}$} & \multirow{2}{*}{$\begin{array}{c}\text { Recelpte } \\
\begin{array}{c}(1,000 \\
\text { Mon })\end{array}\end{array}$} & \multicolumn{2}{|c|}{$\begin{array}{l}\text { Average } \\
\text { Cout" }\end{array}$} & \multirow[b]{2}{*}{ Coal } & & \\
\hline & & $\begin{array}{c}\text { (Cents } \\
\text { per } \\
10^{\circ} \\
\text { Btu) }\end{array}$ & $\begin{array}{l}\text { (S per } \\
\text { chort } \\
\text { ton) }\end{array}$ & & & $\begin{array}{c}\text { (Conts } \\
\text { per } \\
10^{\circ} \\
\text { Btu) }\end{array}$ & s por & & & $\begin{array}{c}\text { (Cents } \\
\text { per } \\
10^{6} \\
\text { Btu) }\end{array}$ & Mor & & tro- & ans \\
\hline Pacific Cas a Electric Co & & & & & & & & & & & & & & \\
\hline 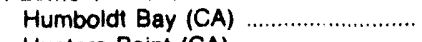 & - & - & -- & - & - & -- & -- & -- & 184 & 274.0 & 2.81 & -- & -- & 100 \\
\hline 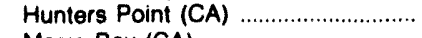 & - & - & - & - & - & -- & -- & - & 771 & 274.0 & 2.78 & - & -- & 100 \\
\hline 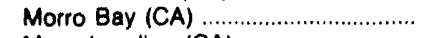 & - & -- & - & -- & - & -- & -- & - & 1,434 & 274.0 & 2.82 & -- & -- & 100 \\
\hline 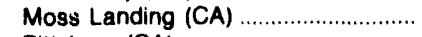 & - & - & -- & - & - & -- & -- & - & 6,739 & 274.0 & 2.81 & - & - & 100 \\
\hline Pittsburg (CA) & - & - & - & - & -- & - & -- & - & 4,825 & 274.0 & 2.79 & - & - & 100 \\
\hline 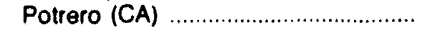 & - & - & -- & - & - & - & - & - & 821 & 274.0 & 2.78 & - & - & 100 \\
\hline 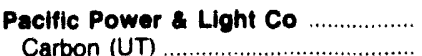 & $\begin{array}{r}2,489 \\
59\end{array}$ & 94.9 & 17.91 & 0.56 & 3 & 458.8 & 26.98 & 0.30 & 380 & 258.9 & 2.81 & 9 & • & \\
\hline & 59 & 70.2 & 16.98 & .50 & -- & -- & - & - & - & -- & - & 100 & - & - \\
\hline Centralia (WA) & 439 & 130.6 & 21.07 & .66 & 2 & 464.3 & 27.30 & .30 & - & - & -- & 100 & $\cdot$ & $-\cdots$ \\
\hline 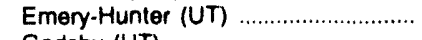 & 324 & 98.9 & 22.20 & .48 & - & - & - & - & -- & -- & - & 100 & - & - \\
\hline 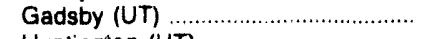 & -- & - & $-\overline{7}$ & - & -- & - & -- & - & 375 & 257.6 & 2.80 & - & -- & 100 \\
\hline 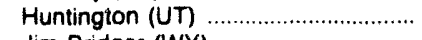 & 253 & 74.1 & 17.14 & .54 & - & - & -- & - & - & - & - & 100 & -- & - \\
\hline 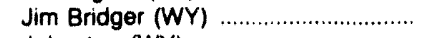 & 744 & 104.4 & 19.89 & .62 & - & -- & -- & - & -- & -- & - & 100 & - & - \\
\hline 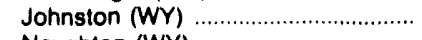 & 378 & 55.4 & 8.90 & .45 & - & - & - & - & - & - & - & 100 & -- & - \\
\hline Naughton (WY) & 120 & 120.4 & 23.41 & .59 & 1 & 447.8 & 26.33 & .30 & 5 & 360.2 & 3.71 & 100 & • & - \\
\hline Wyodak $(W Y)$ & 172 & 65.7 & 10.59 & .51 & - & - & -- & - & - & -- & - & 100 & - & - \\
\hline Painesville City of .............................. & 13 & 138.0 & 34.34 & 2.13 & - & - & - & - & 1 & 479.0 & 4.79 & 100 & - & • \\
\hline Painesville $(\mathrm{OH})$ & 13 & 138.0 & 34.34 & 2.13 & - & - & -- & - & $i$ & 479.0 & 4.79 & 100 & - & • \\
\hline 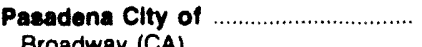 & - & - & - & - & - & - & - & - & 322 & 308.5 & 3.14 & - & - & 100 \\
\hline & - & - & - & - & - & - & - & - & 322 & 306.5 & 3.14 & -- & -- & 100 \\
\hline Pennoylvania Eloctric Co ..................... & 1,244 & $\begin{array}{l}134.7 \\
122.3\end{array}$ & 32.99 & 1.80 & 24 & 399.8 & 23.31 & .05 & 13 & 293.9 & 3.03 & 99 & - & - \\
\hline 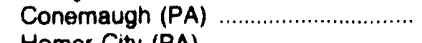 & 271 & 122.3 & 30.49 & 2.19 & 3 & 405.7 & 23.65 & .05 & 13 & 293.9 & 3.03 & 100 & $\cdot$ & - \\
\hline 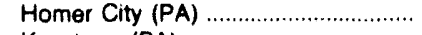 & 406 & 135.5 & 32.35 & 1.93 & 12 & 401.5 & 23.41 & .05 & -- & - & - & 99 & 1 & - \\
\hline 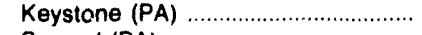 & 421 & 147.8 & 36.66 & 1.91 & 2 & 405.3 & 23.63 & .05 & - & -- & -- & 100 & $\cdot$ & - \\
\hline 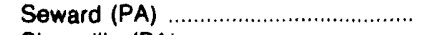 & 38 & 124.6 & 30.43 & 1.51 & 1 & 409.2 & 23.85 & .05 & -- & -- & - & 99 & 1 & -- \\
\hline Shawville (PA) & 87 & 111.0 & 27.19 & 2.01 & 6 & 390.0 & 22.74 & .05 & - & - & - & 98 & 2 & -- \\
\hline 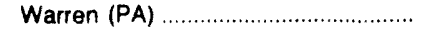 & 21 & 132.1 & 32.83 & 1.45 & - & - & - & - & -- & - & -- & 100 & - & -- \\
\hline Pennaylvania Power a Light Co ..... & 538 & 147.4 & 36.82 & 1.71 & 188 & 272.4 & 17.41 & .92 & - & - & - & 91 & 9 & \\
\hline 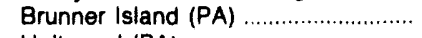 & 196 & 147.1 & 38.98 & 1.81 & 3 & 395.8 & 23.05 & .11 & -- & -- & - & 100 & $\cdot$ & - \\
\hline Holtwood (PA) .................................... & 13 & 100.2 & 15.16 & .59 & - & - & - & -- & -- & -- & -- & 100 & - & -- \\
\hline 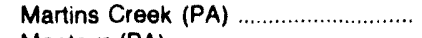 & 61 & 155.2 & 41.07 & 1.61 & - & -- & - & - & - & - & - & 100 & -- & -- \\
\hline Montour (PA) & 189 & 154.8 & 38.47 & 1.87 & -- & - & -- & - & -- & - & -. & 100 & - & -- \\
\hline Storage Facility $\# 1$.............................. & -- & - & $\bar{z}$ & - & 194 & 270.0 & 17.29 & .93 & - & -- & -- & - & 100 & - \\
\hline 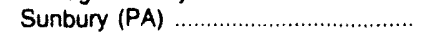 & 79 & 126.1 & 27.77 & 1.33 & 1 & 405.1 & 23.61 & .12 & - & -- & -- & 100 & $\because$ & - \\
\hline Pennsylvania Power Co ..................... & 467 & 159.0 & 38.35 & 2.65 & • & 563.4 & 32.68 & .08 & - & - & - & 100 & • & - \\
\hline Bruce Mansfield (PA) ......................... & 429 & 162.6 & 39.21 & 2.76 & - & - & - & - & -- & - & - & 100 & - & - \\
\hline 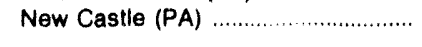 & 37 & 117.6 & 28.46 & 1.43 & • & 563.4 & 32.68 & .08 & - & - & -- & 100 & • & - \\
\hline $\begin{array}{l}\text { Phlladelphla Electric Co } \ldots \ldots \ldots \ldots \ldots \ldots \\
\text { Cromby (PA) }\end{array}$ & $\begin{array}{r}102 \\
22\end{array}$ & $\begin{array}{l}156.8 \\
147.8\end{array}$ & $\begin{array}{l}41.26 \\
39.47\end{array}$ & $\begin{array}{l}1.78 \\
1.75\end{array}$ & $\begin{array}{r}373 \\
71\end{array}$ & 246.1 & $\begin{array}{l}15.73 \\
15.92\end{array}$ & $\begin{array}{l}.55 \\
.89\end{array}$ & $\begin{array}{l}247 \\
247\end{array}$ & $\begin{array}{l}283.8 \\
283.8\end{array}$ & $\begin{array}{l}2.92 \\
2.92\end{array}$ & $\begin{array}{l}50 \\
45\end{array}$ & $\begin{array}{r}45 \\
35\end{array}$ & $\begin{array}{r}5 \\
20\end{array}$ \\
\hline 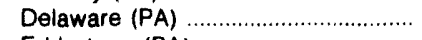 & - & - & - & -- & 81 & 204.8 & 13.11 & .44 & -- & - & - & - & 100 & - \\
\hline 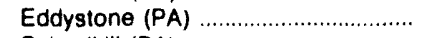 & 80 & 159.4 & 41.75 & 1.79 & 165 & 232.9 & 14.91 & .50 & -- & -- & -- & 66 & 34 & - \\
\hline Schuylkill (PA) & $\cdots$ & - & - & - & 56 & 342.0 & 21.67 & .44 & -- & - & -- & - & 100 & -- \\
\hline Plains Elec GensTrans Coop Inc ... & 68 & 138.4 & 25.05 & .73 & - & - & - & - & - & 437.6 & 3.72 & 100 & - & - \\
\hline 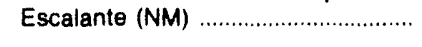 & 66 & 138.4 & 25.05 & .73 & - & - & -- & -- & • & 437.6 & 3.72 & 100 & - & - \\
\hline Platte River Power Authority ........... & 102 & 72.0 & 12.76 & .32 & - & - & - & - & - & - & - & 100 & - & - \\
\hline 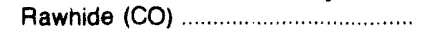 & 102 & 72.0 & 12.76 & .32 & - & -- & - & -- & $-\cdots$ & -- & - & 100 & -- & - \\
\hline Portland General Electric Co ............ & 167 & 113.8 & 19.17 & .37 & & - & - & - & 1,638 & 214.1 & 2.16 & 63 & - & 37 \\
\hline 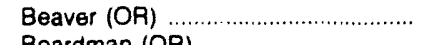 & -- & -- & $-\bar{s}$ & -- & - & -- & $\rightarrow$ & -- & 1,638 & 214.1 & 2.16 & - & -- & 100 \\
\hline Boardman (OR) & 167 & 113.8 & 19.17 & .37 & - & - & - & - & -- & -- & - & 100 & -- & -- \\
\hline Potomac Edison Co $\ldots \ldots \ldots \ldots \ldots \ldots \ldots \ldots$ & 24 & 135.3 & 34.20 & .98 & 1 & 423.5 & 25.08 & .30 & - & - & - & 99 & 1 & - \\
\hline Smith (MD) & 24 & 135.3 & 34.20 & .98 & 1 & 423.5 & 25.08 & .30 & -- & -- & -- & 99 & 1 & - \\
\hline Potomac Electric Power Co ............ & 380 & 166.9 & 42.82 & 1.56 & 467 & 237.9 & 14.87 & 1.62 & 618 & 310.6 & 3.24 & 73 & 22 & 5 \\
\hline
\end{tabular}

See notes and tootnotes at end of table. 
Table 63. Recelpts, Average Cost, and Quality of Fosell Fuels Dellvered to U.S. Electric Utilities by Company and Plant, September 1993 (Continued)

\begin{tabular}{|c|c|c|c|c|c|c|c|c|c|c|c|c|c|c|}
\hline \multirow{3}{*}{$\begin{array}{l}\text { Utilty (Holding Company) } \\
\text { Plant (State) }\end{array}$} & \multicolumn{4}{|c|}{ Coal } & \multicolumn{4}{|c|}{ Potroleum' } & \multicolumn{3}{|c|}{ ans } & \multicolumn{3}{|c|}{ \% of Total Btu } \\
\hline & \multirow{2}{*}{\begin{tabular}{|c|} 
Accolpts \\
$(1,000$ \\
tons)
\end{tabular}} & \multicolumn{2}{|c|}{$\begin{array}{l}\text { Average } \\
\text { Coot }\end{array}$} & \multirow{2}{*}{$\begin{array}{l}\text { Avg. } \\
\text { sut } \\
\text { fur } \\
\%\end{array}$} & \multirow{2}{*}{\begin{tabular}{|c|} 
Recolpte \\
$(1,000$ \\
bth $)$
\end{tabular}} & \multicolumn{2}{|c|}{$\begin{array}{l}\text { Averege } \\
\text { Coat? }\end{array}$} & \multirow{2}{*}{$\begin{array}{l}\text { Avg. } \\
\text { sur } \\
\text { fur } \\
\%\end{array}$} & \multirow{2}{*}{$\begin{array}{c}\text { Recelpte } \\
\left.\begin{array}{c}(1,000 \\
\text { mot }\end{array}\right)\end{array}$} & \multicolumn{2}{|c|}{$\begin{array}{l}\text { Avertope } \\
\text { Coot" }\end{array}$} & \multirow[b]{2}{*}{ Coal } & \multirow{2}{*}{$\begin{array}{l}\text { Po- } \\
\text { tro- } \\
\text { loum }\end{array}$} & \multirow[b]{2}{*}{ Cas } \\
\hline & & $\begin{array}{l}\text { (Conts } \\
\text { per } \\
10^{\circ} \\
\text { Etu) }\end{array}$ & $\begin{array}{l}\text { (S por } \\
\text { chort } \\
\text { ton) }\end{array}$ & & & $\begin{array}{l}\text { (Cents } \\
\text { per } \\
10^{\circ} \\
\text { Dtu) }\end{array}$ & ber & & & $\begin{array}{l}\text { (Cents } \\
\text { per } \\
104 \\
\text { Dtu) }\end{array}$ & Wor & & & \\
\hline 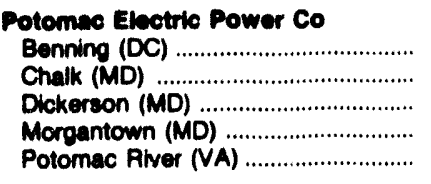 & $\begin{array}{r}- \\
51 \\
97 \\
160 \\
72\end{array}$ & $\begin{array}{l}-169.7 \\
143.1 \\
177.7 \\
172.4\end{array}$ & $\begin{array}{l}-\overline{42.32} \\
36.25 \\
45.38 \\
46.32\end{array}$ & $\begin{array}{l}- \\
1.83 \\
1.46 \\
1.83 \\
.85\end{array}$ & $\begin{array}{r}85 \\
366 \\
-\quad 6 \\
-\quad 10\end{array}$ & $\begin{array}{l}305.8 \\
217.0 \\
387.0 \\
- \\
385.4\end{array}$ & $\begin{array}{c}18.40 \\
13.72 \\
22.44 \\
-32.44\end{array}$ & $\begin{array}{c}0.82 \\
1.84 \\
.20 \\
- \\
.20\end{array}$ & $\begin{array}{l}-618 \\
- \\
-\end{array}$ & $\begin{array}{l}\overline{310.6} \\
- \\
-\end{array}$ & $\begin{array}{l}\overline{3.24} \\
- \\
- \\
-\end{array}$ & $\begin{array}{r}30 \\
99 \\
100 \\
97\end{array}$ & $\begin{array}{r}100 \\
55 \\
1 \\
-- \\
3\end{array}$ & $\begin{array}{c}- \\
15 \\
- \\
-\end{array}$ \\
\hline $\begin{array}{l}\text { Power Authorthy of state of NY } \\
\text { Poletti (NY) }\end{array}$ & - & - & - & - & - & - & - & - & $\begin{array}{l}1,600 \\
1,608\end{array}$ & $\begin{array}{l}372.6 \\
372.6\end{array}$ & $\begin{array}{l}3.85 \\
3.85\end{array}$ & - & - & $\begin{array}{l}100 \\
100\end{array}$ \\
\hline 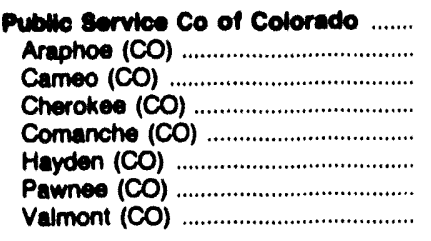 & $\begin{array}{r}630 \\
44 \\
21 \\
63 \\
246 \\
148 \\
59 \\
48\end{array}$ & $\begin{array}{r}108.1 \\
111.2 \\
91.2 \\
109.5 \\
120.3 \\
83.7 \\
108.2 \\
111.8\end{array}$ & $\begin{array}{l}20.72 \\
24.91 \\
20.56 \\
25.11 \\
20.43 \\
17.80 \\
17.87 \\
25.17\end{array}$ & $\begin{array}{l}.37 \\
.49 \\
.57 \\
.46 \\
.27 \\
.39 \\
.30 \\
.54\end{array}$ & $\begin{array}{l}- \\
- \\
- \\
-\end{array}$ & $\begin{array}{l}- \\
- \\
- \\
-\end{array}$ & $\begin{array}{l}- \\
- \\
- \\
- \\
-\end{array}$ & $\begin{array}{l}- \\
- \\
- \\
- \\
-\end{array}$ & $\begin{array}{r}123 \\
5 \\
1 \\
39 \\
2 \\
2 \\
-74\end{array}$ & $\begin{array}{r}241.0 \\
214.3 \\
260.7 \\
225.1 \\
186.5 \\
209.4 \\
251.6 \\
1,582.3\end{array}$ & $\begin{array}{r}2.48 \\
2.10 \\
2.69 \\
2.21 \\
1.84 \\
2.28 \\
2.66 \\
15.66\end{array}$ & $\begin{array}{r}90 \\
100 \\
100 \\
97 \\
100 \\
100 \\
93 \\
100\end{array}$ & $\begin{array}{l}- \\
- \\
- \\
- \\
-\end{array}$ & $:^{1}$ \\
\hline 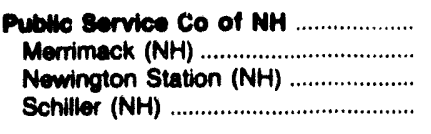 & $\begin{array}{r}107 \\
-\quad 80 \\
-\quad 27\end{array}$ & $\begin{array}{l}158.3 \\
158.8 \\
148.9\end{array}$ & $\begin{array}{l}40.07 \\
41.80 \\
-- \\
38.56\end{array}$ & $\begin{array}{c}1.59 \\
1.93 \\
- \\
.59\end{array}$ & -369 & $\begin{array}{l}171.5 \\
\overline{171.5}\end{array}$ & $\overline{11.27}$ & $\frac{1.80}{1.80}$ & $\overline{-}$ & $\overline{-}$ & $\bar{m}$ & $\begin{array}{l}54 \\
100 \\
\frac{100}{100}\end{array}$ & $\frac{46}{100}$ & $\overline{-}$ \\
\hline 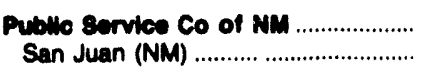 & $\begin{array}{l}466 \\
456\end{array}$ & $\begin{array}{l}165.9 \\
165.9\end{array}$ & $\begin{array}{l}31.10 \\
31.10\end{array}$ & .86 & 6 & $\begin{array}{l}42.1 \\
482.1\end{array}$ & $\begin{array}{l}27.54 \\
27.54\end{array}$ & $\begin{array}{l}1.00 \\
1.00\end{array}$ & $\overline{-}$ & - & - & $\begin{array}{l}100 \\
100\end{array}$ & $\bullet$ & - \\
\hline 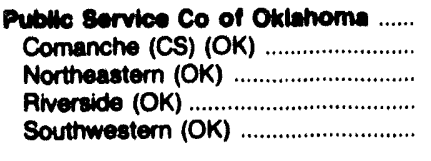 & $\begin{array}{l}363 \\
- \\
-\end{array}$ & $\begin{array}{l}162.5 \\
\overline{162.5} \\
-\end{array}$ & $\begin{array}{l}28.06 \\
\overline{28.06} \\
-\end{array}$ & $\begin{array}{c}.45 \\
- \\
-\end{array}$ & 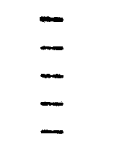 & $\overline{-}$ & $\overline{-}$ & $\begin{array}{l}- \\
- \\
-\end{array}$ & $\begin{array}{r}6,202 \\
910 \\
1,956 \\
2,831 \\
505\end{array}$ & $\begin{array}{l}311.8 \\
311.8 \\
311.8 \\
311.8 \\
311.8\end{array}$ & $\begin{array}{l}3.20 \\
3.41 \\
3.26 \\
3.24 \\
3.29\end{array}$ & $\begin{array}{c}49 \\
- \\
- \\
-\end{array}$ & $\begin{array}{l}- \\
- \\
-\end{array}$ & $\begin{array}{r}51 \\
100 \\
25 \\
100 \\
100\end{array}$ \\
\hline 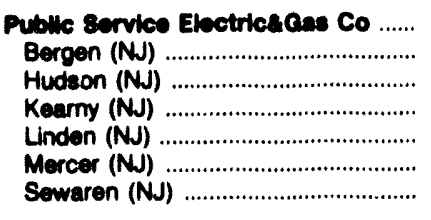 & $\begin{array}{l}12 \\
- \\
- \\
-\end{array}$ & $\begin{array}{l}178.8 \\
- \\
- \\
\overline{178.8} \\
-\end{array}$ & $\begin{array}{l}49.75 \\
- \\
- \\
- \\
- \\
-\end{array}$ & $\begin{array}{l}.77 \\
- \\
- \\
- \\
- \\
-\end{array}$ & $\begin{array}{l}- \\
- \\
- \\
-\end{array}$ & $\begin{array}{l}297.4 \\
- \\
\overline{291.2} \\
299.4 \\
-\end{array}$ & $\begin{array}{l}18.59 \\
\overline{-} \\
18.18 \\
18.72 \\
-\end{array}$ & $\begin{array}{l}.20 \\
- \\
- \\
.28 \\
.29 \\
-\end{array}$ & $\begin{array}{r}2,230 \\
627 \\
857 \\
-\quad \\
-\quad 388 \\
368\end{array}$ & $\begin{array}{l}254.0 \\
254.0 \\
254.0 \\
- \\
- \\
254.0 \\
254.0\end{array}$ & $\begin{array}{l}2.63 \\
2.62 \\
2.63 \\
- \\
- \\
2.62 \\
2.63\end{array}$ & $\begin{array}{l}- \\
- \\
- \\
-\end{array}$ & $\begin{array}{l}17 \\
- \\
- \\
100 \\
- \\
-\end{array}$ & $\begin{array}{r}72 \\
100 \\
100 \\
- \\
- \\
55\end{array}$ \\
\hline 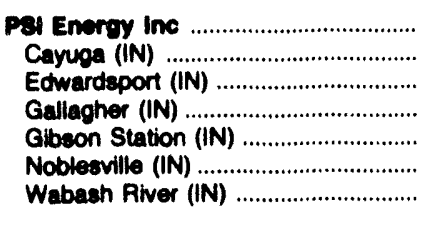 & $\begin{array}{r}959 \\
161 \\
13 \\
72 \\
583 \\
9 \\
121\end{array}$ & $\begin{array}{r}131.4 \\
129.5 \\
86.0 \\
125.6 \\
137.1 \\
156.5 \\
114.1\end{array}$ & $\begin{array}{l}28.98 \\
29.00 \\
19.58 \\
31.45 \\
29.51 \\
35.08 \\
25.48\end{array}$ & $\begin{array}{l}1.97 \\
2.23 \\
2.52 \\
2.23 \\
1.83 \\
2.10 \\
2.04\end{array}$ & $\begin{array}{r}16 \\
5 \\
1 \\
2 \\
3 \\
-\quad 5\end{array}$ & $\begin{array}{l}437.3 \\
486.2 \\
432.0 \\
444.4 \\
384.3 \\
421.0\end{array}$ & $\begin{array}{l}25.18 \\
27.07 \\
24.86 \\
25.57 \\
22.11 \\
- \\
24.22\end{array}$ & $\begin{array}{l}.30 \\
.30 \\
.30 \\
.30 \\
.30 \\
-.30\end{array}$ & $\begin{array}{l}- \\
- \\
- \\
-\end{array}$ & $\begin{array}{l}- \\
- \\
- \\
-\end{array}$ & $\begin{array}{l}- \\
- \\
- \\
-\end{array}$ & $\begin{array}{r}100 \\
99 \\
98 \\
99 \\
100 \\
100 \\
99\end{array}$ & $\begin{array}{r}1 \\
2 \\
1 \\
-1 \\
-1\end{array}$ & $\begin{array}{l}- \\
\bar{z} \\
\overline{-}\end{array}$ \\
\hline $\begin{array}{l}\text { Richmond City of } \\
\text { Whitewater (IN) }\end{array}$ & $\begin{array}{l}24 \\
24\end{array}$ & $\begin{array}{l}146.3 \\
146.3\end{array}$ & $\begin{array}{l}33.64 \\
33.64\end{array}$ & $\begin{array}{l}2.10 \\
2.10\end{array}$ & - & - & - & - & - & - & - & $\begin{array}{l}100 \\
100\end{array}$ & - & - \\
\hline $\begin{array}{l}\text { Rocheoter Clty of } \\
\text { Silver Lake (MN) }\end{array}$ & $\begin{array}{l}5 \\
5\end{array}$ & $\begin{array}{l}174.6 \\
174.6\end{array}$ & $\begin{array}{l}42.16 \\
42.16\end{array}$ & $\begin{array}{l}1.59 \\
1.59\end{array}$ & - & - & - & - & 9 & $\begin{array}{l}244.8 \\
264.8\end{array}$ & $\begin{array}{l}2.69 \\
2.69\end{array}$ & $\begin{array}{l}93 \\
93\end{array}$ & - & $\begin{array}{l}7 \\
7\end{array}$ \\
\hline 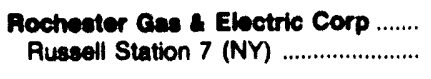 & $\begin{array}{l}19 \\
19\end{array}$ & $\begin{array}{l}146.8 \\
146.8\end{array}$ & $\begin{array}{l}38.10 \\
38.10\end{array}$ & $\begin{array}{l}1.54 \\
1.54\end{array}$ & $\overline{-}$ & - & - & - & $\overline{-}$ & - & - & $\begin{array}{l}100 \\
100\end{array}$ & - & $\overline{-}$ \\
\hline 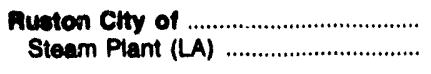 & - & - & $\overline{-}$ & - & $\overline{-}$ & $\overline{-}$ & - & $\overline{-}$ & $\begin{array}{l}160 \\
160\end{array}$ & $\begin{array}{l}224.6 \\
224.6\end{array}$ & $\begin{array}{l}2.37 \\
2.37\end{array}$ & - & - & $\begin{array}{l}100 \\
100\end{array}$ \\
\hline 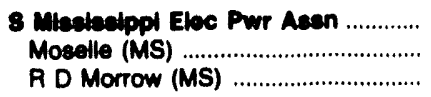 & -54 & $\frac{210.0}{210.0}$ & $\overline{52.23}$ & $\stackrel{.97}{.97}$ & - & - & - & - & $\begin{array}{r}601 \\
601 \\
-\quad\end{array}$ & $\begin{array}{l}247.5 \\
247.5 \\
-\end{array}$ & $\begin{array}{l}2.51 \\
2.51 \\
-\end{array}$ & $\frac{60}{100}$ & - & $\begin{array}{r}31 \\
100 \\
--\end{array}$ \\
\hline
\end{tabular}

See notes and footnotes at end of table. 
Table 63. Recelpts, Average Cost, and Quallty of Fossll Fuels Dellvered to U.S. Electric Utilities by Company and Plant, Soptember 1993 (Continued)

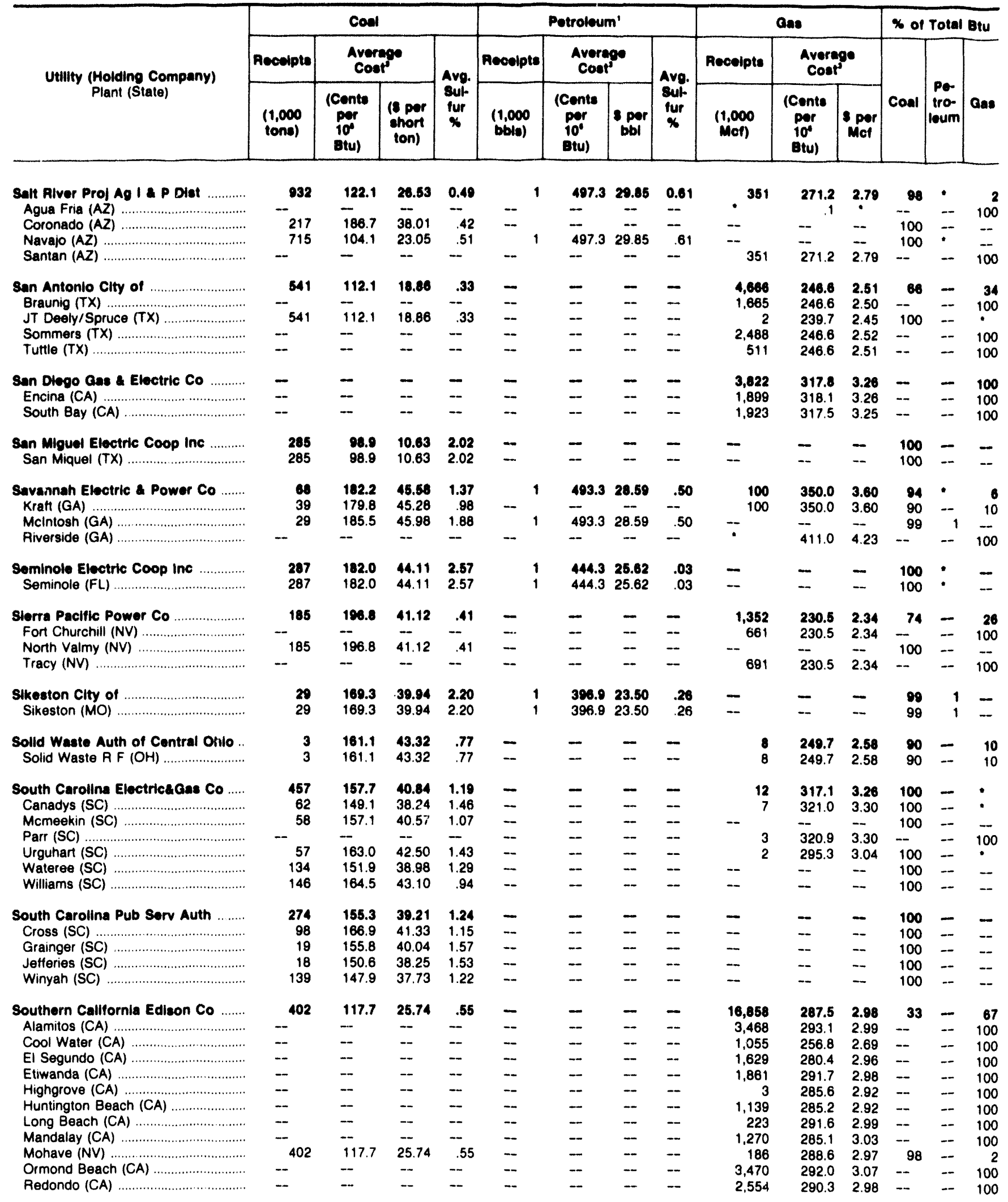

See notes and footnotes at end of table. 
Table 63. Recelpts, Average Cost, and Quality of Fosall Fuele Dellvered to U.S. Electric Utillties by Company and Plant, September 1993 (Continued)

\begin{tabular}{|c|c|c|c|c|c|c|c|c|c|c|c|c|c|c|}
\hline \multirow{3}{*}{$\begin{array}{l}\text { Utility (Holding Company) } \\
\text { Plant (State) }\end{array}$} & \multicolumn{4}{|c|}{ Coal } & \multicolumn{4}{|c|}{ Petroleum' } & \multicolumn{3}{|c|}{ Gas: } & \multicolumn{3}{|c|}{ \% of Total Btu } \\
\hline & \multirow{2}{*}{\begin{tabular}{|c|} 
Aecolpts \\
$\begin{array}{c}(1,000 \\
\text { tons })\end{array}$
\end{tabular}} & \multicolumn{2}{|c|}{$\begin{array}{l}\text { Averape } \\
\text { Cost' }\end{array}$} & \multirow{2}{*}{$\begin{array}{c}\text { Avg. } \\
\text { sul- } \\
\text { fur } \\
\%\end{array}$} & \multirow{2}{*}{\begin{tabular}{|c|} 
Recolpto \\
$\begin{array}{c}(1,000 \\
\text { bblo })\end{array}$
\end{tabular}} & \multicolumn{2}{|c|}{$\begin{array}{l}\text { Average } \\
\text { Cost" }\end{array}$} & \multirow{2}{*}{$\begin{array}{l}\text { Avg. } \\
\text { sul- } \\
\text { fur } \\
\%\end{array}$} & \multirow{2}{*}{\begin{tabular}{|c|} 
Rocolpts \\
$\begin{array}{c}(1,000 \\
\text { Mof })\end{array}$
\end{tabular}} & \multicolumn{2}{|c|}{$\begin{array}{l}\text { Average } \\
\text { Cost }^{3}\end{array}$} & \multirow[b]{2}{*}{ Coal } & \multirow{2}{*}{$\begin{array}{l}\text { Po- } \\
\text { tro- } \\
\text { loum }\end{array}$} & \multirow[b]{2}{*}{ Gas } \\
\hline & & $\begin{array}{l}\text { (Conts } \\
\text { perr } \\
10^{\circ} \\
\text { Btu) }\end{array}$ & $\begin{array}{l}\text { (\$ per } \\
\text { short } \\
\text { ton) }\end{array}$ & & & $\begin{array}{l}\text { (Conts } \\
\text { pert } \\
10^{4} \\
\text { Btu) } \\
\end{array}$ & par & & & $\begin{array}{c}\text { (Conte } \\
\text { por } \\
10^{\circ} \\
\text { Btu) } \\
\end{array}$ & Mor & & & \\
\hline $\begin{array}{l}\text { Bouthern IIIInois Power Coop ............ } \\
\text { Marion (IL) }\end{array}$ & $\begin{array}{l}54 \\
54\end{array}$ & $\begin{array}{l}77.7 \\
77.7\end{array}$ & & $\begin{array}{l}2.59 \\
2.59\end{array}$ & 1 & & $\begin{array}{l}25.33 \\
25.33\end{array}$ & $\begin{array}{r}0.00 \\
.00\end{array}$ & $\overline{-}$ & - & $\ddot{-}$ & $\begin{array}{l}99 \\
99\end{array}$ & $\begin{array}{l}1 \\
1\end{array}$ & $\overline{-}$ \\
\hline 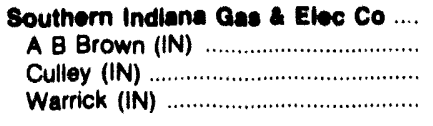 & $\begin{array}{r}187 \\
45 \\
87 \\
54\end{array}$ & $\begin{array}{l}147.4 \\
202.0 \\
131.2 \\
125.9\end{array}$ & $\begin{array}{l}33.23 \\
47.14 \\
29.28 \\
27.89\end{array}$ & $\begin{array}{l}2.51 \\
3.39 \\
2.26 \\
2.18\end{array}$ & $\ddot{-}$ & $\begin{array}{l}174.1 \\
\overline{474.1} \\
-\end{array}$ & $\begin{array}{c}27.68 \\
27.68 \\
--\end{array}$ & $\begin{array}{c}.39 \\
- \\
- \\
-\end{array}$ & - & $\begin{array}{l}342.9 \\
326.1 \\
367.5 \\
363.2\end{array}$ & $\begin{array}{l}3.51 \\
3.34 \\
3.76 \\
3.72\end{array}$ & $\begin{array}{l}100 \\
100 \\
100 \\
100\end{array}$ & $\dot{:}$ & : \\
\hline $\begin{array}{l}\text { Southweatom Electric Power Co } \\
\text { Arsenal Hill (LA) } \\
\text { Flint Creek (AR) } \\
\text { Knox Lee (TX) } \\
\text { Lieberman (LA) } \\
\text { Pirkey (TX) } \\
\text { Welsh Station (TX) } \\
\text { Wilkes (TX) }\end{array}$ & $\begin{array}{l}813 \\
-\quad 67 \\
- \\
- \\
-331 \\
-415\end{array}$ & $\begin{array}{l}162.9 \\
- \\
183.0 \\
-- \\
\overline{100.9} \\
200.0 \\
--\end{array}$ & $\begin{array}{l}24.85 \\
\overline{30.16} \\
\overline{-} \\
\overline{13.56} \\
32.99 \\
--\end{array}$ & $\begin{array}{c}.68 \\
-- \\
.37 \\
-- \\
-- \\
1.15 \\
.36 \\
--\end{array}$ & $\begin{array}{l}\bar{z} \\
\bar{z} \\
\bar{z}\end{array}$ & $\begin{array}{l}- \\
\overline{-} \\
\overline{-} \\
\overline{-} \\
\overline{-}\end{array}$ & $\begin{array}{l}\overline{-} \\
\overline{-} \\
\overline{-} \\
\overline{-}\end{array}$ & $\begin{array}{l}\overline{-} \\
\overline{-} \\
\overline{-} \\
\overline{-} \\
-\end{array}$ & $\begin{array}{r}3,461 \\
35 \\
-\quad \\
944 \\
292 \\
-\quad 2 \\
2,188\end{array}$ & $\begin{array}{l}286.1 \\
257.2 \\
-\overline{247.2} \\
252.6 \\
270.0 \\
\overline{310.1}\end{array}$ & $\begin{array}{l}2.84 \\
2.78 \\
-- \\
2.64 \\
2.58 \\
2.98 \\
-- \\
2.97\end{array}$ & $\begin{array}{l}78 \\
-- \\
100 \\
-- \\
-\overline{100} \\
100 \\
--\end{array}$ & $\begin{array}{l}- \\
\overline{-} \\
\overline{-} \\
\overline{-} \\
\overline{-}\end{array}$ & $\begin{array}{c}22 \\
100 \\
-- \\
100 \\
100 \\
- \\
\overline{100}\end{array}$ \\
\hline 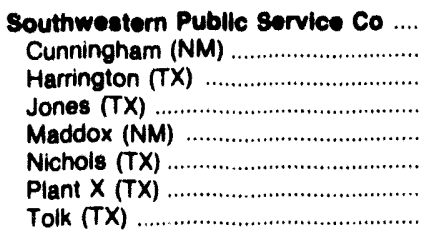 & $\begin{array}{l}737 \\
-- \\
- \\
- \\
- \\
- \\
- \\
-\end{array}$ & $\begin{array}{l}172.4 \\
\overline{147.1} \\
\overline{-} \\
- \\
\overline{200.3}\end{array}$ & $\begin{array}{l}30.29 \\
\overline{25.88} \\
\overline{-} \\
\overline{-} \\
\overline{35.17}\end{array}$ & $\begin{array}{l}.31 \\
-- \\
.30 \\
-- \\
- \\
- \\
-.33\end{array}$ & $\begin{array}{l}\bar{z} \\
\bar{z} \\
\bar{z} \\
\overline{-}\end{array}$ & $\begin{array}{l}\overline{-} \\
\overline{-} \\
\overline{-} \\
\overline{-} \\
\overline{-}\end{array}$ & $\begin{array}{l}\overline{-} \\
\overline{-} \\
\overline{-} \\
\overline{-}\end{array}$ & $\begin{array}{l}- \\
\overline{-} \\
\overline{-} \\
\overline{-} \\
\overline{-}\end{array}$ & $\begin{array}{r}4,384 \\
753 \\
2 \\
1,359 \\
428 \\
1,453 \\
382 \\
7\end{array}$ & $\begin{array}{l}211.9 \\
217.9 \\
264.0 \\
228.0 \\
220.5 \\
183.0 \\
239.2 \\
264.0\end{array}$ & $\begin{array}{l}2.12 \\
2.17 \\
2.54 \\
2.31 \\
2.26 \\
1.80 \\
2.41 \\
2.66\end{array}$ & $\begin{array}{l}75 \\
-- \\
100 \\
-- \\
- \\
- \\
100\end{array}$ & $\begin{array}{l}- \\
\overline{-} \\
= \\
\overline{-} \\
\overline{-}\end{array}$ & $\begin{array}{r}25 \\
100 \\
100 \\
100 \\
100 \\
100 \\
.\end{array}$ \\
\hline $\begin{array}{l}\text { Springtield City of } \\
\text { James River (MO) } \\
\text { Southwest (MO) }\end{array}$ & $\begin{array}{l}70 \\
23 \\
47\end{array}$ & & $\begin{array}{l}33.59 \\
32.69 \\
34.03\end{array}$ & $\begin{array}{r}.02 \\
1.54 \\
.46\end{array}$ & $\overline{-}$ & $\bar{z}$ & $\bar{m}$ & $\overline{-}$ & $\begin{array}{l}85 \\
39 \\
46\end{array}$ & & $\begin{array}{l}2.35 \\
2.35 \\
2.35\end{array}$ & $\begin{array}{l}95 \\
93 \\
96\end{array}$ & $\overline{-}$ & \\
\hline 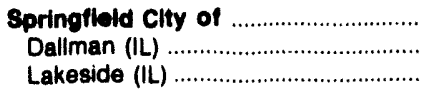 & $\begin{array}{r}79 \\
75 \\
5\end{array}$ & $\begin{array}{l}114.0 \\
114.0 \\
114.0\end{array}$ & $\begin{array}{l}23.72 \\
23.72 \\
23.72\end{array}$ & $\begin{array}{l}3.14 \\
3.14 \\
3.14\end{array}$ & $\overline{-}$ & $\overline{-}$ & $\bar{z}$ & $\overline{-}$ & $\overline{-}$ & $\overline{-}$ & $\overline{-}$ & $\begin{array}{l}100 \\
100 \\
100\end{array}$ & $\bar{z}$ & $\overline{-}$ \\
\hline 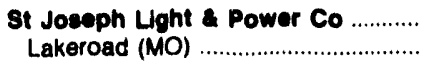 & $\begin{array}{l}11 \\
11\end{array}$ & $\begin{array}{l}139.9 \\
139.8\end{array}$ & $\begin{array}{l}33.41 \\
33.41\end{array}$ & $\begin{array}{l}3.70 \\
3.70\end{array}$ & & $\begin{array}{l}175.5 \\
175.5\end{array}$ & $\begin{array}{l}11.50 \\
11.50\end{array}$ & $\begin{array}{l}2.98 \\
2.98\end{array}$ & $\begin{array}{l}17 \\
17\end{array}$ & $\begin{array}{l}266.9 \\
266.9\end{array}$ & $\begin{array}{l}2.71 \\
2.71\end{array}$ & $\begin{array}{l}73 \\
73\end{array}$ & $\begin{array}{l}22 \\
22\end{array}$ & \\
\hline 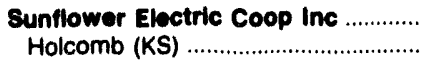 & $\begin{array}{l}68 \\
68\end{array}$ & $\begin{array}{l}108.0 \\
108.0\end{array}$ & $\begin{array}{l}18.16 \\
18.16\end{array}$ & $\begin{array}{l}.39 \\
.39\end{array}$ & $\overline{-}$ & $\overline{-}$ & $\overline{-}$ & $\overline{-}$ & & & $\begin{array}{l}2.78 \\
2.78\end{array}$ & $\begin{array}{l}100 \\
100\end{array}$ & $\overline{-}$ & : \\
\hline $\begin{array}{l}\text { Tacoma Clty of } \\
\text { Steam No.2 (WA) }\end{array}$ & 4 & $\begin{array}{l}182.0 \\
182.0\end{array}$ & $\begin{array}{l}34.61 \\
34.61\end{array}$ & $\begin{array}{l}.30 \\
.30\end{array}$ & : & $\begin{array}{l}698.0 \\
698.0\end{array}$ & $\begin{array}{l}40.46 \\
40.46\end{array}$ & $\begin{array}{l}.50 \\
.50\end{array}$ & $\begin{array}{l}2 \\
2\end{array}$ & & $\begin{array}{l}4.00 \\
4.00\end{array}$ & $\begin{array}{l}98 \\
98\end{array}$ & $"$ & \\
\hline $\begin{array}{l}\text { Tallahasese Clty of } \\
\text { Hopkins (FL) } \\
\text { Purdom (FL) }\end{array}$ & $\overline{-}$ & $\bar{z}$ & $\begin{array}{l}- \\
--\end{array}$ & $\overline{-}$ & $\overline{-}$ & $\bar{z}$ & $\bar{z}$ & $\overline{-}$ & $\begin{array}{r}1,438 \\
972 \\
467\end{array}$ & & $\begin{array}{l}2.66 \\
2.66 \\
2.66\end{array}$ & $\begin{array}{l}- \\
-\end{array}$ & $\overline{-}$ & $\begin{array}{l}10 \\
10 \\
10\end{array}$ \\
\hline 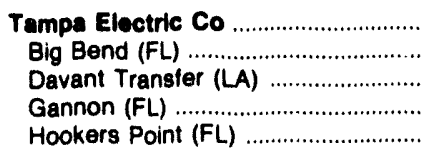 & $\begin{array}{r}463 \\
-\quad 391 \\
-\end{array}$ & $\begin{array}{l}196.7 \\
\overline{192.0} \\
221.6 \\
-\end{array}$ & $\begin{array}{l}48.21 \\
\overline{46.88} \\
55.47 \\
--\end{array}$ & $\begin{array}{l}1.82 \\
- \\
1.94 \\
1.18 \\
-\end{array}$ & $\begin{array}{r}109 \\
-\quad 6 \\
-\quad 2 \\
102\end{array}$ & $\begin{array}{l}233.5 \\
412.9 \\
-- \\
413.7 \\
221.4\end{array}$ & $\begin{array}{l}14.54 \\
24.10 \\
-- \\
24.11 \\
13.85\end{array}$ & $\begin{array}{r}1.13 \\
.39 \\
-- \\
.34 \\
1.18\end{array}$ & $\begin{array}{l}\bar{z} \\
\bar{z}\end{array}$ & $\begin{array}{l}- \\
-- \\
--\end{array}$ & $\begin{array}{l}\overline{-} \\
\overline{-} \\
-\end{array}$ & $\begin{array}{r}94 \\
-\overline{100} \\
99 \\
--\end{array}$ & $\begin{array}{r}6 \\
100 \\
- \\
1 \\
100\end{array}$ & $\overline{-}$ \\
\hline $\begin{array}{l}\text { Taunton Clty of } \\
\text { Cleary (MA) }\end{array}$ & $\overline{-}$ & $\overline{-}$ & $\overline{-}$ & $\overline{-}$ & 7 & $\begin{array}{l}236.5 \\
236.5\end{array}$ & $\begin{array}{l}14.81 \\
14.81\end{array}$ & $\begin{array}{l}2.20 \\
2.20\end{array}$ & $\begin{array}{l}126 \\
126\end{array}$ & $\begin{array}{l}249.7 \\
249.7\end{array}$ & $\begin{array}{l}2.57 \\
2.57\end{array}$ & $\overline{-}$ & 24 & 7 \\
\hline 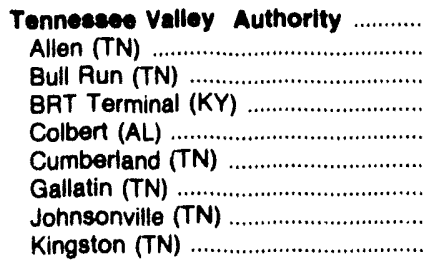 & $\begin{array}{r}3,232 \\
126 \\
162 \\
87 \\
278 \\
584 \\
152 \\
205 \\
223\end{array}$ & $\begin{array}{l}124.4 \\
121.0 \\
119.0 \\
128.7 \\
136.3 \\
129.7 \\
129.9 \\
124.5 \\
126.0\end{array}$ & $\begin{array}{l}29.57 \\
30.14 \\
30.33 \\
27.85 \\
32.62 \\
31.07 \\
32.05 \\
30.40 \\
31.95\end{array}$ & $\begin{array}{l}2.21 \\
2.08 \\
1.43 \\
1.39 \\
1.60 \\
2.18 \\
2.44 \\
1.85 \\
1.15\end{array}$ & $\begin{array}{r}18 \\
1 \\
5\end{array}$ & $\begin{array}{l}423.6 \\
425.3 \\
416.5 \\
478.5 \\
393.2 \\
427.2 \\
452.8 \\
-\end{array}$ & $\begin{array}{c}24.40 \\
24.92 \\
23.97 \\
-- \\
27.31 \\
22.74 \\
24.96 \\
26.57 \\
--\end{array}$ & $\begin{array}{l}.50 \\
.50 \\
.50 \\
- \\
.50 \\
.50 \\
.50 \\
.50 \\
-.\end{array}$ & $\begin{array}{l}\overline{-} \\
\bar{z} \\
\overline{-} \\
\overline{-} \\
\overline{-}\end{array}$ & $\begin{array}{l}- \\
\overline{-} \\
\overline{-} \\
\overline{-} \\
\overline{-} \\
\overline{-}\end{array}$ & $\begin{array}{l}- \\
\overline{-} \\
\overline{-} \\
\overline{-} \\
\overline{-} \\
\overline{-}\end{array}$ & $\begin{array}{r}100 \\
100 \\
99 \\
100 \\
100 \\
100 \\
100 \\
100 \\
100\end{array}$ & $\begin{array}{l}: \\
: 1 \\
:- \\
: \\
--\end{array}$ & $\bar{z}$ \\
\hline
\end{tabular}

See notes and footnotes at end of table. 
Table 63. Recelpts, Average Cost, and Quallty of Fosell Fuels Dellvered to U.S. Electric Utilities by Company and Plant, September 1993 (Continued)

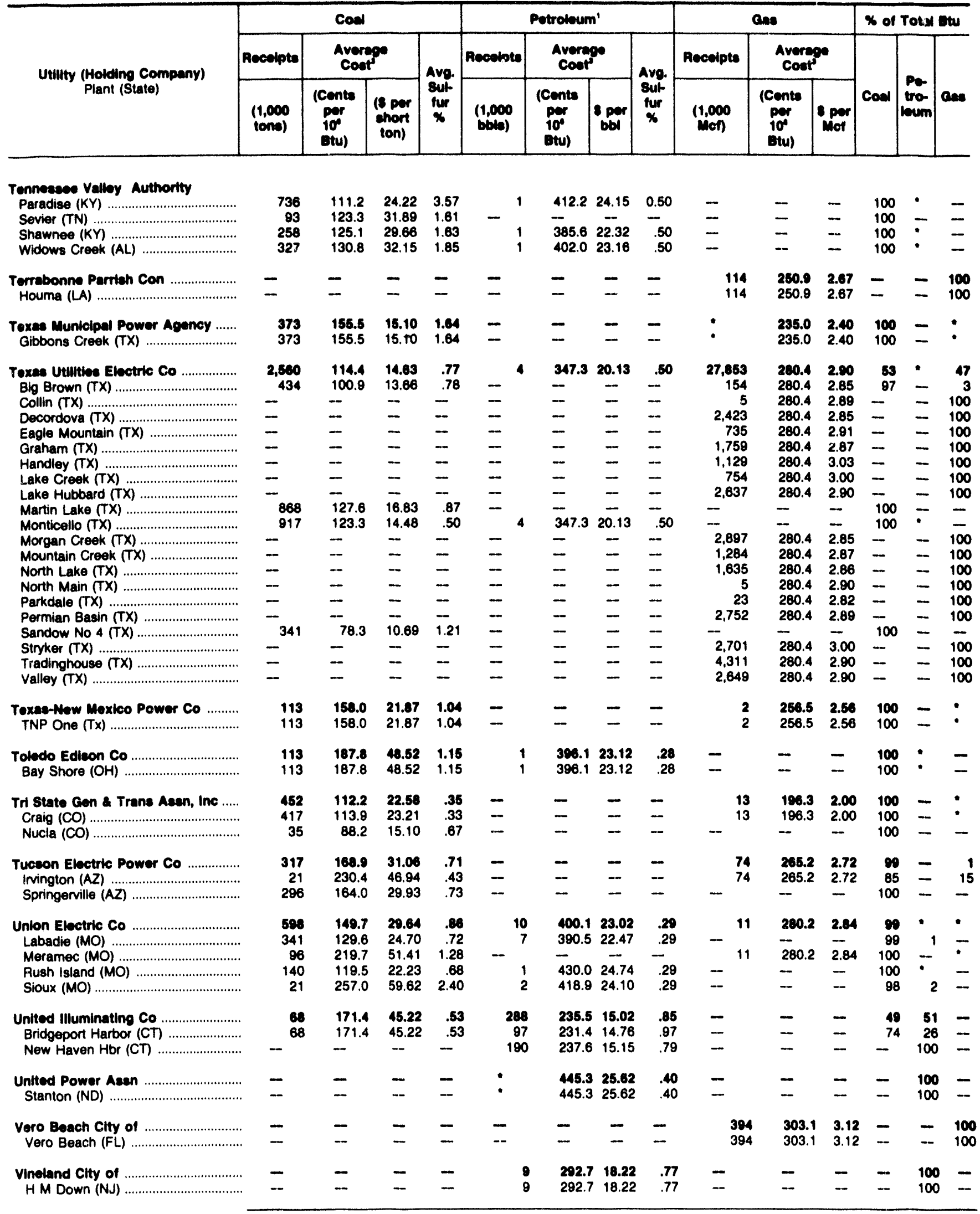

See notes and footnotes at end of table. 
Table 63. Recelpts, Average Cost, and Quality of Fossill Fuels Dellvered to U.S. Electric Util!ties by Company and Plant, September 1993 (Continued)

\begin{tabular}{|c|c|c|c|c|c|c|c|c|c|c|c|c|c|c|}
\hline \multirow{3}{*}{$\begin{array}{l}\text { Utility (Holding Company) } \\
\text { Plant (State) }\end{array}$} & \multicolumn{4}{|c|}{ Coal } & \multicolumn{4}{|c|}{ Potroloum' } & \multicolumn{3}{|c|}{ Cas } & \multicolumn{3}{|c|}{ \% of Total Btu } \\
\hline & \multirow{2}{*}{\begin{tabular}{|c|} 
Aecolpte \\
$(1,000$ \\
tons $)$
\end{tabular}} & \multicolumn{2}{|c|}{$\begin{array}{l}\text { Average } \\
\text { Coot? }\end{array}$} & \multirow{2}{*}{$\begin{array}{c}\text { Avg. } \\
\text { Sul- } \\
\text { fur } \\
\%\end{array}$} & \multirow{2}{*}{\begin{tabular}{|c|} 
Recelpts \\
$(1,000$ \\
bblo $)$
\end{tabular}} & \multicolumn{2}{|c|}{$\begin{array}{l}\text { Average } \\
\text { Coot" }\end{array}$} & \multirow{2}{*}{$\begin{array}{c}\text { Avg. } \\
\text { sut. } \\
\text { fur } \\
\%\end{array}$} & \multirow{2}{*}{$\begin{array}{c}\text { Recelpte } \\
\begin{array}{c}(1,000 \\
\text { Mef })\end{array}\end{array}$} & \multicolumn{2}{|c|}{$\begin{array}{l}\text { Average } \\
\text { Coot? }\end{array}$} & \multirow[b]{2}{*}{ Coal } & \multirow[b]{2}{*}{$\begin{array}{l}\text { Po- } \\
\text { tro- } \\
\text { loum }\end{array}$} & \multirow[b]{2}{*}{ Cas } \\
\hline & & $\begin{array}{l}\text { (Conts } \\
\text { per } \\
10^{\circ} \\
\text { Btu) }\end{array}$ & $\begin{array}{l}\text { (s per } \\
\text { ehort } \\
\text { ton) }\end{array}$ & & & $\begin{array}{l}\text { (Cente } \\
\text { per } \\
10^{4} \\
\text { Btu) }\end{array}$ & par & & & $\begin{array}{l}\text { (Cents } \\
\text { per } \\
10^{\circ} \\
\text { Btu) }\end{array}$ & Mor & & & \\
\hline 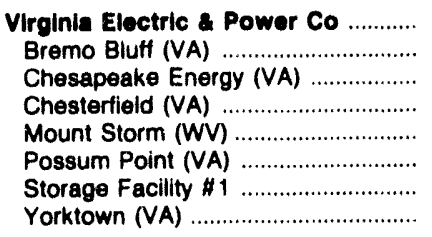 & $\begin{array}{r}1,039 \\
32 \\
185 \\
278 \\
446 \\
-\quad 38 \\
-\quad 59\end{array}$ & $\begin{array}{l}135.5 \\
145.7 \\
152.0 \\
140.4 \\
119.2 \\
147.4 \\
166.4 \\
\end{array}$ & $\begin{array}{l}34.54 \\
37.63 \\
39.78 \\
36.14 \\
29.70 \\
38.23 \\
-- \\
43.16\end{array}$ & $\begin{array}{r}1.45 \\
.92 \\
1.00 \\
1.20 \\
1.81 \\
1.07 \\
-- \\
1.90\end{array}$ & $\begin{array}{r}747 \\
-\quad 10 \\
-\quad 4 \\
40 \\
693 \\
-\quad\end{array}$ & $\begin{array}{l}219.9 \\
-- \\
367.9 \\
\overline{453.1} \\
224.5 \\
216.4 \\
--\end{array}$ & $\begin{array}{c}13.93 \\
-\overline{21.63} \\
- \\
26.64 \\
14.01 \\
13.73 \\
-\end{array}$ & $\begin{array}{l}0.87 \\
-- \\
.20 \\
-- \\
.20 \\
.70 \\
.89 \\
--\end{array}$ & 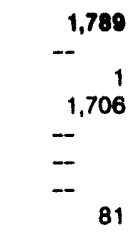 & $\begin{array}{l}281.7 \\
\overline{443.8} \\
285.7 \\
-\overline{-} \\
\overline{1} \\
\overline{191.3}\end{array}$ & $\begin{array}{l}2.95 \\
-- \\
4.68 \\
2.99 \\
-- \\
-- \\
-\overline{1.91}\end{array}$ & $\begin{array}{r}80 \\
100 \\
99 \\
80 \\
100 \\
80 \\
-- \\
95\end{array}$ & $\begin{array}{c}14 \\
-- \\
1 \\
- \\
20 \\
100 \\
--\end{array}$ & $\begin{array}{c}6 \\
-- \\
20 \\
-- \\
- \\
- \\
5\end{array}$ \\
\hline $\begin{array}{l}\text { Weat Penn Power Co } \\
\text { Armstrong (PA) } \\
\text { Hattield (PA) } \\
\text { Mitchell (PA) }\end{array}$ & $\begin{array}{r}346 \\
54 \\
240 \\
52\end{array}$ & $\begin{array}{l}146.4 \\
130.4 \\
152.9 \\
131.2\end{array}$ & $\begin{array}{l}37.16 \\
32.49 \\
39.51 \\
31.18\end{array}$ & $\begin{array}{l}2.24 \\
1.93 \\
2.18 \\
2.86\end{array}$ & $\begin{array}{r}2 \\
-\quad 2 \\
-\quad\end{array}$ & $\begin{array}{l}414.6 \\
409.6 \\
415.2 \\
--\end{array}$ & $\begin{array}{c}24.56 \\
24.26 \\
24.59 \\
-\end{array}$ & $\begin{array}{l}.27 \\
.27 \\
.27 \\
--\end{array}$ & - & $\begin{array}{l}425.6 \\
\overline{425.6}\end{array}$ & $\begin{array}{l}4.26 \\
-- \\
4.26\end{array}$ & $\begin{array}{l}100 \\
100 \\
100 \\
100\end{array}$ & $:$ & $\begin{array}{l}\dot{-} \\
--\end{array}$ \\
\hline $\begin{array}{l}\text { Weat Toxas Utllitios Co } \\
\text { Fort Phantom (TX) } \\
\text { Oak Creek (TX) } \\
\text { Oklaunion (TX) } \\
\text { Paint Creek (TX) } \\
\text { Rio Pecos (TX) } \\
\text { San Angelo (TX) }\end{array}$ & $\begin{array}{l}{ }^{153} \\
- \\
- \\
\overline{-}\end{array}$ & $\begin{array}{l}178.9 \\
\overline{-} \\
\overline{178.9} \\
\overline{-} \\
=-\end{array}$ & $\begin{array}{l}29.63 \\
\overline{-} \\
29.63 \\
\overline{-} \\
-\end{array}$ & $\begin{array}{l}.37 \\
\overline{-} \\
.37 \\
- \\
-\end{array}$ & $\begin{array}{l}\bar{z} \\
\bar{z} \\
\bar{z}\end{array}$ & $\begin{array}{l}\overline{-} \\
\bar{z} \\
\bar{z} \\
\overline{-}\end{array}$ & $\begin{array}{l}\overline{-} \\
\overline{-} \\
\overline{-} \\
\overline{-}\end{array}$ & $\begin{array}{l}\bar{z} \\
\overline{-} \\
\bar{z} \\
\overline{-}\end{array}$ & $\begin{array}{r}4,373 \\
1,800 \\
376 \\
-\quad \\
511 \\
884 \\
802\end{array}$ & $\begin{array}{l}229.4 \\
234.3 \\
234.0 \\
235.4 \\
201.9 \\
242.4\end{array}$ & $\begin{array}{l}2.31 \\
2.38 \\
2.35 \\
- \\
2.38 \\
2.02 \\
2.43\end{array}$ & $\begin{array}{l}36 \\
-- \\
-- \\
100 \\
-- \\
--\end{array}$ & $\begin{array}{l}\overline{-} \\
\bar{z} \\
\bar{z} \\
\bar{m}\end{array}$ & $\begin{array}{r}64 \\
100 \\
100 \\
-100 \\
100 \\
100\end{array}$ \\
\hline $\begin{array}{l}\text { Western Farmers Elec Coop Inc .... } \\
\text { Anadarko (OK) . } \\
\text { Hugo (OK) } \\
\text { Mooreland (OK) . }\end{array}$ & $-{ }_{-}^{151}$ & $\begin{array}{l}165.9 \\
-- \\
165.9 \\
--\end{array}$ & $\begin{array}{l}27.66 \\
-- \\
27.66 \\
--\end{array}$ & $\begin{array}{l}.40 \\
-.40 \\
-\end{array}$ & $\bar{z}$ & $\begin{array}{l}\overline{-} \\
\bar{m}\end{array}$ & $\bar{z}$ & $\begin{array}{l}\overline{-} \\
\overline{-}\end{array}$ & $\begin{array}{r}882 \\
810 \\
-\quad 72\end{array}$ & $\begin{array}{l}256.0 \\
256.0 \\
255.8\end{array}$ & $\begin{array}{l}2.62 \\
2.62 \\
\overline{2.62}\end{array}$ & $\frac{74}{100}$ & $\begin{array}{l}\overline{-} \\
\bar{z}\end{array}$ & $\begin{array}{r}26 \\
100 \\
- \\
100\end{array}$ \\
\hline 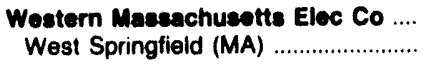 & $\overline{-}$ & $\overline{-}$ & $\overline{-}$ & $\overline{-}$ & $\overline{-}$ & $\overline{-}$ & $\overline{-}$ & $\overline{-}$ & $\begin{array}{l}69 \\
69\end{array}$ & $\begin{array}{l}235.0 \\
235.0\end{array}$ & $\begin{array}{l}2.39 \\
2.39\end{array}$ & $\overline{-}$ & $\overline{-}$ & $\begin{array}{l}100 \\
100\end{array}$ \\
\hline 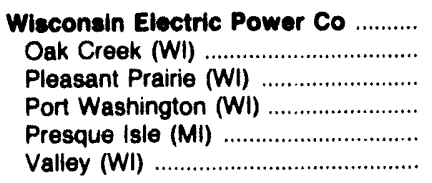 & $\begin{array}{r}764 \\
83 \\
411 \\
-\quad \\
200 \\
70\end{array}$ & $\begin{array}{r}118.1 \\
150.3 \\
67.5 \\
- \\
164.9 \\
170.7\end{array}$ & $\begin{array}{l}23.32 \\
37.71 \\
11.58 \\
\overline{33.83} \\
45.05\end{array}$ & $\begin{array}{r}.52 \\
.49 \\
.34 \\
- \\
.57 \\
1.45\end{array}$ & $\begin{array}{l}\bar{z} \\
\bar{z} \\
\overline{-}\end{array}$ & $\begin{array}{l}- \\
- \\
- \\
-\end{array}$ & $\begin{array}{l}\bar{z} \\
\bar{z} \\
\overline{-}\end{array}$ & $\begin{array}{l}- \\
\overline{-} \\
\overline{-}\end{array}$ & $\begin{array}{r}77 \\
45 \\
25 \\
4\end{array}$ & $\begin{array}{l}323.1 \\
317.6 \\
321.2 \\
345.6 \\
- \\
386.1\end{array}$ & $\begin{array}{l}3.28 \\
3.23 \\
3.27 \\
3.50 \\
\overline{3.91}\end{array}$ & $\begin{array}{r}99 \\
98 \\
100 \\
-100 \\
100\end{array}$ & $\begin{array}{l}- \\
- \\
- \\
-\end{array}$ & $\begin{array}{r}1 \\
2 \\
100 \\
--\end{array}$ \\
\hline $\begin{array}{l}\text { Wieconain Power L Llght Co } . . . . . . . . \\
\text { Blackhawk (WI) } \\
\text { Columbia (WI) } \\
\text { Edgewater (WI) } \\
\text { Nelson Dewey (WI) } \\
\text { Rock River (WI) }\end{array}$ & $\begin{array}{r}520 \\
-\quad 209 \\
203 \\
85 \\
23\end{array}$ & $\begin{array}{l}127.8 \\
125.9 \\
131.4 \\
111.6 \\
168.3\end{array}$ & $\begin{array}{l}23.41 \\
\overline{21.46} \\
24.83 \\
21.64 \\
34.79\end{array}$ & $\begin{array}{r}.54 \\
-.51 \\
.55 \\
.39 \\
1.28\end{array}$ & $\begin{array}{l}\overline{-} \\
\overline{-} \\
\overline{-}\end{array}$ & $\begin{array}{l}\bar{z} \\
\bar{z} \\
\bar{z}\end{array}$ & $\begin{array}{l}\overline{-} \\
\bar{z} \\
\overline{-}\end{array}$ & $\begin{array}{l}- \\
\bar{z} \\
\overline{-}\end{array}$ & $\begin{array}{l}\overline{-} \\
\overline{-}\end{array}$ & $\begin{array}{l}300.9 \\
300.9 \\
- \\
- \\
- \\
--\end{array}$ & $\begin{array}{l}3.06 \\
3.06 \\
- \\
- \\
- \\
-\end{array}$ & $\begin{array}{l}100 \\
-100 \\
100 \\
100 \\
100\end{array}$ & $\begin{array}{l}- \\
\overline{-} \\
\overline{-} \\
-\end{array}$ & $\begin{array}{l}\ddot{100} \\
-- \\
- \\
--\end{array}$ \\
\hline $\begin{array}{l}\text { Wlaconain Public Service Corp ....... } \\
\text { Pulliam (WI) .... } \\
\text { Weston (WI) . }\end{array}$ & $\begin{array}{l}215 \\
114 \\
101\end{array}$ & $\begin{array}{l}120.2 \\
125.7 \\
113.2\end{array}$ & $\begin{array}{l}22.35 \\
24.51 \\
19.90\end{array}$ & $\begin{array}{l}.32 \\
.34 \\
.29\end{array}$ & $\overline{-}$ & $\bar{z}$ & $\bar{z}$ & $\overline{-}$ & $\begin{array}{r}27 \\
19 \\
8\end{array}$ & $\begin{array}{l}414.5 \\
453.1 \\
322.6\end{array}$ & $\begin{array}{l}4.21 \\
4.61 \\
3.27\end{array}$ & $\begin{array}{r}99 \\
99 \\
100\end{array}$ & $\bar{z}$ & $\begin{array}{r}1 \\
.\end{array}$ \\
\hline $\begin{array}{l}\text { Wyandotte Municlpal Serv Comm .. } \\
\text { Wyandotte (MI) }\end{array}$ & $\begin{array}{l}16 \\
16\end{array}$ & $\begin{array}{l}190.0 \\
190.0\end{array}$ & $\begin{array}{l}50.35 \\
50.35\end{array}$ & $\begin{array}{l}.69 \\
.69\end{array}$ & $\overline{-}$ & $\overline{-}$ & $\overline{-}$ & $\overline{-}$ & $\overline{-}$ & $\overline{-}$ & $\overline{-}$ & $\begin{array}{l}100 \\
100\end{array}$ & $\overline{-}$ & - \\
\hline U.S. Total & 65,358 & 138.5 & 28.44 & 1.12 & 15,766 & 231.0 & 14.66 & 1.25 & 249,708 & ${ }^{2} 263.6$ & 2.70 & 79 & 6 & 15 \\
\hline
\end{tabular}

1. The September 1993 petroleum coke receipts were 131,082 short tons and the cost was 70.3 cents per million Btu.

2 Monetary values are expressed in nominal terms.

- The entry includes at least one delivery at a price of 1,000 cents per million etu or greater. High price is frequently caused when fixed costs are averaged into a small quantity.

- Less than 0.05

Notes: - Totals may not equal sum of components because of independent rounding. - Data are for electric generating plants with a total steam-electric and combined-cycle nameplate capacity of 50 or more megawatts. -Data for 1993 are preliminary. $-M c f=x$ thousand cubic feet and bbl=barrel.eHolding Companies are: AEP is American Electric Power, APS is Allegheny Power System, ACE is Atlantic City Electric, CSW is Central \& South West Corporation, CES is Commonwealth Energy System, DMV is Delmarva, EU is Eastern Utilities Associates Company, GPS is General Public Utilities, MSU is Middle South Utilities, NEES is New England Electric System, NU is Northeast Utilities, SC is Southern Company, TU is Texas Utilities.

Source: Federal Energy Regulatory Commission, FERC Form 423, "Monthly Report of Cost and Quality of Fuels for Electric Plants." 


\section{Appendix A}

Major

Disturbances and

Unusual

Occurrences in

U.S. Electric

Power Systems 


\section{Appendix A}

\section{Major Disturbances and Unusual Occurrences in U.S. Electric Power Systems}

Electric power systems are subject to a variety of incidents that, to a smaller or greater degree, may adversely affect the delivery of electricity to consumers. Among these are natural phenomena (such as storms and earthquakes); failure of electric system components; accidental or purposeful activities inimical to continued safe operation of electric power systems; and difficulties associated with the normal operation of large, extremely complex, real-time systems.

Under current Federal regulations, some disturbances are reported to the Federal Government. The legal basis for the requirements and the specifications of information reported are detailed in Title 10, Part 205, Subpart W, of the Code of Federal Regulations, Sections $205.350-205.353$, published in the Federal Register on October 31, 1986.

In general, the incidents to be reported are grouped into two categories: mandatory in all cases and mandatory if the incident meets specified criteria, where the utility involved is permitted to exercise some judgment as to whether the criteria have been met. Underlying the formulation of the reporting criteria, requirements, and procedures was the need for the Federal Government to be aware of potentially dangerous situations, tempered by the desire to minimize burdens on the reporting utilities. Another consideration in the development of the rules was the benefit gained from knowledge of the causes and effects of undesired events that may have been caused by unforeseen system defects or by purposeful adverse actions to system design and operation. The final rules reflect modification of the preliminary rules, as published in the Federal Register, based on comments from the electric power industry and the general public.

A report is mandatory when, for the purpose of maintaining the continuity of the bulk power supply system, a utility (1) initiates a system voltage reduction of 3 percent or more, (2) disconnects circuits supplying over 100 megawatts of firm customer load, or (3) issues an appeal to the public for a voluntary reduction in the use of electricity. A report is also mandatory in regard to any actual or suspected act of sabotage or terrorism directed at the bulk power supply system.

In general, reports are to be made by telephone to the Emergency Operating Center, Department of Energy, in Washington, D.C. as soon as practicable for instances of load shedding or loss of service and, at the latest, within 3 hours of the beginning of a service interruption. For other disturbances, the allowable reporting time ranges from 24 hours to days. Written reports may be required by the Director, Office of Energy Emergency Operations, if the circumstances so indicate.

The operation of the bulk power system in the United States should be as trouble-free as possible. To that end, information is collected, as discussed above, regarding major disturbances to the normal functioning of that system. Events, such as damage to some local distribution circuits by storms or other uncontrollable events, do not greatly affect the supply of bulk power to the sytem as a whole. These events are more properly the concern of local and State authorities.

\section{Data Sources}

The information contained in Table $A 1$ is based on data from the Form OE-417R, "Electric Power System Emergency Report." These data are collected by the Office of Energy Emergency Operations (under the Assistant Secretary for International Affairs and Energy Emergencies). 
Table A1. Major Disturbances and Unusual Occurrences in U.S. Electric Power Systems, 1993

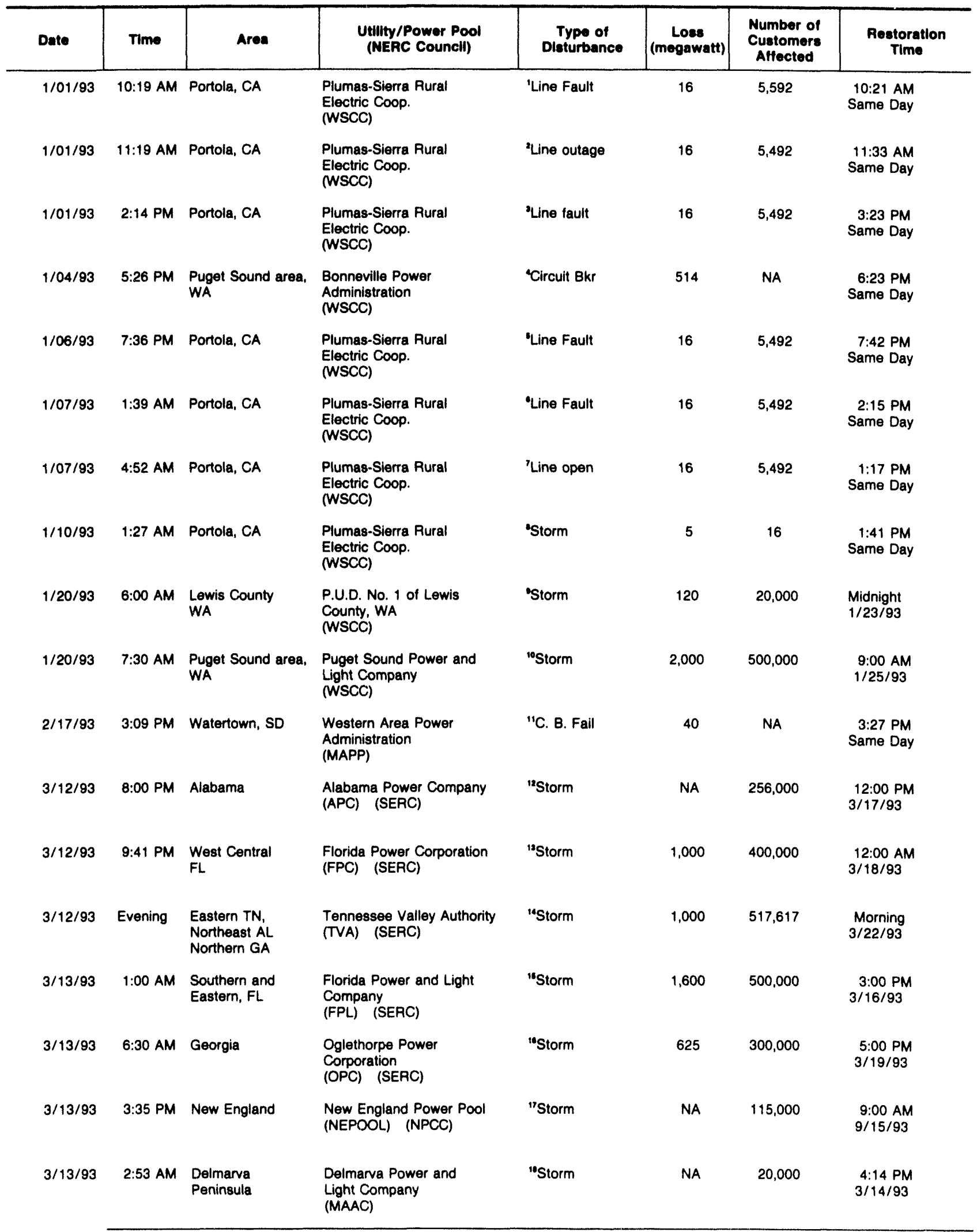

See endnotes on the next page. 
Table A1. Major Disturbances and Unusual Occurrences in U.S. Electric Power Systems, 1993-Continued

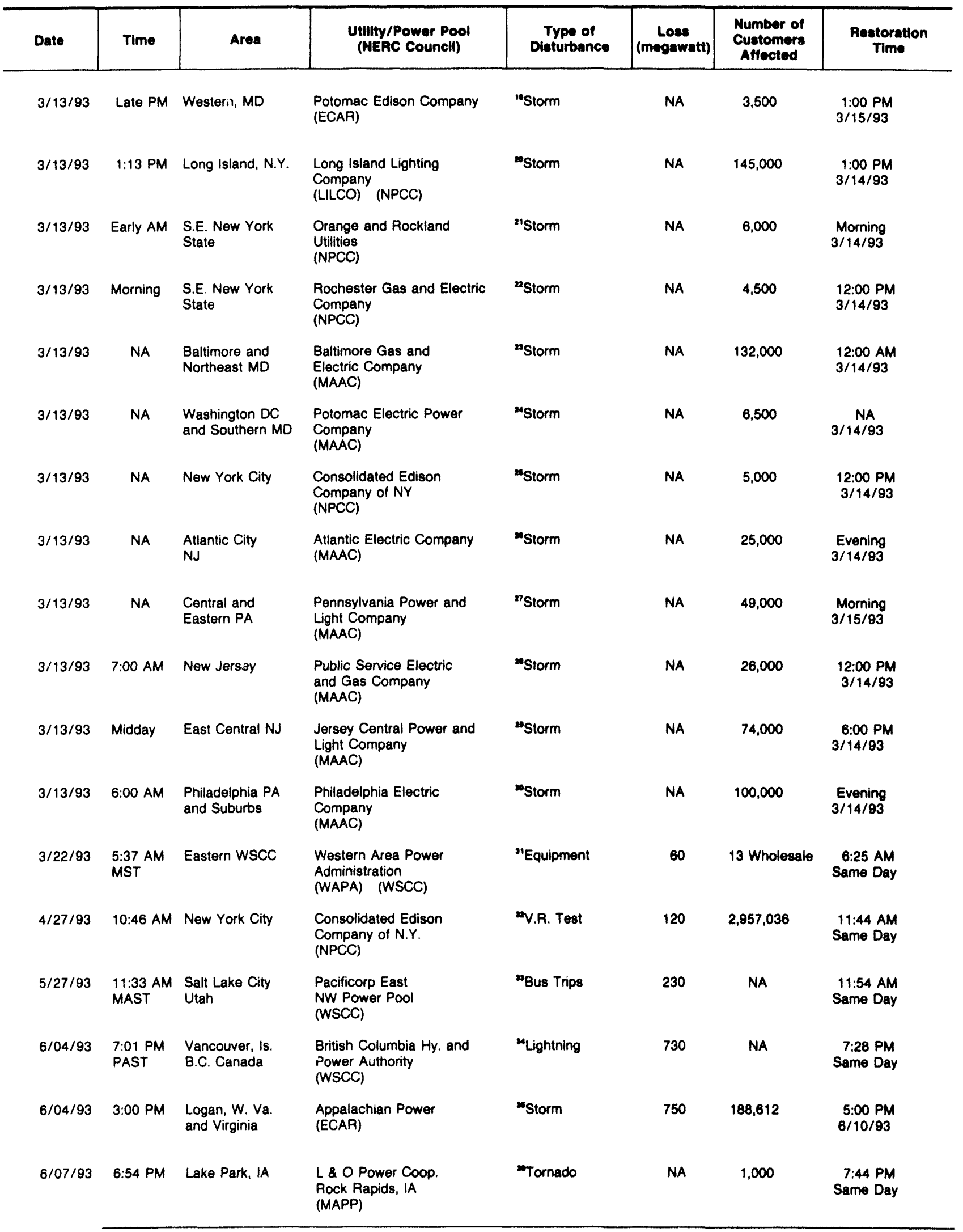

See endnotes on the next page. 
Table A1. Major Disturbances and Unusual Occurrences in U.S. Electric Power Systems, 1993-Continued

\begin{tabular}{|c|c|c|c|c|c|c|c|}
\hline Date & Time & Araa & $\begin{array}{l}\text { Utillty/Power Pool } \\
\text { (NERC Councli) }\end{array}$ & $\begin{array}{c}\text { Type of } \\
\text { Disturbance }\end{array}$ & $\begin{array}{c}\text { Loss } \\
\text { (megawatt) }\end{array}$ & $\begin{array}{l}\text { Number of } \\
\text { Customers } \\
\text { Affected }\end{array}$ & $\begin{array}{l}\text { Restoration } \\
\text { Time }\end{array}$ \\
\hline $6 / 10 / 93$ & 10:30 AM & Pierre, SD & $\begin{array}{l}\text { Western Area Power } \\
\text { Administration } \\
\text { (MAPP) }\end{array}$ & ${ }^{37}$ Accident & 23 & NA & $\begin{array}{l}11: 15 \text { AM } \\
\text { Same Day }\end{array}$ \\
\hline $6 / 27 / 93$ & $\begin{array}{l}\text { 12:41 PM } \\
\text { MAST }\end{array}$ & $\begin{array}{l}\text { Wyoming } \\
\text { Colorado, Border }\end{array}$ & $\begin{array}{l}\text { Tri-State G. \& T. } \\
\text { Association, Inc. } \\
\text { (WSCC) }\end{array}$ & ${ }^{30}$ Forest fire & None & None & NA \\
\hline $7 / 02 / 93$ & $\begin{array}{l}11: 41 \text { PM } \\
\text { MAST }\end{array}$ & $\begin{array}{l}\text { S.W. Wyoming } \\
\text { and S.E. Idaho }\end{array}$ & $\begin{array}{l}\text { Pacificorp-East } \\
\text { (WSCC) }\end{array}$ & ${ }^{*}$ Line Fault & 75 & NA & \\
\hline $7 / 14 / 93$ & $\begin{array}{l}9: 43 \text { AM } \\
\text { PAST }\end{array}$ & $\begin{array}{l}\text { S. Oregon and } \\
\text { N. California }\end{array}$ & $\begin{array}{l}\text { Pacificorp-West } \\
\text { (WSCC) }\end{array}$ & ${ }^{40} \mathrm{Fallen}$ trees & 355 & 100,000 & $\begin{array}{r}11: 18 \text { AM } \\
\text { Same Day }\end{array}$ \\
\hline $7 / 20 / 93$ & $\begin{array}{l}\text { 1:20 PM } \\
\text { CDT }\end{array}$ & S.W. Missouri & $\begin{array}{l}\text { Empire District } \\
\text { Electric Company } \\
\text { (SPP) }\end{array}$ & "High Load & 225 & NA & $\begin{array}{c}\text { 4:30 PM } \\
\text { Same Day }\end{array}$ \\
\hline $7 / 20 / 93$ & 9:40 PM & Southern Illinois & $\begin{array}{l}\text { Southern Illinois Power } \\
\text { Cooperative } \\
\text { (MAIN) }\end{array}$ & ${ }^{42}$ Vandalism & 15 & 14,500 & $\begin{array}{l}1: 17 \text { PM } \\
7 / 21 / 93\end{array}$ \\
\hline $7 / 28 / 93$ & 12:00 PM & $\begin{array}{l}\text { Kansas City, } \\
\text { Missouri }\end{array}$ & $\begin{array}{l}\text { Utilitcorp United Inc } \\
\text { Mo. Pub. Service } \\
\text { (SPP) }\end{array}$ & ${ }^{4}$ Fuel Supply & None & & $\begin{array}{l}\text { Not } \\
\text { Applicable }\end{array}$ \\
\hline $8 / 21 / 93$ & $\begin{array}{l}\text { 6:03 PM } \\
\text { CDT }\end{array}$ & Ordill, Illinois & $\begin{array}{l}\text { Central Illinois Public } \\
\text { Service Company } \\
\text { (MAIN) }\end{array}$ & "Sabotage & NA & 1,000 & $\begin{array}{r}\text { 7:30 PM } \\
\text { Same day }\end{array}$ \\
\hline $8 / 25 / 93$ & $\begin{array}{l}\text { 5:06 AM } \\
\text { HST }\end{array}$ & $\begin{array}{l}\text { Honolulu, } \\
\text { Oahu, Hawaii }\end{array}$ & $\begin{array}{l}\text { Hawaiian Electric Company } \\
\text { Hawaii }\end{array}$ & ${ }^{46}$ Equipment & NA & 79,000 & $\begin{array}{r}\text { 8:00 AM } \\
\text { Same day }\end{array}$ \\
\hline $10 / 04 / 93$ & $2: 14 \mathrm{AM}$ & $\begin{array}{l}\text { Seattle, WA } \\
\text { Business Area }\end{array}$ & $\begin{array}{l}\text { Seattle City Light } \\
\text { (WSCC) }\end{array}$ & "Cable Fire & 10.9 & 1,800 & $\begin{array}{l}12: 00 \mathrm{AM} \\
10 / 08 / 93\end{array}$ \\
\hline $10 / 10 / 93$ & $\begin{array}{l}1: 08 \text { AM } \\
\text { PAST }\end{array}$ & Washington State & $\begin{array}{l}\text { BPA and several P.U.D.S } \\
\text { (WSCC) }\end{array}$ & "Equipment & 713 & NA & $\begin{array}{l}\text { 2:22 AM } \\
\text { Same Day }\end{array}$ \\
\hline $10 / 10 / 93$ & $\begin{array}{l}3: 25 \text { AM } \\
\text { PAST }\end{array}$ & West Columbia, Al & $\begin{array}{l}\text { Alabama Power Company } \\
\text { (SERC) }\end{array}$ & "Sabotage & NA & 100 & $\begin{array}{l}3: 05 \text { AM } \\
10 / 11 / 93\end{array}$ \\
\hline $10 / 12 / 93$ & $\begin{array}{l}\text { 6:04 AM } \\
\text { PAST }\end{array}$ & $\begin{array}{l}\text { Los Angeles } \\
\text { South Nevada } \\
\text { Utah }\end{array}$ & $\begin{array}{l}\text { LA Dept of Wtr and Pwr } \\
\text { Intermt. Pwr. } \\
\text { (WSCC) }\end{array}$ & ${ }^{50}$ Operation & None & None & $\begin{array}{l}\text { Not } \\
\text { Applicable }\end{array}$ \\
\hline $10 / 30 / 93$ & NA & Syimar, CA & $\begin{array}{l}\text { LA Dept of Wtr and Pwr } \\
\text { (WSCC) }\end{array}$ & ${ }^{\text {s1Fire }}$ & NA & NA & NA \\
\hline
\end{tabular}

1 A tree fell into the Pacific Gas and Electric Company line that supplies the cooperative, causing the line to trip.

- The Pacific Gas and Electric Company line supplying the cronerative was out of service. No cause was stated.

- A 21-kilovolt circuit fell into the 60-kilovolt circuit from Pacii:. Fas and Electric that supplies the cooperative; the supply line tripped.

- The 230-kilovolt circuit breaker at the Olympla Substation of Bonnesville Power Administration (BPA) caught fire, causing loss of the east bus. This breaker was on the 230-kilovolt line to Kitsap. The Olympia-to-Shelton No. 4230 -kilovolt line then was tripped by protective relays (reason unknown), separating the Olympic peninsula from the 230-kilovolt system. The resulting overload caused tripping of the Shelton-Kitsap No. 2 115-kilovolt line within 12 seconds of the breaker fire and also resulted in tripping of the 230/115 kilovolt transformer at Fairmount Substation on overload. Load was restored in times ranging from 9 minutes to 57 minutes. Customers without service were Daishowa Paper Mill, Rayonier Paper Mill, U.S. Navy, and an unknown number of customers in the service areas of City of Port Angeles, Puget Sound Power and Light Company and Clallum County (WA) Public Utility District No. 1.

: Outage of supply line from Pacific Gas and Electric Company due to fault on neutral overcurrent.

- Fault on the Pacitic Gas and Electric Company supply line; reason unknown.

- Outage of supply line from Pacific Gas and Electric Sompany; a jumper burned open on the first pole out of Quincy substation on the Pacific Gas and Electric system. 
- Supply line from Pacific Gas and Electric Company locked out due to storm.

- A windstorm caused trees to fall into overhead lines, breaking poles, crossarms and insulators, and damaging distribution transformers. Many miles of line conductor were knocked to the ground.

10 By noon on January 20,1993, the storm which had begun at about 7:30 AM had caused the loss of about 500,000 customers. As of 10:00 AM on January 21, some 340,000 customers were still without power. At 6:30 AM on January 22, about 160,000 customers were still without power, with 350 line crews working on service restoration. There were no significant generation problems but many distribution lines and about 50 transmission lines were down.

11 Failure of a 115-kilovolt bus circuit breaker de-energized the bus and circuits to Waterloo, South Dakota and the East River Cooperative. The total load of the two systems was about 40-megawatts. Watertown has a population of about 17,000. Power to the cooperative was restored in one minute by switching to an alternate feeder. Power to Watertown was restored in 18 minutes.

12 Heavy snowtall and storm conditions damaged distribution facilities and tripped 18 transmission lines. Lines out of service at various times during the storm were: 15 at 115 -kilovolts, 1 at 161 -kilovolts, 2 at 230 -kilovolts. The 500 kilovolts lines did not trip.

is Severe weather with winds gusting to $100 \mathrm{mph}$ and sustained winds of $20-30 \mathrm{mph}$ traversed the 32 -county service area of FPC. Winds damaged microwave facilities at Crystal River Plant and at major load centers in the Orlando area. Salt spray contamination and flooding to the level of switch. yard foundation caps also occurred at the Crystal River plant. Tie-line, substation and generation control and indication circuits were lost. High winds hampered aerial bucket-truck work. An FPC Phase I Capacity Alert was declared at 5:00 PM on March 13 due to severe cold front, 2 large steam units on annual maintenance and one large unit on forced outage. On Sunday March 14, two additional large steam units were forced out due to failure of contaminated insulation, and a Statewide Capacity Alert was declared for Monday morning March 15 because state operating reserve margin was less than the required amount. On March 15, an FPC Phase II Capacity Alert was declared at 6:00 AM because high loads were anticipated and some generating units were unavailable. A Statewide Capacity Emergency was declared because it appeared possible that firm loads might have to be curtailed. An FPC Phase III Capacity Alert was declared at 7:06 AM. Load management interruptible load actions were implemented, a 1.5 percent voltage reduction was instituted and emergencypower purchases were made. Firm loads were not curtailed.At 7:53 AM, the FPC Phase III Capacity Alert was terminated. The Phase II and Phase I Capacity Alerts were ended at 11:00 AM. On March 17, transmission problems were again experienced because of wind-blown salt contamination and misting rain and fog. Problems at the Crystal River plant 230-kilovolt and 500-kilovolt switchyards caused loss of all remaining units at the plant (1,350 megawatt scheduled off and 2,000 megawatt forced out). The last customers were returned to service on March 18.

14 The TVA service area was subjected to blizzard conditions on the evening of March 12 and the morning of March 13 . Winds and falling trees damaged the distribution system, mainly in the area from Scottsboro, Alabama to Bristol, Virginia. Ten transmission and subtransmission lines tripped and were restored in about 2.5 hours on the average. As of March 22 about 2,500 customers, mostly in the mountainous terrain of northern Alabame and northern Georgia, were still without electrical service. About 125 line crews were used to restore service, made up of TVA construction and maintenance personnel and crews from TVA distributors in the central and western portions of TVA's service area. The total customers without power on March 13 were: Tennessee 348,960; Alabama 91,157; Georgia 77,500.

is Heavy winds, lightning and a cold fromt traversed the FPL service area beginning Saturday, March 13. Fifteen transmission lines tripped out of service some of them several times. Thirteen of the lines serve a total of 47 distribution substations. All transmission lines and substations were back in service by the end of the day on March 13. All distribution feeders were back in service by Tuesday morning March 16 . At the height of the storm on March 13 about 500,000 customers were without service. The number was reduced to 140,000 by Sunday morning March 14 . As of 3:00 PM on Tuesday March 16, 13,500 customers were still without service, in northeastern Florida. The company had 1,650 utility and contract working crews on service restoration.

"OPC provides wholesale power to 39 of the 42 Electric Membership Corporations in Georgia. "Tornado-like winds" in southern Georgia and a blizzard in the central and northern parts of the state, beginning early Saturday, March 13 and continuing through Sunday, March 14 damaged distribution facilities. Transmission facilities were mostly intact.

1 A blizzard with 70-mph winds and much snow caused tripping of two 345-kilovolt lines and three generators. In addition, nuclear units Millstone 1 and 3 had to reduce output because their cooling water intakes were clogged by debris due to high seas, Brayton Point 2 had its output reduced be cause of wet coal, the Canal plant had its output reduced because of opacity problems and Merrimack No. 2 unit had its output reduced because of a forced draft fan problem. Customer outages were due to distribution circuit damage.

10 A storm tripped a 69-kilovolt line and aftected numerous distribution circuits. At the Indian River Power Plant, a temporary operating problem occurred when high winds blew water out of the cooling water intake.

1" Customer outages were due to storm effects on distribution circuits. The transmission system and generating plants were not affected.

30 Customer outages were due to storm effects on distribution circuits. There were no transmission problems, although winds gusting to $80 \mathrm{MPH}$ knocked down some trees. At Northport Power Plant, one 380-megawatt unit tripped due to salt contamination on a standoff insulator and at Port Jefferson Power Plant one 190-megawatt unit tripped because of salt contamination on an insulator. Some contractor crews were called in to assist company line crews.

21 Customer outages were caused by storm effects on distribution circuits. There were no generation or transmission problems.

2 The storm affected distribution circuits but not the generation or transmission facilities.

2) Customers were affected by storm damage to distribution facilities but transmission facilities were not affected. Crane Generating Plant tripped when a static wire failed but the Plant was restored to service without difficulty.

24 The storm disrupted service to some customers by damaging distribution circuits. There were no generation or transmission problems and no need to activate the Edison Electric Institute Mutual Assistance Pact. Contractor crews were used to supplement the company's line crews in service restoration work.

28 Some distribution circuits were out of service because of the storm. Several transmission circuits tripped but were restored to service within two hours.

* Distribution circuit damage interrupted service to some customers. No other difficulties were reported.

27 Some distribution circuits were out of service because of the storm, interrupting some customer service.

2 Distribution circuits tripped, interrupting customers. There were no problems with generation or transmission

- The service interruptions were caused by breakage of 35 distribution poles, 315 wire sections down, 2 circuit breaker trips and outage of 38 distribution circuits.

* One transmission substation tripped when hit by lightning but was restored to service. Generating plants were not affected. Customer outages were caused by distribution circuit damage.

"A conductor of the Warren-Skyline 115-kilovolt line fell to the ground (cause unknown). The associated circuit breaker at Archer Substation failed to trip, causing the tripping of four additional 115-kilovolt lines and three 230 kilovolt lines out of Archer, and the Poudre-Richard Lake 115-kilovolt line. The HVDC Convertor Stations at Stegall (110 megawatt) and Sidney (200 megawatt) tripped with 300 megawatt flowing from MAPP to WSCC, and Unit 1 at Hayden Power Plant in Colorado, carrying 180 megawatt, also tripped. Frequency rose to 60.01 cycles and fell to 59.96 . Sixty megawatts of firm load being supplied to 13 wholesale customers was lost. Transmission restoration began within 5 minutes, generation was restored within 17 minutes and load within 48 minutes. There were no adverse effects in MAPP.

32 Consolidated Edison Company instituted a test of its voltage reduction procedures: 8 percent at 10:46 AM, 5 percent at 10:54 AM, 3 percent at at $11: 14$ AM, normal volage at 11:44 AM.

20 The 138-kilovolt Pacificorp East 90th South Substation was in a single bus configuration for maintenance; work was in progress to return it to the normal double bus arrangement. The 138-kilovolt bus differential relaying operated inadvertently to clear the East and West busses, tripping five 138 . kilovolt lines, two 345/138-kilovolt transformers and three 138/46 kilovolt transformers. Two megawatts of small hydro generation (at Stairs and Granite hydro plants) tripped, as well as 230 megawatts of firm load. Restoration began immediately.

* One of the two 138-kilovolt cable from Arnott Substation (mainland) to Vancouver Island Terminal was out of service due to a failed cable. The two 500-kilovolt lines from Cheekye Substation to Malaspina (on the mainland) to Dunsmuir Substation (on Vancouver Island) then tripped and the re. maining 138-kilovolt cable from Arnott opened at the Vancouver Island terminal. One pole of the Arnott-Vancouver Terminal DC line blocked but the other pole maintained the tie in service for the 27 minutes needed to resynchronize. Three synchronous condensers at Vancouver Terminal, 27 megawatts of hydro generation and 230-kilovolt line on the isiand tripped. The frequency on the island dropped to $58.6 \mathrm{HZ}$ and the frequency in the 
portion of the WSCC interconnection rose to $60.09 \mathrm{HZ}$ before conditions returned to normal.

From the afternoon of June 4 through the early morning hours of June 5 a storm traversed the service area of Appalachian Power Company, damaging substransmission lines and distribution circuits. As of June 4 late evening customer outages were 141,680 in Virginia and 46,932 in West Virginia (total was 188,612). By June 5 evening the total was 54,366 and by Sunday evening June 6 it was 22,318 . Total load lost was 750 megawatts. As of Monday, June 7, 17,305 customers were without service. Damage was due to trees, broken and uprooted by the wind, falling on conductors and breaking them, in addition to damage by lightning. One 138/34.5 kilovolt transformer was damaged and was temporarily replaced by by a mobile transformer.

- Distribution circuit poles were knocked over by a tornado near Lake Park, lowa. Power to Lake Park was restored at 7:44 PM CDT that day.

"7 A 115-kilovolt circuit breaker in the WAPA Pierre substation, feeding the City of Pierre, South Dakota tripped and locked out, interrupting about 23 megawatts of the city's load. Inspection of equipment in the city's substation did not reveal any reason for the trip and lockout signal. It is believed telephone company personnel engaged in cable location activities injected a signal into the cables used for relaying and communications, that tripped the WAPA curcuit breaker. All load was restored by 11:15 AM.

- A forest fire northeast of Walden, Colorado on the Colorado-Wyoming border, burning under the 345-kilovolt Craig-Ault transmission line, caused a C-phase to ground fault at 12:41 PM MAST. The line tested at 12:44 PM MAST, the test was satisfactory, and the line was closed via a circuit breaker at Craig substation at 12:46 PM; it tripped in 5 seconds on a ground fault to a A-phase on the line. As the breaker was opening to clear the the fault it developed a ground fault on its fault on its A-phase. The circuit breaker failure relay did not operate, theredby causing tripping of adjacent lines and transformers and all circuits emanating from the Craig 345-kilovolt South Bus. Craig Generating Unit No. 3, carrying 408 megawatts, then tripped, causing frequency to drop to $59.945 \mathrm{HZ}$ before it recovered to normal. Lines were restored to normal by 6:40 PM and Craig Unit No. 3 was restored to service a $3: 21$ AM on June 28. No customers were affected, as spinning reserve picked up the load when Craig No. 3 tripped.

* A three-phase ground fault on the Naughton-Monument 230-kilovolt lines resulted from burning of the center pole of a three-pole wooden struc. ture located 5 miles from Naughton. The line tripped, re-closed and tripped again. When the system dispatcher reclosed the line circuit breaker at Naughton into the fault, the breaker and the associated breaker of the breaker-and a-half scheme tripped in less than 3 cycles to clear the fault. $A$ breaker failure relay then mis-operated, tripping the West bus at Naughton and causing 3 other 230 -kilovolt lines to trip. Three generating units at Naughton then tripped: No. 1 on underexcitation, No. 2 on excessive current and No. 3 on indication of a transformer ground fault; the total generation dropped was 630 megawatts. All lines were returned to transformer service by 10:34 PM MAST on July 3 . Generating Unit No. 1 was restored to service at 3:31 AM on July 3, No. 2 at 5:23 AM on July 3 and No. 3 was kept off line until 1:31 PM on July 4 for for repair of a boiler tube leak. One of the 230-kilovolt lines that tripped (Naughton-Treasureton) did so at maximum phase angle difference on the power swing, causing tailure of a lightning arrestor and subsequent tripping of the 230-kilovolt bus at Treasureton.

The Dixonville-Grants Pass 230-kilovolt line tripped when struck by a fallen tree. This led to tripping of the Klamath Falls-Boyle 230-kilovolt line and the Weed Junction-Cascade 115-kilovolt tie to Pacific Gas and Electric Company. Generating units delivering a total of 205 megawatts (55 U.S. Bureau of Reclamation and 150 megawatts Pacificorp) tripped as follows: J.C. Boyle unit 2; COPCO units 11, 21, 22; Rogue River-Prospect; Bureau of Reclamation Lost Creek; Green Springs; Biomass Cogen; several small hydro units. The customers who lost service were in the area of Mediord and Grants Pass in southeastern Oregon. The transmission circuits were restored by 10:28 AM and the lost generating units by 11:40 AM same day.

Service to customers was restored between 9:56 AM and 11:18 AM same day.

"High temperature and high humidity caused high loads. Empire District could not purchase sufficient capacity to supplement its own capacity because of low voltage in the area due to cascading tripping of 18 transmission lines in the region: Grand River Dam Authority lost a 345-kilovolt interconnection to Southwestern Electric Power Company, this trip was followed by tripping of another 345-kilovolt line, eight 161-kilovolt lines and eight 69kilovolt lines. During this incident nominal 161-kilovolt transmission voltages in the area around Joplin, Missouri sagged as low as $137-\mathrm{kilovolt}$.

12 Vandals cut guy wires on a three-pole dead-end structure and sawed part way through one of the poles immediately east of the Franklin Junction (Illinois) Switching Station of Southern Illinois Power Cooperative. A 138-kilovolt conductor fell and contacted a $69-\mathrm{kilovolt}$ conductor, causing the 138kilovolttie from Franklin Junction to Central Illinois Public Service Company to trip. Loss of the tie and the 69-kilovolt line made it necessary to close the emergency tie between the City of Fairfield and Wayne-White Counties Electric Cooperative and to ask the City of McLeansboro, Illinois to generate. Southern llinois Cooperative lost about 6,000 customers, Fairfield 7,000 and McLeansboro 1,500.

is Coal stocks on hand at the Sibley Power Plant are at less than 50 percent of the normal amount for this time of the year. The company reports that the coal-pile reduction is due to the combined effects of a coal strike and floods in the Midwest. Potential flooding has forced rail yards to close, interrupting coal delivery by normal routes. Power supply to customers has not been interrupted. At this time the 490 megawatt plant ( 3 generating units) has an 18-day supply of coal on hand. This situation was reported in accordance with Section 205.351(d) of the regulations for the reporting of electric power system emergencies (CFR Title 10, Part 205, Subpart W).

4he oil valve on a 138-kilovolt transformers was opened by persons unknown, allowing the oil to drain out and causing the transformer to over. heat. Power was lost to about 1,000 customers including the Federal prison at Marion, Illinois. The prison used its back-up generator to supply power during the outage.

46 Leakage current across insulators caused a pole-top fire on a 138-kilovolt structure, allowing a line conductor to contact a guy wire and a shield wire. The shield wire fell into the substation bus. The circuit breaker that should have opened to isolate the fault did not trip; it was later discovered that the breaker trip coil was open because of previous voltage impulse. The back-up circuit breaker failed to operated because of an open circuit. forcing several other breakers to trip.

The New York Power Pool Manager of Operations reported that coal supply at the C.R. Huntley power plant of Niagara Mohawk Power Corporation, in Tonawanda, New York, had been reduced to 25 days of normal operations. A shipment of coal is expected within several days. The situation is not regarded as critical but was reported under Section d.1 of the disturbance reporting requirements.

${ }^{17}$ A short circuit in 4-kilovolt secondary cables in an underground vault, located in the downtown area of Seattle, caused a fire that destroyed two 13-kilovolt main cables, five 13-kilovolt branch cables, two 4-kilovolt cables and several 120/208-volt bus ties. As cables began tripping under control of protective relays, system operators opened circuits to prevent damage to transiormers and other secondary circuits from overloads. Businesses, high-rise condominiums, the Pacific Science Center and two television stations (KIRO and KOMO) lost power. Partial restoration of power was ef. fected at 8:19 AM on October 4 and at 10:00 PM on October 7. By noon on Friday October 8 service had been restored to all customers.

- A static wire broke and dropped across the Longview-Allston No. 3 230-kilovolt transmission line, three 230 -kilovolt feeders at Longview Substation and one 12.5-kilovolt distribution line owned by Cowlitz County Public Utility District (PUB) No. 1 . The resulting faults caused less of load as follows: Bonnesville Power Administration 275 megawatt, Clatskanie County PUB 90 megawatt, Cowlitz County PUD 330 and Lewis County PUD 18 megawatts. At the Beaver Power Plant of Portland General Electric Company, units 2 and 3 tripped with a total of 90 megawatts of generation and unit 7 was manually taken off line. Frequency rose to $60.103 \mathrm{HZ}$ and returned to normal in about 2 minutes. The Longview-Allston line was carrying 306 megawatts when tripped by the static wire accident; it was restored to service at 11:36 PM PAST the same day. The generating units were returned to service the same day as follows: No. 7 at 2:19 AM, No. 3 at 2:53 AM, No. 2 at 8:23 AM.

49 A lock was cut from the gate of the district substation and all the oil was drained from the transformer. The transformer overheated and the associated 115-kilovolt line (39 miles long) tripped. Four industrial customers and less than 100 residential customers lost power. Most power was restored within 1.5 hours but one industrial was without service for nearly 24 hours.

so The Victorville-Adelanto No. 2 500-kilovolt line tripped when contaminated insulators on two towers flashed over in heavy fog. As a result both poles of the Intermountain-Adelanto D.C. line blocked, causing both Intermountain generating units to trip, dropping 1680 megawatt of generation. Frequency dropped to $59.978 \mathrm{~Hz}$ and recovered to normal in about 15 minuies. Intermountain No. 2 was returned to service at $7: 26$ AM same day and Unit 1 at 8:22 AM same day. The Victorville-Adelanto line was left out of service for later replacement of insulators.

61 According to a report in Electric Utility Week of November 8 a fire in the Sylmar Convertor Station destroyed a thyristor device associated with the Pacific D.C. intertie, reducing the capacity of the line by about 1000 megawatts. The D.C. intertie runs from the Dalles, in north central Oregon near the Washington border, to the Sylmar Converter Station in Los Angeles County. As of December 16 the Department of Energy has not received a report of this incident

Notes: - Under the Code of Federal Regulations (10 CFR Part 205) and published in the Federal Reglster of October 31, 1986, the United States Department of Energy does not receive reports of distribution system outages that are local in nature and affect only a small area or a small number of customers; the following abbreviations may be applicable:

PAST $=$ Pacific Advanced Standard Time, 
$\mathrm{CDT}=$ Central Daylight Time,

EDT = Eastern Daylight Time,

EST = Eastern Standard Time,

HST = Hawaiian Standard Time,

Int $=$ Interruption of service,

$L F=L$ ine Fault (Accidental contact of energized conductors or of energized conductors with ground)

LR $=$ Load Reduction,

LTNG = Lightning Strike,

MAST = Mountain Advanced Standard Time,

$M W=$ Megawatts

$N A=$ Not Available

$\mathrm{PA}=$ Public Appoal,

$E M C=$ Electric Membership Corporation,

UO= Unusual Occurrence,

$V R=$ Voltage Reduction

LS $=$ Load Shodding,

$L C=$ Curtailment and internuptible customers were to drop some load

$\mathrm{IPL}=$ Indianapolis Power and Light Company,

PSI = Public Service of Indiana,

$H E=$ Hoosier Energy Aural Electric Cooperative.

IM = Indiana Michigan Power Company (subsidiary of American Electric Power Company)

Source: International Aftairs and Energy Emergencies, Form IE-417R, "Electric Power System Emergency Report." 


\section{Appendix B}

References 


\section{References}

1. Energy Information Administration, Office of Energy Markets and End Use, Annual Energy Outlook, DOE/EIA-0383(93) (Washington DC, 1992).

2. Energy Information Administration, Office of Energy Markets and End Use, Annual Energy Review, DOE/EIA-0384(91) (Washington DC, 1992).

3. Bishop, Y.M.M. "Imputation, Revision, and Seasonal Adjustment," 1980 Proceedings of the American Statistical Association Meetings, July 1980.

4. Energy Information Administration, Office of Coal, Nuclear, Electric and Alternate Fuels, Cost and Quality of Fuels for Electric Utility Plants Annual, DOE/EIA-0191(91), (Washington DC, 1992).

5. Energy Information Administration, Office of Coal, Nuclear, Electric and Alternate Fuels, Electric Power Annual, DOE/EIA-0348(91), (Washington DC, 1993).

6. Hill, E. and French C. "Editing Very Large Data Bases," 1981 Conference on Information Sciences and Systems, The Johns Hopkins University, March 1981.

7. Energy Information Administration, Office of Coal, Nuclear, Electric and Alternate Fuels, Inventory of Power Plants in the United States, DOE/EIA-0095(91) (Washington DC, 1992).

8. Energy Information Administration, Office of Energy Markets and End Use, Monthly Energy Review, DOE/EIA-0035(93) (Washington DC, 1993).

9. Energy Information Administration, Office of Coal, Nuclear, Electric and Alternate Fuels, Electric Sales and Revenue 1990, DOE/EIA-0540(92) (Washington DC, 1991).

10. Energy Information Administration, Office of Statistical Standards, An Assessment of the Quality of Selected EIA Data Series: Electric Power Data, DOE/EIA-0292(89) (Washington DC, 1989).
11. Kott, P.S., "Nonresponse in a Periodic Sample Survey," Journal of Business and Economic Statistics, April 1987, Volume 5, Number 2, pp. 287-293.

12. Knaub, J.R., Jr., "Ratio Estimation and Approximate Optimum Stratification in Electric Power Surveys," Proceedings of the Section on Survey Research Methods, American Statistical Association, 1989, pp. 848-853.

13. Knaub, J.R., Jr., "More Model Sampling and Analyses Applied to Electric Power Data," Proceedings of the Section on Survey Research Methods, American Statistical Association, 1992.

14. Royall, R.M. (1970), "On Finite Population Sampling Theory Under Certain Linear Regression Models," Biometrika, 57, 377-387.

15. Royall, R.M., and W.G. Cumberland (1978), "Variance Estimation in Finite Population Sampling," Journal of the American Statistical Association, 73, 351-358.

16. Royall, R.M., and W.G. Cumberland (1981), "An Empirical Study of the Ratio Estimator and Estimators of Its Variance," Journal of the American Statistical Association, 76, 66-68.

17. Knaub, J.R., Jr. (1993), “Alternative to the Iterated Reweighted Least Squares Method: Apparent Heteroscedasticity and Linear Regression Model Sampling," Proceedings of the International Conference on Establishment Surveys, American Statistical Association.

18. Royall, R.M., and W.G. Cumberland (1981), "An Empirical Study of the Ratio Estimator and Estimators of Its Variance," Journal of the American Statistical Association, 76, 66-68.

19. Rao, P.S.R.S. (1992), Unpublished notes and model covariance.

20. Hansen, M.H., Hurwitz, W.N. and Madow, W.G. (1953), "Sample Survey Methods and Theory," Volume II, Theory, pp. 56-58.

21. Knaub, J.R., Jr. (1994), in Proceedings of the Section on Survey Research Methods. 
Appendix C

Technical Notes 


\section{Appendix C}

\section{Technical Notes}

\section{Sources of Data}

The Electric Power Monthly (EPM) is prepared by the Survey Management Division, Office of Coal, Nuclear, Electric and Alternate Fuels (CNEAF), Energy Information Administration (EIA), U.S. Department of Energy. Data published in the EPM are compiled from six data sources. Three statistical forms are filed monthly and two forms are filed annually by electric utilities. Those forms are: the Form EIA-759, "Monthly Power Plant Report," the FERC Form 423, "Monthly Report of Cost and Quality of Fuels for Electric Plants," the Form ElA-826, "Monthly Electric Utility Sales and Revenue Report with State Distributions," the Form EIA-861, "Annual Electric Utility Report," and the Form EIA-860, "Annual Electric Generator Report." In addition, the Electric Power Monthly also includes data collected on the Form OE-417R, "Electric Power System Emergency Report." A brief summary of these forms is presented below.

\section{Form EIA-759}

The Form EIA-759 is a census of all operators of electric utility plants producing electric power for public use. The Form EIA-759 is used to collect monthly data on net generation, consumption of coal, petroleum, and natural gas; and end-of-the-month stocks of coal and petroleum for each plant by prime mover and fueltype combination. Summary data from the Form EIA-759 are also contained in the Electric Power Annual (EPA), Monthly Energy Review (MER), and the Annual Energy Review (AER). These reports present aggregated data for electric utilities at the U.S., Census division, and North American Electric Reliability Council Region (NERC) levels.

Instrument and Design History. Prior to 1936, the Bureau of the Census and the U.S. Geological Survey collected, compiled, and published data on the electric power industry. In 1936, the Federal Power Commission (FPC) assumed all data collection and publication responsibilities for the electric power industry and implemented the FPC Form 4. The Federal Power Act, Sections 311 and 312, and FPC Order 141 define the legislative authority to collect power production data.
The Form EIA-759 replaced the FPC Form 4 in January 1982.

Data Processing. The Form EIA-759, along with a return envelope, is mailed to respondents approximately 4 working days before the end of the month. The completed forms are to be returned to the EIA by the 10th day after the end of the reporting month. After receipt, data from the completed forms are manually logged in and edited before being keypunched for automatic data processing. An edit program checks the data for errors not found during manual editing. The electric utilities are telephoned to obtain data in cases of missing reports and to verify data when questions arise during editing. After all forms are received from the respondents, the final automated edit is submitted. Following verification of the data, text and tables of aggregated data are produced for inclusion in the EPM. Following EIA approval of the EPM, the data are made available for public use, on a costrecovery basis, through custom computer runs, data tapes, or in publications.

\section{FERC Form 423}

The Federal Energy Regulatory Commission (FERC) Form 423 is a monthly record of delivered-fuel purchases, submitted by approximately 230 electric utilities for each electric generating plant with a total steamelectric and combined-cycle nameplate capacity of 50 or more megawatis. Summary data from the FERC Form 423 are also contained in the EPA, MER, and the Cost and Quality of Fuels for Electric Utility Plants - Annual. These reports present aggregated data on electric utilities at the U.S., Census division, and State levels.

Instrument and Design History. On July 7, 1972, the FPC issued Order Number 453 enacting the New Code of Federal Regulations, Section 141.61, legally creating the FPC Form 423. Originally, the form was used to collect data only on fossil-steam plants, but was amended in 1974 to include data on internal combustion and combustion turbines. The FERC Form 423 replaced the FPC Form 423 in January 1983. The FERC Form 423 eliminated peaking units, which were previously collected on the FPC Form 423. In addition, the 
generator nameplate capacity threshold was changed from 25 megawatts to 50 megawatts. This reduction in coverage eliminated approximately 50 utilities and 250 plants. All historical FPC Form 423 data in this publication were revised to reflect the new generator nameplate capacity threshold of 50 or more megawatts reported on the FERC Form 423. In January 1991, the collection of data on the FERC Form 423 was extended to include combined-cycle units. Historical data have not been revised to include these units.

Starting with the January 1993 data, the FERC began to collect the data directly from the respondents. The FERC will process the data through edits and each month provide the EIA with a diskette containing the data. The EIA will review the data for accuracy. Publication of the data will not be effected.

Data Processing. Following verification of the data, text and tables of aggregated data are produced for inclusion in the $E P M$. After the $E P M$ is cleared by the EIA, the data become available for public use, on a cost-recovery basis, through custom computer runs or in publications.

\section{Form EIA-826}

The Form EIA-826 is a monthly collection of data from approximately 240 of the largest primarily investor-owned and publicly owned electric utilities. A model is then applied to estimate for the entire universe of U.S. electric utilities. This is the first year (1993) EIA has used a model sample for the Form EIA-826. A stratified-random sanple, employing auxiliary data, was used for each of the 4 previous years. (See previous issues of this publication, and (Knaub, 12) for details.) The Form ElA-826 provides some financial data to the Department of Commerce for use in calculating the Gross Domestic Product and construction costs. The electric power sales data are used by the Federal Reserve Board in their economic analyses.

Instrument and Design History. The collection of electric power sales, revenue, and income data began in the early 1940's and was established as FPC Form 5 by FPC Order 141 in 1947. In 1980, the report was revised with only selected income items remaining and became the FERC Form 5. The Form EIA-826 replaced the FERC Form 5 in January 1983. In January 1987, the Form EIA-826 was changed to the "Monthly Electric Utility Sales and Revenue Report with State Distributions;" it was formerly titled, "Electric Utility Company Monthly Statement." The Form EIA-826 was revised in January 1990, and some data elements were eliminated.

Frame. The current sample for the Form EIA-826, which was designed to obtain estimates of electricity sales and revenue per kilowatthour at the State level by end-use sector, was chosen to be in effect for the
January 1993 data. The frame for the Form EIA-826 was originally based on the 1989 submission of the Form EIA-861 (Section 1.4), which consisted of approximately 3,250 electric utilities selling retail and/or sales for resale. Note that for the Form EIA-826, we are only interested in retail sales. Updates have been made to the frame to reflect mergers that affect data processing. Some electric utilities serve in more than one State. Thus, the State-service area is actually the sampling unit. For each State served by each utility, there is a utility State-part, or "State-service area," This approach allows for an explicit calculation of estimates for State, Census division, and U.S. level sales, revenue and revenue per kilowatthour by end-use sector (residential, commercial, industrial and other). Regressor data came from the Form EIA-861. (Note that estimates at the "State level" are for sales for the entire State, and similarly for "Census division" and "U.S." levels.)

The preponderance of electric power sales to ultimate consumers in each State are made by a few large utilities. Ranking of electric utilities by retail sales on a State-by-State basis revealed a consistent pattern of dominance by a few electric utilities in nearly all 50 States and the District of Columbia. These dominant electric utilities were selected as a model sample. These electric utilities constitute about 8 percent of the population of U.S. electric utilities, but provide threequarters of the total U.S. retail electricity sales. The procedures used to derive electricity sales, revenue, revenue per kilowatthour, and associated coefficient of variation (CV) estimates are provided in the Form EIA-826 subsection of the Formulas Data Section. See (Knaub, 13) for a study of CV estimates for this survey.

Data Processing. The forms are mailed each year to the electric utilities with State-parts selected in the sample. The completed form is to be returned to the EIA by the last calendar day of the month following the reporting month. Nonrespondents are telephoned to obtain the data. Imputation, in model sampling, is an implicit part of the estimation. That is, data that are not available either because it was not part of the sample or because the data are missing are estimated using a model. The data are edited and entered into the computer where additional checks are completed. After all forms have been received from the respondents, the final automated edit is submitted. Following verification, tables and text of the aggregated data are produced for inclusion in the EPM. After the $E P M$ receives clearance from the EIA, the data are made available for public use through custom computer runs, data tapes, or in publications on a cost-recovery basis.

\section{Form EIA-861}

The Form ElA-861 is a mandatory census of electric utilities in the United States. The survey is used to collect information on power production and sales data from approximately 3,250 electric utilities. The data 
collected are used to maintain and update the EIA's electric utility frame data base. This data base supports queries from the Executive Branch, Congress, other public agencies, and the general public. Summary data from the Form EIA-861 are also contained in the Electric Sales and Revenue; the Financial Statistics of Selected Publicly Owned Electric Utilities; the Financial Statistics of Selected Investor-Owned Electric Utilities; and, the Annual Outlook for U.S. Electric Power. These reports present aggregate totals for electric utilities on a national level, by State, and by ownership type.

Instrument and Design History. The Form EIA-861 was implemented in January 1985 to collect data as of year-end 1984. The Federal Administration Act of 1974 (Public Law 93-275) defines the legislative authority to collect these data.

Data Processing. The Form EIA-861 is mailed to the respondents in February of each year to collect data as of the end of the preceding calendar year. The completed forms are to be returned to the EIA by May 1 . The data are manually edited before being entered into the interactive on-line system. Internal edit checks are performed to verify that current data total across and between schedules, and are comparable to data reported the previous year. Edit checks are also performed to compare data reported on the Form EIA-861 and similar data reported on the Forms EIA-826; EIA-412, "Annual Report of Public Electric Utilities;" and FERC Form 1, "Annual Report of Major Electric Utilities, Licensees, and Others." Respondents are telephoned to obtain clarification of reported data and to obtain missing data.

\section{Form EIA-860}

The Form EIA-860 is a mandatory census of electric utilities in the United States and Puerto Rico that operate power plants or plan to operate a power plant within 10 years of the reporting year. The survey is used to collect data on electric utilities' existing power plants and their 10-year plans for constructing new plants, generating unit additions, modifications, and retirements in existing plants. Data on the survey are collected at the generating unit level. These data are then aggregated to provide totals by energy source (coal, petroleum, gas, water, nuclear, other) and geographic area (State, NERC region, Federal region, Census division). Additionally, at the national level, data are aggregated to provide totals by prime mover. Data from the Form EIA-860 are also summarized in the Inventory of Power Plants in the United States, and as input to publications and studies by other offices in the Department of Energy.

Instrument and Design History. The Form EIA-860 was implemented in January 1985 to collect data as of year-end 1984. The Federal Energy Administration Act of 1974 (Public Law 93-275) defines the legislative authority to collect these data.
Data Processing. The Form EIA-860 is mailed to approximately 900 respondents in December to collect data as of the end of the preceding calendar year. The completed forms are to be returned to the ElA by February 15. Data for each respondent are preprinted from the applicable data base. Respondents are instructed to verify all preprinted data and to supply missing data. The data are manually edited before being keypunced for automatic data processing. Computer programs containing additional edit checks are run. Respondents are telephoned to obtain correction or clarification of reported data and to obtain missing data, as a result of the manual and automatic editing process.

\section{Form OE-417R}

Electric utilities or other entities, subject to the provisions of Section 311 of the Federal Power Act (FPA), that are engaged in the generation, transmission, or distribution of electric energy for delivery and/or sale to the public are required to report exeditiously any 1) loss of firm system loads; 2) voltage reductions and public appeals; 3 ) vulnerabilities that could impact bulk electric power system adequacy or reliability; and, 4) fuel supply emergencies to the DOE.

In accordance with Section 202(a) of the Federal Power Act (FPA), the DOE is responsible for encouraging actions to assure an abundant supply of electric energy throughout the country. Under Section 311 of the FPA, the DOE is authorized and directed to collect information regarding the generation, transmission, and distribution of electric energy and to report the problems and developments of the electric utility industry to Congress. The Secretary of Energy has the Federal responsibility of receiving reports of major electric utility system emergencies. The Secretary has delegated that responsibility to the Office of International Affairs and Energy Emergencies (IE) with the DOE.

Instrument and Design History. The collection of outage data was initiated by the FPC prior to the organization of the DOE. After Congress passed legislation creating the DOE, the collection of electric power system outage data became a function of the DOE. Currently the Assistant Secretary of IE is the principal DOE office for this activity. Form IE-417 was activated after public comment on a rule-making procedure (FR 7/6/83). The form was revised to Form IE-417R after public comment under a later rulemaking procedure (FR 10/31/86). This organization is now known as the Office of Emergency Planning and Operations (OE). The form IE-417R was renamed to OE-417R.

Data Processing. Reports of emergencies are usually received by the Alert Coordination Officer vis telephone. The Director, Office of Emergency Operations, 
has the authority to require a full technical report (after notice in the Federal Register).

\section{Quality of Data}

The CNEAF office is responsible for routine data improvement and quality assurance activities. All operations in this office are done in accordance with formal standards established by the EIA. These standards are the measuring rod necessary for quality statistics. Data improvement efforts include verification of data-keyed input by automatic computerized methods, editing by subject matter specialists, and follow-up on nonrespondents. The CNEAF office supports the quality assurance efforts of the data collectors by providing advisory reviews of the structure of information requirements, and of proposed designs for new and revised data collection forms and systems. Once implemented, the actual performance of working data collection systems is also validated. Computerized respon. dent data files are checked to identify those who fail to respond to the survey. By law, nonrespondents may be fined or otherwise penalized for not filing a mandatory EIA data form. Before invoking the law, the EIA tries to obtain the required information by encouraging cooperation of nonrespondents.

Completed forms received by the CNEAF office are sorted, screened for completeness of reported information, and keyed onto computer tapes for storage and transfer to random access data bases for computer processing. The information coded on the computer tapes is manually spot-checked against the forms to certify accuracy of the tapes. To ensure the quality standards established by the EIA, formulas that use the past history of data values in the data base have been designed and implemented to check data input for errors automatically. (See items 3 and 6 in Appendix B). Data values that fall outside the ranges prescribed in the formulas are verified by telephoning respondents to resolve any discrepancies.

Conceptual problems affecting the quality of data are discussed in the report, An Assessment of the Quality of Selected EIA Data Series: Electric Power Data. This report is published by the Energy Information Administration (Office of Statistical Standards). See item 10 in Appendix B.

\section{Data Editing System}

Data from the form surveys are edited on a monthly basis using automated systems. The edit includes both ueterministic checks, in which records are checked for the presence of required fields and their validity; and statistical checks. in which estimation techniques are used to validate data according to their behavior in the past and $\mathrm{i}$ - comparison to other current fields. When all data have passed the edit process, the system builds monthly master files, which are used as input to the $E P M$.

\section{Confidentiality of the Data}

The data collected on the forms used for input to this report are not confidential.

\section{Formulas/Methodologies}

The following formula is used to calculate percent differences.

Percent Difference $=\left(\frac{x\left(t_{2}\right)-x\left(t_{1}\right)}{x\left(t_{1}\right)}\right) \times 100$

where $x\left(t_{1}\right)$ and $x\left(t_{2}\right)$ denote the quantity at year $t_{1}$ and subsequent year $t_{2}$.

Form EIA-759. Data for the Form EIA-759 are collected at the plant level. These data are then aggregated to provide geographic totals at the State, Census division, and U.S. level, or totals by type of -lant. Consumption of fuel(s) is converted from quantities (in short tons, barrels, or thousand cubic feet) to Btu at he plant level. End-of-month fuel stocks for a single erating plant may not equal beginninghe-month stocks, plus receipts, less consumption, i many reasons, including the fact that several plants may share the same fuel stock.

FERC Form 423. Data for the FERC Form 423 are collected at the plant level. These data are then used in the following formulas to produce aggregates and averages for each fuel type at the State, Census division, and U.S. level. For these formulas, receipts and average heat content are at the plant level. For each geographic region, the summation $\Sigma$ represents the sum of all plants in that geographic region. Additionally,

- For coal, units for receipts $(R)$ are in tons, units for average heat content $(A)$ are in Btu per pound, and the unit conversion $(U)$ is 2,000 pounds per ton;

- For petroleum, units for receipts $(R)$ are in barrels, units for average heat content $(A)$ are in Btu per gallon, and the unit conversion $(U)$ is 42 gallons per barrel;

- For gas, units for receipts $(R)$ are in thousand cubic feet (Mcf), average heat content $(A)$ are in Btu per cubic foot, and the unit conversion $(U)$ is 1,000 cubic feet per Mcf.

Total Btu $=\sum_{i}\left(R_{i} \times A_{i} \times U\right)$ 
where $i$ denotes a plant; $R_{i}=$ receipts for plant $i$; $A_{i}=$ average heat content for receipts at plant $i$; and, $U=$ unit conversion;

Weighted Average Btu $=\frac{\sum_{i}\left(R_{i} \times A_{i}\right)}{\sum_{i} R_{i}}$,

where $i$ denotes a plant; $R_{i}=$ receipts for plant $i$; and, $A_{i}=$ average heat content for receipts at plant $i$.

The weighted average cost in cents per million Btu is calculated using the following formula:

Weighted Average Cost $=\frac{\sum_{i}\left(R_{i} \times A_{i} \times C_{i}\right)}{\sum_{i}\left(R_{i} \times A_{i}\right)}$,

where $i$ denotes a plant; $R_{i}=$ receipts for plant $i$; $A_{i}=$ average heat content for receipts at plant $i$; and, $C_{i}=$ cost in cents per million Btu for plant $i$.

The weighted average cost in dollars per unit is calculated using the following formula:

Weighted Average Cost $=\frac{U \sum_{i}\left(R_{i} \times A_{i} \times C_{i}\right)}{\left(10^{8} \frac{\text { cents }}{\text { dollar }}\right) \sum_{i} R_{i}}$

where $i$ denotes a plant; $R_{i}=$ receipts for plant $i$; $A_{i}=$ average heat content for receipts at plant $i$; $U=$ unit conversion; and, $C_{i}=$ cost in cents per million Btu for plant $i$.

Form IIA-826. The Form EIA-826 data are collected at the utility level by sector and State. When a utility has sales in more than one State, the State data that may be required are dependent upon the sample selection that was done for each State independently. Data from the Form EIA-826 are used to determine estimates by sector at the State, Census division, and national level for the entire corresponding State, Census division, or national category. Form EIA-861 data were used as the frame from which the sample was selected, and also as regressor data.

The sample consists of approximately 240 electric utilities. This includes a somewhat larger number of Stateservice areas for electric utilities. Estimation procedures include imputation to account for nonresponse. Nonsampling error must also be considered. The nonsampling error is not estimated, although attempts are made to minimize it.

State-level sales and revenue estimates are calculated. Also, a ratio estimation procedure is used for estimation of revenue per kilowatthour at the State level. These estimates are accumulated separately to produce the Census division and U.S. level estimates.
The coefficient of variation (CV) statistic, usually given as a percent, describes the magnitude of sampling error that might reasonably be incurred. The CV, sometimes referred to as the relative standard error, is the square root of the estimated relative variance of the variable of interest. The variable of interest may be the ratio of two variables (for example, revenue per kilowatthour), or a single variable (for example, sales).

The sampling error may be less than the nonsampling error. Nonsampling errors may be attributed to many sources, including the response errors, definitional difficulties, differences in the interpretation of questions, mistakes in recording or coding data obtained, and other errors of collection, response, or coverage. These nonsampling errors also occur in complete censuses. In a complete census, this problem may become unmanageable.

Coefficients of variation are indicators of error due to sampling. (CVs do not account for nonsampling errors, such as errors of misclassification or transposed digits. However, estimates of CVs, although not designed to measure nonsampling error, are affected by them.) Using the Central Limit Theorem, which applies to sums and means such as are applicable here, there is approximately a 68-percent chance that the true sampling error is less than the corresponding CV. Note that reported CVs are always estimates, themselves, and are usually, as here, reported as percents. As an example, suppose that a revenue-per-kilowatthour value is estimated to be 5.13 cents per kilowatthour with an estimated $\mathrm{CV}$ of 1.6 percent. This means that, ignoring any nonsampling error, there is approximately a 68-percent chance that the true average revenue per kilowatthour is within approximately 1.6 percent of 5.13 cents per kilowatthour (that is, between 5.05 and 5.21 cents per kilowatthour). There is approximately a 95-percent chance of a true sampling error being 2 CVs or less.

The basic approach used is shown in (Royall, 14) with additional discussion of variance estimation in (Royall and Cumberland, 15), (Royall and Cumberland, 16), and (Knaub, 13). From (Royall, 14), for sales or revenue for any sector at the State level, if we let $\mathrm{x}$ represent an observation from the Form EIA-861, y represents an observation from the Form EIA-826, and $y$ represents an estimated value for data not collected, then

Here, $\mathrm{n}$ is the Form EIA-826 sample size for that State, and $\mathrm{b}$ is the factor ('slope') relating $\mathrm{x}$ to $\mathrm{y}$ in the linear regression. $y$ is taken to be $1 / 2$ (see (Knaub, 13)), although more research (Knaub, 17) could refine this. For the Form EIA-826, $\gamma=1 / 22$ has certainly been shown to be adequate (see Knaub, 13), page 878, Table 1). The variance formula for $V_{d}$ found in (Royall and Cumberland, 15 and 16) performs well for sales and for revenue. For revenue per kilowatthour, the model covariance comes from notes provided by Professor 
Poduri S.R. and S. Rao (Rao, 18) of the University of Rochester and the Energy Information Administration. Aggregate level CV estimates for revenue per kilowatthour are calculated as supported by (Hansen, Hurwitz and Madow, 19). Details are planned to be published in (Knaub, 20).

Additional information or clarification can be addressed to the Energy Information Administration as indicated in the "Contacts" section of this publication.

Form EIA-861. Data for the Form EIA-861 are calculated at the utility level from all electric utilities in the United States, its territories, and Puerto Rico. These data are then aggregated to provide nationallevel electricity sales values by consumer class of service.

Form EIA-860. Data from the Form EIA-860 are submitted at the generating unit level and are then aggregated to provide total capacity by energy source and geographic area. In addition, at the national level, data are aggregated by prime mover.

Estimated values for net summer and net winter capability for electric generating units were developed by use of a regression formula. The formula is used to estimate values for existing units where data are missing and for projected units. It was found that a zerointercept linear regression works very well for estimating capability based on nameplate capacity. The only parameter then is the slope $(b)$ that is used to relate capacity to capability as follows: $y=b x$, where $y$ is the estimated capability, and $x$ is the known nameplate capacity. There will be a different value for $b$ for different prime movers and for summer and winter capabilities and different percentages as shown in the tables below.

\section{Average Heat Content}

Heat content values (Table $\mathrm{C} 1$ ) collected on the FERC Form 423 were used to convert the consumption data from the Form EIA-759 into Btu. Respondents to FERC Form 423 represent a subset of all generating plants (steam plants with a capacity of 50 megawatts or larger), while Form EIA-759 respondents represent all generating plants. The results, therefore, may not be completely representative.

\section{Rounding Rules for Data}

Given a number with $r$ digits to the left of the decimal and $\mathrm{d}+\mathrm{t}$ digits in the fraction part, with $\mathrm{d}$ being the place to which the number is to be rounded and $t$ being the remaining digits which will be truncated, this number is rounded to $r+d$ digits by adding 5 to the $(\mathrm{r}+\mathrm{d}+1)$ th digit when the number is positive or by subtracting $5 v$ hen the number is negative. The $t$ digits are then truncated at the $(r+d+1)$ th digit. The symbol for a rounded number truncated to zero is $\left({ }^{*}\right)$.

\section{Data Correction Procedure}

The Office of Coal, Nuclear, Electric and Alternate Fuels has adopted the following policy with respect to the revision and correction of recurrent data in energy publications:

1. Annual survey data collected by this office are published either as preliminary or final when first appearing in a data report. Data initially released as preliminary will be so noted in the report. These data will be revised, if necessary, and declared final in the next publication of the data.

2. All monthly and quarterly survey data collected by this office are published as preliminary. These data are revised only after the completion of the 12-month cycle of the data. No revisions are made to the published data before this.

3. The magnitudes of changes due to revisions experienced in the past will be included in the data reports, so that the reader can assess the accuracy of the data.

4. After data are published as final, corrections will be made only in the event of a greater than one percent difference at the national level. Corrections for differences that are less than the beforementioned threshold are left to the discretion of the Office Director. Note that in this discussion, changes or revisions are referred to as "errors."

In accordance with policy statement number 3 , the mean values (unweighted average) for the 12 monthly revisions of each item are provided at the U.S. level for the past 2 years (Table C2). For example, the mean of the 12 monthly absolute errors (absolute differences between preliminary and final monthly data) for coalfired generation in 1991 was 250 . That is, on average, the absolute value of the change made each month to coal-fired generation was $\mathbf{2 5 0}$ million kilowatthours.

The U.S. total net summer capability, updated monthly in the EPM (Table 1), is based solely on new electric generating units and retirements which come to the attention of the EIA during the year through telephone calls with electric utilities and on the Form EIA-759, "Monthly Power Plant Report." Data on net summer capability, including new electric generating units, are collected annually on the Form EIA-860, "Annual Electric Generator Report." The data are published in the Inventory of Power Plants as preliminary data. Final data for net summer capability are published in the Electric Power Annual (EPA). With respect to net summer capability published in the EPM, the EIA examines the accuracy of that data by comparing the annual total value with the final annual total value published in the EPA. For 1991, the absolute value of the change 
was 143 megawatts. Final data for 1992 are not available at this time.

\section{NERC Aggregation}

Beginning in January 1986, NERC region totals for the Form EIA-759 are aggregates based on membership of the individual electric utilities in NERC. Prior to January 1986, NERC region totals were aggregates defined by the physical location of the power plants generating electricity.

\section{Use of the Glossary}

The terms in the glossary have been defined for general use. Restrictions on the definitions as used in these data collection systems are included in each definition when necessary to define the terms as they are used in this report.

\section{Obtaining Copies of Data}

Upon EIA approval of the $E P M$, the data become available for public use on a cost-recovery basis.
Computer listings are obtained by submitting a written request to:

Energy Information Administration, EI-521

Forrestal Building

U.S. Department of Energy

Washington, DC 20585

These data are also available monthly on machinereadable tapes. Tapes may be purchased by using Visa, Master Card, or American Express cards as well as money orders or checks payable to the National Technical Information Service (NTIS). Purchasers may also use NTIS and Government Printing Office depository accounts. To place an order, contact:

National Technical Information Service (NTIS) Office of Data Base Services

U.S. Department of Commerce 5285 Port Royal Road

Springfield, Virginia 22161

(703) $487-4650$ 
Data for Table $\mathrm{Cl}$ include all quality of fuels. For a detailed breakdown on types of coal, petroleum and gas, see Tables 40,44 , and 48 , respectively.

Table C1. Average Heat Content of Fossil-Fuel Receipts, September 1993

\begin{tabular}{|c|c|c|c|}
\hline $\begin{array}{l}\text { Consus Division } \\
\text { and State }\end{array}$ & $\begin{array}{c}\text { Coal } \\
\text { (Btu per ton) }\end{array}$ & $\begin{array}{l}\text { Potroloum } \\
\text { (Btu per barrel) }\end{array}$ & $\begin{array}{l}\text { Gas } \\
\text { (Btu per thousand } \\
\text { cublc foet) }\end{array}$ \\
\hline $\begin{array}{l}\text { New England } \\
\text { Connecticut }\end{array}$ & $25,917,289$ & $6,381,710$ & $1,036,215$ \\
\hline & $26,381,412$ & $6,361,293$ & $1,030,000$ \\
\hline 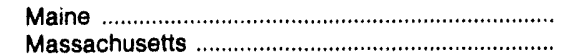 & $25 \overline{703.110}$ & $6,339,386$ & -- \\
\hline $\begin{array}{l}\text { Massachusetts } \\
\text { New Hampshire }\end{array}$ & $\begin{array}{l}25,703,110 \\
26,211694\end{array}$ & $6,359,696$ & $1,036,473$ \\
\hline $\begin{array}{l}\text { New Hampshire } \\
\text { Rhode Island }\end{array}$ & $26,211,694$ & $6,568,541$ & $-\overline{0}$ \\
\hline $\begin{array}{l}\text { Rhode Island } \\
\text { Vermont }\end{array}$ & -- & $5 \overline{440.260}$ & $1,018,000$ \\
\hline 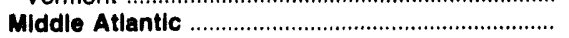 & $24,955,595$ & $6,333,974$ & $\overline{1.031 .829}$ \\
\hline 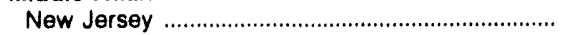 & $26,426,398$ & $6,245,383$ & $1,033,893$ \\
\hline 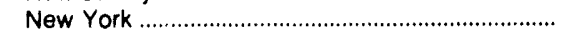 & $25,771,462$ & $6,345,289$ & $1,031,503$ \\
\hline 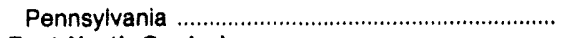 & $24,780,147$ & $6,361,509$ & $1,030,230$ \\
\hline 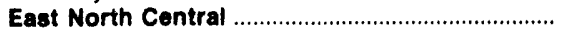 & $21,625,385$ & $6,178,415$ & 805,497 \\
\hline Illinois & $20,373,294$ & $6,287,223$ & $1,015,605$ \\
\hline 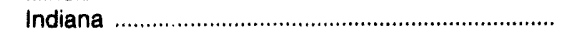 & $20,949,039$ & $5,749,434$ & $1,019,955$ \\
\hline Michigan & $21,843,911$ & $6,247,559$ & - 397,294 \\
\hline Ohio & $24,048,536$ & $5,795,259$ & $1,029,786$ \\
\hline 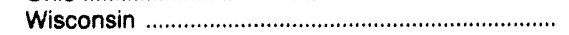 & $19,198,152$ & $5,880,000$ & $1,013,476$ \\
\hline West North Central .................................................. & $16,723,751$ & $6,072,354$ & 989,375 \\
\hline 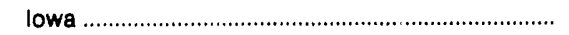 & $17,431,688$ & $5,886,617$ & $1,002,632$ \\
\hline 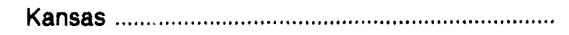 & $17,313,756$ & - & 982,279 \\
\hline 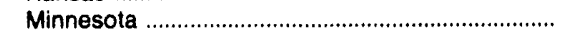 & $17,660,898$ & $5,773,595$ & $1,002,741$ \\
\hline 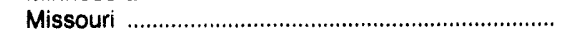 & $19,055,292$ & $6,186,348$ & $1,003,660$ \\
\hline 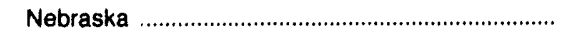 & $17,074,334$ & $5,775,000$ & 966,797 \\
\hline North Dakota ………………………………... & $13,140,164$ & $5,836,710$ & $1,103,000$ \\
\hline South Dakota & $12,460,000$ & - & - \\
\hline 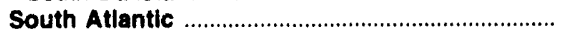 & $25,048,762$ & $6,349,922$ & $1,014,361$ \\
\hline Delaware & $25,930,676$ & $6,366,859$ & $1,033,123$ \\
\hline District of Columbia & - & $6,016,019$ & - \\
\hline 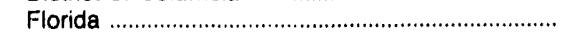 & $24,764,394$ & $6,366,918$ & $1,008,966$ \\
\hline 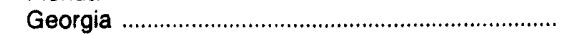 & $24,747,582$ & $5,815,046$ & $1,028,034$ \\
\hline 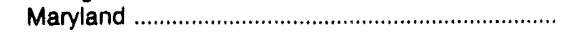 & $25,526,075$ & $6,317,151$ & $1,043,350$ \\
\hline North Carolina & $24,952,336$ & $5,802,178$ & $1,032,000$ \\
\hline 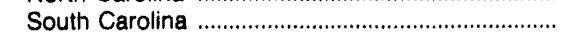 & $25,633,160$ & $5,853,834$ & $1,027,303$ \\
\hline Virginia & $25,867,498$ & $6,326,640$ & $1,045,730$ \\
\hline 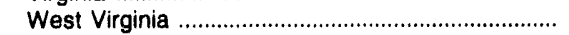 & $24,916,793$ & $5,831,514$ & $1,000,000$ \\
\hline 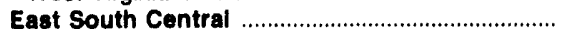 & $23,915,554$ & $6,419,549$ & $1,026,515$ \\
\hline Alabama . . & $24,355,272$ & $5,767,605$ & $1,009,992$ \\
\hline 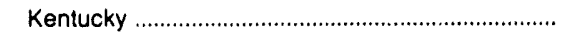 & $23,195,636$ & $5,832,413$ & $1,021,290$ \\
\hline 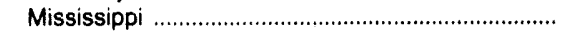 & $24,686,174$ & $6,442,598$ & $1,028,183$ \\
\hline 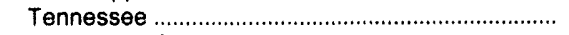 & $24,638,774$ & $5,790,550$ & - \\
\hline West South Central ........................................... & $15,382,349$ & $5,812,982$ & $1,030,835$ \\
\hline 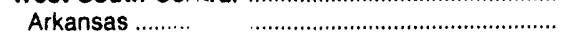 & $17,353,086$ & $5,742,429$ & $1,021,598$ \\
\hline Louisiana .. & $16,225,215$ & $5,880,000$ & $1,042,686$ \\
\hline 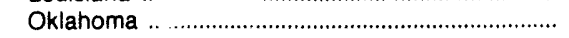 & $17,185,338$ & - & $1,042,603$ \\
\hline 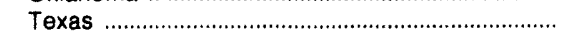 & $14,642,930$ & $5,796,000$ & $1,026,500$ \\
\hline 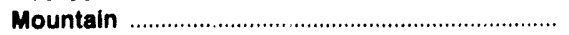 & $19,526,678$ & $5,770,021$ & $1,023,913$ \\
\hline 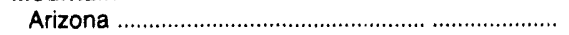 & $20,687,510$ & $6,001,926$ & $1,025,069$ \\
\hline 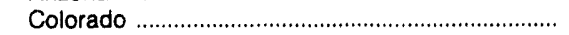 & $19,707,762$ & - & $1,025,993$ \\
\hline Idaho & -- & -- & - \\
\hline Montana & $17,094,857$ & -- & $1,126,000$ \\
\hline 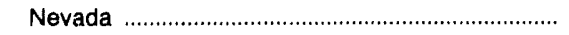 & $21,865,466$ & -- & $1,020,222$ \\
\hline 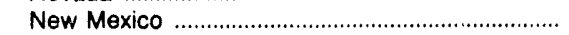 & $17,951,056$ & $5,712,000$ & $1,015,463$ \\
\hline 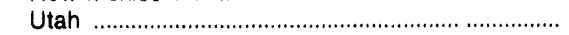 & $22,919,728$ & - & $1,086,000$ \\
\hline 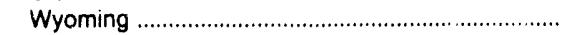 & $17,563,940$ & $5,806,685$ & $1,029,000$ \\
\hline 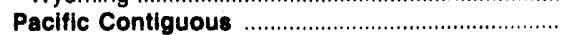 & $16,343,942$ & $5,878,840$ & $1,027,929$ \\
\hline California & -- & - & $1,028,559$ \\
\hline 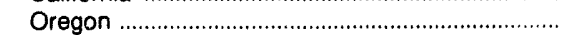 & $16,848,000$ & -- & $1,011,000$ \\
\hline Washington & $16,153,924$ & $5,878,840$ & $1,050,000$ \\
\hline 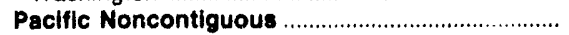 & - & $6,286,521$ & 999,306 \\
\hline Alaska & -- & - & 999,306 \\
\hline Hawaii & -- & $6,286,521$ & -- \\
\hline U.S. Average & $20,529,845$ & $6,348,933$ & $1,025,731$ \\
\hline
\end{tabular}

- Consists mostly of blast furnace gas which has a heat content of 73,000 Btu per thousand cubic feet. Note: Data for 1993 aie preliminary.

Source: Federal Energy Regulatory Commission, FERC Form 423, "Monthly Report of Cost and Quality of Fuels for Electric Plants." 
Table C2. Accuracy of Preliminary Monthly Values Compared With Final Monthly Values at the U.S. Level, 1991 and 1992

\begin{tabular}{|c|c|c|}
\hline \multirow{2}{*}{ Item } & \multicolumn{2}{|c|}{ Mean Absolute Value of Change } \\
\hline & 1991 & 1992 \\
\hline \multicolumn{3}{|l|}{$\begin{array}{l}\text { Goneration (million kllowatthours) } \\
\text { Coal }\end{array}$} \\
\hline 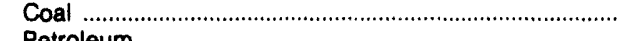 & 250 & 69 \\
\hline $\begin{array}{l}\text { Petroleum } \\
\text { Ggs }\end{array}$ & 27 & 42 \\
\hline $\begin{array}{l}\text { Gas } \\
\text { Hydroelectric }\end{array}$ & 64 & 15 \\
\hline 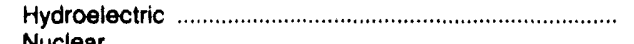 & 5 & 13 \\
\hline 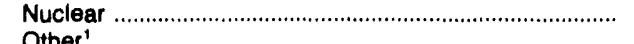 & 3 & 2 \\
\hline 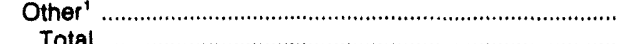 & 3 & 0 \\
\hline 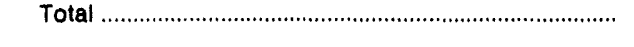 & 288 & 104 \\
\hline \multicolumn{3}{|l|}{ Consumption } \\
\hline 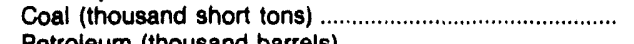 & 88 & 85 \\
\hline Petroleum (thousand barrels) & 35 & 71 \\
\hline Gas (million cubic feet) & 644 & 163 \\
\hline \multicolumn{3}{|l|}{ Stocks² } \\
\hline 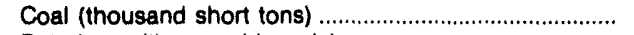 & 1,613 & 345 \\
\hline 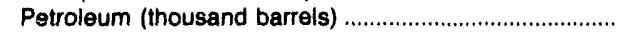 & 131 & 49 \\
\hline \multicolumn{3}{|l|}{ Sales (million kllowatthours) } \\
\hline 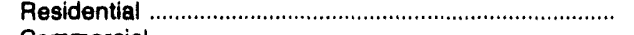 & 114 & 65 \\
\hline 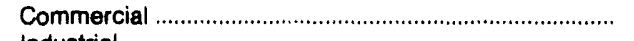 & 83 & 51 \\
\hline 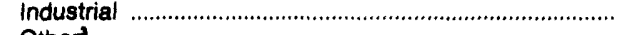 & 334 & 320 \\
\hline Other & 50 & 29 \\
\hline 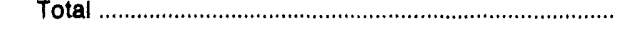 & 423 & 409 \\
\hline \multicolumn{3}{|l|}{ Revenue (million dollars) } \\
\hline 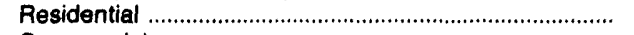 & 11 & 4 \\
\hline 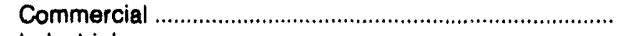 & 4 & 4 \\
\hline Industrial & 10 & 8 \\
\hline Other' & 3 & 2 \\
\hline 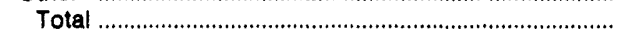 & 18 & 14 \\
\hline \multicolumn{3}{|l|}{ Average Revenue per Kllowatthour (cents) } \\
\hline 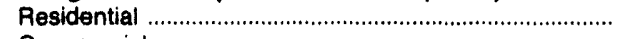 & .0 & .0 \\
\hline 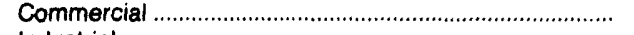 & .0 & .0 \\
\hline 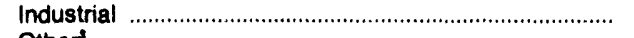 & .0 & .0 \\
\hline Other" & .0 & .0 \\
\hline 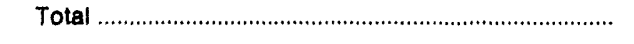 & .0 & .0 \\
\hline \multicolumn{3}{|l|}{ Recolpts } \\
\hline Coal (thousand short tons) & 280 & 59 \\
\hline 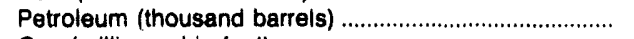 & 8 & 46 \\
\hline 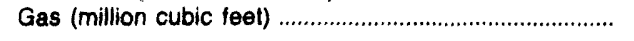 & 59 & 147 \\
\hline \multicolumn{3}{|l|}{ Cost (cents per million Btu) } \\
\hline 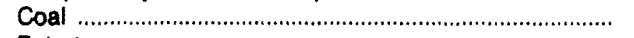 & .0 & .0 \\
\hline Petroleum & .0 & .0 \\
\hline Gas & .0 & .0 \\
\hline
\end{tabular}

1 Includes geothermal, wood, waste, and solar.

Stocks are end of month values.

- Includes public street and highway lighting, other sales to public authorities, sales to railroads and railways, and interdepartmental sales.

Notes: - Change refers to the difference between preliminary monthly data published in the Electric Power Monthly (EPM) and the final monthly data published in the EPM. Electric Power Annual, and the Cost and Quality of Fuels for Electric Utility Plants. - Mean absolute value of change is the unweighted average of the absolute changes.

Sources: "Energy Information Administration: Form EIA-759, "Monthly Power Plant Report" and Form EIA-826, "Monthly Electric IJtility Sales and Revenue Report with State Distributions." -Federal Energy Regulatory Commision: FERC Form 423, "Monthly Report of Cost and wuality of Fuels for
Electric Plants." 
Table C3. Unit-of-Measure Equivalents for Electriclty

\begin{tabular}{|c|c|c|c|}
\hline Unit & & Equivalent & \\
\hline $\begin{array}{l}\text { Kilowatt (kW) } \\
\text { Megawatt (MW) } \\
\text { Gigawatt (GW) } \\
\text { Terawatt (TW) }\end{array}$ & $\begin{array}{r}1,000 \\
1,000,000 \\
1,000,000,000 \\
1,000,000,000,000\end{array}$ & $\begin{array}{l}\text { (One Thousand) } \\
\text { (One Million) } \\
\text { (One Billion) } \\
\text { (One Trillion) }\end{array}$ & $\begin{array}{l}\text { Watts } \\
\text { Watts } \\
\text { Watts } \\
\text { Watts }\end{array}$ \\
\hline $\begin{array}{l}\text { Gigawatt } \\
\text { Thousand Gigawatts }\end{array}$ & $\begin{array}{r}1,000,000 \\
1,000,000,000\end{array}$ & $\begin{array}{l}\text { (One Million) } \\
\text { (One Billion) }\end{array}$ & $\begin{array}{l}\text { Kilowatts } \\
\text { Kilowatts }\end{array}$ \\
\hline $\begin{array}{l}\text { Kilowatthours (kWh) } \\
\text { Megawatthours (MWh) } \\
\text { Gigawatthours (GWh) } \\
\text { Terawatthours (TWh) }\end{array}$ & $\begin{array}{r}1,000 \\
1,000,000 \\
1,000,000,000 \\
1,000,000,000,000\end{array}$ & $\begin{array}{l}\text { (One Thousand) } \\
\text { (One Million) } \\
\text { (One Billion) } \\
\text { (One Trillion) }\end{array}$ & $\begin{array}{l}\text { Watthours } \\
\text { Watthours } \\
\text { Watthours } \\
\text { Watthours }\end{array}$ \\
\hline Gigawatthours & $\begin{array}{r}1,000,000 \\
1,000,000,000\end{array}$ & $\begin{array}{l}\text { (One Million) } \\
\text { (One Billion) }\end{array}$ & $\begin{array}{l}\text { Kilowatthours } \\
\text { Kilowatthours }\end{array}$ \\
\hline
\end{tabular}

Source: Energy Information Administration, Survey Management Division. 
Table C4. Comparison of Published Data at the State Level, 1990 and 1991, by End-use Sector

\begin{tabular}{|c|c|c|c|c|c|c|}
\hline \multirow[b]{2}{*}{ Itom } & \multicolumn{3}{|c|}{1980} & \multicolumn{3}{|c|}{1991} \\
\hline & EIA-826 & EIA-861 & $\begin{array}{l}\text { Difterence } \\
\text { (Percent) }\end{array}$ & EIA-826 & EIA-861 & $\begin{array}{l}\text { Difference } \\
\text { (Percent) }\end{array}$ \\
\hline \multicolumn{7}{|l|}{ Sales (million kllowatthours) } \\
\hline Residential & $\begin{array}{r}921,473 \\
750,835\end{array}$ & $\begin{array}{l}924,019 \\
751,027\end{array}$ & 0.3 & $\begin{array}{l}957,801 \\
765,476\end{array}$ & $\begin{array}{l}955,417 \\
765,664\end{array}$ & -0.2 \\
\hline 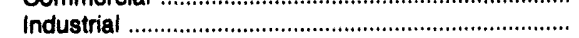 & 936,428 & 945,522 & 1.0 & 944,684 & 946,583 & .2 \\
\hline Other' & 95,936 & 91,988 & -4.3 & 96,513 & 94,339 & -2.3 \\
\hline 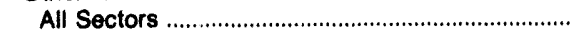 & $2,704,672$ & $2,712,555$ & .3 & $2,764,474$ & $2,762,003$ & -.1 \\
\hline \multicolumn{7}{|l|}{ Revenue (mililion dollare) } \\
\hline 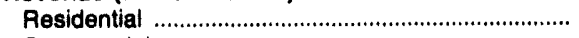 & 72,332 & 72,378 & .1 & 77,142 & 76,828 & -.4 \\
\hline 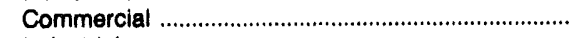 & 55,080 & 55,117 & .1 & 57,471 & 57,655 & .3 \\
\hline 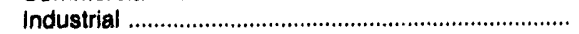 & 44,453 & 44,857 & .9 & 45,803 & 45,737 & -.1 \\
\hline Other' & 5,941 & 5,891 & -.9 & 6,207 & 6,138 & -1.1 \\
\hline 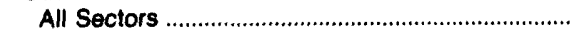 & 177,806 & 178,243 & .2 & 186,624 & 186,359 & -.1 \\
\hline \multicolumn{7}{|l|}{ Average Revenue per Kllowatthour (cents) } \\
\hline 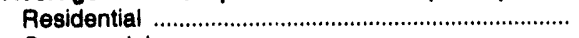 & 7.8 & 7.8 & -.2 & 8.1 & 8.0 & -.2 \\
\hline 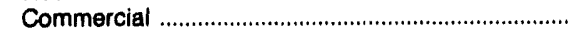 & 7.3 & 7.3 & * & 7.5 & 7.5 & .3 \\
\hline 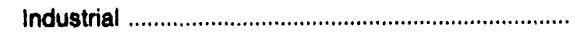 & 4.7 & 4.7 & -.1 & 4.8 & 4.8 & -.3 \\
\hline Other ${ }^{t}$ & 6.2 & 6.4 & 3.3 & 6.4 & 6.5 & 1.2 \\
\hline 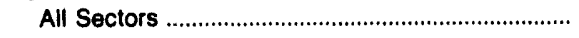 & 6.6 & 6.6 & • & 6.8 & 6.7 & -.1 \\
\hline
\end{tabular}

1 Includes public street and highway lighting, other sales to public authorities, sales to railroads and railways, and interdepartmental sales.

- Value less than 0.1.

Notes: - The average revenue per kilowatthour is calculated by dividing revenue by sales. - Totals may not equal sum of components because of independent rounding. Percent difference is calculated before rounding.

Sources: Energy information Administration, Form ElA-861, "Annual Electric Utility Report," Form ElA-826, "Monthly Electric Utility Sales and Revenue Report with State Distributions." 


\section{Glossary}

Ampere: The unit of measurement of electrical current produced in a circuit by 1 volt acting through a resistance of $1 \mathrm{ohm}$.

Anthracite: A hard, black lustrous coal, often referred to as hard coal, containing a high percentage of fixed carbon and a low percentage of volatile matter. Comprises three groups classified according to the following ASTM Specification D388-84, on a dry mineralmatter-free basis:

$\begin{array}{lllrr} & \begin{array}{l}\text { Fixed } \\ \text { Carbon }\end{array} & & \\ & \text { Volatile } \\ \text { Limits } & \text { Matter }\end{array}$

Average Revenue per Kilowatthour: The average revenue per kilowatthour of electricity sold by sector (residential, commercial, industrial, or other) and geographic area (State, Census division, and national), is calculated by dividing the total monthly revenue by the corresponding total monthly sales for each sector and geographic area.

Barrel: A volumetric unit of measure for crude oil and petroleum products equivalent to 42 U.S. gallons.

Baseload: The minimum amount of electric power delivered or required over a given period of time at a steady rate.

Baseload Capacity: The generating equipment normally operated to serve loads on an around-the-clock basis.

Baseload Plant: A plant, usually housing highefficiency steam-electric units, which is normally operated to take all or part of the minimum load of a system, and which consequently produces electricity at an essentially constant rate and runs continuously. These units are operated to maximize system mechanical and thermal efficiency and minimize system operating costs.

Bcf: The abbreviation for 1 billion cubic feet.

Bituminous Coal: The most common coal. It is dense and black (often with well-defined bands of bright and dull material). Its moisture content usually is less than 20 percent. It is used for generating electricity, making coke, and space heating. Comprises five groups classified according to the following ASTM Specification D388-84, on a dry mineral-matter-free ( $\mathrm{mmf}$ ) basis for fixed-carbon and volatiie matter and a moist $\mathrm{mmf}$ basis for calorific válue.

\begin{tabular}{|c|c|c|c|c|c|c|}
\hline & \multicolumn{2}{|c|}{$\begin{array}{l}\text { Fixed } \\
\text { Carbon } \\
\text { Limits }\end{array}$} & \multicolumn{2}{|c|}{$\begin{array}{l}\text { Volatile } \\
\text { Matter } \\
\text { Limits }\end{array}$} & \multicolumn{2}{|c|}{$\begin{array}{l}\text { Calorific } \\
\text { Value } \\
\text { Limits } \\
\text { Btu/lb }\end{array}$} \\
\hline $\begin{array}{l}\text { LV } \\
\text { MV }\end{array}$ & $\begin{array}{l}\text { GE } \\
78 \\
69\end{array}$ & $\begin{array}{l}\text { LT } \\
86 \\
78\end{array}$ & $\begin{array}{l}G T \\
14 \\
22\end{array}$ & $\begin{array}{l}L T \\
22 \\
31\end{array}$ & $\begin{array}{l}\text { GE } \\
-\end{array}$ & $\begin{array}{l}\text { LE } \\
-\end{array}$ \\
\hline HVA & - & 69 & 31 & - & 14000 & - \\
\hline HVB & - & - & - & - & 13000 & 14000 \\
\hline HVC & - & - & - & - & 10500 & D 13000 \\
\hline $\begin{array}{l}\text { LV } \\
\text { MV } \\
\text { HVA } \\
\text { HVB } \\
\text { HVC }\end{array}$ & $\begin{array}{l}=\mathrm{LO} \\
=\mathrm{Me} \\
=\mathrm{Hi} \\
=\mathrm{Hi} \\
=\mathrm{Hi}\end{array}$ & $\begin{array}{l}\text { um-vo } \\
\text {-vola } \\
\text { i-vola } \\
\text {-vola }\end{array}$ & $\begin{array}{l}\text { atile } \\
\text { ile } \\
\text { ile } \\
\text { ille }\end{array}$ & $\begin{array}{l}\text { umino } \\
\text { bitum } \\
\text { bitun } \\
\text { bitun } \\
\text { bitun }\end{array}$ & $\begin{array}{l}\text { s coal } \\
\text { nous } \\
\text { nous } \\
\text { nous } \\
\text { nous }\end{array}$ & $\begin{array}{l}\text { l } \\
\text { coal } \\
\text { coal } \\
\text { coal } \\
\text { coal }\end{array}$ \\
\hline
\end{tabular}

Boiler: A device for generating steam for power, processing, or heating purposes or for producing hot water for heating purposes or hot water supply. Heat from an external combustion source is transmitted to a fluid contained within the tubes in the boiler shell. This fluid is delivered to an end-use at a desired pressure, temperature, and quality.

Btu (British Thermal Unit): A standard unit for measuring the quantity of heat energy equal to the quantity of heat required to raise the temperature of 1 pound of water by 1 degree Fahrenheit. 
Capability: The maximum load that a generating unit, generating station, or other electrical apparatus can carry under specified conditions for a given period of time without exceeding approved limits of temperature and stress.

Capacity: The full-load continuous rating of a generator, prime mover, or other electric equipment under specified conditions as designated by the manufacturer. It is usually indicated on a nameplate attached to the equipment.

Capacity (Purchased): The amount of energy and capacity available for purchase from outside the system.

Census Divisions: The nine geographic divisions of the United States established by the Bureau of the Census, U.S. Department of Commerce, for the purpose of statistical analysis. The boundaries of Census divisions coincide with State boundaries. The Pacific Division is subdivided into the Pacific Contiguous and Pacific Noncontiguous areas.

Circuit: A conductor or a system of conductors through which electric current flows.

Coal: A black or brownish-black solid combustible substance formed by the partial decomposition of vegetable matter without access to air. The rank of coal, which includes anthracite, bituminous coal, subbituminous coal, and lignite, is based on fixed carbon, volatile matter, and heating value. Coal rank indicates the progressive alteration from lignite to anthracite. Lignite contains approximately 9 to $17 \mathrm{mil}-$ lion Btu per ton. The contents of subbituminous and bituminous coal range from 16 to 24 million Btu per ton and from 19 to 30 million Btu per ton, respectively. Anthracite contains approximately 22 to 28 million Btu per ton.

Coincidental Demand: The sum of two or more demands that occur in the same time interval.

Coincidental Peak Load: The sum of two or more peak loads that occur in the same ime interval.

Coke (Petroleum): A residue high in carbon content and low in hydrogen that is the final product of thermal decomposition in the condensation process in cracking. This product is reported as marketable coke or catalyst coke. The conversion factor is 5 barrels (42 U.S. gallons each) per short ton.
Combined Pumped-Storage Plant: A pumped-storage hydroelectric power plant that uses both pumped water and natural streamflow to produce electricity.

Commercial Operation: Commercial operation begins when control of the loading of the generator is turned over to the system dispatcher.

Compressor: A pump or other type of machine using a turbine to compress a gas by reducing the volume.

Consumption (Fuel): The amount of fuel used for gross generation, providing standby service, start-up and/or flame stabilization.

Contract Receipts: Purchases based on a negotiated agreement that generally covers a period of 1 or more years.

Cost: The amount paid to acquire resources, such as plant and equipment, fuel, or labor services.

Crude Oil (including Lease Condensate): A mixture of hydrocarbons that existed in liquid phase in underground reservoirs and that remains liquid at atmospheric pressure after passing through surface separating facilities. Included are lease condensate and liquid hydrocarbons produced from tar sands, gilsonite, and shale oil. Drip gases are also included, but topped crude oil (residual oil) and other unfinished oils are excluded. Liquids produced at natural gas processing plants and mixed with crude oil are likewise excluded where identifiable.

Current (Electric): A flow of electrons in an electrical conductor. The strength or rate of movement of the electricity is measured in amperes.

Demand (Electric): The rate at which electric energy is delivered to or by a system, part of a system, or piece of equipment, at a given instant or averaged over any designated period of time.

Demand Interval: The time period during which flow of electricity is measured (usually in 15-, 30-, or 60-minute increments.)

Electric Plant (Physical): A facility containing prime movers, electric generators, and auxiliary equipment for converting mechanical, chemical, and/or fission energy into electric energy. 
Electric Utility: An enterprise that is engaged in the generation, transmission, or distribution of electric energy primarily for use by the public and that is the major power supplier within a designated service area. Electric utilities include investor-owned, publicly owned, cooperatively owned, and government-owned (municipals, Federal agencies, State projects, and public power districts) systems.

Energy: The capacity for doing work as measured by the capability of doing work (potential energy) or the conversion of this capability to motion (kinetic energy). Energy has several forms, some of which are easily convertible and can be changed to another form useful for work. Most of the world's convertible energy comes from fossil fuels that are burned to produce heat that is then used as a transfer medium to mechanical or other means in order to accomplish tasks. Electrical energy is usually measured in kilowatthours, while heat energy is usually measured in British thermal units.

Energy Deliveries: Energy generated by one electric utility system and delivered to another system through one or more transmission lines.

Energy Receipts: Energy generated by one electric utility system and received by another system through one or more transmission lines.

Energy Source: The primary source that provides the power that is converted to electricity through chemical, mechanical, or other means. Energy sources include coal, petroleum and petroleum products, gas, water, uranium, wind, sunlight, geothermal, and other sources.

Fahrenheit: A temperature scale on which the boiling point of water is at 212 degrees above zero on the scale and the freezing point is at 32 degrees above zero at standard atmospheric pressure.

Failure or Hazard: Any electric power supply equipment or facility failure or other event that, in the judgment of the reporting entity, constitutes a hazard to maintaining the continuity of the bulk electric power supply system such that a load reduction action may become necessary and a reportable outage may occur. The imposition of a special operating procedure, the extended purchase of emergency power, other bulk power system actions that may be caused by a natural disaster, a major equipment failure that would impact the bulk power supply, and an environmental and/or regulatory action requiring equipment outages are types of abnormal conditions that should be reported.
Firm Gas: Gas sold on a continuous and generally long-term contract.

Fossil Fuel: Any naturally occurring organic fuel, such as petroleum, coal, and natural gas.

Fossil-Fuel Plant: A plant using coal, petroleum, or gas as its source of energy.

Fuel: Any substance that can be burned to produce heat; also, materials that can be fissioned in a chain reaction to produce heat.

Fuel Emergencies: An emergency that exists when supplies of fuels or hydroelectric storage for generation are at a level or estimated to be at a level that would threaten the reliability or adequacy of bulk electric power supply. The following factors should be taken into account to determine that a fuel emergency exists: (1) Fuel stock or hydroelectric project water storage levels are 50 percent or less of normal for that particular time of the year and a continued downward trend in fuel stock or hydroelectric project water storage level are estimated; or (2) Unscheduled dispatch or emergency generation is causing an abnormal use of a particular fuel type, such that the future supply or stocks of that fuel could reach a level which threatens the reliability or adequacy of bulk electric power supply.

Gas: A fuel burned under boilers and by internal combustion engines for electric generation. These include natural, manufactured and waste gas.

Generation (Electricity): The process of producing electric energy by transforming other forms of energy; also, the amount of electric energy produced, expressed in watthours (Wh).

Gross Generation: The total amount of electric energy produced by the generating units at a genera:ing station or stations, measured at the generator terminals.

Net Generation: Gross generation less the electric energy consumed at the generating station for station use.

Generator: A machine that converts mechanical energy into electrical energy.

Generator Nameplate Capacity: The full-load continuous rating of a generator, prime mover, or other electric power production equipment under specific conditions as designated by the manufacturer. Installed generator nameplate rating is usually indicated on a nameplate physically attached to the generator. 
Geothermal Plant: A plant in which the prime mover is a steam turbine. The turbine is driven either by steam produced from hot water or by natural steam that derives its energy from heat found in rocks or fluids at various depths beneath the surface of the earth. The energy is extracted by drilling and/or pumping.

Gigawatt (GW): One billion watts.

Gigawatthour (GWh): One billion watthours.

Gross Generation: The total amount of electric energy produced by a generating facility, as measured at the generator terminals.

Heavy Oil: The fuel oils remaining after the lighter oils have been distilled off during the refining process. Except for start-up and flame stabilization, virtually all petroleum used in steam plants is heavy oil.

Horsepower: A unit for measuring the rate of work (or power) equivalent to 33,000 foot-pounds per minute or 746 watts.

Hydroelectric Plant: A plant in which the turbine generators are driven by falling water.

Instantaneous Peak Demand: The maximum demand at the instant of greatest load.

Integrated Demand: The summation of the continuously varying instantaneous demand averaged over a specified interval of time. The information is usually determined by examining a demand meter.

Internal Combustion Plant: A plant in which the prime mover is an internal combustion engine. An internal combustion engine has one or more cylinders in which the process of combustion takes place, converting energy released from the rapid burning of a fuel-air mixture into mechanical energy. Diesel or gas-fired engines are the principal types used in electric plants. The plant is usually operated during periods of high demand for electricity.

Interruptible Gas: Gas sold to customers with a provision that permits curtailment or cessation of service at the discretion of the distributing company under certain circumstances, as specified in the service contract.
Kilowatt (kW): One thousand watts.

Kilowatthour (kWh): One thousand watthours.

Light Oil: Lighter fuel oils distilled off during the refining process. Virtually all petroleum used in internal combustion and gas-turbine engines is light oil.

Lignite: A brownish-black coal of low rank with high inherent moisture and volatile matter (used almost exclusively for electric power generation). It is also referred to as brown coal. Comprises two groups classified according to the following ASTM Specification D388-84 for calorific values on a moist materialmatter-free basis:

\begin{tabular}{ccc} 
& \multicolumn{2}{c}{ Limits Btu/lb. } \\
& GE & LT \\
Lignite A & 6300 & 8300 \\
Lignite B & - & 6300
\end{tabular}

Maximum Demand: The greatest of all demands of the load that has occurred within a specified period of time.

Mcf: One thousand cubic feet.

Megawatt (MW): One million watts.

Megawatthour (MWh): One million watthours.

MMcf: One million cubic feet.

Natural Gas: A naturally occurring mixture of hydrocarbon and nonhydrocarbon gases found in porous geological formations beneath the earth's surface, often in association with petroleum. The principal constituent is methane.

Net Energy for Load: Net generation of main generating units that are system-owned or system-operated plus energy receipts minus energy deliveries.

Net Generation: Gross generation minus plant use from all electric utility owned plants. The energy required for pumping at a pumped-storage plant is regarded as plant use and must be deducted from the gross generation. 
Net Summer Capability: The steady hourly output, which generating equipment is expected to supply to system load exclusive of auxiliary power, as demonstrated by tests at the time of summer peak demand.

Noncoincidental Peak Load: The sum of two or more peak loads on individual systems that do not occur in the same time interval. Meaningful only when considering loads within a limited period of time, such as a day, week, month, a heating or cooling season, and usually for not more than 1 year.

North American Electric Reliability Council (NERC): A council formed in 1968 by the electric utility industry to promote the reliability and adequacy of bulk power supply in the electric utility systems of North America. NERC consists of nine regional reliability councils and encompasses essentially all the power regional of the contiguous United States, Canada, and Mexico. The NERC Regions are:

ASCC - Alaskan System Coordination Council

ECAR - East Central Area Reliability Coordination Agreement

ERCOT - Electric Reliability Council of Texas

MAIN - Mid-America Interconnected Network

MAAC - Mid-Atlantic Area Council

MAPP - Mid-Continent Area Power Pool

NPCC - Northeast Power Coordinating Council

SERC - Southeastern Electric Reliability Council

SPP - Southwest Power Pool

WSCC - Western Systems Coordinating Council

Nuclear Fuel: Fissionable materials that have been enriched to such a composition that, when placed in a nuclear reactor, will support a self-sustaining fission chain reaction, producing heat in a controlled manner for process use.

Nuclear Power Plant: A facility in which heat produced in a reactor by the fissioning of nuclear fuel is used to drive a steam turbine.

Off-Peak Gas: Gas that is to be delivered and taken on demand when demand is not at its peak.
Ohm: The unit of measurement of electrical resistance. The resistance of a circuit in which a potential difference of 1 volt produces a current of 1 ampere.

Operable Nuclear Unit: A nuclear unit is "operable" after it completes low-power testing and is granted authorization to operate at full power. This occurs when it receives its full power amendment to its operating license from the Nuclear Regulatory Commission.

Other Gas: Includes manufactured gas, coke-oven gas, blast-furnace gas, and refinery gas. Manufactured gas is obtained by distillation of coal, by the thermal decomposition of oil, or by the reaction of steam passing through a bed of heated coal or coke.

Other Generation: Electricity originating from these sources: biomass, fuel cells, geothermal heat, solar power, waste, wind, and wood.

Other Unavailable Capability: Net capability of mair generating units that are unavailable for load for reasons other than full-forced outrage or scheduled maintenance. Legal restrictions or other causes make these units unavailable.

Peak Demand: The maximum load during a specified period of time.

Peak Load Plant: A plant usually housing old, lowefficiency steam units; gas turbines; diesels; or pumpedstorage hydroelectric equipment normally used during the peak-load periods.

Peaking Capacity: Capacity of generating equipment normally reserved for operation during the hours of highest daily, weekly, or seasonal loads. Some generating equipment may be operated at certain times as peaking capacity and at other times to serve loads on an around-the-clock basis.

Percent Difference: The relative change in a quantity over a specified time period. It is calculated as follows: the current value has the previous value subtracted from it; this new number is divided by the absolute value of the previous value; then this new number is multiplied by 100 .

Petroleum: A mixture of hydrocarbons existing in the liquid state found in natural underground reservoirs, often associated with gas. Petroleum includes fuel oil No. 2, No. 4, No. 5, No. 6; topped crude; Kerosene; and jet fuel. 
Petroleum Coke: See Coke (Petroleum).

Petroleum (Crude Oil): A naturally occurring, oily, flammable liquid composed principally of hydrocarbons. Crude oil is occasionally found in springs or pools but usually is drilled from wells beneath the earth's surface.

Plant: A facility at which are located prime movers, electric generators, and auxiliary equipment for converting mechanical, chemical, and/or nuclear energy into electric energy. A plant may contain more than one type of prime mover. Electric utility plants exclude facilities that satisfy the definition of a qualifying facility under the Public Utility Regulatory Policies Act of 1978.

Plant Use: The electric energy used in the operation of a plant. Included in this definition is the energy required for pumping at pumped-storage plants.

Plant-Use Electricity: The electric energy used in the operation of a plant. This energy total is subtracted from the gross energy production of the plant; for reporting purposes the plant energy production is then reported as a net figure. The energy required for pumping at pumped-storage plants is, by definition, subtracted, and the energy production for these plants is then reported as a net figure.

Power: The rate at which energy is transferred. Electrical energy is usually measured in watts. Also used for a measurement of capacity.

Price: The amount of money or consideration-in-kind for which a service is bought, sold, or offered for sale.

Prime Mover: The motive force that drives an electric generator (e.g., steam engine, turtine, or water wheel).

Production (Electric): Act or process of producing electric energy from other forms of energy; also, the amount of electric energy expressed in watthours (Wh).

Pumped-Storage Hydroelectric Plant: A plant thut usually generates electric energy' during peak-load periods by using water previously pumped into an elevated storage reservoir during off-peak periods when excess generating capacity is available to do so. When additional generating capacity is needed, the water can be released from the reservoir through a conduit to turbine generators located in a power plant at a lower level.
Pure Pumped-Storage Hydroelectric Plant: A plant that produces power only from water that has previously been pumped to an upper reservoir.

Qualifying Facility (QF): This is a cogenerator or small power producer that meets certain ownership, operating and efficiency criteria established by the Federal Energy Regulatory Commission (FERC) pursuant to the PURPA, and has filed with the FERC for QF status or has self-certified. For additional information, see the Code of Federal Regulation, Title 18, Part 292.

Railroad and Railway Electric Service: Electricity supplied to railroads and interurban and street railways, for general railroad use, including the propulsion of cars or locomotives, where such electricity is supplied under separate and distinct rate schedules.

Receipts: Purchases of fuel.

Reserve Margin (Operating): The amount of unused available capability of an electric power system at peak load for a utility system as a percentage of total capability.

Restoration Time: The time when the major portion of the interrupted load has been restored and the emergency is considered to be ended. However, some of the loads interrupted may not have been restored due to local problems.

Restricted-Universe Census: This is the complete enumeration of data from a specifically defined subset of entities including, for example, those that exceed a given level of sales or generator nameplate capacity.

Retail: Sales covering electrical energy supplied for residential, commercial, and industrial end-use purposes. Other small classes, such as agriculture and street lighting, also are included in this category.

Running and Quick-Start Capability: The net capability of generating units that carry load or have quick-start capability. In general, quick-start capability refers to generating units that can be available for load within a 30-minute period.

Sales: The amount of kilowatthours sold in a given period of time; usually grouped by classes of service, such as residential, commercial, industrial, and other. Other sales include public street and highway lighting, other sales to public authorities and railways, and interdepartmental sales. 
Scheduled Outage: The shutdown of a generating unit, transmission line, or other facility, for inspection or maintenance, in accordance with an advance schedule.

Short Ton: A unit of weight equal to 2,000 pounds.

Spot Purchases: A single shipment of fuel or volumes of fuel, purchased for delivery within 1 year. Spot purchases are often made by a user to fulfill a certain portion of energy requirements, to meet unanticipated energy needs, or to take advantage of low-fuel prices.

Standby Facility: A facility that supports a utility system and is generally running under no-load. It is available to replace or supplement a facility normally in service.

Standby Service: Support service that is available, as needed, to supplement a consumer, a utility system, or to another utility if a schedule or an agreement authorizes the transaction. The service is not regularly used.

Steam-Electric Plant (Conventional): A plant in which the prime mover is a steam turbine. The steam used to drive the turbine is produced in a boiler where fossil fuels are burned.

Stocks: A supply of fuel accumulated for future use. This includes coal and fuel oil stocks at the plant site, in coal cars, tanks, or barges at the plant site, or at separate storage sites.

Subbituminous Coal: Subbituminous coal, or black lignite, is dull black and generally contains 20 to 30 percent moisture. The heat content of subbituminous coal ranges from 16 to 24 million Btu per ton as received and averages about 18 million Btu per ton. Subbituminous coal, mined in the western coal fields, is used for generating electricity and space heating.

Substation: Facility equipment that switches, changes, or regulates electric voltage.

Sulfur: One of the elements present in varying quantities in coal which contributes to environmental degradation when coal is burned. In terms of sulfur content by weight, coal is generally classified as low (less than or equal to 1 percent), medium (greater than 1 percent and less than or equal to 3 percent), and high (greater than 3 percent). Sulfur content is measured as a percent by weight of coal on an "as received" or a "dry" (moisture-free, usually part of a laboratory analysis) basis.
Switching Station: Facility equipment used to tie together two or more electric circuits through switches. The switches are selectively arranged to permit a circuit to be disconnected, or to change the electric connection between the circuits.

System (Electric): Physically connected generation, transmission, and distribution facilities operated as an integrated unit under one central management, or operating supervision.

Transformer: An electrical device for changing the voltage of alternating current.

Transmission: The movement or transfer of electric energy over an interconnected group of lines and associated equipment between points of supply and points at which it is transformed for delivery to consumers, or is delivered to other electric systems. Transmission is considered to end when the energy is transformed for distribution to the consumer.

Transmission System (Electric): An interconnected group of electric transmission lines and associated equipment for moving or transferring electric energy in bulk between points of supply and points at which it is transformed for delivery over the distribution system lines to consumers, or is delivered to other electric systems.

Turbine: A machine for generating rotary mechanical power from the energy of a stream of fluid (such as water, steam, or hot gas). Turbines convert the kinetic energy of fluids to mechanical energy through the principles of impulse and reaction, or a mixture of the two.

Watt: The electrical unit of power. The rate of energy transfer equivalent to 1 ampere flowing under a pressure of 1 volt at unity power factor.

Watthour (Wh): An electrical energy unit of measure equal to 1 watt of power supplied to, or taken from, an electric circuit steadily for 1 hour.

Wheeling Service: The movement of electricity from one system to another over transmission facilities of intervening systems. Wheeling service contracts can be established between two or more systems.

Year to Date: The cumulative sum of each month's value starting with January and ending with the current month of the data. 


\section{Electronic Publishing System EPTB}

EPUB is an electronic publishing system maintained by the Energy Information Administration (EIA) of the U.S. Department of Energy. EPUB allows the general public to electronically access selected energy data from many of EIA's statistical reports. The system is a menu-driven, bulletin-board-type system with extensive online help capabilities that can be accessed free of charge 24 hours a day by using a terminal or PC with an asynchronous modem. (EPUB will be taken down briefly at midnight for backup.)

PC users must provide the following information to their communications software in order to successfully access the EPUB system:

Communications Parameters:

Baud Rate: $300-2400$ bps

Data Bits: 8 ; Stop Bits: 1

Parity: None; Duplex: Full

Terminal Type: ANSI, ANSI-BBS, VT100, etc.

Once communications software and/or hardware have been configured, EPUB can be accessed by dialing (202)586-2557. When a connection to the system has been made, some users may find that the menu-driven instructions and the online capabilities will provide enough information to effectively use EPUB. If needed, more extensive information may be found in the EPUB User's Guide, which is available online from the EPUB system or from:

National Energy Information Center, EI-231

Energy Information Administration

Forrestal Building, Room 1F-048

Washington, DC 20585

(202)586-8800

TTY: For people who are deaf or

hard of hearing: (202)586-1181

Hours: 9 a.m. to 5 p.m., M-F, eastern time
For communications or technical assistance, call (202)586-8959, 8 a.m. to 5 p.m. eastern time, Monday through Friday. For questions about the contents of EPUB reports and data, call (202)5868800,9 a.m. to 5 p.m., M-F, eastern time.

EPUB provides statistical information, as well as data from selected EIA publications, such as:

Heating fuel data

Updated the 2nd week of the month.

Oxygenates data

Updated approximately the 25 th of the month.

Weekly Petroleum Status Report

Updated on Wednesdays (Thursdays in the event of a holiday) at 5 p.m.

Petroleum Supply Monthly

Updated on the 20th of the month.

Petroleum Marketing Monthly

Updated on the 20th of the month.

Natural Gas Monthly

Updated on the 20th of the month.

Weekly Coal Production

Updated on Fridays at 5 p.m.

Quarterly Coal Report

Updated 60 days after the end of the quarter.

Electric Power Monthly

Updated on the 1st of the month.

Monthly Energy Review

Updated the last week of the month.

Short-Term Energy Outlook

Updated 60 days after the end of the quarter.

Winter Fuels Report (October through April) Updated every Thursday at 5 p.m.

W

Periodically, new items are added to the electronic bulletin board. EIA is now completing implementation of two new subject areas on this system. The first area, "End-Use Consumption Surveys," provides electronic access to selected data and analyses from the residential, commercial, manufacturing, and residential transportation consumption surveys. The second area, "Monthly Energy Review (MER) Features," provides access to special articles from the most recent publication, with a listing of all previous articles. 


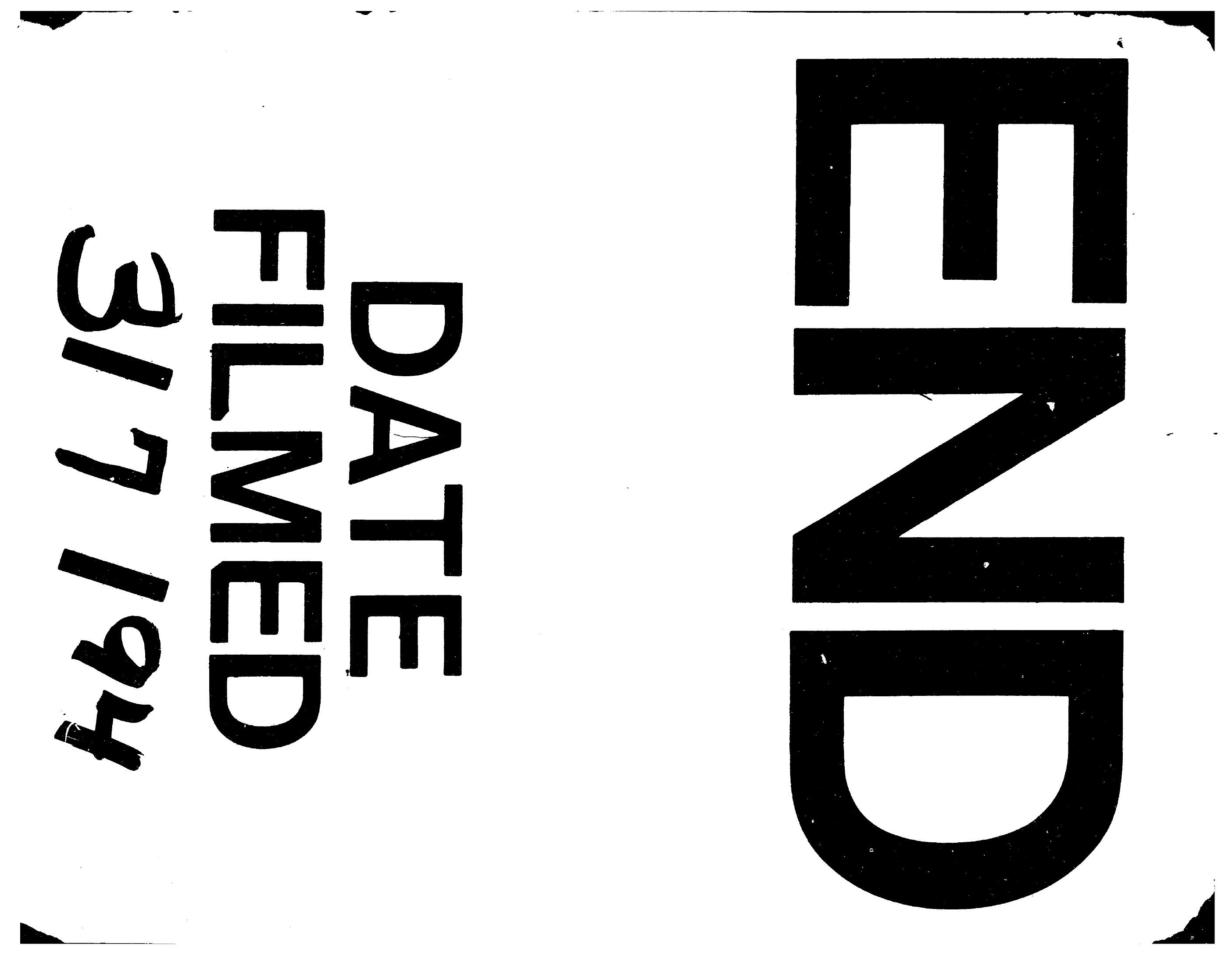


